\title{
Auswirkungen der Gestaltung von verkehrsberuhigten Bereichen auf das Unfallgeschehen
}

Tabea Kesting

Kristin Koros

Mark-Simon Krause

Michael Vieten

Petra Butterwegge

Jörg Ortlepp 


\title{
Auswirkung der Gestaltung von verkehrsberuhigten Bereichen auf das Unfallgeschehen
}

\author{
Dr.-Ing. Tabea Kesting \\ Kristin Koros M. Sc. \\ Dipl.-Ing. Mark-Simon Krause \\ Dipl.-Ing. Michael Vieten \\ Dipl.-Ing. Petra Butterwegge \\ Dipl.-Ing. Jörg Ortlepp
}




\section{Impressum}

Gesamtverband der Deutschen Versicherungswirtschaft e. V. Unfallforschung der Versicherer

Wilhelmstraße 43/43G, 10117 Berlin

Postfach 0802 64, 10002 Berlin

E-Mail: unfallforschung@gdv.de

Internet: www.udv.de

Facebook: www.facebook.com/unfallforschung

Twitter: @unfallforschung

YouTube: www.youtube.com/unfallforschung

ISBN-Nr.: 978-3-939163-62-6

Redaktion: Dipl.-Ing. Petra Butterwegge, Dipl.-Ing. Jörg Ortlepp

Bildnachweis: IGS, UDV und siehe Quellenangaben

Erschienen: 07/2015 


\section{Auswirkung der Gestaltung von verkehrsberuhigten Bereichen auf das Unfallgeschehen}

bearbeitet durch:

IGS Ingenieurgesellschaft Stolz mbH

Dr.-Ing. Tabea Kesting

Kristin Koros M. Sc.

Dipl.-Ing. Michael Vieten

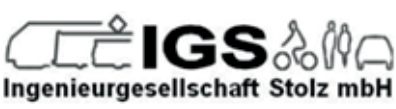

Hochschule Darmstadt

Dipl-Ing. Mark-Simon Krause

h_da

HOCHSCHULE DARMSTAOT
UNIVERSITY OF APPLIEO SCIENCES

|

Bei der UDV betreut von:

Dipl.-Ing. Petra Butterwegge

Dipl.-Ing. Jörg Ortlepp

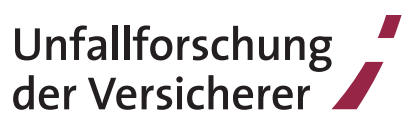





\section{Inhaltsverzeichnis}

Seite

$1 \quad$ Abstract

$2 \quad$ Kurzfassung

$3 \quad$ Ausgangslage

$4 \quad$ Untersuchungsgegenstand

$5 \quad$ Stand der Wissenschaft und Technik

$5.1 \quad$ Entwicklung von Ansätzen zur Verkehrsberuhigung $\quad 12$

$\begin{array}{llr}5.2 & \text { Verkehrsberuhigte Bereiche im Verkehrsrecht } & 17\end{array}$

5.3 Rechtsprechung

$6 \quad$ Untersuchungsmethodik

$7 \quad$ Makroskopische Unfallanalyse

7.1 Definitionen und Erläuterungen

7.1.1 Unfallkategorie / Unfallschwere

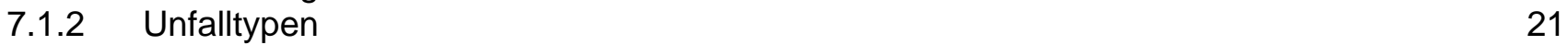

$\begin{array}{ll}7.1 .3 & \text { Unfallarten }\end{array}$

7.1.4 Beteiligte, Fahrzeugbenutzer und Alleinunfälle 24

$\begin{array}{ll}7.1 .5 & \text { Unfallursachen }\end{array}$

$\begin{array}{lll}7.1 .6 & \text { Unfallumstände } & 25\end{array}$

$\begin{array}{lll}7.2 & \text { Datengrundlage } & 26\end{array}$

$\begin{array}{lll}7.3 & \text { Genauigkeit der Unfalldaten } & 27\end{array}$

7.4 Analyse der Unfalldaten des Statistischen Bundesamtes $\quad 30$

7.5 Analyse der Unfalldaten in den Bundesländer 36

7.5.1 Analyse der Unfalldaten aus Baden-Württemberg 36

7.5.2 Analyse der Unfalldaten Hessen 38

7.5.3 Analyse der Unfalldaten Nordrhein-Westfalen 40

7.5.4 Analyse der Unfalldaten Sachsen-Anhalt 41

7.5.5 Analyse der Unfalldaten Thüringen 42

7.6 Zusammenfassung der makroskopischen Unfallanalyse 44

$8 \quad$ Umsetzungspraxis in den Kommunen $\quad 45$

$9 \quad$ Untersuchungsobjekte 48

9.1 Zusammenstellung der Untersuchungsobjekte 48

9.2 Dokumentation der Untersuchungsobjekte 49

9.3 Kategorisierung der Untersuchungsobjekte 52

$10 \quad$ Unfallanalyse der Untersuchungsobjekte $\quad 59$

10.1 Unfallanalysen „Kollektiv Kat. 1-4“ 59

10.1.1 Datengrundlage $\quad 59$

10.1.2 Vorgehensweise und Vergleichskollektiv $\quad 64$

10.1.3 Unfallkategorien $\quad 66$

$\begin{array}{lll}10.1 .4 & \text { Unfalltypen } & 68\end{array}$

$\begin{array}{lll}10.1 .5 & \text { Unfallarten } & 70\end{array}$

$\begin{array}{lll}10.1 .6 & \text { Beteiligte } & 74\end{array}$

10.1.7 Unfallursachen $\quad 82$

$\begin{array}{ll}10.1 .8 & \text { Unfallumstände }\end{array}$ 
10.2 Zusammenfassung der Unfallanalyse der Untersuchungsobjekte

11 Ableitung von Zusammenhängen zwischen Unfällen und Gestaltung 88

11.1 Datengrundlage und Vorgehensweise $\quad 88$

11.2 Unfallanalysen „Kollektiv Kat. 1-4“ nach Straßenfunktionen 88

11.2.1 Unfallkategorien / Unfalldichten / Unfallkostendichten $\quad 88$

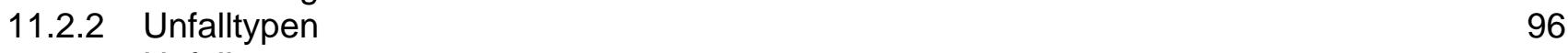

$\begin{array}{llr}11.2 .3 & \text { Unfallart } & 98\end{array}$

$\begin{array}{lll}11.2 .4 & \text { Beteiligte } & 101\end{array}$

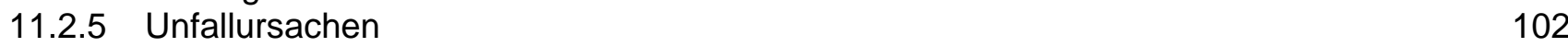

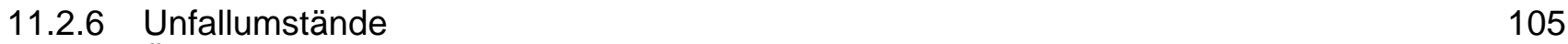

11.2.7 Übergangsbereiche $r 105$

11.2.8 Streckenabschnittslängen 111

11.3 Zusammenfassung der Zusammenhänge zwischen Unfällen und Gestaltung 112

$12 \quad$ Analyse des Verkehrsablaufs $\quad 114$

12.1 Auswahl der Untersuchungsobjekte für die Verkehrsbeobachtung 114

$\begin{array}{lll}12.2 & \text { Erhebungskonzept } & 117\end{array}$

$\begin{array}{lll}12.2 .1 & \text { Flächennutzung } & 118\end{array}$

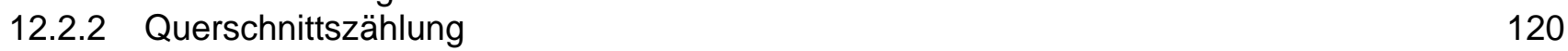

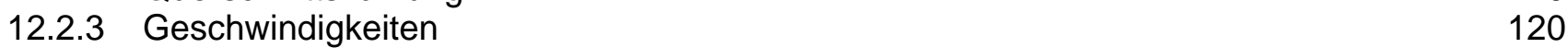

$\begin{array}{ll}12.2 .4 & \text { Interaktionen } \\ 12.322\end{array}$

12.3 Darstellung der Auswertungen 123

$\begin{array}{lll}12.4 & \text { Ergebnisse der Verkehrsbeobachtung } & 126\end{array}$

12.4.1 Querschnittszählung 126

12.4.2 Flächennutzungen $\quad 129$

12.4.3 Geschwindigkeiten $\quad 135$

$\begin{array}{ll}12.4 .4 & 137\end{array}$

$\begin{array}{ll}12.4 .5 & 138\end{array}$

$\begin{array}{lll}12.5 & \text { Zusammenfassung der Ergebnisse } & 140\end{array}$

$13 \quad$ Empfehlung von Gestaltungsmaßnahmen für ausgewiesene VBB 140

ANHANG 1 Unfallursachenverzeichnis 143

ANHANG 2 Fragebogen zur Umsetzungspraxis von Verkehrsberuhigten Bereichen 147

ANHANG 3 Unfallanalysen „Kollektiv 2011“ Kategorie 1-6 152

ANHANG 4 Unfallanalysen „Kollektiv 2011“ nach Straßenfunktionen 164

$\begin{array}{ll}\text { ANHANG } 5 \text { Unfallkosten } & 174\end{array}$

ANHANG 6 Beschreibung der Kameratechnik und Fehleranfälligkeit 176

$\begin{array}{ll}\text { Literaturverzeichnis } & 177\end{array}$

$\begin{array}{ll}\text { Abbildungsverzeichnis } & 179\end{array}$

$\begin{array}{lr}\text { Tabellenverzeichnis } & 184\end{array}$ 


\section{Abstract}

Since 1980, roads are labelled traffic calming zones (Verkehrsberuhigter Bereich, VBB) with traffic sign 325 of the German Highway Code (Straßenverkehrsordnung, StVO). Increasing road safety, improving quality of stay, and environmental aspects have remained topical reasons for this type of traffic calming measure until this day.

Traffic calming zones aim to achieve equality among road users. Both the speed of vehicles and the amount of motor traffic should be reduced. Consistent design rules for traffic calming zones were previously inexistent, whereby design elements such as mixed traffic areas and a level expansion within the Administrative Regulation of the German Highway Code (Verwaltungsvorschrift zur Straßenverkehrsordnung, VwV-StVO) - amongst other things - are recommended.

In practice, there is a wide field of application for traffic calming zones. Alongside the application area of residential streets that was originally envisaged, nowadays, traffic calming zones with traffic sign 325 StVO can also be found in inner city residential areas, access roads and, more and more frequently, in commercial streets. Furthermore, there are numerous special application areas for traffic sign 325 StVO in practice, such as parking areas, for example.

Although traffic sign 325 StVO was introduced into the StVO as early as 1980, the only scientific studies on the impact on traffic flow and the occurrence of accidents date back to the early 1980s. More recent studies are not available, despite traffic sign 325 StVO finding more and more widespread use.

The current study initially observed accidents in traffic calming zones with traffic sign 325 StVO based on official nationwide statistics. Furthermore, detailed accident analyses of almost 300 practical VBB examples as well as traffic flow analyses from more than 200 hours monitoring in 50 selected traffic calming zones were performed.

Overall, it was established that the occurrence of accidents in all areas monitored is inconspicuous, and that as a rule, traffic calming zones with traffic sign 325 StVO can be classified as safe for traffic. Equally, the analyses of traffic flow monitoring demonstrated that there were no significant uncertainties to be observed.

However, it must be stated that the average speed in traffic calming zones in parts significantly exceeds the permissible walking speed of $7 \mathrm{~km} / \mathrm{h}$. This applies to both motor and bicycle traffic. In addition, traffic flow regulations regarding the transition from traffic calming zones to adjacent road networks do not seem to be clear-cut for some road users. Here, depending on local conditions, different "right-of-way regulations" are implemented by road users.

In conclusion, it can be said that in practice, due to its diverse design possibilities, a traffic calming zone with traffic sign 325 StVO is often even difficult to recognise within the same municipality on the basis of its design. 


\section{Kurzfassung}

Die Kennzeichnung von Straßen mit dem Verkehrszeichen 325 der Straßenverkehrsordnung (StVO) zu einem Verkehrsberuhigten Bereich (VBB) erfolgt seit 1980. Die Steigerung der Verkehrssicherheit, die Verbesserung der Aufenthaltsqualität sowie ökologische Aspekte sind bis heute aktuelle Gründe für diese Art der Verkehrsberuhigungsmaßnahme.

Mit Verkehrsberuhigten Bereichen wird das Ziel verfolgt, eine Gleichberechtigung unter den Verkehrsteilnehmern zu erreichen. Die Fahrgeschwindigkeiten der Fahrzeuge und auch der Kraftfahrzeugverkehr in seiner Anzahl sollen reduziert werden. Einheitliche Gestaltungsregeln für Verkehrsberuhigte Bereiche gibt es bislang nicht, wobei unter anderem Gestaltungselemente wie eine Mischverkehrsfläche und ein niveaugleicher Ausbau in der Verwaltungsvorschrift zur Straßenverkehrsordnung (VwV-StVO) empfohlen werden.

Die Anwendungsbereiche von Verkehrsberuhigten Bereichen in der Praxis sind sehr vielfältig. Neben dem ursprünglich angedachten Einsatzbereich der Wohnstraßen sind Verkehrsberuhigte Bereiche mit Verkehrszeichen 325 StVO heutzutage auch in innerstädtischen Quartieren, in Erschließungsstraßen und immer häufiger in Geschäftsstraßen vorzufinden. Darüber hinaus sind auch zahlreiche Sondereinsatzbereiche des Verkehrszeichens 325 StVO in der Praxis, wie z.B. auf Parkplätzen, vorhanden.

Obwohl das Verkehrszeichen 325 StVO schon 1980 in die StVO aufgenommen wurde, liegen wissenschaftliche Studien zu den Auswirkungen auf den Verkehrsablauf und das Unfallgeschehen nur aus den Anfangsjahren der 1980er Jahre vor. Aktuellere Untersuchungen liegen nicht vor, obwohl das Verkehrszeichen immer größere Verbreitung findet.

In der vorliegenden Studie wurde zunächst das Unfallgeschehen in Verkehrsberuhigten Bereichen mit Verkehrszeichen 325 StVO auf Grundlage der amtlichen bundesweiten Statistik betrachtet. Des Weiteren wurden detaillierte Auswertungen des Unfallgeschehens von fast 300 Praxisbeispielen in VBB sowie Analysen des Verkehrsablaufs durch Beobachtungen von über 200 Stunden in 50 ausgewählten Verkehrsberuhigten Bereichen durchgeführt.

Insgesamt konnte festgestellt werden, dass das Unfallgeschehen in allen betrachteten Bereichen unauffällig ist und die Verkehrsberuhigten Bereiche mit Verkehrszeichen 325 StVO in der Regel als verkehrssicher eingestuft werden können. Ebenso zeigten die Auswertungen der Verkehrsablaufbeobachtung, dass keine besonderen Unsicherheiten zu beobachten waren.

Festgehalten werden muss allerdings, dass die durchschnittliche Fahrtgeschwindigkeit durch die Verkehrsberuhigten Bereiche zum Teil deutlich oberhalb der zulässigen Schrittgeschwindigkeit (7 km/h) liegt. Dies betrifft sowohl den Kfz-Verkehr als auch den Radverkehr. Zusätzlich scheint der Übergang aus dem Verkehrsberuhigten Bereich auf das angrenzende Straßennetz einem Teil der Verkehrsteilnehmer in seiner Verkehrsregelung nicht eindeutig klar zu sein. Hier werden je nach örtlicher Situation unterschiedliche „Vorfahrtsregelungen“ durch die Verkehrsteilnehmer umgesetzt.

Abschließend lässt sich festhalten, dass aufgrund der vielfältigen Gestaltungsvarianten in der Praxis ein Verkehrsberuhigter Bereich mit Verkehrszeichen 325 StVO häufig sogar in der gleichen Kommune nicht eindeutig anhand seiner Gestaltung zu erkennen ist. 


\section{Ausgangslage}

Mit der Kennzeichnung einer Straße als Verkehrsberuhigter Bereich (VBB) mittels Verkehrszeichen (VZ) 325 der Straßenverkehrsordnung (StVO) (Stand 2013) wird das Ziel verfolgt, die Dominanz des Kfz-Verkehrs auf dieser Straße zu verringern. Durch die Maßnahme zur Verkehrsberuhigung soll die Bedeutung der Erschließungs- und Aufenthaltsfunktion einer Straße hervorgehoben werden. Dies soll einerseits durch die Verringerung des Geschwindigkeitsniveaus des Kfz-Verkehrs erreicht werden und gleichzeitig soll die Maßnahme zur Verkehrsberuhigung ggf. auch zur Verminderung der Kfz-Verkehrsstärke beitragen. Die grundlegenden Ziele der Verkehrsberuhigung liegen in der Erhöhung der Verkehrssicherheit und der Verbesserung der Aufenthaltsqualität und damit verbunden in der Attraktivierung des Wohnumfeldes entlang der Straße. Erste Modellprojekte zur Verkehrsberuhigung wurden Ende der 1970er Jahre in Deutschland realisiert. Eine offizielle Einführung beschilderter Verkehrsberuhigter Bereiche erfolgte in Deutschland 1980. Seitdem ist eine stetige Zunahme der Anzahl ausgewiesener Verkehrsberuhigter Bereiche in Deutschland zu verzeichnen ${ }^{1}$.

In der jüngeren Vergangenheit ist das Thema Verkehrsberuhigung durch die Diskussion über "Begegnungszonen“ und „Shared Space“ wieder deutlicher in den Fokus der Öffentlichkeit, aber auch der Planer und Verantwortlichen vor Ort gerückt. Auch hier ist das Ziel, die Dominanz des Kfz-Verkehrs im Straßenraum zu mindern, um so einerseits eine höhere Umfeldattraktivität zu erzielen, aber auch andererseits ein höheres Maß an Verkehrssicherheit zu gewährleisten.

In der amtlichen Statistik wird für die Verkehrsberuhigten Bereiche, die mit VZ 325 StVO gekennzeichnet sind, für den Zeitraum von 1995 bis 2000 ein Rückgang der Unfälle mit Personenschaden $(U(P))$ um rd. ein Viertel von $2.053 U(P)$ auf $1.524 U(P)$ ausgewiesen. Für den Zeitbereich 2000 bis 2012 liegt die Anzahl der Unfälle mit Personenschaden in Verkehrsberuhigten Bereichen bei rd. $1.500 \mathrm{U}(\mathrm{P})$ pro Jahr (Destatis, 2013). In einer detaillierteren Analyse der Unfallstatistik zu Unfällen in ausgewiesenen VBB zeigte sich, dass unter dem Begriff Verkehrsberuhigte Bereiche auch Unfälle aus anderen geschwindigkeitsreduzierten Bereichen (GRB) aufgenommen wurden. Es handelt sich demnach nur bei einem Teil der in der Unfallstatistik aufgeführten VBB um mit dem VZ 325 StVO ausgewiesene Bereiche. Die übrigen Unfallstellen befinden sich auf Straßenabschnitten oder an Knotenpunkten, die anderweitig verkehrsberuhigt sind (z.B. Tempo 30Zone, Fußgängerzone). Eine belastbare Aussage zum Unfallgeschehen in VBB mit VZ 325 StVO ist somit auf Grundlage der amtlichen Statistiken derzeit nicht möglich.

Vor dem Hintergrund der wachsenden Bedeutung der Verkehrsberuhigung als Mittel der Verkehrsplanung, der älter werdenden Bevölkerung, des wachsenden Anspruchs eines attraktiven Wohnumfeldes und der stagnierenden Unfallzahlen in Verkehrsberuhigten Bereichen stellt sich die Frage, wie die Gestaltung in Verkehrsberuhigten Bereichen verbessert werden kann, um den genannten Anforderungen gerecht zu werden. Hierzu bedarf es einer belastbaren Analyse des Unfallgeschehens in Verkehrsberuhigten Bereichen, aktueller Kenntnisse zum Verkehrsablauf in ausgewiesenen Verkehrsberuhigten Bereichen, eines Überblicks über die Umsetzungspraxis bei der Ausweisung von Verkehrsberuhigten Bereichen und einer Ableitung von Zusammenhängen zwischen baulicher Gestaltung einerseits und dem Verkehrsablauf und dem Unfallgeschehen andererseits.

Bereits Anfang der 1980er Jahre wurden insbesondere durch die Beratungsstelle für Schadensverhütung eine Vielzahl von Untersuchungen veröffentlicht, wie Verkehrsberuhigte Bereiche (nicht nur mit VZ 325) allgemein gestaltet sein müssen und welche weiteren Maßnahmen dazu geeignet

1 Eine Statistik zur Anzahl der Verkehrsberuhigten Bereiche mit Verkehrszeichen 325 gemäß StVO existiert nicht. Aufgrund der Recherchen im Rahmen dieser Studie konnte allerdings festgestellt werden, dass die Kommunen regelmäßig VBB einrichten und nur in Einzelfällen diese später wieder umbeschildern. 
sind, damit ein niedriges Geschwindigkeitsniveau einerseits erreicht und die Verkehrssicherheit andererseits verbessert werden kann. Ziel der vorliegenden Studie ist es u.a., die inzwischen 30 Jahre alten Erkenntnisse zu aktualisieren und zu überprüfen.

\section{$4 \quad$ Untersuchungsgegenstand}

Untersuchungsgegenstand der vorliegenden Studie sind ausgewiesene Verkehrsberuhigte Bereiche. Gemäß StVO ist eine Straße oder ein zusammenhängender Bereich von Straßen ein Verkehrsberuhigter Bereich, dessen Anfang mit dem VZ 325.1 gemäß §42 - Anlage 3 zu § 42 Abs.2 der StVO und dessen Ende mit dem VZ 325.2 gemäß §42 - Anlage 3 zu §42 Abs. 2 der StVO gekennzeichnet ist (vgl. Abbildung 1).
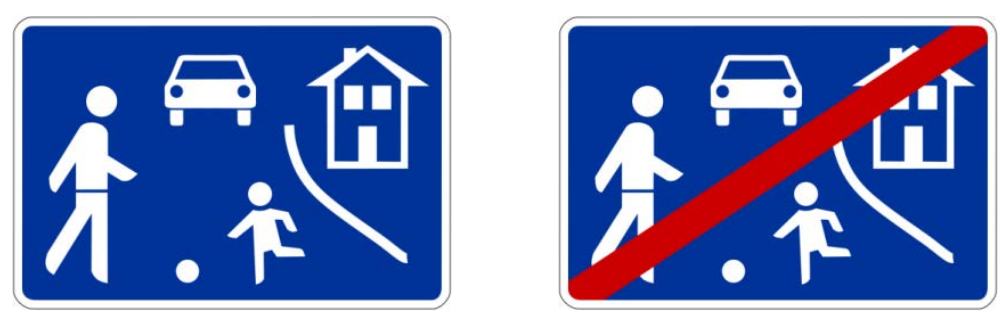

Abbildung 1: Verkehrszeichen 325.1 und 325.2 gemäß StVO, Stand 2013 (Beginn und Ende eines Verkehrsberuhigten Bereichs)

Durch die StVO ist die Bezeichnung des Verkehrsberuhigten Bereichs eindeutig durch die Verwendung der Verkehrszeichen 325 gegeben. Die Ge- und Verbote, die gemäß Anlage 3 (zu §42 Absatz 2) der StVO gelten, sind folgende:

„(1) Wer ein Fahrzeug führt, muss mit Schrittgeschwindigkeit fahren.

(2) Wer ein Fahrzeug führt, darf den Fußgängerverkehr weder gefährden noch behindern; wenn nötig, muss gewartet werden.

(3) Wer zu Fuß geht, darf den Fahrverkehr nicht unnötig behindern.

(4) Wer ein Fahrzeug führt, darf außerhalb der dafür gekennzeichneten Flächen nicht parken, ausgenommen zum Ein- oder Aussteigen und zum Be- oder Entladen.

(5) Wer zu Fuß geht, darf die Straße in ihrer ganzen Breite benutzen; Kinderspiele sind überall erlaubt."

Planerisch wird ein Verkehrsberuhigter Bereich durch die Begriffsbestimmungen der Forschungsgesellschaft für Straßen- und Verkehrswesen (FGSV) folgendermaßen definiert:

„Straßen innerhalb eines abgegrenzten Gebietes, einzelne Straßen oder Straßenabschnitte, die überwiegend eine Aufenthalts- und Erschließungsfunktion aufweisen und auf denen nach Straßenverkehrsordnung der Fußgängerverkehr Vorrang hat" (FGSV, 2012).

Mit dem Verkehrszeichen 325 der StVO sind neben den o.g. bestimmte Ge- und Verbote verbunden (Kapitel 5.2). In der Verkehrsplanung selbst wird der Begriff der Verkehrsberuhigung über die gemäß StVO getroffene Definition für einen Verkehrsberuhigten Bereich hinaus verwendet. Entsprechend heißt es in den Begriffsbestimmungen zum Begriff „Verkehrsberuhigung“ (FGSV, 2012):

„Verdrängung des quartiersfremden und Verlangsamung des verbleibenden motorisierten Verkehrs zur Erhöhung der Verkehrssicherheit und Verbesserung des Wohnumfeldes." 
Dementsprechend gibt es für den Planer weitere Möglichkeiten gemäß der o.g. Definition, den Verkehr auf Straßen zu beruhigen. Hierzu sieht die StVO die Einrichtung eines sog. verkehrsberuhigten Geschäftsbereichs (\$45, Abs. 1d) vor oder die Einrichtung von sog. Tempo 30-Zonen (Zeichen 274, §41) (vgl. Abbildung 2).

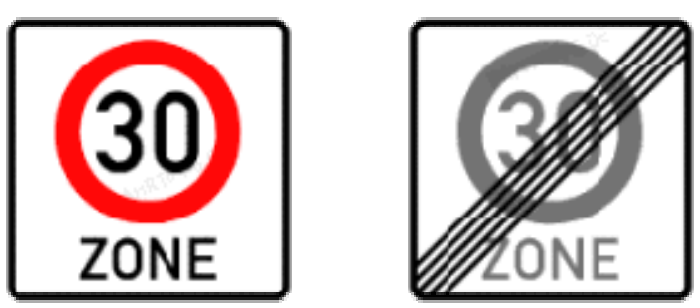

Abbildung 2: Verkehrszeichen 274.1 und 274.2 gemäß StVO, Stand 2013 (Beginn und Ende einer Tempo 30 Zone)

Ein Verkehrsberuhigter Geschäftsbereich (VGB) kann in zentralen städtischen Bereichen mit hohem Fußgängeraufkommen und überwiegender Aufenthaltsfunktion angeordnet werden. Im Gegensatz zu den Verkehrsberuhigten Bereichen werden Verkehrsberuhigte Geschäftsbereiche mit dem VZ 274 StVO ausgewiesen. Die Zonengeschwindigkeitsbeschränkung von VGB liegt dabei in der Regel bei $20 \mathrm{~km} / \mathrm{h}^{2}$. In diesen Zonen ist der nichtmotorisierte Verkehr gegenüber dem motorisierten Verkehr jedoch nicht bevorrechtigt. D.h. in Verkehrsberuhigten Geschäftsbereichen dürfen Fußgänger die Straße nicht in ihrer ganzen Breite benutzen und der Fahrzeugverkehr hat auf der Straße Vorrang.

Aufgrund der in der jüngeren Vergangenheit wieder aufkommenden Diskussion zur Verkehrsberuhigung innerorts werden die Maßnahmen „Verkehrsberuhigter Bereich mit VZ 325“, „Verkehrsberuhigter Geschäftsbereich“ und „Tempo 30-Zone“ häufiger im Zusammenhang behandelt. Diese drei Möglichkeiten zur Verkehrsberuhigung werden in unterschiedlichen Konzepten wie zum Beispiel „Shared Space, „Gemeinschaftsstraßen“ oder „SimpliCity“ häufig zusammengefasst oder bei der örtlichen Umsetzung unterschiedlich verwendet.

Untersuchungsgegenstand der vorliegenden Studie ist nicht das allgemeine Thema „Verkehrsberuhigung innerorts“, sondern ausschließlich Straßen oder Straßennetzabschnitte, die mittels Verkehrszeichen 325 der StVO als Verkehrsberuhigter Bereich definiert sind. Die Studie folgt damit nicht der Fragestellung, welche Methode oder Maßnahme zur Verkehrsberuhigung die geeignetste Methode bzw. Maßnahme ist, sondern wie ein Verkehrsberuhigter Bereich laut VZ 325 der StVO verkehrssicher gemäß den vorliegenden Rahmenbedingungen des Einsatzortes zu gestalten ist. Dementsprechend ist im Folgenden, wenn von Verkehrsberuhigten Bereichen (VBB) gesprochen wird, immer eine Straße oder ein Straßenabschnitt gemeint, der mit den Verkehrszeichen 325.1 und 325.2 gekennzeichnet ist.

2 Gemäß StVO Anlage 2 (zu § 41Absatz 1) können mit dem Zeichen 274 in Verkehrsberuhigten Geschäftsbereichen auch Zonengeschwindigkeitsbeschränkungen von weniger als $30 \mathrm{~km} / \mathrm{h}$ angeordnet sein. 


\section{$5 \quad$ Stand der Wissenschaft und Technik}

\subsection{Entwicklung von Ansätzen zur Verkehrsberuhigung}

Das Thema Verkehrsberuhigung wurde in den 1970er Jahren in Deutschland zum ersten Mal in den Fokus der Verkehrsplanung gerückt. Ausgangspunkt war ein durch das Verkehrsministerium NRW im Jahr 1976 initiierter Großversuch zur Verkehrsberuhigung von Wohnstraßen (MWMV, 1979).

Im Rahmen dieses Großversuches wurden neben der Ausweisung von Tempo 30 in Wohnstraßen auch punktuell niveaugleiche Mischflächen (vgl. Abbildung 3) umgesetzt, die für alle Verkehrsteilnehmer und für Aufenthaltsaktivitäten gleichberechtigt zur Verfügung stehen sollten. Diese Versuche mit Mischflächen basierten dabei auf Erkenntnissen, die mit ähnlichen Ansätzen in Skandinavien und in den Niederlanden (woonerf-Projekte) gewonnen wurden (Topp, 2011).

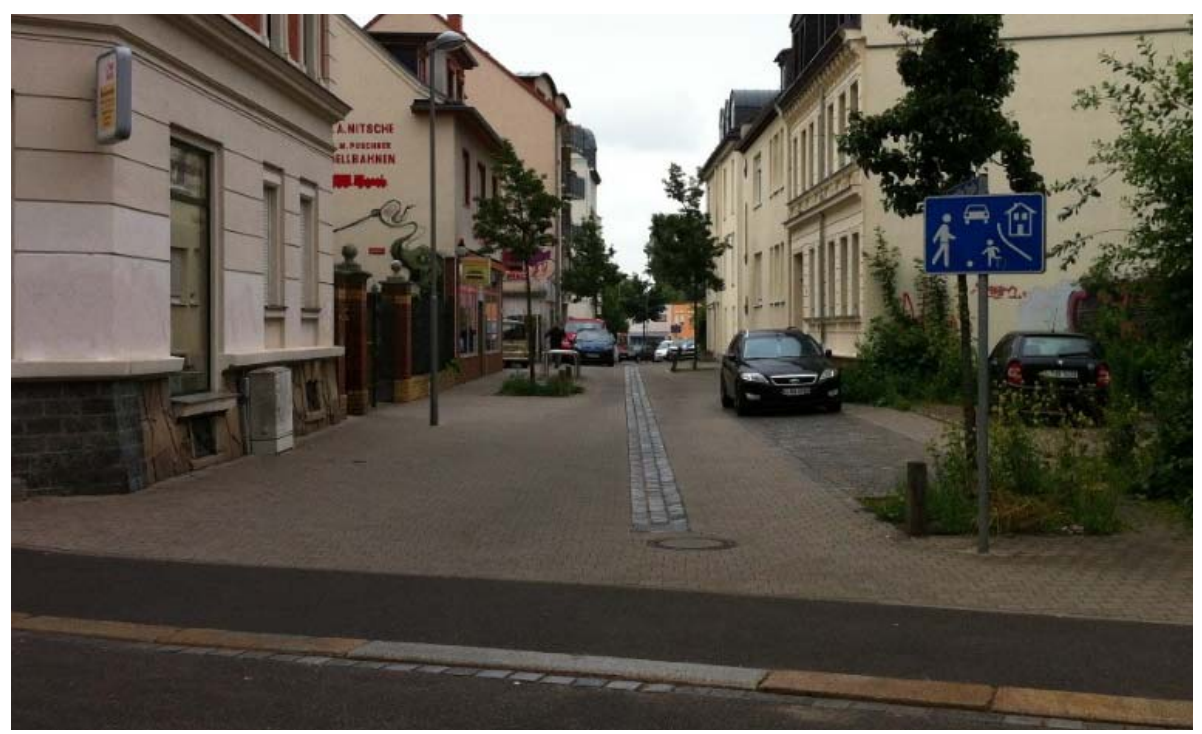

Abbildung 3: Beispielhafte Darstellung einer Mischfläche (Foto: IGS mbH 2013)

Die im Großversuch gewonnenen Erkenntnisse fanden 1980 unter dem Begriff „Verkehrsberuhigter Bereich" Eingang in die StVO (Kapitel 5.2).

Aufbauend auf den Erkenntnissen des Großversuches wurden verschiedene Hilfestellungen zur praktischen Umsetzung der Verkehrsberuhigung abgeleitet. So erschienen beispielsweise 1980 die Empfehlungen der Beratungsstelle für Schadensverhütung zur Verkehrsberuhigung in Wohnbereichen (BfS, 1980).

In diesen Empfehlungen wird das Ziel der Verkehrsberuhigung wie folgt beschrieben:

„Durch Maßnahmen der Verkehrsberuhigung sollen die Straßen in einem Wohnbereich so verändert werden, dass die Verkehrsstärken vermindert und die Kraftfahrer zu langsamer und rücksichtsvoller Fahrweise veranlasst werden. Zugleich kann das Wohnumfeld verbessert werden und es sollen nach Möglichkeit mehr Freiflächen entstehen, insbesondere dort, wo entsprechende Mängel herrschen. So soll erreicht werden, dass die Verkehrssicherheit verbessert und das Leben in der Stadt angenehmer wird." (BfS, 1980).

Als Maßnahmen zur Umsetzung der Verkehrsberuhigung werden in diesen Empfehlungen Maßnahmen aufgeführt, die entweder eine Reduzierung der Kraftfahrzeugverkehrsstärke oder eine 
„Drosselung der Fahrweise“ (Verringerung der Fahrgeschwindigkeit) im gesamten Wohnbereich bewirken. Dabei kommen ebenso Maßnahmen im gesamten Straßennetz in Betracht, wie z.B. die Unterbindung der Durchfahrtmöglichkeit (Sackgassen, Diagonalsperren) oder wie Maßnahmen im Straßenraum, z.B. Fahrgassenversätze, Einengungen (vgl. Abbildung 4), Schwellen, Blinkzeichenanlagen, Teilaufpflasterungen oder eine Beschränkung der zulässigen Höchstgeschwindigkeit auf Tempo 30.

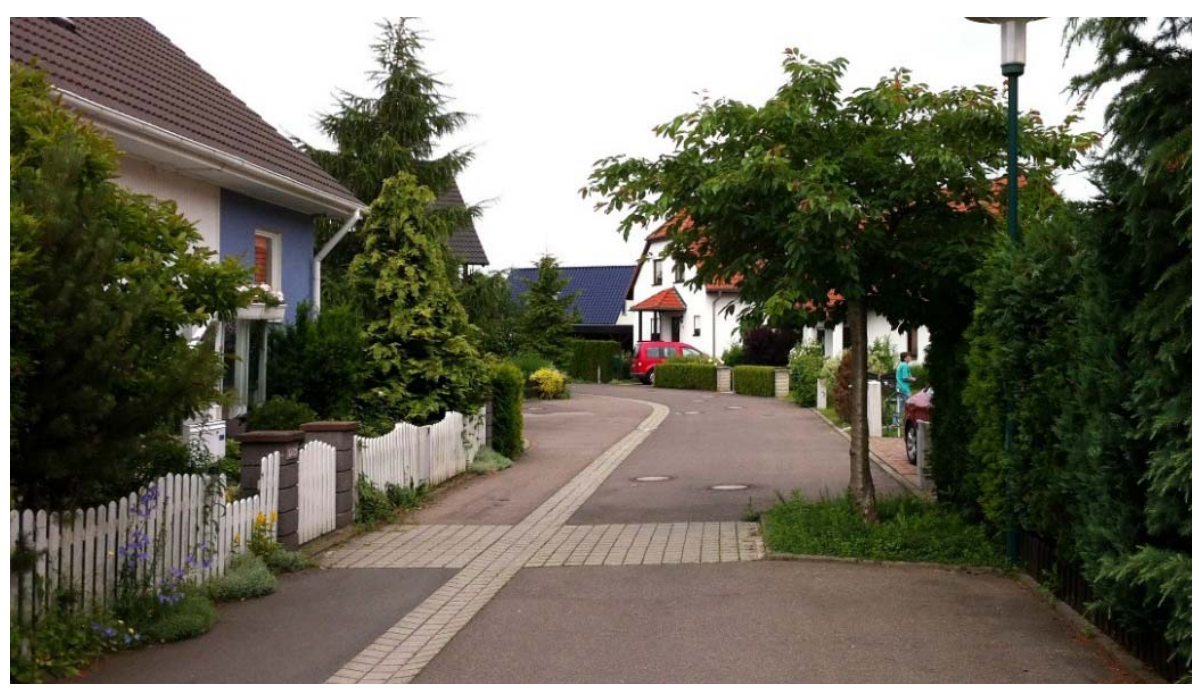

Abbildung 4: Beispielhafte Darstellung einer punktuellen Einengung im Straßenraum zur Reduzierung der Fahrgeschwindigkeit (Foto: IGS mbH 2013)

Zusätzlich wird als Maßnahme in Teilbereichen der Verkehrsberuhigten Bereiche die Einrichtung von Mischflächen vorgeschlagen. Allerdings kommt dabei die Anlage von Mischflächen gemäß den Empfehlungen nur dann in Frage, wenn aus Gründen der Verkehrssicherheit sichergestellt ist, dass diese Bereiche, in denen die Mischflächen eingerichtet werden, nur von Anwohnern befahren werden (z.B. Sackgassen) und Fahrtlängen sowie das Verkehrsaufkommen begrenzt sind. Die Empfehlungen gehen dabei davon aus, dass 85\% der Kraftfahrzeuge nicht schneller als $20 \mathrm{~km} / \mathrm{h}$ fahren, wenn auf der Mischfläche wirklich nur Anwohner fahren und die Fahrbereiche für Kraftfahrzeuge eng gehalten werden und im Abstand von spätestens $30 \mathrm{~m}$ bis $40 \mathrm{~m}$ Fahrgassenversätze oder Schwellen angeordnet werden (BfS 1980).

Darüber hinaus werden in den Empfehlungen folgende Bedingungen genannt, die erfüllt sein müssen, wenn Straßenabschnitte als Mischfläche gestaltet werden sollen (BfS, 1980, S.41):

"- Sie dürfen nicht unmittelbar an Verkehrsstraßen angrenzen, da Mischflächen in der Regel Spielbereiche sind und verhindert werden muss, dass Kinderspiele direkt neben einer Verkehrsstraße stattfinden.

- $\quad$ Durch die Lage der Straße im Netz muss sichergestellt sein, dass sie nur von Anwohnern befahren wird (z.B. in Sackgassen).

- $\quad$ Über die Straße dürfen keine gewerblichen Betriebe, Geschäfte usw. erschlossen sein.

- Der Kraftfahrzeugverkehr muss zu allen Zeiten gering sein (welche Verkehrsstärken in Kauf genommen werden können, hängt auch von den örtlichen Gegebenheiten ab; selbst bei besonders günstigen örtlichen Voraussetzungen sind 100 Kraftfahrzeuge in der Spitzenstunde aber die allenfalls noch zulässige Obergrenze).

- $\quad$ Der Streckenabschnitt darf nicht lang sein, weil die Einhaltung von Schrittgeschwindigkeit über Strecken von z.B. wesentlich mehr als $100 \mathrm{~m}$ als unzumutbar empfunden wird.

- Es dürfen keine Linienbusse verkehren." 
Als Hinweise zur Gestaltung der Mischverkehrsflächen nennen die Empfehlungen auf S.41 zusammengefasst (BfS, 1980):

\author{
"- Die Befestigungen der Mischflächen sind deutlich von dem anderer Straßen zu \\ unterscheiden;
}

- Die von Kraftfahrzeugen befahrbaren Bereiche sollen nicht länger als auf einer Strecke von maximal 30m bis 40m geradeaus verlaufen; ggf. sind auch Schwellen einzurichten;

- Diejenigen Flächen, die nicht als befahrbarer Bereich oder als Stellplätze dienen, sollen farblich hervorgehoben und durch Poller oder Bordsteine abgegrenzt werden;

- Den Fußgängern vorbehaltene Bereiche können bepflanzt oder möbliert werden; dabei empfiehlt es sich aber, mit der Möblierung sparsam zu sein und noch ausreichende Freiflächen, die Kinderspiele ermöglichen, offen zu lassen;

- Bei der Möblierung und Bepflanzung ist dafür Sorge zu tragen, dass keine gefährlichen Sichthindernisse geschaffen werden;

- Der Verlauf des befahrbaren Bereiches in einer Mischfläche muss dem Fahrer z.B. durch farbliche Gestaltung der Oberfläche deutlich gemacht werden; es darf nur eine einzige Fahrgasse vorhanden sein;

- Anfang und Ende von Mischflächen sind durch entsprechende Ausführung als solche kenntlich zu machen."

Im Jahre 1981 wird durch die Beratungsstelle für Schadenverhütung eine Studie zum Thema "Erfahrungen mit Verkehrsberuhigten Bereichen“ (Zeichen 325/326 StVO) veröffentlicht (BfS, 1981). Diese Studie beinhaltet Ergebnisse von Geschwindigkeitsmessungen in Straßen, die nach dem Inkrafttreten der StVO mit dem Verkehrszeichen 325 ausgeschildert wurden. Die Ergebnisse lassen sich wie folgt zusammenfassen (BfS, 1981, S.1):

„- In Straßen, die baulich nicht entsprechend umgestaltet wurden und bei denen es weiterhin deutliche Unterschiede zwischen Gehwegen und Fahrbahn gibt, wird auch nach Aufstellung von Zeichen 325 zu schnell gefahren. Es werden Geschwindigkeiten von $50 \mathrm{~km} / \mathrm{h}$ und mehr erreicht. Von Schrittgeschwindigkeit kann in solchen Straßen nirgendwo die Rede sein.

- $\quad$ Auch in Mischflächen wird wesentlich schneller als Schrittgeschwindigkeit gefahren, wenn die Straße eine gewisse Sammelfunktion hat und also nicht ausschließlich Anlieger dort verkehren. Auch die Tiefe der Fahrgassenversätze in solchen Straßen spielt eine Rolle.

- Vergleiche der Geschwindigkeiten vor und nach Aufstellung der Zeichen 325 in Straßen, die vor der Beschilderung nicht umgebaut wurden, machen deutlich, dass die Beschilderung keinen Einfluss auf die Geschwindigkeit hat."

Die Empfehlungen „Verkehrsberuhigung in Wohnbereichen“ (BfS, 1980) wurden im Jahre 1986 durch die Empfehlungen „Verkehrserschließung von Wohnbereichen“ ersetzt (BfS, 1986). Diese neuen Empfehlungen basieren im Wesentlichen auf den durch die Beratungsstelle für Schadensverhütung gesammelten Erfahrungen mit Verkehrserschließung und Verkehrsberuhigung in Wohngebieten. Sie weichen von den Empfehlungen von Mischverkehrsflächen ab. Im Gegensatz zu den im Jahre 1985 durch die FGSV veröffentlichten „Empfehlungen für die Anlage von Erschließungsstraßen“ (EAE 85), die das „Mischungsprinzip der Verkehrsarten“ favorisierte, welches zur Schaffung von „Mischverkehrsflächen“ in ausgewiesenen VBB führte, wird nun wieder über eine Trennung der Verkehrsarten diskutiert. Die Empfehlungen der Beratungsstelle für Schadensverhütung gehen nunmehr davon aus, dass - ausgenommen in kurzen Wohnwegen neuer Wohngebiete - die Gehwegbereiche für Fußgänger immer durch Borde abgetrennt werden und eine Beschilderung durch Zeichen 325 StVO (Verkehrsberuhigter Bereich) nur in bestimmten Einzel- oder Ausnahmefällen in Frage kommt. 
Als Begründung wird aufgeführt (BfS, 1986, S.8):

„- Erfahrungen haben gezeigt, dass Flächen, die in einem Niveau befestigt sind, in problematischen Straßenräumen mit hohem Freiflächendefizit und hohem Parkdruck (vgl. Abbildung 5) nicht funktionieren: Alle für das Fahrzeug erreichbaren Flächen werden zum Fahren oder zum Parken benutzt; Schutzräume für Fußgänger werden - wenn überhaupt meist nachträglich durch Reihen von Pollern geschaffen, die Zweiradfahrer aber nicht fernhalten können und das Straßenbild nicht aufwerten.

- Das Weglassen der Bordsteine führt weder zu einer Geschwindigkeitsdämpfung noch zu einer Aufwertung des Straßenbildes.

- $\quad$ Auch in eigentlich unproblematischen Straßenräumen sind Flächen, bei denen Gehwege nicht durch Bordsteine abgetrennt sind, umstritten: Eltern von Kindern und ältere Menschen wünschen für Fußgänger Flächen, die von fahrenden und parkenden Kraftfahrzeugen nicht erreicht werden können und die sich deutlich von den Fahrflächen abheben."

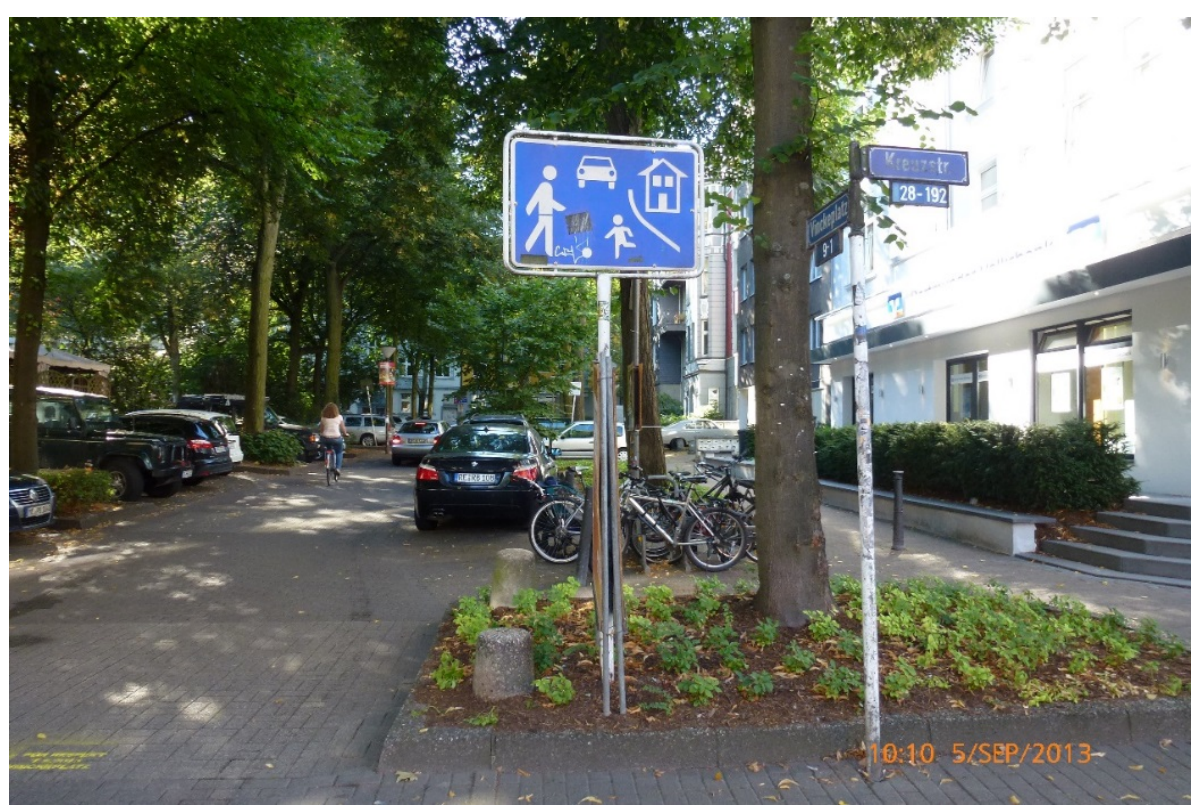

Abbildung 5: Beispielhafte Darstellung eines Verkehrsberuhigten Bereiches mit hohem Parkdruck

Die Empfehlungen aus dem Jahre 1986 setzen dabei vielmehr auf den §31 der StVO bzw. der zugehörigen Verwaltungsvorschrift zur Straßenverkehrsordnung (VWV-StVO) zu §31, die unter Absatz 4 Folgendes zusammenfasst:

"- Wohnstraßen und auch andere Straßen ohne Verkehrsbedeutung, auf denen der Kraftfahrer mit spielenden Kindern rechnen muss, brauchen nach der Erfahrung nicht zu „Spielstraßen“ erklärt werden. Auch das Zeichen 136 (,Kinder") ist dort i.d.R. entbehrlich. Gegen Kinderspiele sollte dort nicht eingeschritten werden."

Daher sind gemäß den Empfehlungen von 1986 alle Erschließungsstraßen in Wohngebieten nach dem „Mischungsprinzip“ zu konzipieren, d.h. es muss für die Verträglichkeit der Nutzung gesorgt werden. Die Kennzeichnung einer Straße als Verkehrsberuhigten Bereich mit StVO-Zeichen 325 wird in den Empfehlungen nur für den Fall geraten, wenn sehr geringer Kraftfahrzeugverkehr herrscht und durch bauliche Maßnahmen bewirkt wird, dass 85\% der Kraftfahrer auch ohne Beschilderung nicht schneller als mit $25 \mathrm{~km} / \mathrm{h}$ fahren. In den Empfehlungen von 1986 wird ebenfalls festgehalten, dass die für Verkehrsberuhigte Bereiche vorgeschriebene Schrittgeschwindigkeit sich nicht durch bauliche Maßnahmen erzwingen lässt. 
Aus den gewonnenen Erfahrungen zum punktuellen Ansatz der Verkehrsberuhigung, folgten die Untersuchungen zur flächenhaften Verkehrsberuhigung, im Rahmen des Großversuchs. Hierzu wurde in sechs verschiedenen Modellstädten ein jeweils unterschiedlich strukturiertes Modellgebiet ausgewählt. Im Rahmen von Vorher/Nachher-Untersuchungen wurde dabei die Wirkung einer flächenhaften Verkehrsberuhigung betrachtet. Die gewonnenen Erkenntnisse wurden im Rahmen eines Forschungsvorhabens zur flächenhaften Verkehrsberuhigung zusammengetragen (BRBS, 1992).

In den 1980er und 1990er Jahren dehnte sich das Thema der Verkehrsberuhigung vom punktuellen Straßenumbau zu einem stadtweiten Konzept aus. So wurde nunmehr unter dem Thema Verkehrsberuhigung ein Konzept angesehen, das die folgenden drei Zielrichtungen beinhaltet:

- Umverteilung von Verkehrsflächen zugunsten von Fußgängern, Radfahrern, Aufenthalt, Straßenraumgestaltung und Begrünung,

- Erhöhung des Modal-Split-Anteils des Umweltverbundes,

- umweltschonende Abwicklung des verbleibenden Kfz-Verkehrs, zum Schutze und zur Sicherheit aller Straßenbenutzer.

Die drei zentralen Maßnahmenbereiche hießen dabei:

- Straßenraumgestaltung und Geschwindigkeitsdämpfung,

- Förderung der Verkehrsmittel des Umweltverbundes sowie

- Verkehrsmanagement.

Unter Verkehrsmanagement wurden dabei u.a. die Parkraumbewirtschaftung einschließlich Park+Ride sowie das Staumanagement (z.B. durch Pförtnereinrichtung) und die räumliche Verkehrslenkung zusammengefasst.

Ab den 1990er Jahren rückte das Thema Verkehrsberuhigung wieder in den Hintergrund. Erst mit Beginn des neuen Jahrtausends trat es wieder in den Fokus der Verkehrsplaner. Dabei steht die Verkehrsberuhigung hinter Begriffen wie Begegnungszonen, Shared-Space, SimpliCity und ähnlichen Begriffen zurück. Vielmehr geht es in der aktuellen Diskussion zur Verkehrsberuhigung eher um eine Planungskultur, die zwar auch dem Ziel der Steigerung der Aufenthaltsqualität und der Verkehrssicherheit im Straßenraum folgt, aber auch eine verstärkte Einbeziehung der Öffentlichkeit und eine Veränderung des Verkehrsverhaltens mit in die Betrachtung einbezieht.

Viele dieser Konzepte beziehen eine Ausweisung von Verkehrsberuhigten Bereichen mit dem Zeichen 325 gemäß StVO ein. Da sich diese Anwendungsbeispiele im Regelfall jedoch auf Straßenabschnitte mit sehr hoher Verkehrsbelastung beziehen, kommt die Ausweisung eines Verkehrsberuhigten Bereiches gemäß Zeichen 325 der StVO eigentlich nicht in Betracht, da es in der Verwaltungsvorschrift zur StVO heißt, dass ein VBB nur auf einer Straße ausgewiesen werden darf, auf der sehr geringer Verkehr vorhanden ist. Auch wenn der Begriff "sehr geringer Verkehr“ nicht eindeutig definiert wird, ist davon auszugehen, dass es sich hierbei um eine Verkehrsbelastung von deutlich weniger als 400 Kraftfahrzeugen (Kfz) in der Spitzenstunde handelt. In den ersten Städten wird daher schon die Beschilderung dieser Bereiche mit dem Verkehrszeichen 325 gemäß StVO zurückgenommen. So wird beispielsweise der als Verkehrsberuhigter Bereich ausgewiesene Abschnitt des Hochemmericher Marktes in Duisburg in einen Verkehrsberuhigten Geschäftsbereich umgewandelt. 


\subsection{Verkehrsberuhigte Bereiche im Verkehrsrecht}

Der Verkehrsberuhigte Bereich wurde erstmals mit der Verordnung zur Änderung der Straßenverkehrsordnung vom 21. Juli 1980 im Verkehrsrecht eingeführt ${ }^{3}$. Bis zur heutigen gültigen Straßenverkehrsordnung (Fassung vom 06.03.2013) und der dazugehörigen Verwaltungsvorschrift (Fassung vom 11.11.2014) wurden die Inhalte und Ausführungen zu Verkehrsberuhigten Bereichen mehrfach in Teilbereichen geändert. Die derzeit gültigen Fassungen sehen für Verkehrsberuhigte Bereiche die nachfolgenden Regelungen vor.

Laut der aktuellen Straßenverkehrsordnung (Stand 2013) sind Verkehrsberuhigte Bereiche mit den Richtzeichen 325.1 und 325.2 auszuweisen. Die Ausführungen zu Verkehrsberuhigten Bereichen werden in der StVO in $\$ 42$ (Richtzeichen) unter Anlage 3, Abschnitt 4, Ifd. Nr. 12 und 13 beschrieben. Zu Zeichen 325.1 (Beginn eines Verkehrsberuhigten Bereiches) werden die folgenden Ge- oder Verbote genannt:

„1. Wer ein Fahrzeug führt, muss Schrittgeschwindigkeit fahren.

2. Wer ein Fahrzeug führt, darf den Fußgängerverkehr weder gefährden noch behindern; wenn nötig, muss gewartet werden.

3. Wer zu Fuß geht, darf den Fahrverkehr nicht unnötig behindern.

4. Wer ein Fahrzeug führt, darf außerhalb der dafür gekennzeichneten Flächen nicht parken, ausgenommen zum Ein- oder Aussteigen und zum Be- oder Entladen.

5. Wer zu Fuß geht, darf die Straße in ihrer ganzen Breite benutzen; Kinderspiele sind überall erlaubt."

Diese Ge- und Verbote können und werden in der Praxis unterschiedlich interpretiert und ausgelegt. Eine Hilfestellung zur Auslegung kann aus der amtlichen Begründung zur Änderung der Straßenverkehrsordnung (allerdings der Änderung der StVO von 1980) entnommen werden ${ }^{4}$. Hier steht zu den einzelnen 5 Punkten:

\section{Zu Punkt 1:}

Der Begriff „Fahrzeugverkehr" stellt klar, dass hiermit nicht nur Kraftwagen gemeint sind. Auch Radfahrer, Mofas und Mopeds müssen Schritt fahren. Der Begriff "Schrittgeschwindigkeit" deckt sich mit dem in \$24, Abs. 2 aufgeführten gleichnamigen Begriff. Es ist dies eine sehr langsame Geschwindigkeit, die der eines normal gehenden Fußgängers entspricht, sie muss jedenfalls wesentlich unter $20 \mathrm{~km} / \mathrm{h}$ liegen.

Zu Punkt 2:

Hier wird im Ergebnis der Vorrang des Fußgängers - vor dem Fahrzeugverkehr - normiert. Dies kommt in der Formulierung zum Ausdruck: „Wenn nötig, müssen sie (die Fahrzeuge) warten“. Diese Formulierung findet sich in $\S 9$, Abs. 3, §20, Abs. 1, 1a und 2 sowie in $\S 26$, Abs. 1.

Zu Punkt 3:

Diese Vorschrift soll verhindern, dass die Fußgänger einen unangemessenen Gebrauch von ihrem Vorrang machen.

31980 wurden die Verkehrszeichen „VZ 325“ (heute VZ 325.1) und „VZ 326“ (heute VZ 325.2 vgl. Abbildung 1) eingeführt.

4 In der StVO von 1980 sind die fünf Punkte lediglich anders gruppiert und etwas umformuliert worden. Sinngemäß wurden sie beibehalten. Die Erläuterungen/Interpretationen wurden entsprechend der Rangfolge der StVO von 2013 zugeordnet. 


\title{
Zu Punkt 4:
}

Die zum Parken bestimmten Flächen innerhalb des verkehrsberuhigten Bereiches brauchen nicht durch Parkplatzschilder gekennzeichnet zu sein. Es genügt auch eine Bodenmarkierung ( $\$ 41, A b s$. $3 \mathrm{Nr} .7$ ) oder, wenn dies ausreichend deutlich möglich ist, eine besondere Art der Pflasterung.

\section{Zu Punkt 5:}

Diese Vorschrift hebt die Differenzierung der einzelnen Straßenteile nach Benutzungsarten (Gehweg, Radweg, Fahrbahn) auf. Es ist klar, dass eine solche Regelung ohne eine erhebliche bauliche Umgestaltung der Straße nicht möglich ist. Dies wäre im Interesse der Verkehrssicherheit nicht zu verantworten.

Zu Zeichen 325.2 (Ende eines Verkehrsberuhigten Bereiches) wird in der StVO (§42, Anlage 3, Abschnitt 4, Ifd. 13) unter der Erläuterung genannt, dass beim Ausfahren $\$ 10$ zu beachten ist. In $\S 10$ der StVO (2013) ist Folgendes geregelt:

\begin{abstract}
„Wer aus einem Grundstück, aus einer Fußgängerzone (Zeichen 242.1 und 242.2), aus einem verkehrsberuhigten Bereich (Zeichen 325.1 und 325.2) auf die Straße oder von anderen Straßenteilen oder über einen abgesenkten Bordstein hinweg auf die Fahrbahn einfahren oder vom Fahrbahnrand anfahren will, hat sich dabei so zu verhalten, dass eine Gefährdung anderer Verkehrsteilnehmer ausgeschlossen ist; erforderlichenfalls muss man sich einweisen lassen. Die Absicht, einzufahren oder anzufahren, ist rechtzeitig und deutlich anzukündigen; dabei sind die Fahrtrichtungsanzeiger zu benutzen. Dort, wo eine Klarstellung notwendig ist, kann Zeichen 205 stehen."
\end{abstract}

Die zugehörige Verwaltungsvorschrift zu den Zeichen 325.1 und 325.2 (Verkehrsberuhigte Bereiche) umfasst folgende Ausführungen (VwV-StVO, zu §42, zu den Zeichen 325.1 und 325.2):

„I. Ein verkehrsberuhigter Bereich kommt nur für einzelne Straßen oder für Bereiche mit überwiegender Aufenthaltsfunktion und sehr geringem Verkehr in Betracht. Solche Bereiche können auch in Tempo-30-Zonen integriert werden.

II. Die mit Zeichen 325.1 gekennzeichneten Straßen müssen durch ihre besondere Gestaltung den Eindruck vermitteln, dass die Aufenthaltsfunktion überwiegt und der Fahrzeugverkehr eine untergeordnete Bedeutung hat. In der Regel wird ein niveaugleicher Ausbau für die ganze Straßenbreite erforderlich sein.

III. Zeichen 325.1 darf nur angeordnet werden, wenn Vorsorge für den ruhenden Verkehr getroffen ist.

IV. Zeichen 325.1 ist so aufzustellen, dass es aus ausreichender Entfernung wahrgenommen werden kann; erforderlichenfalls ist es von der Einmündung in die Hauptverkehrsstraße abzurücken oder beidseitig aufzustellen.

V. Mit Ausnahme von Parkflächenmarkierungen sollen in verkehrsberuhigten Bereichen keine weiteren Verkehrszeichen angeordnet werden. Die zum Parken bestimmten Flächen sollen nicht durch Zeichen 314 gekennzeichnet werden, sondern durch Markierung, die auch durch Pflasterwechsel erzielt werden kann."

\subsection{Rechtsprechung}

Trotz der ausführlichen Beschreibung der Rahmenbedingungen für die Einrichtung eines ausgewiesenen Verkehrsberuhigten Bereiches gemäß VZ 325 der StVO, bleiben in der StVO und der zugehörigen VwV-StVO (Kapitel 5.2) dennoch einige Aspekte nicht abschließend geklärt. Der Begriff der Schrittgeschwindigkeit ist in der StVO nicht genauer definiert. Daher soll eine Erläuterung nach Müller (Fachanwaltskommentar Verkehrsrecht; 2009) folgen. Der Begriff der Schrittgeschwindigkeit gilt in seiner Auslegung immer noch als umstritten (Müller, 2009). 
Gemäß der einfachsten juristischen Auslegungsmethode, der sogenannten Wortauslegung, ist unter Schrittgeschwindigkeit eine langsame Geschwindigkeit zu verstehen, die der eines normal gehenden Fußgängers entspricht (Müller, 2009). Entsprechend haben die Oberlandesgerichte Köln, Stuttgart und Brandenburg die Schrittgeschwindigkeit zu etwa 4 bis $7 \mathrm{~km} / \mathrm{h}$ bzw. als Obergrenze die Geschwindigkeit von $7 \mathrm{~km} / \mathrm{h}$ festgelegt. Das Amtsgericht Leipzig hingegen empfiehlt als Schrittgeschwindigkeit eine Geschwindigkeit von 15km/h (Müller, 2009).

Gemäß des Bund-Länder-Fachausschusses Straßenverkehrsordnungswidrigkeiten gilt die Schrittgeschwindigkeit in ausgewiesenen Verkehrsberuhigten Bereichen bei $10 \mathrm{~km} / \mathrm{h}$ als noch eingehalten. Dies hat der Fachausschuss auf seiner Sitzung vom 29./30.09.1998 festgehalten. Weiterhin wurde dabei festgehalten, dass eine Ahndung der Überschreitung der Schrittgeschwindigkeit erst ab einer vorwerfbaren Geschwindigkeit von 16km/h erfolgt (Müller, 2009).

Aufbauend auf der Begründung, dass eine Schrittgeschwindigkeit von 4 bis $7 \mathrm{~km} / \mathrm{h}$ von Kraftfahrzeugführern mittels Tachometer nicht zuverlässig zu überprüfen sei und Radfahrer bei dieser Geschwindigkeit instabil fahren würden, wird eine deutlich über 4 bis $7 \mathrm{~km} / \mathrm{h}$ liegende Schrittgeschwindigkeit als angemessen betrachtet (Henschel, 2013).

Insgesamt bleibt festzuhalten, dass rechtlich gesehen offensichtlich noch keine abschließende Klärung des Begriffes der Schrittgeschwindigkeit erfolgt ist.

\section{Untersuchungsmethodik}

Untersuchungsgegenstand der Studie waren, wie in Kapitel 4 dargelegt, Verkehrsberuhigte Bereiche, die mit Verkehrszeichen 325 StVO (StVO 2013) gekennzeichnet sind. Die Auswertung der vorhandenen Erkenntnisse zum Unfallgeschehen und Verkehrsablauf in diesen Verkehrsberuhigten Bereichen zeigte, dass keine aktuellen Untersuchungen zum Verkehrsablauf und zur Verkehrssicherheit vorliegen. Ebenso wenig war die aktuelle Umsetzungspraxis in den Kommunen von ausgewiesenen Verkehrsberuhigten Bereichen bekannt. Daher bildete die Grundlage dieser Studie eine umfangreiche Bestandsaufnahme.

Der Ablauf und die Untersuchungsmethodik der Studie können der Abbildung 6 entnommen werden.

Die Voruntersuchung (Stufe 1) umfasste eine makroskopische Unfallanalyse, bei der eine Auswertung der amtlichen Unfallstatistik für einzelne Bundesländer durchgeführt wurde. Ziel dieser makroskopischen Unfallanalyse war es, einen Überblick über die Struktur (Unfalltyp, Unfallart) des Unfallgeschehens in ausgewiesenen Verkehrsberuhigten Bereichen und deren Entwicklung zu erlangen. Es wurden die Unfalldaten für einen 6-Jahres-Zeitraum ausgewertet.

Ein weiterer Teil der ersten Stufe war die Durchführung einer Umfrage zur Umsetzungspraxis in den Kommunen. Hierzu wurde ein Fragebogen zur Umsetzungspraxis von ausgewiesenen Verkehrsberuhigten Bereichen entwickelt. Dieser Fragebogen enthielt unterschiedliche Fragen wie zum Beispiel:

- die Gründen für die Entscheidung zur Umsetzung eines ausgewiesenen VBBs,

- $\quad$ eine Auskunft über die mit der Umsetzung verbundenen Zielsetzungen oder

- $\quad$ auf welche Art und Weise die Bevölkerung bei der Realisierung einbezogen wurde. 


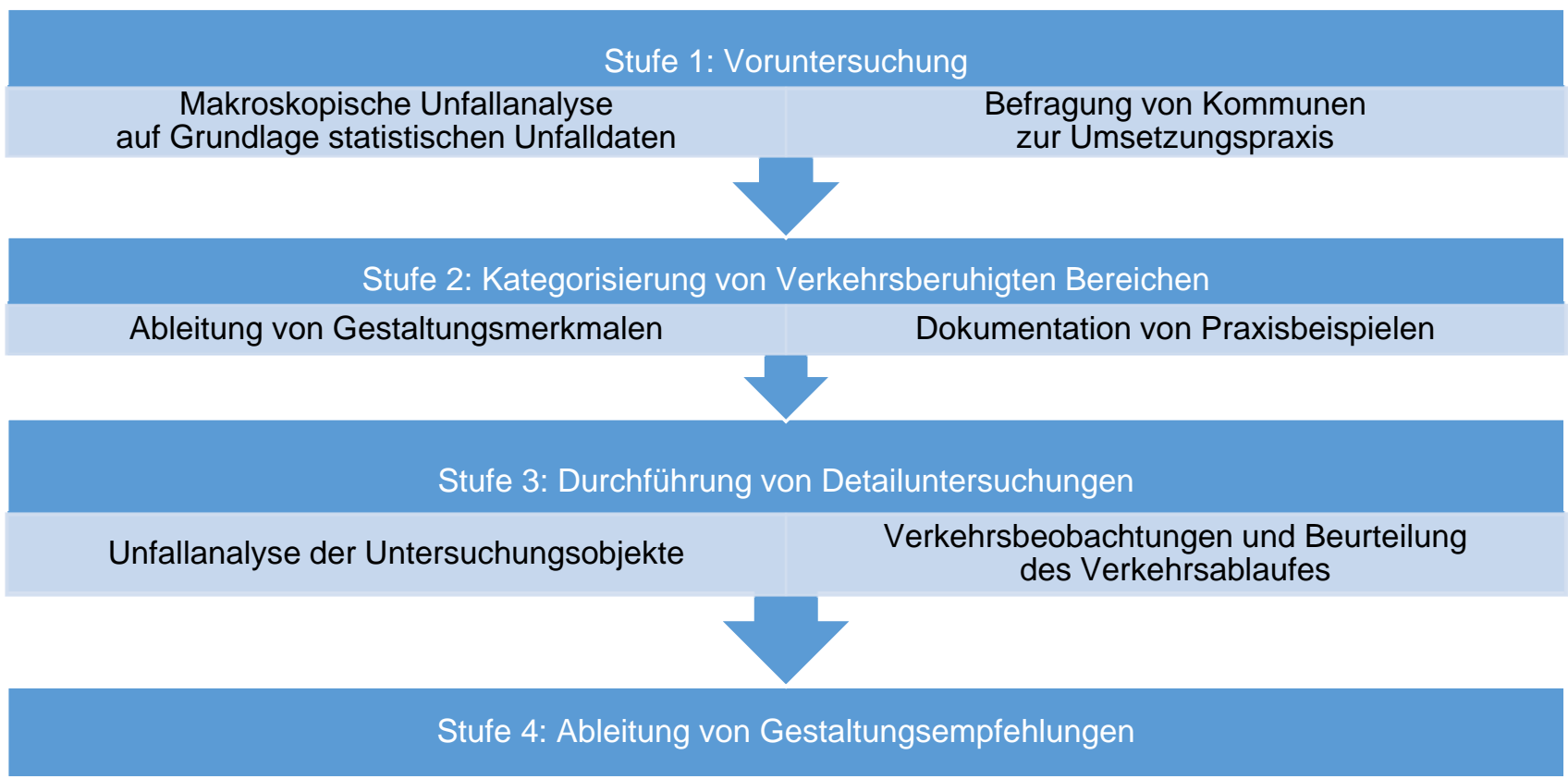

Abbildung 6: Untersuchungsmethodik

Aus den Erkenntnissen der makroskopischen Unfallanalyse, der Umfrage zur Umsetzungspraxis in den Kommunen und der Aufbereitung des derzeitigen Kenntnisstandes wurde eine Kategorisierung von ausgewiesenen Verkehrsberuhigten Bereichen vorgenommen (Stufe 2). Diese Kategorisierung diente dazu, Rahmenbedingungen für den Einsatz von ausgewiesenen VBB abzuleiten, um später einheitliche Gestaltungsgrundsätze für entsprechende Einsatzbereiche festlegen zu können. Insgesamt konnten 302 ausgewiesene Verkehrsberuhigte Bereiche deutschlandweit erhoben werden, die kategorisiert wurden.

Nach der Kategorisierung und Dokumentation der erhobenen VBB wurden Detailuntersuchungen durchgeführt (Stufe 3). Zum einen waren dies Unfallanalysen und zum anderen Verkehrsbeobachtungen.

Für 278 der 302 Untersuchungsobjekte konnten Unfalldaten über einen Zeitraum von 6 Jahren erhoben werden, anhand derer eine detaillierte Unfallanalyse vorgenommen wurde. Hierzu wurden die Unfalldaten aus elektronischen Datenbanken oder auch anonymisierte Verkehrsunfallanzeigen bei den zuständigen Polizeidienststellen erhoben und ausgewertet. Ziel war es, typische Unfallsituationen von VBB zu identifizieren.

Aus den 278 Untersuchungsobjekten, die einer detaillierten Unfallanalyse unterzogen wurden, sind wiederum 50 Objekte für eine vertiefende Analyse des Verkehrsablaufs ausgewählt worden. An diesen 50 Untersuchungsobjekten wurden u.a. Geschwindigkeitsmessungen und Verhaltensbeobachtungen durchgeführt. Die Verhaltensbeobachtungen, die mittels Videoerfassung dokumentiert worden sind, dienten zur Aufdeckung von Interaktionen und auch Konflikten, die zwischen den verschiedenen Verkehrsteilnehmern stattfanden. Ziel der Verhaltensbeobachtung war es, u.a. zu ermitteln, welche Gestaltungselemente innerhalb der ausgewiesenen Verkehrsberuhigten Bereiche zu Fehlverhalten der Verkehrsteilnehmer beitragen können. Ebenso sollte untersucht werden, ob gewisse Gestaltungsmaßnahmen einen positiven Einfluss auf das Geschwindigkeitsniveau haben.

Abschließend wurde untersucht, ob es Zusammenhänge zwischen dem Verkehrsablauf bzw. dem Unfallgeschehen und der Gestaltung von ausgewiesenen Verkehrsberuhigten Bereichen gibt (Stufe 4). 


\section{$7 \quad$ Makroskopische Unfallanalyse}

\subsection{Definitionen und Erläuterungen}

In dem vorliegenden Kapitel werden zunächst die für die Unfallanalyse relevanten Begrifflichkeiten und Abkürzungen erläutert.

\subsubsection{Unfallkategorie / Unfallschwere}

Anhand von Unfall-Kategorien wird die Unfallschwere beschrieben. Eine Übersicht zeigt Abbildung 7. Unfälle $(U)$ der Kategorie (Kat.) 1-4 sind also solche, die schwere Unfallfolgen besitzen.

\begin{tabular}{|c|c|c|c|}
\hline $\begin{array}{l}\text { Schwerste } \\
\text { Unfallfolge }\end{array}$ & & $\begin{array}{l}\text { Unfall- } \\
\text { Kategorie }\end{array}$ & Beschreibung* \\
\hline $\begin{array}{l}\text { Unfall mit } \\
\text { Getöteten }\end{array}$ & $\mathrm{U}(\mathrm{GT})$ & Kat. 1 & Mindestens ein getöteter Verkehrsteilnehm \\
\hline $\begin{array}{l}\text { Unfall mit } \\
\text { Schwerverletzten }\end{array}$ & $\mathrm{U}(\mathrm{SV})$ & Kat. 2 & $\begin{array}{l}\text { Mindestens ein schwerverletzter Verkehrsteilnehmier, } \\
\text { aber keine Getöteten }\end{array}$ \\
\hline $\begin{array}{l}\text { Unfall mit } \\
\text { Leichtverletzten }\end{array}$ & $\mathrm{U}(\mathrm{LV})$ & Kat. 3 & $\begin{array}{l}\text { Mindestens ein leichtverletzter Verkehrsteilnehmer, } \\
\text { aber keine Getöteten und keine Schwerverletzten }\end{array}$ \\
\hline \multirow[t]{2}{*}{$\begin{array}{l}\text { schwerwiegender } \\
\text { Unfall mit } \\
\text { Sachschaden }\end{array}$} & $\mathrm{U}(\mathrm{SS})$ & Kat. 4 & $\begin{array}{l}\text { Unfälle mit Sachschaden und Straftatbestand oder Ordnungswidrigkeits- } \\
\text { Anzeige (unfallursächlich), bei denen mindestens ein Kraftfahrzeug } \\
\text { nicht mehr fahrbereit ist (abschleppen) }\end{array}$ \\
\hline & & Kat. 6 & Alle übrigen Sachschadenunfälle unter Einfluß berauschender Mitt \\
\hline $\begin{array}{l}\text { sonstiger Unfall mit } \\
\text { Sachschaden } \\
\text { U-Kategonie_but }\end{array}$ & $\mathrm{U}(\mathrm{LS})$ & Kat. 5 & $\begin{array}{l}\text { Sachschadenunfälle } \\
\text { - mit Straftatbestand oder Owi-Anzeige ohne Einfluß berauschender Mittel, } \\
\text { bei denen alle Kraftfahrzeuge fahrbereit sind, } \\
\text { - mit lediglich geringfügiger Ordnungswidrigkeit (Verwarnung), } \\
\text { unabhängig, ob Kfz fahrbereit oder nicht fahrbereit }\end{array}$ \\
\hline
\end{tabular}

Abbildung 7: Definition der Unfallkategorien (Quelle: UDV)

\subsubsection{Unfalltypen}

Die Unfalltypen beschreiben die Konfliktsituationen, in denen es zur Folge zum Unfall gekommen ist. Es wird zwischen 7 Unfalltypen unterschieden (vgl. Abbildung 8).

Unfalltyp 1 ist der Fahrunfall (F), Unfalltyp 2 der Abbiege-Unfall (AB), Unfalltyp 3 der EinbiegenKreuzen-Unfall (EK), Unfalltyp 4 der Überschreiten-Unfall (ÜS), Unfalltyp 5 der Unfall durch ruhenden Verkehr (RV), Unfalltyp 6 der Unfall im Längsverkehr (LV) und Unfalltyp 7 der Sonstiger Unfall (SO). 


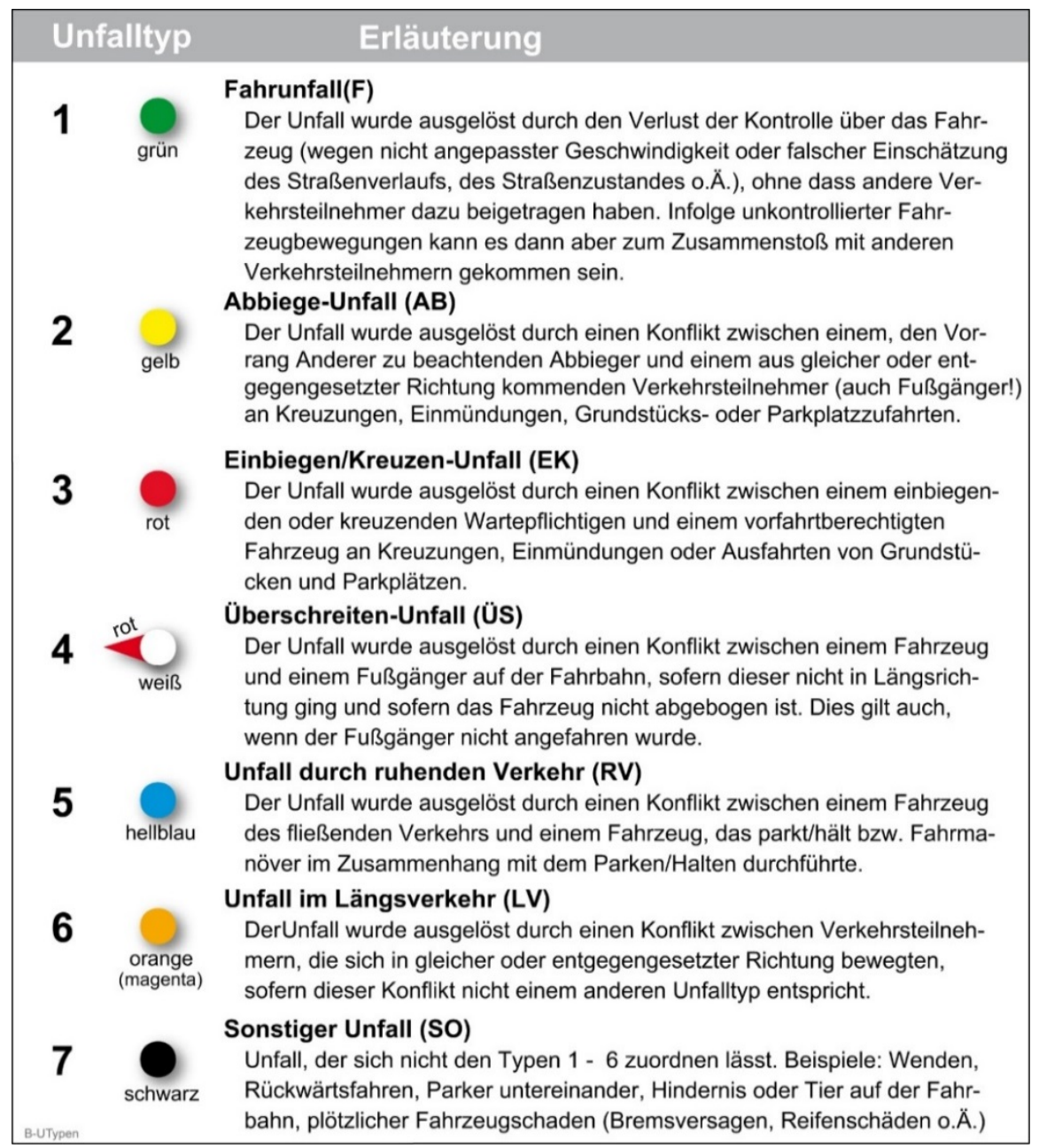

Abbildung 8: Definition der Unfalltypen (Quelle: UDV)

\subsubsection{Unfallarten}

Die Unfallart beschreibt vom gesamten Unfallablauf die Bewegungsrichtung der beteiligten Fahrzeuge zueinander beim ersten Zusammenstoß auf der Fahrbahn. Es werden 10 Unfallarten unterschieden (Statistisches Bundesamt „Destatis“ 2011) (vgl. Tabelle 1), die wie folgt definiert sind:

„1. Zusammenstoß mit anderem Fahrzeug, das anfährt, anhält oder im ruhenden Verkehr steht. Anfahren oder Anhalten ist hier im Zusammenhang mit einer gewollten Fahrtunterbrechung zu sehen, die nicht durch die Verkehrslage veranlasst ist. Ruhender Verkehr im Sinne dieser Unfallart ist das Halten oder Parken am Fahrbahnrand, auf Seitenstreifen, auf den markierten Parkstellen unmittelbar am Fahrbahnrand, auf Gehwegen oder auf Parkplätzen. Der Verkehr von und zu Parkplätzen mit eigenen Zufahrten gehört zur Unfallart 5.

2. Zusammenstoß mit anderem Fahrzeug, das vorausfährt oder wartet. Unfälle durch Auffahren auf ein Fahrzeug, das selbst noch fuhr oder verkehrsbedingt hielt. Auffahren auf anfahrende bzw. anhaltende Fahrzeuge gehören zur Unfallart 1.

3. Zusammenstoß mit anderem Fahrzeug, das seitlich in gleicher Richtung fährt. Unfälle beim Nebeneinanderfahren (Streifen) oder beim Fahrstreifenwechsel (Schneiden). 
4. Zusammenstoß mit anderem Fahrzeug, das entgegenkommt. Zusammenstöße im Begegnungsverkehr, ohne dass ein Kollisionspartner die Absicht hatte, über die Gegenspur abzubiegen.

5. Zusammenstoß mit anderem Fahrzeug, das einbiegt oder kreuzt. Zu dieser Unfallart gehören Zusammenstöße mit dem Querverkehr und Kollisionen mit Fahrzeugen die aus anderen Straßen, Wegen oder Grundstücken einbiegen oder dorthin abbiegen wollen. Das Auffahren auf wartende Abbieger gehört zur Unfallart 2.

6. Zusammenstoß zwischen Fahrzeug und Fußgänger. Personen, die sich arbeitsbedingt auf der Fahrbahn aufhalten oder noch in enger Verbindung zu einem Fahrzeug stehen, wie Straßenarbeiter, Polizeibeamte bei der Verkehrsregelung oder ausgestiegene Fahrzeuginsassen bei Pannen zählen nicht als Fußgänger. Zusammenstöße mit ihnen gehören zur Unfallart 10.

7. Aufprall auf ein Hindernis auf der Fahrbahn. Zu den Hindernissen zählen z.B. umgestürzte Bäume, Steine, verlorene Fracht sowie freilaufende Tiere oder Wild. Zusammenstöße mit geführten Tieren oder Reitern gehören zur Unfallart 10.

8./9. Abkommen von der Fahrbahn nach rechts/links. Bei diesen Unfallarten ist es nicht zu einem Zusammenstoß mit anderen Verkehrsteilnehmern gekommen. Es kann jedoch weitere Unfallbeteiligte geben, z.B. wenn das verunglückte Fahrzeug von der Straße abgekommen ist, weil es einem anderen Verkehrsteilnehmer ausgewichen ist, ohne ihn zu berühren.

10. Unfall anderer Art. Hier werden alle Unfälle erfasst, die sich nicht einer der Unfallarten von 1 bis 9 zuordnen lassen."

Tabelle 1: Definition Unfallarten

\begin{tabular}{|c|l|}
\hline Unfallart & Beschreibung \\
\hline 1 & Zusammenstoß mit anderem Fahrzeug, das anfährt, anhält oder im ruhenden Verkehr steht \\
\hline 2 & Zusammenstoß mit anderem Fahrzeug, das vorausfährt oder wartet \\
\hline 3 & Zusammenstoß mit anderem Fahrzeug, das seitlich in gleicher Richtung fährt \\
\hline 4 & Zusammenstoß mit anderem Fahrzeug, das entgegenkommt \\
\hline 5 & Zusammenstoß mit anderem Fahrzeug, das einbiegt oder kreuzt \\
\hline 6 & Zusammenstoß zwischen Fahrzeug und Fußgänger \\
\hline 7 & Aufprall auf ein Hindernis auf der Fahrbahn \\
\hline 8 & Abkommen von der Fahrbahn nach rechts \\
\hline 9 & Abkommen von der Fahrbahn nach links \\
\hline 10 & Unfall anderer Art \\
\hline
\end{tabular}




\subsubsection{Beteiligte, Fahrzeugbenutzer und Alleinunfälle}

Die Definitionen für Beteiligte, Fahrzeugbenutzer und Alleinunfälle können aus (Destatis 2011) entnommen werden:

„Beteiligte: Als Beteiligte an einem Straßenverkehrsunfall werden alle Fahrzeugführer oder Fußgänger erfasst, die selbst - oder deren Fahrzeug - Schäden erlitten oder hervorgerufen haben. Verunglückte Mitfahrer zählen somit nicht zu den Unfallbeteiligten. Der Hauptverursacher (1. Beteiligter) ist der Beteiligte, der nach Einschätzung der Polizei die Hauptschuld am Unfall trägt. Beteiligte an Alleinunfällen gelten immer als Hauptverursacher."

„Fahrzeugbenutzer: Es werden die Fahrzeugbenutzer der unfallbeteiligten Fahrzeuge, also die verletzten und unverletzten Personen in oder auf dem Fahrzeug, ausgezählt. Mit diesen Angaben lassen sich nicht nur durchschnittliche Fahrzeugbesetzungen errechnen. Das Verhältnis von potentiell gefährdeten (Fahrzeugbenutzer) zu wirklich verletzten oder getöteten Verkehrsteilnehmern gibt Aufschluss über die Verletzungsrisiken bei den einzelnen Verkehrsbeteiligungsarten."

„Alleinunfälle: Als Alleinunfälle gelten Unfälle, an denen nur ein Fahrzeug beteiligt ist. Es können jedoch mehrere Insassen verunglücken."

\subsubsection{Unfallursachen}

Es gibt insgesamt rund 80 Unfallursachen, die in einem Unfallursachenkatalog aufgelistet sind. Das Verzeichnis der Unfallursachen kann dem Anhang 1 entnommen werden.

„Es wird unterschieden nach allgemeinen Ursachen (u. a. Straßenverhältnisse, Witterungseinflüsse, Hindernisse), die dem Unfall und nicht einzelnen Beteiligten zugeordnet werden, sowie personenbezogenem Fehlverhalten (wie Vorfahrtsmissachtung, zu schnelles Fahren usw.), das bestimmten Fahrzeugführern oder Fußgängern - d.h. den Beteiligten zugeschrieben wird. Je Unfall können bis zu zwei allgemeine Ursachen angegeben werden." (Destatis 2011)

Die Unfallursachen werden von den Polizeibeamten, die den Unfall aufnehmen, entsprechend ihrer Einschätzung festgehalten.

Für die Auswertungen der vorliegenden Studie war es wegen der Übersichtlichkeit und Stichprobengröße sinnvoll, die Unfallursachen nach den jeweiligen Unterüberschriften des Unfallursachenverzeichnisses zu gruppieren. Die gruppierten Unfallursachen können der Tabelle 2 entnommen werden. 
Tabelle 2: $\quad$ Unfallursachen (in Gruppen zusammengefasst)

\begin{tabular}{|c|l|}
\hline Unfallursache & Beschreibung \\
\hline $01-04$ & Verkehrstüchtigkeit \\
\hline Fehler der Fahrzeugführer \\
\hline $10-11$ & Straßenbenutzung \\
\hline $12-13$ & Geschwindigkeit \\
\hline $14-15$ & Abstand \\
\hline $16-23$ & Überholen \\
\hline $24-25$ & Vorbeifahren \\
\hline 26 & Nebeneinanderfahren \\
\hline $27-33$ & Vorfahrt, Vorrang \\
\hline $35-37$ & Abbiegen, Wenden, Rückwärtsfahren, Ein- und Anfahren \\
\hline $38-42$ & Falsches Verhalten gegenüber Fußgängern \\
\hline $43-45$ & Ruhender Verkehr, Verkehrssicherung \\
\hline 46 & Nichtbeachten der Beleuchtungsvorschriften \\
\hline $47-48$ & Ladung, Besetzung \\
\hline 49 & Andere Fehler beim Fahrzeugführer \\
\hline $50-55$ & Technische Mängel, Wartungsmängel \\
\hline $60-69$ & Falsches Verhalten der Fußgänger \\
\hline Allgemeine Unfallursachen \\
\hline $70-79$ & Straßenverhältnisse \\
\hline $80-84$ & Witterungseinflüsse \\
\hline $85-88$ & Hindernisse \\
\hline 89 & Sonstige Ursachen \\
\hline
\end{tabular}

\subsubsection{Unfallumstände}

Als Unfallumstände werden Unfallmerkmale wie z.B. die Lichtverhältnisse, der Straßenzustand, der Aufprall auf ein Hindernis oder Alkoholeinwirkung bezeichnet. Für ausgewählte Unfallumstände liegen Werte für Deutschland vor, die die durchschnittliche Ausprägung quantifizieren. Diese können z.B. dem Merkblatt zur Örtlichen Unfalluntersuchung in Unfallkommissionen (FGSV 316/1, 2012) entnommen werden (vgl. Abbildung 9).

\begin{tabular}{|lcccl|}
\hline \multicolumn{4}{|c|}{$\begin{array}{c}\text { Ausprägung der Unfallumstände } \\
\text { (Durchschnittswerte Deutschland)*) }\end{array}$} \\
\hline & innerorts & Landstraße & BAB \\
\hline Winter & $30 \%$ & $35 \%$ & $35 \%$ & Dez - März \\
\hline Wochenende & $25 \%$ & $30 \%$ & $30 \%$ & Sa/So \\
\hline Spitzenzeiten & $45 \%$ & $45 \%$ & $45 \%$ & $6-9 / 16-19$ \\
\hline Nacht & $30 \%$ & $35 \%$ & $40 \%$ & dä/du \\
\hline Nässe/Glätte & $30 \%$ & $40 \%$ & $45 \%$ & $\mathrm{na} / \mathrm{wgl}$ \\
\hline
\end{tabular}

*) Unfallgeschehen 2007-2009

Abbildung 9: Ausprägung der Unfallumstände (Durchschnittswerte Deutschland) (Quelle MUko) 
Werden die Durchschnittswerte mit dem vorhandenen Unfallgeschehen verglichen, so kann festgestellt werden, ob es von den Erwartungswerten für Deutschland abweicht.

„Ein höherer Prozentsatz einer Unfallausprägung im Untersuchungskollektiv gegenüber dem Erwartungswert weist dieses Unfallmerkmal als Unfall begünstigend aus. Ist der Anteil für die Unfallhäufung kleiner, handelt es sich nicht um eine strukturelle Gleichartigkeit im engeren Sinn. Auch das "Nichterreichen" der Erwartungswerte kann Hinweise für die weitere Analyse geben: Treten z.B. schwere Unfälle am Knotenpunkt häufig außerhalb der Spitzenzeiten auf, ist dies ein Indiz für unangemessen hohe Geschwindigkeiten oder schlechte Erkennbarkeit." (FGSV 316/1, 2012)

\subsection{Datengrundlage}

Für die makroskopische Analyse der Unfälle in ausgewiesenen Verkehrsberuhigten Bereichen konnten folgende Datengrundlagen herangezogen werden:

- zum einen die Unfalldaten des Statistischen Bundesamtes und

- zum anderen die polizeilich registrierten Unfälle aus den Bundesländern Baden-Württemberg, Hessen, Nordrhein-Westfalen, Sachsen, Sachsen-Anhalt und Thüringen.

In den o.g. Bundesländern werden die Unfalldaten Mithilfe der Software EUSKA (Elektronische Unfalltypensteckkarte) gesammelt und ausgewertet. Die Unfalldaten werden in EUSKA mit einer digitalen Straßenkarte verknüpft. Auf diese Weise lassen sich sehr einfach Auffälligkeiten im Unfallgeschehen (Unfallhäufungen) erkennen oder auch andere Analysen und Abfragen schnell durchführen. Im Rahmen der vorliegenden Studie wurden anonyme Unfalldaten als ExcelDatensatz von den in Tabelle 3 dargestellten Bundesländern entweder direkt oder über die Unfallforschung der Versicherer (UDV) zur Verfügung gestellt.

Tabelle 3: Datengrundlage für die Unfallanalysen

\begin{tabular}{|l|l|c|l|}
\hline Bundesland & Jahre & U Kat. & Anmerkungen \\
\hline $\begin{array}{l}\text { Baden- } \\
\text { Württemberg }\end{array}$ & 2007 bis 2012 & $1-6$ & $\begin{array}{l}\text { Reduzierter EUSKA-Datensatz als Excel-Export aus- } \\
\text { schließlich für ausgewiesene Verkehrsberuhigte Bereiche }\end{array}$ \\
\hline Hessen & 2007 bis 2012 & $1-6$ & $\begin{array}{l}\text { Reduzierter EUSKA-Datensatz als Excel-Export } \\
\text { ausschließlich für ausgewiesene Verkehrsberuhigte } \\
\text { Bereiche }\end{array}$ \\
\hline $\begin{array}{l}\text { Nordrhein- } \\
\text { Westfalen }\end{array}$ & 2007 bis 2012 & $1-4$ & Excel-Datensatz aller innerörtlichen Unfälle \\
\hline Sachsen & 2006 bis 2012 & $1-6$ & $\begin{array}{l}\text { Reduzierter EUSKA-Datensatz als Excel-Export } \\
\text { ausschließlich für ausgewiesene Verkehrsberuhigte } \\
\text { Bereiche, als auch Datensatz zum Einlesen in EUSKA }\end{array}$ \\
\hline Sachsen-Anhalt & 2006 bis 2012 & $1-6$ & $\begin{array}{l}\text { Reduzierter EUSKA-Datensatz als Excel-Export } \\
\text { ausschließlich für ausgewiesene Verkehrsberuhigte } \\
\text { Bereiche, als auch Datensatz zum Einlesen in EUSKA }\end{array}$ \\
\hline Thüringen & 2007 bis 2012 & $1-6$ & $\begin{array}{l}\text { Reduzierter EUSKA-Datensatz als Excel-Export } \\
\text { ausschließlich für ausgewiesene Verkehrsberuhigte } \\
\text { Bereiche }\end{array}$ \\
\hline
\end{tabular}

Ausgewertet wurden aus diesen Datensätzen im Wesentlichen die Unfall-Kategorien 1-4 für insgesamt jeweils 6 Jahre. Die Unfall-Kategorien 5 und 6 wurden ergänzend für ein Jahr betrachtet, da sie nicht für alle Bundesländer vorlagen. 
Ein Abgleich der Datensätze der Länder mit den Angaben aus der amtlichen Statistik zeigte geringfügige Abweichungen bei der Anzahl der Unfälle pro Jahr. Da es sich allerdings um zahlenmäßig unwesentliche Abweichungen handelte und deren Ursache sich zudem nicht ermitteln ließ, wurde dieser Unterschied im Weiteren nicht gesondert berücksichtigt. Zum Thema Genauigkeit der Unfalldaten wird auf das nachfolgende Kapitel 7.3 verwiesen.

Im Rahmen von Unfallanalysen werden in der Regel Unfallkenngrößen wie z.B. die Unfallrate oder Unfallkostenraten ermittelt. Hierzu ist die Kenntnis über die Verkehrsleistung auf dem betrachteten Straßennetzabschnitt erforderlich. Angaben zu Verkehrsmengen in ausgewiesenen Verkehrsberuhigten Bereichen lagen jedoch nicht vor. Ebenso wenig lagen Angaben zur Netzlänge bzw. zum Anteil von ausgewiesenen Verkehrsberuhigten Bereichen am gesamten innerstädtischen Straßennetz vor. Damit entfiel die Möglichkeit der Einbeziehung einer relativierenden Größe bei der Analyse der Unfalldaten. Es kann lediglich davon ausgegangen werden, dass aufgrund der Charakteristik der VBB die Verkehrsbelastungen in diesen Bereichen deutlich geringer sind als im übrigen Teil des Straßennetzes.

Eine beispielhafte Auswertung der Straßennetzlänge der VBB innerorts zeigt für die Stadt Mönchengladbach eine Gesamtlänge von rd. $31 \mathrm{~km}$ bei rd. $900 \mathrm{~km}$ Gesamtstraßennetzlänge. Dies entspricht einem Anteil von etwa 3,5\%. Im Vergleich hierzu umfasst das Hauptstraßennetz der Stadt Mönchengladbach rd. $120 \mathrm{~km}$ und damit einen Anteil von etwa $13 \%$.

\subsection{Genauigkeit der Unfalldaten}

Neben den aggregierten Unfalldaten des Statistischen Bundesamtes, die keinerlei Möglichkeit einer Einzelauswertung von Unfalldaten zulassen, konnte im Rahmen der vorliegenden Studie auf anonymisierte Unfalldaten einiger Bundesländer zurückgegriffen werden.

Mit Hilfe des Merkmals „Besonderheit der Unfallstelle“ wird u.a. in der Unfallerfassung festgehalten, ob es sich bei der Unfallstelle um einen Ort innerhalb eines ausgewiesenen Verkehrsberuhigten Bereiches gemäß VZ325 StVO handelt oder nicht. Anhand dieses Vermerks werden die Unfälle in den amtlichen Statistiken dann den „Verkehrsberuhigten Bereichen“ zugeordnet. Anhand der in den EUSKA-Datensätzen vorhandenen Koordinaten der Unfallstelle wurde stichprobenartig überprüft, ob die Unfälle, die einen entsprechenden Vermerk aufwiesen, tatsächlich innerhalb eines VBB gemäß VZ325 StVO stattgefunden haben.

Bei dieser Analyse wurde festgestellt, dass das Merkmal „Besonderheit der Unfallstelle“ zum Teil fehlerhaft ausgefüllt wurde. Eine Überprüfung dieses Merkmals für die Stadt Leipzig ergab, dass lediglich 15 von 85 betrachteten Unfällen mit dem Kriterium „Verkehrsberuhigter Bereich“ tatsächlich in Verkehrsberuhigten Bereichen gemäß VZ325 StVO stattfanden. Rund die Hälfte dieser Unfälle fand in Tempo 30-Zonen statt. Darüber hinaus sind Parkplätze, Fußgängerzonen, Anliegerstraßen und Straßen mit sonstiger Beschränkung der zulässigen Höchstgeschwindigkeit als „Verkehrsberuhigter Bereich“ unter dem Merkmal „Besonderheit der Unfallstelle: Verkehrsberuhigter Bereich" gekennzeichnet worden.

Die Größenordnung der fehlerhaften Kennzeichnung der ausgewiesenen Verkehrsberuhigten Bereiche konnte nicht festgestellt werden, da keine offizielle Statistik zu Verkehrsberuhigten Bereichen gemäß VZ325 StVO und deren Lage existiert. Um dennoch eine Abschätzung der Abweichung vornehmen zu können, wurde im Rahmen der Studie auf ein Hilfsmittel zurückgegriffen. Dabei handelte es sich um den internetbasierten Kartendienst „OpenStreetMap“. Dieser bietet die Möglichkeit, dass ausgewiesene Verkehrsberuhigte Bereiche in den Karten gesondert markiert werden können. Ein entsprechendes Beispiel ist in Abbildung $\mathbf{1 0}$ dargestellt. 


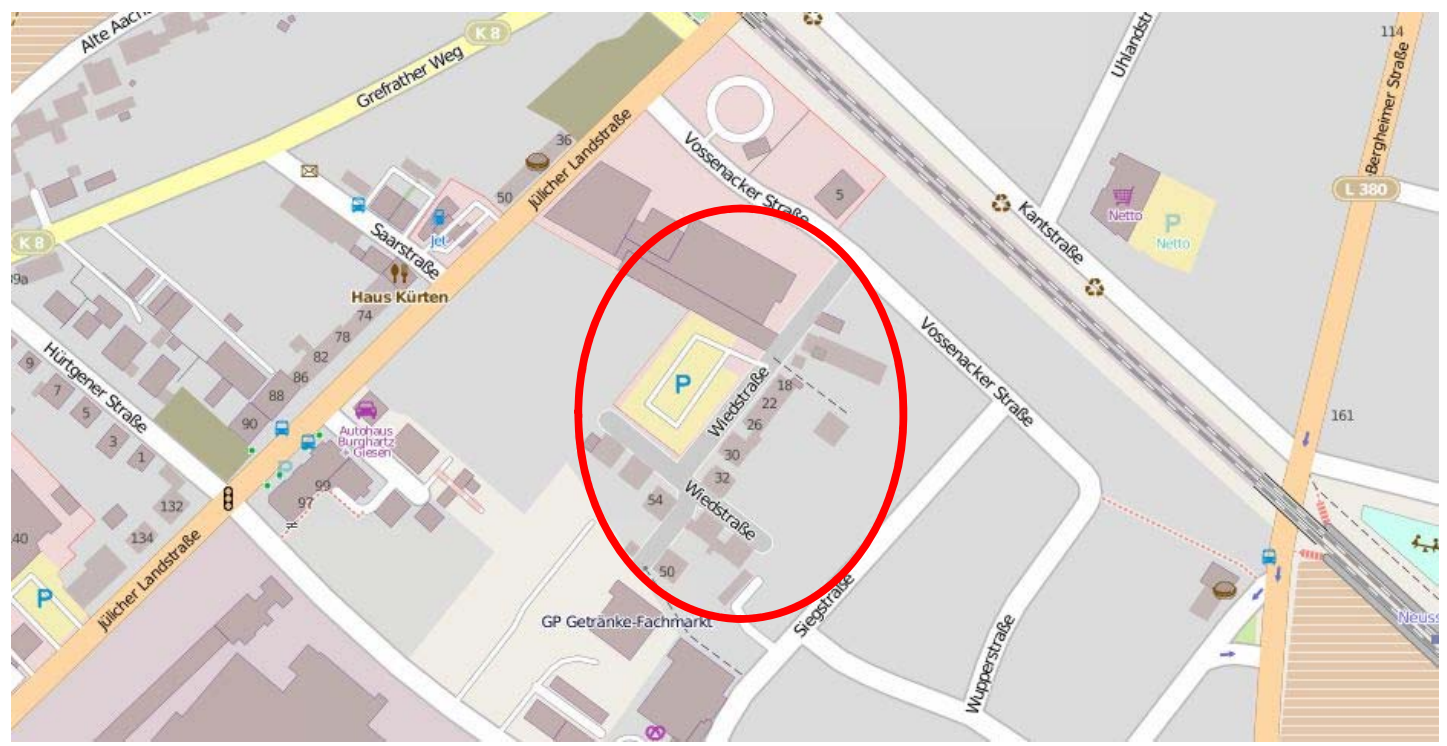

Abbildung 10: Darstellung von Verkehrsberuhigten Bereichen (grau dargestellt) im Kartendienst OpenStreetMap

Vergleiche der Angaben in OpenStreetMap wurden beispielhaft in Nordrhein-Westfalen und Hessen durchgeführt. Dazu wurde auf Grundlage der Angabe in OpenStreetMap eine Liste von ausgewiesenen Verkehrsberuhigten Bereichen in zwei Beispielstädten erstellt und mit der Situation der Straße vor Ort verglichen. Dabei konnten Übereinstimmungen von über 95\% festgestellt werden. Daher wurden für die Bundesländer Baden-Württemberg, Hessen, Sachsen-Anhalt und Thüringen beispielhaft Gegenüberstellungen der Angaben in den Unfalldatenbanken und der Information von OpenStreetMap durchgeführt. Die Ergebnisse sind in den nachfolgenden Tabellen (Tabelle 4 bis Tabelle 7) zusammengestellt.

Aus der Tabelle 4 kann z.B. für die Stadt Baden-Baden entnommen werden, dass rund 82\% der Unfälle Kat. 1-3 (9 Stück), die in den Unfalldatenbanken mit dem Merkmal „VBB“ ausgewiesen wurden, laut OpenStreetMap auch wirklich in ausgewiesenen VBB stattfanden. 18\% der Unfälle Kat. 1-3 (2 Stück) lagen laut OpenStreetMap nicht in Verkehrsberuhigten Bereichen.

Tabelle 4: $\quad$ Gegenüberstellung der Unfälle Kat. 1-3 mit dem Merkmal „VBB“ aus den Unfalldaten im Vergleich zu OpenStreetMap beispielhaft für Kommunen in Baden-Württemberg

\begin{tabular}{|l|c|c|c|c|}
\hline \multirow{2}{*}{ Stadt } & \multicolumn{2}{|c|}{ Übereinstimmung } & \multicolumn{2}{c|}{ Abweichung } \\
\cline { 2 - 5 } & Unfälle Kat. 1-3 & Anteil & Unfälle Kat. 1-3 & Anteil \\
\hline Baden-Baden & 9 & $81,8 \%$ & 2 & $18,2 \%$ \\
\hline Böblingen & 18 & $66,7 \%$ & 9 & $33,3 \%$ \\
\hline Friedrichshafen & 4 & $36,4 \%$ & 7 & $63,6 \%$ \\
\hline Heidelberg & 30 & $65,2 \%$ & 16 & $34,8 \%$ \\
\hline Ulm & 25 & $73,5 \%$ & 9 & $26,5 \%$ \\
\hline Emmendingen & 19 & $59,4 \%$ & 13 & $40,6 \%$ \\
\hline Heilbronn & 5 & $38,5 \%$ & 8 & $61,5 \%$ \\
\hline Mannheim & 9 & $42,9 \%$ & 12 & $57,1 \%$ \\
\hline Weil am Rhein & 4 & $57,1 \%$ & 3 & $42,9 \%$ \\
\hline Raststatt & 18 & $90,0 \%$ & 2 & $10,0 \%$ \\
\hline $\begin{array}{l}\text { Durchschnittswerte für } \\
\text { Baden-Württemberg }\end{array}$ & $\mathbf{1 4 1}$ & $\mathbf{6 3 , 5 \%}$ & $\mathbf{8 1}$ & $\mathbf{3 6 , 5 \%}$ \\
\hline
\end{tabular}


Tabelle 5: Gegenüberstellung der Unfälle Kat. 1-3 mit dem Merkmal „VBB“ aus den Unfalldaten im Vergleich zu OpenStreetMap beispielhaft für Kommunen in Hessen

\begin{tabular}{|l|c|c|c|c|}
\hline \multirow{2}{*}{ Stadt } & \multicolumn{2}{|c|}{ Übereinstimmung } & \multicolumn{2}{c|}{ Abweichung } \\
\cline { 2 - 5 } & Unfälle Kat. 1-3 & Anteil & Unfälle Kat. 1-3 & Anteil \\
\hline Darmstadt & $\mathbf{4}$ & $33,3 \%$ & $\mathbf{8}$ & $66,7 \%$ \\
\hline Gießen & $\mathbf{5}$ & $35,7 \%$ & $\mathbf{9}$ & $64,3 \%$ \\
\hline Limburg a. d. Lahn & $\mathbf{0}$ & $0,0 \%$ & $\mathbf{5}$ & $100,0 \%$ \\
\hline Offenbach am Main & $\mathbf{1 3}$ & $65,0 \%$ & $\mathbf{7}$ & $35,0 \%$ \\
\hline Rodgau & $\mathbf{4}$ & $33,3 \%$ & $\mathbf{8}$ & $66,7 \%$ \\
\hline Wiesbaden & $\mathbf{2}$ & $33,3 \%$ & $\mathbf{4}$ & $66,7 \%$ \\
\hline Griesheim & $\mathbf{7}$ & $77,8 \%$ & $\mathbf{2}$ & $22,2 \%$ \\
\hline Bensheim & $\mathbf{7}$ & $77,8 \%$ & $\mathbf{2}$ & $22,2 \%$ \\
\hline Dietzenbach & $\mathbf{4}$ & $66,7 \%$ & $\mathbf{2}$ & $33,3 \%$ \\
\hline Ober-Ursel & $\mathbf{5}$ & $55,6 \%$ & $\mathbf{4}$ & $44,4 \%$ \\
\hline $\begin{array}{l}\text { Durchschnittswert für } \\
\text { Hessen }\end{array}$ & $\mathbf{5 1}$ & $\mathbf{5 0 , 0 \%}$ & $\mathbf{5 1}$ & $\mathbf{5 0 , 0 \%}$ \\
\hline
\end{tabular}

Tabelle 6: $\quad$ Gegenüberstellung der Unfälle Kat. 1-3 mit dem Merkmal „VBB“ aus den Unfalldaten im Vergleich zu OpenStreetMap beispielhaft für Kommen in Sachsen-Anhalt

\begin{tabular}{|l|c|c|c|c|}
\hline \multirow{2}{*}{ Stadt } & \multicolumn{2}{|c|}{ Übereinstimmung } & \multicolumn{2}{c|}{ Abweichung } \\
\cline { 2 - 5 } & Unfälle Kat. 1-3 & Anteil & Unfälle Kat. 1-3 & Anteil \\
\hline Bernburg & 5 & $45,5 \%$ & 6 & $54,5 \%$ \\
\hline Bitterfeld-Wolfen & 4 & $44,4 \%$ & 5 & $55,6 \%$ \\
\hline Dessau & 5 & $50,0 \%$ & 5 & $50,0 \%$ \\
\hline Halle & 7 & $25,9 \%$ & 20 & $74,1 \%$ \\
\hline Sandersdorf & 12 & $92,3 \%$ & 1 & $7,7 \%$ \\
\hline Sangershausen & 0 & $0,0 \%$ & 17 & $100,0 \%$ \\
\hline Wittenberg, Lutherstadt & 1 & $7,7 \%$ & 12 & $92,3 \%$ \\
\hline Magdeburg & 6 & $25,0 \%$ & 18 & $75,0 \%$ \\
\hline Haberstadt & 2 & $25,0 \%$ & 6 & $75,0 \%$ \\
\hline Aschersleben & 0 & $0,0 \%$ & 8 & $100,0 \%$ \\
\hline $\begin{array}{l}\text { Durchschnittswerte für } \\
\text { Sachsen-Anhalt }\end{array}$ & $\mathbf{4 2}$ & $\mathbf{3 0 , 0 \%}$ & $\mathbf{9 8}$ & $\mathbf{7 0 , 0 \%}$ \\
\hline
\end{tabular}

Tabelle 7: Gegenüberstellung der Unfälle Kat. 1-3 mit dem Merkmal „VBB“ aus den Unfalldaten im Vergleich zu OpenStreetMap beispielhaft für Kommen in Thüringen

\begin{tabular}{|l|c|c|c|c|}
\hline \multirow{2}{*}{ Stadt } & \multicolumn{2}{|c|}{ Übereinstimmung } & \multicolumn{2}{c|}{ Abweichung } \\
\cline { 2 - 5 } & Unfälle Kat. 1-3 & Anteil & Unfälle Kat. 1-3 & Anteil \\
\hline Erfurt & 13 & $61,9 \%$ & 8 & $38,1 \%$ \\
\hline Sömmerda & 1 & $12,5 \%$ & 7 & $87,5 \%$ \\
\hline Weimar & 4 & $66,7 \%$ & 2 & $33,3 \%$ \\
\hline Arnstadt & 0 & $0,0 \%$ & 3 & $100,0 \%$ \\
\hline Altenburg & 0 & $0,0 \%$ & 9 & $100,0 \%$ \\
\hline Gera & 4 & $44,4 \%$ & 5 & $55,6 \%$ \\
\hline Durchschnittswerte für & $\mathbf{2 2}$ & $\mathbf{3 9 , 3 \%}$ & $\mathbf{3 4}$ & $\mathbf{6 0 , 7 \%}$ \\
\hline
\end{tabular}


Aus den beispielhaften Gegenüberstellungen von Kommunen aus vier Bundesländer wurde ersichtlich, dass - vorausgesetzt die Angaben im Kartendienst OpenStreetMap entsprechen der Realität - z.T. eine erhebliche Anzahl an Unfällen der Kategorie 1-3 (Unfälle mit Personenschaden) mit dem Merkmal ausgewiesener Verkehrsberuhigter Bereich versehen wurden, die nicht in Bereichen stattgefunden haben, die tatsächlich mit dem VZ 325 StVO als Verkehrsberuhigter Bereich ausgewiesenen waren. Die durchschnittliche Abweichung aller überprüften Unfälle der Kat. 1-3 (insg. 514 Stück) lag bei 51\% (vgl. Tabelle 8).

Da viele dieser Unfälle dennoch in Tempo 30-Zonen, auf Parkplätzen, in Fußgängerzonen, in Anliegerstraßen und Straßen mit sonstiger Beschränkung der zulässigen Höchstgeschwindigkeit stadtfanden, werden die Unfälle des Statistischen Bundesamtes und der Bundesländer die als ausgewiesener Verkehrsberuhigter Bereich unter dem Merkmal „Besonderheit der Unfallstelle“ gekennzeichnet wurden, im Folgenden als "geschwindigkeitsreduzierte Bereiche" (GRB) bezeichnet. Dadurch soll eine Verwechslung der Begrifflichkeit vermieden werden.

Tabelle 8: Gegenüberstellung aller Unfälle der Kat. 1-3 mit dem Merkmal „VBB“ aus den Unfalldaten im Vergleich zu OpenStreetMap (durchschnittliche Abweichung)

\begin{tabular}{|l|c|c|}
\hline \multirow{2}{*}{ Bundesland } & \multicolumn{2}{|c|}{ Unfälle der Kat. 1-3 } \\
\cline { 2 - 3 } & Übereinstimmung & Abweichung \\
\hline Baden-Württemberg & 141 & 81 \\
\hline Hessen & 51 & 51 \\
\hline Sachsen-Anhalt & 42 & 98 \\
\hline Thüringen & 22 & 34 \\
\hline Summe & $\mathbf{2 5 6}$ & $\mathbf{2 6 4}$ \\
\hline Anteil & $\mathbf{4 9 \%}$ & $\mathbf{5 1 \%}$ \\
\hline
\end{tabular}

\subsection{Analyse der Unfalldaten des Statistischen Bundesamtes}

Die Analyse der Unfalldaten des Statistischen Bundesamtes basierte auf den in Tabelle 9 und Tabelle 10 angegebenen Daten, die aus der Zeitreihendarstellung der Verkehrsunfälle in Deutschland entnommen wurden (Destatis, 2013). Ein Vergleich dieser Daten mit detaillierteren Datensätzen aus den Unfalldaten der Bundesländer zeigte, dass in der Statistik des Bundesamtes entgegen der Definition nicht ausschließlich ausgewiesene Verkehrsberuhigte Bereiche mit VZ325 StVO aufgenommen wurden (vgl. Kapitel 7.3). Im Folgenden wird daher für das Kollektiv des Statistischen Bundesamtes der Begriff "Geschwindigkeitsreduzierte Bereiche“ (GRB) gewählt, um diese vom eigentlichen Untersuchungsgegenstand der Verkehrsberuhigten Bereiche mit VZ325 StVO (VBB) eindeutig abzugrenzen.

Aus den Unfalldaten des Statistischen Bundesamtes konnte ausgewertet werden, dass die Anzahl der Unfälle mit Personenschaden in GRB seit dem Jahr 2000 im Mittel bei 1.500 Unfällen pro Jahr lag. Der Höchstwert bei der Anzahl der Unfälle mit Personenschaden lag in diesem Zeitraum im Jahre 2008 bei $1.643 \mathrm{U}(\mathrm{P})$ und der niedrigste Wert im Jahre 2010 bei $1.384 \mathrm{U}(\mathrm{P})$ (vgl. Tabelle 9). Die Anzahl der Verunglückten sind in Tabelle 10 festgehalten. Ab dem Jahr 2000 existierte die höchste Anzahl auch im Jahr 2008 (1.786 Verunglückte), die niedrigste Anzahl im Jahr 2010 (1.499 Verunglückte). In Abbildung $\mathbf{1 1}$ ist der Verlauf der Unfälle mit Personenschaden und der dabei Verunglückten über den Zeitraum von 1995 bis 2012 dargestellt.

Aus der Entwicklung der absoluten Anzahl der Unfälle mit Personenschaden in GRB ließen sich generell auf Bundesebene folgende Punkte feststellen:

- Bis zum Jahr 2000 ist insgesamt ein rückläufiger Trend der Unfallzahlen in GRB zu erkennen. Besonders stabil zeigt sich dieser Trend bei der Anzahl der schwerverletzten Verkehrsteilnehmer. 
- Ab dem Jahr 2001 stagnieren die Unfallzahlen in GRB. Die Gesamtzahl der Unfälle mit Personenschaden in GRB pendelt um den Wert von 1.500 Unfälle pro Jahr. Die Zahl der schwerverletzten Verkehrsteilnehmer schwankt seit 2001 zwischen 220 und 240 Verunglückten pro Jahr.

Im Vergleichszeitraum von 1995 bis 2012 ließen sich bei Straßenverkehrsunfällen der UnfallKategorien 1-4 innerorts folgende Entwicklungen erkennen (vgl. Abbildung 12):

- Im Zeitraum 1995 bis 2000 ist eine Stagnation der Unfälle mit Personenschaden festzustellen. Hier liegt die Anzahl der Unfälle im Bereich zwischen 240.208 und 252.087 $\mathrm{U}(\mathrm{P})$ pro Jahr.

- Ab dem Jahr 2001 ist ein stabiler Rückgang der Unfallzahlen bei den U(P) bis zum Jahr $2010 \mathrm{zu}$ verzeichnen. Im Jahr 2010 wurden innerorts insgesamt weniger als 200.000 Unfälle mit Personenschaden registriert. Seitdem ist wieder eine Stagnation der Unfallzahlen zu erkennen.

Tabelle 9: $\quad$ Unfälle mit Personenschaden und schwerwiegende Unfälle mit Sachschaden in GRB im Zeitraum 1995-2012 (Quelle: Destatis, 2013)

\begin{tabular}{|c|c|c|c|c|c|}
\hline \multirow{2}{*}{ Jahr } & \multicolumn{4}{|c|}{ Unfälle mit Personenschaden } & \multirow{2}{*}{$\begin{array}{c}\text { Schwerwiegende } \\
\text { Unfälle mit } \\
\text { Sachschaden }\end{array}$} \\
\cline { 2 - 5 } & $\begin{array}{c}\text { mit } \\
\text { Getöteten }\end{array}$ & $\begin{array}{c}\text { mit Schwer- } \\
\text { verletzten }\end{array}$ & $\begin{array}{c}\text { mit Leicht- } \\
\text { verletzten }\end{array}$ & Summe & 430 \\
\hline 1995 & 10 & 460 & 1.583 & 2.053 & 317 \\
\hline 1996 & 2 & 320 & 1.404 & 1.726 & 266 \\
\hline 1997 & 8 & 382 & 1.477 & 1.867 & 231 \\
\hline 1998 & 4 & 318 & 1.310 & 1.632 & 213 \\
\hline 1999 & 8 & 308 & 1.428 & 1.744 & 179 \\
\hline 2000 & 4 & 255 & 1.265 & 1.524 & 173 \\
\hline 2001 & 6 & 237 & 1.311 & 1.554 & 205 \\
\hline 2002 & 3 & 232 & 1.230 & 1.465 & 201 \\
\hline 2003 & 4 & 221 & 1.284 & 1.509 & 179 \\
\hline 2004 & 2 & 217 & 1.269 & 1.488 & 167 \\
\hline 2005 & 4 & 238 & 1.291 & 1.533 & 176 \\
\hline 2006 & 4 & 210 & 1.243 & 1.457 & 194 \\
\hline 2007 & 4 & 229 & 1.296 & 1.529 & 188 \\
\hline 2008 & 5 & 229 & 1.409 & 1.643 & 188 \\
\hline 2009 & 3 & 232 & 1.353 & 1.588 & 171 \\
\hline 2010 & 3 & 215 & 1.166 & 1.384 & 156 \\
\hline 2011 & 4 & 243 & 1.279 & 1.526 & 181 \\
\hline 2012 & 1 & 216 & 1.276 & 1.493 & \\
\hline
\end{tabular}


Tabelle 10: Verunglückte in GRB im Zeitraum 1995-2012 (Quelle: Destatis, 2013)

\begin{tabular}{|c|c|c|c|c|}
\hline \multirow{2}{*}{ Jahr } & \multicolumn{4}{|c|}{ Verunglückte } \\
\cline { 2 - 5 } & Getötete & Schwerverletzte & Leichtverletzte & Gesamt \\
\hline 1995 & 10 & 476 & 1.803 & 2.289 \\
\hline 1996 & 2 & 331 & 1.566 & 1.899 \\
\hline 1997 & 8 & 394 & 1.657 & 2.059 \\
\hline 1998 & 4 & 331 & 1.462 & 1.797 \\
\hline 1999 & 8 & 315 & 1.593 & 1.916 \\
\hline 2000 & 4 & 263 & 1.401 & 1.668 \\
\hline 2001 & 6 & 241 & 1.469 & 1.716 \\
\hline 2002 & 3 & 239 & 1.361 & 1.603 \\
\hline 2003 & 4 & 227 & 1.422 & 1.653 \\
\hline 2004 & 2 & 222 & 1.404 & 1.628 \\
\hline 2005 & 4 & 242 & 1.405 & 1.651 \\
\hline 2006 & 4 & 216 & 1.354 & 1.574 \\
\hline 2007 & 4 & 235 & 1.437 & 1.676 \\
\hline 2008 & 5 & 234 & 1.547 & 1.786 \\
\hline 2009 & 3 & 241 & 1.488 & 1.732 \\
\hline 2010 & 6 & 220 & 1.273 & 1.499 \\
\hline 2011 & 4 & 249 & 1.396 & 1.649 \\
\hline 2012 & 1 & 219 & 1.390 & 1.610 \\
\hline
\end{tabular}

Entwicklung Unfallzahlen (Bundesweit) in GRB Anzahl der Unfälle mit Personenschaden und Anzahl der Verunglückten

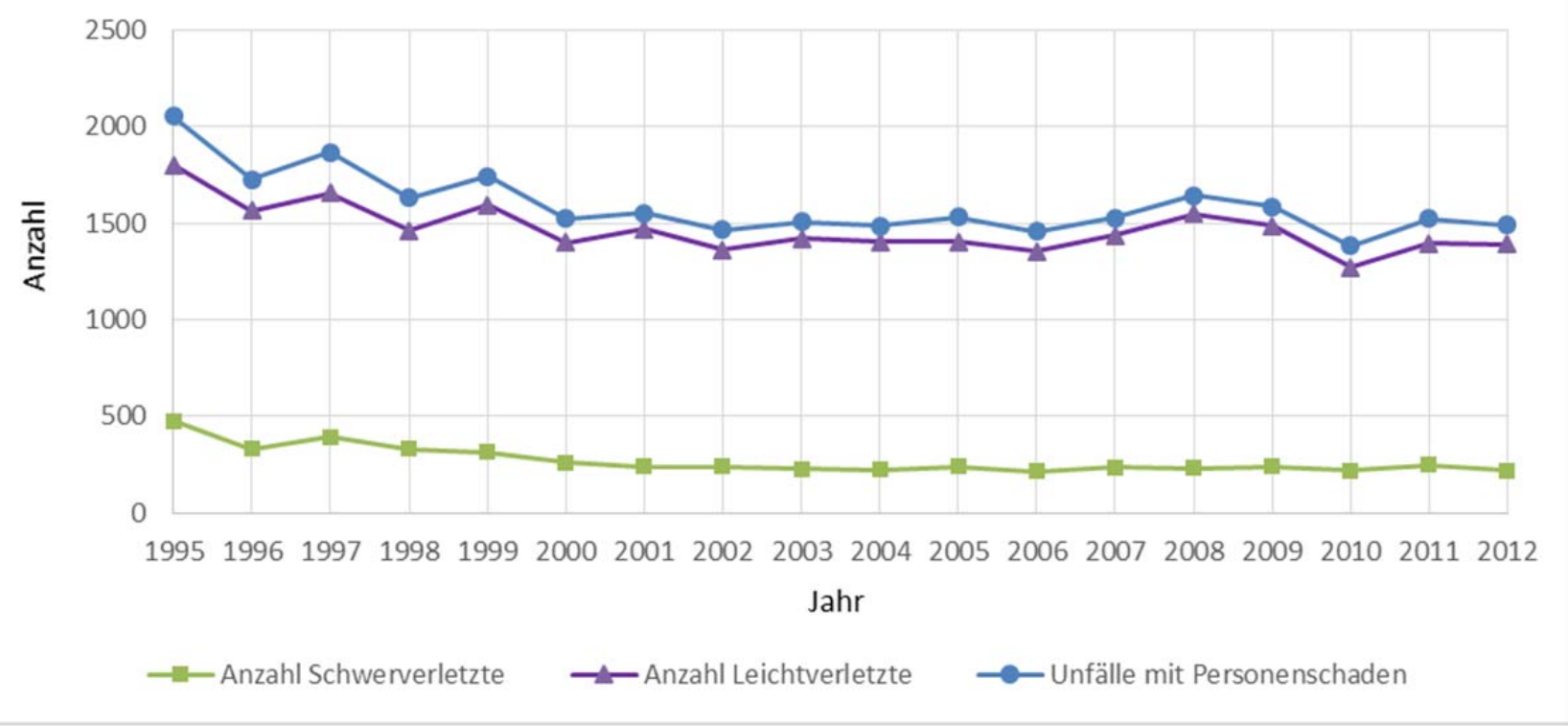

Abbildung 11: Entwicklung der Anzahl der Unfälle mit Personenschaden und der dabei Verunglückten in GRB im Zeitraum 1995 bis 2012 


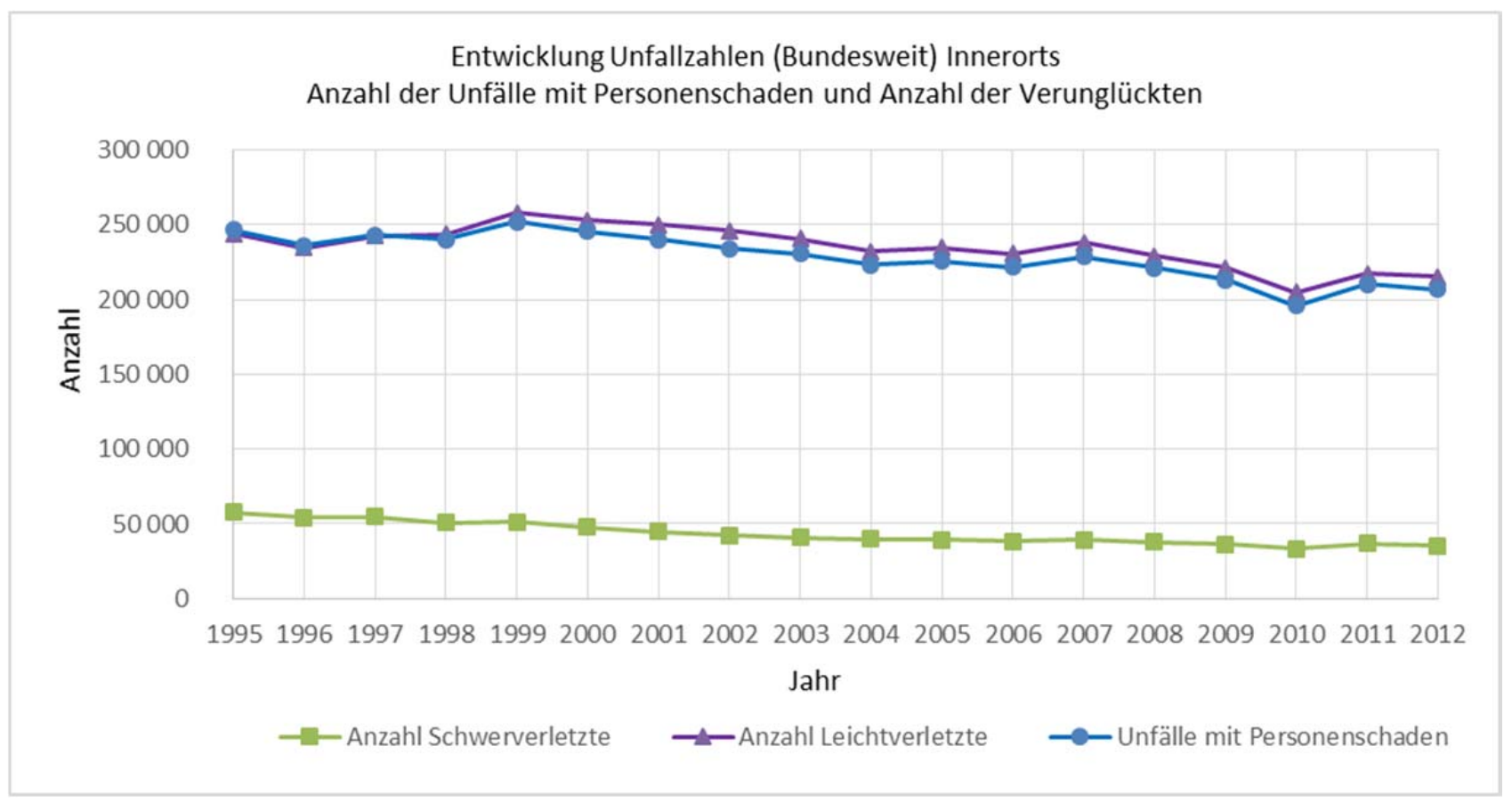

Abbildung 12: Entwicklung der Anzahl der Unfälle mit Personenschaden und der dabei Verunglückten Innerorts im Zeitraum 1995 bis 2012

Es ließ sich somit ein entgegengesetzter Trend bei der Entwicklung der Anzahl der Unfälle mit Personenschaden innerorts insgesamt und in den geschwindigkeitsreduzierten Bereichen feststellen. Während die Unfallzahlen im Zeitraum 1995 bis 2000/01 in den GRB rückläufig waren, waren insgesamt innerorts stagnierende Unfallzahlen bei den $U(P)$ zu verzeichnen. Ab dem Jahr 2001 sind die Unfallzahlen bei den $U(P)$ insgesamt innerorts rückläufig gewesen und in den GRB stagnierten diese.

Eine Ursache für diese gegenläufige Entwicklung kann nur vermutet werden. So ist beispielsweise das öffentliche Interesse an GRB seit dem Jahr 2000 wieder gestiegen, was eine Zunahme der Straßennetzlänge bei den GRB zur Folge gehabt haben könnte, was sich jedoch aufgrund der fehlenden statistischen Datengrundlagen nicht belegen lässt.

Die gegenläufige Entwicklung war auch bei der Entwicklung des prozentualen Anteils der Unfälle mit Personenschaden in GRB am gesamten Unfallgeschehen innerorts zu erkennen. So nahm dieser bis zum Jahr 2001 zunächst ab und anschließend kontinuierlich wieder zu (Abbildung 13). Insgesamt lag dieser Anteil jedoch mit weniger als 0,8\% sehr niedrig. Da keine Angaben zur Fahrleistung in den GRB und im weiteren innerstädtischen Straßennetz vorliegen, konnte dieser geringe Anteil nicht abschließend bewertet werden. Es kann zwar davon ausgegangen werden, dass der Anteil der Straßennetzlänge der GRB oberhalb von 0,8\% liegt (vgl. Beispiel Mönchengladbach), für das Unfallgeschehen ist jedoch die Fahrleistung relevant. 


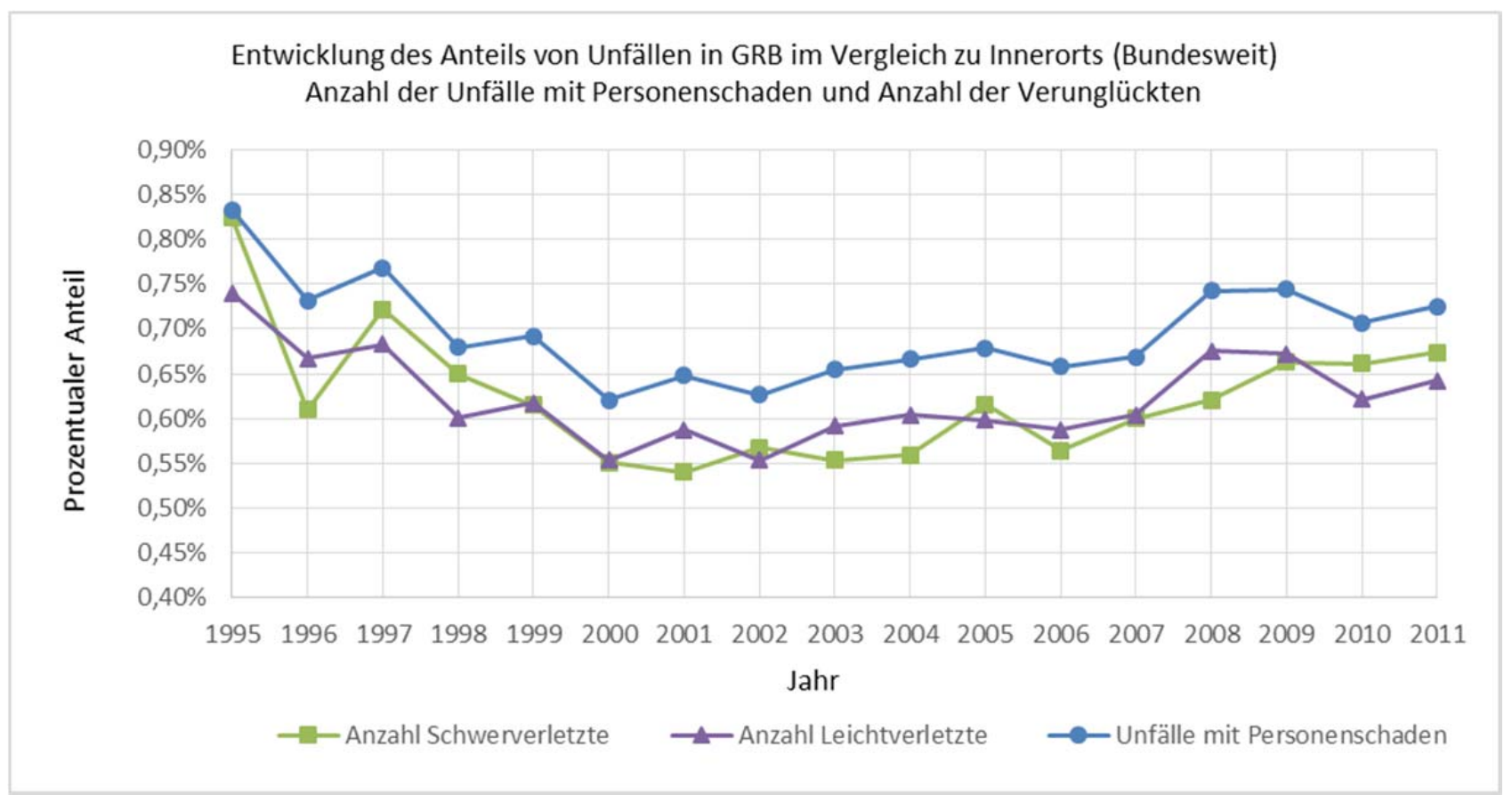

Abbildung 13: Entwicklung des prozentualen Anteils von Unfällen mit Personenschaden und der Zahl der Verunglückten in GRB am Unfallgeschehen mit Personenschaden innerorts gesamt

Betrachtet wurde auch die Anzahl der Unfälle mit Personenschaden in den einzelnen Bundesländern untereinander über den Zeitverlauf der Jahre 2007 bis 2011. Es konnte erkannt werden, dass sich das Unfallgeschehen in den betrachteten fünf Jahren sehr unterschiedlich entwickelte. Während sich in den meisten Bundesländern eine eher schwankende Anzahl an Unfällen mit Personenschaden über den Zeitraum 2007 bis 2011 herausstellte, war für den Stadtstaat Berlin eine kontinuierliche Abnahme der Unfallzahlen bei den Unfällen mit Personenschaden im Zeitraum 2007 bis 2010 und eine Stagnation im Zeitraum 2010 bis 2011 zu verzeichnen. Dementgegen steht die Entwicklung der Anzahl der Unfälle mit Personenschaden in Baden-Württemberg. Hier war eine kontinuierliche Zunahme der Anzahl der Unfälle mit Personenschaden im Zeitraum 2007 bis 2011 zu verzeichnen (vgl. Tabelle 11 und Abbildung 14). 
Tabelle 11: Unfallzahlen der Unfälle mit Personenschaden in GRB Unterschieden nach Bundesländern im Zeitraum 2007 bis 2011 (Quelle: Destatis 2013)

\begin{tabular}{|l|c|c|c|c|c|}
\hline Land & $\mathbf{2 0 0 7}$ & $\mathbf{2 0 0 8}$ & $\mathbf{2 0 0 9}$ & $\mathbf{2 0 1 0}$ & $\mathbf{2 0 1 1}$ \\
\hline Baden-Württemberg & 122 & 167 & 174 & 191 & 240 \\
\hline Bayern & 146 & 168 & 165 & 134 & 162 \\
\hline Berlin & 102 & 97 & 82 & 68 & 69 \\
\hline Brandenburg & 41 & 42 & 46 & 33 & 34 \\
\hline Bremen & 16 & 24 & 16 & 10 & 5 \\
\hline Hamburg & 11 & 11 & 19 & 12 & 9 \\
\hline Hessen & 81 & 79 & 92 & 92 & 63 \\
\hline Mecklenburg-Vorpommern & 42 & 41 & 46 & 29 & 41 \\
\hline Niedersachsen & 133 & 185 & 170 & 154 & 156 \\
\hline Nordrhein-Westfalen & 411 & 437 & 430 & 346 & 409 \\
\hline Rheinland-Pfalz & 111 & 99 & 85 & 84 & 95 \\
\hline Saarland & 26 & 23 & 16 & 5 & 20 \\
\hline Sachsen & 108 & 96 & 87 & 66 & 77 \\
\hline Sachsen-Anhalt & 44 & 44 & 40 & 33 & 39 \\
\hline Schleswig-Holstein & 113 & 99 & 95 & 107 & 81 \\
\hline Thüringen & 22 & 31 & 25 & 20 & 26 \\
\hline
\end{tabular}

Entwicklung der Unfälle mit Personenschaden in GBR in (Flächen-)Bundesländern (Index)

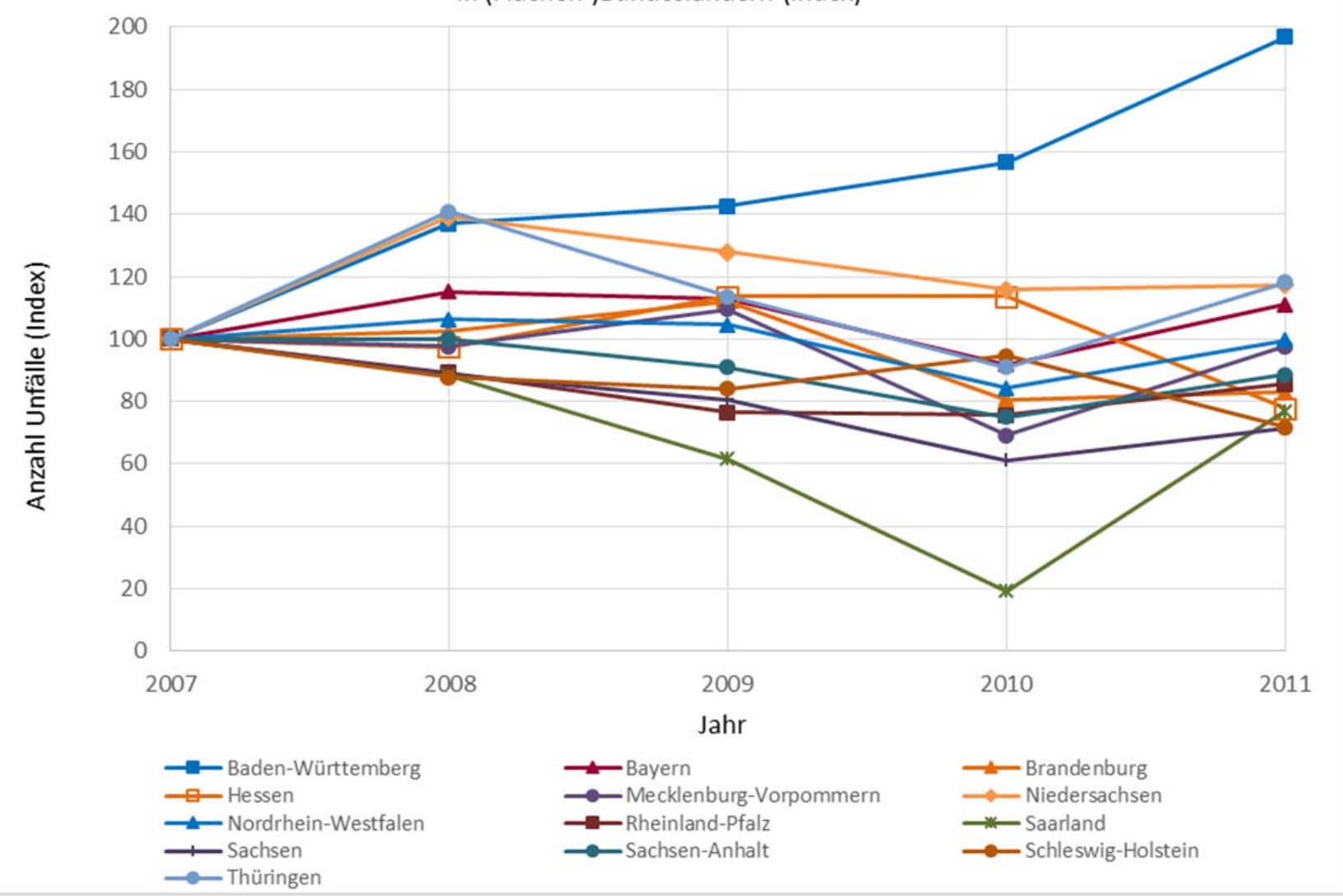

Abbildung 14: Entwicklung Anzahl der Unfälle mit Personenschaden in GRB im Zeitraum 2007 bis 2011, unterschieden nach (Flächen-)Bundesländern (Indexwert 100 für Jahr 2007) 


\subsection{Analyse der Unfalldaten in den Bundesländer}

Die nachfolgenden Analysen der Unfalldaten aus den Ländern (vgl. Tabelle 3) umfassten alle Unfälle der Kategorie 1-4, die als Unfälle in Verkehrsberuhigten Bereichen gekennzeichnet waren. Es handelte sich hiermit, wie bereits erläutert, um die Unfälle, die in sogenannten geschwindigkeitsreduzierten Bereichen (GRB) erfolgten.

\subsubsection{Analyse der Unfalldaten aus Baden-Württemberg}

Die auffälligste Entwicklung der Unfallanzahl (Kategorie 1-4) für die Jahre 2007 bis 2011 im Vergleich der Bundesländer wiesen die Unfallzahlen in Baden-Württemberg auf. Hier war für die GRB ein kontinuierlicher Anstieg der Unfallzahlen bei den Unfällen mit Personenschaden (Unfälle der Kategorie 1-3) im betrachteten Zeitraum zu verzeichnen. Auffallend war hierbei zusätzlich neben einem stabilen Trend in den Jahren 2007 bis 2011, dass im gleichen Zeitraum die Unfälle mit Personenschaden insgesamt in Baden-Württemberg um rd. 10\% von $40.634 \mathrm{U}(\mathrm{P})$ im Jahre 2007 auf $36.531 \mathrm{U}(\mathrm{P})$ im Jahre 2011 abgenommen haben (Destatis 2013).

Es wurde deutlich, dass der Anstieg der Unfallzahlen bei den $U(P)$ ausschließlich durch eine Zunahme der Unfälle der Kategorie 3 erfolgte. Die Unfallzahlen der Kategorie 2 waren hingegen schwankend und erfreulicherweise kam in fünf Jahren kein Unfall der Kategorie 1 vor. Auch bei den Unfällen der Kategorie 4, konnte in den GRB kein Trend erkannt werden (Abbildung 15). Sie spielten aber mit einem Anteil von etwa 10\% (122U(SS)) am betrachteten Unfallgeschehen nur eine untergeordnete Rolle.

Im Jahr 2012 war in Baden-Württemberg wieder eine Abnahme der Unfallzahlen der Kategorie 3 zu verzeichnen. Mit 169 Unfällen der Kategorie 3 wurde 2012 wieder in etwa das Niveau des Jahres 2010 erreicht.

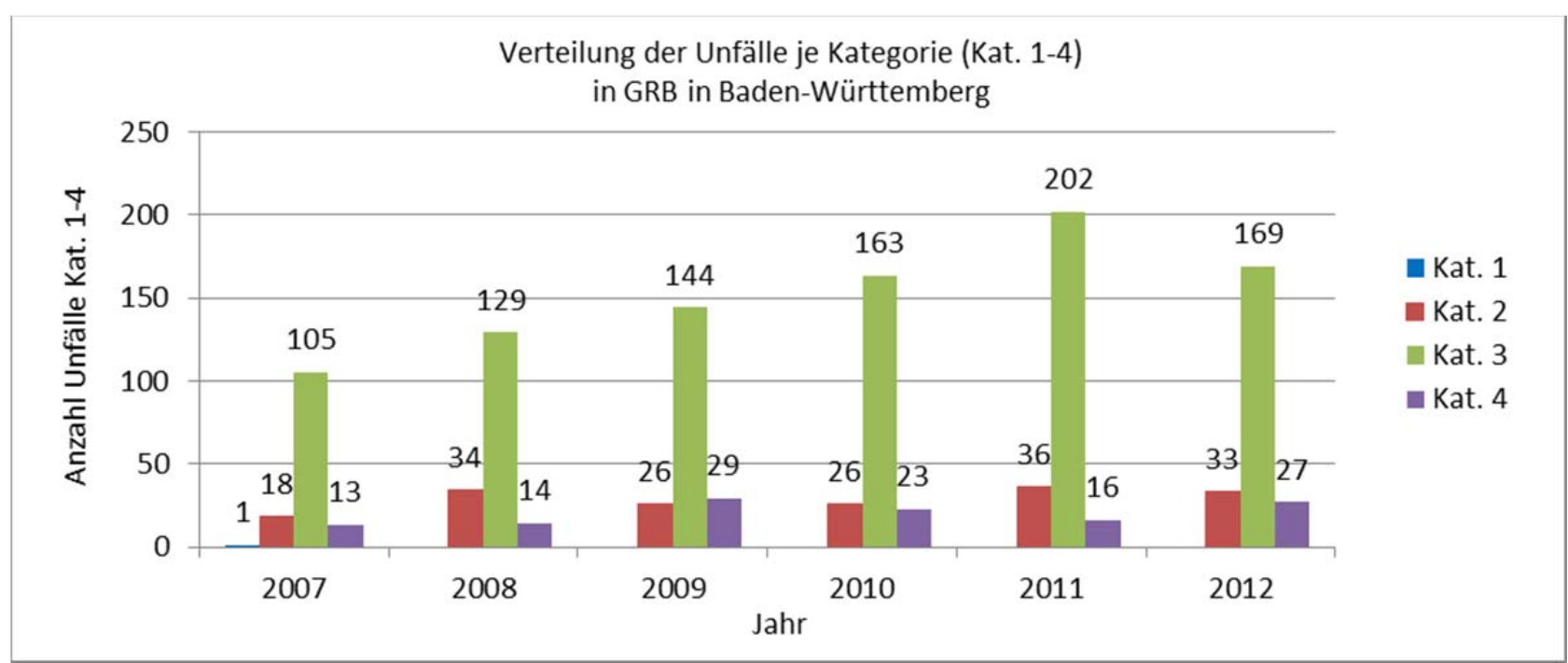

Abbildung 15: Verteilung der Unfälle je Kategorie (Kat. 1-4) in GRB in Baden-Württemberg im Zeitraum 2007 bis 2012 (Angabe der Anzahl der Unfälle in Absolutzahlen; Unfälle Kat. 1-4 gesamt: 1.208)

Eine Analyse der Unfalltypen der Unfälle in GRB der Kategorie 1-4 zeigte, dass in BadenWürttemberg der Hauptunfalltyp der Unfalltyp 3 (Einbiegen/Kreuzen-Unfall) war. Knapp 3 von 10 Unfällen wurden diesem Typ zugeordnet. Einen Anteil von rund $25 \%$ machte der Unfalltyp 7 (Sonstiger Unfall) aus. Dieser Unfalltyp umfasste insbesondere auch die Unfälle im ruhenden Verkehr (Abbildung 16). 


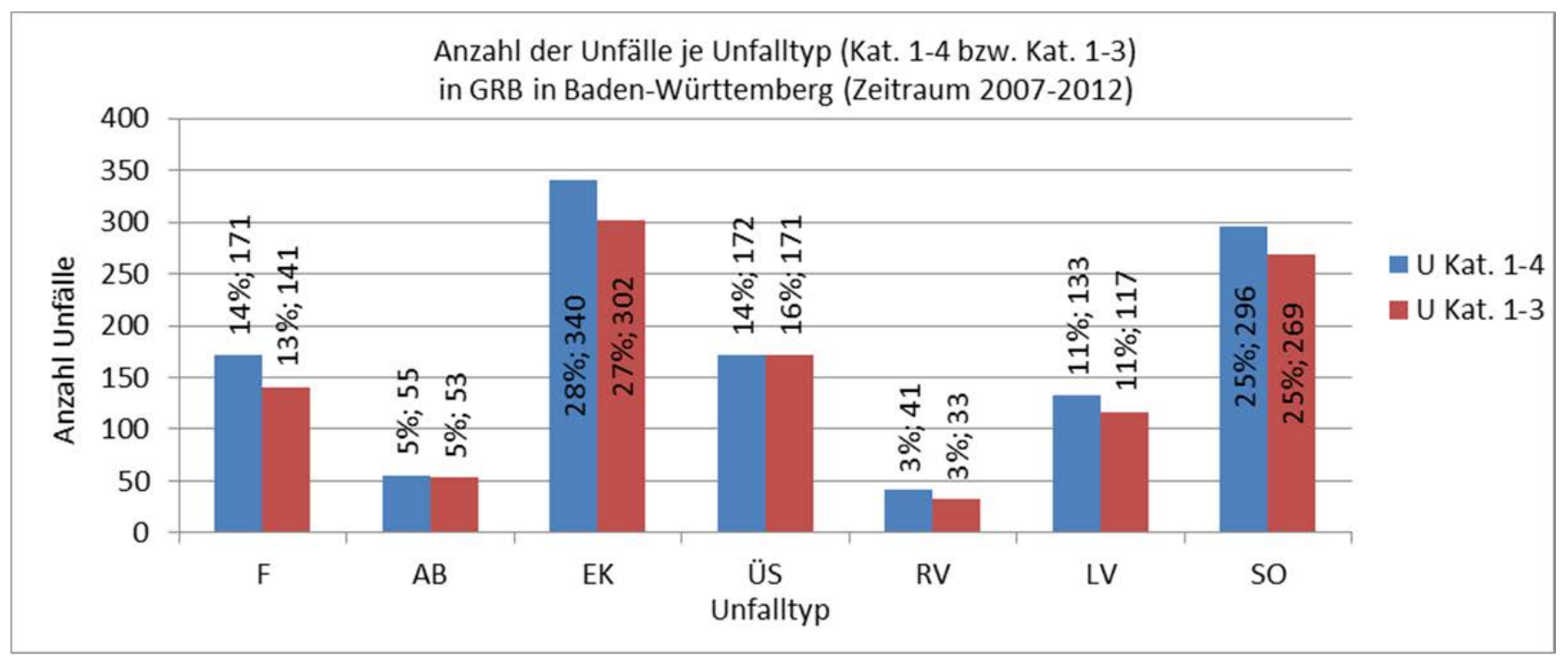

Abbildung 16: Verteilung der Unfälle der Kat. 1-4 bzw. Kat. 1-3 je Unfalltyp in GRB in Baden-Württemberg im Zeitraum 2007 bis 2012 (Angabe des prozentualen Anteils des jeweiligen Unfalltyps an der Gesamtzahl der Unfälle und Angabe der Anzahl der Unfälle in Absolutzahlen; Unfälle Kat. 1-4 gesamt: 1.208; Unfälle Kat. 1-3 gesamt: 1.086)

Verglichen wurden zudem die Verteilung der Unfalltypen in den GRB in Baden-Württemberg im Zeitraum 2007 bis 2012 mit der Verteilung der Unfalltypen der Unfälle der Kat. 1-4 innerorts im gesamten Bundesgebiet für das Jahr 2011. Es fiel auf, dass zwar auch hier der Unfalltyp 3 mit knapp 30\% den Hauptunfalltyp darstellte, der Unfalltyp 7 jedoch einen nur etwa halb so großen Anteil ausmachte (Abbildung 17). Ebenso wurde deutlich, dass der Unfalltyp 2 (Abbiege-Unfall) innerorts insgesamt etwa dreimal so häufig auftrat, wie in den GRB, wo dieser nur einen Anteil von etwa $5 \%$ aufwies.

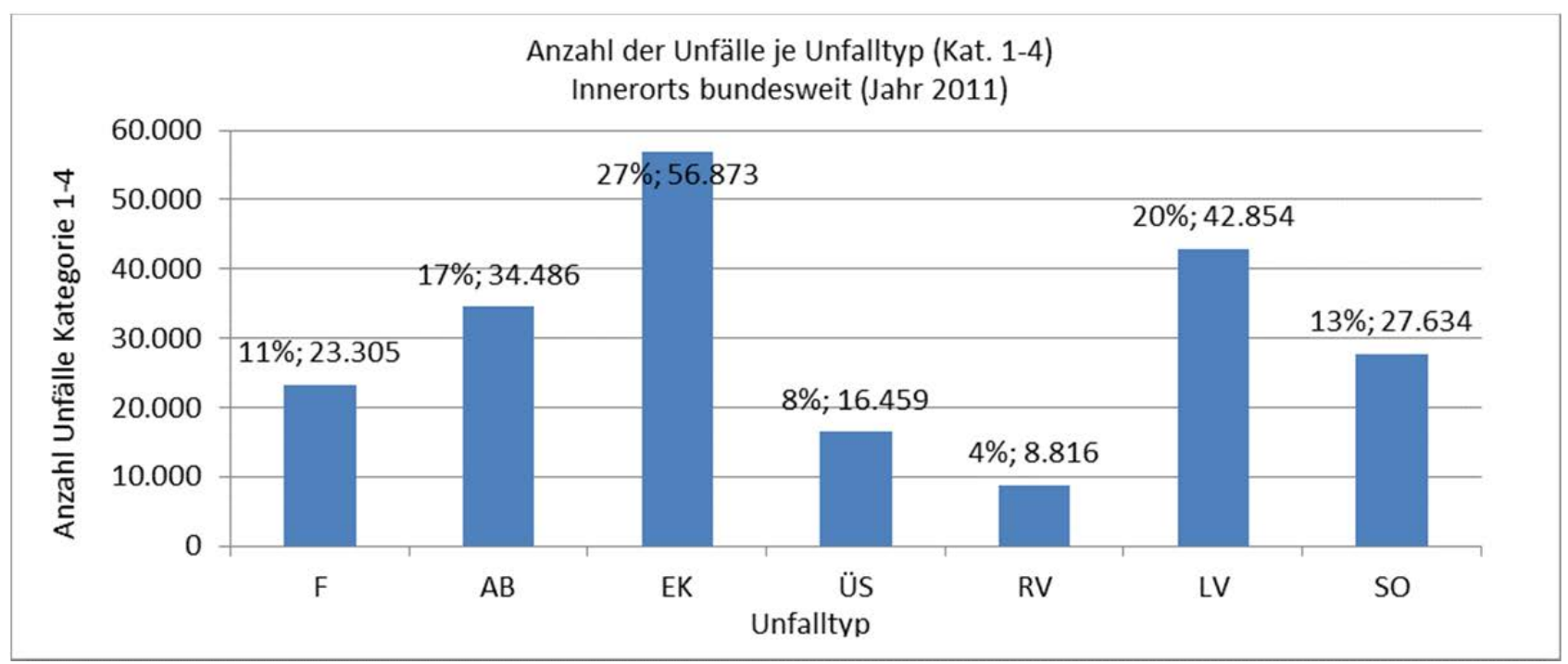

Abbildung 17: Verteilung der Unfälle der Kat. 1-4 je Unfalltyp Innerorts bundesweit im Jahr 2011 (Angabe des prozentualen Anteils des jeweiligen Unfalltyps an der Gesamtzahl der Unfälle und Angabe der Anzahl der Unfälle in Absolutzahlen; Unfälle Kat. 1-4 gesamt: 210.427)

Bei der Betrachtung der Beteiligung nach Verkehrsarten bei den Unfällen in GRB, war zu erkennen, dass etwas mehr als zwei Drittel aller Unfälle zwischen motorisierten (MV) und nichtmotorisierten 
Verkehrsteilnehmern (NMV) stattfanden. An rund einem Fünftel der Unfälle waren ausschließlich motorisierte Verkehrsteilnehmer beteiligt. Die restlichen Unfälle fanden zwischen Fußgängern und Radfahrern bzw. Radfahrern und Radfahrern statt (Abbildung 18).

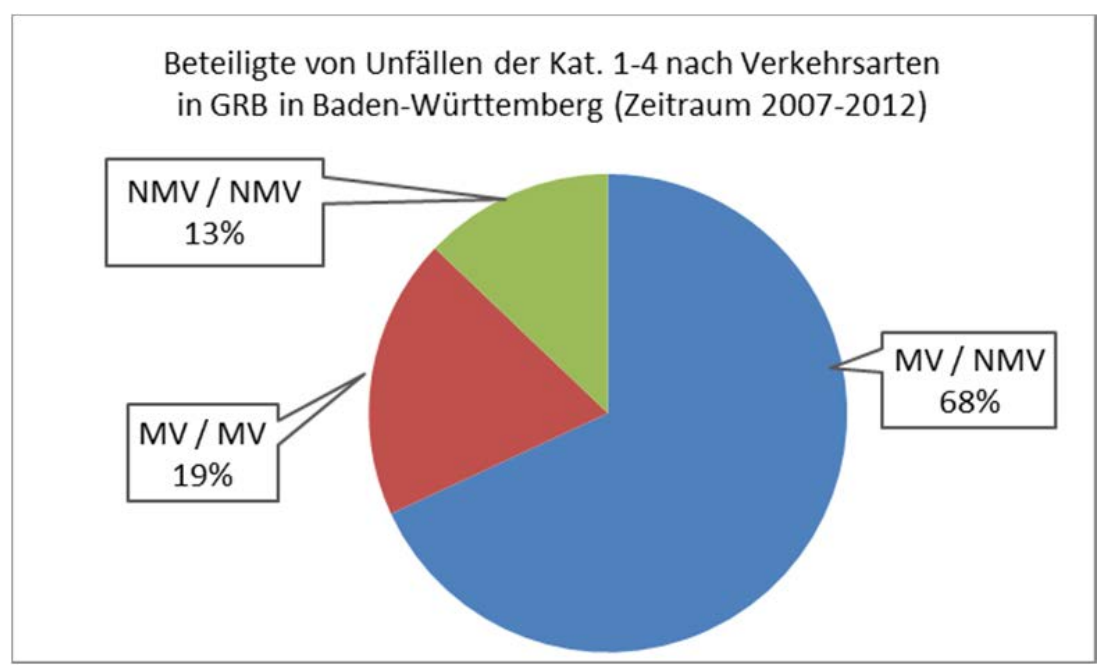

Abbildung 18: Beteiligte von Unfällen der Kat. 1-4 nach Verkehrsarten in GRB in Baden-Württemberg im Zeitraum 2007 bis 2012 (Angabe des Anteils der Beteiligung nach Verkehrsart an der Gesamtzahl an Unfällen; Unfälle Kat. 1-4 gesamt: $1.025^{5}$ )

\subsubsection{Analyse der Unfalldaten Hessen}

Die Verteilung der Unfälle je Kategorie in GRB in Hessen in den Jahren 2007 bis 2012 zeigte ein ähnliches Bild auf wie die Verteilung in Baden-Württemberg. Auch hier dominierten die Unfälle der Kategorie 3 und alle Unfallkategorien wiesen unregelmäßige Schwankungen auf (Abbildung 19).

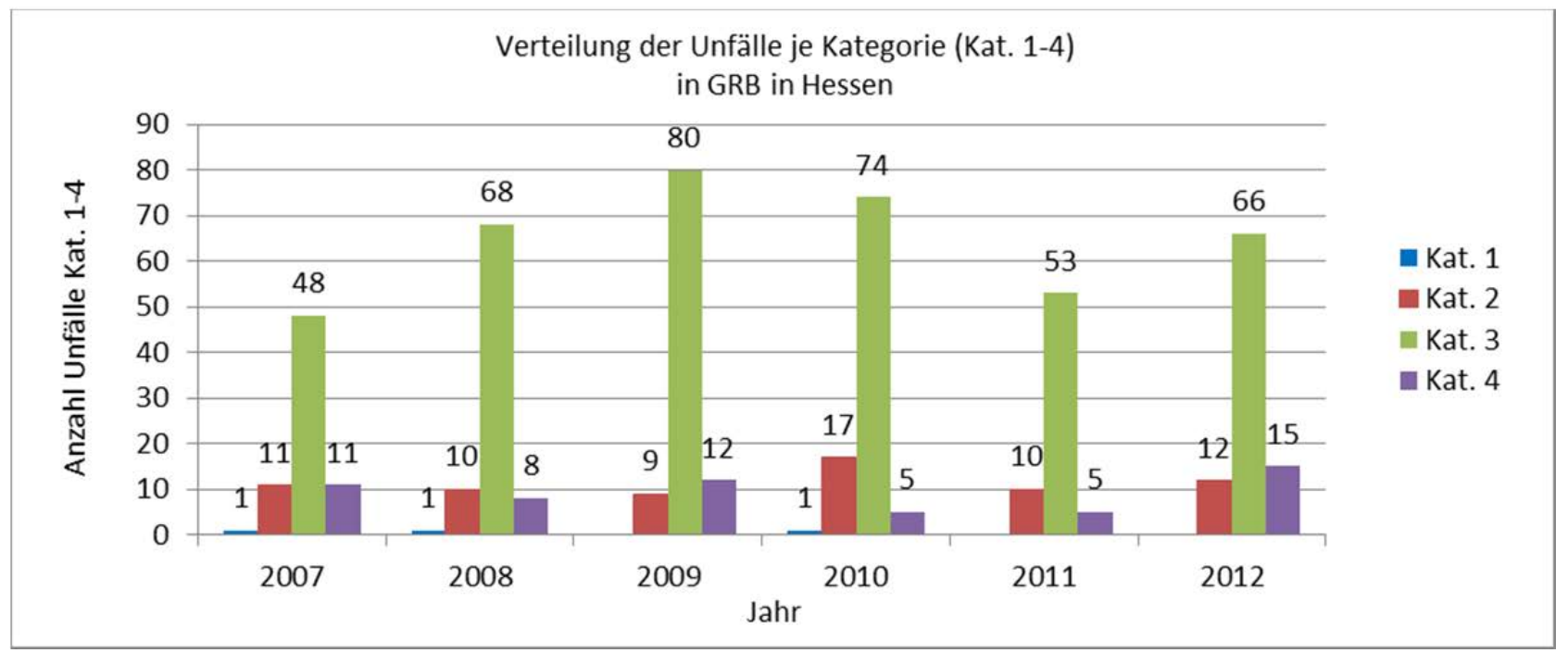

Abbildung 19: Verteilung der Unfälle je Kategorie (Kat. 1-4) in GRB in Hessen im Zeitraum 2007 bis 2012 (Angabe der Anzahl der Unfälle in Absolutzahlen; Unfälle Kat. 1-4 gesamt: 517)

5 In den Unfalldaten der Länder lagen nicht immer vollständige Angaben zu allen Unfallmerkmalen vor, so dass bei den Auswertungen häufig auf unterschiedliche Stichprobengrößen zurückgegriffen wurde. Daher kommt es vor, dass die Gesamtanzahl der Unfälle je Auswertung variiert. 
Auch die Verteilung der Unfalltypen der Unfälle der Kategorie 1-4 in GRB in Hessen in den Jahren 2007 bis 2012 zeigt wieder deutlich, dass die vorherrschenden Unfalltypen die Unfalltypen 3 und 7 sind. Diesen beiden Unfalltypen sind in GRB über die Hälfte alle Unfälle zuzuordnen (Abbildung 20).

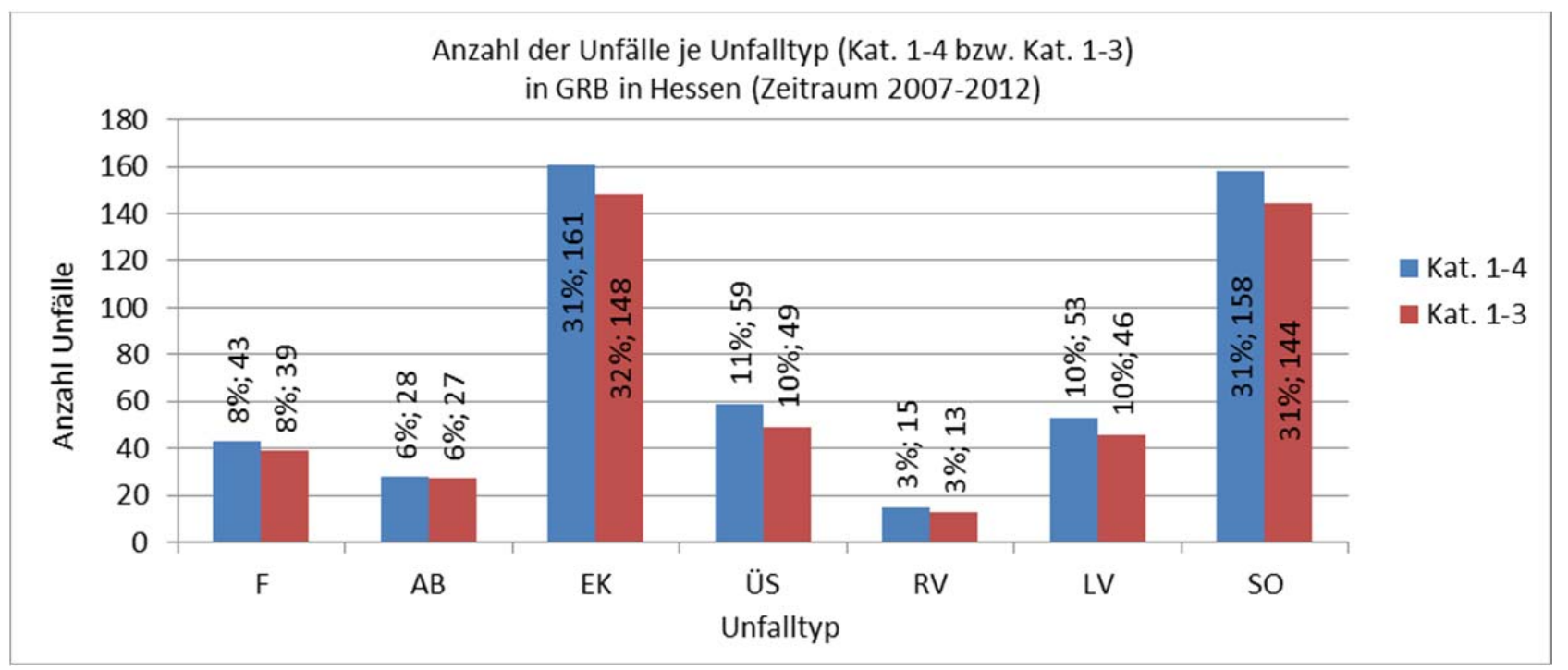

Abbildung 20: Verteilung der Unfälle der Kat. 1-4 bzw. Kat. 1-3 je Unfalltyp in GRB in Hessen im Zeitraum 2007 bis 2012 (Angabe des prozentualen Anteils des jeweiligen Unfalltyps an der Gesamtzahl der Unfälle und Angabe der Anzahl der Unfälle in Absolutzahlen; Unfälle Kat. 1-4 gesamt: 517; Unfälle Kat. 1-3 gesamt: 466)

Bei der Verteilung der Unfälle nach der Beteiligung der Verkehrsarten bestätigt sich auch bei den Unfällen in Hessen für GRB der Trend aus Baden-Württemberg. Knapp 20\% der Unfälle fanden zwischen motorisierten Verkehrsteilnehmern statt, während der Großteil der Unfälle zwischen MV und NMV stattfanden (Abbildung 21).

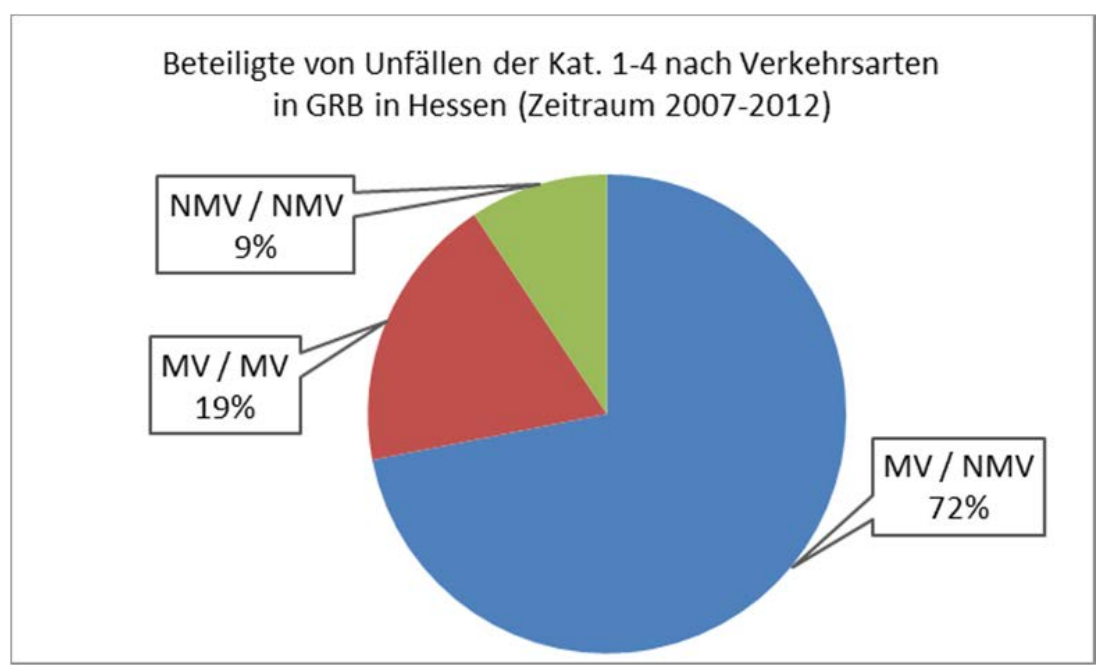

Abbildung 21: Beteiligte von Unfällen der Kat. 1-4 nach Verkehrsarten in GRB in Hessen im Zeitraum 2007 bis 2012 (Angabe des Anteils der Beteiligung nach Verkehrsart an der Gesamtzahl an Unfällen; Unfälle Kat. 1-4 gesamt: 438) 


\subsubsection{Analyse der Unfalldaten Nordrhein-Westfalen}

Bei der Verteilung der Unfälle in geschwindigkeitsreduzierten Bereichen in Nordrhein-Westfalen (NRW) nach Unfallkategorien ergab sich ein ähnliches Bild zu den übrigen betrachteten Bundesländern. Hier dominierten ebenso die Unfälle der Kategorie 3 und die Unfälle der Kategorie 4 lagen in etwa auf dem Niveau der Unfälle der Kategorie 2. Unfälle der Kategorie 1 waren nur wenige zu verzeichnen (Abbildung 22).

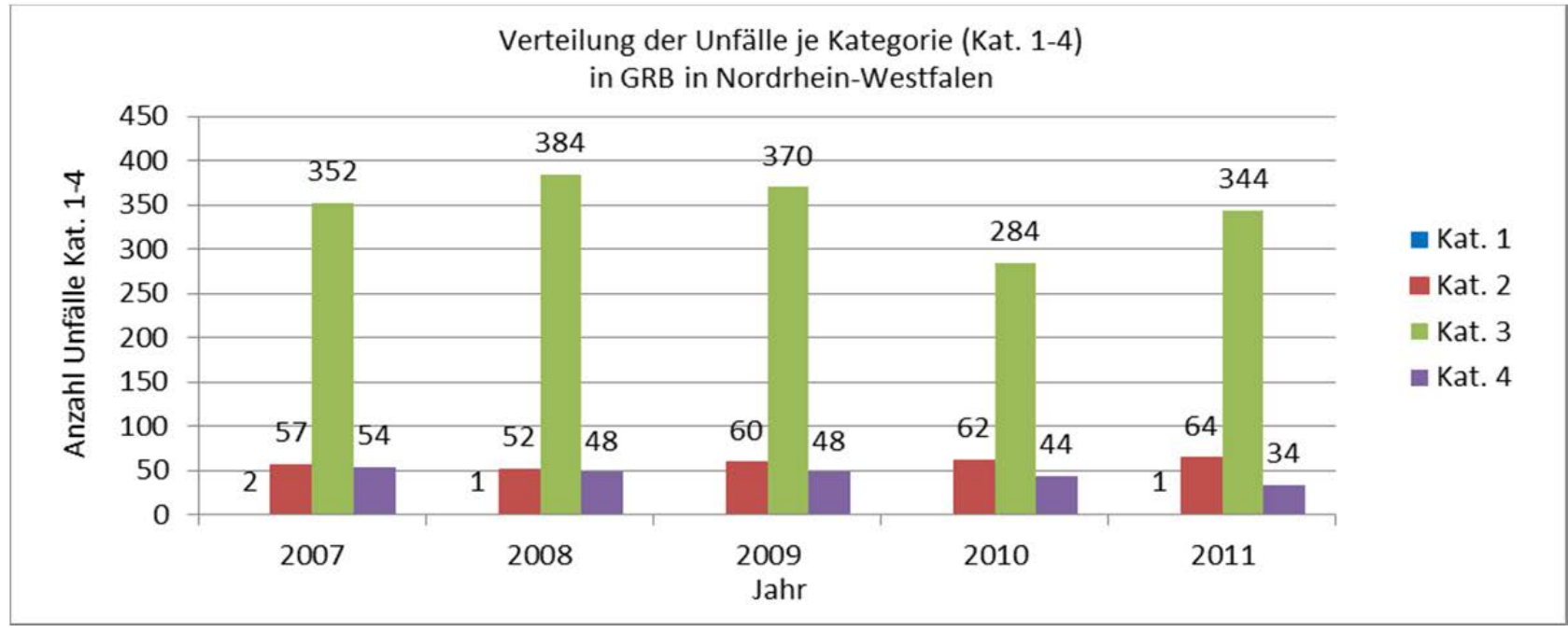

Abbildung 22: Verteilung der Unfälle je Kategorie (Kat. 1-4) in GRB in NRW im Zeitraum 2007 bis 2011 (Angabe der Anzahl der Unfälle in Absolutzahlen; Unfälle Kat. 1-4 gesamt: 2.261)

Die Verteilung der Unfälle in GRB nach Unfalltypen zeigte in Nordrhein-Westfalen ein ähnliches Bild wie bei den Bundesländern Baden-Württemberg und Hessen. In Nordrhein-Westfalen war ebenfalls der Unfalltyp 3 (Einbiegen/Kreuzen) der häufigste Unfalltyp (Abbildung 23). Allerdings wurde bei der Unfallbeteiligung der Verkehrsarten eine deutliche Abweichung gegenüber den übrigen betrachteten Bundesländern erkannt. In NRW lag der Anteil der Unfälle zwischen motorisierten Verkehrsteilnehmern bei 46\% (Abbildung 24). Eine Ursache für diese Abweichung konnte nicht unmittelbar geklärt werden.

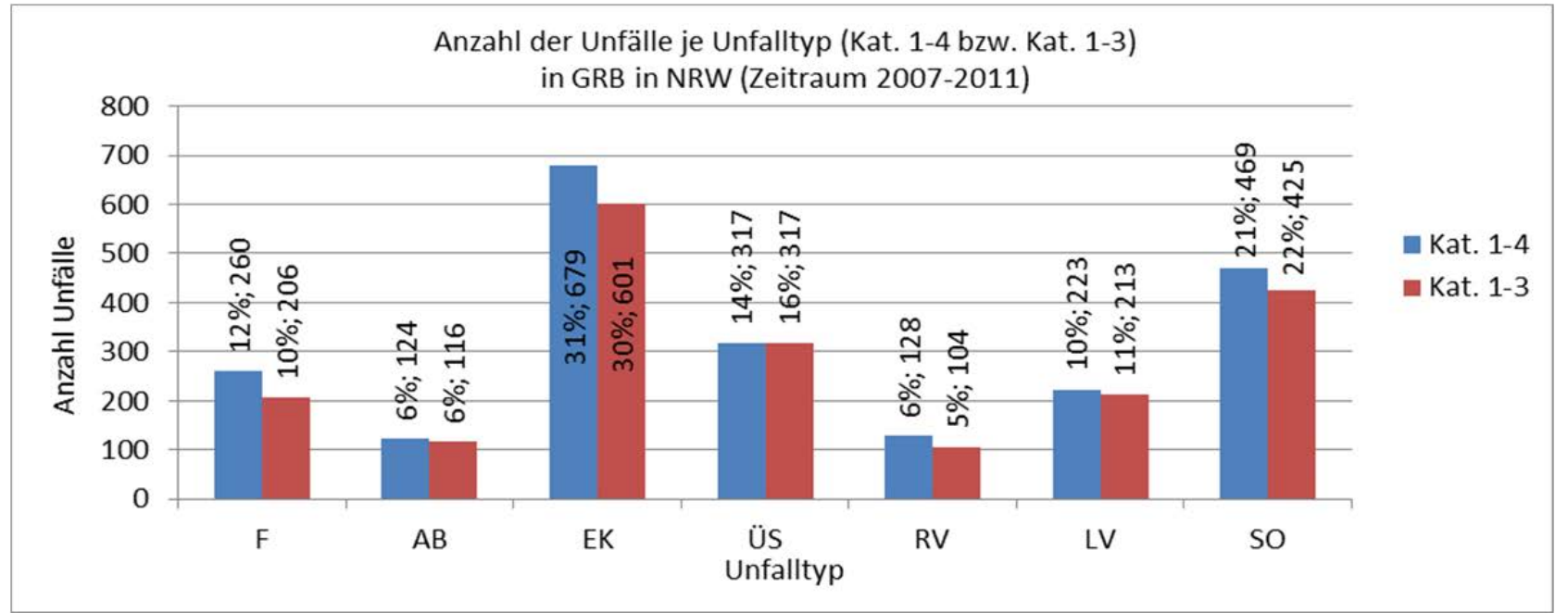

Abbildung 23: Verteilung der Unfälle der Kat. 1-4 bzw. Kat. 1-3 je Unfalltyp in GRB in NRW im Zeitraum 2007 bis 2011 (Angabe des prozentualen Anteils des jeweiligen Unfalltyps an der Gesamtzahl der Unfälle und Angabe der Anzahl der Unfälle in Absolutzahlen; Unfälle Kat. 1-4 gesamt: 2.200; Unfälle Kat. 1-3 gesamt: 1.982) 


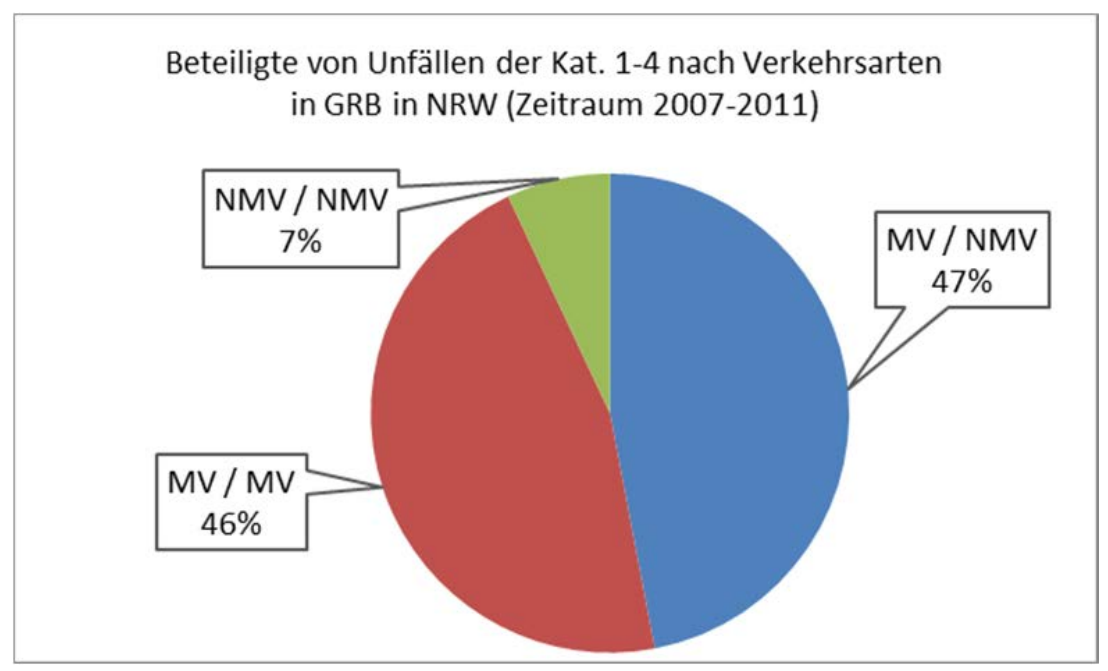

Abbildung 24: Beteiligte von Unfällen der Kat. 1-4 nach Verkehrsarten in GRB in NRW im Zeitraum 2007 bis 2011 (Angabe des Anteils der Beteiligung nach Verkehrsart an der Gesamtzahl an Unfällen; Unfälle Kat. 1-4 gesamt: 2.200)

\subsubsection{Analyse der Unfalldaten Sachsen-Anhalt}

Bei der Verteilung der Unfälle in geschwindigkeitsreduzierten Bereichen in Sachsen-Anhalt nach Unfallkategorien ergab sich ebenfalls wie in NRW ein ähnliches Bild zu den übrigen betrachteten Bundesländern. Hier dominierten ebenso die Unfälle der Kategorie 3 und die Unfälle der Kategorie 4 lagen in etwa auf dem Niveau der Unfälle der Kategorie 2. Unfälle der Kategorie 1 waren nur wenige zu verzeichnen (Abbildung 25).

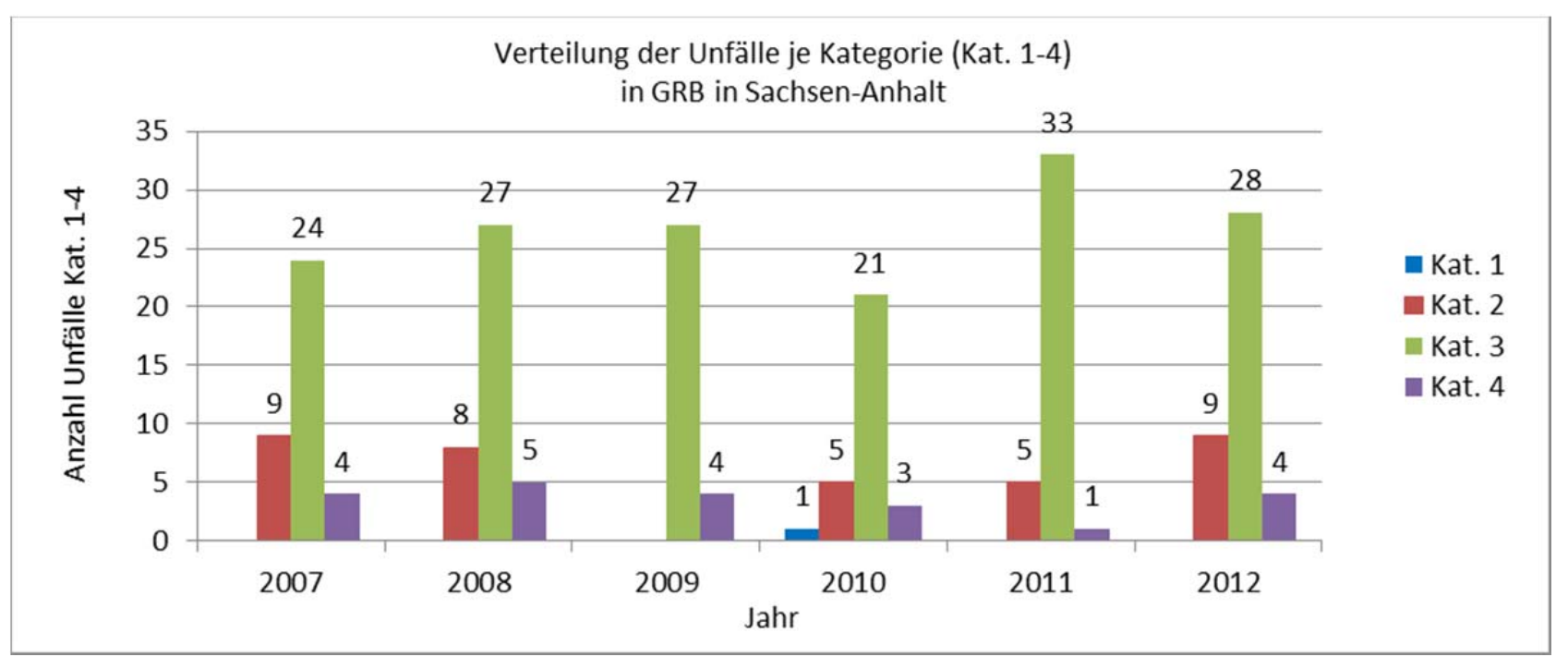

Abbildung 25: Verteilung der Unfälle je Kategorie (Kat. 1-4) in GRB in Sachsen-Anhalt im Zeitraum 2007 bis 2012 (Angabe der Anzahl der Unfälle in Absolutzahlen; Unfälle Kat. 1-4 gesamt: 218)

Bei der Verteilung der Unfalltypen der Unfälle der Kategorie 1-4 in den GRB in Sachsen-Anhalt war wiederum der Unfalltyp 3 derjenigen, dem die meisten Unfälle zugeordnet wurden. Auffallend in Sachsen-Anhalt hingegen war, dass der Unfalltyp 7 hier keine besondere Rolle spielte, sondern in etwa auf dem Niveau der übrigen Unfalltypen lag (Abbildung 26). 


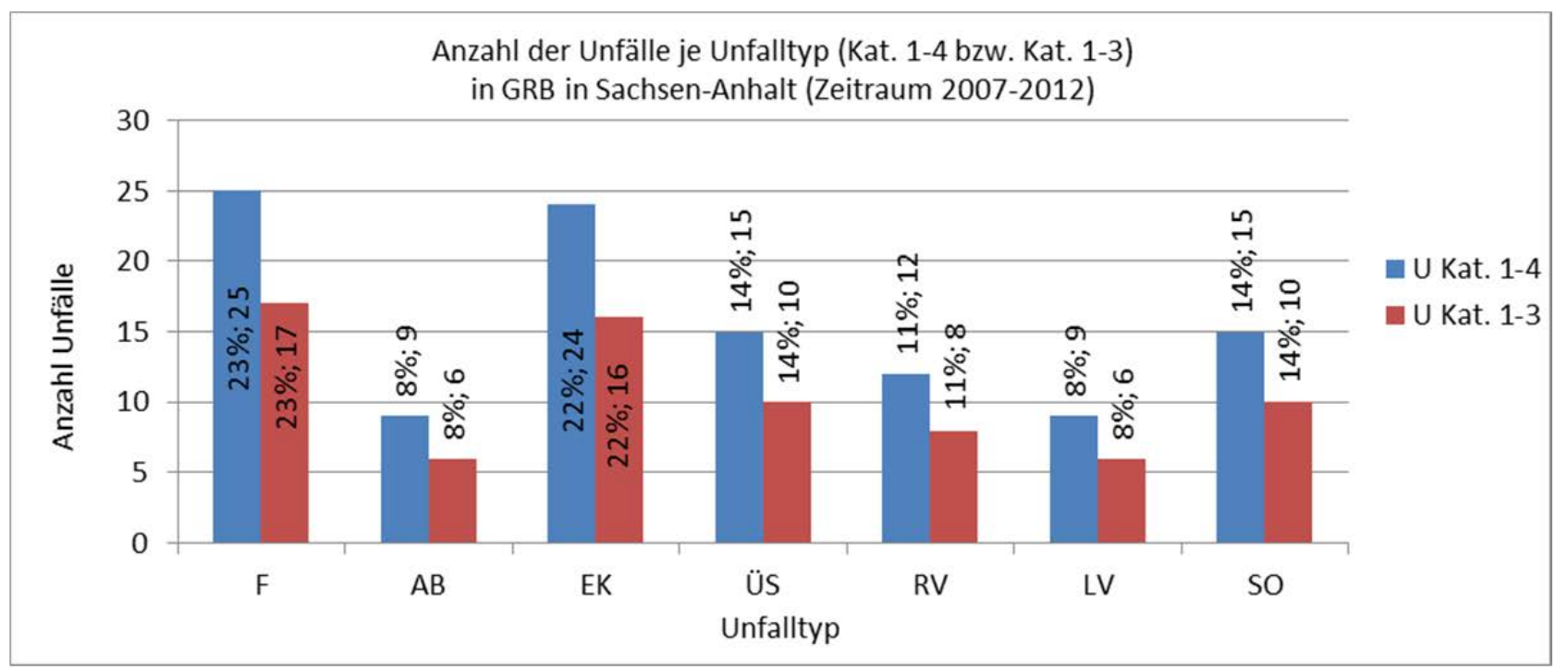

Abbildung 26: Verteilung der Unfälle der Kat. 1-4 bzw. Kat. 1-3 je Unfalltyp in GRB in Sachsen-Anhalt im Zeitraum 2007-2012 (Angabe des prozentualen Anteils des jeweiligen Unfalltyps an der Gesamtzahl der Unfälle und Angabe der Anzahl der Unfälle in Absolutzahlen; Unfälle Kat. 1-4 gesamt: 109; Unfälle Kat. 1-3 gesamt: 73)

In Sachsen-Anhalt ereigneten sich rd. drei Viertel aller Unfälle in GRB zwischen motorisierten und nichtmotorisierten Verkehrsteilnehmern. Der Anteil der Unfälle zwischen motorisierten Verkehrsteilnehmern lag hier nur bei 6\% (vgl. Abbildung 27).

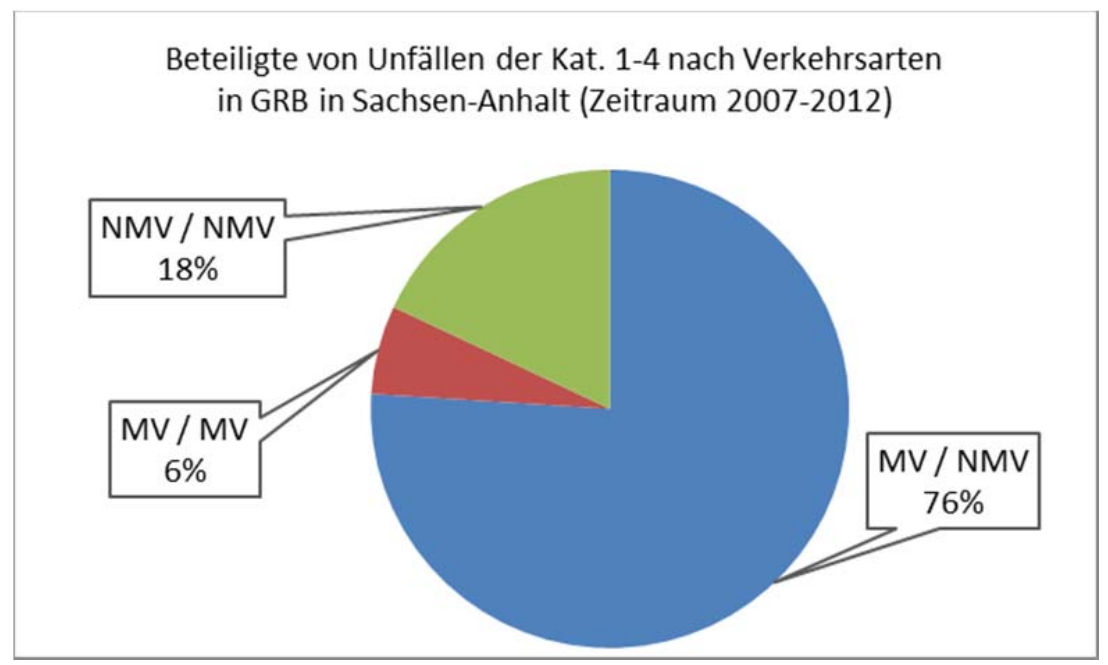

Abbildung 27: Beteiligte von Unfällen der Kat. 1-4 nach Verkehrsarten in GRB in Sachsen-Anhalt im Zeitraum 2007 bis 2012 (Angabe des Anteils der Beteiligung nach Verkehrsart an der Gesamtzahl an Unfällen; Unfälle Kat. 1-4 gesamt: 109)

\subsubsection{Analyse der Unfalldaten Thüringen}

Auch in Thüringen dominierten die Unfälle der Kategorie 3 bei den Unfällen der Kategorien 1-4 in den geschwindigkeitsreduzierten Bereichen in den Jahren 2007 bis 2012. Aufgrund der geringen Unfallanzahl in den VBB lag die Anzahl der Unfälle der Kategorie 1, 2 und 4 sehr niedrig (Abbildung 28). 


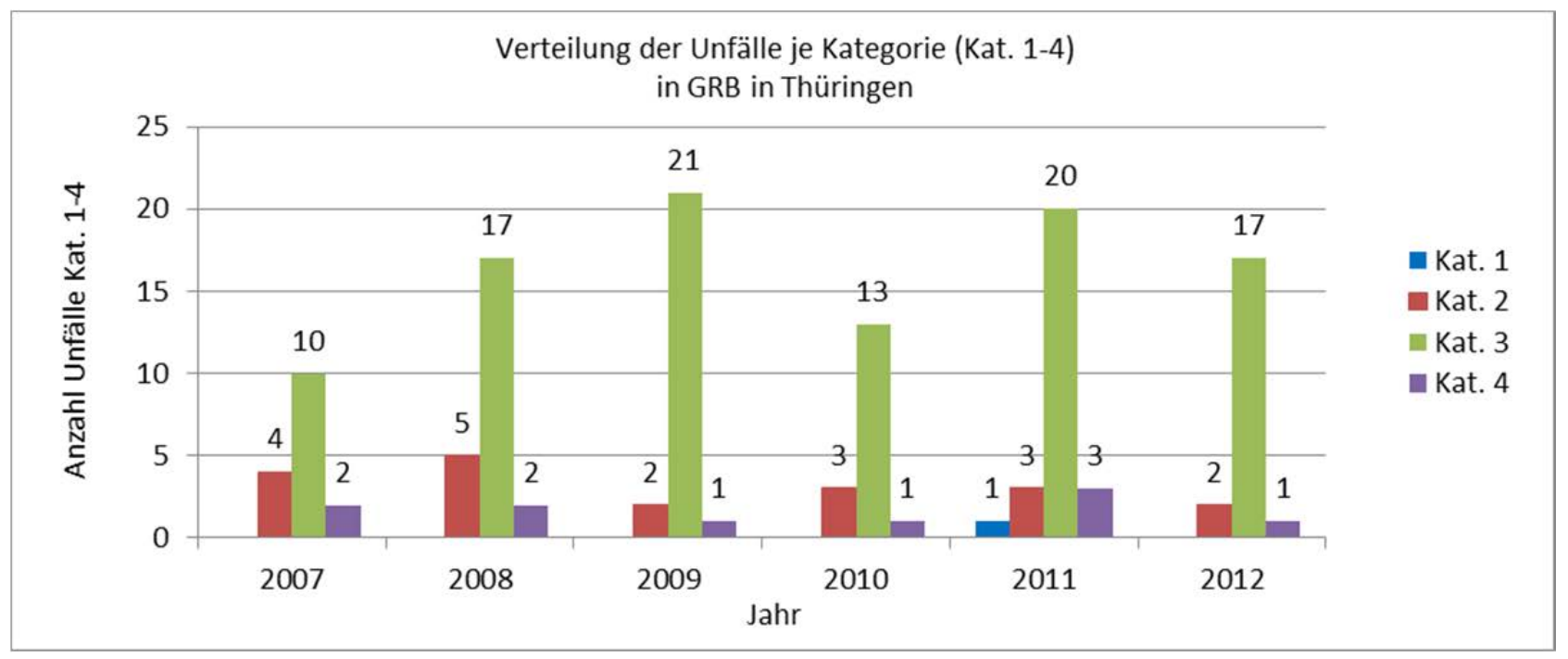

Abbildung 28: Verteilung der Unfälle je Kategorie (Kat. 1-4) in GRB in Thüringen im Zeitraum 2007 bis 2012 (Angabe der Anzahl der Unfälle in Absolutzahlen; Unfälle Kat. 1-4 gesamt: 128)

Bei der Verteilung der Unfälle nach Unfalltypen ergab sich für das Bundesland Thüringen ein ähnliches Bild wie für die Bundesländer Baden-Württemberg, Hessen und Sachsen-Anhalt. Auch hier dominierten die Unfalltypen 3 und 7 und machten in der Summe rd. die Hälfte aller Unfälle aus. Auffällig war lediglich, dass der Unfalltyp 7 einen größeren Anteil ausmachte als in den anderen betrachteten Bundesländern (Abbildung 29).

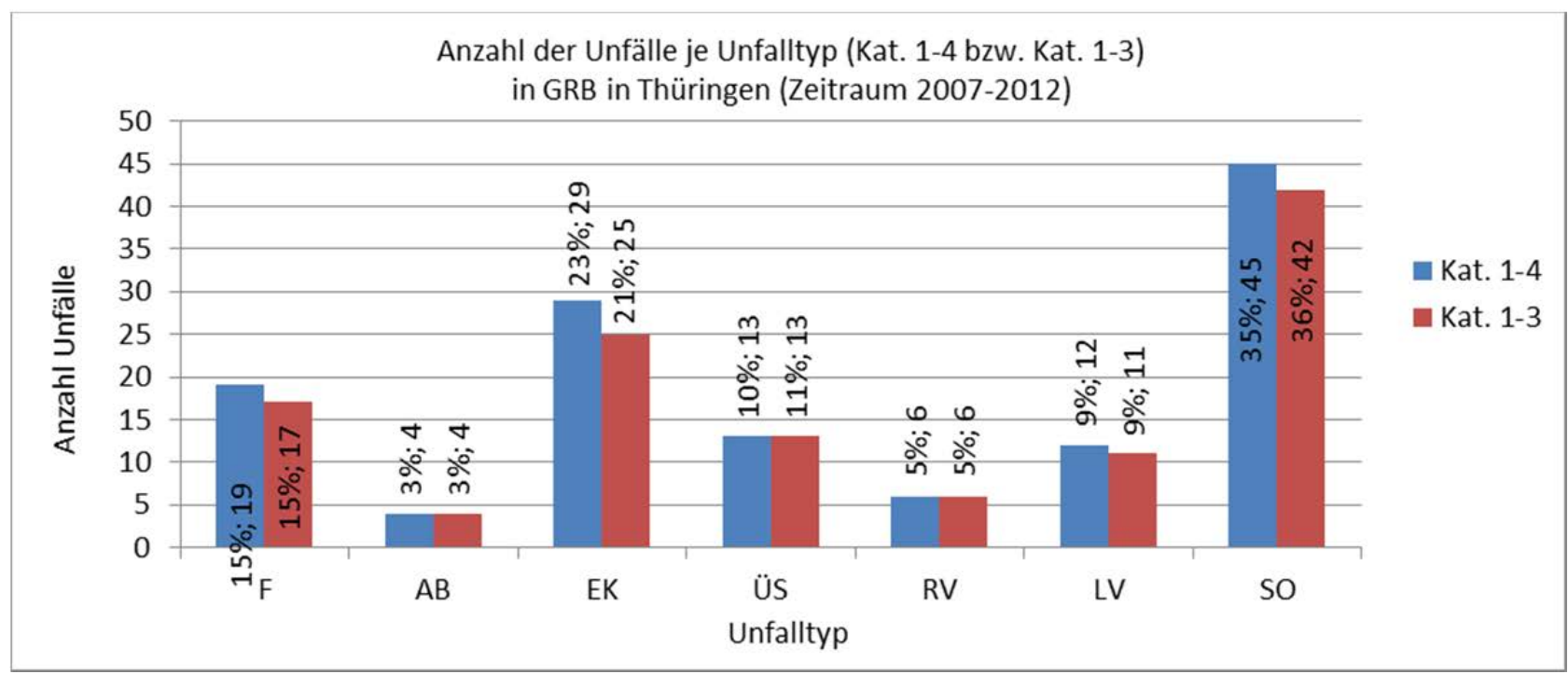

Abbildung 29: Verteilung der Unfälle der Kat. 1-4 bzw. Kat. 1-3 je Unfalltyp in GRB in Thüringen im Zeitraum 2007-2012 (Angabe des prozentualen Anteils des jeweiligen Unfalltyps an der Gesamtzahl der Unfälle und Angabe der Anzahl der Unfälle in Absolutzahlen; Unfälle Kat. 1-4 gesamt: 128; Unfälle Kat. 1-3 gesamt: 118)

Bei der Beteiligung der Verkehrsarten ergab sich auch in Thüringen das gewohnte Bild, wonach der Anteil der Unfälle zwischen motorisierten und nichtmotorisierten Verkehrsteilnehmern mit fast drei Viertel den größten Anteil einnahm (vgl. Abbildung 30). 
Beteiligte von Unfällen der Kat. 1-4 nach Verkehrsarten

in GRB in Thüringen (Zeitraum 2007-2012)

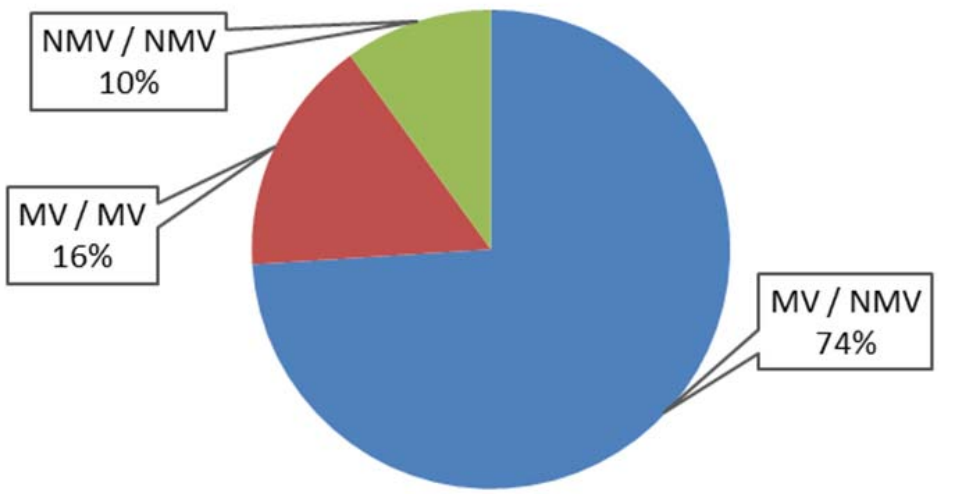

Abbildung 30: Unfallbeteiligung nach Verkehrsarten in GRB in Sachsen-Anhalt im Zeitraum 2007 bis 2012 (nur Unfälle der Kategorie 1 bis 4) (Angabe des Anteils der Beteiligung nach Verkehrsart an der Gesamtzahl an Unfällen; Unfälle gesamt: 128)

\subsection{Zusammenfassung der makroskopischen Unfallanalyse}

Aus der Auswertung der Unfalldaten der Länder sowie der Bundesstatistik ließen sich folgende Erkenntnisse zusammenfassen:

- Die Anzahl der Unfälle mit Personenschaden in geschwindigkeitsreduzierten Bereichen stagnieren in den letzten zehn Jahren bei etwa 1.500 Unfällen pro Jahr.

- Die in den Statistiken gemeldeten Unfälle, die den ausgewiesenen Verkehrsberuhigten Bereichen mit VZ325 StVO zugeordnet wurden, passierten nur zum Teil tatsächlich in VBB mit VZ 325 StVO. Nur etwa die Hälfte der in den Statistiken als VBB mit VZ325 StVO bezeichneten Bereiche wies tatsächlich eine Beschilderung mit VZ325 auf.

- Die Unfalltypen 3 (EK) und 7 (SO) machen rund die Hälfte aller Unfälle in den GRB aus.

- Etwa 20\% dieser Unfälle in GRB fanden zwischen motorisierten Verkehrsteilnehmern statt, rund 70\% der Unfälle zwischen motorisierten und nicht motorisierten Verkehrsteilnehmern.

- Zu erkennen ist, dass das Unfallgeschehen in GRB in NRW, zumindest hinsichtlich der Unfallbeteiligung, von dem in anderen Bundesländern abwich. 


\section{Umsetzungspraxis in den Kommunen}

Die Literaturanalyse zeigte, dass derzeit kein Überblick über die Praxis der Kommunen bei der Planung und Umsetzung von ausgewiesenen Verkehrsberuhigten Bereichen existiert. Dies liegt unter anderem auch daran, dass kein Richtlinienwerk existiert, das einheitliche Gestaltungsgrundsätze für VBB umfasst. Ebenso wurden in den vergangenen Jahrzehnten immer wieder neue und unterschiedliche Hinweise zur Gestaltung von VBB veröffentlicht. Somit hat sich mittlerweile ein sehr unterschiedlicher Kenntnisstand zur Thematik "Verkehrsberuhigte Bereiche" in den Kommunen eingestellt.

Um den vorhandenen Kenntnisstand zu erfassen, wurde eine Umfrage bei den 500 einwohnerstärksten Kommunen in Deutschland durchgeführt. Diese Befragung fand mittels eines OnlineFragebogens, der im Anhang 2 dargestellt ist, im Zeitraum von Juni bis September 2013 statt. Insgesamt haben 148 Kommunen (30\%) diesen Fragebogen ausgefüllt. Die Kommunen, die an der Befragung teilgenommen haben, können der Abbildung 31 entnommen werden.

In allen 148 befragten Kommunen existieren ausgewiesene Verkehrsberuhigte Bereiche, die mit dem VZ 325 StVO gekennzeichnet sind. Dabei existieren in 42 Kommunen jeweils mehr als 25 dieser Bereiche. 145 Kommunen gaben an, dass bei innen punktuelle oder streckenbezogenen VBB mit VZ 325 StVO vorhanden sind und 113 Kommunen gaben an, dass flächenhafte bzw. gebietsbezogene VBB bei innen existieren.

Darüber hinaus existieren in 76 der befragten Kommunen Verkehrsberuhigte Geschäftsbereiche gemäß §45 StVO. 18 Kommunen gaben an, dass bei innen Bereiche nach dem Prinzip „SharedSpace" oder mit Begegnungszonen-Charakter vorhanden sind.

In 98\% aller befragten Kommunen werden die VBB mit VZ 325 StVO in Wohngebieten angewendet. Bei rd. einem Fünftel allerdings auch in Geschäftsstraßen. Darüber hinaus gaben knapp unter $10 \%$ der Kommunen an, die ausgewiesenen VBB auch in sonstigen Fällen, z.B. vor Schulen, anzuwenden.

Etwa ein Drittel der Kommunen haben schon seit über 20 Jahren Erfahrung mit VBB mit VZ 325 StVO. Diese gaben an, dass schon im Zeitraum 1980-1989 ausgewiesene VBB in ihrer Kommunen umgesetzt wurden. Im Großteil der Kommunen wurde auch in der jüngeren Vergangenheit ein VBB mit VZ 325 eingerichtet. Nur 13 Kommunen gaben an, dass seit 2000 bei Ihnen kein VBB mehr umgesetzt wurde.

Die Umsetzung erfolgte im Regelfall im Zuge von Neubaumaßnahmen (rd. 85\% der Kommunen) und in bestehenden Straßen mit zusätzlichen Gestaltungsmaßnahmen (ca. 55\% der Kommunen).

Etwa die Hälfte der Kommunen bestimmte die Anwendungsbereiche der auszuweisenden VBB u.a. im Rahmen der Verkehrsentwicklungsplanung. Allerdings wurden auch im Rahmen von Stadtteilkonzepten (rd. 30\%) und im Rahmen eigenständiger Konzepte zur Verkehrsberuhigung (ca. 23\%) die Einsatzorte des VZ 325 genannt. Die Umsetzung eines VBB als Ergebnis der Lärmaktionsplanung gaben immerhin $13,5 \%$ der Kommunen an. 


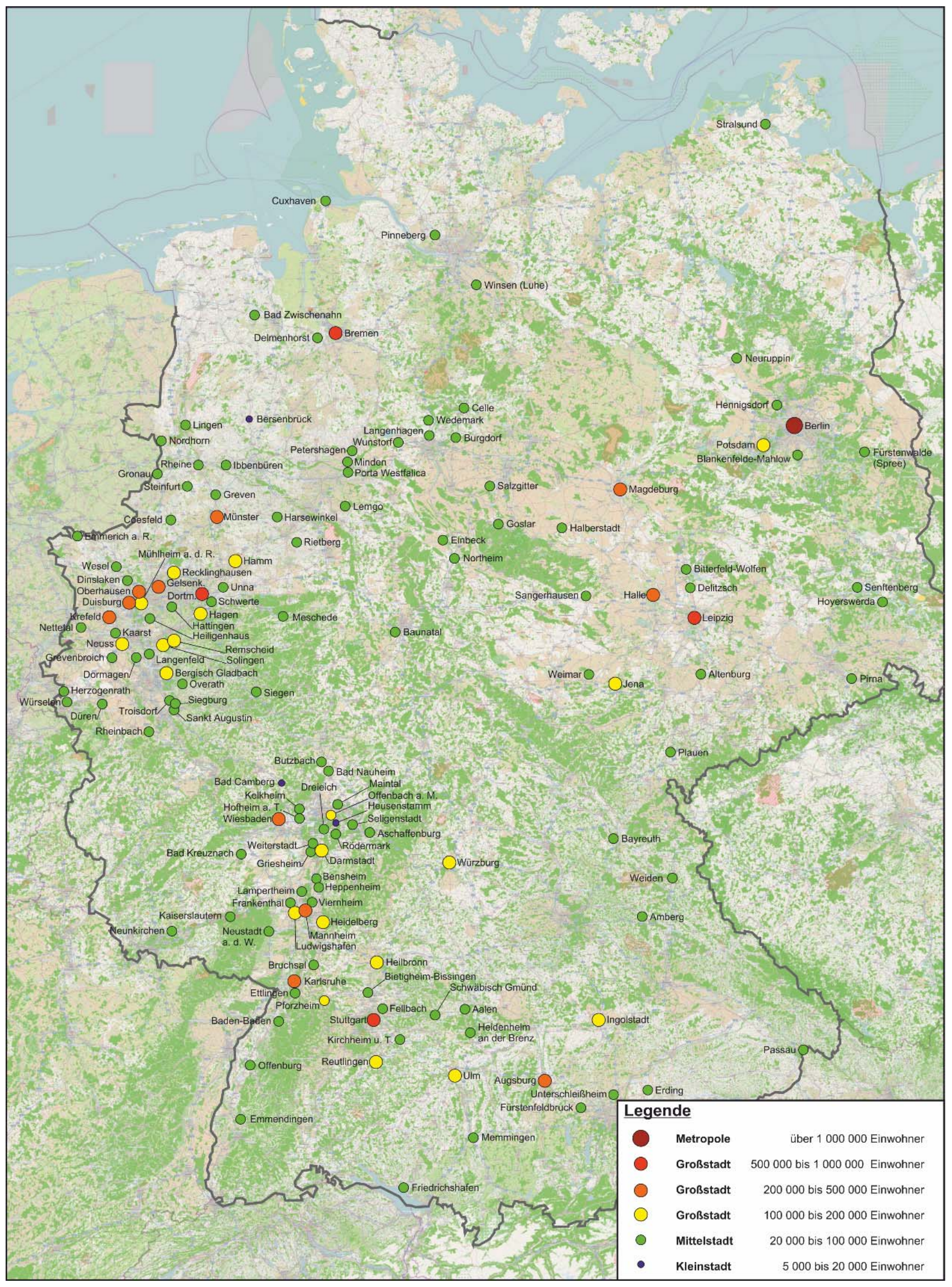

Abbildung 31: Übersicht über die befragten Kommunen 
Die wesentlichen Ziele, die mit der Einrichtung von VBB mit VZ 325 StVO bei den Kommunen verbunden wurden, waren die Verbesserung der Aufenthaltsqualität bzw. Verringerung der Dominanz des Kfz-Verkehrs im Straßenraum und die Verbesserung der Verkehrssicherheit. Während die Verbesserung der Straßen- und Stadtgestaltung ein weiteres wichtiges Ziel war, stand die Verbesserung der Überquerungsmöglichkeiten für Fußgänger, die Reduzierung der Lärmbelastung und die Verdrängung vom Kfz-Verkehr nur in Einzelfällen im Vordergrund (Abbildung 32).

zur Verbesserung der Verkehrssicherheit

zur Verbesserung der Aufenthaltsqualität / Verringerung der Dominanz des Kfz-Verkehrs im Straßenraum

Verbesserung der Straßen und Stadtgestaltung

Verbesserung der Querungsmöglichkeiten für Fußgänger

Verbesserung der gemeinsamen Abwicklung von nicht motorisiertem Verkehr und Kfz-Verkehr

Reduzierung der Lärmbelastungen

Verdrängung vom Kfz-(Durchgangs-)Verkehr
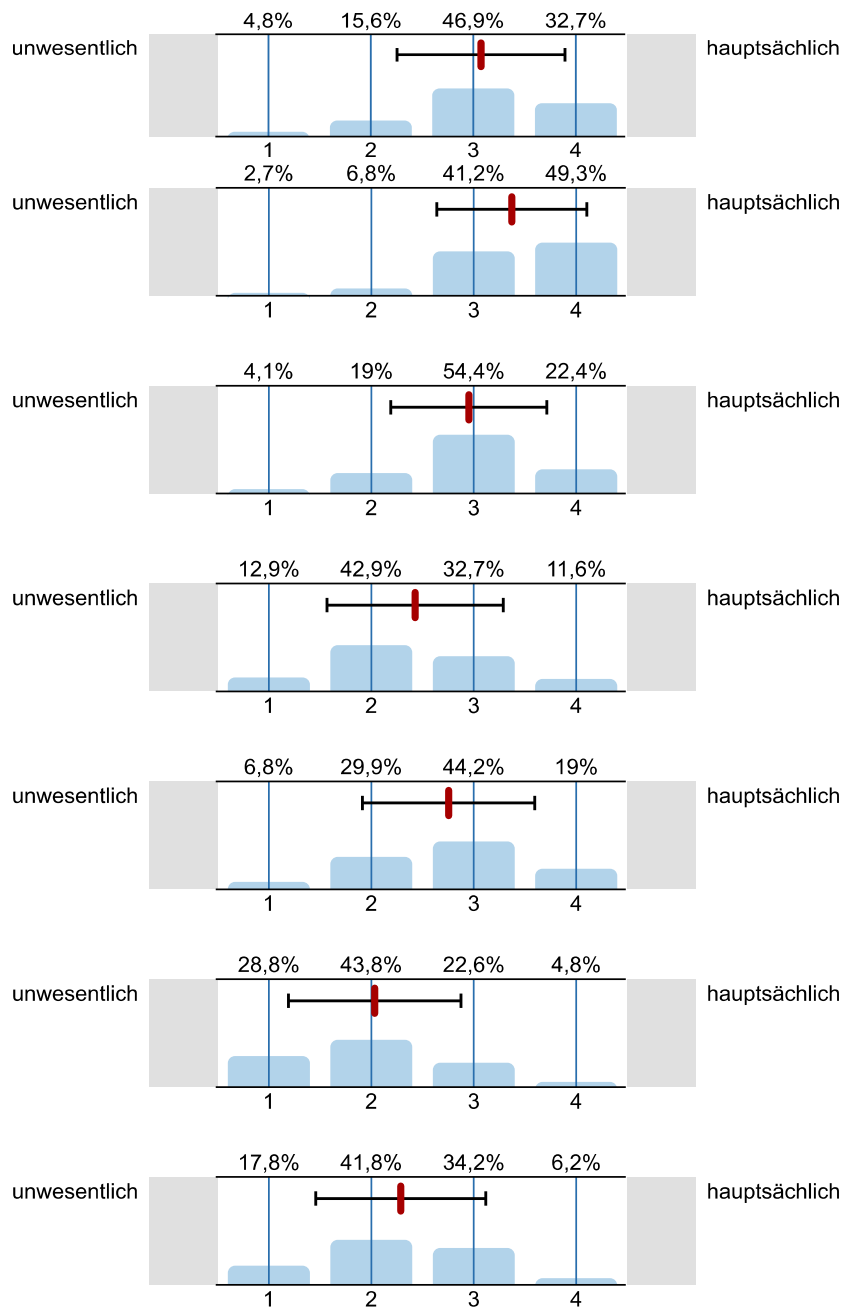

Abbildung 32: Ziel, das mit der Einrichtung von VBB mit VZ 325 StVO bei den Kommunen verbunden ist

Besonders positive Erfahrungen mit Gestaltungsmaßnahmen in ausgewiesenen Verkehrsberuhigten Bereichen haben $\mathrm{rd}$. $80 \%$ der Kommunen bei den Auswirkungen auf das Geschwindigkeitsniveau und die Aufenthaltsqualität gemacht. Hinsichtlich der Auswirkungen auf den Unterhaltungsbedarf/-aufwand gaben rd. $20 \%$ der Kommunen an, dass sie hier negative Erfahrungen gemacht haben (Abbildung 33). 
Auswirkungen auf das Unfallgeschehen/ Verkehrssicherheit

Auswirkungen auf das Geschwindigkeitsniveau

Auswirkungen auf den Durchgangsverkehr

Auswirkungen auf den Unterhaltungsbedarf

Auswirkungen auf die Aufenthaltsqualität

Auswirkungen auf die Querungsmöglichkeiten
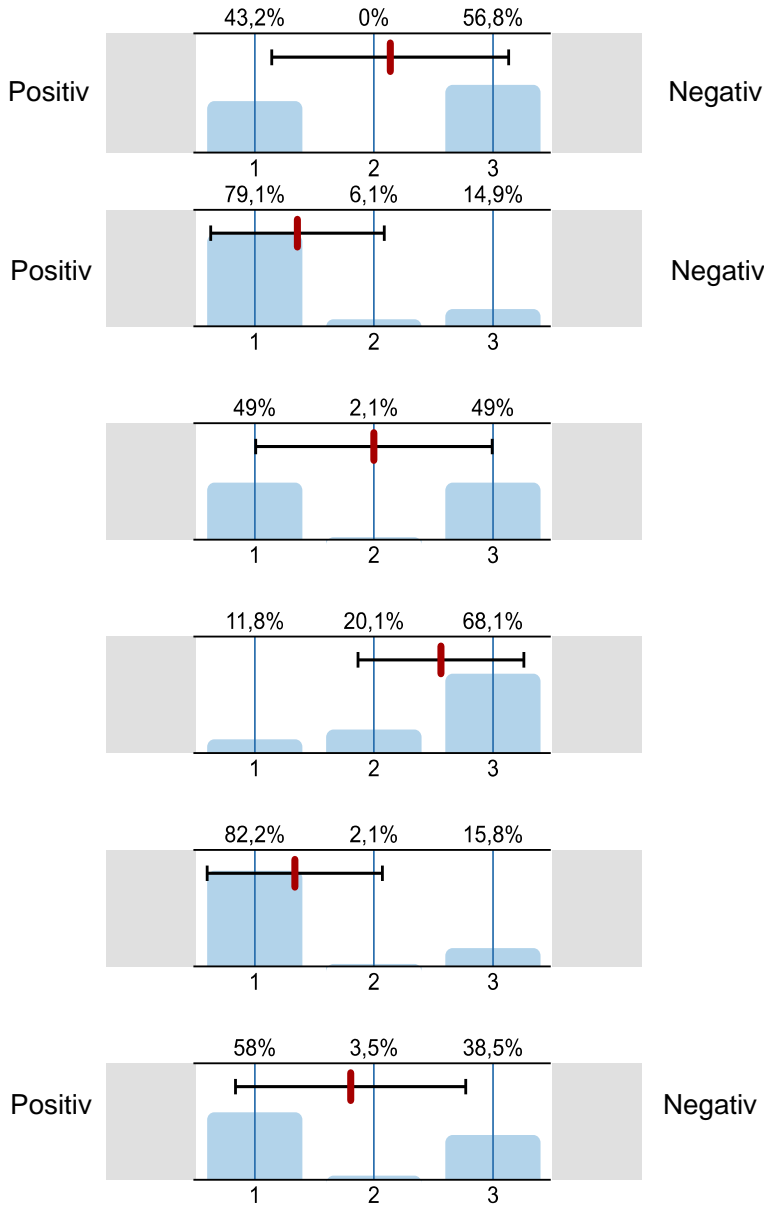

Abbildung 33: Angaben zu positiven (1), neutralen (2) und negativen (3) Erfahrungen mit Gestaltungsmaßnahmen in ausgewiesenen Verkehrsberuhigten Bereichen durch die befragten Kommunen

\section{Untersuchungsobjekte}

In den folgenden Kapiteln wird dargestellt, wie die Untersuchungsobjekte bzw. Untersuchungsbeispiele ausgewählt wurden. Es wird erläutert wie sich der zur Dokumentation entwickelte Steckbrief für die aufgenommenen VBB darstellt, und erläutert, wie die Kategorisierung der dokumentierten Bereiche vorgenommen wurde.

Alle erhobenen Untersuchungsobjekte (Steckbriefe) wurden aufgrund ihres Umfangs in einem separaten Anlagenband dokumentiert.

\subsection{Zusammenstellung der Untersuchungsobjekte}

Für die vorliegende Studie sind in mehreren Bundesländern ausgewiesene Verkehrsberuhigte Bereiche (beschildert mit VZ 325) erhoben worden. Diese sind hinsichtlich ihrer Gestaltungsform und den zugehörigen Rahmenbedingungen dokumentiert worden. Bei der Zusammenstellung der Untersuchungsobjekte wurde darauf geachtet, dass:

- unter den Beispielen auch besonders unfallauffällige Beispiele berücksichtigt wurden und

- ausschließlich Bereiche, die mit dem VZ 325 ausgewiesen sind, aufgenommen wurden. 
Die Untersuchungsobjekte setzen sich aus mehreren Informationsquellen zusammen. Als erstes wurden zur Identifikation von möglichen „unfallauffälligen“ Straßen oder Bereichen auf die vorhandenen Unfalldatensätze der Länder (Kapitel 7.3) zurückgegriffen. Berücksichtigt wurden in dieser Auswertung ausschließlich die Datensätze zu den Unfällen der Kategorien 1-4. Zum Zwecke der Identifizierung von Beispielen mit einer Häufung von Unfallereignissen wurden die Datensätze nach der Angabe von Gemeinde, Straße und Verkehrsberuhigten Bereichen gefiltert. Mit diesem Vorgehen konnten im ersten Ergebnis keine Unfallhäufungen im klassischen Sinn erhoben werden. Somit wurden auch Beispiele mit drei Unfallereignissen in sechs Jahren mit in die Liste aufgenommen, um ein größeres Auswahlspektrum zu erhalten.

Den zweiten Teil an möglichen Untersuchungsobjekten für die Studie, stellt eine Stichprobe der durch Kommunen und Städte vorgeschlagenen Beispiele dar. Da Kommunen und Städte für die Umsetzung von Verkehrsberuhigten Bereichen zuständig sind, sollten diese auch aus ihrer Sicht interessante Beispiele benennen können. Zu diesem Zweck wurden die Teilnehmer - in der Kapitel 0 beschriebenen Umfrage - aufgefordert bzw. innen die Chance gegeben, aus ihrer Sicht "geeignete" Beispiele, die sie für optimal gestaltet oder für unfallauffällig halten, zu benennen. Es blieb dabei jedoch offen, nach welchen Kriterien die Kommunen, die benannten Straßen ausgesucht und angegeben haben. Dadurch können sowohl unfallauffällige Bereiche, aber auch gestaltungstechnisch positive und negative Beispiele im Kollektiv enthalten sein. Die Beantwortung dieser Frage war ein freiwilliger Zusatz und kein Muss (Frage 8.3 der Umfrage im Anhang 2). Nichts desto trotz haben von den insgesamt rund 150 Teilnehmern, 82 Kommunen und Städte freiwillig ausgewiesenen Verkehrsberuhigte Bereiche genannt, die für die Studie weiter verwendet werden konnten. Diese wurden in das Kollektiv mit aufgenommen und stellen somit einen großen Anteil der zusammengetragenen Nennungen bzw. Beispiele für die Auswahl der zu dokumentierenden Untersuchungsobjekte dar.

Ergänzt wurde das Kollektiv potenzieller Untersuchungsobjekte durch weitere Beispiele, die dem Forschungsnehmer anderweitig bekannt, vor Ort aufgefallen oder aus anderen Studien bekannt waren.

Insgesamt lagen somit 302 Untersuchungsobjekte vor, die erhoben und dokumentiert wurden:

- 46 Beispiele, die aus den Unfalldatenbanken der Länder unfallauffällig sind,

- 100 Beispiele, die aus der Umfrage der Kommunen benannt wurden,

- 148 Beispiele, die dem Auftragnehmer bekannt sind oder vor Ort entdeckt wurden und

- 8 Beispiele, die aus vorherigen Studien bekannt sind.

Bei den dokumentierten Untersuchungsobjekten handelte es sich in der überwiegenden Mehrzahl um eigenständige und abgeschlossene Beispiele. Aufgrund von örtlichen Gegebenheiten oder auftretenden Besonderheiten, wie beispielsweise einer Unterbrechung eines Verkehrsberuhigten Bereichs im Verlauf durch die Anordnung einer Fußgängerzone, wurde es notwendig diese Untersuchungsobjekte in mehrere Teilabschnitte aufzuteilen.

\subsection{Dokumentation der Untersuchungsobjekte}

Ein Ziel der Dokumentation bestehender ausgewiesener Verkehrsberuhigter Bereiche war es, darzustellen, wo ausgewiesene Verkehrsberuhigte Bereiche zum Einsatz kommen und wie diese gestaltet wurden. Hierzu wurde eine Reihe von unterschiedlichen Aspekten der VBB erfasst.

Zur Durchführung der Erhebung und späteren vergleichbaren Dokumentation, bot sich die Erstellung eines Erhebungs- bzw. Erfassungsbogens an. Zu diesem Zweck wurde in einem iterativen Prozess ein Formular in Form eines Steckbriefes erarbeitet. Um die Handhabung vor Ort zu vereinfachen und der erhebenden Person die Möglichkeit zu geben, sich Auffälligkeiten eines 
Bereiches bereits vor Ort zu notieren, wurde bei der Erstellung des Bogens auf ein handliches Format geachtet.

Die nachstehende Abbildung 34 zeigt die Vorlage für Steckbriefe, die im Rahmen des Projektes erstellt wurden. Der Steckbrief sollte ein möglichst breites und objektives Spektrum von ausgewiesenen VBB einheitlich darstellen können. Um dies zu erreichen, war es notwendig, die Straßencharakteristik vor Ort ausreichend genau zu beschreiben. Im Wesentlichen lässt sich der Steckbrief grob in drei Bereiche unterteilen (vgl. farbig eingefasste Bereiche in der Abbildung 34).

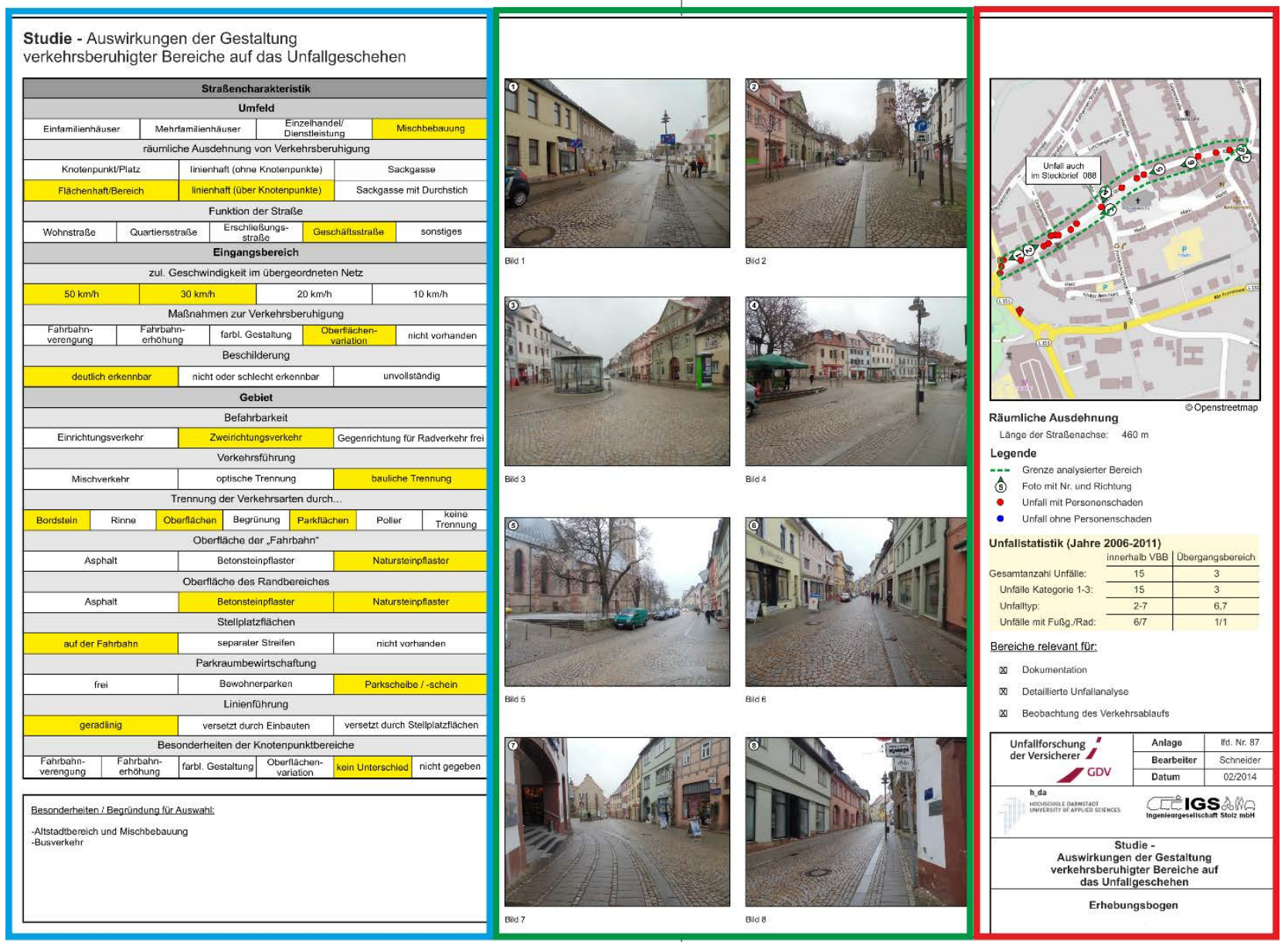

Abbildung 34: Steckbrief für die erhobenen Untersuchungsobjekte

Im linken (blauen umrandeten) Bereich des Steckbriefs wurden verschiedene Merkmale von VBB dargestellt. Bei der Aufnahme vor Ort konnte mittels Ankreuzen des jeweils zutreffenden Kriteriums eine schnelle Bestandsaufnahme erfolgen. Die aufgeführten Merkmale beruhen auf den Erkenntnissen der zu Beginn dieser Studie durchgeführten Literaturrecherche über Gestaltungsmaßnahmen und Empfehlungen für die Gestaltung und Anlage von ausgewiesenen Verkehrsberuhigten Bereichen, sowie Erfahrungen die aus den ersten Erhebungen von Straßen vor Ort gewonnen werden konnten. Diese zusammengestellten Merkmale ließen sich in drei Gruppen unterteilen:

- das Umfeld,

- den Eingangsbereich und 
- Aussagen zur Gestaltung des Gebietes.

Zusammenfassend beschreiben die so dokumentierten Merkmale die Straßencharakteristik vor Ort und werden nachfolgend genauer erläutert.

Im Themenbereich „Umfeld“, wurde neben der Randbebauung auch die jeweilige räumliche Ausdehnung des ausgewiesenen Verkehrsberuhigten Bereiches erhoben. Hier wurde dokumentiert, ob es sich beispielsweise um einen Platz, eine einzelne Straße oder gar um einen Knotenpunkt/Platz handelte. Als dritter Punkt wurde in dieser Gruppe die Funktion der Straße erfasst. Mit Hilfe dieser Aussage ließen sich erste Rückschlüsse auf ein mögliches Fußgängeraufkommen oder den zu erwartenden Durchgangsverkehr ziehen. Mit Hilfe der Angaben zur Funktion der Straße wurden die zu dokumentierenden Beispiele im weiteren Verlauf der Studie kategorisiert und in verschiedenen Gruppen zusammengefasst (hierzu Näheres im nachfolgenden Kapitel 9.3).

Der „Eingangsbereich“ stellte einen wichtigen Teil eines ausgewiesenen Verkehrsberuhigten Bereichs dar. Mit der Gestaltung des Eingangsbereichs soll der Kfz-Verkehr darauf aufmerksam gemacht werden, dass er sich nun in einem mit VZ325 gekennzeichnetem Verkehrsberuhigten Bereich befindet, in diesem eine zulässige Höchstgeschwindigkeit von $7 \mathrm{~km} / \mathrm{h}$ gilt und er seine Geschwindigkeit anzupassen hat. Deshalb wurde - neben Abfragen zur zulässigen Geschwindigkeit im übergeordneten Netz und Maßnahmen zur Verkehrsberuhigung im Eingangsbereich - im Steckbrief auch die Erkennbarkeit der Beschilderung abgefragt. Zwar stellt ein Eingangsbereich auch gleichzeitig einen Ausgangbereich dar, jedoch sollte die Aufmerksamkeit beim Einfahren liegen und der damit verbundenen Reduzierung der Geschwindigkeiten und dem Wiedererkennungswert des ausgewiesenen VBB.

Der Themenbereich „Gebiet“ stellte den dritten Block der Aufnahmemerkmale dar. In diesem wurde die Beschreibung aller charakteristischen Merkmale zusammengefasst, die ein Verkehrsberuhigter Bereich innerhalb eines Gebietes aufwies. VBB mit VZ 325 sind sowohl in Einbahnstraßen als auch in Straßen mit Zweirichtungsverkehr eingerichtet worden. Ebenfalls wurde die Situation des Radverkehrs aufgenommen. Zum Beispiel konnte dieser in Einbahnstraßen auch durch eine Zusatzbeschilderung in beide Richtungen geführt werden. Für die VBB sind auch die Gestaltung sowie die verwendeten Materialien aufgenommen worden. Hier gab es beispielsweise Unterschiede in baulicher Hinsicht.

In der VwV zur StVO ist zunächst einmal vorgesehen, dass die Verkehrsfläche in Verkehrsberuhigten Bereichen als Mischverkehrsfläche ausgebaut wird und es keine Separation von Fahrbahn und Gehweg geben soll. Erste Aufnahmen, sowie die Erkenntnis aus der Umfrage haben gezeigt, dass dies nicht durchgehend der Fall ist. Daher wurden auftretende Separationseffekte in optischer oder auch baulicher Form durch erhaltene Bordsteine, Bänke, Bepflanzungen, Stellplatzflächen oder verwendete Materialien ebenfalls dokumentiert.

Neben den bereits angesprochenen Merkmalen sind auch Informationen zur Stellplatzsituation erhoben worden. Dabei war es interessant zu wissen, wo Parkflächen angeordnet und ggf. auch bewirtschaftet wurden. Grundsätzlich gilt für das Parken in einem ausgewiesenen Verkehrsberuhigten Bereich, dass dies nur in gekennzeichneten Flächen erlaubt ist. Diese Regel ist an das VZ 325 gekoppelt und nicht mit einer Zusatzbeschilderung auszuweisen. Gesonderte Regeln zum Parken sind über eine Zusatzbeschilderung anzuordnen, wie beispielsweise Anwohnerparken oder das Parken mit Parkschein/-scheibe.

Als letzte Aspekte wurden die Form der Linienführung oder das Vorhandensein von Knotenpunkten innerhalb des Bereichs erfasst. Im Zuge der Linienführung kam es vor, dass der Kfz-Verkehr durch Engstellen, Einbauten oder Versätze zur Geschwindigkeitsreduzierung gezwungen oder auch daran erinnert wurde. Für vorhandene Knotenpunkte innerhalb des Bereichs wurde geprüft, ob diese durch weitere Gestaltungsmerkmale hervorgehoben wurden und wie diese gegebenenfalls ausgesehen haben. 
Im Anschluss an den „Textteil“, mit den spezifischen Merkmalen des dokumentierten Bereiches, schließt sich ein freies Kommentarfeld an. In diesem konnten und sollten Besonderheiten festgehalten werden. Hierzu zählten beispielweise die Gründe für eine Auswahl des Bereichs, aber auch ob besondere Gestaltungsmaßnahmen vorhanden waren, die eine Aufenthaltsqualität steigerten, besondere Maßnahmen zur Verbesserung der Überquerungsmöglichkeiten oder besondere Bebauungen vorhanden waren wie z.B. Schulen, Kindergärten oder andere öffentliche Einrichtungen.

Der mittlere Bereich, der in Abbildung $\mathbf{3 4}$ grün umrandet wurde, diente zur bildlichen Veranschaulichung des Bereiches. Zum besseren Verständnis des erhobenen Bereiches wurde hier eine Fotostrecke angelegt, die die vorhandenen Untersuchungsaspekte wiedergibt. Dabei wurden die Bilder mit Nummern gekennzeichnet. Zur Orientierung sind diese Nummern mit Blickrichtung in eine Übersichtskarte eingetragen worden. Diese Übersichtskarte ist im rechten Bereich des Steckbriefes angeordnet worden.

Der rechte (rot umrandete) Bereich stellte ergänzende Informationen bereit (Abbildung 34). Hier wurde im oberen Bereich eine Übersichtkarte angeordnet. Diese sollte in erster Linie einen Überblick über Lage und Verlauf des erhobenen Bereichs geben. Darüber hinaus konnten besondere Auffälligkeiten vermerkt und markante Punkte erfasst werden. Als Kartengrundlage wurden hier Bildausschnitte aus dem Kartendienst OpenStreetMap verwendet. Bei der späteren Nachbearbeitung konnten aufgenommene Fotos mit Lage und Blickrichtung eingetragen werden. Lagen zu den aufgenommenen Steckbriefen Unfalldaten der Kategorie 1-4 vor, wurden diese ebenfalls auf der Übersichtskarte eingetragen.

An die Übersichtskarte wurde eine Tabelle mit näheren Angaben zu den in diesem Bereich dokumentierten Unfallgeschehen ergänzt. Diese Angaben bezogen sich auf die Auswertung der zur Verfügung stehenden Unfalldatensätze der einzelnen Länder oder auf Unfalldaten, die bei den zuständigen Polizeidienststellen erhoben wurden. Diese Angaben bildeten im Weiteren auch die Grundlage der detaillierten Unfallanalyse aller Unfälle der Kategorie 1-4 sämtlicher dokumentierter Bereiche. Die Angaben auf dem Steckbrief dienten in erster Linie dazu, dem Betrachter einen ersten Eindruck über das vorherrschende Unfallgeschehen zu vermitteln und ermöglichten eine erste Verknüpfung mit Gestaltungsaspekten. In die Tabelle wurden die Anzahl der aufgetretenen Unfälle innerhalb des ausgewiesenen Verkehrsberuhigten Bereichs und solchen, die im Übergangsbereich geschehen sind, eingetragen. Ergänzend wurden die Unfallkategorie, der Unfalltyp und die Anzahl der Beteiligten Radfahrer und Fußgänger aufgeführt.

Abgerundet wurde der Steckbrief durch eine Legende inklusive einer Längenangabe des Bereichs. Mit der Längenangabe konnte im Zuge der Unfallanalysen z.B. die Unfalldichte bestimmt werden. Auf dem Steckbrief ist ein Feld für einen Vermerk vorgesehen worden, ob der Bereich nur für Dokumentationszwecke erhoben wurde, sich für die Unfallanalyse oder zur weiteren Beobachtung des Verkehrsablaufs eignete. Den Abschluss des Steckbriefs bildete der obligatorische Planstempel.

\subsection{Kategorisierung der Untersuchungsobjekte}

Es sind insgesamt 302 Untersuchungsobjekte mit der Beschilderung gemäß VZ325 erhoben worden. Diese wurden in Form des unter Kapitel 9.2 beschriebenen Steckbriefs dokumentiert.

Die Erhebung zeigte, dass Verkehrsberuhigten Bereiche in der Praxis sehr unterschiedlich gestaltet wurden und in sehr unterschiedlichen Straßenräumen zum Einsatz kamen. Daher war eine Kategorisierung der VBB erforderlich, um im weiteren Verlauf der Projektbearbeitung Gestaltungshinweise und Empfehlungen für Einsatzbereiche ableiten zu können. 
In erster Linie wurden die ausgewiesenen Verkehrsberuhigten Bereiche nach der Funktion der Straße bzw. des Straßenabschnitts, der mit VZ 325 beschildert war, kategorisiert (Kategorisierungsstufe 1). Als Orientierung für die vorgenommene Kategorisierung, dienten die typischen Entwurfssituationen nach den Richtlinien für die Anlage von Stadtstraßen RASt 06 (vgl. FGSV 200, 2006). Dabei wurde nach den Straßenfunktionen Wohnstraße, Quartiersstraße, Sammelstraße, Erschließungsstraße, Geschäftsstraße und Sonstige unterschieden.

Bei einer detaillierteren Betrachtung und nach Anwendung der Kategorisierung fiel auf, dass in einigen Untergruppen nur eine sehr geringe Stichprobengröße vorhanden war. Darüber hinaus zeigte sich, dass die Straßentypen „Sammelstraße“ und „Erschließungsstraße“ bezüglich ihrer Verbindungsfunktion und den damit verbundenen Durchgangsverkehrsanteilen Ähnlichkeiten aufwiesen. Aufgrund der geringen Anzahl an Untersuchungsobjekten rechtfertige dies keine getrennte Erfassung und es wurde in Abstimmung mit dem UDV entschieden, dass diese beiden Kategorien unter dem Namen „Erschließungsstraße“ zusammengefasst wurden. Im Folgenden wird die Kategorisierung der VBB näher erläutert.

\section{Kategorisierungsstufe: Straßenfunktion}

Es wurden folgende Gruppen in der 1. Kategorie festgelegt bzw. unterschieden:

- Wohnstraßen,

- Quartierstraßen,

- Erschließungsstraßen (inkl. Sammelstraßen),

- Geschäftsstraßen sowie

- Sonstige Bereiche.

\section{Wohnstraße mit ausgewiesenem Verkehrsberuhigtem Bereich}

Straße, die ausschließlich von Wohnbebauung (im Regelfall Einfamilienhäusern) umgeben ist und sich in reinen Wohngebieten befindet, die i.d.R. in Randlage liegen. Die Straße wird ausschließlich durch Quell- und Zielverkehren genutzt (Abbildung 35).
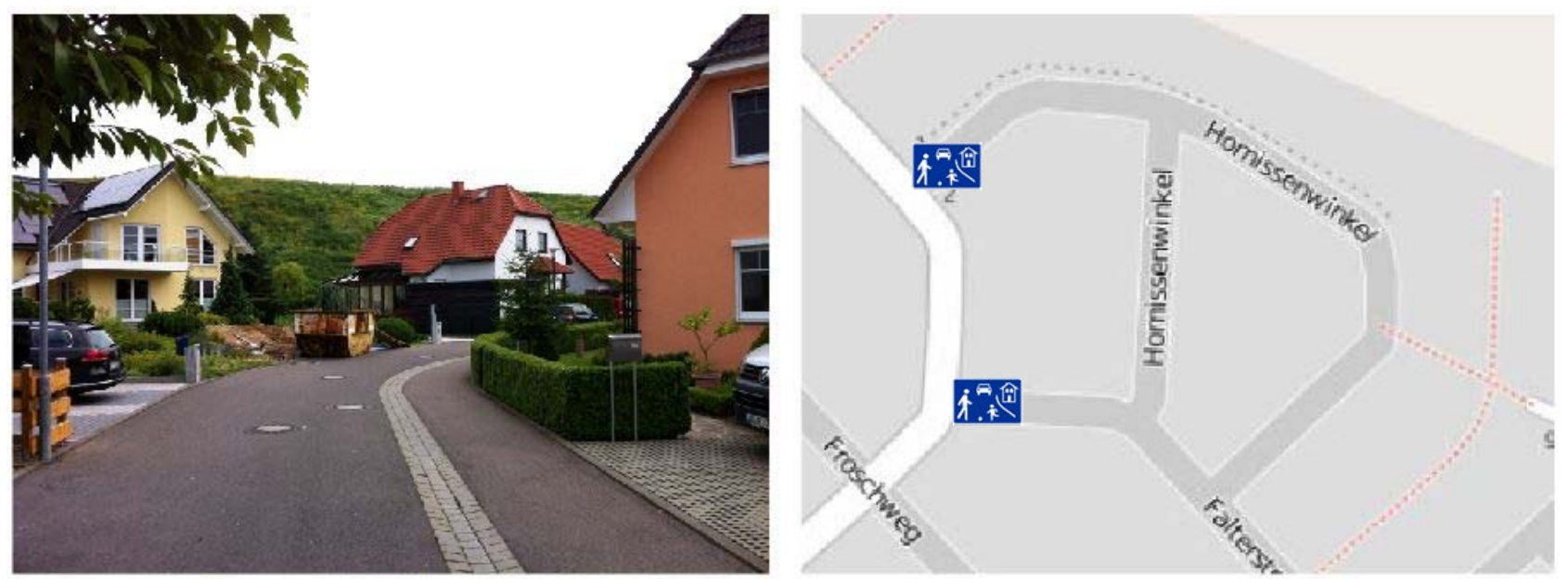

Abbildung 35: Beispiel einer Wohnstraße mit ausgewiesenem Verkehrsberuhigtem Bereich 


\section{Quartierstraße mit ausgewiesenem Verkehrsberuhigtem Bereich}

Straße, die ausschließlich von Wohnbebauung (im Regelfall Mehrfamilienhäusern) umgeben ist und sich in zentraler Lage von Stadtteilen befindet. Diese schließt direkt an das Erschließungsoder Hauptverkehrsstraßennetz an. Aufgrund der Lage im Straßennetz ist gegebenenfalls sogar Durchgangsverkehr vorhanden (Abbildung 36).
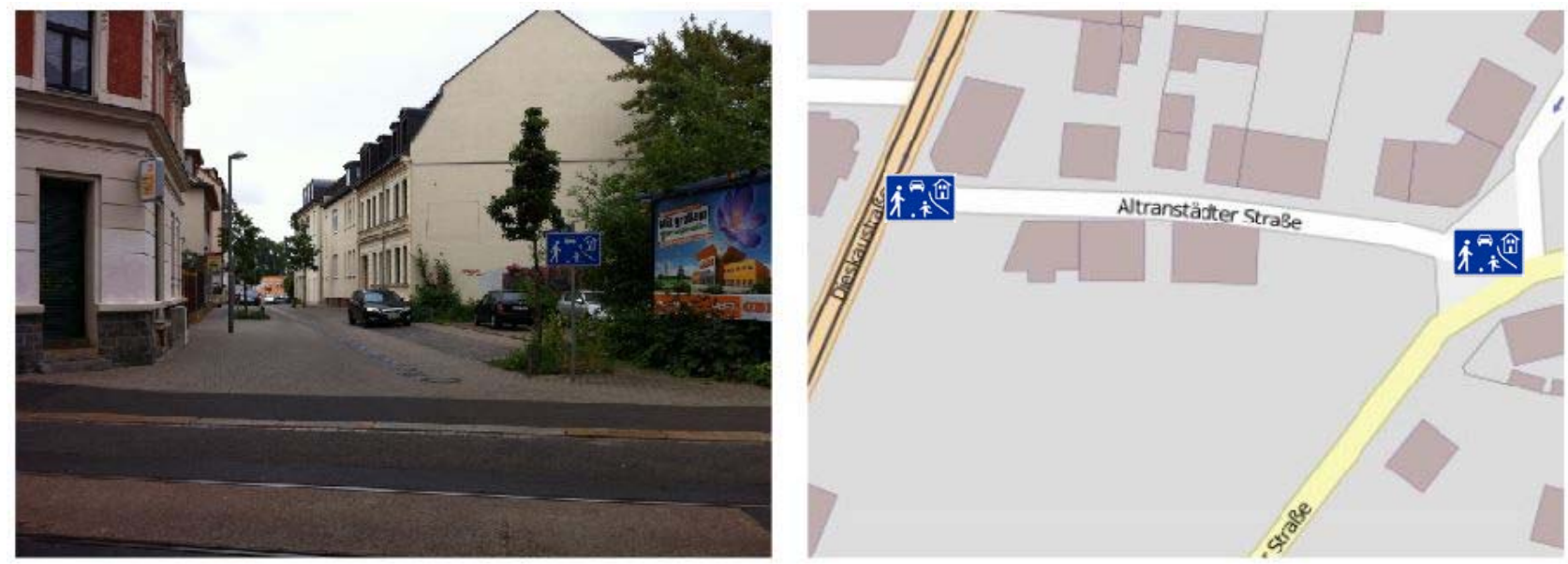

Abbildung 36: Beispiel einer Quartiersstraße mit ausgewiesenem Verkehrsberuhigtem Bereich

\section{Sammelstraße mit ausgewiesenem Verkehrsberuhigtem Bereich}

Straße, die mehrere Wohnstraßen mit dem übergeordneten Straßennetz verbindet. Im Regelfall ist diese ebenfalls von Wohnbebauung umgeben (Abbildung 37).
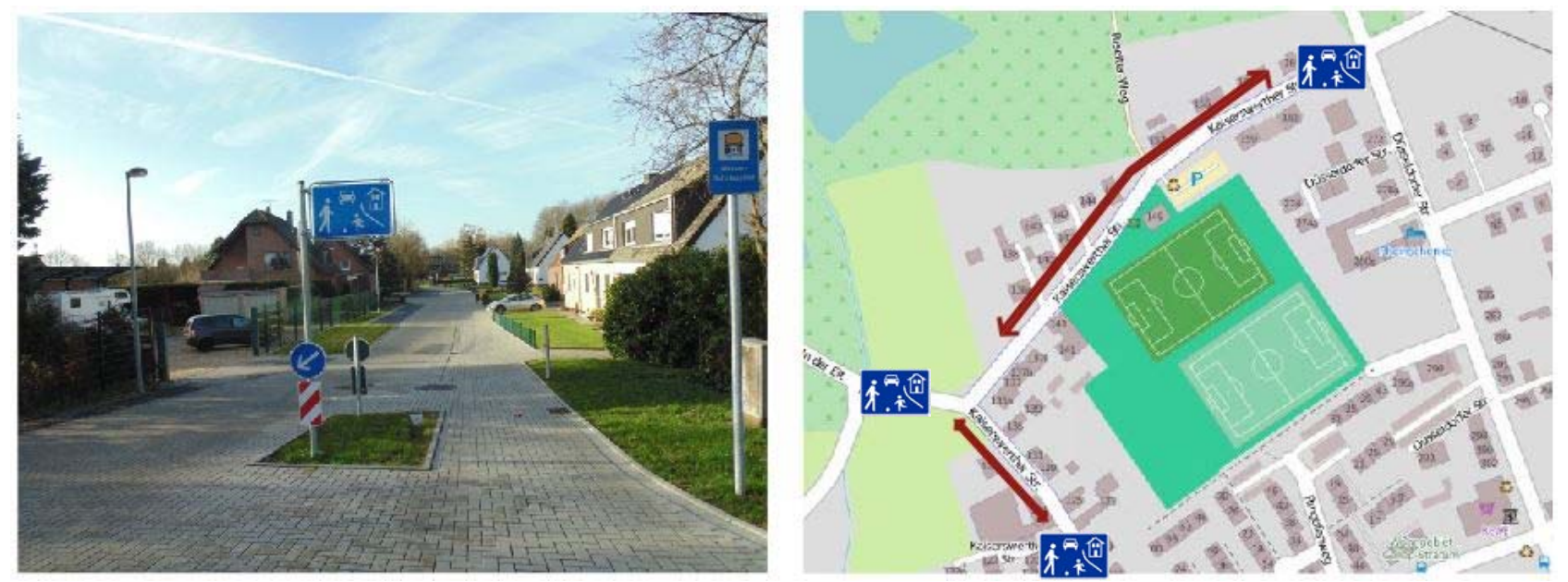

Abbildung 37: Beispiel einer Sammelstraße mit ausgewiesenem Verkehrsberuhigtem Bereich 


\section{Erschließungsstraße mit ausgewiesenem Verkehrsberuhigtem Bereich}

Straße, die das Hauptverkehrsstraßennetz mit dem nachgeordneten Straßennetz verbindet. In der Randnutzung tritt sowohl Wohnnutzung als auch gewerbliche Nutzung auf (Abbildung 38).
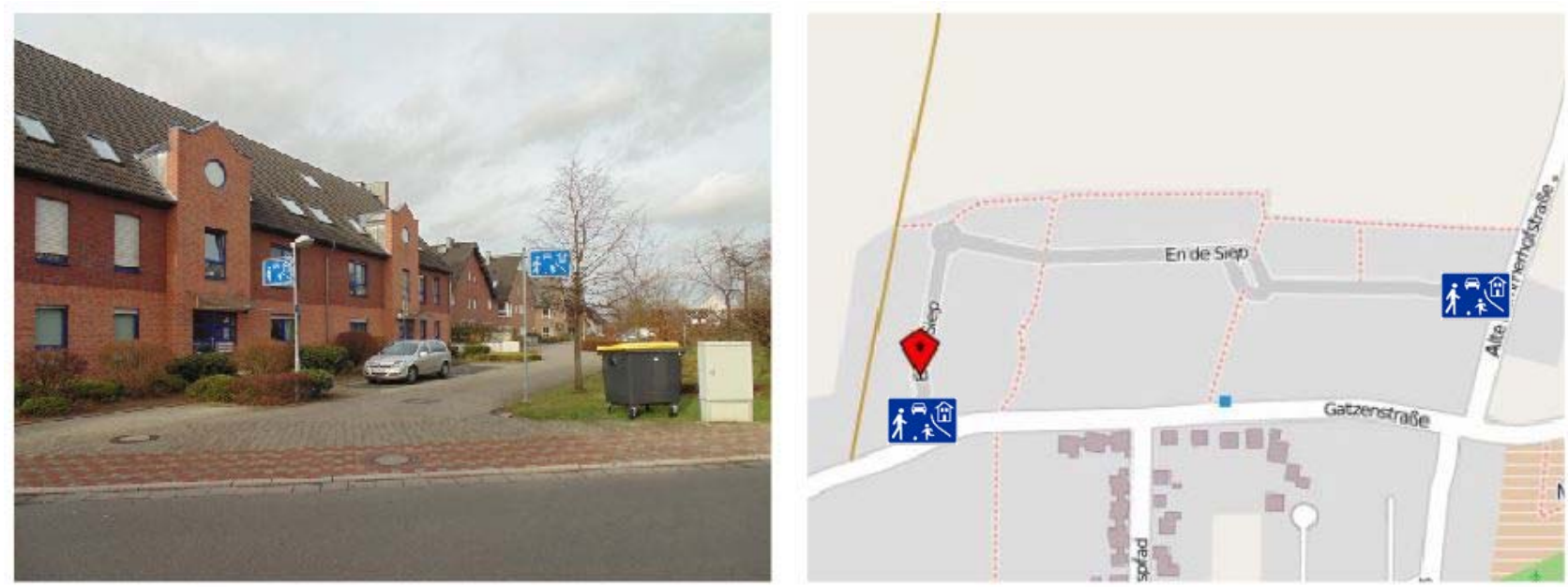

Abbildung 38: Beispiel einer Erschließungsstraße mit ausgewiesenem Verkehrsberuhigtem Bereich

\section{Geschäftsstraße mit ausgewiesenem Verkehrsberuhigtem Bereich}

Straße, deren Randbebauung überwiegend eine gewerbliche Nutzung (vorrangig Einzelhandel) aufweist. In kleineren Kommunen handelt es sich häufig um die zentrale Einkaufsstraße (Abbildung 39).
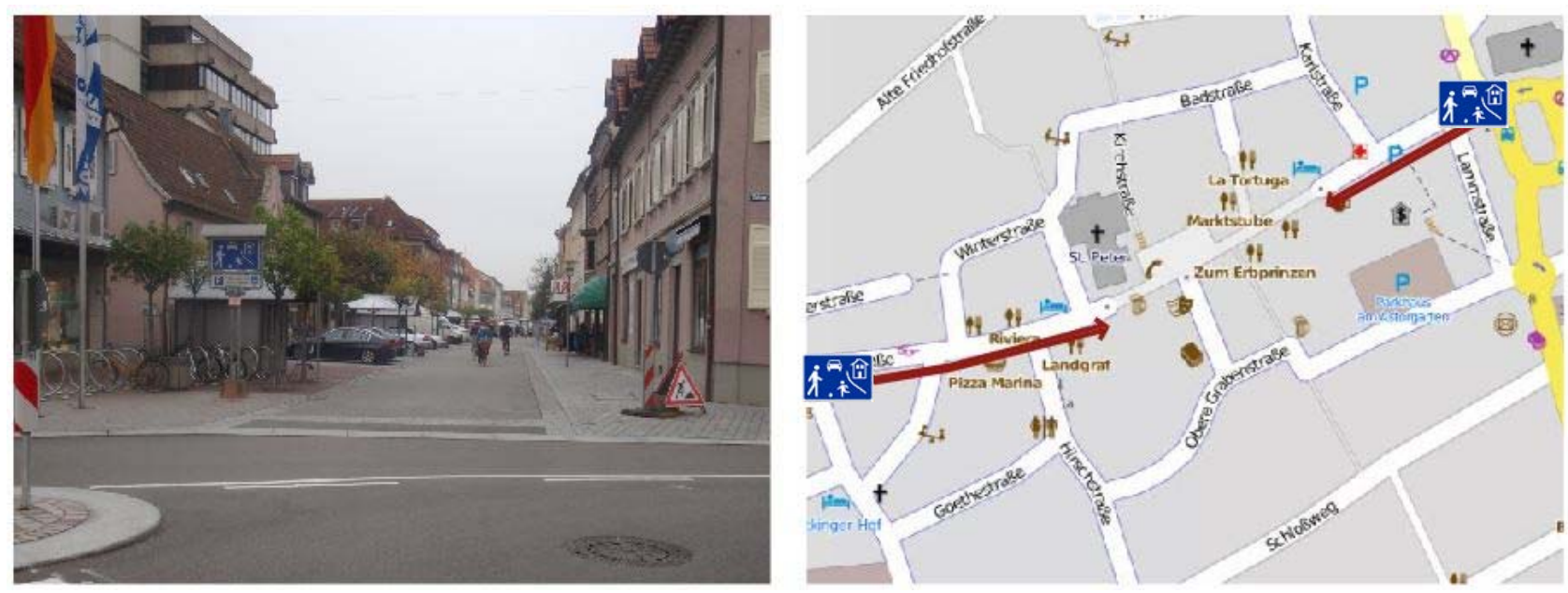

Abbildung 39: Beispiel einer Geschäftsstraße mit ausgewiesenem Verkehrsberuhigtem Bereich

\section{Sonstige Straße mit ausgewiesenem Verkehrsberuhigtem Bereich}

Einzelne Knotenpunkte, Plätze, Parkplätze, Zufahrten, (Wohn-)Straße mit öffentlichen Einrichtungen oder sonstige Bereiche im öffentlichen Straßenraum, die sich nicht den o.g. Kategorien zuordnen lassen (Abbildung 40). 

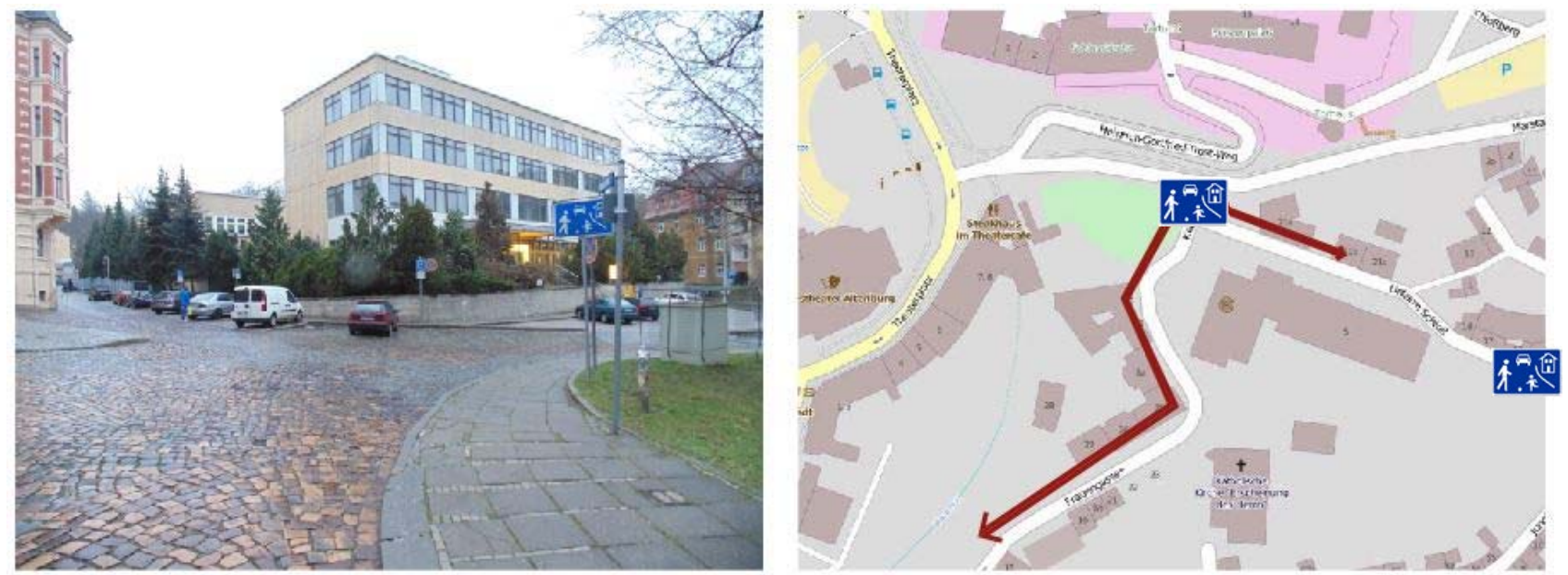

Abbildung 40: Beispielbereich Sonstige Straße mit ausgewiesenem Verkehrsberuhigtem Bereich

Auf eine detaillierte Betrachtung der Kategorie „Hauptverkehrsstraße“ wurde verzichtet. Hintergrund war die Tatsache, dass zwar ein Untersuchungsobjekt im Kollektiv mit diesen Merkmalen vorhanden war, allerdings die Funktion "Hauptverkehrsstraße“ kein geeigneter Einsatzbereich des VZ 325 darstellt. Der erstellte Steckbrief blieb mit der Straßenfunktion „Sonstiges“ aber Bestandteil der Dokumentationsreihe.

Die Straßen bzw. Straßenabschnitte der Gruppe „Sonstige“ wurden aufgrund ihrer vielfältigen Unterschiede untereinander keiner vertiefenden Betrachtung unterzogen. Zur allgemeinen Veranschaulichung, in welchen Bereichen VBB in Deutschland umgesetzt wurden, sind die Steckbriefe dieser Untersuchungsobjekte der Studie beigefügt worden.

Das erhobene Kollektiv setzte sich gemäß Abbildung 41 zusammen. Danach ließen sich 132 der 302 Bereiche eindeutig als Wohnstraße kategorisieren, 54 als Quartierstraße, 12 als Erschließungs- und Sammelstraße und 48 als Geschäftsstraßen. Weitere 50 Untersuchungsbereiche wurden der Kategorie „Sonstige“ zugeordnet.

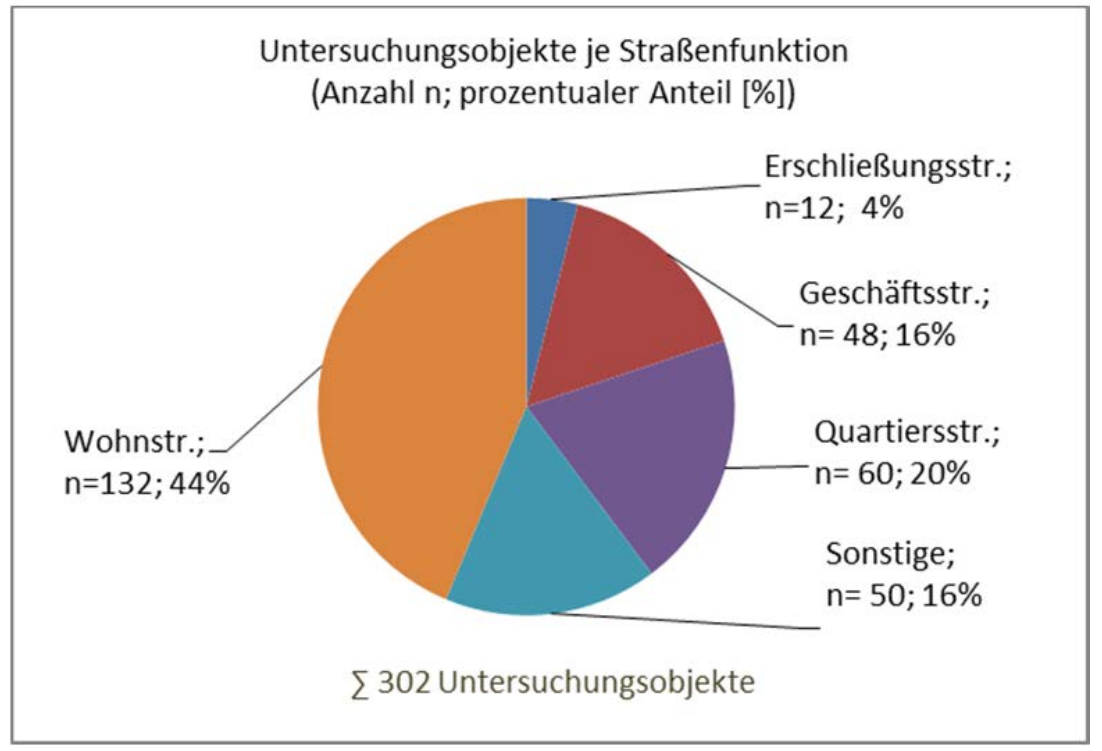

Abbildung 41: Aufteilung der erhobenen Untersuchungsobjekte je Straßenfunktion 
Die ersten Auswertungen der Untersuchungsobjekte nach der Kategorisierungsstufe 1 (Straßenfunktion) zeigten, dass eine weitere Kategorisierungsstufe sinnvoll war, nämlich die nach der Art der Verkehrsführung (s.u. Kategorisierungsstufe 2). Weitere Stichproben waren aufgrund der großen Vielfalt der Ausgestaltungsmerkmale in den erhobenen VBB zu klein, so dass aussagekräftige Rückschlüsse nicht gezogen werden konnten. Deshalb beschränkten sich die Auswertungen auf die beiden Hauptkategorien Straßenfunktion und Verkehrsführung.

\section{Kategorisierungsstufe: Verkehrsführung}

Die zweite Kategorisierungsstufe umfasste die „Verkehrsführung“, die im ausgewiesenen Verkehrsberuhigten Bereich vorlag. Hier wurde zwischen einer Mischverkehrsfläche, einer optischen Trennung und einer baulichen Trennung der Verkehrsarten unterschieden. Dabei konnten in einem ausgewiesenen Verkehrsberuhigten Bereich natürlich auch zwei oder mehr Verkehrsführungen umgesetzt worden sein. Es kam bspw. vor, dass eine bauliche Trennung im Übergangsbereich umgesetzt wurde und diese im Laufe des Bereichs zu einer optisch getrennten Fläche wurde. Bei den 302 Untersuchungsobjekten konnten demnach die folgenden „reinen“ und "kombinierten“ Verkehrsführungen festgestellt werden:

- Mischverkehr (Mi)

- Optische Trennung (oT)

- Bauliche Trennung (bT)

- Mischverkehr mit optischer Trennung (Mi und oT)

- Mischverkehr mit baulicher Trennung (Mi und oT)

- Optische und bauliche Trennung (OT und bT)

- Mischverkehr, optische und bauliche Trennung (Mi, oT und bT)

\section{Bauliche Trennung}

Die bauliche Trennung kann entweder durch die Ausstattung des ausgewiesenen VBBs, wie bspw. Bänke, Poller, Parkstände oder Begrünung erzeugt werden, aber auch durch Rund- oder Hochborde. Durch die bauliche Trennung wird stets eine Art Gehweg geschaffen, auch wenn dieser Abschnittsweise unterbrochen sein kann (Abbildung 42).
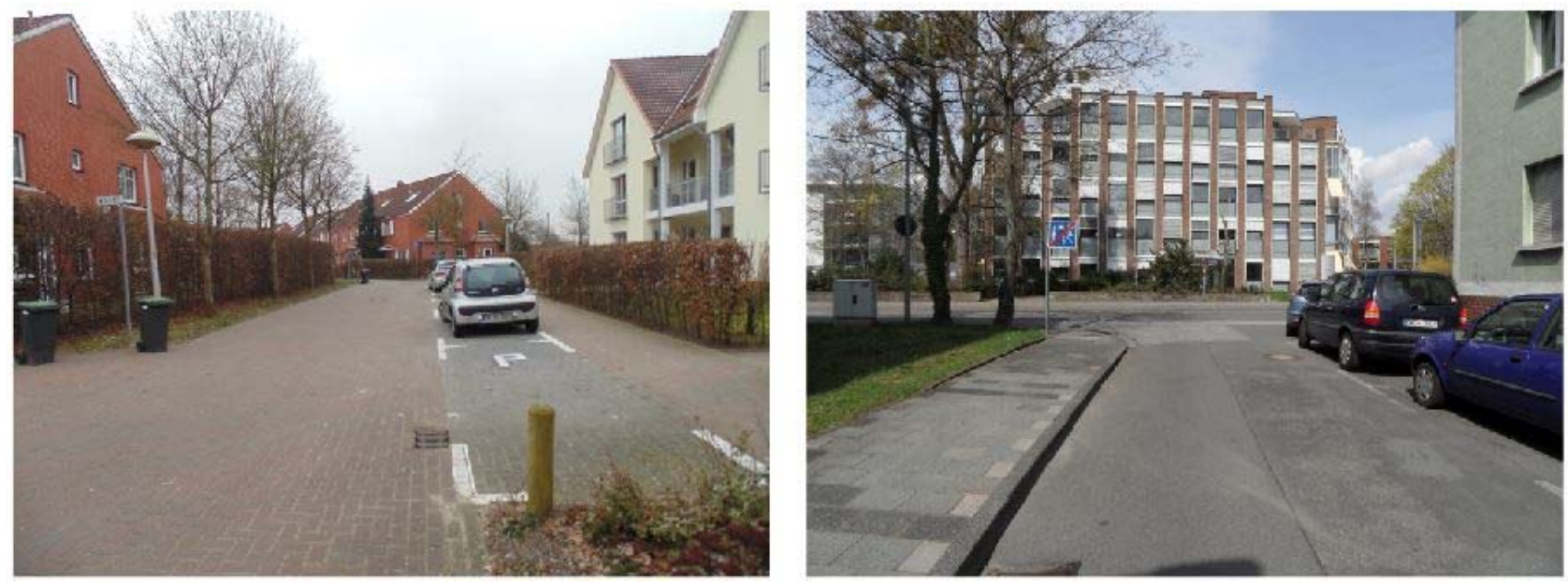

Abbildung 42: Beispiele für bauliche Trennungen (Fotos: IGS mbH 2013) 


\section{Optische Trennung}

Die optische Trennung wird, wie der Name schon sagt, optisch erzeugt. Eine optische Trennung ist immer niveaugleich ausgebaut und somit zu jedem Zeitpunkt überfahrbar. Zumeist wird die optische Trennung, durch eine Rinne, Materialwechsel, Farbwechsel oder Musteränderung des Pflasterverlaufs eingerichtet (Abbildung 43). Durch die optische Trennung wird ebenfalls optisch ein (überfahrbarer) Randbereich geschaffen.
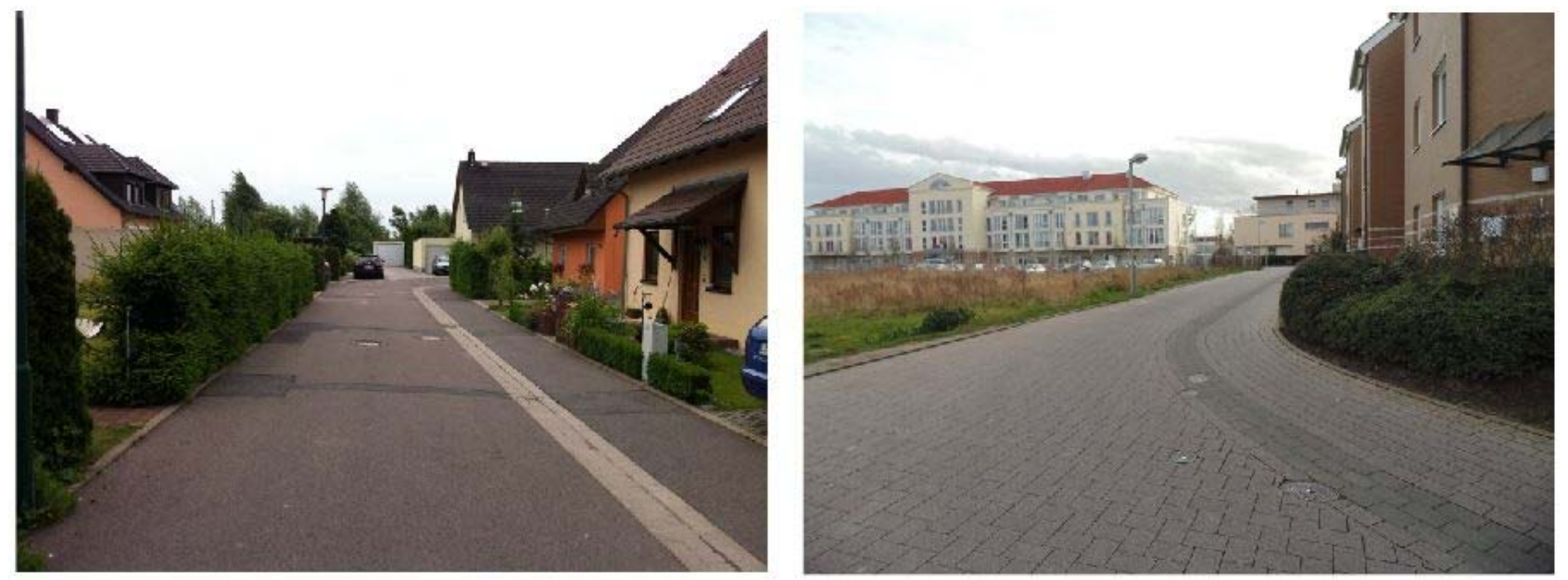

Abbildung 43: Beispiele für optische Trennungen (Fotos: IGS mbH 2013)

\section{Mischverkehr}

Bei einer Mischverkehrsfläche werden die Verkehrsflächen verschiedener Verkehrsteilnehmer zusammengelegt, so dass es keine Randbereiche/Gehwege oder Fahrbahnen/Fahrgassen mehr gibt. Das Parken findet in diesen Bereichen ausschließlich in den Randbereichen statt, so dass auch die Fußgänger und Radfahrer zur Benutzung der mittleren Fläche aufgefordert werden. Der Verkehr kann sich „frei“ im Straßenraum bewegen (Abbildung 44).
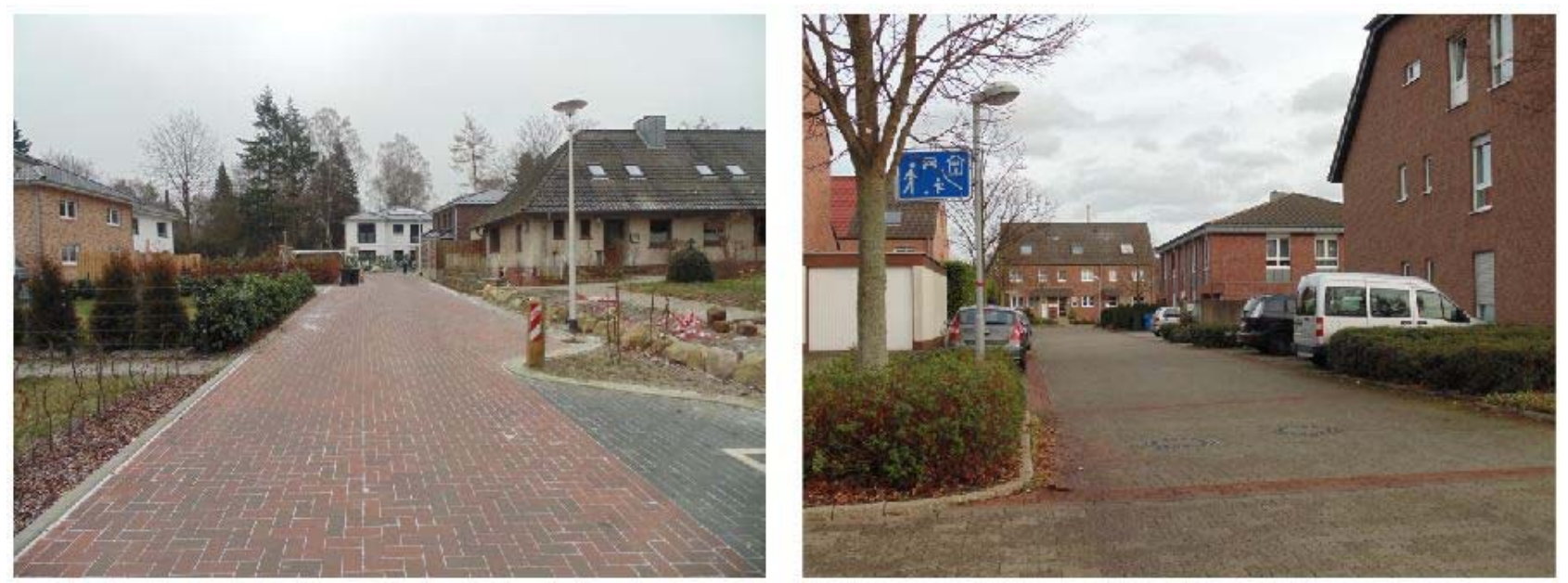

Abbildung 44: Beispiele für Mischverkehrsflächen (Fotos: IGS mbH 2013) 


\section{Unfallanalyse der Untersuchungsobjekte}

Das vorliegende Kapitel dokumentiert die Ergebnisse der Unfallanalysen, die für die 302 erhobenen und ausgewiesenen Verkehrsberuhigten Bereiche durchgeführt wurden. Die Unfallanalysen konzentrierten sich darauf, die Besonderheiten des Unfallgeschehens von VBB im Vergleich zu innerörtlichen Straßen herauszuarbeiten.

Als Vergleichskollektiv wurden die innerörtlichen Unfalldaten des Statistischen Bundesamtes (Destatis 2011) herangezogen. Insofern die Unfalldaten für die 302 Untersuchungsobjekte erhoben werden konnten, lagen diese flächendeckend für 6 Jahre vor. Die Analyse der Unfallmerkmale erfolgte ausschließlich nach Unfällen der Kategorie 1-4. Dies lag zum einen daran, dass keine Unfalldaten für die Unfälle der Kategorien 5 und 6 für das Vergleichskollektiv vorlagen. Zum anderen konnten diese auch nicht flächendeckend für die VBB erhoben werden. Aufgrund dessen wurde das Kollektiv der Unfalldaten der VBB, in denen alle Unfälle der Kategorie 1-4 flächendeckend für 6 Jahre vorlagen, als „Kollektiv Kat. 1-4“ bezeichnet.

Für eine weitere Analyse zu Unfällen der Kategorie 5 und 6 wurde ein Unfallkollektiv der unfallauffälligsten Untersuchungsobjekte aus dem oben genannten „Kollektivs Kat. 1-4“ gebildet. In diesem Kollektiv wurden alle Untersuchungsobjekte zusammengefasst, die mindestens drei oder mehr Unfälle aufzeigten. Die Unfalldaten konnten ausschließlich für das Jahr 2011 vollständig erhoben werden, weshalb das Unfallkollektiv daher als „Kollektiv 2011“ bezeichnet wurde. Das Ziel dieser weiteren Auswertung war es, einen Überblick zu erhalten, wie sich das Unfallgeschehen aller Unfälle der Untersuchungsobjekte verteilt und ob wesentliche Unterschiede im Vergleich zum Unfallgeschehen der Kategorie 1-4 (,Kollektiv Kat. 1-4“ und Unfälle innerorts Kategorie 1-4) gibt. Die Ergebnisse der Unfallanalyse des „Kollektiv 2011“ sind in Anhang 3 dokumentiert.

\subsection{Unfallanalysen „Kollektiv Kat. 1-4“}

\subsubsection{Datengrundlage}

Von den 302 vorliegenden Untersuchungsobjekten, konnten die Unfälle der Kategorie 1-4 für 278 Beispiele flächendeckend über 6 Jahre erhoben werden.

Für Sachsen und Sachsen-Anhalt lagen Unfalldaten aus den Jahren 2006-2011 vor, für BadenWürttemberg, Hessen, Nordrhein-Westfalen und Thüringen aus den Jahren 2007-2012.

Die Unfalldaten der einzelnen Beispiele wurden in den Steckbriefen (Anlagenband) dokumentiert und in eine gemeinsame „Unfalliste“ eingetragen. Für besonders unfallauffällige Untersuchungsbeispiele war ursprünglich angedacht, eigenständige Unfallisten je VBB zu erstellen. Da die Unfallanzahlen allerdings für die einzelnen Beispiele sehr gering waren, wurde eine Unfallliste erstellt, in der alle Unfalldaten zu den VBB enthalten waren. Die zusammengefasste Unfalliste hatte zusätzlich den Vorteil, dass Unfälle in Übergangsbereichen nicht doppelt gezählt wurden. Dies konnte der Fall sein, wenn zwei ausgewiesene Verkehrsberuhigte Bereiche unmittelbar aneinander grenzten und im Übergangsbereich Unfälle zu verzeichnen waren. Diese wurden in den Steckbriefen jeweils beiden Untersuchungsobjekten zugeordnet.

Es lagen insgesamt 244 Unfälle der Kategorie 1-4 vor, die in einer Datenbank zusammengetragen wurden und deren Auswertungsergebnisse in den nachfolgenden Kapiteln beschrieben werden. Die Verteilung der Untersuchungsobjekte je Bundesland ist der Tabelle 12 zu entnehmen. Die 278 Beispiele konnten in 9 Bundesländern und 69 Kommunen erhoben werden. 
Tabelle 12: Zusammensetzung der Untersuchungsobjekte je Bundesland

\begin{tabular}{|l|c|c|}
\hline & Anzahl der Kommunen & Anzahl der Untersuchungsobjekte \\
\hline Baden-Württemberg & 20 & 49 \\
\hline Hessen & 31 & 136 \\
\hline Niedersachsen & 1 & 2 \\
\hline Nordrhein-Westfalen & 5 & 23 \\
\hline Rheinland-Pfalz & 3 & 19 \\
\hline Sachsen & 3 & 19 \\
\hline Sachsen-Anhalt & 4 & 8 \\
\hline Schleswig-Holstein & 1 & 4 \\
\hline Thüringen & 1 & $\mathbf{2 7 8}$ \\
\hline Summe & 69 & 23 \\
\hline
\end{tabular}

Die Unfallliste enthält die klassischen Unfallattribute, die - gemäß dem Merkblatt zur Örtlichen Unfalluntersuchung in Unfallkommissionen (MUko) (FGSV 316/1, 2012) - zur Anwendung empfohlen werden. Da es sich im vorliegenden Fall nicht um Unfallhäufungen handelte, entfielen die Angaben zur Unfallhäufung und der Vorgangsnummer. Nachfolgende Daten zu den Unfällen sind in der Unfalliste enthalten:

- Beschreibung der Örtlichkeit (Ort, Straße, Ortslage),

- Unfalldatum, Wochentag, Uhrzeit,

- Lichtverhältnisse, Straßenzustand,

- Anzahl der Getöteten, Schwerverletzten, Leichtverletzten,

- Anzahl der Beteiligten am Unfall,

- Art der Verkehrsbeteiligung des ersten und des zweiten Beteiligten,

- Unfallkategorie, Unfalltyp, Unfallart,

- Wichtigste Unfallursache,

- Besondere Unfallumstände. 
Die Unfalldaten wurden in der Liste zusätzlich um Angaben zu der Straßenkategorie und der Verkehrsführung ergänzt. Hieraus konnten erste Rückschlüsse zwischen dem Unfallgeschehen und der Örtlichkeit gezogen werden. Gemäß der Kategorisierung (Kapitel 9) wurden die nachfolgenden Angaben aus jedem Steckbrief zu den einzelnen Unfällen ergänzt:

- Straßenkategorie (Erschließungsstraße, Geschäftsstraße, Hauptverkehrsstraße, Quartiersstraße, Wohnstraße und Sonstiges),

- Verkehrsführung (bauliche Trennung, optische Trennung, Mischverkehr) und

- die Länge des ausgewiesenen Verkehrsberuhigten Bereichs.

Aus der Abbildung $\mathbf{4 5}$ kann entnommen werden, dass der Anteil der Verkehrsberuhigten Bereiche, in denen unterschiedliche Verkehrsführungen umgesetzt wurden, sehr hoch ist. In der Summe waren es 44\% (12\% bauliche Trennung und Mischverkehr, 19\% optische und bauliche Trennung, 9\% optische Trennung und Mischverkehr, 4\% Mischverkehr und optische und bauliche Trennung). 23\% der Beispiele wiesen ausschließlich Mischverkehr auf, 19\% eine reine bauliche Trennung bzw. 14\% eine optische Trennung auf.

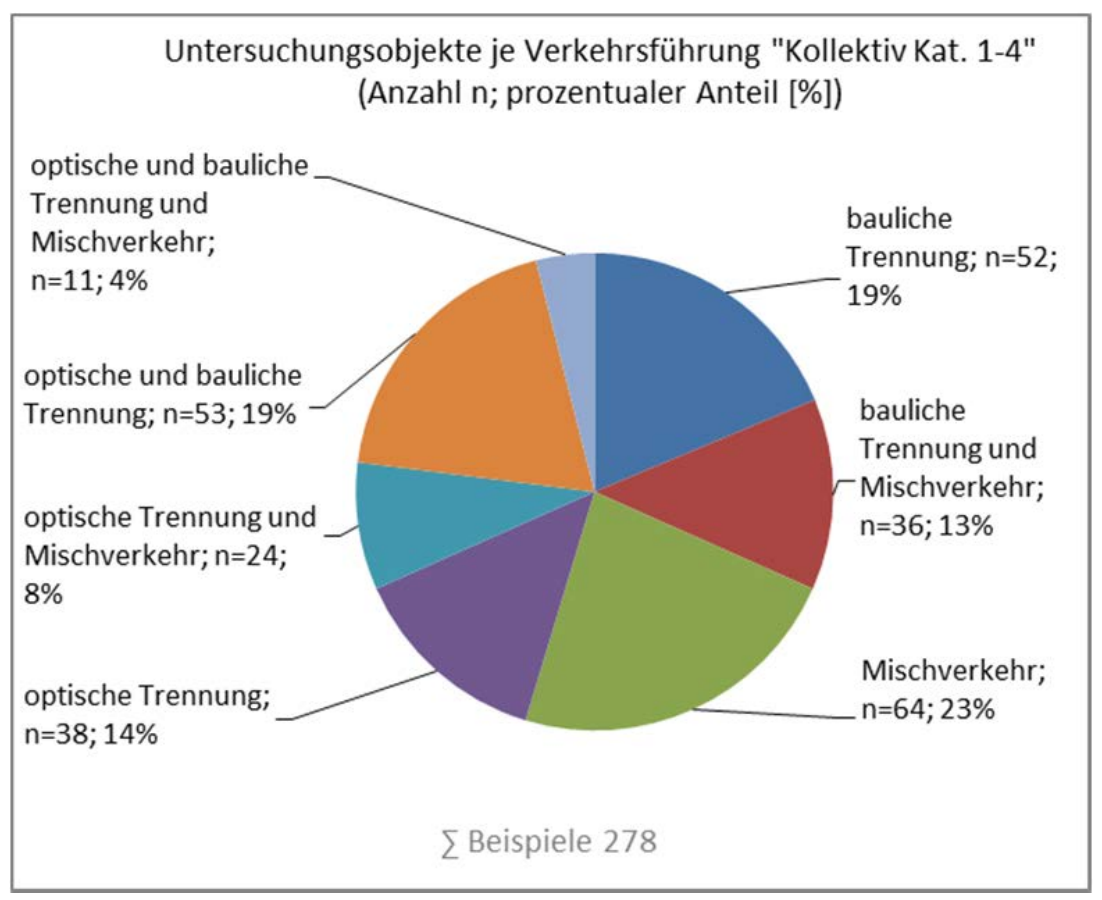

Abbildung 45: Verteilung der Untersuchungsobjekte des „Kollektivs Kat. 1-4“ je Verkehrsführung

Bei der Betrachtung der Verteilung nach der Straßenfunktion, fiel auf, dass $44 \%$ der Unfälle der Kategorie 1-4 in Wohnstraßen lagen. 16\% lagen in Geschäftsstraßen und 20\% in Quartiersstraßen. Die restlichen 20\% verteilten sich auf Erschließungsstraßen und sonstige Straßen (Abbildung 46). 


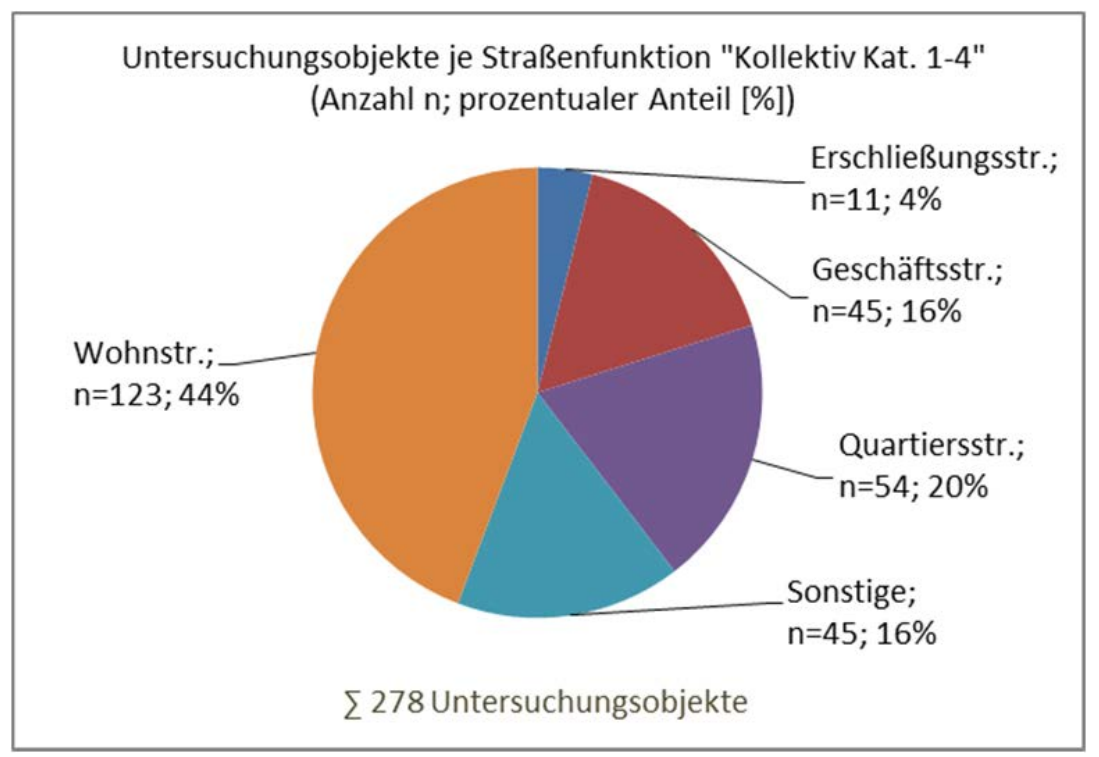

Abbildung 46: Verteilung der Untersuchungsobjekte des "Kollektivs Kat. 1-4" je Straßenfunktion

Auffällig war, dass der Anteil der Geschäftsstraßen, die als Verkehrsberuhigte Bereiche ausgewiesen wurden, $16 \%$ der erhobenen Untersuchungsobjekte ausmachte. Diese Straßen waren jedoch nicht "zufällig“ in der Auswahl enthalten. Rund die Hälfte der erhobenen Geschäftsstraßen war im Kollektiv, weil sie aus den Unfalldaten der Länder als unfallauffällig erkannt wurden. Die Beschilderung einer Geschäftsstraße als VBB ist im klassischen Sinne eigentlich nicht vorgesehen. Hierzu sieht die StVO die Einrichtung eines sog. verkehrsberuhigten Geschäftsbereichs gemäß $\S 45$, Abs. 1d, vor, indem die Fußgänger die Straße nicht in ihrer ganzen Breite benutzen dürfen und der Fahrzeugverkehr auf der Straße Vorrang hat.

Entsprechend hoch war auch der Anteil der „Sonstigen Straßen“ mit 16\%. Diese wurden in über 85\% der Fälle zufällig erhoben (Bearbeiter bekannt, vor Ort entdeckt oder sie wurden in der Umfrage als Beispiel genannt). In dieser Gruppe waren durchaus Streckenabschnitte enthalten, bei denen die Beschilderung als VBB sinnvoll war (z.B. in Wohnstraßen mit Kindergärten oder Schulen, Altstadtgebieten, etc.), jedoch waren auch Beispiele enthalten, bei denen die Ausweisung eines VBB keine geeignete Maßnahme zur Verkehrsberuhigung darstellte (vgl. Abbildung 47).

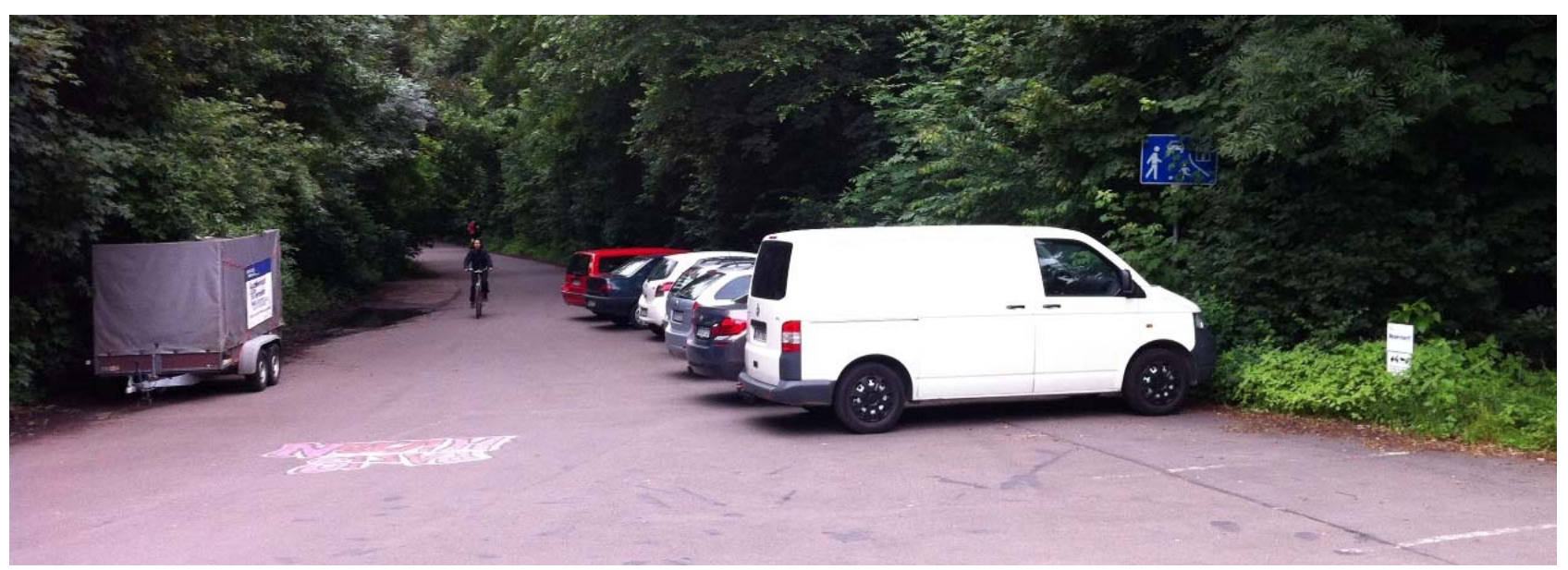

Abbildung 47: Untersuchungsobjekte „Sonstige Straßen“ (Sackgasse zum Park) (Foto: IGS mbH 2013) 
Die jeweiligen Abschnittslängen der ausgewiesenen Verkehrsberuhigten Bereiche variierten je Beispiel erheblich. Die kürzesten Bereiche, die erhoben wurden, lagen bei rund $30 \mathrm{~m}$. Einer davon lag in einer Geschäftsstraße, er fungierte als Zugang zu einem Krankenhaus und einem Park. Der andere nur 30m lange ausgewiesene Verkehrsberuhigte Bereich lag am Ende einer Sackgasse in einer Wohnstraße (mit einem Durchstich zu einer anderen Wohnstraße). Die längsten Verkehrsberuhigten Bereiche, die erhoben wurden, besaßen eine Abschnittslänge von 1,2km und $1,8 \mathrm{~km}$. Es handelte sich hierbei um komplette Wohngebiete, in denen flächendeckend ein ausgewiesenes Verkehrsberuhigtes Gebiet umgesetzt wurde. Aus der Tabelle 12 kann die durchschnittliche Länge je Straßenfunktion oder aller erhobenen VBB abgelesen werden.

Die durchschnittliche Länge aller erhobenen Verkehrsberuhigten Bereiche des Kollektivs Kat. 1-4 lag bei $285 \mathrm{~m}$. Dadurch, dass in Wohnstraßen häufig ganze Gebiete als Verkehrsberuhigte Bereiche ausgewiesen wurden, lag die Durchschnittslänge über dem gesamten Durchschnittswert (Wohnstraßen 330m). Die Geschäftsstraßen besaßen eine durchschnittliche Länge von rund $250 \mathrm{~m}$ und lagen somit unter der Durchschnittslänge aller Beispiele. Die Erschließungsstraßen lagen mit einer Länge von rund 450m auch über dem Durchschnitt. Da es sich bei der Gruppe jedoch nur um eine Anzahl von elf Beispielen handelte, ist dieser Wert nicht aussagekräftig.

Tabelle 13: Durchschnittliche Länge der Untersuchungsobjekte des "Kollektivs Kat. 1-4" je Straßenfunktion

\begin{tabular}{|l|c|c|c|}
\hline Straßenfunktion & $\begin{array}{c}\text { Länge [m] aller } \\
\text { Untersuchungsobjekte }\end{array}$ & Anzahl & $\begin{array}{c}\text { Durchschnittliche } \\
\text { Länge [m] }\end{array}$ \\
\hline Erschließungsstraße & 4.983 & 11 & 453 \\
\hline Geschäftsstraße & 11.208 & 45 & 249 \\
\hline Quartiersstraße & 12.301 & 54 & 228 \\
\hline Sonstige & 10.197 & 45 & 227 \\
\hline Wohnstraße & 40.581 & 123 & 330 \\
\hline Summe & 79.270 & 278 & 285 \\
\hline
\end{tabular}

In der Datenbank lagen 187 Beispiele vor (68\%), in denen kein Unfall der Kategorie 1-4 in sechs Jahren auftrat (vgl. Tabelle 14), obwohl vor der Auswahl des Kollektivs, explizit auch nach unfallauffälligen Verkehrsberuhigten Bereichen gesucht wurde (vgl. Kapitel 9).

Bei der näheren Betrachtung der Bereiche, in denen Unfälle der Kategorie 1-4 vorlagen, konnte erkannt werden, dass die überwiegende Anzahl höchstens 1-3 Unfälle der Kategorie 1-4 in 6 Jahren aufwies. Ausgewiesene Verkehrsberuhigte Bereiche, in denen mehr als drei Unfälle der Kategorie 1-4 in sechs Jahren auftraten, waren Einzelfälle.

Tabelle 14: Anzahl der Unfälle des "Kollektivs Kat. 1-4" je Untersuchungsobjekt

\begin{tabular}{|l|c|c|c|c|c|c|c|c|c|c|c|c|}
\hline $\begin{array}{l}\text { Anzahl der Unfälle der } \\
\text { Kat. 1-4 in 6 Jahren }\end{array}$ & 0 & 1 & 2 & 3 & 4 & 5 & 6 & 7 & 8 & 9 & 12 & 19 \\
\hline Anzahl der VBB & 187 & 40 & 19 & 17 & 1 & 2 & 5 & 1 & 3 & 1 & 1 & 1 \\
\hline Prozentuale Verteilung & $68 \%$ & $14 \%$ & $7 \%$ & $7 \%$ & $0 \%$ & $1 \%$ & $2 \%$ & $0 \%$ & $1 \%$ & $0 \%$ & $0 \%$ & $0 \%$ \\
\hline
\end{tabular}


Die Verteilung der Unfallkategorien je Jahr, kann aus der nachfolgenden Abbildung 48 entnommen werden. Die Unfälle aus dem Jahr 2013 lagen deshalb vor, weil bei drei Beispielen die Unfalldaten nur für den Zeitraum 2008-2013 erhoben werden konnten. Für fast alle Beispiele konnten die Unfälle aus den Jahren 2007-2011 erhoben werden (fünf Jahre). Zudem wurden bei 38 Beispielen das Jahr 2006 zusätzlich erhoben und bei 237 Beispielen das Jahr 2012. Somit lagen zu allen Beispielen Unfalldaten aus sechs Jahren vor.

Die Verteilung der Unfälle der Kategorie 1-4, die in den ausgewiesenen Verkehrsberuhigten Bereichen auftraten, weicht von den Verteilungen, die für die jeweiligen Bundesländer gemacht wurden, ab (Kapitel 7.5.1 bis 7.5.5). Hier dominierten zwar ebenso die Unfälle der Kategorie 3, jedoch lag die Anzahl der Unfälle der Kategorie 4 nicht in etwa auf dem gleichen Niveau wie die Anzahl der Unfälle der Kategorie 2. In der Datenbank der ausgewiesenen Verkehrsberuhigten Bereiche lagen deutlich weniger Unfälle der Kategorie 4 vor, als in den Bundesländern, in denen neben den VBB auch weitere geschwindigkeitsreduzierte Bereiche (GRB) in der Datengrundlage enthalten waren.

Eine Erklärung hierfür könnte sein, dass schwerwiegende Unfälle mit Sachschaden, bei denen ein Kraftfahrzeug aufgrund eines Unfallschadens von der Unfallstelle abgeschleppt werden muss (Kfz nicht fahrbereit), aufgrund des geringen Geschwindigkeitsniveaus in VBB seltener auftreten.

In den nachfolgenden Kapiteln werden die Ergebnisse der Auswertungen zu den Unfallkategorien, Unfalltypen, Unfallarten, Beteiligten und Unfallursachen dargestellt. Bei den Auswertungen zu den Unfallkategorien wurde genauer analysiert, in welchen Fällen Unfälle der Kategorie 4 in ausgewiesenen Verkehrsberuhigten Bereichen überhaupt auftraten.

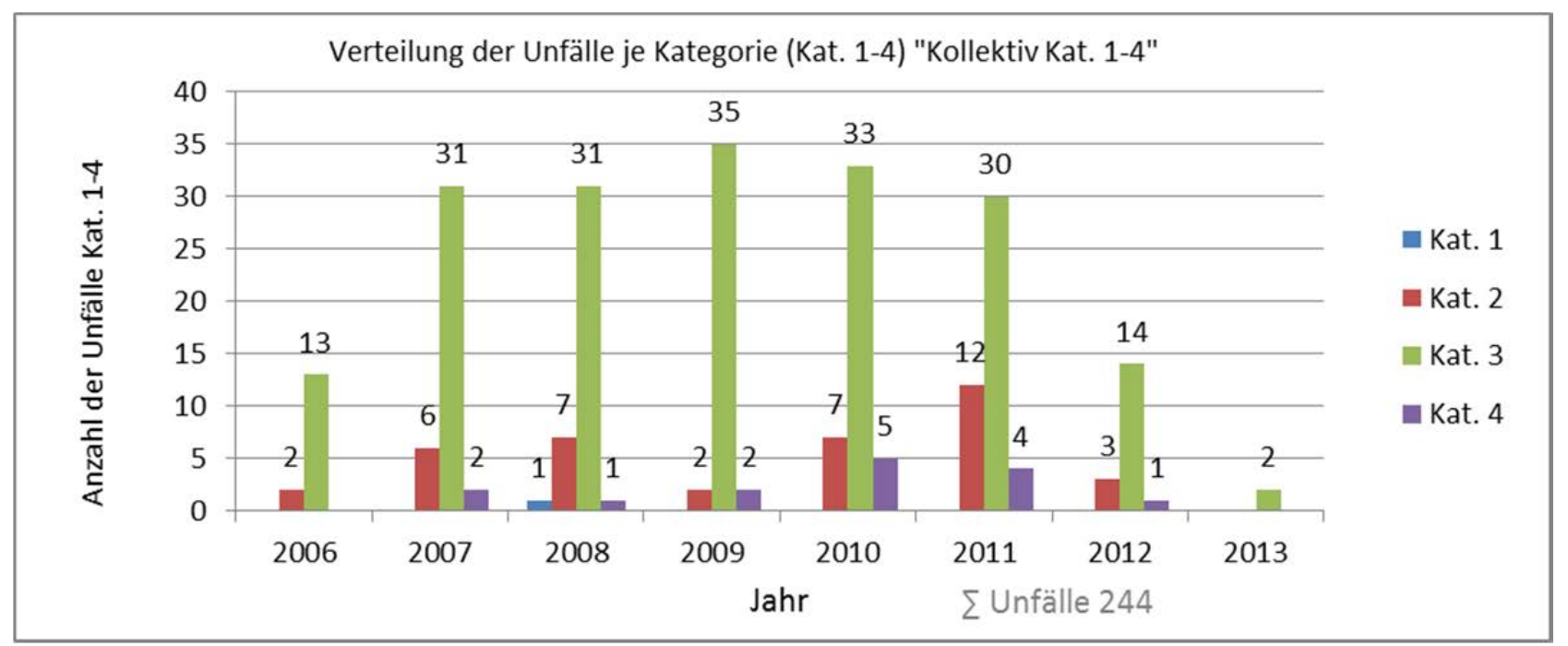

Abbildung 48: Verteilung der Unfälle je Kategorie (Kat. 1-4) des "Kollektivs Kat. 1-4" im Zeitraum 2006-2013

\subsubsection{Vorgehensweise und Vergleichskollektiv}

Um die Besonderheiten der Unfälle in ausgewiesenen Verkehrsberuhigten Bereichen erkennen zu können, wurden sie mit den innerörtlichen Unfalldaten des Statistischen Bundesamtes aus dem Jahr 2011 verglichen. Hierin sind alle innerörtlichen Unfälle der Kategorie 1-4 enthalten (auch die Unfälle in ausgewiesenen Verkehrsberuhigten Bereichen). Im Idealfall sollte das Vergleichskollektiv keine Unfälle aus ausgewiesenen Verkehrsberuhigten Bereichen enthalten, damit die Merkmale dieser Unfälle nicht das Ergebnis beeinflussen. Der Einfluss der Unfälle aus den ausgewiesenen Verkehrsberuhigten Bereichen war jedoch unwesentlich, da schon der Anteil 
der Unfälle mit Personenschaden aus den geschwindigkeitsreduzierten Bereichen an den innerörtlichen Unfällen mit Personenschaden nur ca. 0,8\% ausmachte (Abbildung 13). Werden zudem noch die Unfälle der Kategorie 4 mit in die Betrachtung hineingezogen, so war der Anteil der Unfälle in geschwindigkeitsreduzierten Bereichen am innerörtlichen Unfallgeschehen noch geringer (nur 0,6\%, Tabelle 15).

Tabelle 15: Anzahl und Anteil von Unfällen der Kategorie 1-4 von GRB im Vergleich zu allen innerörtlichen Unfällen (Quelle: Destatis, Reihe 7, 2012)

\begin{tabular}{|c|c|c|c|}
\hline \multirow{2}{*}{ Jahr } & \multicolumn{2}{|c|}{ Unfälle der Kat. 1-4 } & \multirow{2}{*}{ \%-Anteil } \\
\cline { 2 - 3 } & Innerorts & GRB & 0,59 \\
\hline 2006 & 276.444 & 1.633 & 0,61 \\
\hline 2007 & 284.581 & 1.723 & 0,67 \\
\hline 2008 & 275.036 & 1.831 & 0,67 \\
\hline 2009 & 264.962 & 1.776 & 0,63 \\
\hline 2010 & 247.221 & 1.555 & 0,65 \\
\hline 2011 & 258.862 & 1.682 & 0,66 \\
\hline 2012 & 255.267 & 1.674 & \\
\hline
\end{tabular}

Zu erwähnen sind hier noch einmal die Ergebnisse aus Kapitel 7.3, die aufzeigten, dass im Mittel nur 49\% der Unfälle (aus GRB) in ausgewiesenen Verkehrsberuhigten Bereichen geschehen sind (vgl. Tabelle 8). Alle anderen Unfälle fanden in Straßen mit sonstiger Beschränkung der zul. Höchstgeschwindigkeit statt. Deshalb kann davon ausgegangen werden, dass weniger als 0,3\% der innerörtlichen Unfälle in Verkehrsberuhigten Bereichen stattfanden. Da der Einfluss der Unfälle aus ausgewiesenen Verkehrsberuhigten Bereichen am innerörtlichen Unfallgeschehen sehr gering war, wurde das gesamte innerörtliche Unfallgeschehen als ausreichend genau angesehen, um Rückschlüsse auf die Besonderheiten von Unfällen in ausgewiesenen Verkehrsberuhigten Bereichen ziehen zu können.

Wie erwähnt, wurden als Vergleichsdaten die innerörtlichen Unfalldaten des Statistischen Bundesamtes aus dem Jahr 2011 herangezogen, da die Unfalldaten aus den erhobenen Untersuchungsobjekten für fast alle Beispiele aus dem Jahr 2011 vorlagen. Das Jahr 2011 war als Vergleichsjahr geeignet, da es sich nicht um ein Jahr mit besonderem Unfallgeschehen (in Bezug auf die innerörtlichen Unfälle) handelte (vgl. Abbildung 49).

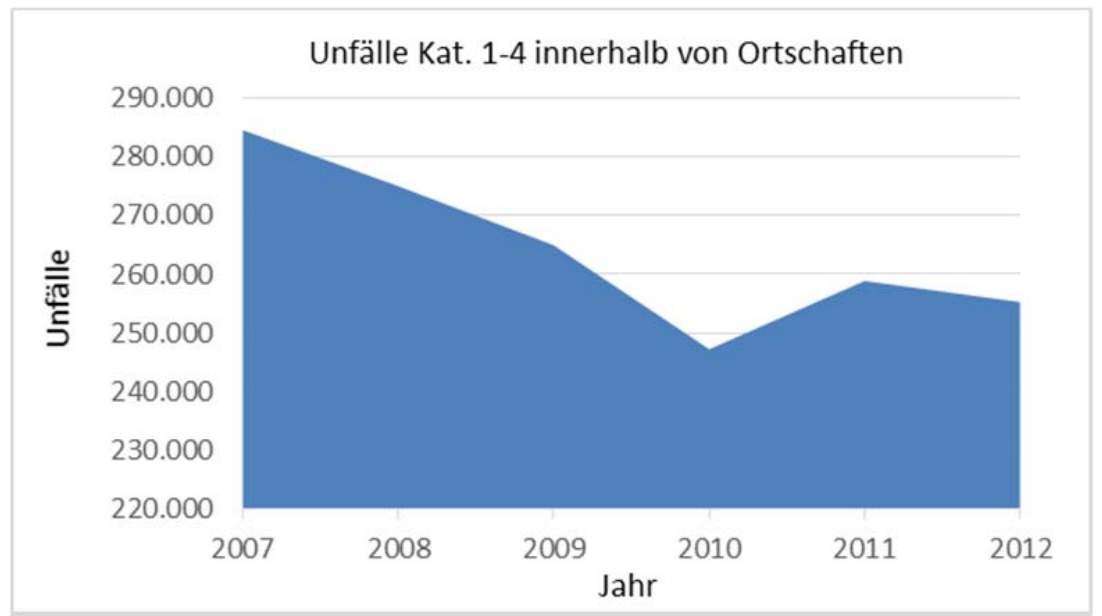

Abbildung 49: Unfälle der Kat. 1-4 innerhalb von Ortschaften (Quelle: Destatis, Reihe 7, 2012) 
Der Aufbau der nachfolgenden Kapitel (Ergebnisse der Unfallanalysen) orientierte sich an folgendem Vorgehen:

\section{Schritt:}

Die in den Überschriften benannten Unfallattribute (Unfallkategorien, Unfalltypen, etc.) des „Kollektivs Kat. 1-4“ wurden ausgewertet und mit den innerörtlichen Unfalldaten des Statistischen Bundesamtes verglichen.

\section{Schritt:}

Lagen Auffälligkeiten in Bezug auf ein bestimmtes Unfallmerkmal vor, so wurde dieses noch einmal genauer betrachtet. Als Beispiel kann an dieser Stelle genannt werden, dass z.B. Auffälligkeiten beim Unfalltyp 7 „Sonstiger Unfall“ in Kapitel 10.1.4 vorlagen. Der Unfalltyp 7 wurde dann in einem zweiten Schritt (im selben Kapitel) detaillierter in Bezug auf seine Unfallarten und Unfallursachen hin untersucht. An diesen Stellen kommt es daher vor, dass auch weitere Unfallattribute ausgewertet wurden, die nicht in der Überschrift der Kapitel benannt sind. Anzumerken ist, dass nicht in jedem Kapitel detaillierte Auswertungen (2. Schritt) durchgeführt wurden, z.B. wenn es keine Auffälligkeiten gab oder eine weitere Untergliederung - auch aus Gründen der Übersichtlichkeit - nicht sinnvoll waren.

\subsubsection{Unfallkategorien}

\section{Schritt}

In den 278 Untersuchungsobjekten lagen insgesamt 244 Unfälle der Kategorie 1-4 vor (vgl. Abbildung 50). Davon lagen 189 Unfälle der Kategorie 3 vor, das waren $77 \%$ aller erhobenen Unfälle. Weitere 39 Unfälle waren solche der Kategorie 2 (16\%). Es wurde nur ein Unfall der Kategorie $1^{6}$ aufgenommen. Alle weiteren Unfälle waren solche der Kategorie 4 (15 Unfälle, 6\%).

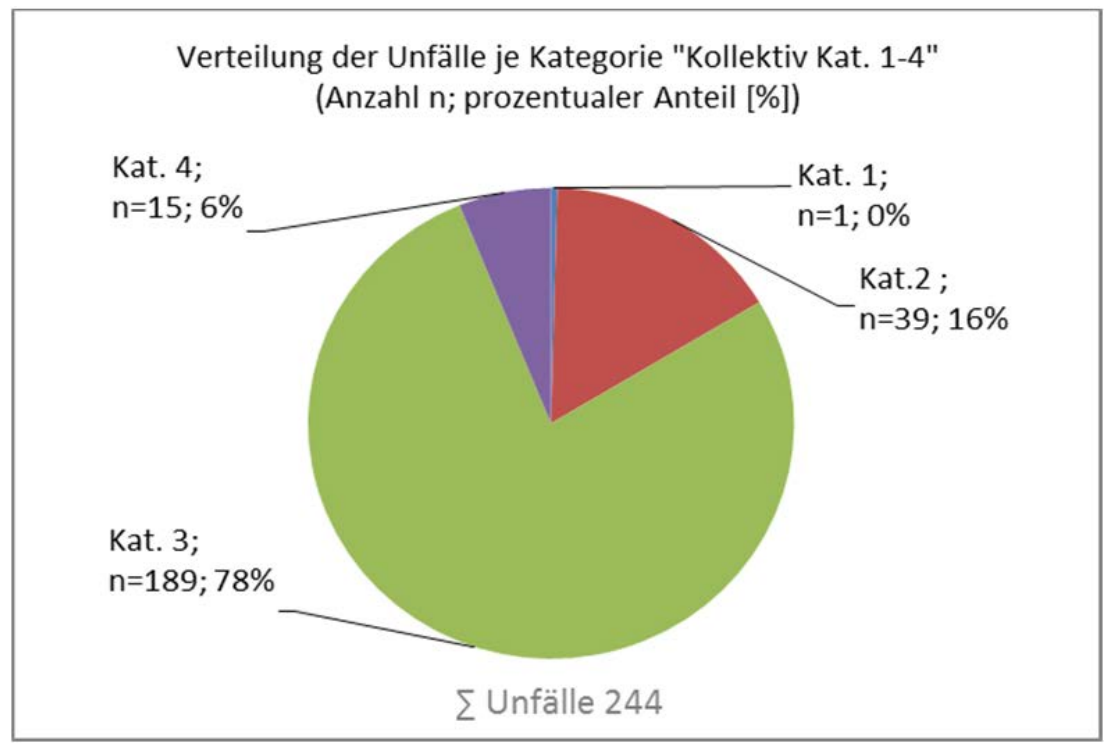

Abbildung 50: Verteilung der Unfälle je Kategorie (Kat. 1-4) „Kollektivs Kat. 1-4“

6 Der Unfall der Kategorie 1 (Unfall mit Getöteten) trat in einer Geschäftsstraße auf (Mischprinzip). Der Unfall ereignete sich in den frühen Morgenstunden (5:08 Uhr) an einer Kreuzung, als es noch dunkel war. Beteiligt waren ein Pkw und ein Fußgänger, es kam zu einem Zusammenstoß zwischen ihnen (Unfallart 6). Klassifiziert wurde der Unfall als Unfalltyp 7 „Sonstiger Unfall“. Als Unfallursache wurde ein Fehler beim Fahrzeugführer festgestellt (Unfallursache 49). 
Bei dem Vergleich der Verteilung der Unfälle je Kategorie in den ausgewiesenen Verkehrsberuhigten Bereichen mit der Verteilung der innerörtlichen Unfälle war deutlich zu erkennen, dass der Anteil der Unfälle der Kategorie 4 in den VBB sehr gering war (Abbildung 51). Der Wert lag im Gegensatz zu innerörtlichen Straßen $(18,7 \%)$ bei 6,1\%. Dies deckt sich mit den Ergebnissen aus Kapitel 10.1.1.

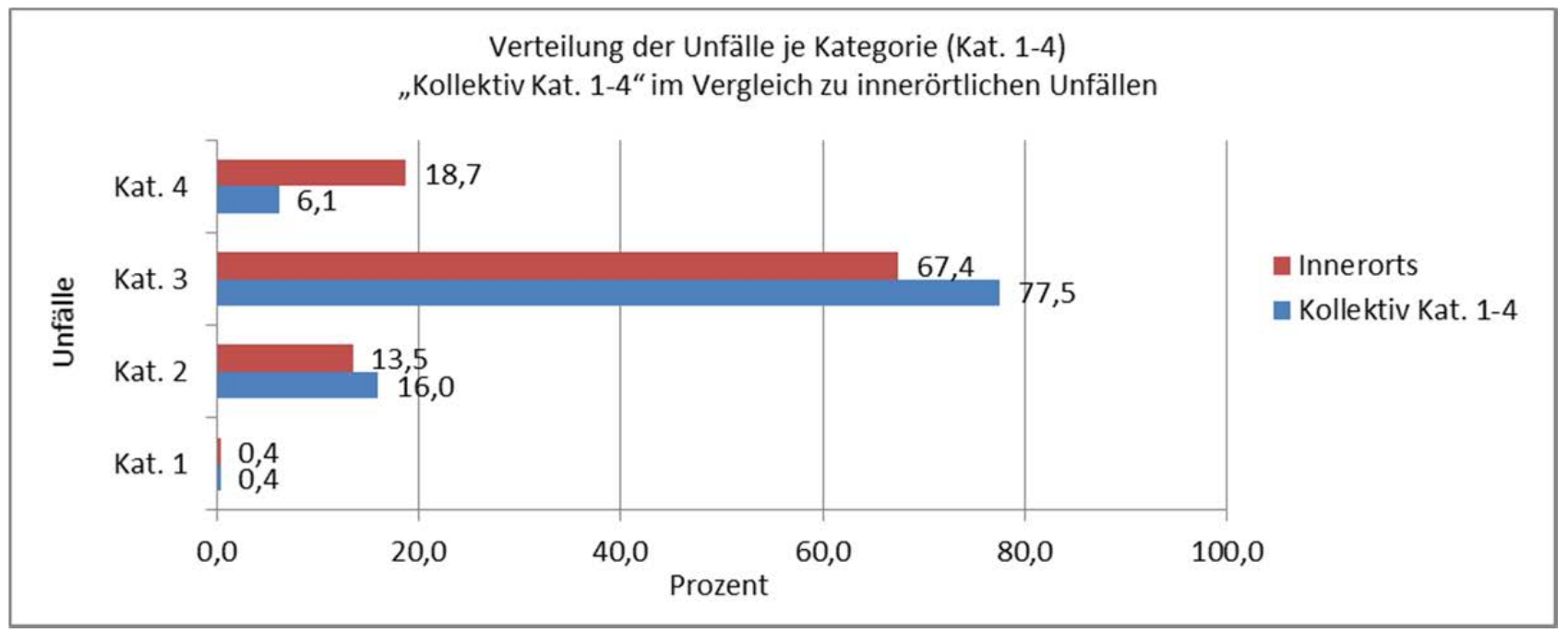

Abbildung 51: Prozentuale Verteilung der Unfälle je Kategorie (Kat. 1-4), „Kollektivs Kat. 1-4“ im Vergleich zu innerörtlichen Unfällen

\section{Schritt}

Dadurch, dass der Anteil der Unfälle der Kategorie 4 in ausgewiesenen Verkehrsberuhigten Bereichen so gering war, fiel die prozentuale Verteilung der Unfälle der Kategorie 1-3 anteilig entsprechend höher aus. Aus diesem Grund wurden in einer weiteren Auswertung (2. Schritt) ausschließlich die Unfälle der Kategorie 1-3 zwischen ausgewiesenen Verkehrsberuhigten Bereichen und innerörtlichen Unfällen untersucht (Abbildung 52). Daraus war eindeutig zu erkennen, dass sich die Verteilung der Unfälle der Kategorie 1-3 in dieser Gruppe nicht wesentlich voneinander unterscheidet.

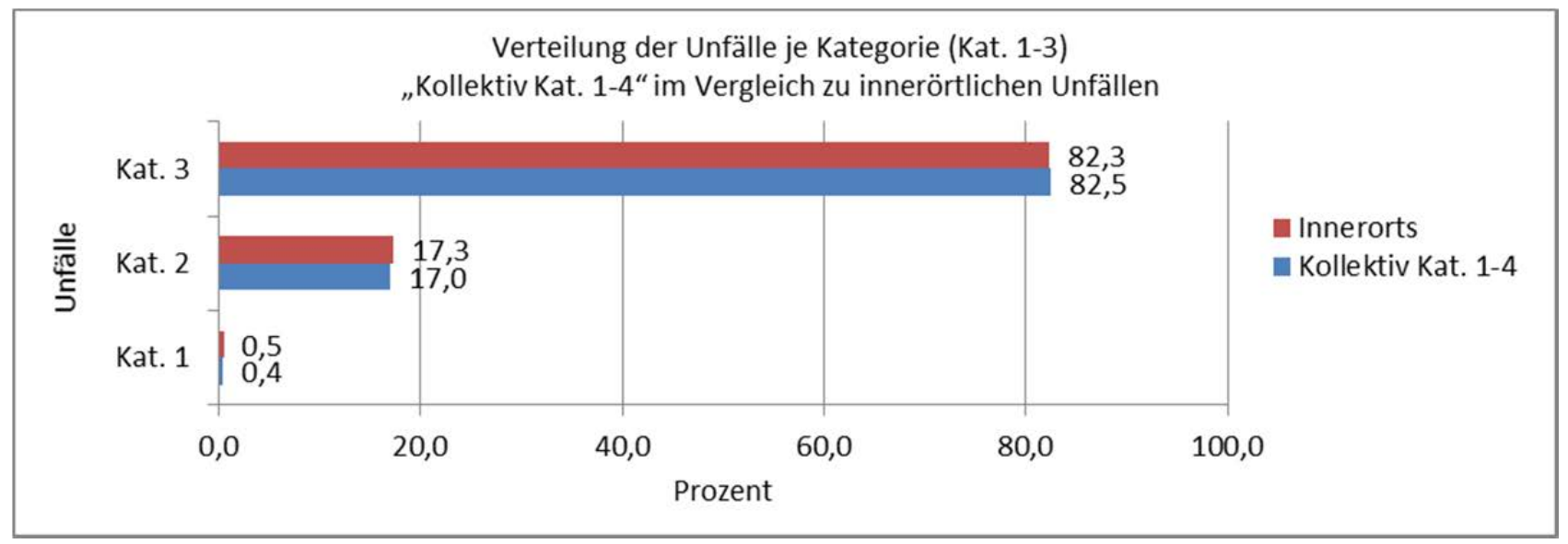

Abbildung 52: Prozentuale Verteilung der Unfälle je Kategorie (Kat. 1-3), „Kollektivs Kat. 1-4“ im Vergleich zu innerörtlichen Unfällen 
Aufgrund des geringen Geschwindigkeitsniveaus in ausgewiesenen VBB war zu vermuten, dass Unfälle der Kategorie 4 in ausgewiesenen Verkehrsberuhigten Bereichen seltene Ereignisse darstellen sollten. Insofern sich die Verkehrsteilnehmer an die zul. Höchstgeschwindigkeit („Schrittgeschwindigkeit“) halten, sollten die Unfallfolgen für die Fahrzeuge i.d.R. sehr gering sein. Warum es in den vorliegenden Fällen dennoch zu schwerwiegenden Unfällen mit Sachschaden gekommen ist, wird nachfolgend näher untersucht.

Insgesamt liegen 15 Unfälle der Kategorie 4 vor. Fünf davon sind Alleinunfälle von Pkw die zumeist aufgrund einer überhöhten oder nicht angepassten Geschwindigkeit stattfanden (Unfallursache 12, 13). Die Fahrzeuge sind häufig von der Fahrbahn nach rechts/links abgekommen oder gegen ein Hindernis geprallt. Sieben Unfälle der Kategorie 4 ereigneten sich zwischen zwei Pkw. Am häufigsten kommt der Unfalltyp 2 und 3 vor, also Unfälle im Kreuzungs- oder Einmündungsbereich. Dementsprechend oft wurden als Unfallart die Ziffern 27 „Nichtbeachten der Regel rechts vor links" und 28 „Nichtbeachten der die Vorfahrt regelnden Verkehrszeichen" angegeben. Zwei Unfälle der Kategorie 4 passierten unter Beteiligung eines Lkw ohne Anhänger, einmal mit einem Pkw, das andere Mal mit einem Kraftrad. Beide Unfälle fanden bei Parkvorgängen statt. Hier konnten die schweren Unfallfolgen auf die Größe und das Gewicht der Lkw zurückgeführt werden. Es lagen also hauptsächlich Unfälle der Kategorie 4 vor, bei denen Geschwindigkeitsübertretungen stattgefunden haben, die an Kreuzungen/Einmündungen passiert sind oder an denen ein Lkw beteiligt war. Dadurch lässt sich erklären, dass in den Verkehrsberuhigten Bereichen Unfälle vorlagen, bei denen ein Kraftfahrzeug aufgrund eines Unfallschadens von der Unfallstelle abgeschleppt werden musste.

\subsubsection{Unfalltypen}

\section{Schritt}

Insgesamt 39\% der Unfälle der Kategorie 1-4 in ausgewiesenen Verkehrsberuhigten Bereichen entstanden in Einmündungs- und Kreuzungsbereichen (31\% Einbiegen/Kreuzen-Unfälle, 8\% Abbiege-Unfälle). Mit 24\% war der nächst größte Anteil der Unfalltyp 7 „Sonstiger Unfall". In $12 \%$ der Fälle traten „Unfälle im Längsverkehr“ auf, in 11\% „Überschreiten-Unfälle“ (vgl. Abbildung 53).

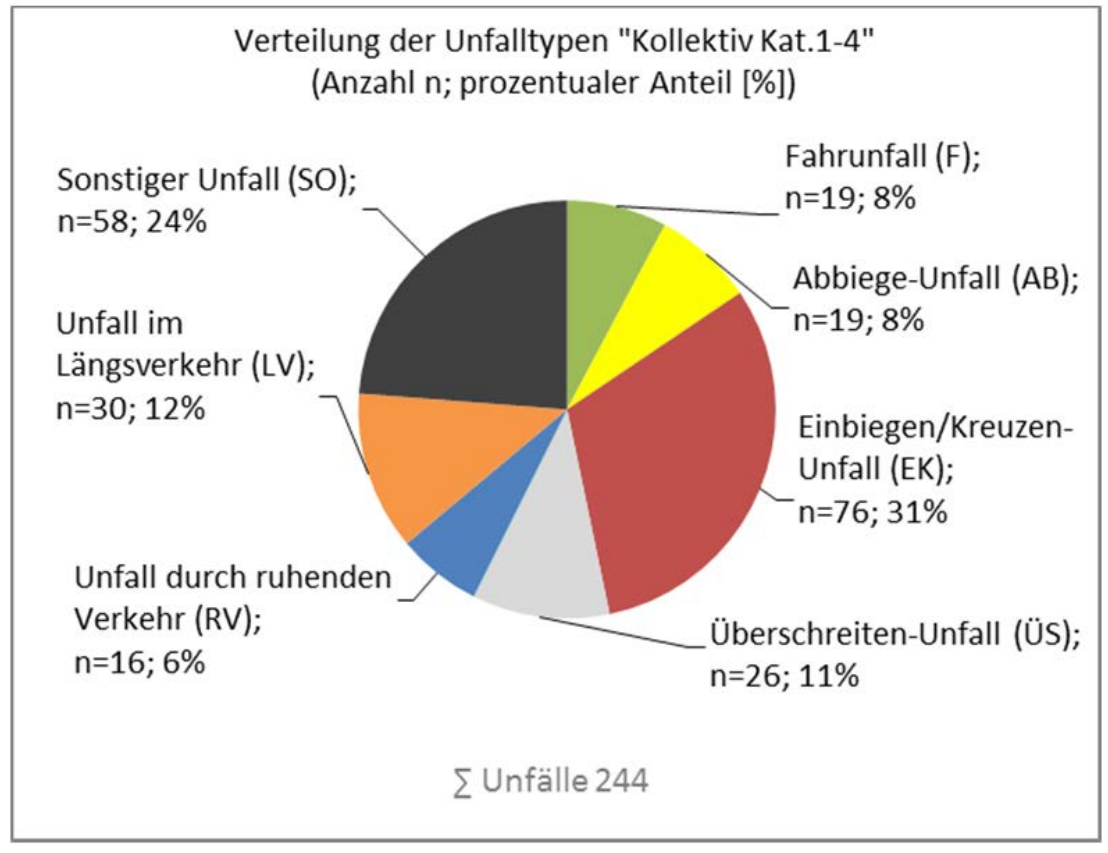

Abbildung 53: Verteilung der Unfalltypen des „Kollektivs Kat. 1-4“ 
Der Unfalltyp 7 "Sonstiger Unfall" lag in den ausgewiesenen Verkehrsberuhigten Bereichen mit 24\% deutlich höher als bei den innerörtlichen Unfällen (13\%). Dafür lagen in VBB die Unfälle mit den Typen „Unfall im Längsverkehr“ und „Abbiege-Unfall“ deutlich unter dem Schnitt von innerörtlichen Unfällen (-8\%) (vgl. Abbildung 54).

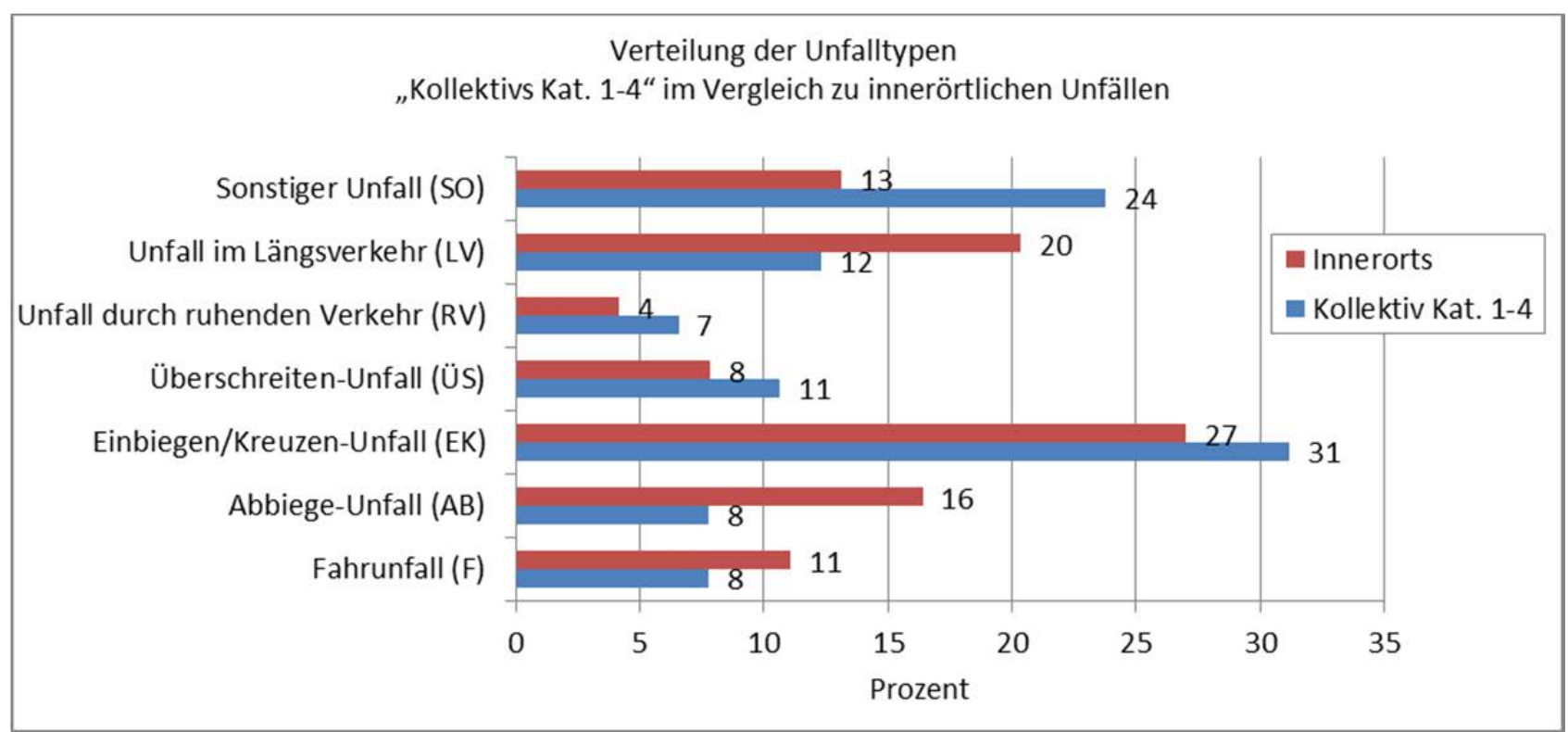

Abbildung 54: Prozentuale Verteilung der Unfalltypen des „Kollektivs Kat. 1-4“ im Vergleich zu innerörtlichen Unfällen

\section{Schritt}

An dieser Stelle wurden die Unfälle des Unfalltyps 7 "Sonstiger Unfall“ der ausgewiesenen Verkehrsberuhigten Bereiche genauer analysiert. Es sollte die Frage geklärt werden, um welche Art von Unfällen und welche Ursachen es sich handelte, um begründen zu können, weshalb der Anteil mit rund $24 \%$ so deutlich über dem Anteil der innerörtlichen Unfälle lag (Faktor: 1,8).

46\% der "Sonstigen Unfälle" sind der Unfallart 6 "Zusammenstoß zwischen Fahrzeug und Fußgänger" zuzuschreiben. Weitere 33\% sind Unfälle anderer Art (Abbildung 55).

Zunächst wurden die Zusammenstöße zwischen Fahrzeugen und Fußgängern (46\% des Unfalltyps 7) näher betrachtet. Es handelte sich hierbei um 27 Stück. Die Unfallursachen lagen hier bei knapp der Hälfte der Unfälle bei einem Fehler der Fahrzeugführer gegenüber Fußgängern an anderen Stellen (12 Unfälle). 10 Zusammenstöße waren von Fahrzeugführern beim Wenden oder Rückwärtsfahren mit Fußgängern geschehen (vgl.

Tabelle 16). Diese Unfälle sind durchaus als typisch für ausgewiesene Verkehrsberuhigte Bereiche anzusehen.

Auch die Unfallursache „Unfälle anderer Art“ war anteilig mit 33\% (19 Unfälle) beim Unfalltyp 7 häufig vertreten. Sie verteilten sich über 7 unterschiedliche Unfallursachen (vgl. Tabelle 17). Einzig 
die Unfallursache „Wenden-/Rückwärtsfahren“ trat hier wieder hervor mit acht Unfällen, die alle unter Beteiligung eines Fußgängers stattfanden.

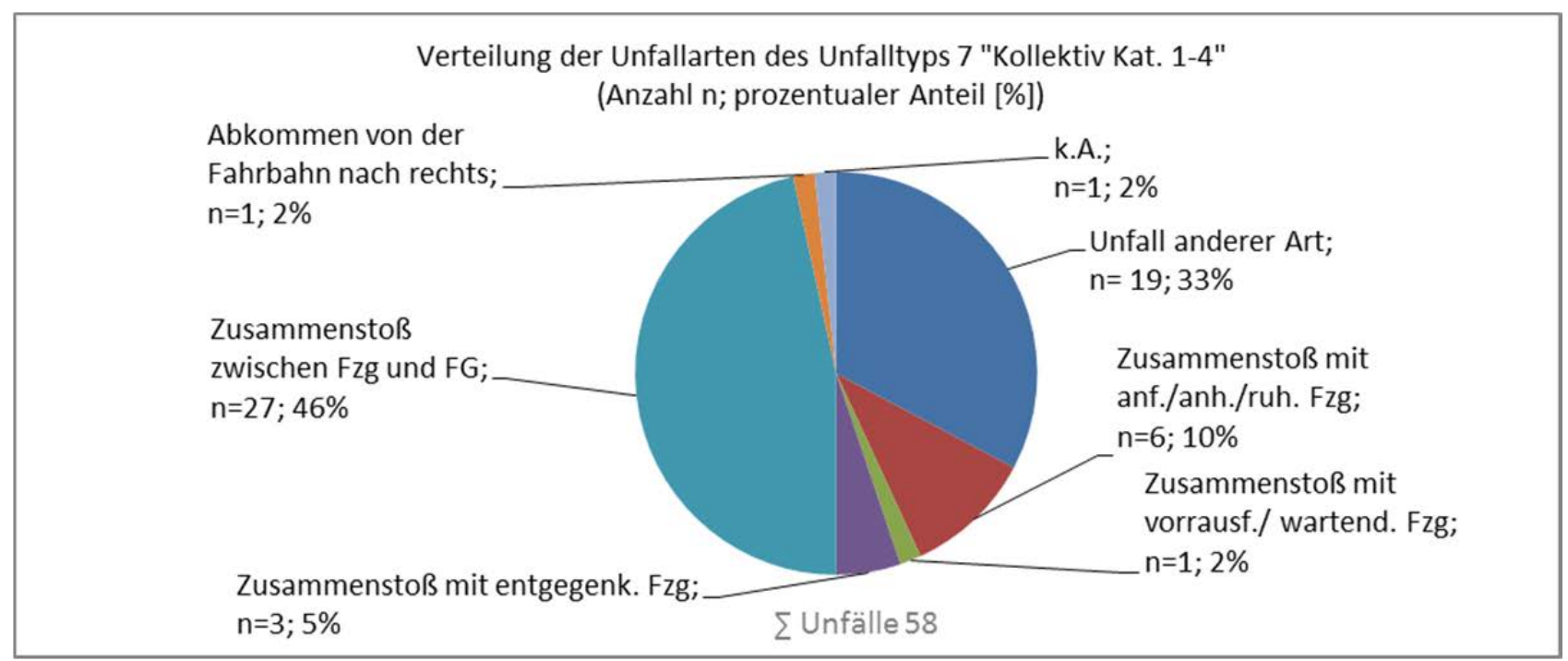

Abbildung 55: Verteilung der Unfallarten des Unfalltyps 7 „Sonstiger Unfall“ des „Kollektivs Kat. 1-4“

Tabelle 16: Unfallursachen der Unfallart „Zusammenstoß zwischen Fahrzeug und Fußgänger" des Unfalltyps 7 „Sonstiger Unfall“ des „Kollektivs Kat. 1-4“

\begin{tabular}{|l|c|}
\hline Unfallursache & Anzahl der Unfälle \\
\hline Nr. 10 Falsche Fahrbahn-/Straßenbenutzung & 2 \\
\hline Nr. 13 Nichtangepasste Geschwindigkeit & 1 \\
\hline Nr. 36 Wenden/Rückwärtsfahren & 10 \\
\hline Nr. 42 Fehler ggn. Fußgänger an anderen Stellen & 12 \\
\hline Nr. 49 andere Fehler beim Fzg-Führer & 1 \\
\hline k.A. & 1 \\
\hline Summe \#nfallursachen der Unfallart „Unfälle anderer Art“ des Unfalltyp 7 „Sonstiger Unfall“ des \\
\hline Tabelle 17: „Kollektivs Kat. 1-4“
\end{tabular}

\begin{tabular}{|l|c|}
\hline Unfallursache & Anzahl der Unfälle \\
\hline Nr. 4 Sonstige körperl. oder geistige Mängel & 1 \\
\hline Nr. 13 Nichtangepasste Geschwindigkeit & 1 \\
\hline Nr. 36 Wenden/Rückwärtsfahren & 8 \\
\hline Nr. 37 Fehler beim Einfahren in den fließenden Verkehr & 2 \\
\hline Nr. 49 Andere Fehler beim Fzg-Führer & 3 \\
\hline Nr. 55 Andere technische Mängel & 2 \\
\hline Nr. 69 Andere Fehler beim Fußgänger & 2 \\
\hline Summe & 19 \\
\hline
\end{tabular}

\subsubsection{Unfallarten}

1. Schritt 
In gut über einem Viertel der Unfälle in den ausgewiesenen Verkehrsberuhigten Bereichen (28\%) wurde die Unfallart „Zusammenstoß zwischen Fahrzeug und Fußgänger" angegeben. Die andere häufig auftretende Unfallart war mit 29\% der „Zusammenstoß mit anderem Fahrzeug, das einbiegt oder kreuzt“. In 15\% der Fälle liegt ein „Unfall anderer Art“ vor. Alle weiteren Unfallarten treten nur mit geringen Anteilen auf (Abbildung 56).

Im Vergleich zu den innerörtlichen Unfällen, lag die Unfallart „Zusammenstoß zwischen Fahrzeug und Fußgänger" auch deutlich höher (Innerorts 13\%, VBB 28\% vgl. Abbildung 57). Deutlich niedriger war der Anteil der Unfälle in Verkehrsberuhigten Bereichen mit der Unfallart „Zusammenstoß mit anderem Fahrzeug, das vorausfährt oder wartet“" (Innerorts 14\%, VBB 2\%). Kein großer Unterschied lag bei der Unfallart „Zusammenstoß mit anderem Fahrzeug, das einbiegt oder kreuzt" vor, als beide Kollektive miteinander verglichen wurden. In ausgewiesenen Verkehrsberuhigten Bereichen trat diese Unfallart mit 29\% seltener auf, als bei innerörtlichen Straßen (34\%).

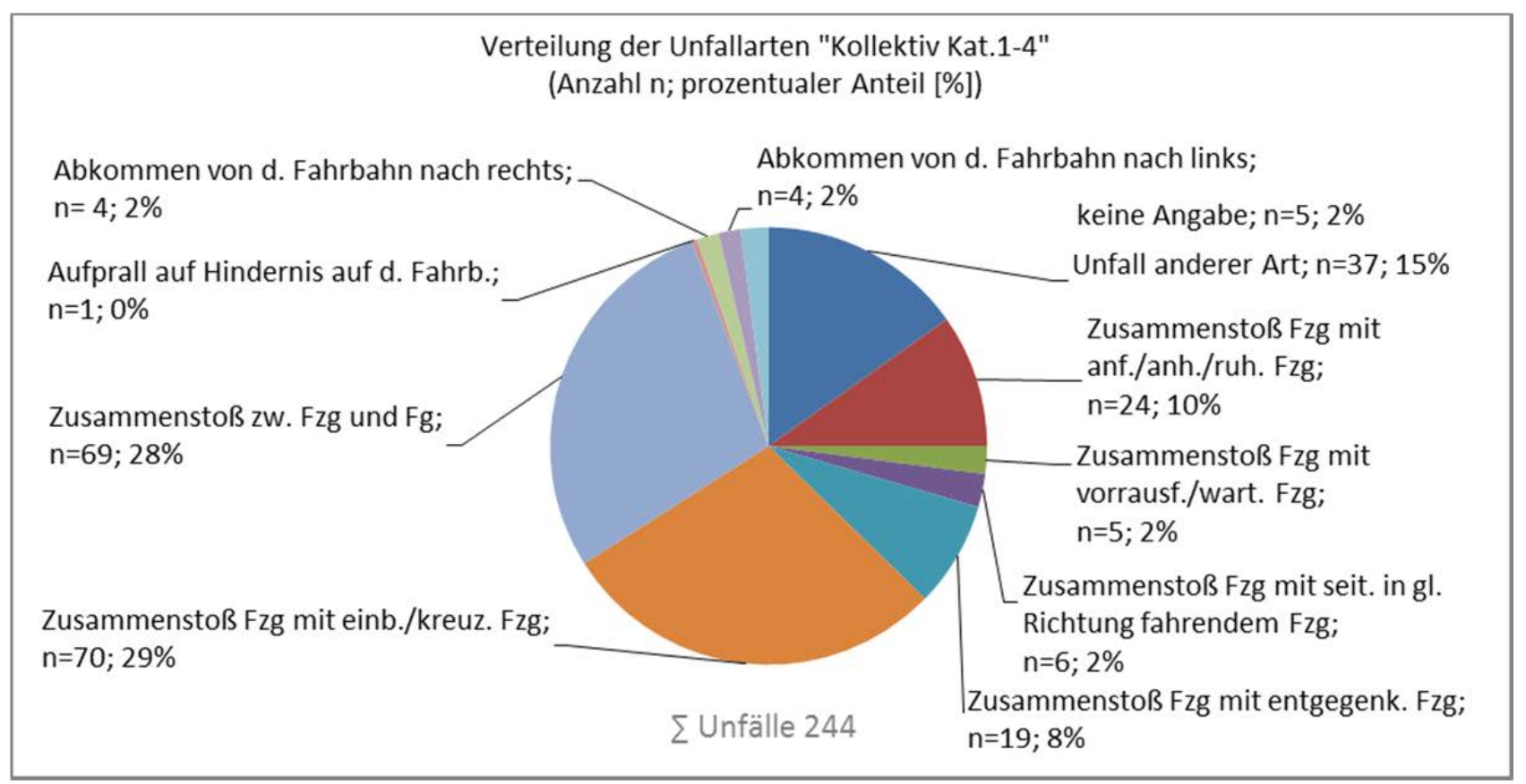

Abbildung 56: Verteilung der Unfallarten des „Kollektivs Kat. 1-4“ 


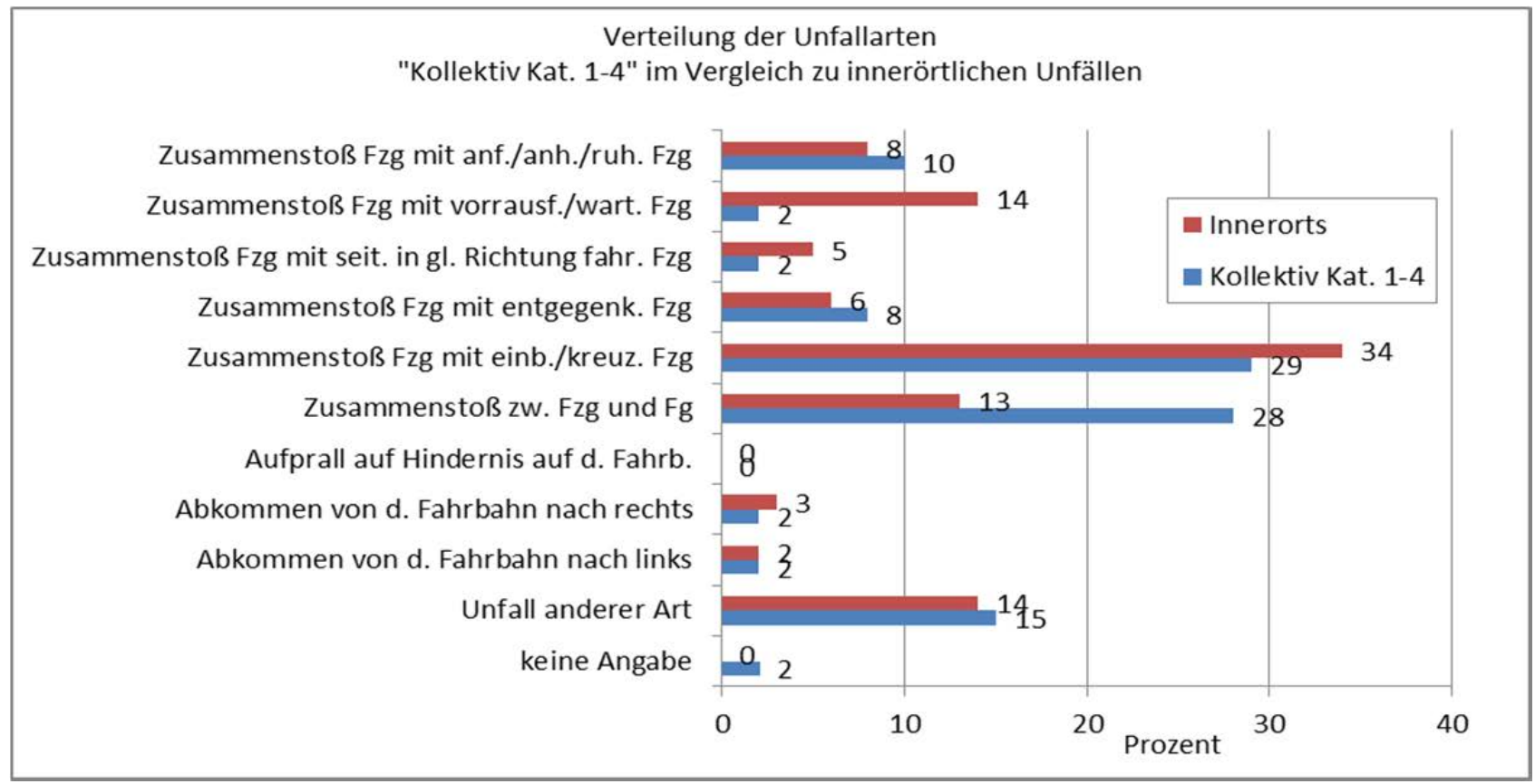

Abbildung 57: Prozentuale Verteilung der Unfallarten des „Kollektivs Kat. 1-4“ im Vergleich zu innerörtlichen Unfällen

\section{Schritt}

Die Unfälle der Unfallart „Zusammenstoß zwischen Fahrzeug und Fußgänger“ wurden aufgrund ihres deutlich höheren Anteils (im Vergleich zu innerörtlichen Unfällen) genauer untersucht. Die häufigste Unfallursache der Unfallart „Zusammenstoß zwischen Fahrzeug und Fußgänger" lag bei einem „Falschen Verhalten gegenüber Fußgängern an anderer Stelle“ (vgl. Abbildung 58). Das heißt, dass diese Unfälle nicht an Fußgängerüberwegen, nicht an Fußgängerfurten und nicht beim Abbiegen oder an Haltestellen vorlagen (diese besitzen eine andere Unfallursache). Von den 35 Unfällen waren 15 Überschreiten-Unfälle (Unfalltyp 4) und 12 Sonstige Unfälle (Unfalltyp 7). Als Sonstige Unfälle kamen in den ausgewiesenen Verkehrsberuhigten Bereichen hauptsächlich Unfälle infolge von Wenden, Rückwärtsfahren oder Rangieren in Frage. Dies bestätigte auch der hohe Anteil von 20\% der Unfallursache „Fehler beim Wenden oder Rückwärtsfahren“.

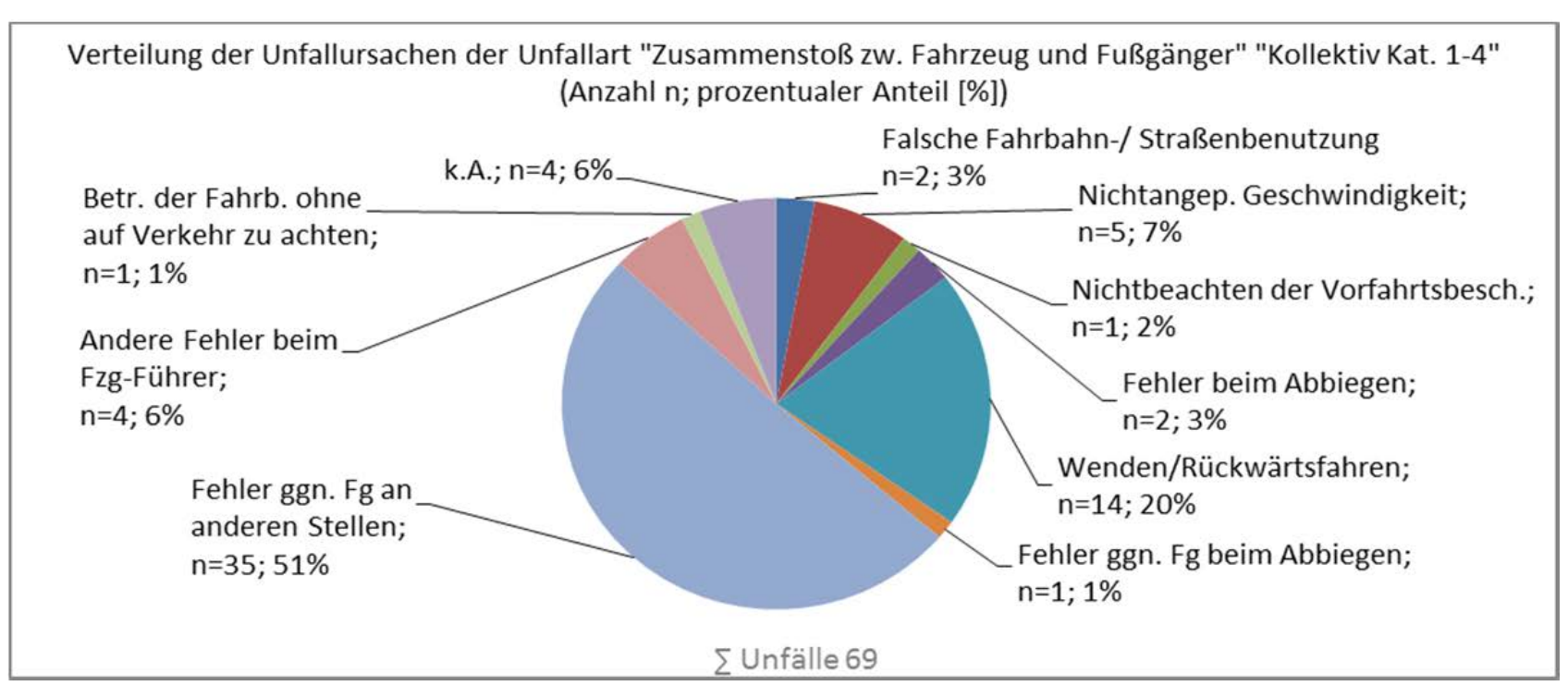

Abbildung 58: Verteilung der Unfallursachen der Unfallart „Zusammenstoß zwischen Fahrzeug und Fußgänger" des „Kollektivs Kat. 1-4“ 
Der deutlich niedrigere Anteil von Unfällen der Unfallart „Zusammenstoß mit anderem Fahrzeug, das vorausfährt oder wartet" lag voraussichtlich darin begründet, dass es wesentlich weniger straßenbauliche sowie verkehrsregelnde Merkmale in ausgewiesenen Verkehrsberuhigten Bereichen gibt, die einen Zusammenstoß mit einem vorausfahrenden/wartenden Fahrzeug fördern. Z.B. ist das Geschwindigkeitsniveau deutlich geringer als auf anderen innerörtlichen Straßen, so dass ein zu geringer Sicherheitsabstand auf Vorrausfahrende nicht häufig zu Kollisionen führen kann.

Die Unfallart „Zusammenstoß mit anderem Fahrzeug, das einbiegt oder kreuzt“ besaß am Unfallgeschehen in ausgewiesenen Verkehrsberuhigten Bereichen mit 28\% einen großen Anteil. Bei der Auswertung dieser Unfälle hinsichtlich der Unfallursachen (Abbildung 59) war folgendes zu erkennen:

- Erwartungsgemäß war der größte Anteil (31\%) die Unfallursache „Nichtbeachten der Regel rechts-vor-links“, denn diese Verkehrsregelung (rechts-vor-links) kommt hauptsächlich in Erschließungsstraßen mit Tempo 30 Zonen (oder in VBB) zum Einsatz.

- Die Unfallursache „Nichtbeachten der die Vorfahrt regelnden Verkehrszeichen“ trat in $16 \%$ der Fälle auf. Hierbei handelte es sich im Wesentlichen um Unfälle im Übergangsbereich zu einer übergeordneten Straße (9 von 11 Unfällen).

- Besonders hoch lag mit 29\% auch der „Fehler beim Einfahren in den fließenden Verkehr“. Dies geschieht gemäß der Definition beim Abbiegen, Wenden, Rückwärtsfahren, Ein- und Anfahren aus einem Grundstück, von einem anderen Straßenteil oder beim Anfahren vom Fahrbahnrand. Da aber alle dieser 20 Unfälle den Unfalltyp 3 besaßen, handelte es sich bei den vorliegenden Fällen ausschließlich um Unfälle, bei denen ein Fahrzeug aus einem Grundstück oder z.B. einem Parkplatz einbog. Ein- und Auspark-Unfälle oder Wenden und Rückwärtsfahren wären sonst den Unfalltypen 5 und 7 zugeordnet worden.

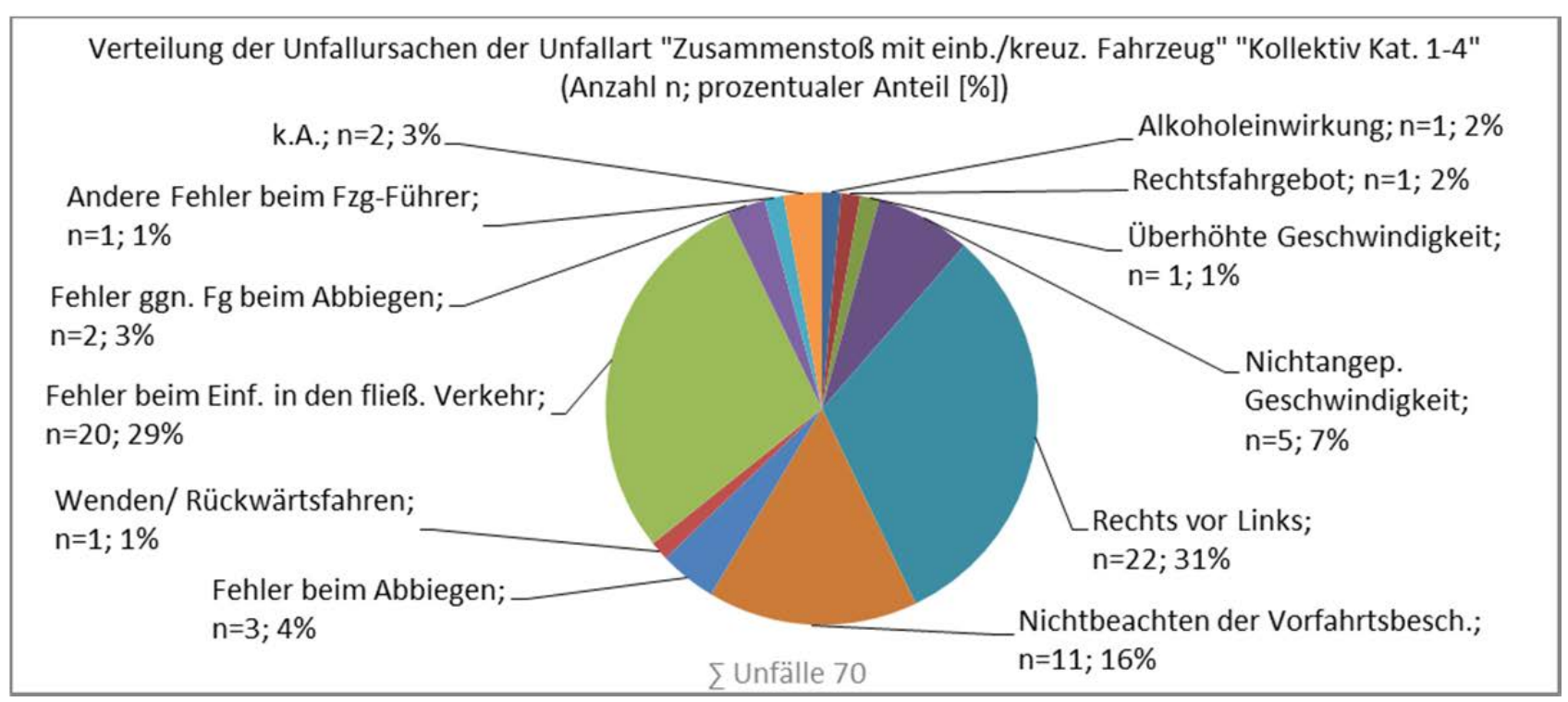

Abbildung 59: Verteilung der Unfallursachen der Unfallart „Zusammenstoß mit einbiegendem/- kreuzendem Fahrzeug“ des „Kollektivs Kat. 1-4“

Der Vollständigkeit halber wurden (bei den Analysen im 2. Schritt) auch die 38 Unfälle mit der Unfallart „Unfall anderer Art" näher betrachtet. Hier stellte sich jedoch keine Auffälligkeit dar. Bei den 38 Unfällen kamen 16 verschiedene Unfallursachen vor und bis auf den Unfalltyp 2 auch alle Unfalltypen. 


\subsubsection{Beteiligte}

\subsubsection{Grundgesamtheit aller Beteiligten}

\section{Schritt}

An den insgesamt 244 Unfällen in Verkehrsberuhigten Bereichen waren 478 Personen beteiligt (244 Hauptverursacher, 224 Beteiligte 2 und 10 Beteiligte 3). An den 244 Unfällen der Kategorie 1-4 waren im Wesentlichen beteiligt (Abbildung 60):

- 220 Pkw (46\%),

- 125 Fahrradfahrer (26\%) und

- 73 Fußgänger (15\%).

Bei dem Vergleich der Verteilung der Beteiligten an innerörtlichen Unfällen war zu erkennen, dass der Anteil von Pkw als Beteiligter höher lag und die Beteiligung von Radfahrern und Fußgängern wesentlich niedriger waren. An den 237.523 Unfällen der Kategorie 1-4 innerorts im Jahr 2011 waren insgesamt beteiligt (Abbildung 61):

- $272.820 \mathrm{Pkw}(62 \%)$,

- $\quad 72.138$ Fahrradfahrer (16\%) und

- 29.011 Fußgänger (7\%).

Die Unfälle unter Beteiligung von Radfahrern und Fußgängern traten in ausgewiesenen Verkehrsberuhigten Bereichen demnach häufiger auf als bei innerörtlichen Unfällen, und Unfälle unter Beteiligung von Pkws waren deutlich seltener. Natürlich ist an dieser Stelle nicht zu vergessen, dass sich das Verkehrsaufkommen in ausgewiesenen Verkehrsberuhigten Bereichen anders als bei innerörtlichen Straßen zusammensetzt. Der Anteil von nicht motorisierten Verkehr ist in VBB höher, so dass auch die Wahrscheinlichkeit für Radfahrer und Fußgänger steigt, in einen Unfall verwickelt zu werden. Die Wahrscheinlichkeit mit einem Lkw oder Sattelschlepper zu verunfallen ist sehr gering, da sich vermuten lässt, dass das Verkehrsaufkommen in ausgewiesenen Verkehrsberuhigten Bereichen kaum einen nennenswerten Lkw-Anteil besitzt.

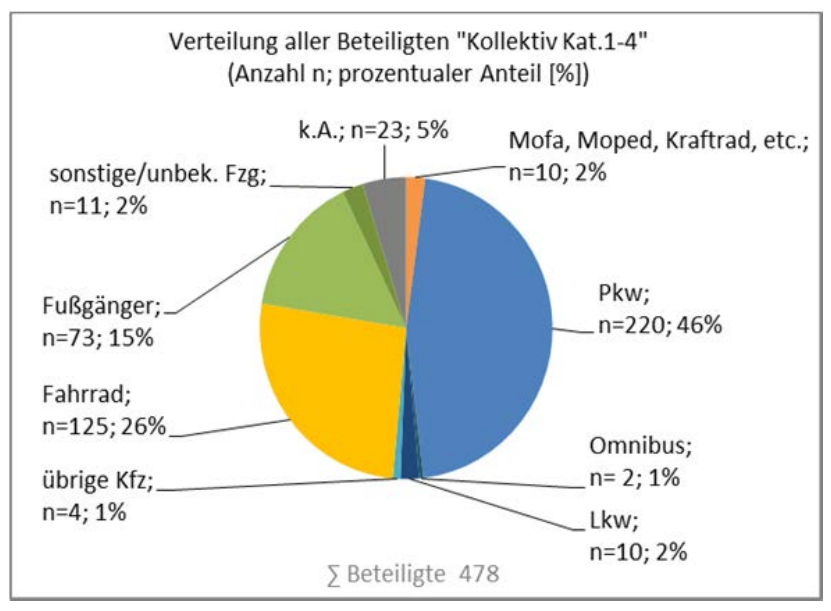

Abbildung 60: Verteilung der Beteiligten an Unfällen des „Kollektivs Kat. 1-4“

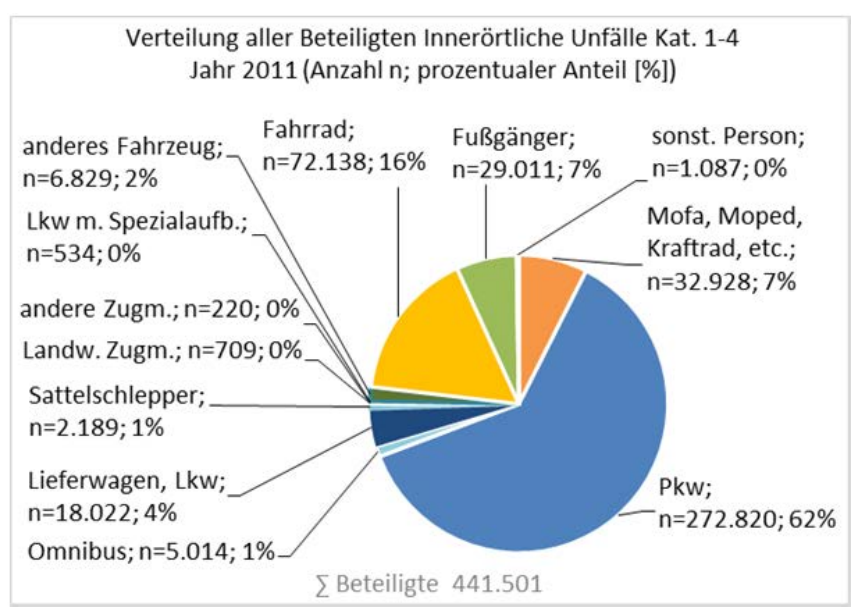

Abbildung 61: Verteilung der Beteiligten an innerörtlichen Unfällen Kat. 1-4 


\section{Schritt}

Weiterführende differenzierte Betrachtungen zu den jeweiligen Beteiligten (1, 2 und ggf. auch 3) konnten Aufschluss darüber geben, welche Verkehrsteilnehmer die Hauptverursacher waren. Für die ausgewiesenen Verkehrsberuhigten Bereiche ist die Verteilung der Beteiligten nach Hauptverursacher und Beteiligter 2 und 3 in Abbildung 62 dargestellt. Die prozentuale Verteilung für die wichtigsten Verkehrsteilnehmer in VBB (Pkw, Fahrradfahrer und Fußgänger) ist in Tabelle 18 enthalten. In 59\% der Unfälle war ein Pkw-Fahrer der Hauptverursacher der Unfälle, in knapp $29 \%$ ein Fahrradfahrer und in gut 3\% ein Fußgänger. Pkw-Fahrer sind (im Vergleich als Hauptverursacher) fast in nur der Hälfte der Fälle als Beteiligter 2 in Unfälle verwickelt gewesen (34\%). Fahrradfahrer waren jedoch nahezu gleich oft Hauptverursacher von Unfällen (29\%) wie auch Beteiligter 2 (24\%). Fußgänger sind in den seltensten Fällen Hauptverursacher von Unfällen aber wesentlich häufiger als zweiter Beteiligter in Unfälle verwickelt gewesen (28\%).

Die Verteilung der Beteiligten nach Hauptverursacher und Beteiligter 2 der innerörtlichen Unfälle der Kategorie 1-4 ist in Abbildung 62. Die prozentuale Verteilung für die wichtigsten Verkehrsteilnehmer (Pkw, Fahrradfahrer, Fußgänger, Motorradfahrer und Lkw) ist in Tabelle 19 enthalten. In gut 69\% der Unfälle war ein Pkw-Fahrer der Hauptverursacher von innerörtlichen Unfällen der Kat. 1-4, in knapp 3\% war es ein Fußgänger. Diese Anteile lagen bei den VBB ähnlich hoch. Als Hauptverursacher waren Fahrradfahrer auf innerörtlichen Straßen wesentlich seltener $(12,5 \%)$ als in VBB an Unfällen beteiligt (29\%). Im innerörtlichen Unfallgeschehen spielten auch die Unfälle unter Beteiligung von Motorradfahrern und Lkw eine Rolle. Motorradfahrer sind z.B. in 6\% der Fälle Hauptverursacher und Lkw in 5\%. Als zweiter Beteiligter waren Pkw zu 46\% in innerörtliche Unfälle involviert, deutlich seltener als in Unfälle in VBB als zweiter Beteiligter (34\%). Die Fahrradfahrer und Fußgänger verunfallten auf innerörtlichen Straßen zu 18\% und 9\% als zweiter Beteiligter, sie verunfallten hingegen anteilig wesentlich häufiger in VBB als zweiter Beteiligter (24\% und 28\%).

Aus dem Vergleich der Hauptverursacher und zweiten Beteiligten war zu erkennen, dass:

- Fußgänger in ausgewiesenen Verkehrsberuhigten Bereichen öfter in einen Unfall als „nicht“ Hauptverursacher verwickelt waren und

- Radfahrer in ausgewiesenen Verkehrsberuhigten Bereichen deutlich öfter als Hauptverursacher an Unfällen der Kat. 1-4 beteiligt waren als an allen innerörtlichen Kat. 1-4-Unfällen.

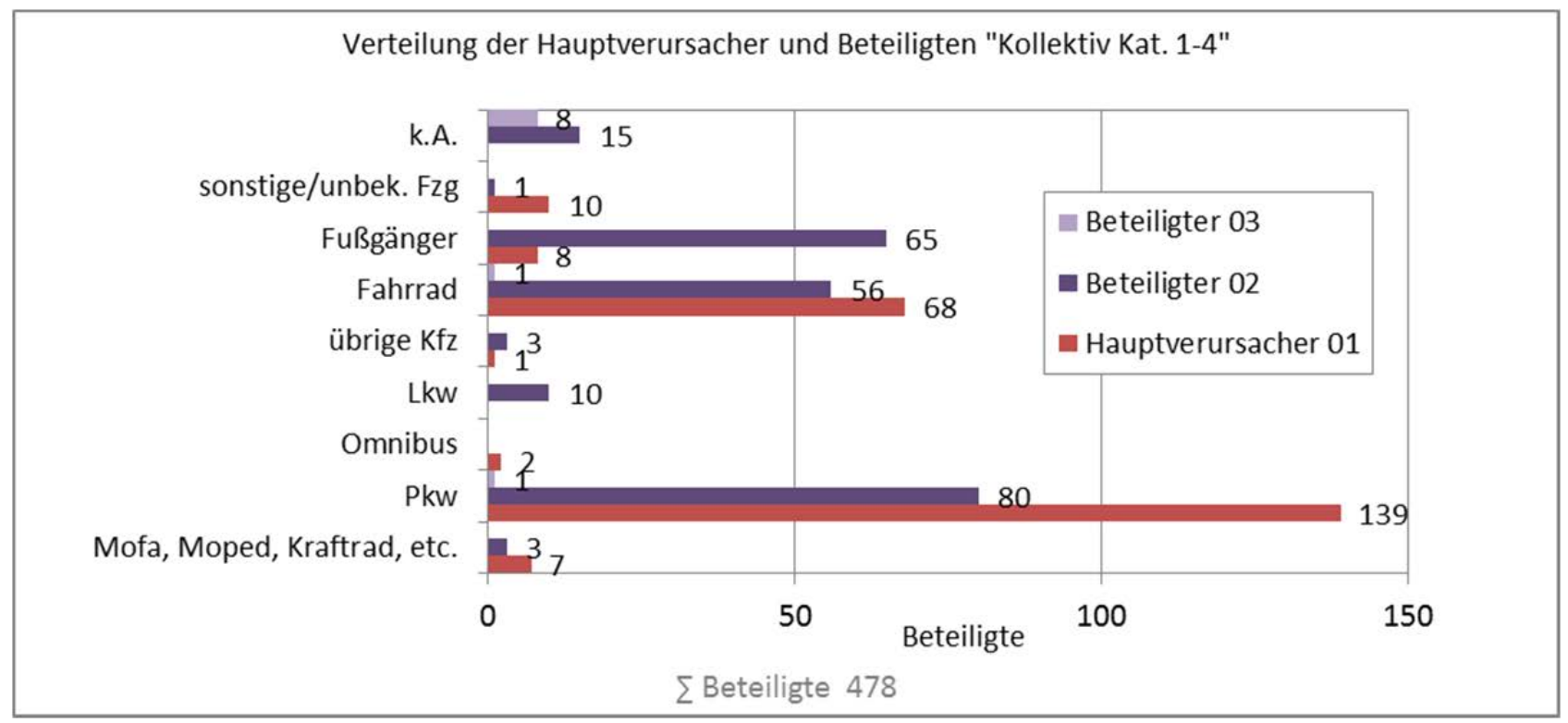

Abbildung 62: Verteilung der Hauptverursacher und Beteiligte von Unfällen des „Kollektivs Kat. 1-4“ 
Tabelle 18: Prozentuale Verteilung der Hauptverursacher und Beteiligten des „Kollektivs Kat. 1-4“

\begin{tabular}{|l|c|c|c|}
\hline Verkehrsart & Hauptverursacher 01 & Beteiligter 02 & Beteiligter 03 \\
\hline Pkw & $59,2 \%$ & $34,3 \%$ & $0,0 \%$ \\
\hline Fahrrad & $28,9 \%$ & $24,0 \%$ & $0,0 \%$ \\
\hline Fußgänger & $3,4 \%$ & $27,9 \%$ & $0,0 \%$ \\
\hline
\end{tabular}

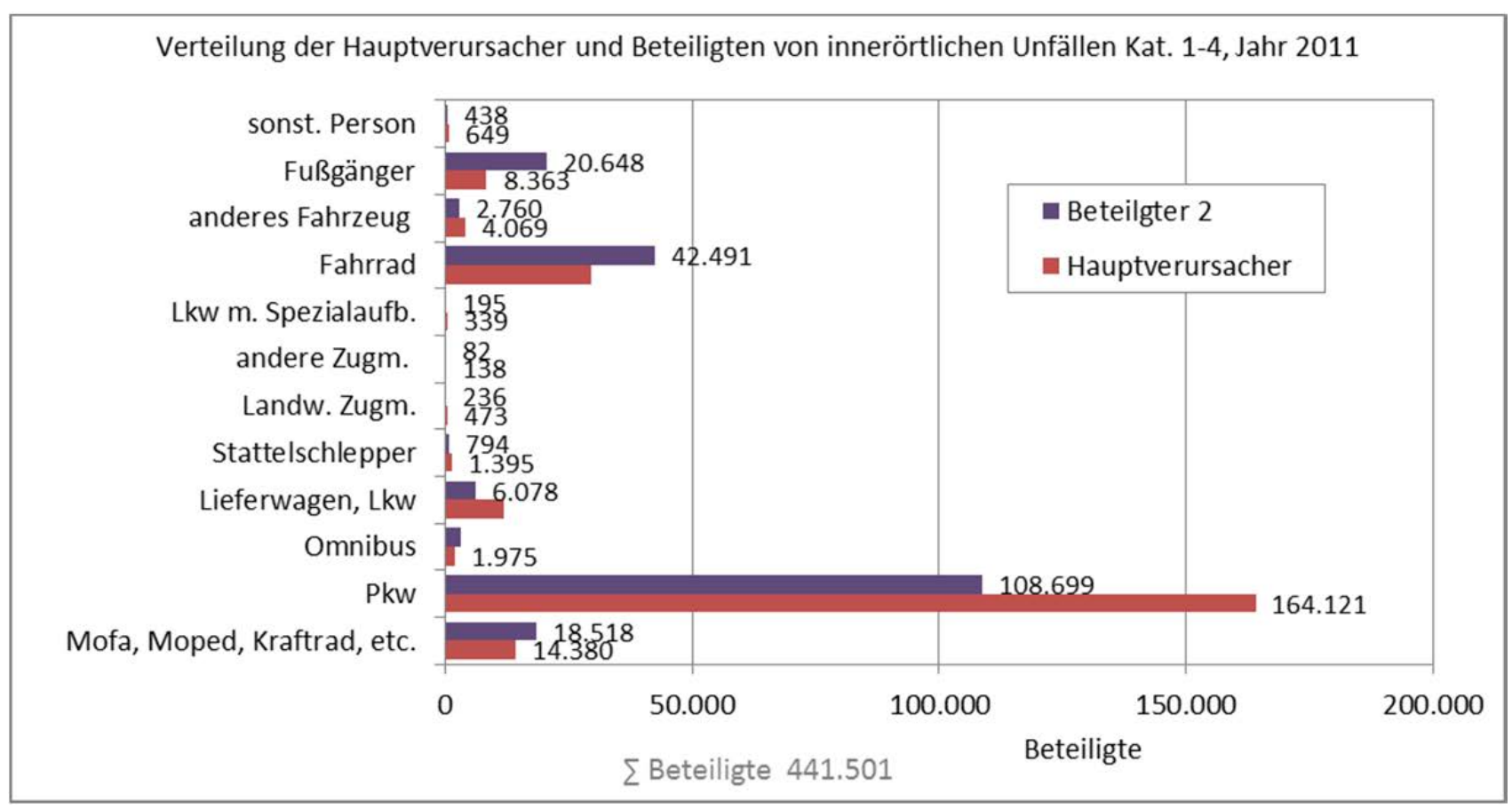

Abbildung 63: Verteilung der Hauptverursacher und Beteiligte von innerörtlichen Unfällen 2011

Tabelle 19: Prozentuale Verteilung der Hauptverursacher und Beteiligten an innerörtlichen Unfällen

\begin{tabular}{|l|c|c|}
\hline Verkehrsart & Hauptverursacher $\mathbf{0 1}$ & Beteiligter 02 \\
\hline Pkw & $69,1 \%$ & $45,8 \%$ \\
\hline Fahrrad & $12,5 \%$ & $17,9 \%$ \\
\hline Fußgänger & $3,5 \%$ & $8,7 \%$ \\
\hline Mofa, Moped, Kraftrad, etc. & $6,1 \%$ & $7,8 \%$ \\
\hline Lieferwagen, Lkw & $5,0 \%$ & $2,6 \%$ \\
\hline
\end{tabular}

Des Weiteren wurden untersucht, wie sich die Beteiligung von Fußgängern, Fahrradfahrern und Pkw in Bezug auf die Unfallschwere (Unfallkategorie), den Unfalltyp und die Unfallarten an Unfällen der Kategorie 1-4 in ausgewiesenen Verkehrsberuhigten Bereichen verhielt. Dabei wurde nicht mehr zwischen dem Hauptverursacher und den weiteren Beteiligten unterschieden. Die Ergebnisse sind in den Abbildung 64 bis Abbildung 66 dargestellt. Zusammengefasst können folgende Punkte festgehalten werden:

Fußgänger

- Von den insgesamt 73 Unfällen, die unter Beteiligung eines Fußgängers stattgefunden haben, waren 86\% Unfälle der Kategorie 3 und 12\% der Kategorie 2. 
- Bei den Unfalltypen trat am häufigsten der Unfalltyp „Sonstiger Unfall“ auf (44\%), gefolgt vom Unfalltyp „Überschreiten-Unfall“ (34\%).

- Als Unfallart wurde in $89 \%$ der Fälle der „Zusammenstoß zwischen Fahrzeug und Fußgänger" angegeben.

Radfahrer

- Von den insgesamt 119 Unfällen unter Beteiligung eines Radfahrers waren 80\% Unfälle der Kategorie 3 und 19\% Unfälle der Kategorie 2.

- Der Haupt-Unfalltyp, der bei Radfahrern vorlag, war der „Einbiegen/Kreuzen-Unfall“ mit $45 \%$.

- Die Unfälle verteilten sich über mehrere Unfallarten, mit $41 \%$ lag die Unfallart „Zusammenstoß mit anderem Fahrzeug, das einbiegt oder kreuzt" am höchsten.

Pkw

- Von insgesamt 195 Unfällen an denen Pkws beteiligt waren, lagen zu 79\% Unfälle der Kategorie 3 und zu 14\% Unfälle der Kategorie 2 vor.

- Die Unfälle verteilten sich über mehrere Unfalltypen. Mit $35 \%$ war der Unfalltyp „Einbiegen/Kreuzen-Unfall“ am meisten vertreten.

- Bei der Unfallart lagen die Unfälle „Zusammenstoß mit anderem Fahrzeug, das einbiegt oder kreuzt" (33\%) und "Zusammenstoß zwischen Fahrzeug und Fußgänger" (28\%) am häufigsten vor.

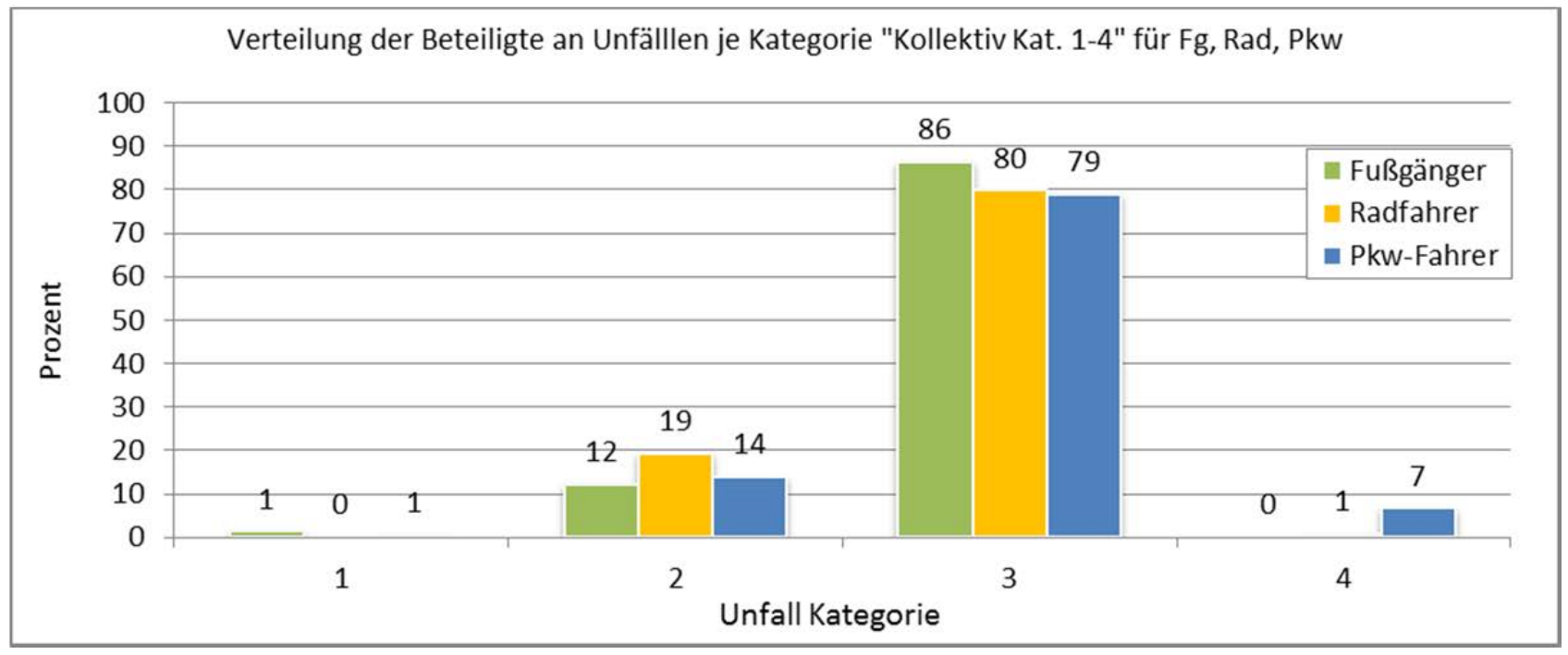

Abbildung 64: Prozentuale Verteilung der Beteiligten Verkehrsarten (für Fußgänger, Radfahrer und Pkw-Fahrer) je Kategorie des „Kollektivs Kat. 1-4“ (478 Beteiligte insgesamt) 


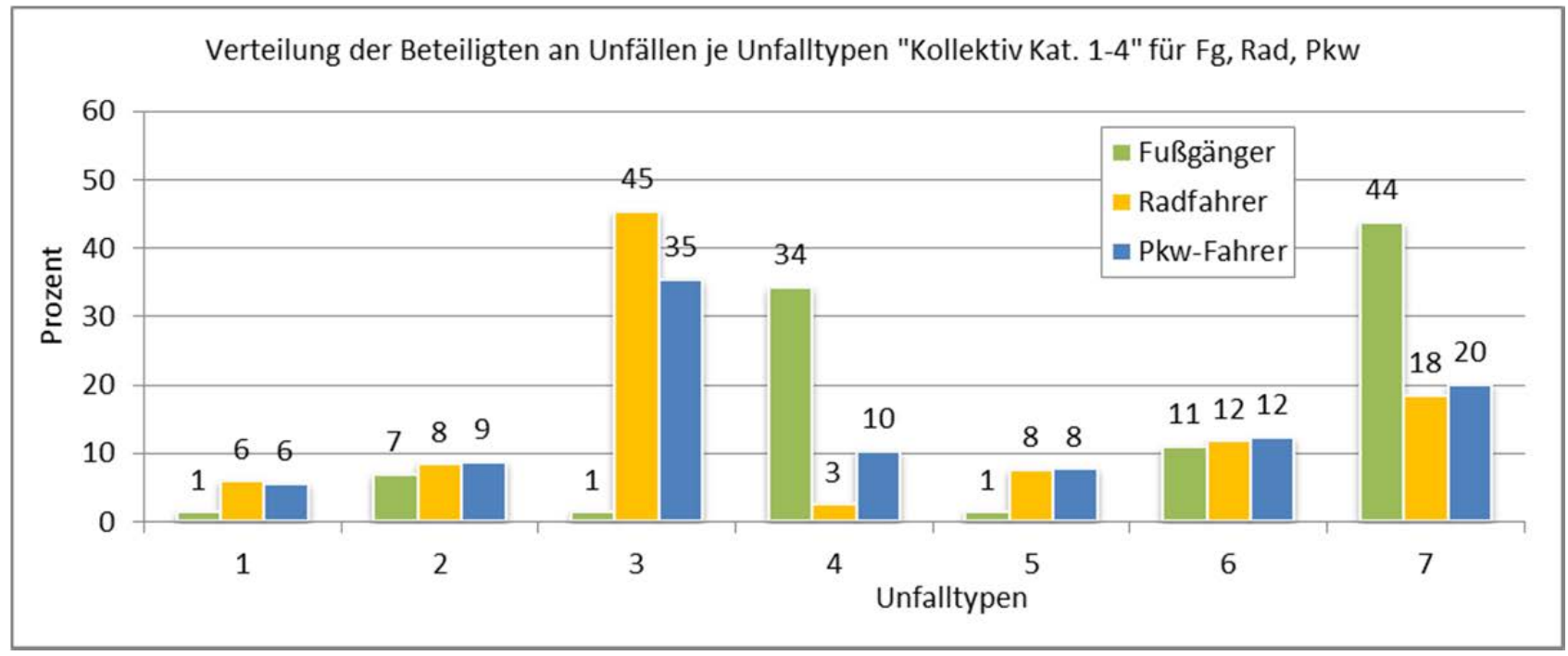

Abbildung 65: Prozentuale Verteilung der Beteiligten Verkehrsarten (für Fußgänger, Radfahrer und Pkw-Fahrer) je Unfalltyp des „Kollektivs Kat. 1-4“ (478 Beteiligte insgesamt)

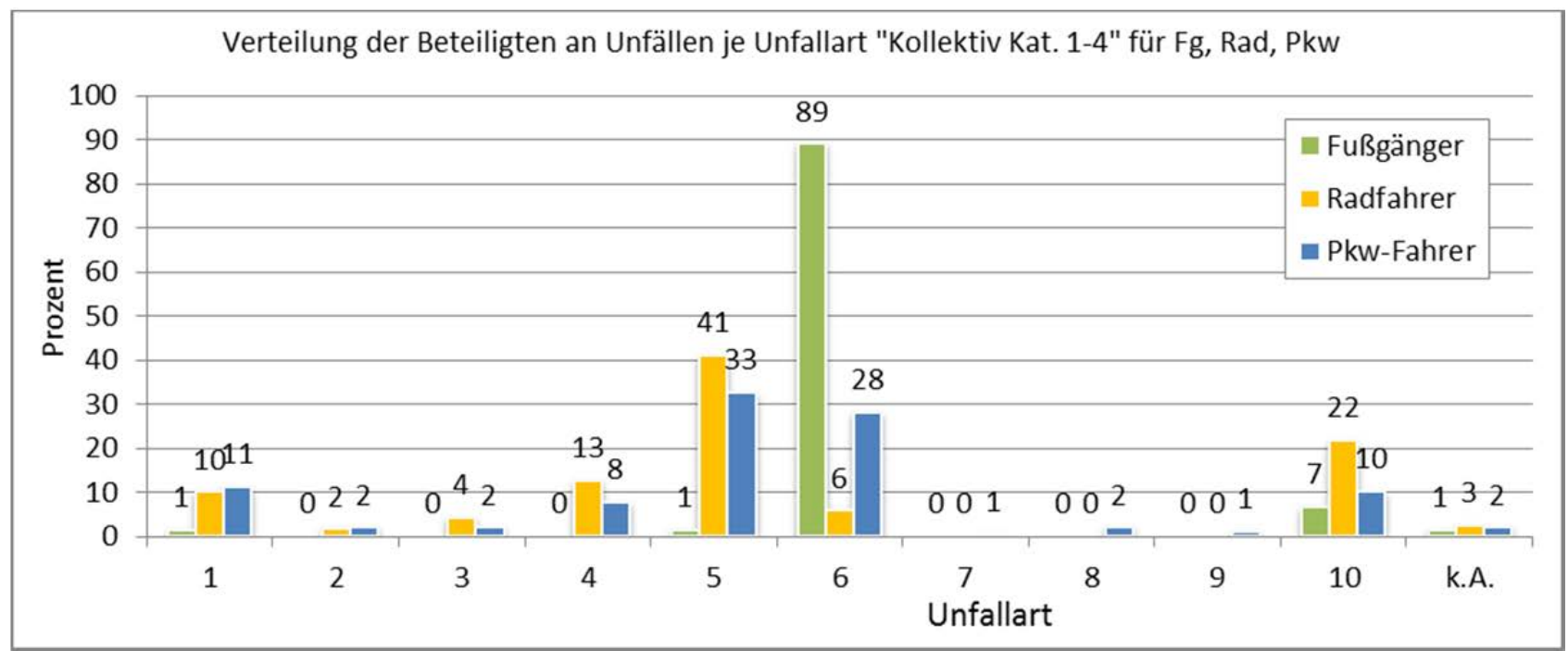

Abbildung 66: Prozentuale Verteilung der Beteiligten Verkehrsarten (für Fußgänger, Radfahrer und Pkw-Fahrer) je Unfallart des „Kollektivs Kat. 1-4“ (478 Beteiligte insgesamt)

\subsubsection{Kombination der Beteiligten}

Die Kombination der einzelnen Beteiligten (Verkehrsarten) der Unfälle der Kategorie 1-4 wurde genauer betrachtet. Für ausgewiesene VBB sind sie der Abbildung $67 \mathrm{zu}$ entnehmen.

In ausgewiesenen Verkehrsberuhigen Bereichen waren besonders zahlreiche Unfälle zwischen einem Pkw als Hauptverursacher und einem Fußgänger vertreten. 50 von insgesamt 244 Unfällen (20,5\%) wurden in dieser Kombination aufgenommen. Der Anteil von Pkw-Rad Unfällen lag bei $16,8 \%$ und der von Rad-Pkw Unfällen bei 18,4\%. Die Kombination von Pkw-Pkw trat mit 9,8\% relativ selten in ausgewiesenen VBB auf. Die wichtigsten Kombinationen der Verkehrsarten sind je Anzahl und prozentualer Verteilung in Tabelle 20 für Unfälle in ausgewiesenen Verkehrsberuhigten Bereichen zusammengefasst. 


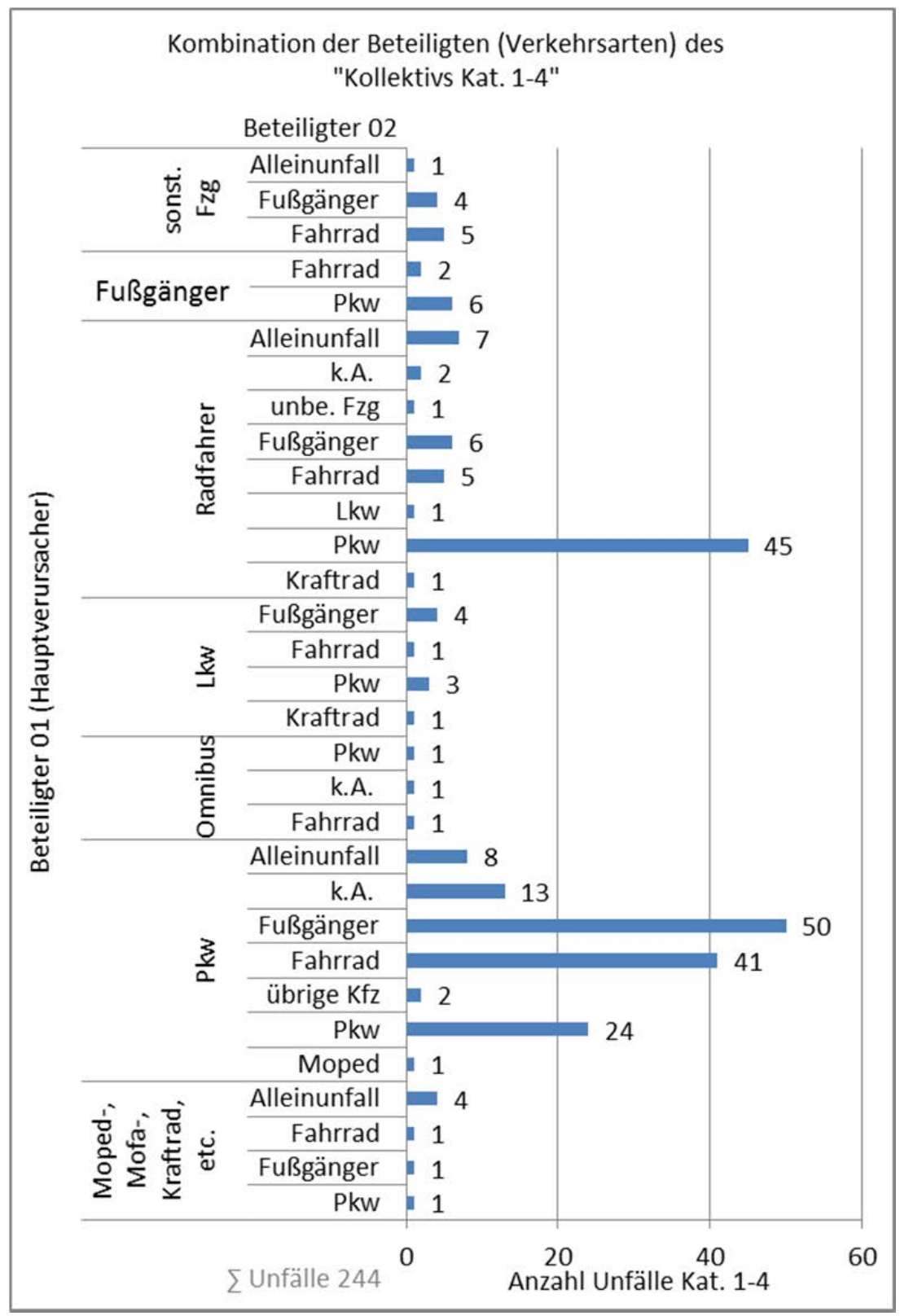

Abbildung 67: Kombination der Beteiligten (Verkehrsarten) des „Kollektivs Kat. 1-4“

Tabelle 20: Häufigste Kombinationen der Beteiligten (Verkehrsarten) des „Kollektivs Kat. 1-4“

\begin{tabular}{|l|l|c|c|}
\hline Hauptverursacher 01 & Beteiligter 02 & Anzahl & Prozent \\
\hline Pkw & Fußgänger & 50 & $20,5 \%$ \\
\hline Pkw & Rad & 41 & $16,8 \%$ \\
\hline Rad & Pkw & 45 & $18,4 \%$ \\
\hline Pkw & Pkw & 24 & $9,8 \%$ \\
\hline
\end{tabular}

Im Vergleich zum innerörtlichen Unfallgeschehen (Kat. 1-4) wurden bei der Kombinationen der Beteiligten bei ausgewiesenen VBB deutliche Unterschiede festgestellt. Dies lag auch daran, dass Innerorts mehr unterschiedliche Verkehrsteilnehmer vorlagen. Aufgrund der ungleichen Kollektive, wurde auf eine gemeinsame Darstellung der Daten in einer Abbildung verzichtet. Die häufigste Kombination, die bei innerörtlichen Unfällen der Kat. 1-4 auftrat, war die zwischen zwei Pkw mit 
32,4\% (in ausgewiesenen VBB lag dieser Wert bei nur 9,8\%), gefolgt von der Kombination Pkw (Hauptverursacher) mit Fahrrad (13,6\%) (vgl. Abbildung 68 und Tabelle 21).

Der Anteil der innerörtlichen Unfälle der Kat. 1-4 zwischen einem Pkw (Hauptverursacher) und Fußgänger lag bei 6,6\% und somit deutlich unter dem Anteil der ausgewiesenen VBB (20,5\%). Unfälle in denen Fahrradfahrer als Hauptverursacher mit einem Pkw verunfallten lagen innerorts bei nur knapp 4,5\%.

Tabelle 21: Häufigste Kombinationen der Beteiligten (Verkehrsarten) von Innerörtlichen Unfällen Kat. 1-4

\begin{tabular}{|l|l|c|c|}
\hline Hauptverursacher 01 & Beteiligter 02 & Anzahl & Prozent \\
\hline Pkw & Pkw & 76.870 & $32,4 \%$ \\
\hline Pkw & Rad & 32.207 & $13,6 \%$ \\
\hline Pkw & Fußgänger & 15.703 & $6,6 \%$ \\
\hline Rad & Pkw & 10.617 & $4,5 \%$ \\
\hline
\end{tabular}

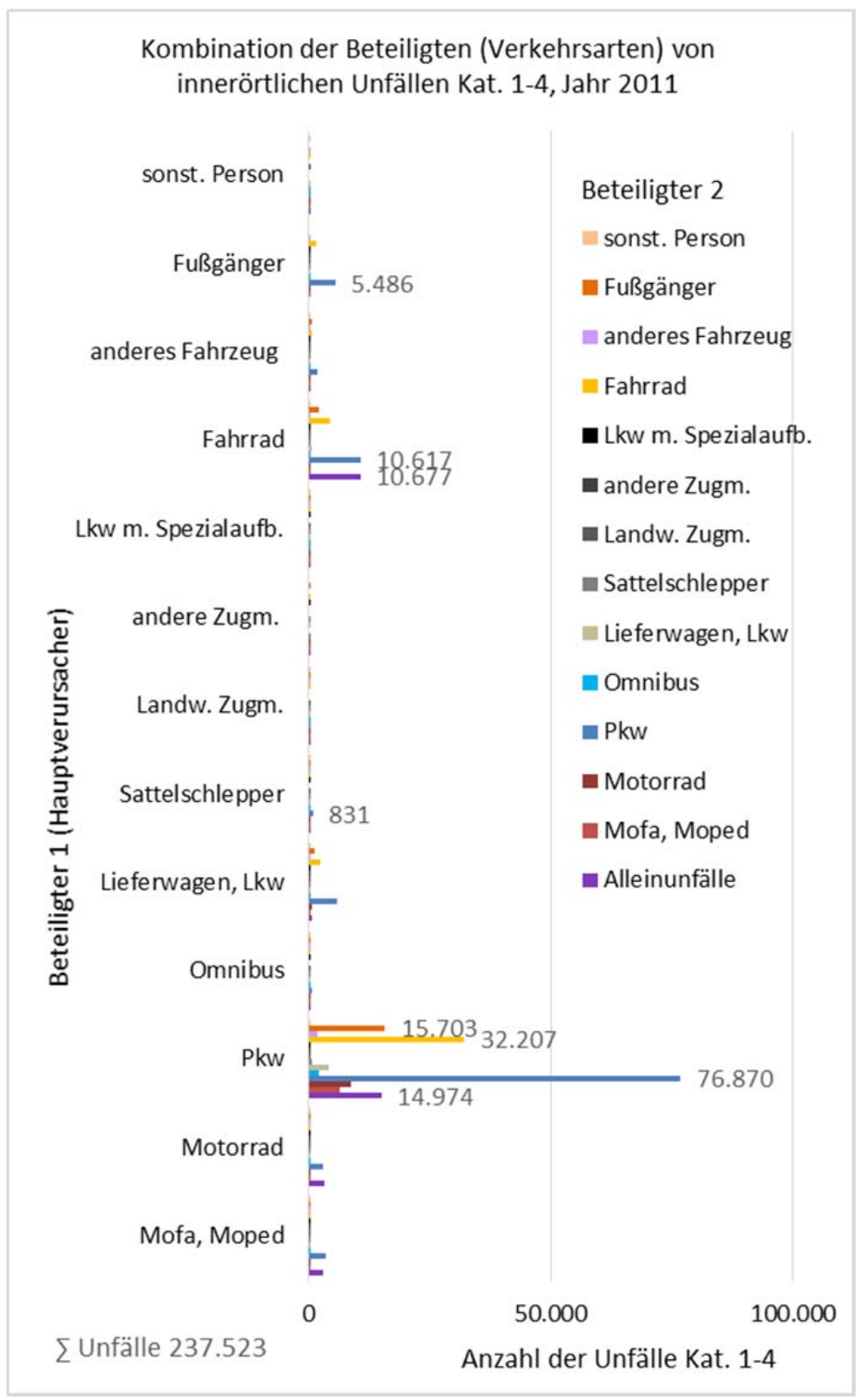

Abbildung 68: Kombination der Beteiligten (Verkehrsarten) von innerörtlichen Unfällen Kat. 1-4, Jahr 2011 


\subsubsection{Alleinunfälle}

\section{Schritt}

Aus Abbildung 67 ist zu erkennen, dass von den 244 Unfällen in den ausgewiesenen Verkehrsberuhigten Bereichen 20 Alleinunfälle vorlagen. Dabei wurden acht Unfälle von Pkw Fahrern, vier Unfälle von Motorradfahrern, sieben Unfälle von Radfahrern und ein Unfall von einem sonstigen Fahrzeug verursacht. Die Anzahl und prozentuale Verteilung wurde für ausgewiesene VBB für die wichtigsten Verkehrsarten in Tabelle 20 zusammengefasst. Vergleicht man die Verteilung der Pkw, Radfahrer und Motorrad Alleinunfälle des innerörtlichen Unfallgeschehens (Tabelle 21), so kann festgehalten werden, dass der Anteil von Alleinunfällen in beiden Fällen sehr gering war (zwischen 2-5\%). Bei innerörtlichen Unfällen der Kat. 1-4 lag der Anteil der Alleinunfälle von Pkw etwas höher als im „Kollektiv Kat. 1-4“.

Tabelle 22: $\quad$ Pkw, Rad und Motorrad Alleinunfälle des „Kollektivs Kat. 1-4“

\begin{tabular}{|l|c|c|}
\hline Alleinunfälle & Anzahl & Prozent \\
\hline Moped, Motorrad, & 4 & $2 \%$ \\
\hline Pkw & 8 & $3 \%$ \\
\hline Radfahrer & 7 & $3 \%$ \\
\hline
\end{tabular}

Tabelle 23: Pkw, Rad und Motorrad Alleinunfälle von innerörtlichen Unfällen

\begin{tabular}{|l|c|c|}
\hline Alleinunfälle & Anzahl & Prozent \\
\hline Pkw & 14.974 & $5 \%$ \\
\hline Rad & 10.677 & $4 \%$ \\
\hline Mofa, Motorrad,... & 6.160 & $2 \%$ \\
\hline
\end{tabular}

\section{Schritt}

Bei genauerer Betrachtung der Alleinunfälle der Radfahrer in ausgewiesenen VBB fiel auf, dass viele verschiedene Ursachen vorlagen (vgl. Tabelle 24). Ein Unfall war aufgrund von Alkoholeinwirkung entstanden, einer wegen eines körperlichen oder geistigen Mangels, einer aufgrund von nichtangepasster Geschwindigkeit, weitere zwei durch andere Fehler beim FahrzeugFührer und zwei durch technische Mängel. Ähnlich verhielt es sich bei der Auswertung der Unfallart, hier waren ebenfalls keine Auffälligkeiten zu erkennen. Die Unfalltypen beschränkten sich auf vier Unfälle als „Fahrunfall“ und drei „Sonstige Unfälle“.

Tabelle 24: Unfallursachen der Alleinunfälle von Radfahrern des „Kollektivs Kat. 1-4“

\begin{tabular}{|l|c|}
\hline Unfallursache & Anzahl Unfälle \\
\hline Nr. 1 Alkoholeinwirkung & 1 \\
\hline Nr. 4 Sonst. körperl. o. geist. Mängel & 1 \\
\hline Nr. 13 Nichtangepasste Geschwindigkeit & 1 \\
\hline Nr. 49 Andere Fehler beim Fzg-Führer & 2 \\
\hline Nr. 55 Andere techn. Mängel & 2 \\
\hline Summe & 7 \\
\hline
\end{tabular}


Die Aufteilung der Unfalltypen für die Alleinunfälle von Pkw war ähnlich. Es lagen vier Unfälle als „Fahrunfall“ vor und drei als „Sonstiger Unfall“. Die Unfallursachen lagen bei den Alleinunfällen von Pkw eindeutig am häufigsten bei den nichtangepassten oder überhöhten Geschwindigkeiten (sechs Unfälle). Ein Unfall war aufgrund eines anderen Fehlers beim Fahrzeug-Führer entstanden und einer aufgrund von Eis oder Schnee (vgl. Tabelle 25).

Tabelle 25: $\quad$ Unfallursachen der Alleinunfälle von Pkw-Fahrern des „Kollektivs Kat. 1-4

\begin{tabular}{|l|c|}
\hline Unfallursache & Anzahl Unfälle \\
\hline Nr. 12 Überhöhte Geschwindigkeit & 1 \\
\hline Nr. 13 Nichtangepasste Geschwindigkeit & 5 \\
\hline Nr. 49 Andere Fehler beim Fzg-Führer & 1 \\
\hline Nr. 72 Eis / Schnee & 1 \\
\hline Summe & 8 \\
\hline
\end{tabular}

\subsubsection{Unfallursachen}

\section{Schritt}

Es gibt insgesamt rund 80 Unfallursachen, die in einem Unfallursachenkatalog aufgelistet sind (vgl. Anhang 1). Für die Auswertungen der vorliegenden Studie war es wegen der Übersichtlichkeit und Stichprobengröße sinnvoll, die Unfallursachen nach den jeweiligen Unterüberschriften des Unfallursachenverzeichnisses zu gruppieren. Die gruppierten Unfallursachen können der Tabelle 2 in Kapitel 7.1.5 entnommen werden. Die Gruppierung orientiert sich an dem Unfallursachenverzeichnis des Statistischen Bundesamtes.

In den nachfolgenden Auswertungen zur Unfallursache wurden die (Haupt-) Unfallursachen bzw. die des ersten Beteiligten (Hauptverursachers) näher betrachtet. Unfallursachen der zweiten Beteiligten lagen nur unvollständig vor und wurden deshalb nicht betrachtet. Von den insgesamt 244 erhobenen Unfällen der Kat. 1-4 in ausgewiesenen VBB lagen in 8 Fällen gar keine Angaben zur Unfallursache vor und in 196 Fällen keine Unfallursachen für die zweiten Beteiligten vor.

Insgesamt 25\% der Unfälle der Kategorie 1-4 entstanden in ausgewiesenen Verkehrsberuhigten Bereichen wegen der Unfallursache „Abbiegen, Wenden, Rückwärtsfahren, Ein- und Anfahren“. $16 \%$ der Unfälle passierten aufgrund eines Fehlverhaltens gegenüber Fußgängern und weitere $16 \%$ aufgrund von Vorfahrts- bzw. Vorrang-Verletzungen (Abbildung 69).

Beim Vergleich der Verteilung der Unfallursachen in den ausgewiesenen Verkehrsberuhigten Bereichen mit der Verteilung der innerörtlichen Unfälle fiel auf, dass in ausgewiesenen VBB wesentlich mehr Unfälle aufgrund eines falschen Verhaltens gegenüber Fußgängern auftraten, als bei innerörtlichen Unfällen (Abbildung 70). Auch der Unfallanteil, der aufgrund von „Abbiegen, Wenden, Rückwärtsfahren, Ein- und Anfahren“ geschah, lag deutlich höher. Dafür lagen die Unfallursachen „Sonstiger Fehler beim Fahrzeugführer" und „Abstand“ bei den innerörtlichen Straßen höher. 


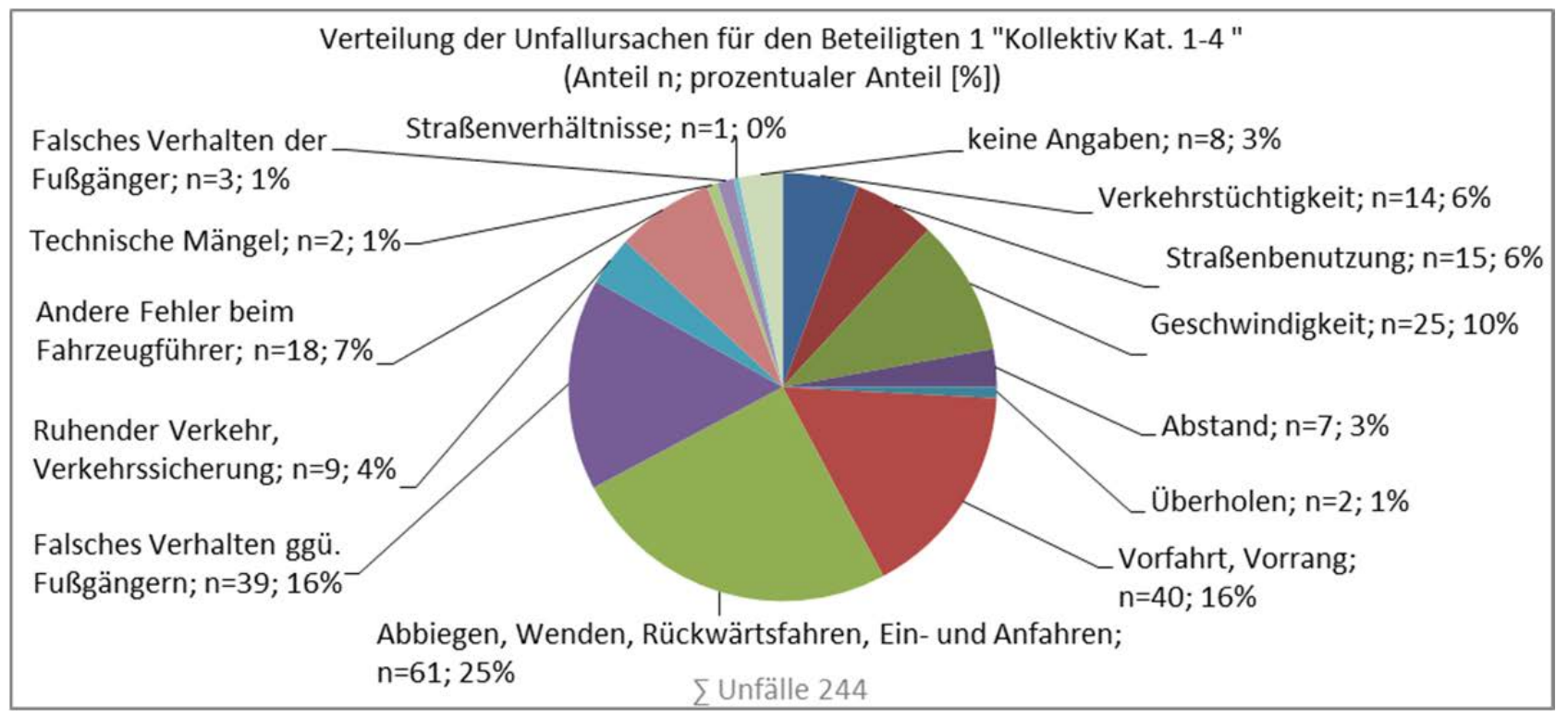

Abbildung 69: Verteilung der Unfallursachen für den Beteiligten 1 des „Kollektivs Kat. 1-4“

\section{Verteilung der Unfallursachen der Beteiligten 1}

"Kollektiv Kat. 1-4" im Vergleich zu innerörtlichen Unfällen

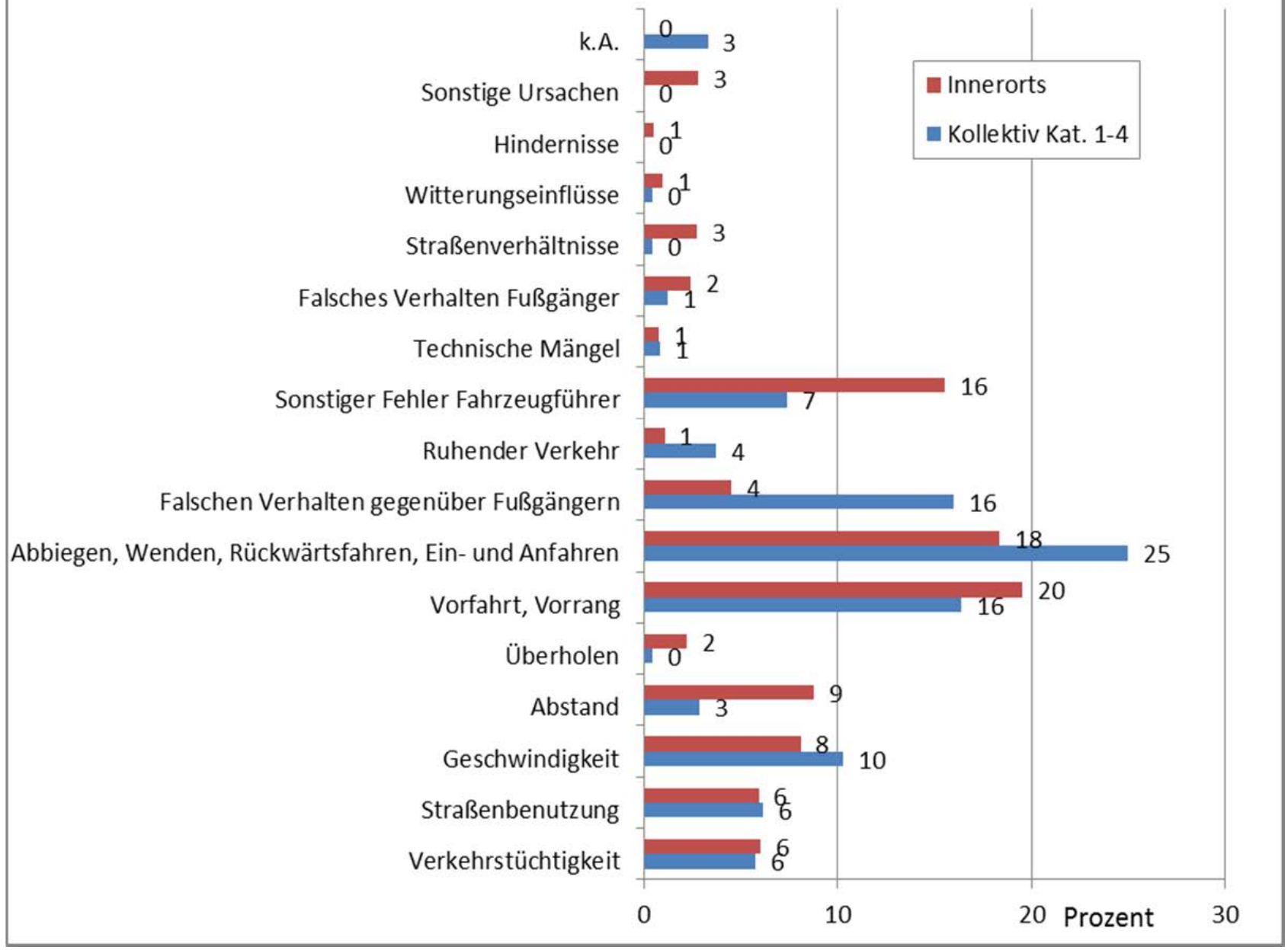

Abbildung 70: Prozentuale Verteilung der Unfallursachen des Beteiligten 1 des „Kollektivs Kat. 1-4“ im Vergleich zu innerörtlichen Unfällen 


\section{Schritt}

Bei den weiterführenden Auswertungen von den insgesamt 40 Unfällen in ausgewiesenen VBB mit der Unfallursache „Vorfahrt, Vorrang“ lagen 37 Unfälle vom Unfalltyp „Einbiegen/KreuzenUnfall“ vor (Abbildung 71). 32 Unfälle davon besaßen die Unfallart „Zusammenstoß mit anderem Fahrzeug, das einbiegt oder kreuzt".

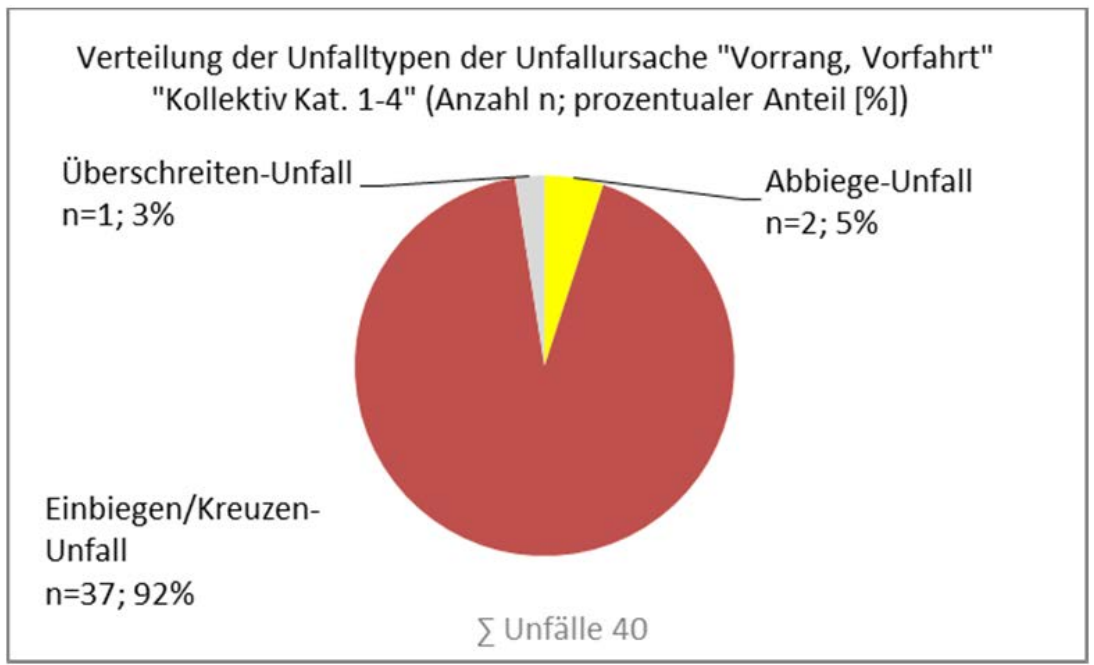

Abbildung 71: Verteilung der Unfalltypen der Unfallursache „Vorrang, Vorfahrt“ des „Kollektivs Kat. 1-4“

Die 61 Unfälle in ausgewiesenen VBB aufgrund der Unfallursache „Abbiegen, Wenden, Rückwärtsfahren, Ein- und Anfahren“ verteilten sich hauptsächlich auf die Unfalltypen „Einbiegen/Kreuzen-Unfall“ (25 Unfälle) und „Sonstiger Unfall“ (24 Unfälle) (Abbildung 72). Die Unfallarten lagen entsprechend hoch bei „Zusammenstoß mit anderem Fahrzeug, das einbiegt oder kreuzt“ (22 Unfälle des Unfalltyps „Einbiegen/Kreuzen-Kreuzen“). Die Unfallarten des Unfalltyps „Sonstiger Unfall“ teilten sich hauptsächlich auf in zehn Unfälle „Zusammenstoß zwischen Fahrzeug und Fußgänger" und zehn „Unfälle anderer Art".

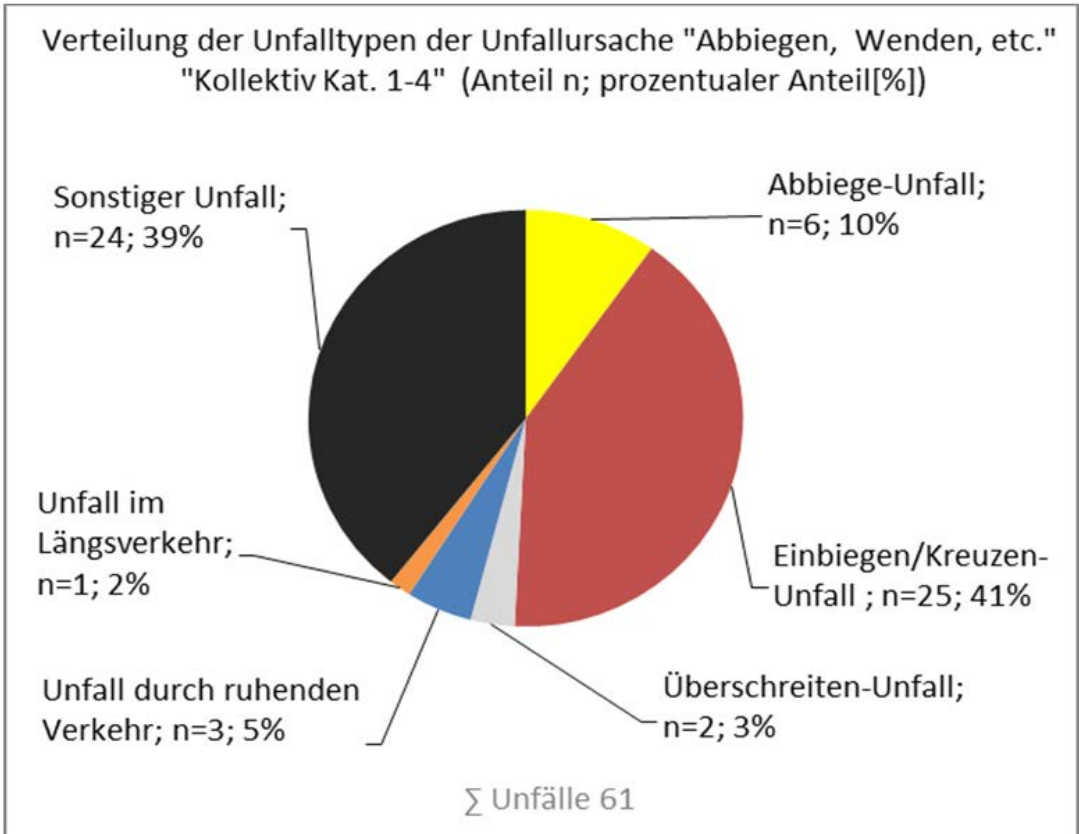

Abbildung 72: Verteilung der Unfalltypen der Unfallursache „Abbiegen, Wenden, Rückwärtsfahren, Ein- und Anfahren“ des „Kollektivs Kat. 1-4“ 
Bei der Unfallursache „falsches Verhalten gegenüber Fußgängern“ (39 Unfälle) lagen 15 Unfälle des Unfalltyps „Überschreiten-Unfall“ vor (alle mit der Unfallart „Zusammenstoß zwischen Fahrzeug und Fußgänger") und 13 des Unfalltyps "Sonstiger Unfall (hiervon sind 12 von der Unfallart „Zusammenstoß zwischen Fahrzeug und Fußgänger“ (Abbildung 73).

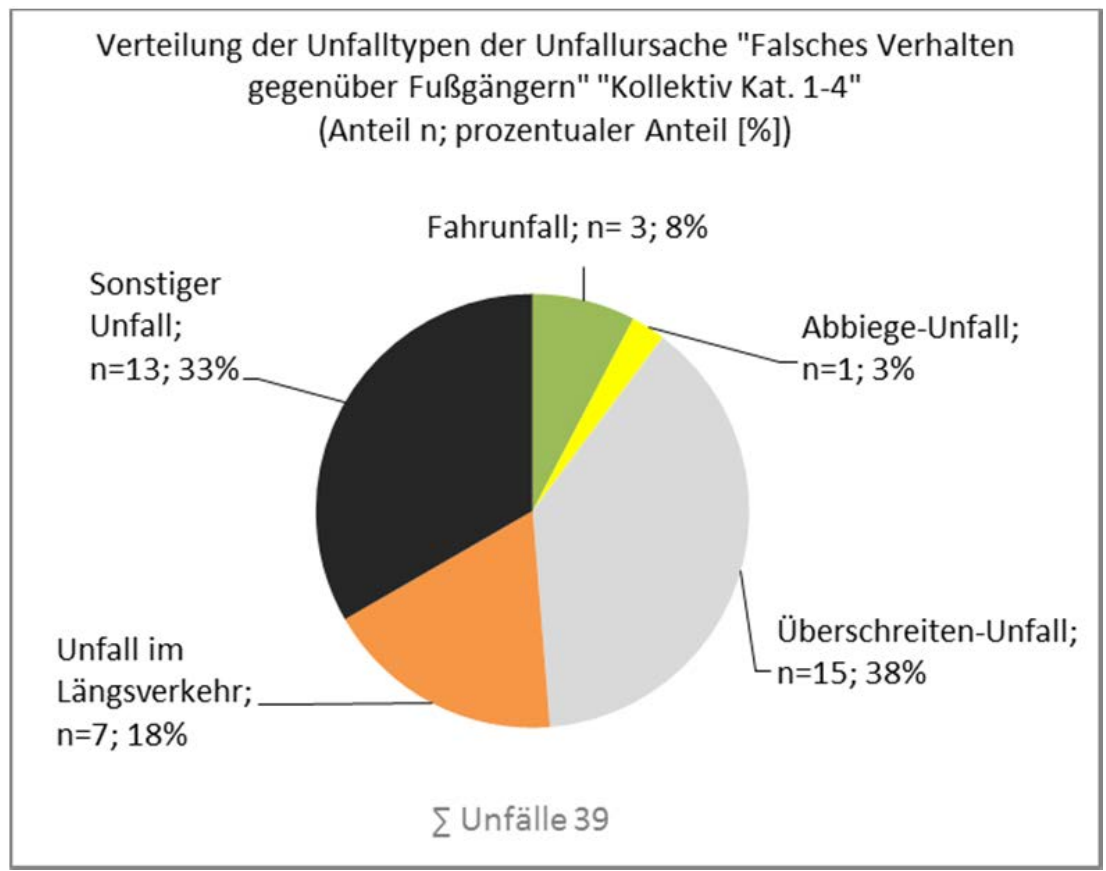

Abbildung 73: Verteilung der Unfalltypen der Unfallursache „Falsches Verhalten gegenüber Fußgängern“ des „Kollektivs Kat. 1-4“

\subsubsection{Unfallumstände}

$\mathrm{Zu}$ den Auswertungen der Unfallumstände der Unfälle der Kategorie 1-4 in ausgewiesenen Verkehrsberuhigten Bereichen liegen folgende Ergebnisse vor:

- 53 Unfälle fanden im Winter von Dezember bis März statt (22\%),

- 50 Unfälle sind am Wochenende (Samstag oder Sonntag) passiert (20\%),

- 64 Unfälle liegen in den Spitzenzeiten vor (6-9/16-19 Uhr) (26\%),

- 50 Unfälle fanden in der Nacht statt, bei Dämmerung oder Dunkelheit (20\%),

- 40 Unfälle sind bei nasser, feuchter oder glatter Fahrbahn passiert (16\%).

Es konnten keine Auffälligkeiten hinsichtlich der Unfallumstände abgeleitet werden. Der Anteil der Unfälle lag jeweils deutlich unter dem durchschnittlichen Erwartungswert (vgl. Abbildung 9). 


\subsection{Zusammenfassung der Unfallanalyse der Untersuchungsobjekte}

Zusammengefasst kann für das Unfallgeschehen der Kategorie 1-4 von ausgewiesenen Verkehrsberuhigten Bereichen festgehalten werden:

\section{Unfallkategorie}

- Unfälle der Kategorie 4 waren in ausgewiesenen Verkehrsberuhigten Bereichen seltene Ereignisse (6\% der Unfälle Kategorie 1-4). Bei innerörtlichen Straßen traten sie wesentlich häufiger auf (19\%).

- Die Verteilung der Unfälle nach Kategorie 1-3 war in ausgewiesenen Verkehrsberuhigten Bereichen ähnlich wie in innerörtlichen Straßen.

\section{Unfalltyp}

- Der Haupt-Unfalltyp der in ausgewiesenen Verkehrsberuhigten Bereichen auftrat, war der Unfalltyp 3 „Einbiegen/Kreuzen-Unfall“. Im Vergleich zu innerörtlichen Unfällen lag der Anteil von $31 \%$ jedoch nicht auffällig hoch.

- Der zweithäufigste Unfalltyp in ausgewiesenen Verkehrsberuhigten Bereichen war der Unfalltyp 7 „Sonstiger Unfall“. Mit 24\% war er deutlich häufiger vertreten als bei innerörtlichen Unfällen. Aus der Analyse der Unfallarten war zu erkennen, dass hiervon:

o 46\% „Zusammenstöße zwischen Fahrzeug und Fußgänger“ sind und die Ursachen in Fehlern der Fahrzeugführer gegenüber Fußgängern an anderen Stellen oder beim Wenden oder Rückwärtsfahren lagen und

o 33\% Unfälle anderer Art waren, bei denen die Haupt-Unfallursache auch im Wenden oder Rückwärtsfahren lag.

Diese Unfallarten und Ursachen können als typische Unfälle in ausgewiesenen Verkehrsberuhigten Beriechen angesehen werden.

- Unfälle mit den Unfalltypen 6 „Unfall im Längsverkehr“ und 2 „Abbiege-Unfall“ waren in ausgewiesenen Verkehrsberuhigten Bereichen deutlich seltener vertreten als auf innerörtlichen Straßen.

\section{Unfallart}

- Die Haupt-Unfallarten von Unfällen der Kategorie 1-4 in ausgewiesenen Verkehrsberuhigten Bereichen waren der „Zusammenstoß zwischen Fahrzeug und Fußgänger" (28\%), der „Zusammenstoß mit anderem Fahrzeug, das einbiegt oder kreuzt“ (29\%) und der „Unfall anderer Art“ (15\%). Diese Erkenntnisse stimmen inhaltlich mit den Auswertungen der Unfallarten und Unfallursachen zu den Haupt-Unfalltypen überein.

- Der Anteil der Unfälle mit der Unfallart „Zusammenstoß zwischen Fahrzeug und Fußgänger" lag deutlich über dem Durchschnitt der innerörtlichen Unfälle Kategorie 1-4. Deutlich niedriger war der Anteil der Unfälle in ausgewiesenen Verkehrsberuhigten Bereichen mit der Unfallart „Zusammenstoß mit anderem Fahrzeug, das vorrausfährt oder wartet".

- Die Zusammenstöße zwischen Fahrzeug und Fußgänger traten ursächlich häufig durch „Falsches Verhalten gegenüber Fußgängern an anderer Stelle" (nicht an Fußgängerüberwegen, Furten, Haltestellen oder beim Abbiegen) auf.

- Die Unfallursachen für die Unfallart „Zusammenstoß mit anderem Fahrzeug, das einbiegt oder kreuzt“ lagen oft bei „Nichtbeachten der Regel rechts-vor-links“, „Nichtbeachten der die Vorfahrt regelnden Verkehrszeichen“ und „Fehler beim Einfahren in den fließenden Verkehr". 


\section{Beteiligte}

- An Unfällen der Kategorie 1-4 waren in ausgewiesenen Verkehrsberuhigten Bereichen seltener Pkw beteiligt als an innerörtlichen Unfällen (46\% VBB, 62\% IO). Der Anteil der Fußgänger- und Radfahrerbeteiligung lag in ausgewiesenen VBB deutlich höher. Fußgänger waren zu $15 \%$ in Unfälle in VBB verwickelt und nur zu 7\% auf innerörtlichen Straßen. Radfahrer sind in ausgewiesenen VBB häufiger in Unfälle verwickelt gewesen (26\%) als auf innerörtlichen Straßen (16\%).

- Fußgänger waren nur selten Hauptverursacher von Unfällen, jedoch in über einem Viertel der Unfälle als zweiter Beteiligter involviert. Fahrradfahrer sind nahezu gleich oft als Hauptverursacher wie auch als zweiter Beteiligter in einen Unfall der Kategorie 1-4 in VBB verwickelt gewesen.

- Unfälle unter Beteiligung eines Fußgängers waren zumeist vom Unfalltyp „Sonstiger Unfall“ oder Unfalltyp „Überschreiten-Unfall“. Als Unfallart wurde in $89 \%$ der Fälle der „Zusammenstoß zwischen Fahrzeug und Fußgänger“ angegeben.

- Der Haupt-Unfalltyp von Unfällen unter Beteiligung eines Radfahrers war der „Einbiegen/Kreuzen-Unfall“. Die Unfälle verteilten sich über mehrere Unfallarten, am häufigsten vertreten war die Unfallart „Zusammenstoß mit anderem Fahrzeug, das einbiegt oder kreuzt".

- Die Kombinationen der Verkehrsbeteiligten an einem Unfall der Kategorie 1-4 unterschieden sich wesentlich von den innerörtlichen Unfällen. In ausgewiesenen Verkehrsberuhigten Bereichen war der Anteil der Unfälle zwischen einem Pkw (Hauptverursacher) und einem Fußgänger mit über 20\% am höchsten. Auf innerörtlichen Straßen lag diese Kombination nur in ca. $7 \%$ der Fälle vor. In innerörtlichen Straßen spielte die Beteiligung bzw. Kollision zweier Pkw an einem Unfall eine wesentlich größere Rolle (32\%) im Vergleich zu VBB (10\%). Auffallend hoch lagen in ausgewiesenen VBB auch die Anteile von Unfällen mit einem Radfahrer (Pkw-Rad 17\% und Rad-Pkw 18\%). Innerorts lagen die Anteile viel geringer (Pkw-Rad 14\% und Rad-Pkw 5\%).

- Der prozentuale Anteil der Alleinunfälle war in ausgewiesenen Verkehrsberuhigten Bereichen ähnlich niedrig wie bei innerörtlichen Straßen (zwischen 2-5\%). Der Anteil der Alleinunfälle lag von Pkws auf innerörtlichen Straßen etwas höher als in VBB.

\section{Unfallursachen}

- Die Haupt-Unfallursache der Unfälle der Kategorie 1-4 in ausgewiesenen Verkehrsberuhigten Bereichen war das „Abbiegen, Wenden, Rückwärtsfahren, Ein- und Anfahren“ (26\%). Weitere $16 \%$ der Unfälle passierten aufgrund eines falschen Verhaltens gegenüber Fußgängern und weitere $16 \%$ aufgrund von Vorfahrts- bzw. VorrangVerletzungen. Diese Erkenntnisse stimmen inhaltlich mit den Auswertungen zu den Unfalltypen, Unfallarten und Unfallursachen überein.

- In ausgewiesenen VBB traten wesentlich mehr Unfälle aufgrund eines falschen Verhaltens gegenüber Fußgängern auf, als bei innerörtlichen Unfällen. Auch der Anteil der Unfälle aufgrund von „Abbiegen, Wenden, Rückwärtsfahren, Ein- und Anfahren“ lag deutlich höher. Dafür waren bei allen innerörtlichen Straßen die Unfallursachen „Sonstiger Fehler beim Fahrzeugführer" und „Abstand“ bei innerörtlichen Straßen häufiger vertreten.

\section{Unfallumstände}

- Es konnten keine Auffälligkeiten hinsichtlich der Unfallumstände erkannt werden. 


\section{Ableitung von Zusammenhängen zwischen Unfällen und Gestaltung}

In Kapitel 10 wurde das Unfallgeschehen von 278 Untersuchungsobjekten (ausgewiesene VBB) ausgewertet. Dabei wurde nicht nach einzelnen charakteristischen Merkmalen wie zum Beispiel der Straßenfunktion, der Gestaltung oder den Abschnittslängen von ausgewiesenen VBB unterschieden. Die nachfolgenden Auswertungen sollten Aufschluss darüber geben, ob sich die Struktur der Unfälle wesentlich ändert, wenn nach den unterschiedlichen Gestaltungsmerkmalen unterschieden wird. Hierzu wurden die Unfallfolgen, die Unfalltypen, Unfallarten etc. in den jeweiligen Straßenfunktionen genauer betrachtet.

Für Analysen zu Unfällen der Kategorie 5 und 6 wurde - analog zu Kapitel 10 - auf das Unfallkollektiv der unfallauffälligsten Untersuchungsobjekte des oben genannten „Kollektivs Kat. 14“ zurückgegriffen. Da die Unfalldaten dieses Kollektivs ausschließlich für das Jahr 2011 vollständig erhoben werden konnten, wurde es „Kollektiv 2011“ genannt. Auch für das „Kollektiv 2011" ist untersucht worden, ob Zusammenhänge zwischen Unfällen (der Kat. 1-6 von unfallauffälligen Bereichen) und der Gestaltung erkennbar sind. Die Ergebnisse der Unfallanalyse des „Kollektiv 2011“ in Bezug auf die Gestaltungsmerkmale sind in Anhang 4 dokumentiert.

\subsection{Datengrundlage und Vorgehensweise}

Für die Ableitung von Zusammenhänge zwischen Unfällen und Gestaltungsmerkmalen von ausgewiesenen Verkehrsberuhigte Bereiche wurde - wie in Kapitel 10 - das „Kollektiv Kat. 1-4“ ausgewertet, in dem die Unfalldaten für 278 VBB aus 6 Jahren vorlagen. Dabei wurde geklärt, ob es Merkmale gab, die sich in den jeweiligen Straßenfunktionen unterschiedlich stark ausprägten. Dementsprechend wurde nach Auffälligkeiten im Unfallgeschehen gesucht und die Unfälle ggf. im Detail betrachtet.

Aufgrund der unterschiedlichen Anzahl von Unfällen in den jeweiligen Untergruppen (Stichprobengrößen), war es schwierig, eine Auffälligkeit bei der Verteilung zwischen den jeweiligen Straßenfunktionen ausfindig zu machen. Dafür musste an einigen Stellen die prozentuale Verteilung der Unfälle innerhalb der Straßenkategorien miteinander verglichen werden. D.h., dass in der Untergruppe einer Straßenfunktion die Gesamtanzahl der Unfälle jeweils $100 \%$ darstellen. Mit dieser Art der Darstellung konnten die Stichproben besser miteinander verglichen werden.

\subsection{Unfallanalysen „Kollektiv Kat. 1-4“ nach Straßenfunktionen}

\subsubsection{Unfallkategorien / Unfalldichten / Unfallkostendichten}

Zuerst wurde die Verteilung der Unfallkategorien je Straßenfunktion ausgewertet, um die Frage beantworten zu können, ob sich die Schwere der Unfallfolgen je Straßenfunktion ähnlich verteilten (Abbildung 74). Bei Betrachtung der Anzahl der Unfälle je Straßenfunktion fiel sofort auf, dass in Geschäftsbereichen wesentlich häufiger Unfälle auftraten als in den anderen Straßenfunktionen. Und das, obwohl die erhobenen Abschnittslängen in der Summe von Geschäftsstraßen im Vergleich zu z.B. Wohnstraßen deutlich niedriger waren (vgl. Tabelle 13, Kapitel 10.1.1). Insgesamt wurden ca. $79 \mathrm{~km}$ Abschnittslängen in den ausgewiesenen VBB erhoben, davon waren „nur" $11 \mathrm{~km}$ (also ca. 14\%) in Geschäftsstraßen. Die Anzahl der Unfälle in Wohnstraßen war hingegen deutlich niedriger, obwohl hier in der Summe Abschnittslängen von 40,5km (ca. 51\%) vorlagen. Daraus ließ sich an dieser Stelle schon ableiten, dass Geschäftsstraßen im betrachteten Kollektiv eine höhere Unfalldichte im Vergleich zu Wohnstraßen besaßen. Die Auswertungen zu den Unfalldichten und Unfallkosten sind am Ende des Kapitels dokumentiert. 


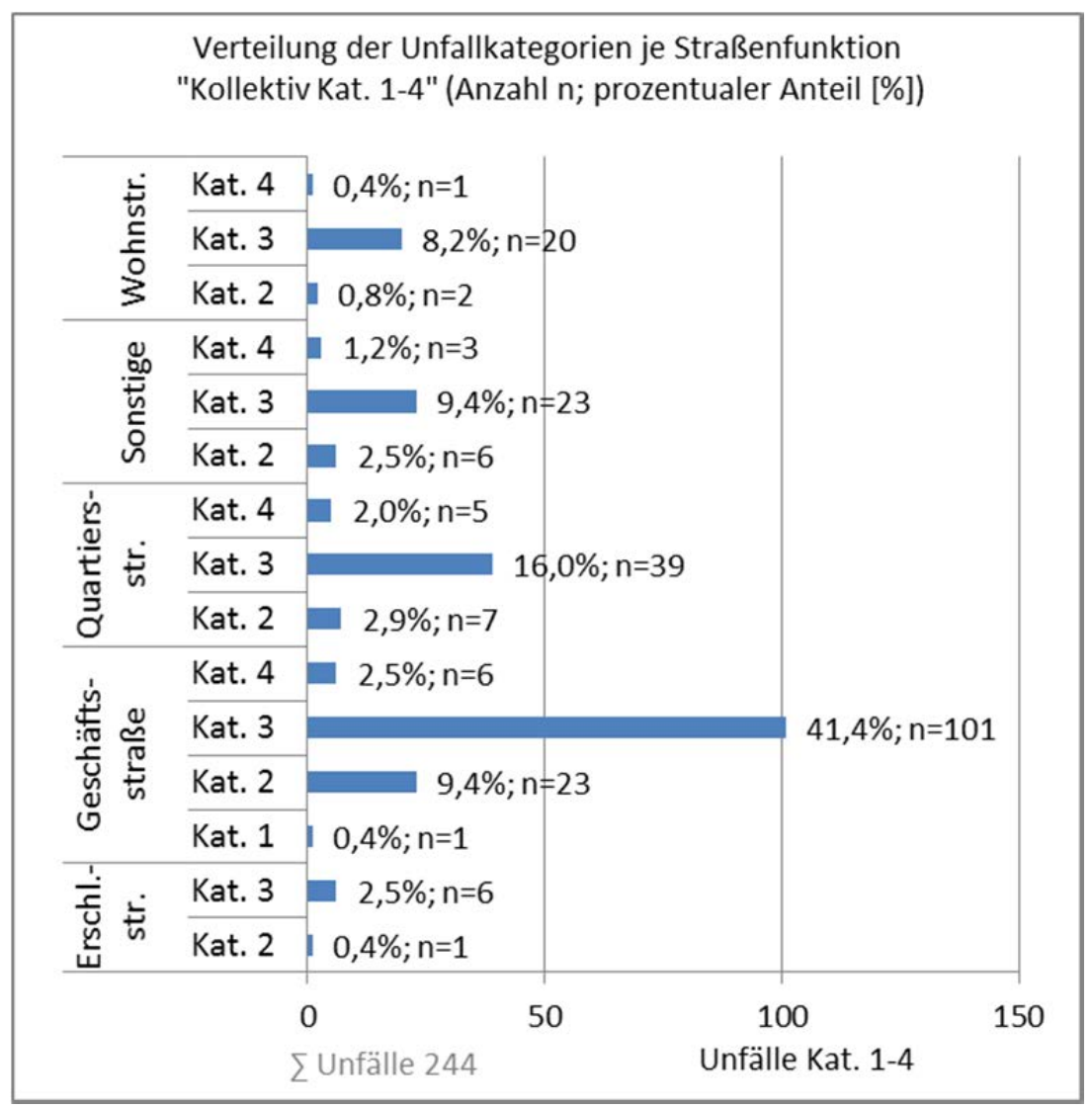

Abbildung 74: Verteilung der Unfallkategorien je Straßenfunktion des „Kollektivs Kat. 1-4“ (Anzahl)

Damit Auffälligkeit bei der Verteilung der Unfallkategorien in den jeweiligen Straßenfunktionen ersichtlich wurden, musste die prozentuale Verteilung der Unfälle je Straßenkategorie miteinander verglichen werden. Diese kann der Abbildung 75 entnommen werden.

Daraus lässt sich ablesen, dass in Wohnstraßen nur knapp 9\% Unfälle der Kategorie 2 waren (Unfälle mit Schwerverletzten). In Geschäftsstraßen waren es knapp 18\%. Der Anteil der Unfälle der Kategorie 4 war in Geschäftsstraßen und Wohnstraßen ähnlich niedrig. Daraus konnte abgeleitet werden, dass die Unfallfolgen in Geschäftsstraßen schwerer waren, als in Wohnstraßen.

In Quartiersstraßen traten Unfälle der Kategorie 2 in ca. 14\% der Fälle auf und damit etwas häufiger als bei Wohnstraßen. Unfälle der Kategorie 3 waren in Quartiersstraßen hingegen seltener als in Wohnstraßen. Aber der Anteil der Kategorie 4 Unfälle in Quartiersstraßen lag deutlich höher (10\%) als bei Wohnstraßen (4\%), so dass sich hier ein anderes Bild bezüglich der Unfallschwere abzeichnete. Abschließend konnte nur durch den Vergleich der Unfallkosten in den jeweiligen Straßenfunktionen korrekte Rückschlüsse auf die durchschnittliche Unfallschwere getroffen werden (am Ende des Kapitels).

Die schwersten Unfallfolgen besaß die Gruppe der „Sonstigen“ Straßen. Hier lag mit der größte Anteil der Kategorie 2 Unfälle vor. Bei einer genauen Betrachtung fiel auf, dass sich vier von den sieben Unfällen der Kategorie 2 in zwei unmittelbar aneinander angrenzenden Beispielen ereignet haben. Alle fanden unter Beteiligung von Radfahrern statt und lagen entweder in einer Einmündung oder Kurve. Eine allgemeine Aussage zu der Straßenfunktion „Sonstige“ wurde daher nicht getroffen. Auch bei den Erschließungsstraßen wurde auf eine weitere Betrachtung verzichtet. Da insgesamt nur sieben Unfälle in dieser Straßenfunktion aufgenommen wurden, ist die Auswertung für diese Gruppe nicht aussagekräftig. 


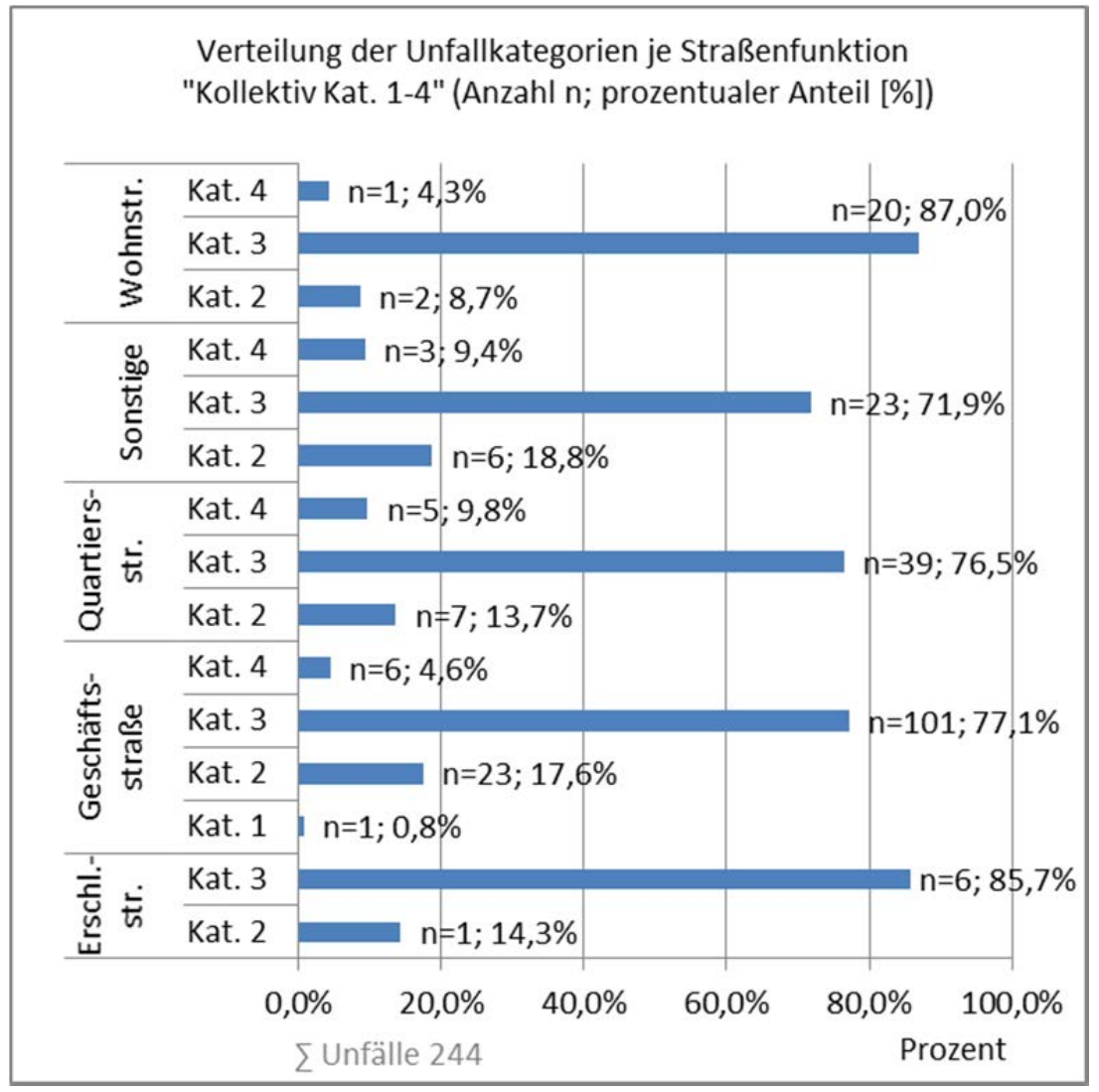

Abbildung 75: Verteilung der Unfallkategorien je Straßenfunktionen „Kollektivs Kat. 1-4“

\section{Unfalldichten}

Die Unfalldichte (UD) beschreibt die durchschnittliche Anzahl der Unfälle, die in einem Betrachtungszeitraum auf $1 \mathrm{~km}$ Straßenlänge existieren. Die Unfalldichte aller Unfälle der Kategorie 1-4 der ausgewiesenen Verkehrsberuhigten Bereiche lag bei 0,51 Unfällen je $\mathrm{km}$ und Jahr (Abbildung 76). Die Unfalldichte der Geschäftsstraßen war mit 1,95 U/(kmª) mit Abstand am höchsten. Die Wohnstraßen haben die niedrigste Unfalldichte aufgezeigt $\left(0,09 \mathrm{U} /\left(\mathrm{km}{ }^{\star} \mathrm{a}\right)\right)$.

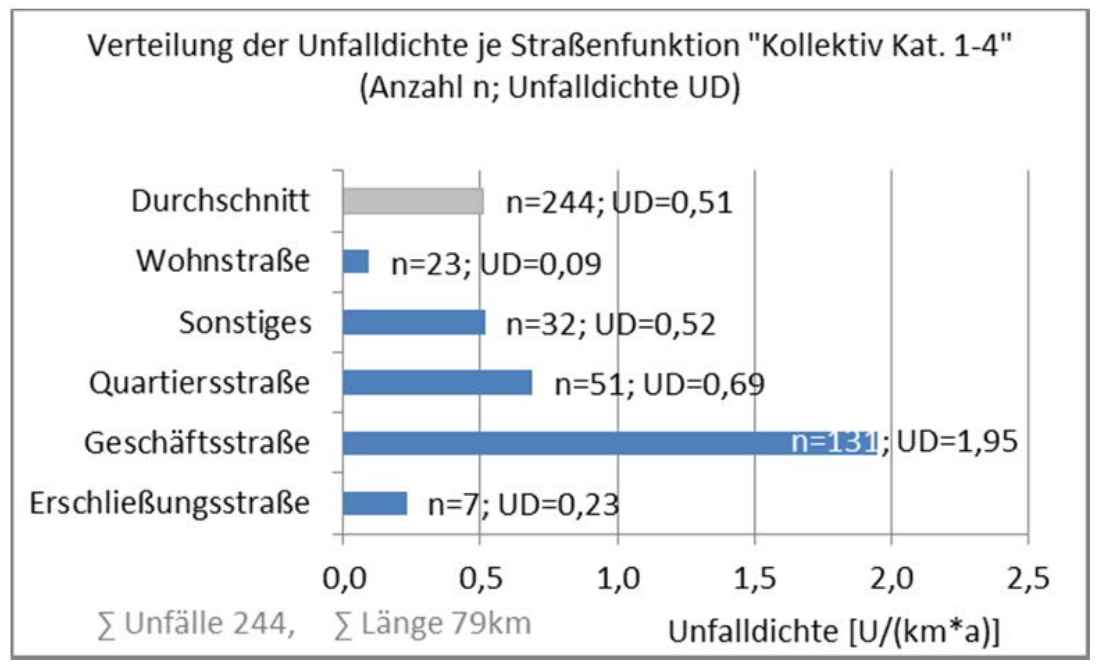

Abbildung 76: Verteilung der Unfalldichte je Straßenfunktion des „Kollektivs Kat. 1-4“ 
Innerhalb der Straßenfunktionen wurde die Unfalldichte weiter nach der Verkehrsführung differenziert (Abbildung 77). Dabei fiel zunächst einmal auf, dass in den jeweiligen Straßenfunktionen immer unterschiedliche Verkehrsführungen die höchsten Unfalldichten aufwiesen. In den Wohnstraßen lag die höchste Unfalldichte bei Bereichen mit optischer und baulicher Trennung $\left(20 \mathrm{U} /\left(\mathrm{km}^{\star} \mathrm{a}\right)\right)$ und mit baulicher Trennung und Mischverkehr am zweithöchsten $\left(0,17 \mathrm{U} /\left(\mathrm{km}^{\star} \mathrm{a}\right)\right)$. In Geschäftsstraßen lag die höchste Unfalldichte bei Verkehrsführungen vor, die baulich getrennt waren. Sowohl bei den Wohnstraßen, als auch bei den Geschäftsstraßen waren die Abschnittslängen und auch die Anzahl an erhobenen Untersuchungsobjekten ausreichend groß, um sicherstellen zu können, dass die gewonnenen Erkenntnisse aussagekräftig sind.

Die jeweiligen Abschnittslängen, die vorliegende Anzahl von Unfällen, die Anzahl der Untersuchungsobjekte und die Unfalldichte können je Straßenfunktion und Verkehrsführung der Tabelle 26 entnommen werden. Hier sind alle Zeilen grün hinterlegt, die mehr als acht Untersuchungsobjekte $^{7}$ enthalten und deren Streckenabschnitte in der Summe mind. $2,7 \mathrm{~km}^{8}$ lang sind. Die Unfalldichten dieser Untergruppen können als aussagekräftig angesehen werden, da sichergestellt ist, dass eine ausreichende Anzahl an unterschiedlichen Beispielen und ausreichend lange Streckenabschnitte betrachtet wurden. Hierbei ist allerdings zu erwähnen, dass die "Grenzwerte" pragmatisch gewählt wurden und keine statistische Größe darstellen.

Zusammenfassend konnte festgehalten werden:

- Geschäftsstraßen mit baulicher Trennung besaßen die höchsten Unfalldichten (3,63 $\left.\mathrm{U} /\left(\mathrm{km}^{\star} \mathrm{a}\right)\right)$.

- Geschäftsstraßen, die eine optische und bauliche Trennung besaßen, wiesen im Vergleich zu solchen, die eine rein bauliche Trennung besaßen deutlich niedrigere Unfalldichten auf $\left(1,27 \mathrm{U} /\left(\mathrm{km}^{\star} \mathrm{a}\right)\right)$.

- Die Wohnstraßen wiesen die niedrigsten Unfalldichten auf, wenn bei ihnen Mischverkehr oder Mischverkehr mit optischer Trennung vorlag $\left(0,04-0,07 \mathrm{U} /\left(\mathrm{km}^{\star} \mathrm{a}\right)\right)$. Wohnstraßen in denen eine optische und bauliche Trennung vorlag, wiesen deutlich höhere Unfalldichten auf $\left(0,20 \mathrm{U} /\left(\mathrm{km}^{\star} \mathrm{a}\right)\right)$

7 Insgesamt liegen 34 unterschiedliche Kombinationen zwischen Straßenkategorie und Verkehrsführung vor. Bei der Gesamtanzahl von 278 Untersuchungsobjekten, sind im Durchschnitt 8,4 Beispiele je Untergruppe enthalten. Gruppen, die mehr als acht Untersuchungsobjekte enthalten, sollen daher als aussagekräftig gelten.

8 Die durchschnittliche Länge eines erhobenen Untersuchungsobjektes beträgt knapp 300m. Wird davon ausgegangen, dass über acht Untersuchungsobjekte (also neun) in einer Gruppe enthalten sein sollen, so sollte die Mindestlänge von $9 \times 300 m=2.700 m$ betragen, um von einem aussagekräftigen Ergebnis sprechen zu können. 


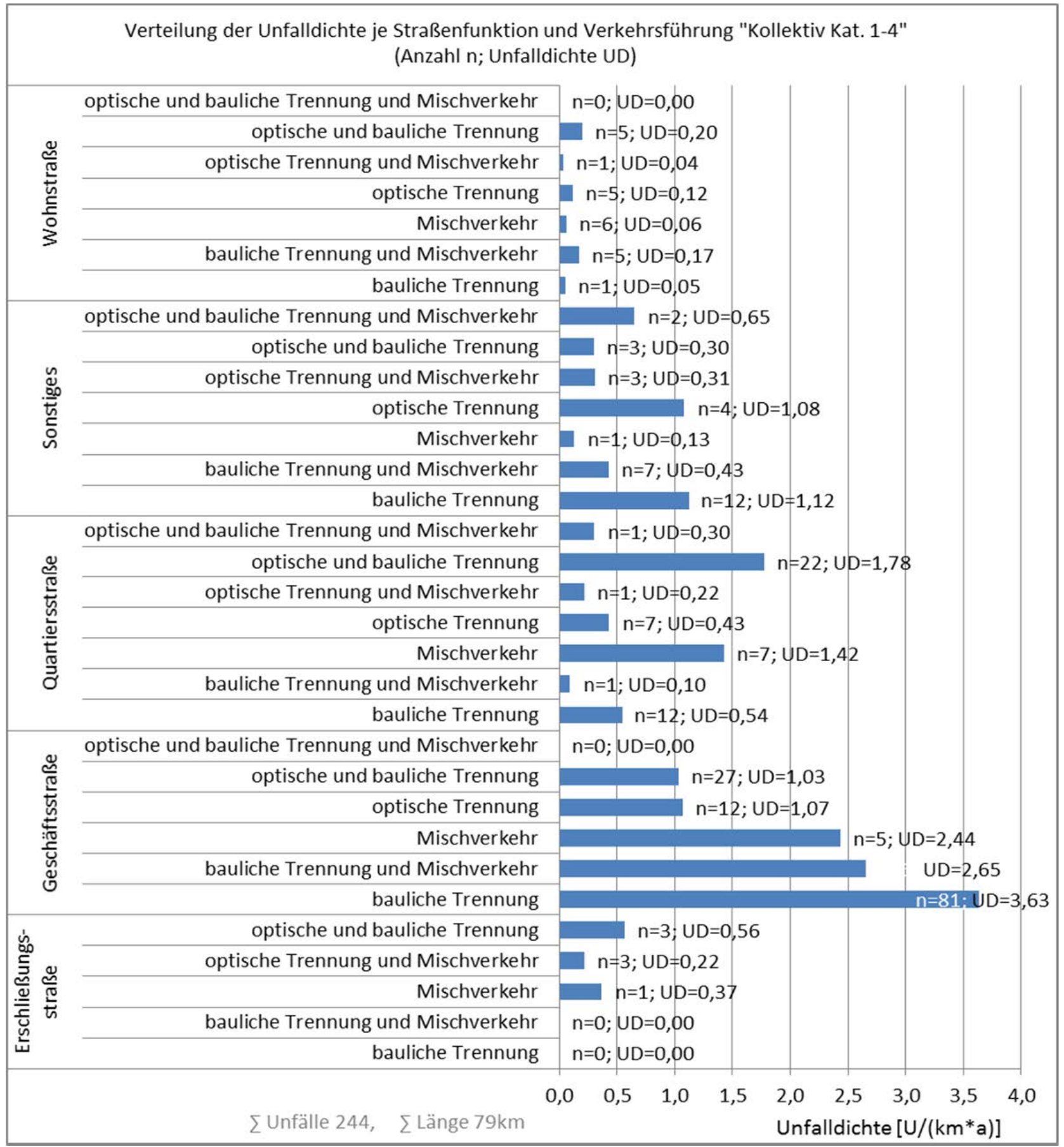

Abbildung 77: Verteilung der Unfalldichte je Straßenfunktion und Verkehrsführung des „Kollektivs Kat. 1-4“ 
Tabelle 26: Unfallanzahl, Abschnittslängen und Unfalldichten je Straßenfunktion und Verkehrsführung des „Kollektivs Kat. 1-4“

\begin{tabular}{|c|c|c|c|c|c|}
\hline $\begin{array}{l}\text { Straßen- } \\
\text { funktion }\end{array}$ & Verkehrsführung & $\begin{array}{l}\text { Unfälle } \\
\text { Kat. 1-4 }\end{array}$ & $\begin{array}{l}\text { Länge } \\
{[\mathrm{m}]}\end{array}$ & VBB & $\begin{array}{c}\text { Unfalldichte } \\
{[\mathrm{U} /(\mathbf{k m * a})]}\end{array}$ \\
\hline \multirow{6}{*}{ 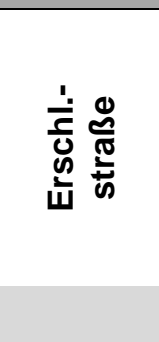 } & bauliche Trennung & 0 & 1.060 & 3 & 0,00 \\
\hline & bauliche Trennung und MV & 0 & 301 & 1 & 0,00 \\
\hline & Mischverkehr & 1 & 452 & 2 & 0,37 \\
\hline & optische Trennung und MV & 3 & 2.285 & 2 & 0,22 \\
\hline & optische und bauliche Trennung & 3 & 885 & 3 & 0,56 \\
\hline & Summe/Mittel & 7 & 4.983 & 11 & 0,23 \\
\hline \multirow{7}{*}{ 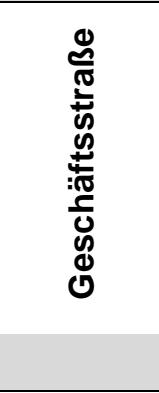 } & bauliche Trennung & 81 & 3.715 & 17 & 3,63 \\
\hline & bauliche Trennung und MV & 6 & 377 & 2 & 2,65 \\
\hline & Mischverkehr & 5 & 342 & 2 & 2,44 \\
\hline & optische Trennung & 12 & 1.862 & 7 & 1,07 \\
\hline & optische und bauliche Trennung & 27 & 4.349 & 16 & 1,03 \\
\hline & optische und bauliche Trennung und MV & 0 & 563 & 1 & 0,00 \\
\hline & Summe/Mittel & 131 & 11.208 & 45 & 1,95 \\
\hline \multirow{8}{*}{ 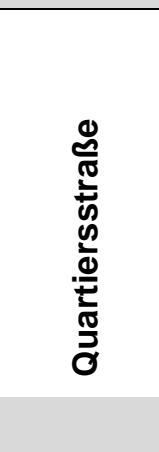 } & bauliche Trennung & 12 & 3.673 & 15 & 0,54 \\
\hline & bauliche Trennung und Mischverkehr & 1 & 1.753 & 9 & 0,10 \\
\hline & Mischverkehr & 7 & 819 & 3 & 1,42 \\
\hline & optische Trennung & 7 & 2.694 & 10 & 0,43 \\
\hline & optische Trennung und MV & 1 & 755 & 3 & 0,22 \\
\hline & optische und bauliche Trennung & 22 & 2.060 & 12 & 1,78 \\
\hline & optische und bauliche Trennung und MV & 1 & 547 & 2 & 0,30 \\
\hline & Summe/Mittel & 51 & 12.301 & 54 & 0,69 \\
\hline \multirow{8}{*}{ 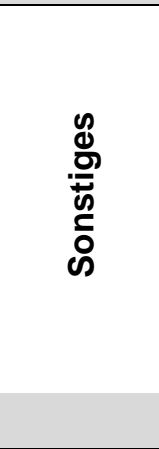 } & bauliche Trennung & 12 & 1.781 & 7 & 1,12 \\
\hline & bauliche Trennung und MV & 7 & 2.729 & 10 & 0,43 \\
\hline & Mischverkehr & 1 & 1.266 & 10 & 0,13 \\
\hline & optische Trennung & 4 & 615 & 5 & 1,08 \\
\hline & optische Trennung und MV & 3 & 1.621 & 5 & 0,31 \\
\hline & optische und bauliche Trennung & 3 & 1.674 & 5 & 0,30 \\
\hline & optische und bauliche Trennung und MV & 2 & 511 & 3 & 0,65 \\
\hline & Summe/Mittel & 32 & 10.197 & 45 & 0,52 \\
\hline \multirow{9}{*}{ 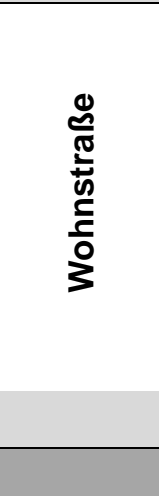 } & bauliche Trennung & 1 & 3.093 & 10 & 0,05 \\
\hline & bauliche Trennung und MV & 5 & 4.851 & 14 & 0,17 \\
\hline & Mischverkehr & 6 & 15.558 & 47 & 0,06 \\
\hline & optische Trennung & 5 & 7.052 & 16 & 0,12 \\
\hline & optische Trennung und MV & 1 & 4.405 & 14 & 0,04 \\
\hline & optische und bauliche Trennung & 5 & 4.184 & 17 & 0,20 \\
\hline & optische und bauliche Trennung und MV & 0 & 1.438 & 5 & 0,00 \\
\hline & Summe/Mittel & 23 & 40.581 & 123 & 0,09 \\
\hline & SummelMittel & 244 & 79.270 & 278 & 0,51 \\
\hline
\end{tabular}




\section{Unfallkostendichten}

Die Unfallkosten fassen die Anzahl und Schwere der Unfälle zusammen und ermöglichen den Vergleich der Verkehrssicherheit zu einem bestimmten Preisstand. Für die jeweiligen Unfallkategorien liegen pauschale Unfallkostensätze für innerörtliche Erschließungsstraßen aus dem Jahr 2009 im Merkblatt zur Örtlichen Unfalluntersuchung in Unfallkommissionen (FGSV 316/1, 2012) vor $^{9}$. Die mittlere Unfallkostendichte beziffert die durchschnittlichen volkswirtschaftlichen Kosten in 1.000 Euro je Jahr durch Straßenverkehrsunfälle, die auf $1 \mathrm{~km}$ Länge des Straßenbereichs entstanden sind. Die volkswirtschaftlichen Kosten fassen die Anzahl und die Schwere der Unfälle zusammen.

Die Unfallkostendichte aller Unfälle der Kategorie 1-4 der Verkehrsberuhigten Bereiche lag bei $19.100 € /\left(\mathrm{km}^{\star} \mathrm{a}\right)$ (Abbildung 78). Die Unfallkostendichten verteilten sich nahezu identisch zu den Unfalldichten. Die Unfallkostendichte der Geschäftsstraßen lag mit $77.800 € /\left(\mathrm{km}^{\star} \mathrm{a}\right)$ mit Abstand auch hier am höchsten. Die Wohnstraßen wiesen die niedrigste Unfallkostendichte mit $2.500 € /\left(\mathrm{km}^{\star} \mathrm{a}\right)$ auf.

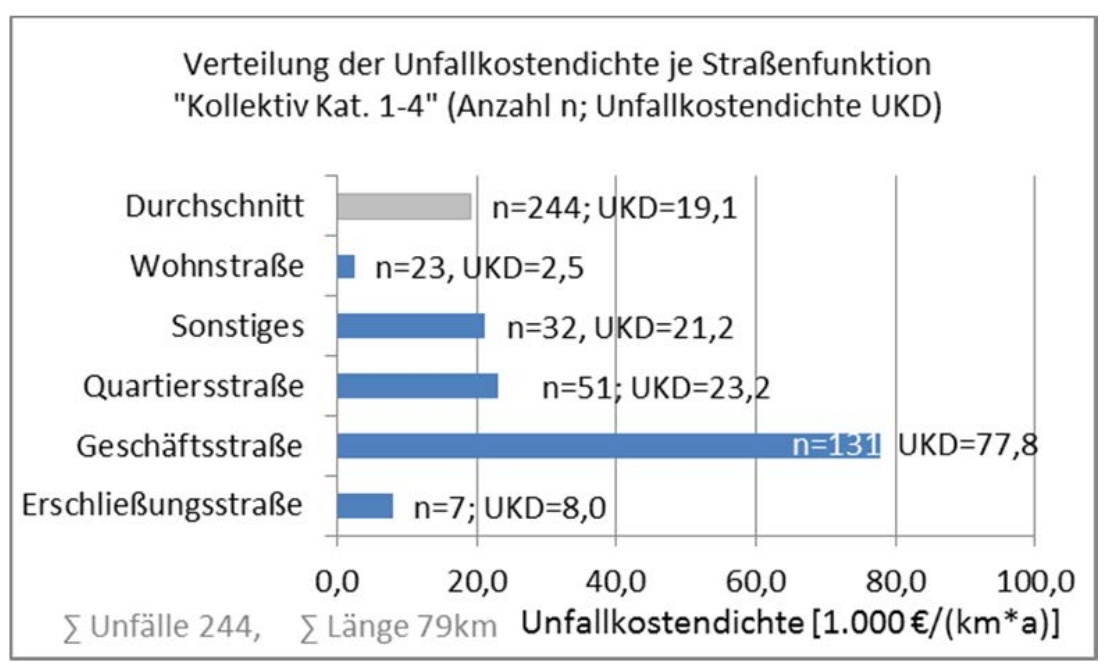

Abbildung 78: Verteilung der Unfallkostendichte je Straßenfunktion des „Kollektivs Kat. 1-4“, Preisstand 2009

Auch eine weitere Differenzierung der Unfallkostendichte je Straßenfunktion und Verkehrsführung zeigte keine große Abweichung im Vergleich zu der Verteilung der Unfalldichten auf. Die jeweiligen Unfallkostendichten können der Abbildung $\mathbf{7 9}$ entnommen werden. Aussagekräftig waren wiederum nur solche Gruppen, die eine ausreichende Anzahl an Untersuchungsobjekten und ausreichend lange Streckenabschnitte beinhalteten. Es wird auf Tabelle $\mathbf{2 6}$ verwiesen.

Resümierend konnte festgehalten werden:

- Geschäftsstraßen mit baulicher Trennung besaßen die höchsten Unfallkostendichten $\left(146.200 € /\left(\mathrm{km}^{\star} \mathrm{a}\right)\right.$.

- Geschäftsstraßen, die eine optische und bauliche Trennung besaßen, wiesen im Vergleich zu solchen, die eine rein bauliche Trennung besaßen deutlich niedrigere Unfallkostendichten auf $\left(41.600 € /\left(\mathrm{km}^{\star} \mathrm{a}\right)\right)$.

9 Im Rahmen der vorliegenden Studie wurde untersucht, ob es aufgrund einer ggf. unterschiedlichen Verunglücktenstruktur bei den Unfällen der Kat. 1-4 von ausgewiesenen VBB sinnvoll gewesen wäre, angepassten Unfallkostensätzen anzuwenden. Die Ergebnisse sind im Anhang 5 dokumentiert. 
- Die Wohnstraßen wiesen die niedrigsten Unfallkostendichten auf, wenn bei innen Mischverkehr mit optischer Trennung oder ausschließlich Mischverkehr vorlag (500$\left.2.400 € /\left(\mathrm{km}^{\star} \mathrm{a}\right)\right)$

- Wohnstraßen in denen eine bauliche Trennung vorlag, wiesen deutlich höhere Unfalldichten auf $\left(800 € /\left(\mathrm{km}^{\star} \mathrm{a}\right)\right)$.

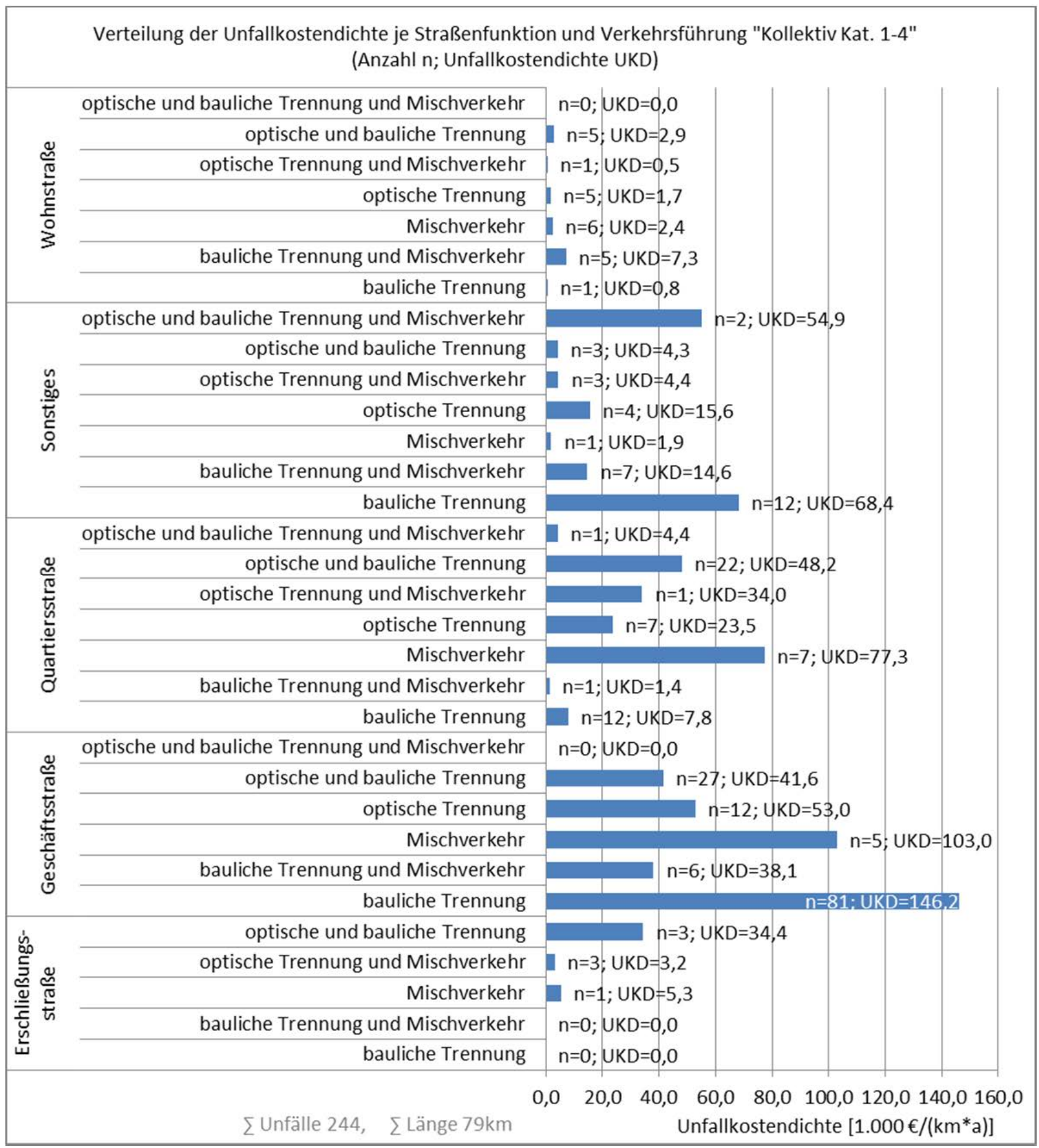

Abbildung 79: Verteilung der Unfallkostendichte je Straßenfunktion und Verkehrsführung des „Kollektivs Kat. $1-4$ ", Preisstand 2009 


\subsubsection{Unfalltypen}

Die Verteilung der Unfalltypen je Straßenfunktion kann der Abbildung $\mathbf{8 0}$ entnommen werden. Hierbei wurde wieder die prozentuale Verteilung der Unfalltypen je Straßenfunktion dargestellt, da durch die unterschiedliche Anzahl der Unfälle in den jeweiligen Gruppen ein Vergleich schwierig war.

Auffällig war, dass in Wohnstraßen und Quartiersstraßen der Haupt-Unfalltyp der Einbiegen/Kreuzen-Unfall war, während in den Geschäftsstraßen sich die Anteile über mehrere Unfalltypen verteilten. Es lagen in Geschäftsstraßen 24\% Einbiegen/Kreuzen- Unfälle und 23\% Sonstige Unfälle vor. Darüber hinaus lagen 16\% Überschreiten-Unfälle vor. Die Verteilung der Unfalltypen in Geschäftsstraßen wich erheblich von denen in Wohnstraßen und Quartiersstraßen ab.

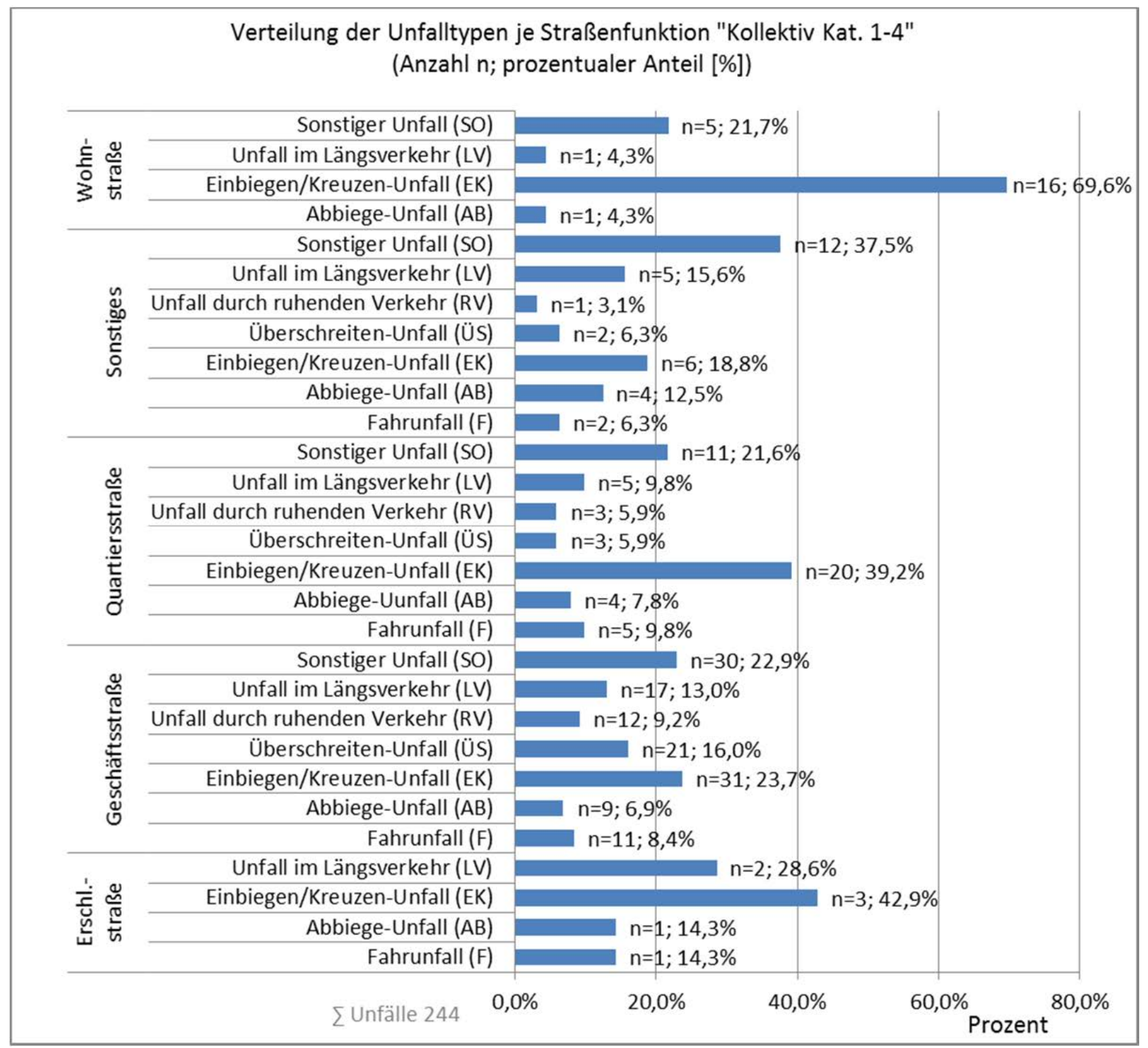

Abbildung 80: Verteilung der Unfalltypen je Straßenfunktion des „Kollektivs Kat. 1-4“ 
Die Unfalltypen wurden aufgrund der geringen Anzahlen in den Untergruppen nicht weiter ausgewertet. Aufgrund der geringen Stichprobengrößen konnten keine aussagekräftigen Ergebnisse mehr erzielt werden.

Nachfolgend wurde noch betrachtet, wie häufig Radfahrer und Fußgänger je Unfalltyp in den Straßenfunktionen Geschäftsstraßen, Quartiersstraßen und Wohnstraßen an Unfällen beteiligt waren. Es wurden nur diese drei Straßenfunktionen betrachtet, da die Erschließungsstraßen ein zu geringes Kollektiv besaßen und die "Sonstigen Straßen" aufgrund ihrer unterschiedlichen Gestaltungen keine aussagekräftigen Rückschlüsse zulassen. In Tabelle 27 sind die Ergebnisse dargestellt. Unfalltypen mit einer geringen Anzahl an Unfällen wurden nicht weiter betrachtet. Aussagekräftig wurden ausschließlich die Untergruppen angesehen, die mind. 15 Unfälle aufwiesen ${ }^{10}$. Sie sind in der Tabelle grün hinterlegt. Auch an dieser Stelle ist zu erwähnen, dass dieser "Grenzwert" willkürlich gesetzt wurde und keine statistische Größe darstellt.

Tabelle 27: Beteiligte Radfahrer und Fußgänger je Straßenfunktion und Unfalltyp des „Kollektivs Kat. 1-4“

\begin{tabular}{|c|c|c|c|c|c|c|}
\hline \multirow{3}{*}{ Straßenfunktion } & \multirow{3}{*}{ Unfalltyp } & \multicolumn{4}{|c|}{ Beteiligte } & \multirow{3}{*}{$\begin{array}{l}\text { Anzahl } \\
\text { U Kat. 1-4 } \\
\text { gesamt }\end{array}$} \\
\hline & & \multicolumn{2}{|c|}{ Radfahrer } & \multicolumn{2}{|c|}{ Fußgänger } & \\
\hline & & Anzahl U & Prozent & Anzahl U & Prozent & \\
\hline \multirow{8}{*}{ Geschäftsstraße } & $1 \mathrm{~F}$ & 4 & $36 \%$ & 1 & $9 \%$ & 11 \\
\hline & $2 A B$ & 5 & $56 \%$ & 4 & $44 \%$ & 9 \\
\hline & $3 \mathrm{EK}$ & 26 & $84 \%$ & 0 & $0 \%$ & 31 \\
\hline & 4 ÜS & 2 & $10 \%$ & 20 & $95 \%$ & 21 \\
\hline & $5 \mathrm{RV}$ & 7 & $58 \%$ & 0 & $0 \%$ & 12 \\
\hline & $6 \mathrm{LV}$ & 9 & $53 \%$ & 4 & $24 \%$ & 17 \\
\hline & $7 \mathrm{SO}$ & 9 & $30 \%$ & 19 & $63 \%$ & 30 \\
\hline & Summe & 62 & $47 \%$ & 48 & $37 \%$ & 131 \\
\hline \multirow{8}{*}{ Quartiersstraße } & $1 \mathrm{~F}$ & 2 & $40 \%$ & 0 & $0 \%$ & 5 \\
\hline & $2 \mathrm{AB}$ & 3 & $75 \%$ & 0 & $0 \%$ & 4 \\
\hline & $3 \mathrm{EK}$ & 12 & $60 \%$ & 0 & $0 \%$ & 20 \\
\hline & 4 ÜS & 1 & $33 \%$ & 3 & $100 \%$ & 3 \\
\hline & $5 \mathrm{RV}$ & 1 & $33 \%$ & 1 & $33 \%$ & 3 \\
\hline & $6 \mathrm{LV}$ & 0 & $0 \%$ & 3 & $60 \%$ & 5 \\
\hline & $7 \mathrm{SO}$ & 4 & $36 \%$ & 7 & $64 \%$ & 11 \\
\hline & Summe & 23 & $45 \%$ & 14 & $27 \%$ & 51 \\
\hline \multirow{7}{*}{ Wohnstraße } & $1 \mathrm{~F}$ & 0 & $0 \%$ & 0 & $0 \%$ & 0 \\
\hline & $2 A B$ & 0 & $0 \%$ & 0 & $0 \%$ & 0 \\
\hline & $3 \mathrm{EK}$ & 10 & $63 \%$ & 1 & $6 \%$ & 16 \\
\hline & $5 \mathrm{RV}$ & 0 & $0 \%$ & 0 & $0 \%$ & 0 \\
\hline & $6 \mathrm{LV}$ & 0 & $0 \%$ & 0 & $0 \%$ & 0 \\
\hline & $7 \mathrm{SO}$ & 4 & $80 \%$ & 1 & $20 \%$ & 5 \\
\hline & Summe & 14 & $67 \%$ & 2 & $10 \%$ & 21 \\
\hline
\end{tabular}

10 Betrachtet man die grün hinterlegten Zeilen so fällt auf, dass in den 6 „aussagekräftigen“ Untergruppen, die ausreichende Abschnittslängen und Anzahlen von Beispielen aufweisen, i.d.R. um die 15 oder mehr Unfälle aufweisen. Daher wurde für die nachfolgenden Auswertungen festgesetzt, dass Untergruppen mit einer Mindestanzahl von 15 Unfällen als insgesamt aussagekräftig gelten können. 
In Geschäftsstraßen waren Radfahrer an Einbiegen/Kreuzen-Unfällen in 84\% der Unfälle dieses Unfalltyps involviert. Fußgänger waren am häufigsten in Geschäftsstraßen in „ÜberschreitenUnfälle“ (95\%) oder „Sonstige Unfälle“ (63\%) verwickelt.

In Quartiersstraßen waren Radfahrer zu 60\% an Einbiegen-Kreuzen-Unfälle involviert. Ebenso häufig sind sie in Wohnstraßen an „Einbiegen-Kreuzen“ Unfällen beteiligt gewesen (63\%).

In Quartiersstraßen waren Fußgänger an mehreren Unfalltypen beteiligt, aufgrund der geringen Anzahl von Unfällen lag hier jedoch kein aussagekräftiges Ergebnis vor.

Die Beteiligung von Fußgängern an Unfällen in Wohnstraßen spielte eher eine untergeordnete Rolle. In nur 10\% der Fälle sind sie überhaupt in einen Unfall verwickelt gewesen (2 Stück).

\subsubsection{Unfallart}

In der Abbildung $\mathbf{8 1}$ ist die Verteilung der Unfallarten je Straßenfunktion des „Kollektivs Kat. 1-4“ dargestellt. Folgende Erkenntnisse konnten gewonnen werden:

- In Wohnstraßen ist die Haupt-Unfallart der „Zusammenstoß mit anderem Fahrzeug, das einbiegt oder kreuzt" (51,9\%). Alle anderen Unfallarten spielen eine untergeordnete Rolle. Die Unfallart Zusammenstoß zwischen Fahrzeug und Fußgänger" tritt beispielsweise nicht auf.

- Die Haupt-Unfallart in Geschäftsstraßen ist der „Zusammenstoß zwischen Fahrzeug und Fußgänger" (35,6\%). In Geschäftsstraßen sind nur rund 20,5\% der Unfälle „Zusammenstöße mit anderem Fahrzeug, das einbiegt oder kreuzt“.

- Bei den Quartiersstraßen lieg der Anteil der „Zusammenstöße zwischen Fahrzeug und Fußgänger" mit 28,8\% niedriger als der Anteil der „Zusammenstöße mit anderem Fahrzeug, das einbiegt oder kreuzt" $(38,5 \%)$.

Auch für die Unfallarten wurde die Beteiligung von Radfahrern und Fußgängern in den jeweiligen Straßenfunktionen ausgewertet. Aus den bekannten Gründen wurden hierbei nur die Geschäftsstraßen, Wohnstraßen und Quartiersstraßen untersucht. Wie im vorherigen Kapitel wurden auch hier nur die Untergruppen kommentiert, die etwa 15 Unfälle besaßen. Diese sind Tabelle 28 grün hinterlegt.

In Geschäftsstraßen waren Radfahrer in knapp 80\% der Unfälle „anderer Art“ und knapp 80\% der „Zusammenstöße mit anderem Fahrzeug, das einbiegt oder kreuzt“ beteiligt. Darüber hinaus waren sie in jeden Unfall verwickelt, der die Unfallart „Zusammenstoß mit entgegenkommendem Fahrzeug“ besaß. Fußgänger sind in Geschäftsstraßen hauptsächlich in „Zusammenstöße zwischen Fahrzeug und Fußgänger" (96\%) involviert gewesen.

In Quartiers- und Wohnstraßen waren Radfahrer ebenfalls am häufigsten in „Zusammenstöße mit anderem Fahrzeug, das einbiegt oder kreuzt" involviert (65\% bzw. 71\%). In Quartiersstraßen geschahen 87\% der „Zusammenstöße zwischen Fahrzeug und Fußgänger“ unter Beteiligung eines Fußgängers. 


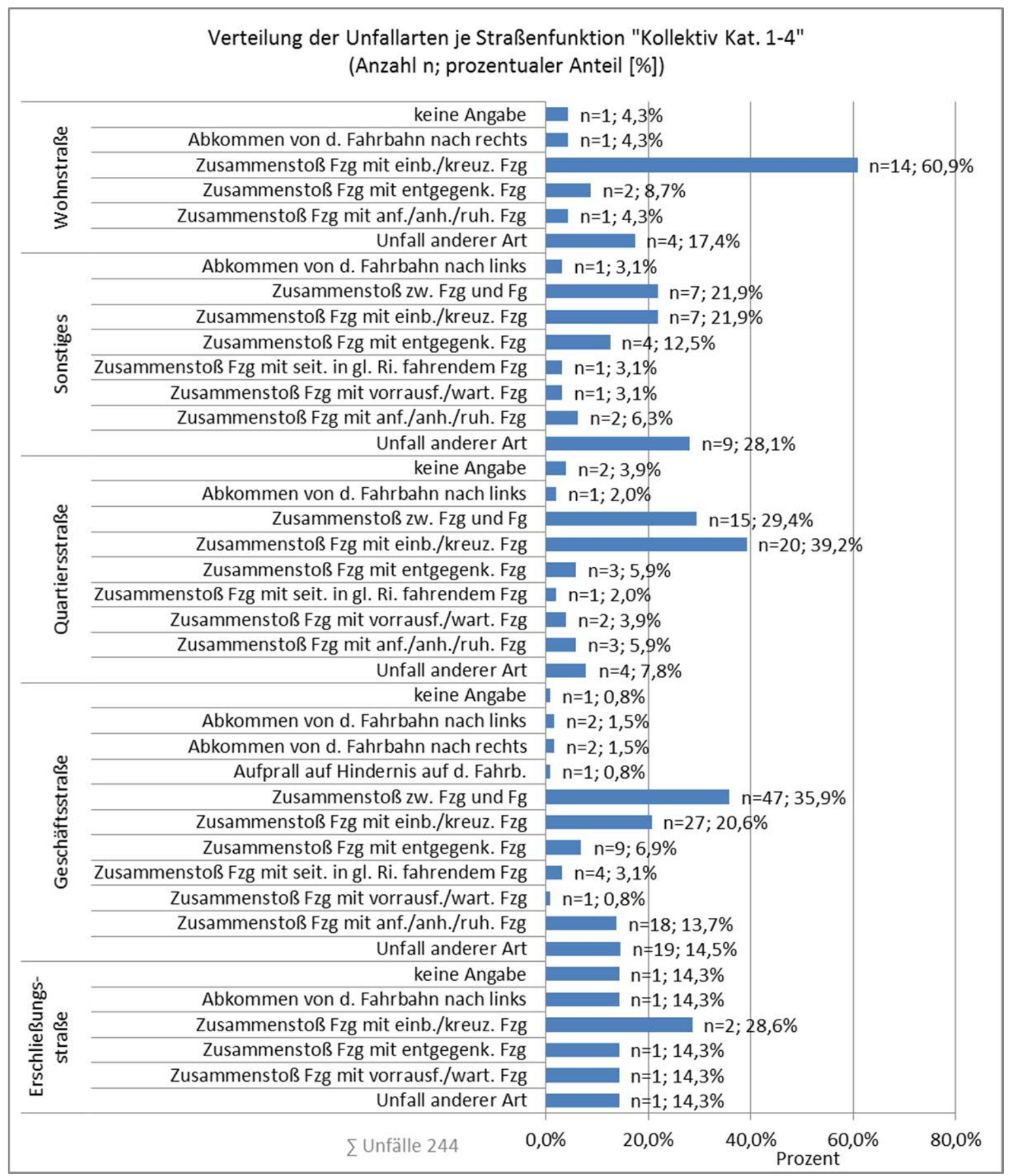

Abbildung 81: Verteilung der Unfallarten je Straßenfunktion des „Kollektivs Kat. 1-4“ 
Tabelle 28: Beteiligte Radfahrer und Fußgänger des „Kollektivs Kat. 1-4“ je Straßenfunktion und Unfallart

\begin{tabular}{|c|c|c|c|c|c|c|}
\hline \multirow[b]{3}{*}{ 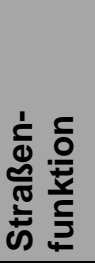 } & \multirow[b]{3}{*}{ Unfallart } & \multicolumn{4}{|c|}{ Beteiligte } & \multirow[b]{3}{*}{ 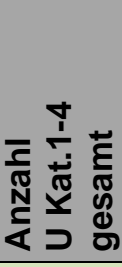 } \\
\hline & & \multicolumn{2}{|c|}{ Radfahrer } & \multicolumn{2}{|c|}{ Fußgänger } & \\
\hline & & 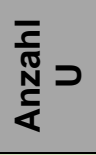 & $\begin{array}{l}\text { ¿ू } \\
\text { N } \\
0 \\
0 \\
0\end{array}$ & $\begin{array}{l}\text { ㄹ } \\
\text { N̦ } \\
\frac{\mathrm{C}}{4}\end{array}$ & \begin{tabular}{l} 
Е \\
\multirow{2}{N}{} \\
0 \\
0 \\
0
\end{tabular} & \\
\hline \multirow{12}{*}{ 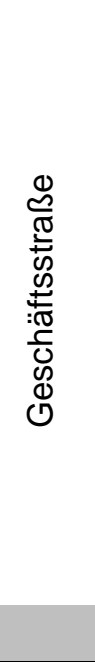 } & Unfall anderer Art & 15 & $79 \%$ & 2 & $11 \%$ & 19 \\
\hline & Zs Fzg mit anf./anh./ruh. Fzg & 9 & $50 \%$ & 1 & $6 \%$ & 18 \\
\hline & Zs Fzg mit vorrausf./wart. Fzg & 0 & $0 \%$ & 0 & $0 \%$ & 1 \\
\hline & Zs Fzg mit seit. in gl. Ri. fahrendem Fzg & 3 & $75 \%$ & 0 & $0 \%$ & 4 \\
\hline & Zs Fzg mit entgegenk. Fzg & 9 & $100 \%$ & 0 & $0 \%$ & 9 \\
\hline & Zs Fzg mit einb./kreuz. Fzg & 21 & $78 \%$ & 0 & $0 \%$ & 27 \\
\hline & Zs zw. Fzg und Fg & 4 & $9 \%$ & $45^{11}$ & $96 \%$ & 47 \\
\hline & Aufprall auf Hindernis auf d. Fahrb. & 0 & $0 \%$ & 0 & $0 \%$ & 1 \\
\hline & Abkommen von d. Fahrbahn nach rechts & 0 & $0 \%$ & 0 & $0 \%$ & 2 \\
\hline & Abkommen von d. Fahrbahn nach links & 0 & $0 \%$ & 0 & $0 \%$ & 2 \\
\hline & keine Angabe & 1 & $100 \%$ & 0 & $0 \%$ & 1 \\
\hline & Summe & 62 & $48 \%$ & 48 & $47 \%$ & 131 \\
\hline \multirow{10}{*}{ 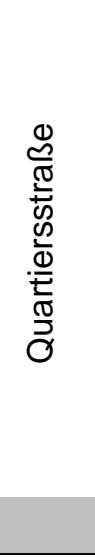 } & Unfall anderer Art & 3 & $75 \%$ & 1 & $25 \%$ & 4 \\
\hline & Zs Fzg mit anf./anh./ruh. Fzg & 1 & $33 \%$ & 0 & $0 \%$ & 3 \\
\hline & Zs Fzg mit vorrausf./wart. Fzg & 1 & $50 \%$ & 0 & $0 \%$ & 2 \\
\hline & Zs Fzg mit seit. in gl. Ri. fahrendem Fzg & 1 & $100 \%$ & 0 & $0 \%$ & 1 \\
\hline & Zs Fzg mit entgegenk. Fzg & 1 & $33 \%$ & 0 & $0 \%$ & 3 \\
\hline & Zs Fzg mit einb./kreuz. Fzg & 13 & $65 \%$ & 0 & $0 \%$ & 20 \\
\hline & Zs zw. Fzg und Fg & 3 & $20 \%$ & $13^{12}$ & $0 \%$ & 15 \\
\hline & Abkommen von d. Fahrbahn nach links & 0 & $0 \%$ & 0 & $0 \%$ & 1 \\
\hline & keine Angaben & 0 & $0 \%$ & 0 & $0 \%$ & 2 \\
\hline & Summe & 23 & $45 \%$ & 14 & $28 \%$ & 51 \\
\hline \multirow{7}{*}{ 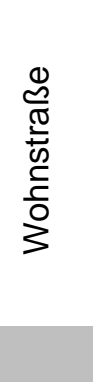 } & Unfall anderer Art & 1 & $25 \%$ & 0 & $0 \%$ & 4 \\
\hline & Zs Fzg mit anf./anh./ruh. Fzg & 1 & $100 \%$ & 0 & $0 \%$ & 1 \\
\hline & Zs Fzg mit entgegenk. Fzg & 1 & $50 \%$ & 0 & $0 \%$ & 2 \\
\hline & Zs Fzg mit einb./kreuz. Fzg & 10 & $71 \%$ & 1 & $7 \%$ & 14 \\
\hline & Abkommen von d. Fahrbahn nach rechts & 0 & $0 \%$ & 0 & $0 \%$ & 1 \\
\hline & keine Angaben & 1 & $100 \%$ & 1 & $100 \%$ & 1 \\
\hline & Summe & 14 & $61 \%$ & 2 & $9 \%$ & 23 \\
\hline
\end{tabular}

11 Zwei Unfälle der Unfallart „Zusammenstoß zwischen Fahrzeug und Fußgänger“ besaßen keine Angaben zum Beteiligten 2 (wobei es sich vermutlich um einen Fußgänger handelte)

12 Ein Unfall war unter Beteiligung eines Radfahrers vermutlich der Unfallart „Zusammenstoß zwischen Fahrzeug und Fußgänger" falsch zugeordnet worden, und ein Unfall besaß keine Angaben zum Beteiligten 2 (der vermutlich ein Fußgänger war)" 


\subsubsection{Beteiligte}

Aus Gründen der Übersichtlichkeit wurden in diesem Kapitel nur die drei Beteiligten Fußgänger, Radfahrer und Pkw untersucht. Um eruieren zu können, bei welchen Straßenfunktionen Radfahrer, Fußgänger oder Pkw besonders häufig in einen Unfall verwickelt wurden, ist die prozentuale Verteilung betrachtet worden. Dies ließ einen Vergleich zwischen den Straßenfunktionen zu.

Für die drei aussagekräftigen Straßenfunktionen Geschäftsstraße, Quartiersstraße und Wohnstraße sind die Ergebnisse in Tabelle 29 festgehalten worden. Radfahrer waren am häufigsten an Unfällen der Kategorie 1-4 in Wohnstraßen beteiligt. Fußgänger hingegen sind am häufigsten an Unfällen der Kategorie 1-4 in Geschäftsstraßen verwickelt gewesen. In Wohnstraßen verunfallten sie am seltensten (9\%). Pkw waren am meisten in Unfälle der Kategorie 1-4 in Wohnstraßen beteiligt.

Tabelle 29: Beteiligte Radfahrer, Fußgänger und Pkw an Unfällen je Straßenfunktion des „Kollektivs Kat. 14“"

\begin{tabular}{|c|c|c|c|c|c|c|c|}
\hline \multirow{3}{*}{ Straßenfunktion } & \multicolumn{6}{|c|}{ Beteiligte } & \multirow{3}{*}{$\begin{array}{c}\text { Anzahl U } \\
\text { Kat.1-4 }\end{array}$} \\
\hline & \multicolumn{2}{|c|}{ Radfahrer } & \multicolumn{2}{|c|}{ Fußgänger } & \multicolumn{2}{|c|}{ Pkw } & \\
\hline & Anzahl U & Prozent & Anzahl U & Prozent & Anzahl U & Prozent & \\
\hline Geschäftsstraße & 62 & $47 \%$ & 48 & $37 \%$ & 106 & $81 \%$ & 131 \\
\hline Quartiersstraße & 23 & $45 \%$ & 14 & $27 \%$ & 42 & $82 \%$ & 51 \\
\hline Wohnstraße & 14 & $61 \%$ & 2 & $9 \%$ & 21 & $91 \%$ & 23 \\
\hline
\end{tabular}

Es wurde untersucht, wie die Beteiligung je Verkehrsführung in den einzelnen Straßenfunktionen aussah. Analog zu vorherigen Kapiteln sind jedoch auch hier nur die Untergruppen aussagekräftig, die eine ausreichend große Anzahl an Unfällen aufwiesen (mind. 15 Stück, in der entsprechenden Tabelle grün hinterlegt) (Tabelle 30). Für Wohnstraßen lag in keiner Untergruppe eine ausreichende Anzahl an Unfällen vor, so dass hier nur die Geschäfts- und Quartiersstraßen dargestellt wurden.

Radfahrer waren häufiger an Unfällen der Kategorie 1-4 in Geschäftsstraßen beteiligt, wenn eine optische oder eine optische und bauliche Trennung vorlag als bei einer reinen baulichen Trennung. Diese Tendenz konnte für Fußgänger nicht festgestellt werden.

Aufgrund der geringen Anzahl der Unfälle in den Untergruppen der anderen Straßenfunktionen waren keine weiteren Aussagen möglich. 
Tabelle 30: Beteiligte Radfahrer, Fußgänger und Pkw an Unfällen des „Kollektivs Kat. 1-4“ je Straßenfunktion und Verkehrsführung

\begin{tabular}{|c|c|c|c|c|c|c|c|c|}
\hline \multirow[b]{3}{*}{$\begin{array}{l}\text { Straßen- } \\
\text { funktion }\end{array}$} & \multirow[b]{3}{*}{ Verkehrsführung } & \multicolumn{6}{|c|}{ Beteiligte } & \multirow{3}{*}{ 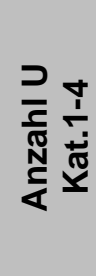 } \\
\hline & & \multicolumn{2}{|c|}{ Radfahrer } & \multicolumn{2}{|c|}{ Fußgänger } & \multicolumn{2}{|c|}{ Pkw } & \\
\hline & & $\begin{array}{l}\supset \\
\frac{D}{c} \\
\frac{1}{\mathbb{N}} \\
\stackrel{2}{<}\end{array}$ & 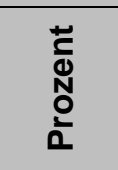 & $\begin{array}{l}\frac{D}{2} \\
\frac{\sqrt{\pi}}{\sqrt{n}} \\
\frac{\mathfrak{\alpha}}{\alpha}\end{array}$ & 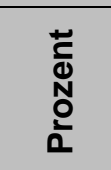 & 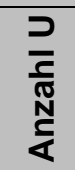 & 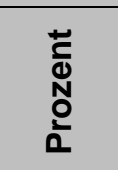 & \\
\hline \multirow{6}{*}{ 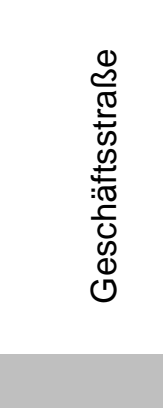 } & Bauliche Trennung & 34 & $42 \%$ & 32 & $40 \%$ & 64 & $79 \%$ & 81 \\
\hline & $\begin{array}{l}\text { Bauliche Trennung und } \\
\text { Mischverkehr }\end{array}$ & 4 & $67 \%$ & 1 & $17 \%$ & 6 & $100 \%$ & 6 \\
\hline & Mischverkehr & 1 & $20 \%$ & 2 & $40 \%$ & 5 & $100 \%$ & 5 \\
\hline & Optische Trennung & 7 & $58 \%$ & 5 & $42 \%$ & 9 & $75 \%$ & 12 \\
\hline & $\begin{array}{l}\text { Optische und bauliche } \\
\text { Trennung }\end{array}$ & 16 & $59 \%$ & 8 & $30 \%$ & 22 & $81 \%$ & 27 \\
\hline & Summe & 62 & $47 \%$ & 48 & $37 \%$ & 106 & $81 \%$ & 131 \\
\hline \multirow{8}{*}{ 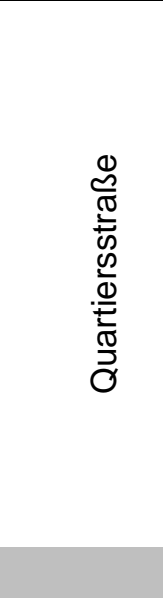 } & Bauliche Trennung & 6 & $50 \%$ & 1 & $8 \%$ & 9 & $75 \%$ & 12 \\
\hline & $\begin{array}{l}\text { Bauliche Trennung und } \\
\text { Mischverkehr }\end{array}$ & 0 & $0 \%$ & 1 & $100 \%$ & 1 & $100 \%$ & 1 \\
\hline & Mischverkehr & 3 & $43 \%$ & 1 & $14 \%$ & 6 & $86 \%$ & 7 \\
\hline & $\begin{array}{l}\text { Mischverkehr, optische } \\
\text { und bauliche Trennung }\end{array}$ & 1 & $100 \%$ & 0 & $0 \%$ & 1 & $100 \%$ & 1 \\
\hline & Optische Trennung & 2 & $29 \%$ & 4 & $57 \%$ & 6 & $86 \%$ & 7 \\
\hline & $\begin{array}{l}\text { optische Trennung und } \\
\text { Mischverkehr }\end{array}$ & 1 & $100 \%$ & 0 & $0 \%$ & 1 & $100 \%$ & 1 \\
\hline & $\begin{array}{l}\text { bauliche und optische } \\
\text { Trennung }\end{array}$ & 10 & $45 \%$ & 7 & $32 \%$ & 18 & $82 \%$ & 22 \\
\hline & Summe & 23 & $45 \%$ & 14 & $27 \%$ & 42 & $82 \%$ & 51 \\
\hline
\end{tabular}

\subsubsection{Unfallursachen}

Wie auch in Kapitel 10.1.6 wurden im vorliegenden Kapitel nur die (Haupt-) Unfallursachen bzw. die des ersten Beteiligten (Hauptverursachers) näher betrachtet. Unfallursachen der zweiten Beteiligten lagen nur unvollständig vor und wurden deshalb nicht betrachtet. Von den insgesamt 244 erhobenen Unfällen der Kat. 1-4 in ausgewiesenen VBB lagen in 8 Fällen gar keine Angaben zur Unfallursache vor und in 196 Fällen keine Unfallursachen für die zweiten Beteiligten vor.

Jeweils ca. ein Viertel der Unfälle der Kategorie 1-4 besaßen in den Straßenfunktionen Wohnstraßen, Quartiersstraßen und Geschäftsstraßen die Unfallursache „Abbiegen, Wenden, Rückwärtsfahren, Ein- und Anfahren“. Dies war die Unfallursache, die anteilig in den jeweiligen Straßenfunktionen am häufigsten auftrat.

Bei den Geschäftsstraßen sind darüber hinaus häufig Unfälle aufgrund von „Falschem Verhalten gegenüber Fußgängern“ entstanden. In Quartiersstraßen und Wohnstraßen lagen viele Unfälle infolge von „Vorfahrt, Vorrang" Verletzungen vor. In Wohnstraßen spielten auch „Unangepasste oder überhöhte Geschwindigkeiten“ eine Rolle. ${ }^{13}$

13 Die Straßenfunktion Erschließungsstraße wurde nicht kommentiert, da in den jeweiligen Untergruppen nur vereinzelt Unfälle der Kat. 1-4 vorlagen 


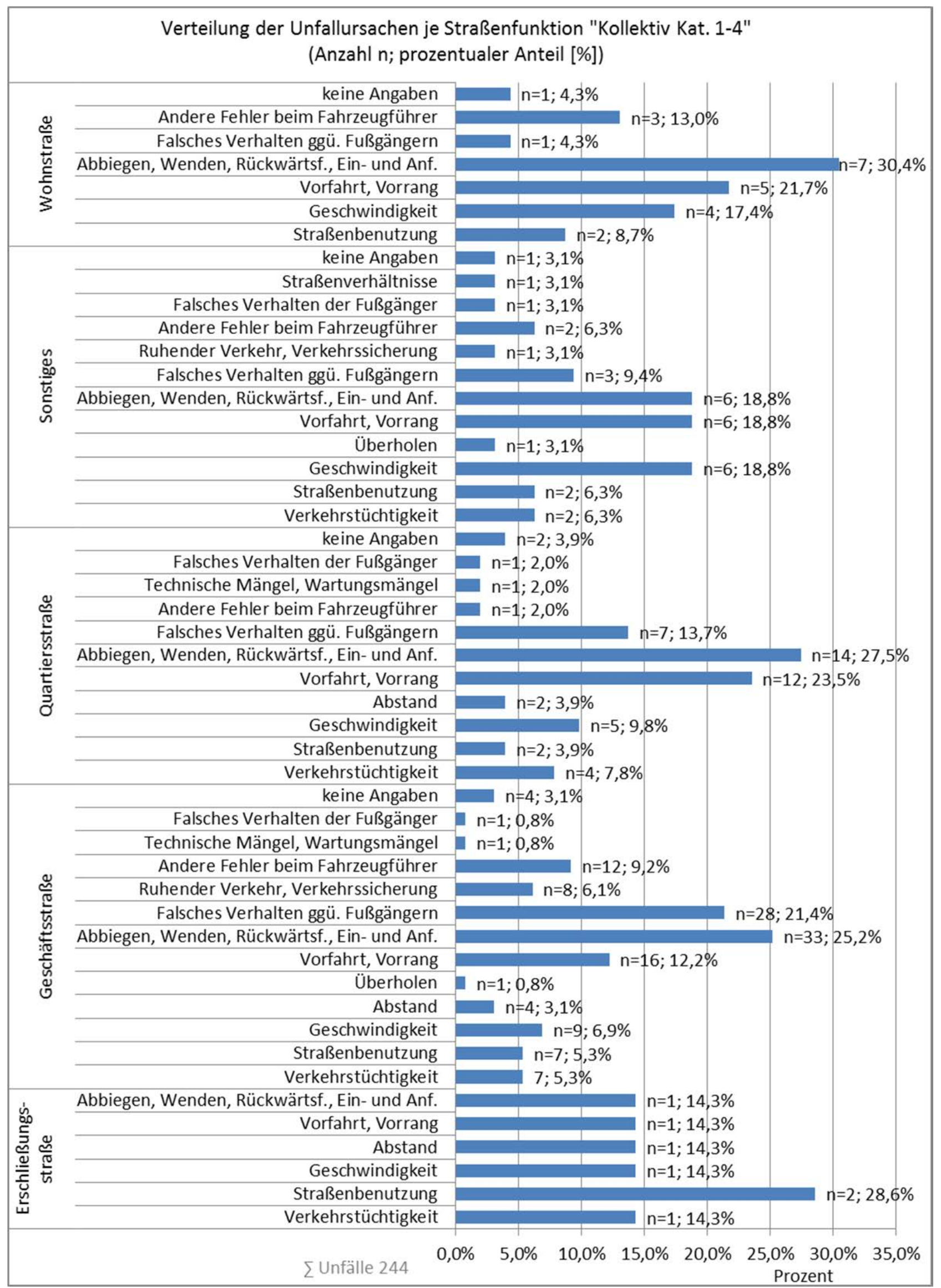

Abbildung 82: Verteilung der Unfallursachen je Straßenfunktion des „Kollektivs Kat. 1-4“ 
Eine separate Betrachtung der Beteiligung von Radfahrern und Fußgängern sollte aufzeigen, ob es bestimmte Unfallursachen gab, an denen nichtmotorisierte Verkehrsteilnehmer besonders häufig involviert waren. Wie in den vorherigen Kapiteln wurden nur die Geschäftsstraßen, Wohnstraßen und Quartiersstraßen untersucht und die Untergruppen kommentiert, die um die 15 oder mehr Unfälle besitzen. Diese sind in Tabelle 31 grün hinterlegt.

In Geschäftsstraßen waren Radfahrer in $75 \%$ der Unfälle verwickelt, deren Unfallursache in der Verletzung von Vorfahrt oder Vorrang lag. Fußgänger sind in Geschäftsstraßen in $86 \%$ der Fälle mit in einen Unfall involviert gewesen, der die Hauptunfallursache „Falsches Verhalten gegenüber Fußgängern“ besaß. Bei 40-50\% der Unfälle mit der Ursache „Abbiegen, Wenden, Rückwärtsfahren, Ein- und Anfahren“ waren Radfahrer in Geschäfts- und Quartiersstraßen beteiligt.

Tabelle 31: Beteiligte Radfahrer und Fußgänger des „Kollektivs Kat. 1-4“ je Straßenfunktion und Unfallursache

\begin{tabular}{|c|c|c|c|c|c|c|}
\hline \multirow{3}{*}{ 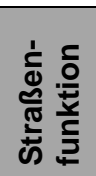 } & \multirow{3}{*}{ Unfallart } & \multicolumn{4}{|c|}{ Beteiligte } & \multirow{3}{*}{ 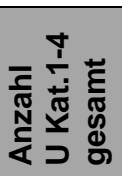 } \\
\hline & & \multicolumn{2}{|c|}{ Radfahrer } & \multicolumn{2}{|c|}{ Fußgänger } & \\
\hline & & $\begin{array}{c}\text { Anzahl } \\
U\end{array}$ & $\begin{array}{l}\text { Pro- } \\
\text { zent }\end{array}$ & $\begin{array}{c}\text { Anzahl } \\
\mathbf{U}\end{array}$ & $\begin{array}{l}\text { Pro- } \\
\text { zent }\end{array}$ & \\
\hline \multirow{13}{*}{ 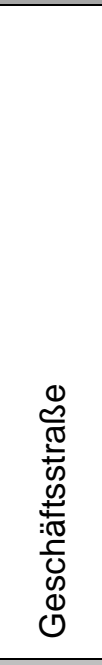 } & Verkehrstüchtigkeit & 6 & $86 \%$ & 0 & $0 \%$ & 7 \\
\hline & Straßenbenutzung & 6 & $86 \%$ & 2 & $29 \%$ & 7 \\
\hline & Geschwindigkeit & 3 & $33 \%$ & 3 & $33 \%$ & 9 \\
\hline & Abstand & 2 & $50 \%$ & 0 & $0 \%$ & 4 \\
\hline & Überholen & 1 & $100 \%$ & 0 & $0 \%$ & 1 \\
\hline & Vorfahrt, Vorrang & 12 & $75 \%$ & 1 & $6 \%$ & 16 \\
\hline & $\begin{array}{l}\text { Abbiegen, Wenden, Rückwf., Ein- und } \\
\text { Anfahren }\end{array}$ & 14 & $42 \%$ & 12 & $36 \%$ & 33 \\
\hline & Falsches Verhalten ggü. Fußgängern & 2 & $7 \%$ & 24 & $86 \%$ & 28 \\
\hline & Ruhender Verkehr, Verkehrssicherung & 5 & $63 \%$ & 0 & $0 \%$ & 8 \\
\hline & Sonstiger Fehler Fahrzeugführer & 7 & $58 \%$ & 3 & $25 \%$ & 12 \\
\hline & Technische Mängel, Wartungsmängel & 1 & $100 \%$ & 0 & $0 \%$ & 1 \\
\hline & Falsches Verhalten der Fußgänger & 1 & $100 \%$ & 1 & $100 \%$ & 1 \\
\hline & Keine Angaben & 2 & $50 \%$ & 2 & $50 \%$ & 4 \\
\hline & Summe & 62 & $48 \%$ & 48 & $37 \%$ & 131 \\
\hline \multirow{12}{*}{ 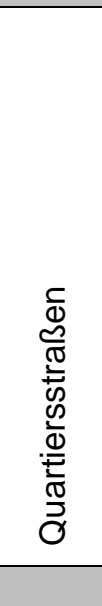 } & Verkehrstüchtigkeit & 1 & $25 \%$ & 0 & $0 \%$ & 4 \\
\hline & Straßenbenutzung & 1 & $50 \%$ & 0 & $0 \%$ & 2 \\
\hline & Geschwindigkeit & 2 & $40 \%$ & 1 & $20 \%$ & 5 \\
\hline & Abstand & 1 & $50 \%$ & 1 & $50 \%$ & 2 \\
\hline & Vorfahrt, Vorrang & 7 & $58 \%$ & 0 & $0 \%$ & 12 \\
\hline & $\begin{array}{l}\text { Abbiegen, Wenden, Rückwf., Ein- und } \\
\text { Anfahren }\end{array}$ & 7 & $50 \%$ & 7 & $50 \%$ & 14 \\
\hline & Falsches Verhalten ggü Fußgängern & 2 & $29 \%$ & 2 & $29 \%$ & 7 \\
\hline & Sonstiger Fehler Fahrzeugführer & 0 & $0 \%$ & 0 & $0 \%$ & 1 \\
\hline & Technische Mängel, Wartungsmängel & 1 & $100 \%$ & 1 & $100 \%$ & 1 \\
\hline & Falsches Verhalten der Fußgänger & 0 & $0 \%$ & 0 & $0 \%$ & 1 \\
\hline & Keine Angaben & 1 & $50 \%$ & 1 & $50 \%$ & 2 \\
\hline & Summe & 23 & $45 \%$ & 13 & $26 \%$ & 51 \\
\hline \multirow{4}{*}{ 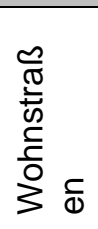 } & Geschwindigkeit & 3 & $75 \%$ & 0 & $0 \%$ & 4 \\
\hline & Vorfahrt, Vorrang & 1 & $20 \%$ & 0 & $0 \%$ & 5 \\
\hline & $\begin{array}{l}\text { Abbiegen, Wenden, Rückwf., Ein- und } \\
\text { Anfahren }\end{array}$ & 7 & $100 \%$ & 0 & $0 \%$ & 7 \\
\hline & Falsches Verhalten ggü Fußgängern & 1 & $100 \%$ & 1 & $100 \%$ & 1 \\
\hline
\end{tabular}




\begin{tabular}{|l|l|c|c|c|c|c|}
\hline & Fehler Fahrzeugführer & 2 & $67 \%$ & 0 & $0 \%$ & 3 \\
\cline { 2 - 6 } & Keine Angaben & 0 & $0 \%$ & 1 & $100 \%$ & 1 \\
\hline & Summe & $\mathbf{1 4}$ & $\mathbf{6 1 \%}$ & $\mathbf{2}$ & $\mathbf{9 \%}$ & 23 \\
\hline
\end{tabular}

\subsubsection{Unfallumstände}

Die einzelnen Straßenfunktionen wurden hinsichtlich der Ausprägung der Unfallumstände untersucht. Die Ergebnisse der Auswertung des „Kollektivs Kat. 1-4“ können je Straßenkategorie der Tabelle 32 entnommen werden. Der Anteil der Unfälle am Wochenende (Samstag und Sonntag) lag in den Wohnstraßen etwas höher, als der durchschnittliche Erwartungswert. Von insgesamt 23 Unfällen in Wohnstraßen haben 6 am Wochenende stattgefunden, das waren 26\%. Der Erwartungswert liegt bei 25\%. Bei genauerer Betrachtung der Beteiligten und der Unfallursachen waren jedoch keine Hinweise darauf zu erkennen, dass die Unfälle aufgrund von Freizeit oder Ausflugsverkehr stattgefunden haben. Es konnten auch keine weiteren Ausprägungen festgestellt werden.

Auffällig hoch war auch der Anteil der Unfälle der Kat. 1-4 in Wohnstraßen. Mit 43\% liegt dieser sehr nahe an dem Erwartungswert innerorts (45\%). Anzumerken ist hierbei, dass die Anzahl der Unfälle der Kategorie 1-4 in den ausgewiesenen VBB in Wohnstraßen sehr gering war (23 Stück). Hiervon lagen 10 Stück in den Spitzenzeiten. Auffällig war, dass sich (alle 10) Unfälle der Kat. 1-4 am Nachmittag (16-19Uhr) ereigneten und in 8 Fällen Fahrradfahrer mit verunfallten; 5 davon waren Kinder und 2 Senioren.

Tabelle 32: Ausprägung der Unfallumstände des „Kollektivs Kat. 1-4“ je Straßenfunktion

\begin{tabular}{|l|c|c|c|c|c|}
\hline Straßenfunktion & Winter & Wochenende & Spitzenzeiten & Nacht & Nässe/Glätte \\
\hline Geschäftsstraße & $22 \%$ & $17 \%$ & $26 \%$ & $12 \%$ & $10 \%$ \\
\hline Quartiersstraße & $25 \%$ & $24 \%$ & $27 \%$ & $4 \%$ & $8 \%$ \\
\hline Wohnstraße & $17 \%$ & $26 \%$ & $43 \%$ & $0 \%$ & $1 \%$ \\
\hline Erwartungswert Innerorts & $\mathbf{3 0 \%}$ & $\mathbf{2 5 \%}$ & $\mathbf{4 5 \%}$ & $\mathbf{3 0 \%}$ & $\mathbf{3 0 \%}$ \\
\hline
\end{tabular}

\subsection{7 Übergangsbereiche}

Bei der Verortung der Unfälle in den Streckenabschnitten der erhobenen ausgewiesenen Verkehrsberuhigten Bereiche ist aufgefallen, dass ein großer Anteil der Unfälle in den Übergangsbereichen zum übergeordneten Netz lag. Die Übergangsbereiche stellen den Anfang und das Ende eines ausgewiesenen Verkehrsberuhigten Bereichs dar. Sie sind räumlich begrenzt und befinden sich unmittelbar außerhalb des eigentlichen Verkehrsberuhigten Bereiches, der hinter dem VZ 325 beginnt. Die Übergangsbereiche befanden sich zumeist an Kreuzungen/Einmündungen, nur in einzelnen Fällen begann ein ausgewiesener VBB innerhalb eines Streckenabschnitts (vgl. Abbildung 83 und auch Abbildung 116). 


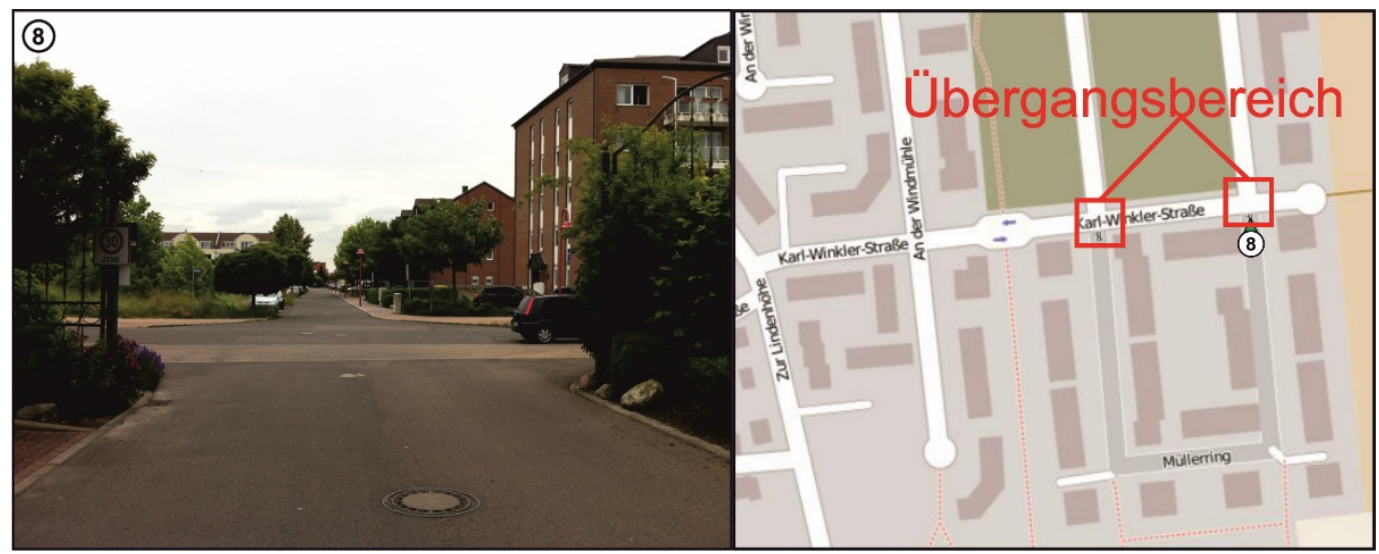

Abbildung 83: Beispiel für einen Übergangsbereich (Foto: IGS mbH; Kartengrundlage: OpenStreetMap)

Fragestellungen, die bei der Auswertung der Unfälle im Übergangsbereich untersucht wurden, waren:

- Lagen in den Übergangsbereichen Unfälle mit schwerwiegenderen Folgen vor?

- Unterscheiden sich die Unfalltypen wesentlich von denen, die in ausgewiesenen Verkehrsberuhigten Bereichen auftraten?

- Wie sah die Beteiligung der Verkehrsarten aus?

Insgesamt lagen rund 40\% aller Unfälle der Kategorie 1-4 in den Übergangsbereichen. Der Tabelle 33 können die Anteile je Straßenfunktion entnommen werden.

Tabelle 33: Unfälle Kat.1-4 in Übergangsbereichen und innerhalb von VBB des „Kollektivs Kat. 1-4“

\begin{tabular}{|c|c|c|c|c|c|}
\hline \multirow{2}{*}{ Straßenfunktion } & \multicolumn{2}{|c|}{ Übergangsbereiche } & \multicolumn{2}{|c|}{ Innerhalb VBB } & \multirow{2}{*}{$\begin{array}{c}\text { gesamt } \\
\text { Anzahl U }\end{array}$} \\
\hline & Anzahl U & Prozent & Anzahl U & Prozent & \\
\hline Erschließungsstraße & 2 & $29 \%$ & 5 & $71 \%$ & 7 \\
\hline Geschäftsstraße & 43 & $33 \%$ & 88 & $67 \%$ & 131 \\
\hline Quartiersstraße & 28 & $55 \%$ & 23 & $45 \%$ & 51 \\
\hline Sonstiges & 12 & $38 \%$ & 20 & $62 \%$ & 32 \\
\hline Wohnstraße & 11 & $48 \%$ & 12 & $52 \%$ & 23 \\
\hline Summe & 96 & $40 \%$ & 148 & $60 \%$ & 244 \\
\hline
\end{tabular}

Die Unfalldichten von den Unfällen in den ausgewiesenen Verkehrsberuhigten Bereichen und denen von den Übergangsbereichen konnten nicht direkt miteinander verglichen werden, da die Unfälle der Übergangsbereiche nur punktuelle Ereignisse sind. Unfalldichten werden für Streckenabschnitte je laufenden Kilometer berechnet und für Stellen (Knoten) je Punkt. Aus diesem Grund wurden an dieser Stelle die Anzahlen bzw. die prozentuale Verteilung der Unfallkategorien zwischen den Unfällen in Übergangsbereichen und den Unfällen innerhalb eines VBB verglichen.

Aus Abbildung $\mathbf{8 4}$ ist erkennbar, dass es hinsichtlich der Verteilung der Unfallkategorien keinen wesentlichen Unterschied in der Unfallschwere gibt. Tendenziell liegen in den Übergangsbereichen im Verhältnis mehr Unfälle der Kategorie 2 vor, als bei Unfällen, die in den ausgewiesenen Verkehrsberuhigten Bereichen selber geschehen. 


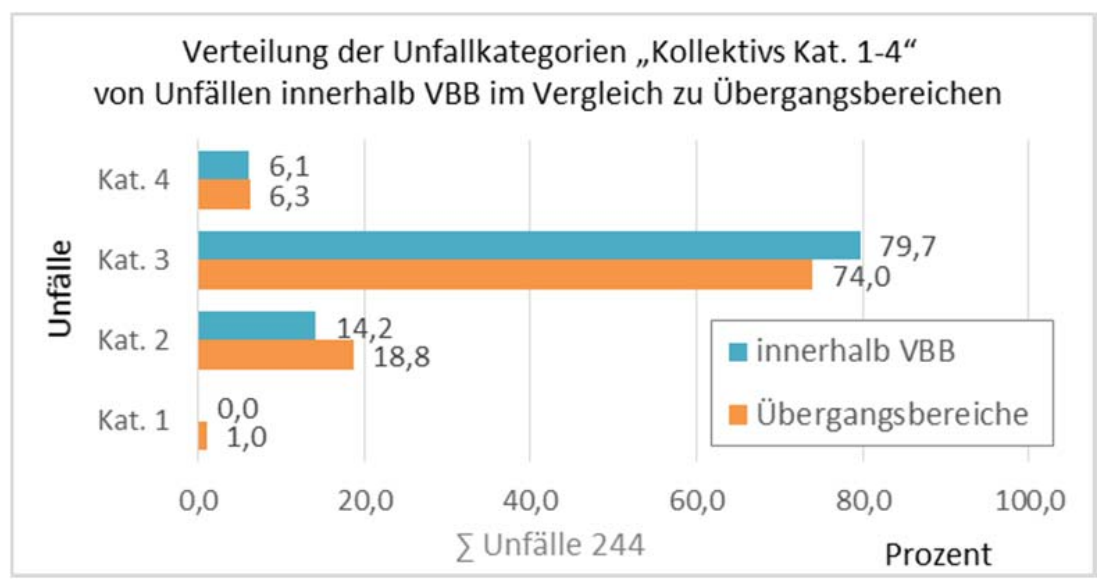

Abbildung 84: Verteilung der Unfallkategorien in Übergangsbereichen und innerhalb von VBB des „Kollektivs Kat. 1-4"

Bei der differenzierten Auswertung der Unfälle in Übergangsbereichen je Straßenfunktion fiel auf, dass der oben beschriebene Trend sich insbesondere in Wohn- und Quartiersstraßen ausprägt. Hier lagen anteilig häufiger Unfälle mit schwerem Personenschaden (Kategorie 2) in den Übergangsbereichen vor (Abbildung 85).

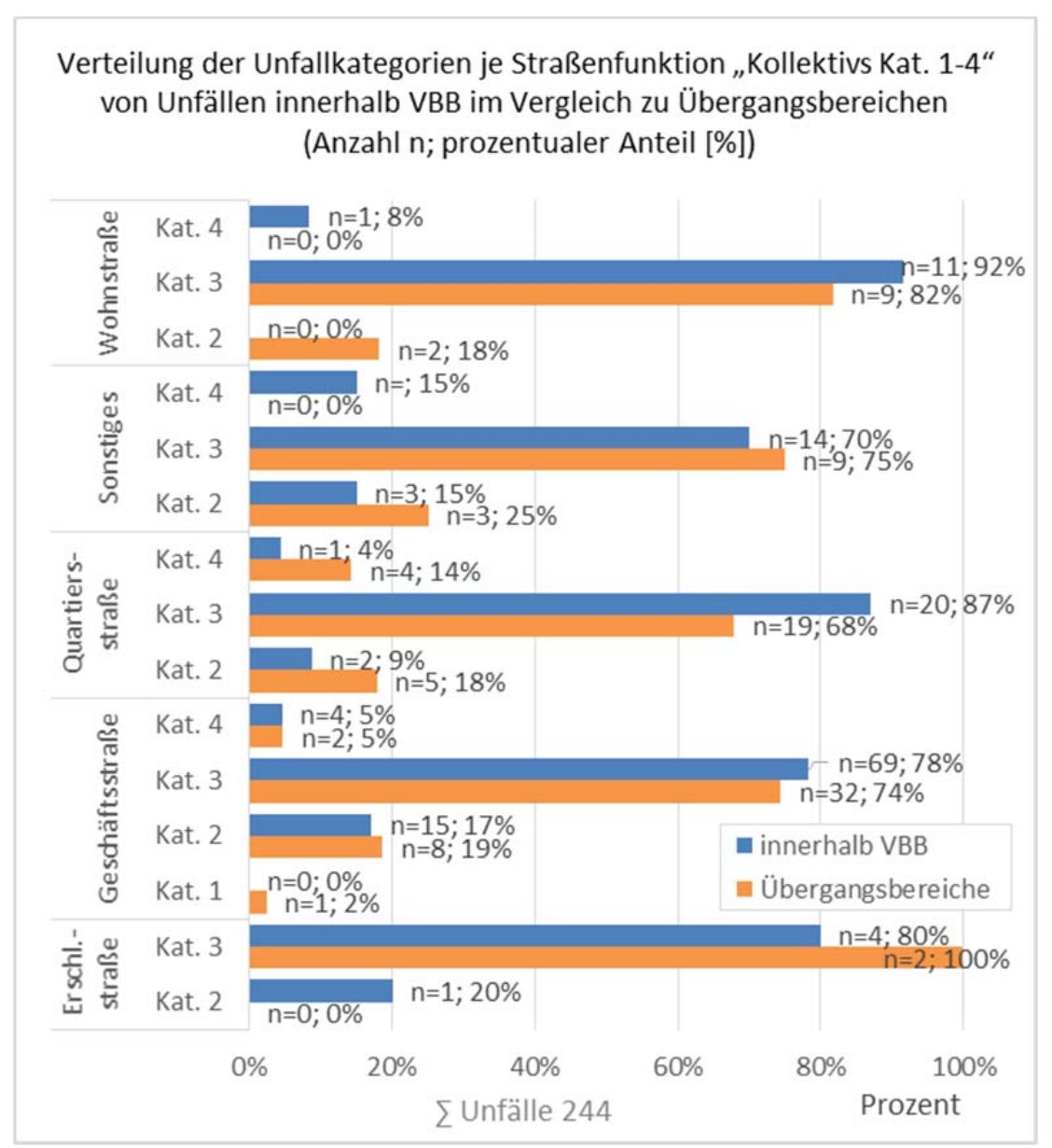

Abbildung 85: Verteilung der Unfallkategorien in Übergangsbereichen und innerhalb von VBB je Straßenfunktion des „Kollektivs Kat. 1-4“ 
Bei der Betrachtung der Verteilung der Unfalltypen je Straßenfunktion konnte erkannt werden, dass in Übergangsbereichen anteilig deutlich häufiger Unfälle des Unfalltyps 3 „Einbiegen/Kreuzen-Unfall“ auftraten (Abbildung 86). Dies ist logisch, da die meisten Übergangsbereiche der Verkehrsberuhigten Bereiche Kreuzungen oder Einmündungen darstellten. In den seltensten Fällen wurde das Verkehrszeichen 325 innerhalb eines Streckenabschnitts angeordnet. Dementsprechend traten die typischen Unfälle für Streckenabschnitte häufiger innerhalb von ausgewiesenen Verkehrsberuhigten Bereichen auf (Unfalltyp „Sonstiger Unfall“, „Unfall im Längsverkehr“, „Unfall durch ruhenden Verkehr“ und „Überschreiten-Unfall“).

Die Analyse der Verteilung der Unfalltypen in den Übergangsbereichen je Straßenfunktion zeigte auf, dass insbesondere in Geschäftsstraßen der Unfalltyp 3 „Einbiegen/Kreuzen-Unfall“ in den Übergangsbereichen stark von denen innerhalb von ausgewiesenen Verkehrsberuhigten Bereichen abwich. Hier lagen doppelt so viele Unfälle des Typ 3 in den Übergangsbereichen vor (Abbildung 87).

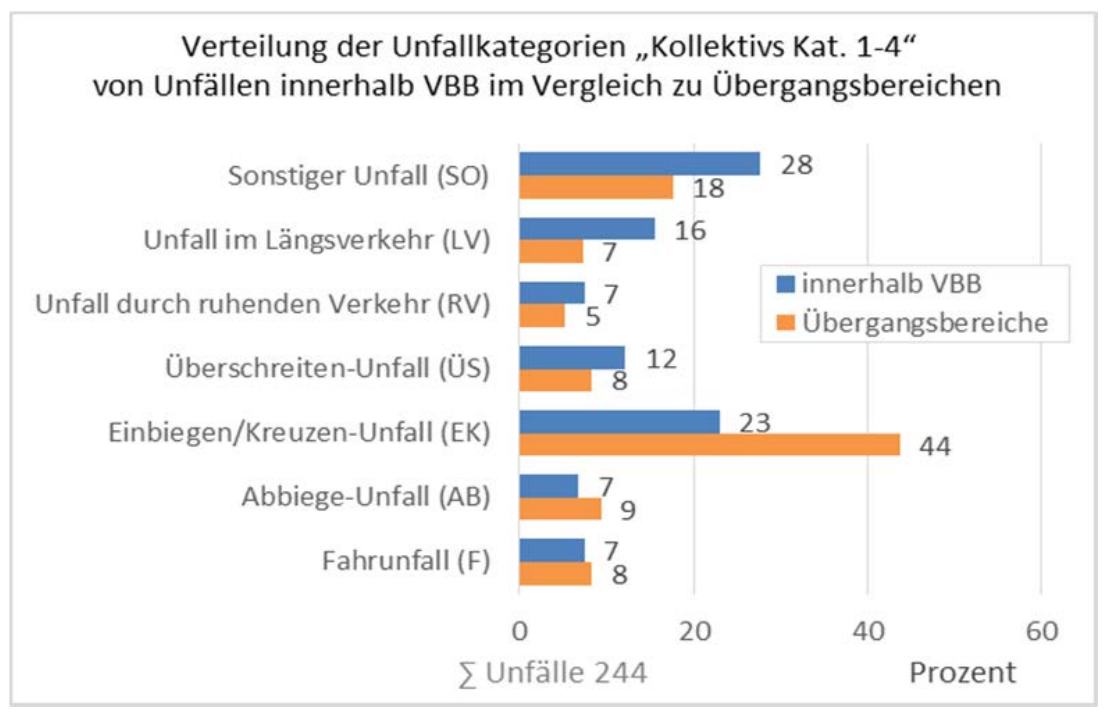

Abbildung 86: Verteilung der Unfalltypen in Übergangsbereichen und innerhalb von VBB des „Kollektivs Kat. $1-4$ " 


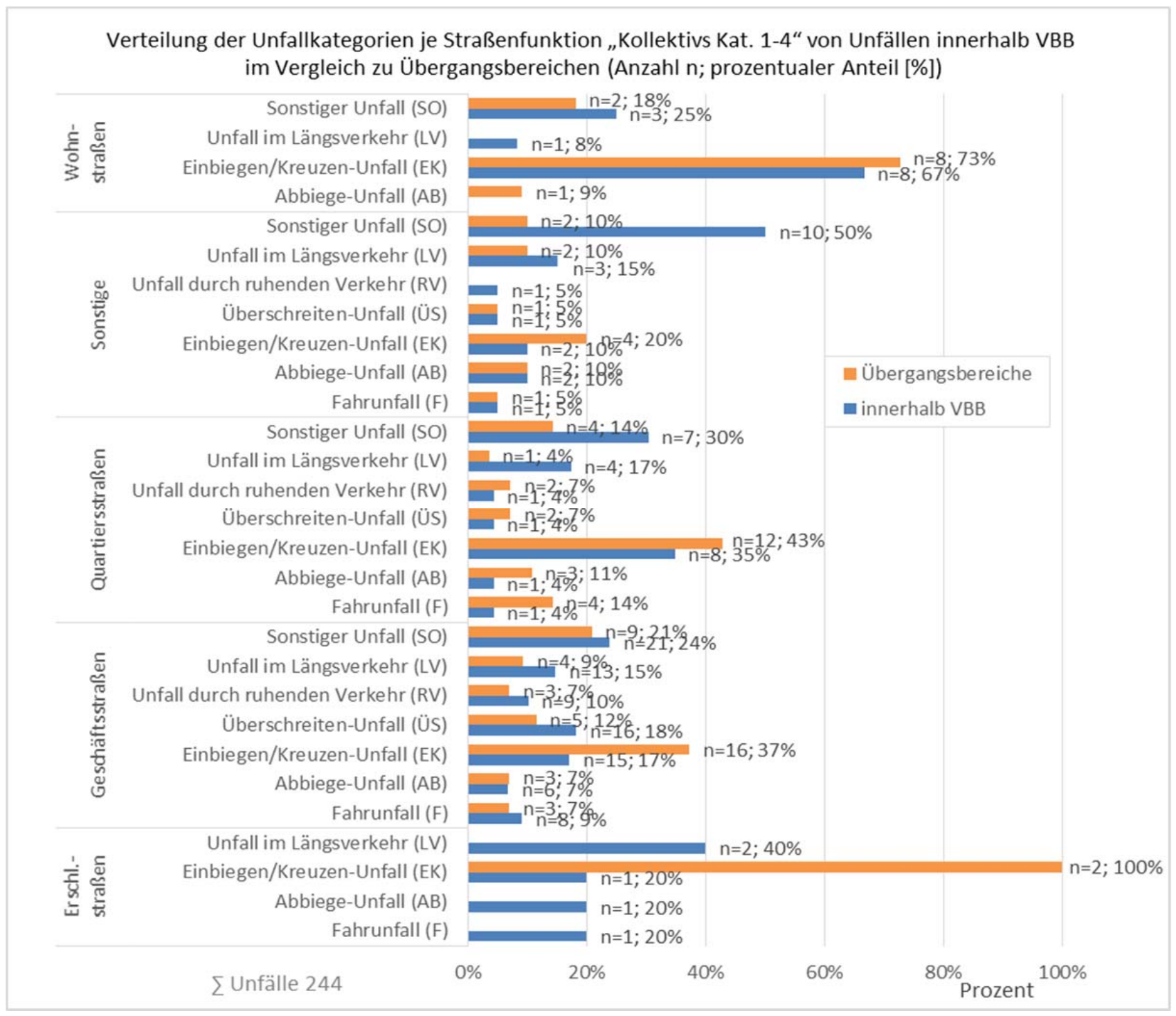

Abbildung 87: Verteilung der Unfalltypen in Übergangsbereichen und innerhalb von VBB je Straßenfunktion des „Kollektivs Kat. 1-4“

Bei der Auswertung zu den Beteiligten in Übergangsbereichen wurden aus Gründen der Übersichtlichkeit wieder nur die drei Beteiligten Fußgänger, Radfahrer und Pkw untersucht. An Unfällen in Übergangsbereichen sind im Verhältnis zu den Unfällen innerhalb von ausgewiesenen Verkehrsberuhigten Bereichen wesentlich seltener Fußgänger (-11\%) und häufiger Pkw (+9\%) beteiligt gewesen (Abbildung 88). Radfahrer verunglückten in Übergangsbereichen genau so häufig wie innerhalb von ausgewiesenen Verkehrsberuhigten Bereichen. 


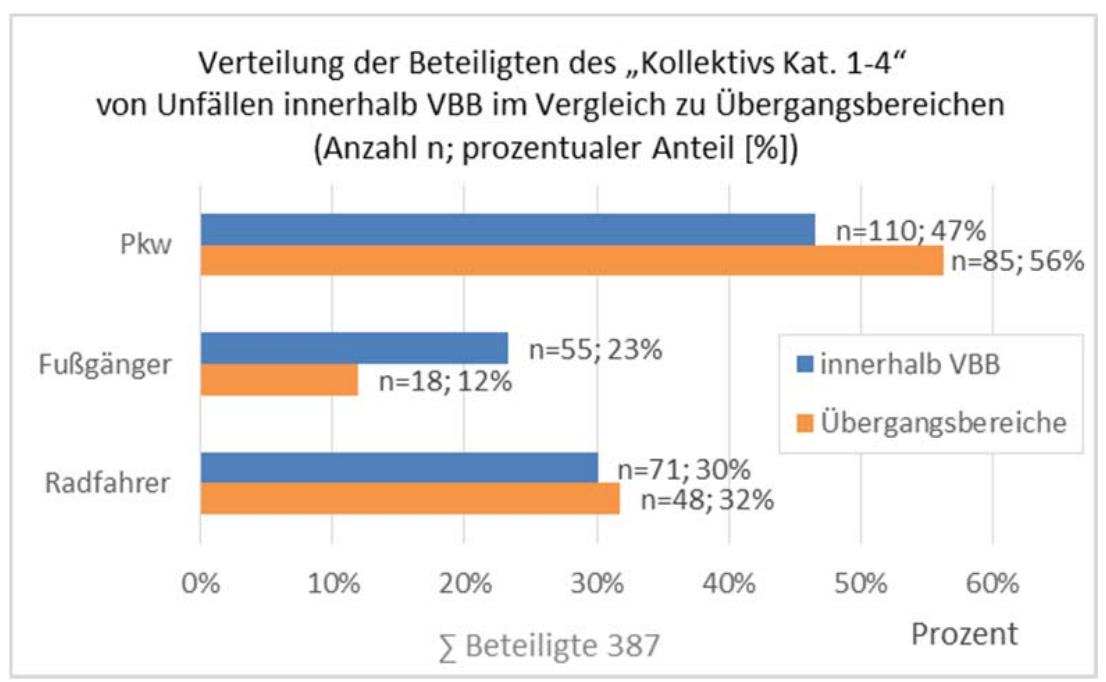

Abbildung 88: Beteiligte in Übergangsbereichen \& innerhalb von VBB des „Kollektivs Kat. 1- 4“

Diese Verteilungen prägten sich insbesondere in den Wohn- und Quartiersstraßen aus. Hier lag der Anteil der Unfälle an denen Pkw beteiligt waren bei 8-19\% über dem Anteil der beteiligten Pkw an Unfällen innerhalb von VBB (Abbildung 89). In Geschäftsstraßen lag die Differenz bei nur 7\% der Unfälle. In Quartiersstraßen war der Anteil der Unfälle mit Beteiligung eines Fußgängers innerhalb eines VBB um zwei Drittel höher als bei den Unfällen in Übergangsbereichen.

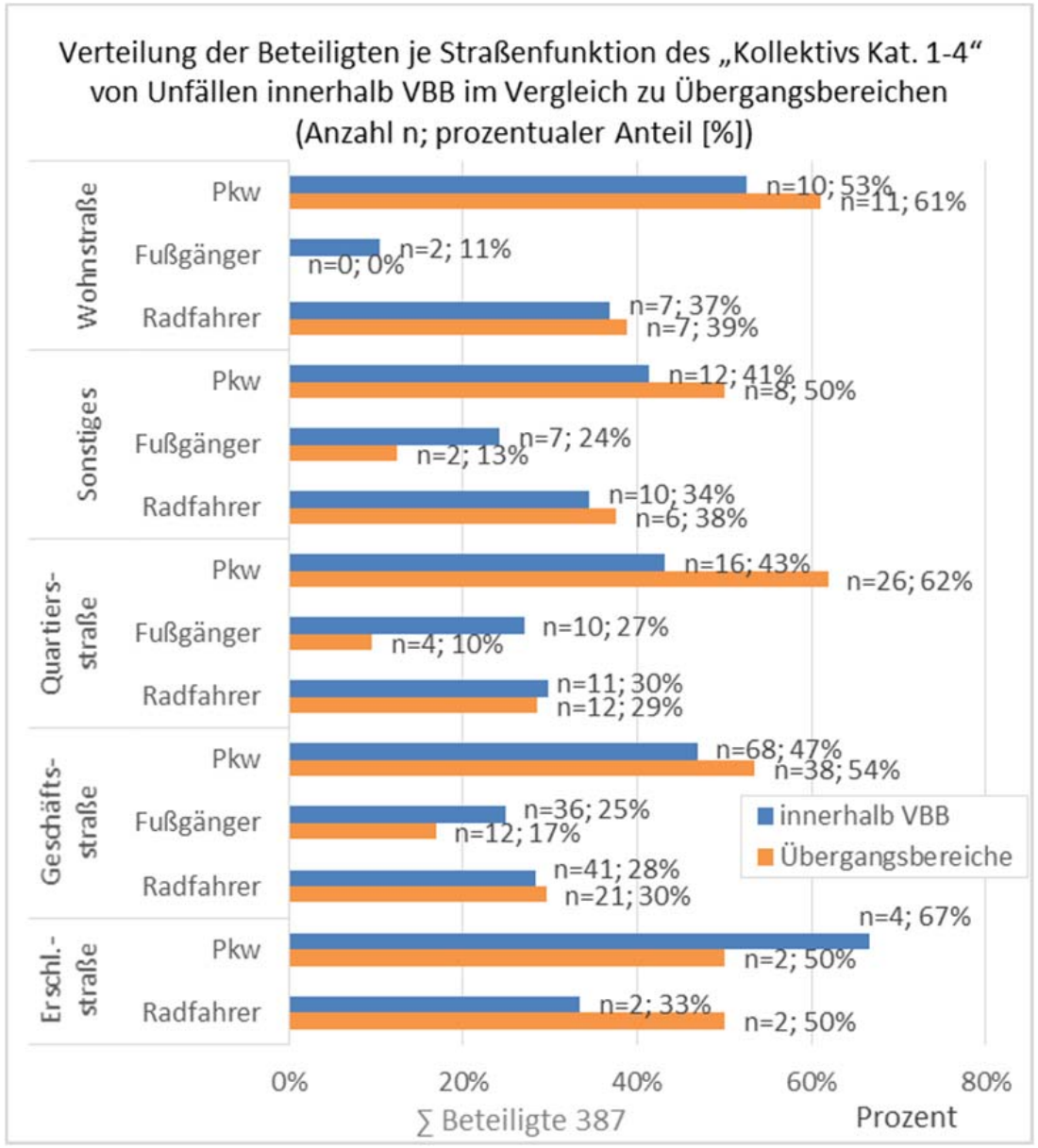

Abbildung 89: Beteiligte in Übergangsbereichen und innerhalb von VBB des „Kollektivs Kat. 1-4“ je Straßenfunktion 


\subsubsection{Streckenabschnittslängen}

Für die Fragestellung, ob die Abschnittslänge von ausgewiesenen Verkehrsberuhigten Bereichen Einfluss auf das Unfallgeschehen hat, wurden nur die Unfälle ausgewertet, die innerhalb eines VBB (auf der Strecke) geschehen sind. Bei der Erhebung der Untersuchungsobjekte war der subjektive Eindruck vorhanden, dass die Verkehrsregeln in ausgewiesenen Verkehrsberuhigten Bereichen eher eingehalten werden, je kürzer die Abschnitte sind. Es lag nahe, dass die Akzeptanz Schrittgeschwindigkeit zu fahren bei längeren Abschnitten geringer war und sich dadurch unter Umständen eine höhere Unfalldichte einstellen konnte. Aus diesem Grund wurden die Unfalldichten je Abschnittslänge untersucht. Hierzu wurden die Beispiele je Abschnittslänge in Intervallen zusammengefasst (0m bis 99m, 100m bis 199m, etc.).

Die Vermutung, dass lange Verkehrsberuhigte Bereiche zwingend konfliktbehafteter (unfallauffälliger) waren, als kürzere Bereiche, konnte nicht bestätigt werden. Aus Abbildung $\mathbf{9 0}$ ist zu erkennen, dass tendenziell längere Abschnitte geringere Unfalldichten aufwiesen (mit Ausnahme der Geschäftsstraßen).

Aus der Abbildung ist jedoch auch zu erkennen, dass Geschäftsstraßen die höchste Unfalldichte besaßen. Ausgewiesene Verkehrsberuhigte Bereiche, in Quartiers- oder Geschäftsstraße, mit einer Länge zwischen 200 und 400m hatten hingegen die geringsten Unfalldichten. Bei der Wohnstraße war die Unfalldichte bei einer Länge von 100 bis 200m am geringsten. Ausgewiesene Verkehrsberuhigte Bereiche mit Abschnittslängen über $600 \mathrm{~m}$ wurden nicht näher betrachtet, da es sich hierbei um nur vereinzelte Bereiche handelte.

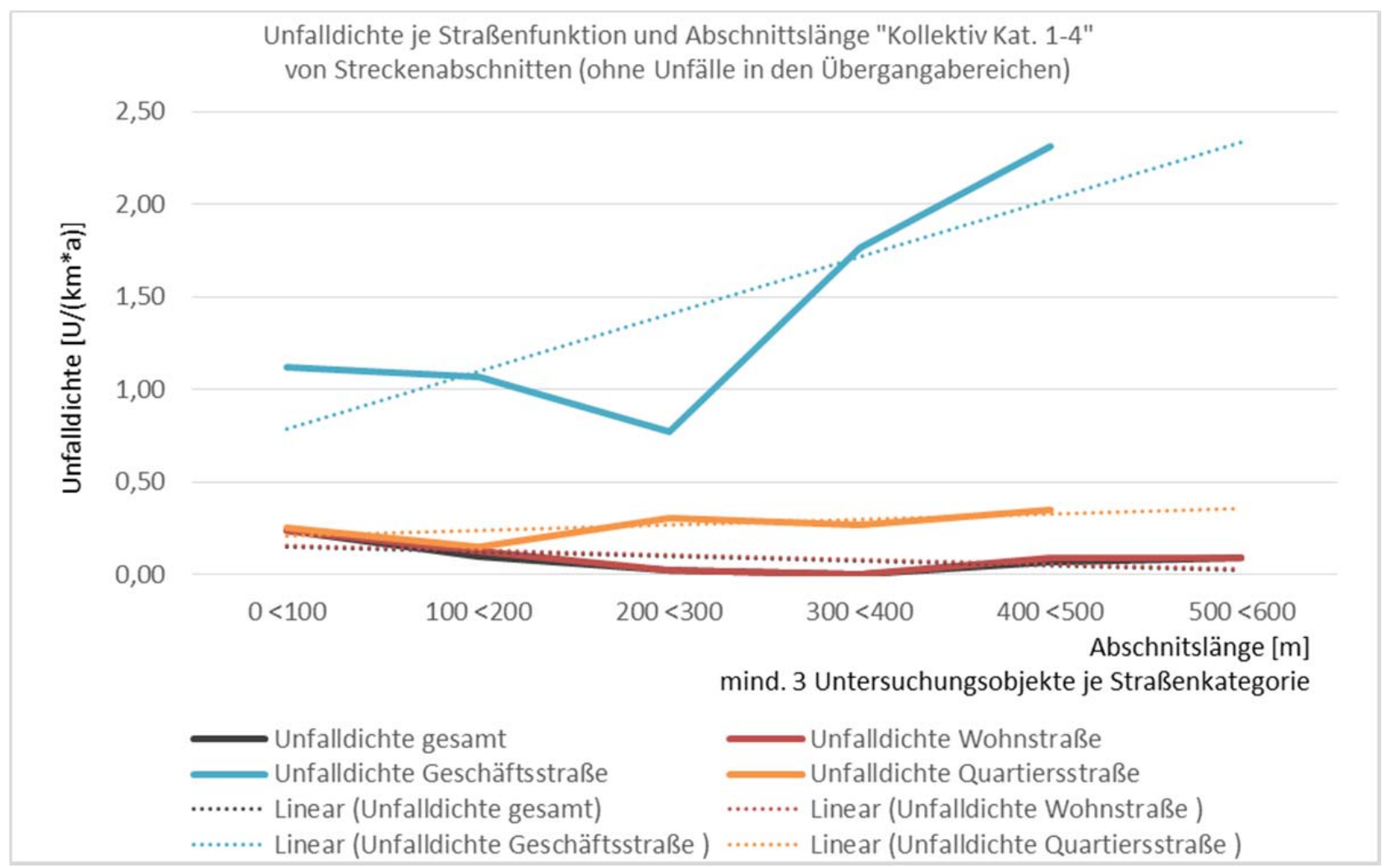

Abbildung 90: Unfalldichte je Straßenfunktion und Abschnittslänge von Streckenabschnitten des „Kollektivs Kat. 1-4"

Die Streckenlänge hatte also keinen (linearen) Einfluss auf das Unfallgeschehen in ausgewiesenen Verkehrsberuhigten Bereichen. Bei den Analysen zum Verkehrsablauf (Kapitel 12) 
wurde dieser Aspekt noch einmal aufgegriffen, um zu untersuchen, ob der Einfluss der Länge auf die Akzeptanz der Verkehrsteilnehmer, sich an Verkehrsregeln zu halten, hat.

\subsection{Zusammenfassung der Zusammenhänge zwischen Unfällen und Gestaltung}

Zusammenfassend konnten folgende Zusammenhänge zwischen Unfällen der Kategorie 1-4 in ausgewiesenen Verkehrsberuhigten Bereichen und der Gestaltung festgehalten werden:

\section{Unfallkategorien / Unfalldichten / Unfallkostendichten}

- Die Unfalldichte aller Unfälle der Kategorie 1-4 der ausgewiesenen Verkehrsberuhigten Bereiche liegt bei 0,51 Unfällen je km und Jahr. Die Unfalldichte der Geschäftsstraßen liegt mit 1,95 U/(km*a) mit Abstand am höchsten. Die Wohnstraßen weisen die niedrigste Unfalldichte auf $\left(0,09 \mathrm{U} /\left(\mathrm{km}^{*} \mathrm{a}\right)\right)$.

- Geschäftsstraßen mit baulicher Trennung besitzen die höchsten Unfalldichten (3,63 $\mathrm{U} /(\mathrm{km} * \mathrm{a}))$ und Unfallkostendichten $(146.220 € /(\mathrm{km} * \mathrm{a}))$.

- Geschäftsstraßen, die eine optische und bauliche Trennung besitzen, weisen im Vergleich zu solchen, die eine rein bauliche Trennung besitzen deutlich niedrigere Unfalldichten $(1,26$ $\left.\mathrm{U} /\left(\mathrm{km}^{\star} \mathrm{a}\right)\right)$ und Unfallkostendichten (51.050 €/(kmª)) auf.

- Die Wohnstraßen weisen die niedrigsten Unfalldichten / Unfallkostendichten auf, wenn bei ihnen Mischverkehr oder Mischverehr mit optischer Trennung vorliegt $(0,06-0,1 \mathrm{U} /(\mathrm{km} * \mathrm{a}))$.

- Wohnstraßen in denen eine bauliche Trennung und Mischverkehr vorliegt, weisen höhere Unfalldichten $(0,17 \mathrm{U} /(\mathrm{km} * \mathrm{a}))$ und Unfallkostendichten $(7.270 € /(\mathrm{km} * \mathrm{a}))$ auf.

\section{Unfalltyp}

- In Wohnstraßen, Quartiersstraßen und Erschließungsstraßen ist der Haupt- Unfalltyp der Einbiegen-/Kreuzen-Unfall. In den Geschäftsstraßen liegen fast zu gleichen Anteilen hauptsächlich Einbiegen-/Kreuzen-Unfälle, Überschreiten-Unfälle und Sonstigen Unfälle vor. Die Verteilung der Unfalltypen in Geschäftsstraßen und Wohnstraßen weicht erheblich voneinander ab.

\section{Unfallart}

- In Wohnstraßen ist die Haupt-Unfallart der „Zusammenstoß mit anderem Fahrzeug, das einbiegt oder kreuzt“. Alle anderen Unfallarten spielen eine untergeordnete Rolle.

- Die Haupt-Unfallart in Geschäftsstraßen ist der „Zusammenstoß zwischen Fahrzeug und Fußgänger". In Geschäftsstraßen sind deutlich weniger Unfälle von der Unfallart „Zusammenstoß mit anderem Fahrzeug, das einbiegt oder kreuzt" als in Wohnstraßen.

\section{Beteiligte}

- Radfahrer sind häufiger an Unfällen der Kategorie 1-4 in Geschäftsstraßen beteiligt, wenn eine optische und bauliche Trennung vorliegt (als bei einer reinen baulichen Trennung).

- Radfahrer sind bei einer baulichen Trennung in Wohnstraßen besonders häufig in Unfälle der Kategorie 1-4 verwickelt. Seltener sind sie in Unfälle verwickelt, wenn Mischverkehr und/oder eine optische Trennung vorliegt.

- Fußgänger sind sehr selten an Unfällen in Wohnstraßen beteiligt. 


\section{Unfallursache}

- Jeweils ca. ein Viertel der Unfälle der Kategorie 1-4 besitzt in den Straßenfunktionen Wohnstraßen, Quartiersstraßen und Geschäftsstraßen die Unfallursache „Abbiegen, Wenden, Rückwärtsfahren, Ein- und Anfahren". Dies ist die Unfallursache, die anteilig in den jeweiligen Straßenfunktionen am häufigsten auftritt.

- Am häufigsten sind Radfahrer in Geschäftsstraßen an Unfällen mit der Unfallursache „Vorfahrt, Vorrang“ beteiligt, Fußgänger an Unfällen bei denen „Falsches Verhalten gegenüber Fußgängern" als Unfallursache angegeben waren.

- Bei den Geschäftsstraßen treten am zweit häufigsten Unfälle aufgrund von „Falschem Verhalten gegenüber Fußgängern" auf. In Quartiersstraßen und Wohnstraßen liegen viele Unfälle infolge von „Vorfahrt/Vorrang“-Verletzungen vor.

- In Wohnstraßen spielen auch „Unangepasste oder überhöhte Geschwindigkeiten“ eine Rolle.

\section{Unfallumstände}

- Es liegen keine Auffälligkeiten bei den Unfallumständen vor. Lediglich der Anteil der Unfälle am Wochenende (Samstag und Sonntag) liegt in den Wohnstraßen etwas höher als der durchschnittliche Erwartungswert. Bei genauerer Betrachtung der Beteiligten und der Unfallursachen sind jedoch keine Hinweise darauf $\mathrm{zu}$ erkennen, dass die Unfälle aufgrund von Freizeit oder Ausflugsverkehr stattgefunden haben. Es konnten auch keine weiteren Ausprägungen festgestellt werden.

- Der Anteil der Unfälle der Kat. 1-4 in Wohnstraßen lag mit 43\% nahe an dem Erwartungswert von $45 \%$. Auffällig war, dass sich alle diese Unfälle am Nachmittag (1619Uhr) ereigneten und in 8 von 10 Fällen Fahrradfahrer mit verunfallten; 5 davon waren Kinder und 2 Senioren.

\section{Unfälle in Übergangsbereichen}

- Vergleicht man die Verteilung der Unfallkategorien je Straßenfunktion, so ist tendenziell zu erkennen, dass in Wohn- und Quartiersstraßen im Verhältnis mehr Unfälle der Kategorie 2 in den Übergangsbereichen vorliegen, als innerhalb der ausgewiesenen Verkehrsberuhigten Bereiche selber.

- Bei der Betrachtung der Verteilung der Unfalltypen je Straßenfunktion fällt auf, dass in Übergangsbereichen anteilig deutlich häufiger Unfälle des Unfalltyps 3 „Einbiegen/Kreuzen Unfall) auftreten. In Geschäftsstraßen liegen in den Übergangsbereichen doppelt so viele Unfälle des Typ 3 „Einbiegen/Kreuzen“ vor wie innerhalb von VBB.

- In Übergangsbereichen sind häufiger Pkw und seltener Fußgänger in Unfälle verwickelt als innerhalb von VBB. Diese Verteilung prägt sich insbesondere in den Wohn- und Quartiersstraßen aus.

\section{Streckenabschnittslängen}

- Ausgewiesene Verkehrsberuhigte Bereiche mit einer Länge zwischen 200 und 300m weisen die geringsten Unfalldichten auf.

- Die höchsten Unfalldichten liegen in sehr kurzen ausgewiesenen Verkehrsberuhigten Bereichen vor (bis zu 100m) und in solchen, deren Abschnittslänge zwischen 400 und $500 \mathrm{~m}$ lang ist. 


\section{Unfallkollektiv 2011 Kategorie 1-6}

Die ausführlichen Ergebnisse der Auswertungen zum „Kollektiv 2011“, in dem für das Jahr 2011 auch die sonstigen Unfälle mit Sachschaden (Unfallkategorien 5 und 6) mitbetrachtet wurden, befinden sich im Anhang 4. Es konnten weder Auffälligkeiten/Unfallhäufungsstellen noch weitere Erkenntnisse festgestellt werden. Zusammenfassend ließen sich folgende Unterschiede zum „Kollektiv Kat. 1-4“ feststellen:

- Unterschiede stellten sich in erster Linie für den Vergleich der Geschäftsstraßen-Kollektive ein.

- Für Geschäftsstraßen wurde der Haupt-Unfalltyp „Einbiegen/Kreuzen-Unfall“ vom „Unfall durch ruhenden Verkehr" verdrängt.

- Die Unfallart „Zusammenstoß zwischen Fahrzeug und Fußgänger“ spielte unter Berücksichtigung der sonstigen Sachschadenunfälle in Geschäfts- und Quartiersstraßen nur noch eine untergeordnete Rolle.

- Der Anteil der Unfallbeteiligung von Radfahrern ging im Unfallkollektiv 2011 für Geschäftsstraßen deutlich zurück.

\section{Analyse des Verkehrsablaufs}

Aus den 302 dokumentierten und der Unfallanalyse unterzogenen Bereichen wurden für 50 Verkehrsberuhigte Bereiche Verkehrsbeobachtungen durchgeführt. Gegenstand der Aufnahme waren Geschwindigkeitsmessungen und Verhaltensbeobachtungen innerhalb der ausgewiesenen Verkehrsberuhigten Bereiche.

Die Erhebungen, die mittels Videoerfassung dokumentiert wurden (im Anhang 6 ist die Kameratechnik und Fehleranfälligkeit der Videoerfassung beschrieben), dienten der Identifikation von Interaktionen zwischen den verschiedenen Verkehrsteilnehmern. Ziel der Verhaltensbeobachtung war es unter anderem zu ermitteln, welche Gestaltungsmaßnahmen innerhalb der VBB zu Fehlverhalten der Verkehrsteilnehmer beitragen können. Ebenso sollte herausgefunden werden, ob gewisse Gestaltungselemente auf das Geschwindigkeitsniveau förderlichen Einfluss nehmen. Durch die Ableitungen von Zusammenhängen zwischen dem Verkehrsablauf und der -gestaltung sollten verkehrssicherheitsfördernde oder -mindernde Gestaltungsmaßnahmen in VBB identifiziert werden.

Die Verkehrsbeobachtungen umfassten in den 50 Bereichen jeweils 4 Stunden und wurden werktags von 14-18 Uhr durchgeführt. Insgesamt wurden über 200 Stunden Verhaltensbeobachtungen an den Untersuchungsobjekten durchgeführt.

\subsection{Auswahl der Untersuchungsobjekte für die Verkehrsbeobachtung}

Im Folgenden wird dargelegt, welche der zuvor dokumentierten und analysierten Untersuchungsobjekte für die vertiefenden Betrachtungen mittels einer Verkehrsablaufbeobachtung herangezogen wurden, sowie die jeweiligen Hintergründe erläutert, die zur Auswahl geführt haben.

Zu Beginn der Studie war es das Ziel, die ergänzenden Verkehrsablaufbeobachtungen an denjenigen Untersuchungsobjekten durchzuführen, die die größte Auffälligkeit im Unfallgeschehen aufweisen. Aufgrund der Tatsache, dass keine unfallauffälligen Bereiche (im Sinne einer 
Unfallhäufung) in dem vorhandenen Untersuchungskollektiv existieren, wurden zunächst die unfallträchtigsten Bereiche aus den Kategorien Wohn-, Quartiers- und Geschäftsstraße ausgewählt.

Insgesamt existieren im gesamten Kollektiv der 302 betrachteten verkehrsberuhigten Bereiche lediglich 32 Untersuchungsobjekte, die mindestens 3 Unfälle im Betrachtungszeitraum von 6 Jahren aufweisen, wovon 17 Untersuchungsobjekte genau 3 Unfälle in 6 Jahren aufwiesen. Aus dem ausgewählten Untersuchungskollektiv von 32 Untersuchungsobjekten konnten für 13 verkehrsberuhigte Bereiche die Genehmigungen für die Videobeobachtungen eingeholt werden und wurden somit in das Kollektiv der VBB aufgenommen, die einer ergänzenden Verkehrsablaufbeobachtung unterzogen wurden.

Die ausgewählten, unfallträchtigen VBB weisen in der Summe 82 Unfälle der Kategorie 1-4 in den 6 betrachteten Jahren auf. Davon haben sich alleine 19 Unfälle (23\% aller Unfälle) in einem verkehrsberuhigten Bereich ereignet. Bei den 82 Unfällen handelt es sich um 1 Unfall der Kategorie 1, 12 Unfälle der Kategorie 2, 64 Unfälle der Kategorie 3 und 5 Unfälle der Kategorie 4.

Um gegebenenfalls Besonderheiten dieser unfallträchtigsten Bereiche identifizieren zu können, wurden aus dem übrigen Untersuchungskollektiv Beispiele ähnlicher Gestaltung („Pendants“) herausgesucht, die nach Möglichkeit keinen Unfall in den 6 betrachteten Jahren aufwiesen. Die Verkehrsberuhigten Bereiche, die keinen Unfall aufweisen, wurden nur dann in die Beobachtung mit einbezogen, wenn diese mit einem der zuvor ausgewählten unfallträchtigeren VBB in der Gestaltung in weiten Teilen übereinstimmen. Bei der Suche nach passenden Pendants ließen sich leichte Abweichungen in der Gestaltung nicht vermeiden.

Es wurden 22 unfallauffällige VBB mit insgesamt 82 Unfällen der Kat. 1-4 und 28 VBB, die keine Unfälle aufweisen, untersucht. Es wurde darauf geachtet, dass die Besetzung der Straßenfunktionsgruppen Geschäfts-, Quartiers- und Wohnstraßen annähernd gleich groß sein sollte. In die Auswertungen konnten nach genauer Prüfung für Geschäftsstraßen sowie für Quartiersstraßen jeweils 20 Untersuchungsobjekte berücksichtigt werden, für Wohnstraßen lediglich 9 Bereiche. Zudem wurde ein als „sonstiger“ eingestufter Verkehrsberuhigter Bereich beobachtet.

Folgende Gestaltungsmaßnahmen spielten für die Auswahl eine wichtige Rolle:

- Zumindest die Bereiche, die miteinander verglichen werden sollten, benötigten möglichst ähnliche Streckenlängen.

- Zudem sollte die Art der Verkehrsführung übereinstimmen.

- Auch geschwindigkeitsreduzierende Maßnahmen wie Aufpflasterungen, Engstellen oder Versätze sollten möglichst ähnlich sein. Ferner wurden auch Bereiche, die entweder einige oder gar keine Gestaltungsmaßnahmen aufweisen, vergleichend betrachtet. Damit konnte untersucht werden, ob z.B. das Geschwindigkeitsniveau in Bereichen mit Gestaltungsmaßnahmen niedriger ist als ohne.

- Ein weiterer Aspekt war die Unterscheidung zwischen Einbahnstraßen und solchen mit Zweirichtungsverkehr.

- Die Parksituation in einem VBB spielte ebenfalls eine Rolle. Zum einen sollte beobachtet werden, ob z.B. durch häufige Parkvorgänge die Geschwindigkeiten reduziert werden können oder die Anordnung von Parkständen eher zu Sichtbehinderungen führen.

- In einigen, wenigen Bereichen ist der ÖPNV zugelassen. Da nur eine geringe Anzahl dieser VBB vorlag, wurde von einer Berücksichtigung dieses Merkmals in den Auswertungen daher Abstand genommen.

In der abschließenden Auswahl befinden sich 20 Geschäftsstraßen (neun Untersuchungsobjekte mit insg. 50 Unfällen der Kategorie 1-4), 20 Quartiersstraßen (sechs mit insg. 24 Unfällen der 
Kategorie 1-4), neun Wohnstraßen mit insg. 6 Unfällen der Kategorie 1-4 und ein Sonstiger Bereich mit zwei Unfällen der Kategorie 1-4 (vgl. Tabelle 34).

Tabelle 34: Übersicht Untersuchungsobjekte

\begin{tabular}{|c|c|c|c|c|c|c|c|c|}
\hline \multirow[b]{2}{*}{$\begin{array}{l}\text { Verkehrs- } \\
\text { führung }\end{array}$} & \multicolumn{2}{|c|}{ Wohnstraße } & \multicolumn{2}{|c|}{ Geschäftsstraße } & \multicolumn{2}{|c|}{ Quartiersstraße } & \multicolumn{2}{|c|}{ Sonstige } \\
\hline & VBB & 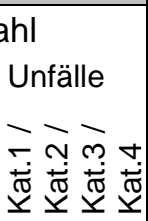 & VBB & 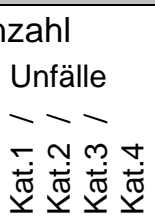 & VBB & 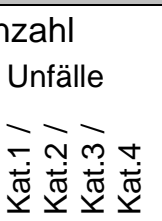 & VBB & 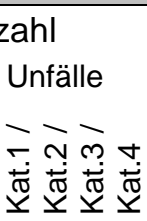 \\
\hline Mischverkehr & 3 & $0 / 0 / 1 / 0$ & 1 & $1 / 0 / 1 / 1$ & 2 & $0 / 2 / 3 / 1$ & & \\
\hline Optische Trennung & 1 & $0 / 0 / 2 / 0$ & 1 & $0 / 1 / 2 / 0$ & 2 & $0 / 0 / 1 / 0$ & & \\
\hline Bauliche Trennung & 1 & & 7 & $0 / 5 / 26 / 1$ & 4 & $0 / 0 / 2 / 1$ & & \\
\hline $\begin{array}{l}\text { Optische Trennung } \\
\text { und Mischverkehr }\end{array}$ & & & & & 1 & & & \\
\hline $\begin{array}{l}\text { Optische Trennung } \\
\text { und bauliche } \\
\text { Trennung }\end{array}$ & 4 & $0 / 0 / 3 / 0$ & 10 & 0/3/9/0 & 9 & 0/1/13/0 & 1 & 0/0/1/1 \\
\hline $\begin{array}{l}\text { Bauliche Trennung } \\
\text { und Mischverkehr }\end{array}$ & & & 1 & & 2 & & & \\
\hline Summe & 9 & $0 / 0 / 6 / 0$ & 20 & $1 / 9 / 38 / 2$ & 20 & $0 / 3 / 19 / 2$ & 1 & $0 / 0 / 1 / 1$ \\
\hline
\end{tabular}

Hinzuzufügen ist, dass es sich bei der Auswahl von vier Quartiersstraßen um jeweils zwei in unmittelbarer Nähe zueinander befindliche VBB handelt, die jedoch aufgrund ihrer unterschiedlichen Gestaltung oder einer Unterbrechung (z.B. Tempo-30 Zone) als eigene Bereiche betrachtet wurden.

Die als „Sonstige“ kategorisierten Bereiche sind weitestgehend nicht berücksichtigt worden, da es sich dabei zumeist um Einzelfälle handelt oder ihr Einsatz diskussionswürdig ist. In der Karte (vgl. Abbildung 91) ist erkennbar in welchen Bundesländern und Städten, das Verkehrsverhalten in VBB untersucht wurde. In den „Fahnen“ der Abbildung 91 ist die Anzahl der Verkehrsberuhigten Bereiche abzulesen, die in den jeweiligen Kommunen untersucht wurden. 


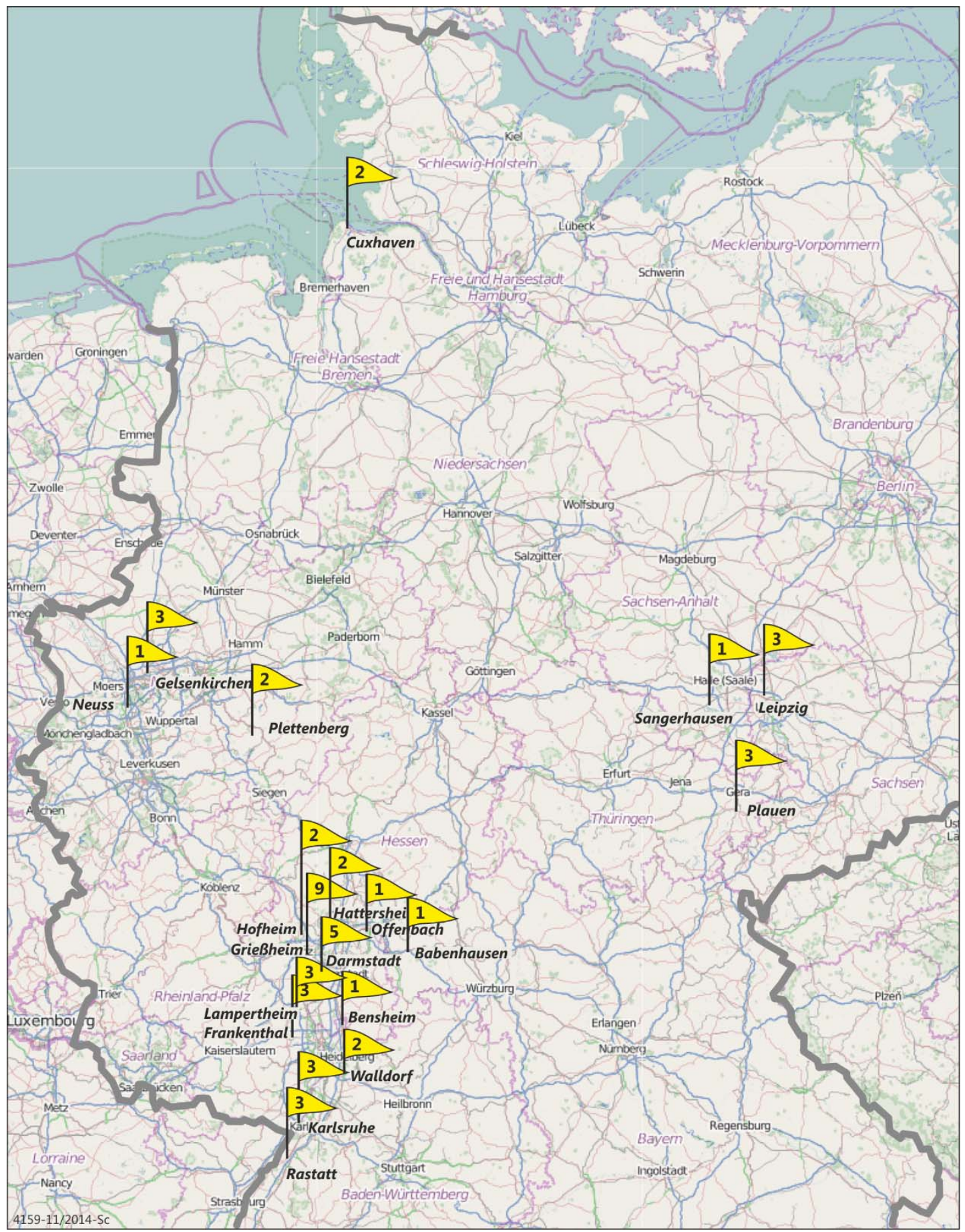

Abbildung 91: Übersichtskarte über die Städte in denen insg. 50 Bereiche untersucht wurden

\subsection{Erhebungskonzept}

Für die Entwicklung des Erhebungskonzept wurden als Basis die Regelungen der StVO §42 herangezogen, da kein anderes Regelwerk bzw. Richtlinienwerk existiert, das einheitliche Gestaltungsgrundsätze der VBB umfasst (vgl. hierzu Kapitel 5.2). Auf Grundlage der fünf, in der VwV-StVO aufgeführten Regelungen zu §42, Anlage 3, Abschnitt 4 wurde ein Konzept aufgestellt, das Querschnittszählungen, Flächennutzungen, Geschwindigkeitsmessungen sowie Interaktionen erfasst.

Für die Auswertung der Aufnahmen wurden Erhebungsbögen angefertigt, die die jeweiligen Auswertungen zu den Messungen (vgl. Kapitel 12.2.1 bis 12.2.4) enthalten. Die wichtigsten vier 
Untersuchungen sowie die dazugehörigen Informationen zum Erhebungsbogen werden im Folgenden näher erläutert.

\subsubsection{Flächennutzung}

Zur Erhebung der durch die Verkehrsteilnehmer in Anspruch genommenen Flächen innerhalb des Verkehrsberuhigten Bereiches wurde erfasst, auf welchen Flächen sich die Verkehrsteilnehmer aufhalten bzw. bewegen. Unter dem Begriff „Flächen“ wurden hierbei zum einen die Randbereiche (Flächen in denen sich in Straßen mit dem Separationsprinzip die Gehwege befinden) und zum anderen die „Fahrgasse/Fahrbahn“ verstanden.

Untersucht wurde, ob die Verkehrsteilnehmer die gleichen Flächen nutzen, unabhängig davon, ob eine Mischverkehrsfläche oder eine optische oder bauliche Trennungen vorlag. Dazu wurden die Verkehrsflächen innerhalb der Verkehrsberuhigten Bereiche im Querschnitt immer in drei Flächen unterteilt. Lag baulich oder optisch bedingt bereits eine Fahrgasse vor, wurde diese auch für die Einteilung der Flächen beibehalten (vgl. Abbildung 92). War beim Untersuchungsobjekt eine Mischverkehrsfläche vorhanden, wurde der Bereich so eingeteilt, dass die Fahrgasse ca.1/3 bis max. die Hälfte der gesamten Straßenraumbreite ausmacht (vgl. Abbildung 93).

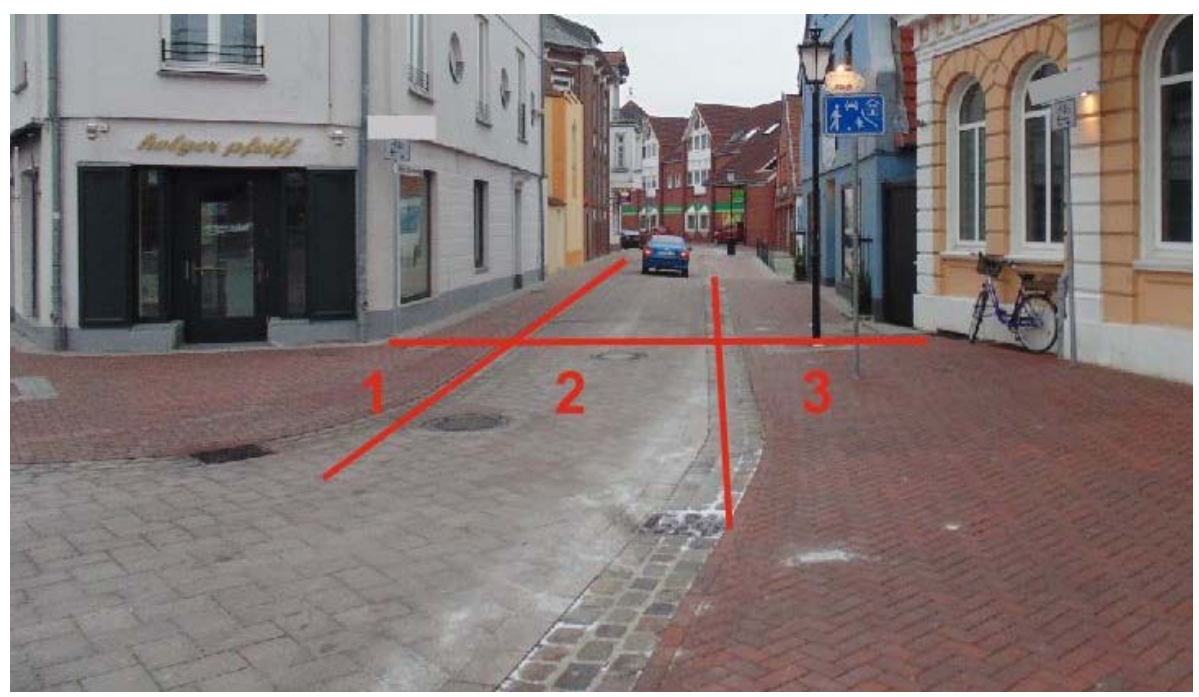

Abbildung 92: Flächeneinteilung eines Verkehrsberuhigten Bereichs (hier mit einer Fahrgasse) 


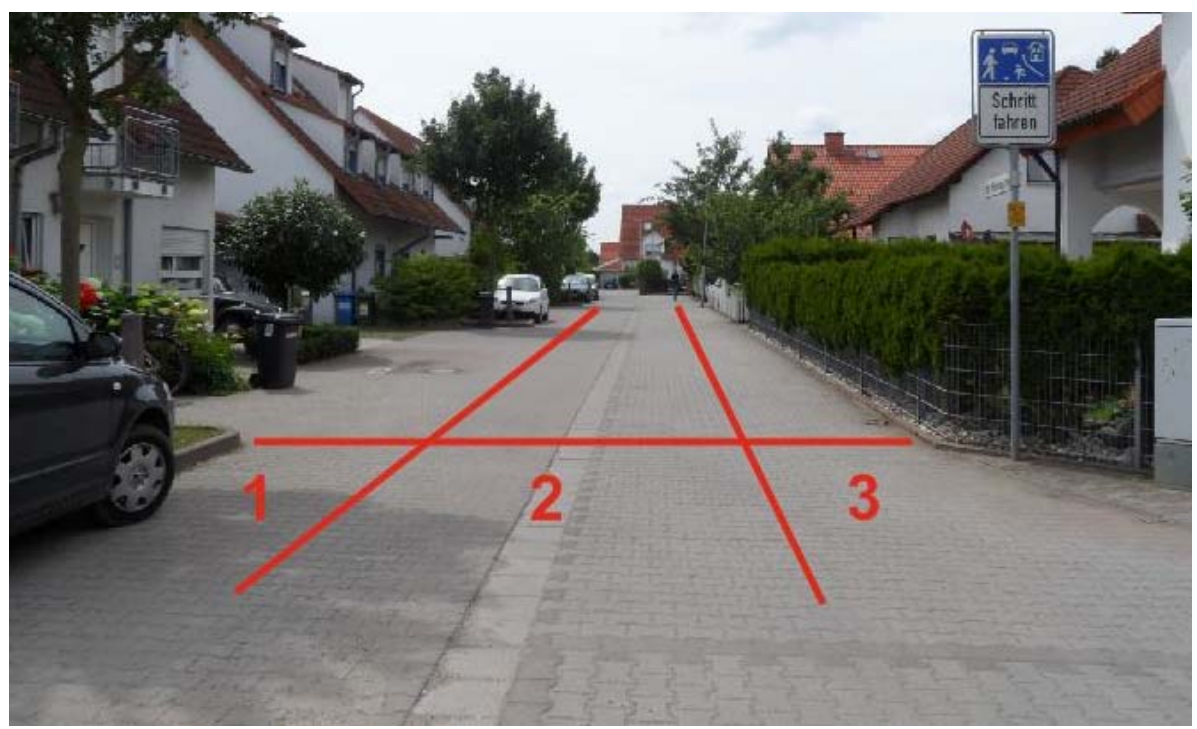

Abbildung 93: Flächeneinteilung eines Verkehrsberuhigten Bereichs (hier mit einer Mischverkehrsfläche)

Es existieren auch Untersuchungsobjekte, in denen die Randbereiche zwar vorgegeben, aber zum Teil nur sehr schmal oder einseitig ausgebaut worden sind. Hierbei wurde während der Auswertung des Videomaterials darauf geachtet, dass bspw. die Randbereiche so breit gewählt wurden, dass eine Person mit Kinderwagen oder mit einer Gehhilfe, ausreichend Platz zur Verfügung stand.

War nur ein einseitiger Randbereich vorhanden oder fand dort Parken statt, galt das gleiche Prinzip wie bei schmalen Rändern, so dass der VBB immer in drei Flächen eingeteilt wurde (Abbildung 94). Nur durch die konsequente Unterteilung der Straßenräume in drei Flächen bei allen 50 VBB, konnte untersucht werden, ob und wie bspw. Fußgänger die Seiträume nutzen und ob sie sich durch angedeutete "Gehwege" leiten lassen oder nicht.

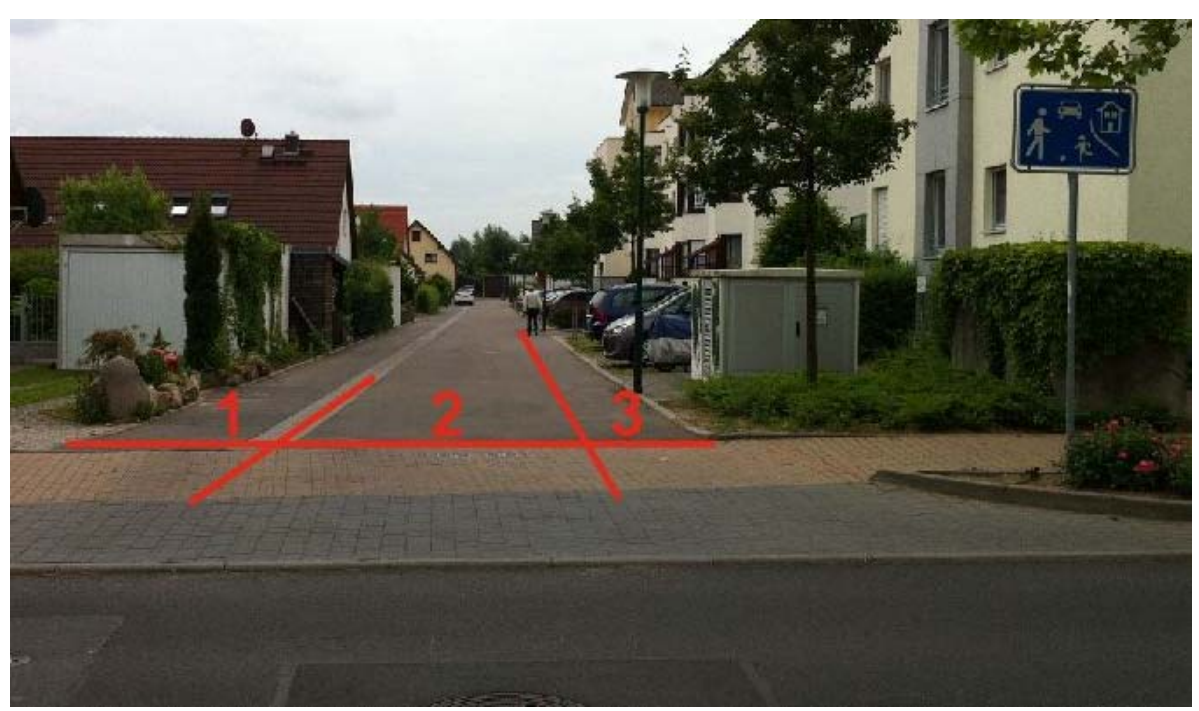

Abbildung 94: Flächeneinteilung eines Verkehrsberuhigten Bereichs (hier mit einem einseitigen Rand) 


\subsubsection{Querschnittszählung}

Für die Erfassung der Verkehrsmengen innerhalb der Verkehrsberuhigten Bereiche wurden die Untersuchungsobjekte individuell in Abschnitte unterteilt, an deren Anfangs- bzw. Endquerschnitt die Verkehrsteilnehmer gezählt werden sollten. Anzumerken ist, dass diese Anfangs- bzw. Endquerschnitte durch die Möglichkeiten zur Anbringung der Kameras im Regelfall vorgegeben waren. Es wurde darauf geachtet, hauptsächlich die Übergangsbereiche zu erfassen. Zusätzlich wurden teilweise weitere Querschnitte innerhalb der Bereiche ausgewählt.

Da der Ort der Querschnittszählung dem Ort der Messung zur Erfassung der Flächennutzung entsprach, erfolgte die Erhebung zeitgleich. Es wurde das Betreten/Einfahren sowie das Verlassen/Ausfahren der Verkehrsteilnehmer je Fläche erhoben (vgl. Abbildung 95). Für die Flächennutzung und die Querschnittszählung wurde demnach nur ein Zählformular je Querschnitt benötigt (vgl. Abbildung 96). Die Zählung wurde per Strichliste durchgeführt.

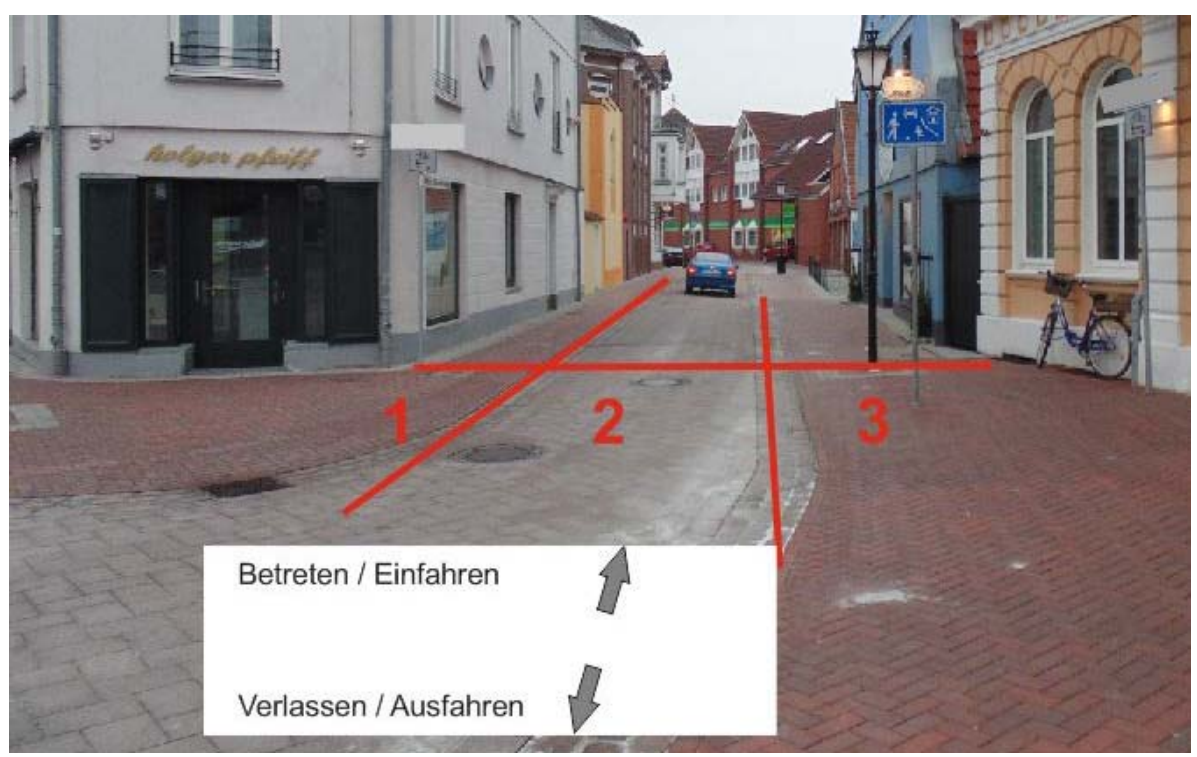

Abbildung 95: Querschnitt, Einteilung des Flächenrasters

\begin{tabular}{|c|c|c|c|c|c|c|c|c|c|c|c|c|c|c|c|c|c|}
\hline \multicolumn{1}{|c|}{ Anzahl Verkehrsteilnehmer } \\
\hline
\end{tabular}

Abbildung 96: Ausschnitt Zählformular

\subsubsection{Geschwindigkeiten}

Die Geschwindigkeiten wurden über die Kamerazeitangaben (Std.-Min.-Sek.) ermittelt. Das bedeutet, dass der Zeitpunkt eines Fahrzeugs sowohl bei der Einfahrt als auch bei der Ausfahrt erfasst wurde. Aufgrund der Datenschutzbedingungen waren bei den Fahrzeugen keine Kennzeichen erkennbar (verpixelt, farbverändert), so dass die Fahrzeuge während der Durchfahrt 
„verfolgt" werden mussten (vgl. Abbildung 97). Da die Verkehrsbelastungen und die Länge der Untersuchungsbereiche gering waren, konnten alle Fahrzeuge problemlos bei der Ein- und Ausfahrt identifiziert werden.

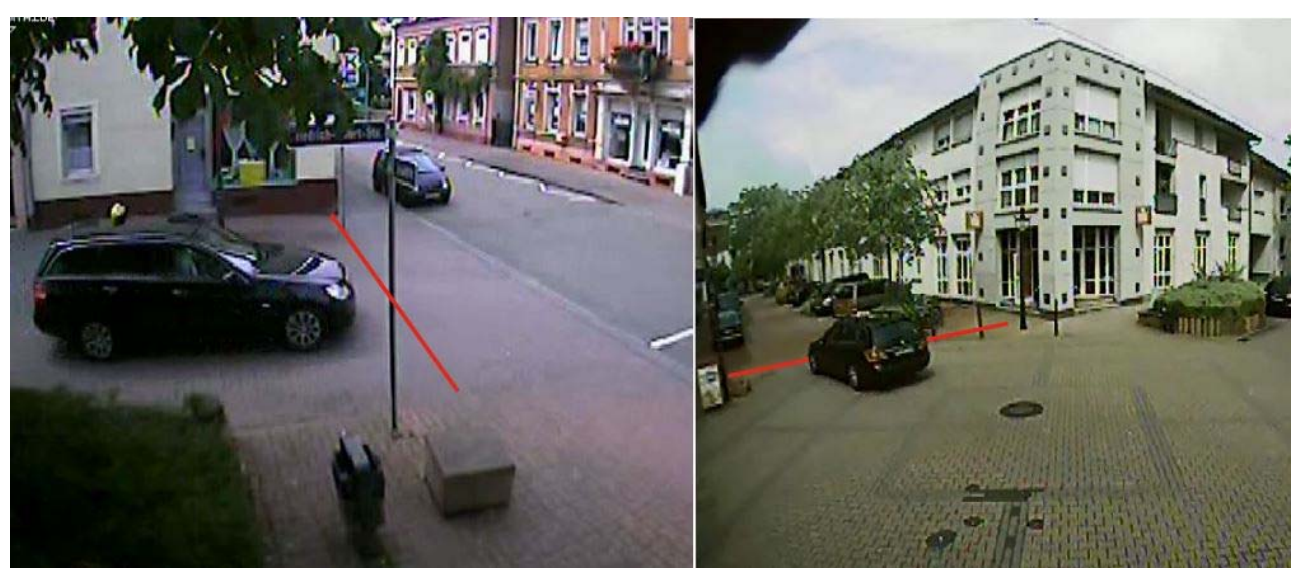

Abbildung 97: Beispiel Geschwindigkeitsmessung

Die Geschwindigkeiten wurden aufgrund der Kamerastandorte nur teilweise über die gesamte Länge des Untersuchungsobjekts gemessen und stimmen daher nicht immer mit den angegebenen Längen im Steckbrief überein. Aufgrund von Gestaltungsmaßnahmen, der Kamerapositionen, kurvigen Streckenführungen innerhalb eines Bereiches oder auch bspw. Parkvorgängen wurden die Geschwindigkeiten abschnittsweise gemessen. Damit sollte auch eine Verfälschung der Ergebnisse durch „zufälliges“ Warten (bei Park-/Haltevorgängen) o.ä. vermieden werden. Die Abbildung 98 zeigt ein Beispiel für die Anbringung von Kameras in einem VBB, wobei die gelben Markierungen die Kameras + Identifikationsnummer (der Kamera) darstellen.

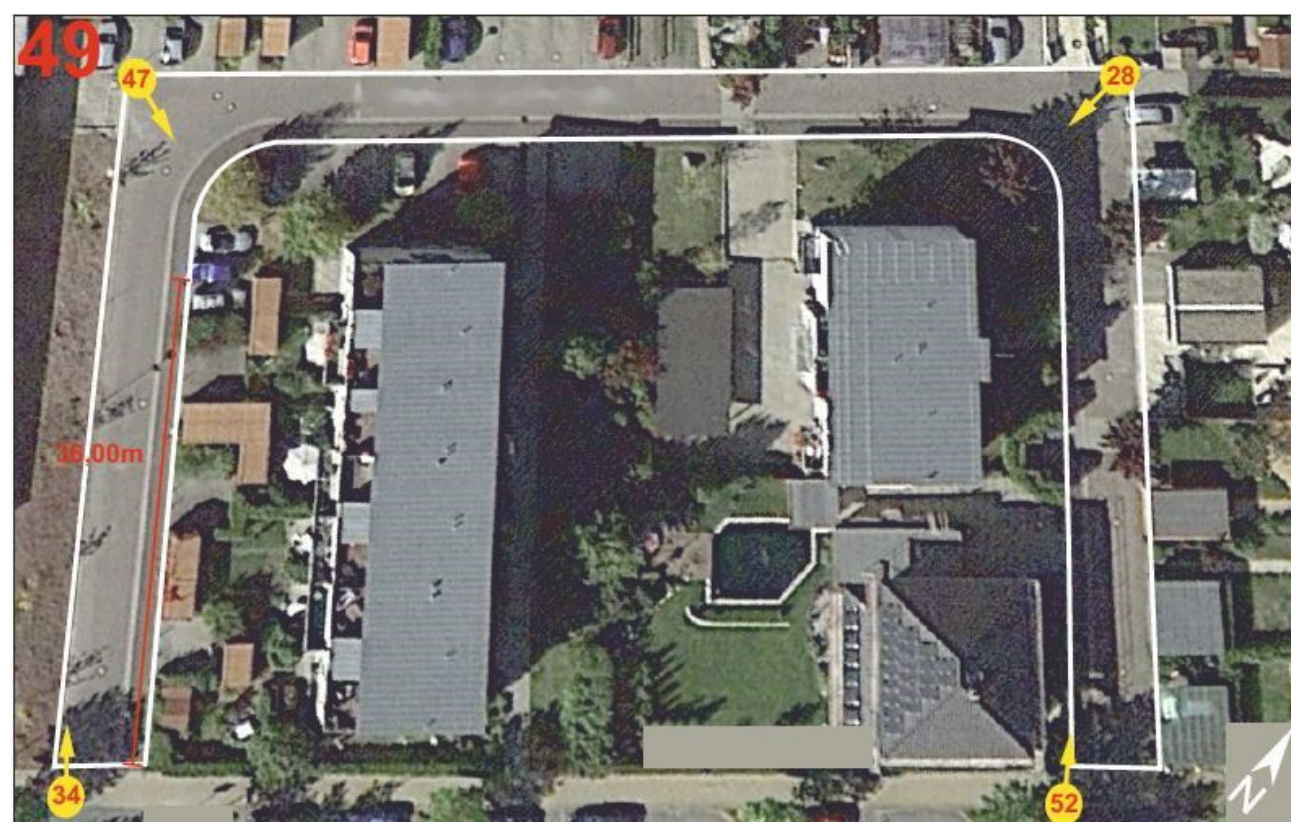

Abbildung 98: Beispiel zur Anbringung von Kameras innerhalb der VBB 
$\mathrm{Da}$ in einigen Bereichen mehrere Kameras angebracht waren, konnten auch Geschwindigkeitsangaben zu mehreren Abschnitten innerhalb eines VBBs angegeben werden. Hieran ist im Anschluss erkennbar, welche Geschwindigkeiten im Übergangsbereich oder innerhalb des VBB gefahren werden und es konnten zusätzliche Zusammenhänge zwischen Gestaltung und Fahrverhalten abgeleitet werden. Für insgesamt 11 Untersuchungsobjekte konnte eine Zeitmessung über mehrere Abschnitte erfolgen.

\subsubsection{Interaktionen}

Unter Interaktionen wird ein wechselseitiges aufeinander Einwirken von verschiedenen oder gleichen Verkehrsteilnehmern verstanden. Dies kann bspw. bedeuten, dass sich ein $\mathrm{Kfz}$ und ein $\mathrm{Rad}$ oder ein $\mathrm{Kfz}$ und ein $\mathrm{Kfz}$ begegnen und es zu verschiedenen Reaktionen der Akteure kommt. Dabei kann es grundsätzlich zu „StVO-konformen“ und nicht „StVO-konformen“ Situationen kommen.

Unter „StVO-konformen“ Situationen sind Verhaltensweisen zwischen zwei Verkehrsteilnehmern zu verstehen, die den Regeln der StVO entsprechen, d.h., dass bspw. die Vorfahrtsregelungen von den beteiligten Verkehrsteilnehmern bei der Zusammenkunft berücksichtigt wurden. Entsprechend wird unter nicht „StVO-konformen“ Situationen ein Verhalten verstanden, bei dem die Regeln der StVO von mindestens einem Verkehrsteilnehmer nicht beachtet wurden.

Zusätzlich wurden die Interaktionen zwischen den Verkehrsteilnehmern hinsichtlich ihrer risikobehafteten Ausprägung bewertet. Hierzu wurden die Interaktionen zwischen den Verkehrsteilnehmern als „nicht kritisch“ bewertet, wenn zu jederzeit der Interaktion ersichtlich war, dass alle Verkehrsteilnehmer sich gegenseitig erkannt haben und entsprechend der Regelungen der StVO agiert haben. Als „kritisch“ wurde eine Interaktion beurteilt, wenn bei dieser nur durch eine aktive Aktion eines der Verkehrsteilnehmer ein Unfall verhindert werden konnte. So stellt eine abrupte Bremsung eines $\mathrm{Kfz}$ (da er einen anderen Verkehrsteilnehmer bspw. zu spät wahrgenommen hat), um einen anderem Verkehrsteilnehmer die Vorfahrt zu gewähren, eine solche kritisch zu beurteilende Situation dar. Die Einstufung einer Interaktion als „kritisch“ umfasst bspw. auch den aktiven Verzicht eines Verkehrsteilnehmers auf sein Vorfahrtrecht. Dabei handelt es sich dann allerdings zusätzlich auch um eine nicht „StVO-konforme“ Situation. Entsprechend sind alle nicht „StVOkonformen" Interaktionen als kritisch einzustufen.

Erfasst wurden nur die als kritisch zu bewertenden Interaktionen zwischen den Verkehrsteilnehmern. Entsprechend wurden die "StVO-konformen“ Interaktionen, die als „nicht kritisch" beurteilt wurden nicht erfasst, da es sich hierbei ausschließlich um übliche Verkehrs- und Begegnungsvorgänge handelt. Die nicht „StVO-konformen“ Interaktionen sollten alle erfasst, da es sich bei diesen gemäß der vorherigen Festlegung prinzipiell um „kritische“ Interaktionen handelt.

Die Auswertungen erfolgten parallel zu den Querschnittszählungen und den Geschwindigkeitsmessungen. Jedem Erhebungsbogen war eine schematische Übersicht über die Gestaltung im Untersuchungsobjekt beigefügt, in dem die Interaktionen eingezeichnet wurden (vgl. Abbildung 99). Durch den Lageplan konnten Konfliktpunkte verdeutlicht werden, da sie sich während der Videoauswertungen beobachten ließen. Je mehr Markierungen an einer Stelle vermerkt wurden, desto höher war das Konfliktpotenzial. Durch die schematische Darstellung der Gestaltung, konnten „auf den ersten Blick“ Zusammenhänge zwischen dem Verhalten und der Gestaltung erkannt werden. 


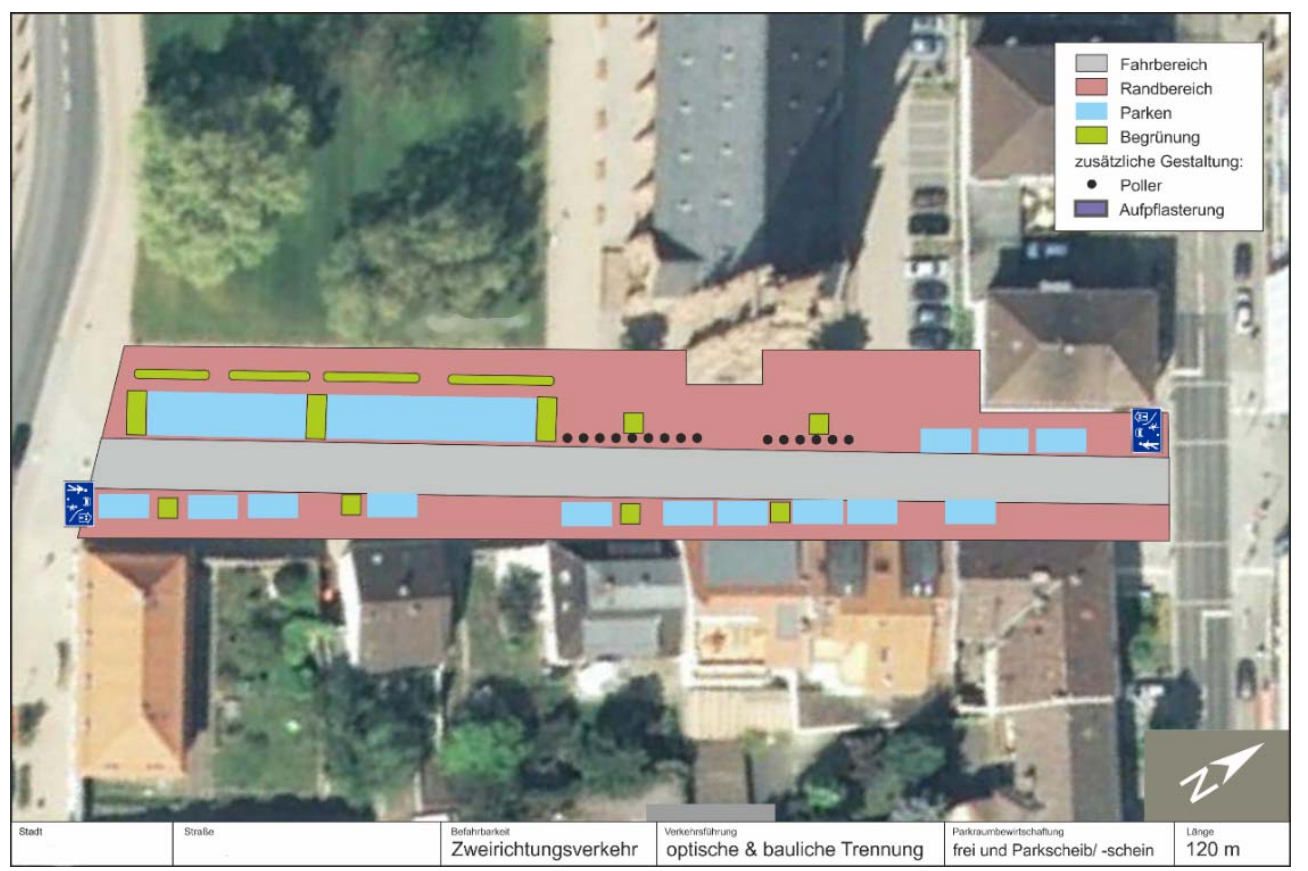

Abbildung 99: Schematische Übersicht der Gestaltung und der Streckenführung eines VBB

\subsection{Darstellung der Auswertungen}

Die Auswertungen der Verkehrsbeobachtungen sind im separaten Anlagenband zur Studie, der auch die Steckbriefe der Untersuchungsobjekte beinhaltet (vgl. Kapitel 9), festgehalten worden. Da in der Steckbriefergänzung nur ein kurzer inhaltlicher Überblick über das Untersuchungsobjekt, mit einer schematischen Übersichtskarte gegeben wird (vgl. Abbildung 100), muss der Steckbrief für die charakterisierenden Details und zum Verständnis hinzugezogen werden. In der Ergänzung wurden Informationen zum Untersuchungsobjekt (Straßenname) sowie zur Stadt, zur Straßenfunktion, der Verkehrsführung, der Länge des Gesamtbereichs sowie zur Anzahl der Abschnitte, in die der Bereich aufgeteilt wurde, festgehalten.

Die Übersichtskarte dient zur Orientierung und gibt unter anderem Auskünfte zum Straßenverlauf und der Ein- und Ausfahrtssituation (Z.B. $90^{\circ}$ Winkel beim Einbiegen). Mit den roten Abgrenzungen wird aufgezeigt, wo sich der gewählte Abschnitt im VBB befindet, wie lang er ist und wo die Querschnittszählungen durchgeführt wurden. Um ggf. die Gesamtsituation im VBB besser zu verstehen, wurden je nach Notwendigkeit auch örtliche Gegebenheiten wie Kindergärten und Marktplätze eingezeichnet.

Die Darstellung der Querschnittszählung und der Flächennutzung kann der Abbildung 101 entnommen werden. Zum einen ist in der Darstellung die genaue Verkehrsteilnehmeranzahl je Verkehrsart zu erkennen, die den VBB innerhalb der 4 beobachteten Stunden betreten oder verlassen hat. Zum anderen kann die genaue Flächennutzung [in \%] der ermittelten Verkehrsteilnehmer abgelesen werden. Da Motorräder nur einen sehr geringen Anteil ausmachten, wurden sie der Übersicht halber nicht eingetragen. Der ÖPNV hat zwar auch nur einen geringen Anteil in Bezug auf alle 50 Untersuchungsobjekte, wird jedoch dargestellt, da der Einsatz von ÖPNV in Straßen eines VBB umstritten ist. 


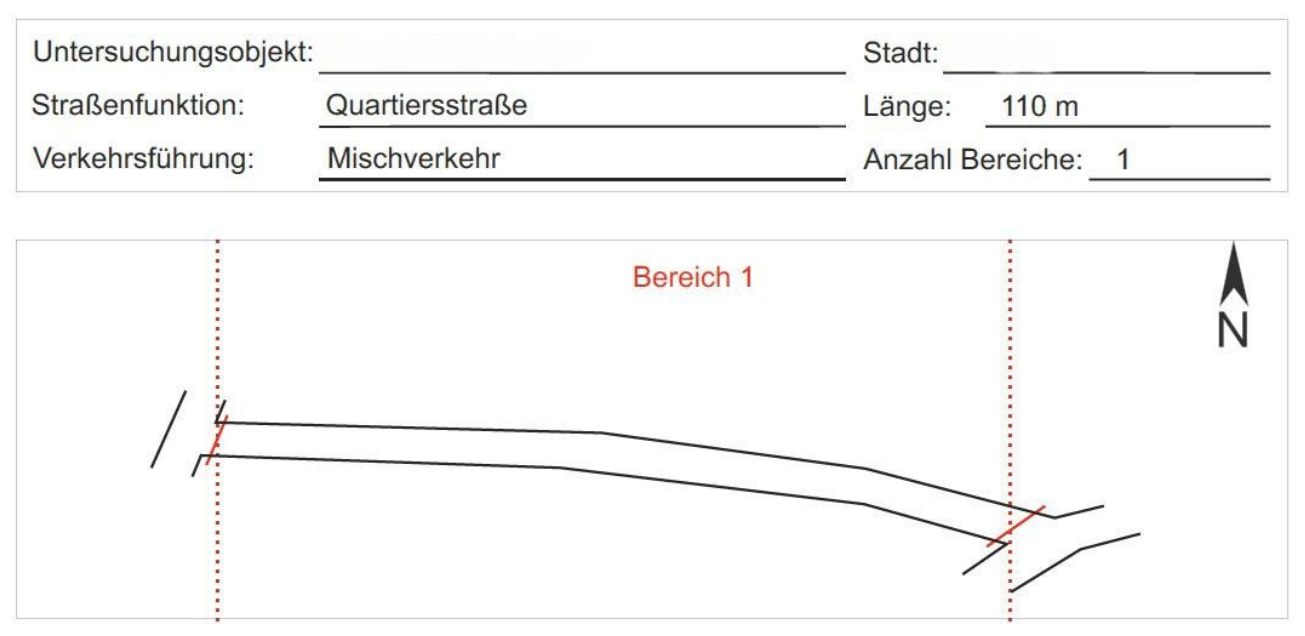

Abbildung 100: Infos Untersuchungsobjekt und schematische Übersichtskarte

In der beispielhaften Auswertung in Abbildung 101 ist z.B. zu erkennen, dass 101 Fußgänger den Bereich auf der westlichen Seite betreten oder verlassen haben und dafür zu $74 \%$ den Randbereich 1 benutzt haben und zu 25\% die mittlere Fläche. Außerdem ist zu erkennen, dass 305 Pkw auf der westlichen Seite ein- oder ausgefahren sind und 312 Pkw auf der östlichen Seite. Dies bedeutet aber nicht, dass es sich bei beiden Übergangsbereichen um die gleichen Fahrzeuge handelt, da manche den VBB z.B. mit einem Pkw befahren, im Bereich Parken und z.B. als Fußgänger den Bereich wieder verlassen.

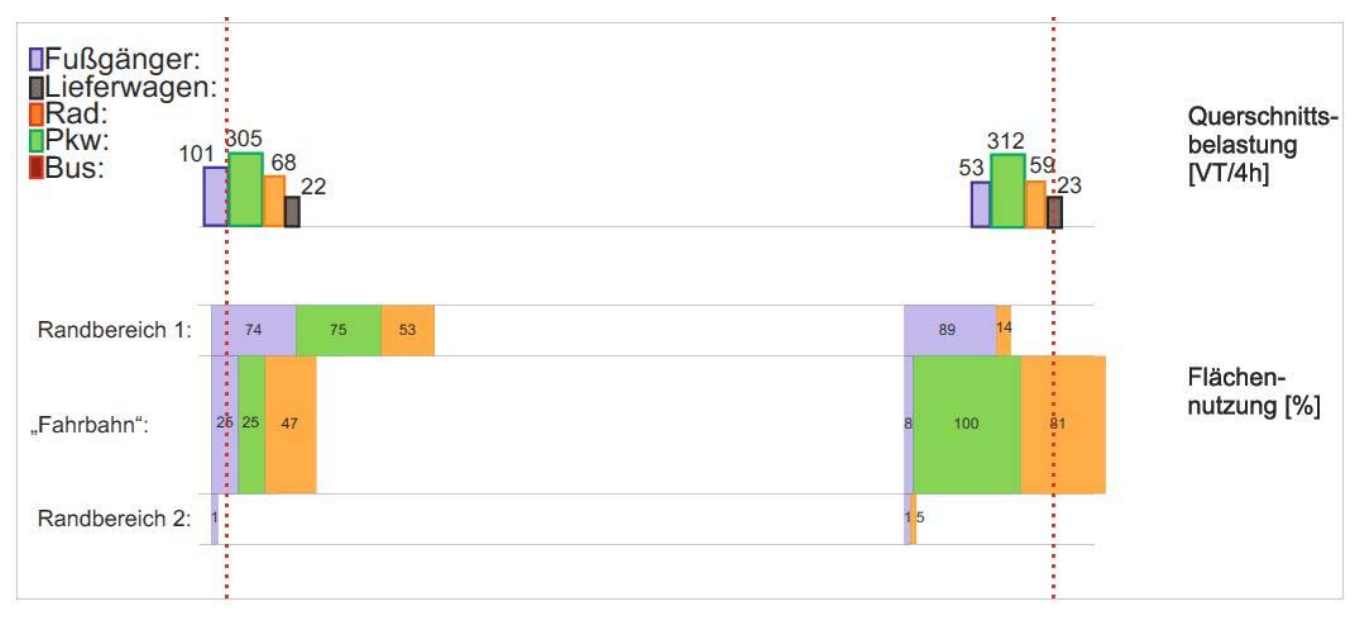

Abbildung 101: Darstellung zur Auswertung der Querschnittszählung und Flächennutzung

Für die Darstellung der Geschwindigkeitsmessungen war es entscheidend, ob nur auf einem oder mehreren Abschnitten gemessen wurde. Wurde die Geschwindigkeit auf nur einem Abschnitt ermittelt, wie beim Großteil der untersuchten Verkehrsberuhigten Bereiche, wurden die Verkehrsteilnehmer Pkw, Rad, Lieferverkehr und der ÖPNV (falls vorhanden) dargestellt. Dabei wurde die Mindestanzahl der Verkehrsteilnehmer (n), die den Abschnitt durchfahren haben, mit $\mathrm{n}>10$ festgelegt. Die Festlegung auf eine Mindestzahl von 10 Verkehrsteilnehmern erfolgte pragmatisch und diente ausschließlich dazu, zumindest eine Tendenz des Kurvenverlaufes zu erkennen, die bei einer geringeren Anzahl als 10 Verkehrsteilnehmer nahezu nicht zu erkennen war. Fuhren nicht mindestens 10 Teilnehmer einer Verkehrsart innerhalb der 4 aufgenommenen Stunden durch den Bereich, wurden sie bei der Darstellung vernachlässigt. Unabhängig von der 
Anzahl der erfassten Abschnitte, wurden bei jeder Geschwindigkeitsauswertung die Kontrollgrößen V15, V50, V85 und Vmax abgebildet, was bedeutet, dass z.B. 15\% aller Verkehrsteilnehmer (n) eine Geschwindigkeit von bis $\mathrm{zu}$ „x"km/h haben oder $50 \%$ von $\mathrm{n}$ eine Geschwindigkeit von bis zu "y" $\mathrm{km} / \mathrm{h}$ haben ( $\mathrm{V}_{85}$ wird äquivalent wie $V_{15}$ und $V_{50}$ bestimmt) (vgl. Abbildung 102).

Aus der Darstellung der Geschwindigkeitsverteilung auf einem Abschnitt können neben den Kenngrößen der Geschwindigkeit ebenso der Verlauf der Verteilungskurve und damit die Spannweite der gefahrenen Geschwindigkeiten abgelesen werden. An dieser Stelle sei noch einmal darauf hingewiesen, dass es sich bei den dargestellten Geschwindigkeiten um Durchfahrtgeschwindigkeiten handelt. An einzelnen Stellen im Untersuchungsbereich können somit auch höhere lokale Geschwindigkeiten gefahren worden sein. Allerdings ließen sich diese mit der angewendeten Erhebungsmethode nicht erfassen.

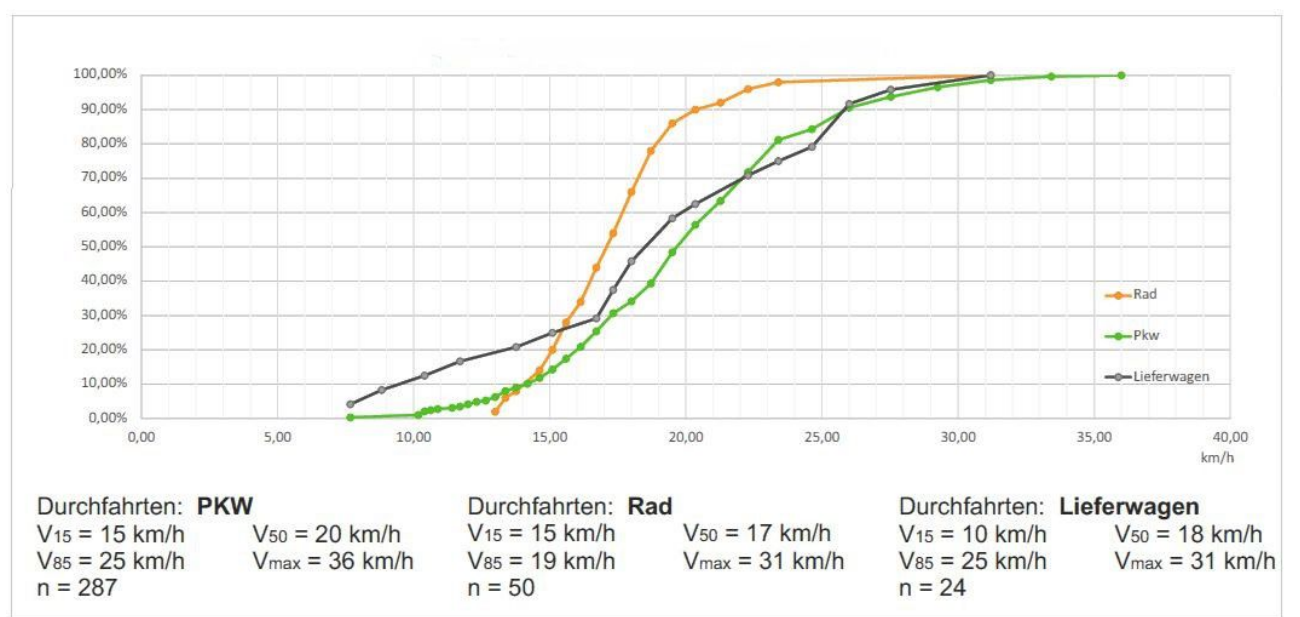

Abbildung 102: Geschwindigkeitsverteilung auf einem Abschnitt nach Verkehrsteilnehmern

Wurde die Geschwindigkeit auf mehreren Abschnitten innerhalb eines VBBs erfasst, sind nur die Pkw-Geschwindigkeiten je Bereich sowie eine Ganglinie für die Gesamtgeschwindigkeit ausgewiesen worden (Abbildung 103). Dies liegt zum einen darin begründet, dass die sonstigen Verkehrsteilnehmer wie Radfahrer und Lieferwagen nur zu einem sehr geringen Anteil die Untersuchungsbereiche in ihrer vollen Länge durchfahren. Zum anderen ließen sich aus stichprobenhaften Auswertungen für die Radfahrer keine zusätzlichen Erkenntnisse über das Geschwindigkeitsverhalten erkenne, wenn diese den gesamten Untersuchungsbereich durchfahren.

Die Durchfahrtgeschwindigkeit der Pkw für den gesamten Bereich errechnete sich aus der insgesamt gefahrenen Zeit und der Gesamtlänge aller Abschnitte. Sie wurde somit nur aus Messungen von Pkws ermittelt, die den Untersuchungsbereich in seiner gesamten Länge vollständig und ohne Unterbrechung durchfahren haben. Somit ist die Anzahl $n$ der Pkw, die der Ermittlung der Durchfahrtgeschwindigkeit zugrunde liegen, kleiner als die Anzahl $n$ der Pkw, die den Untersuchungsbereich befahren, da ein Teil der Pkw, den Untersuchungsbereich nicht ohne Unterbrechung der Fahrt befahren. 


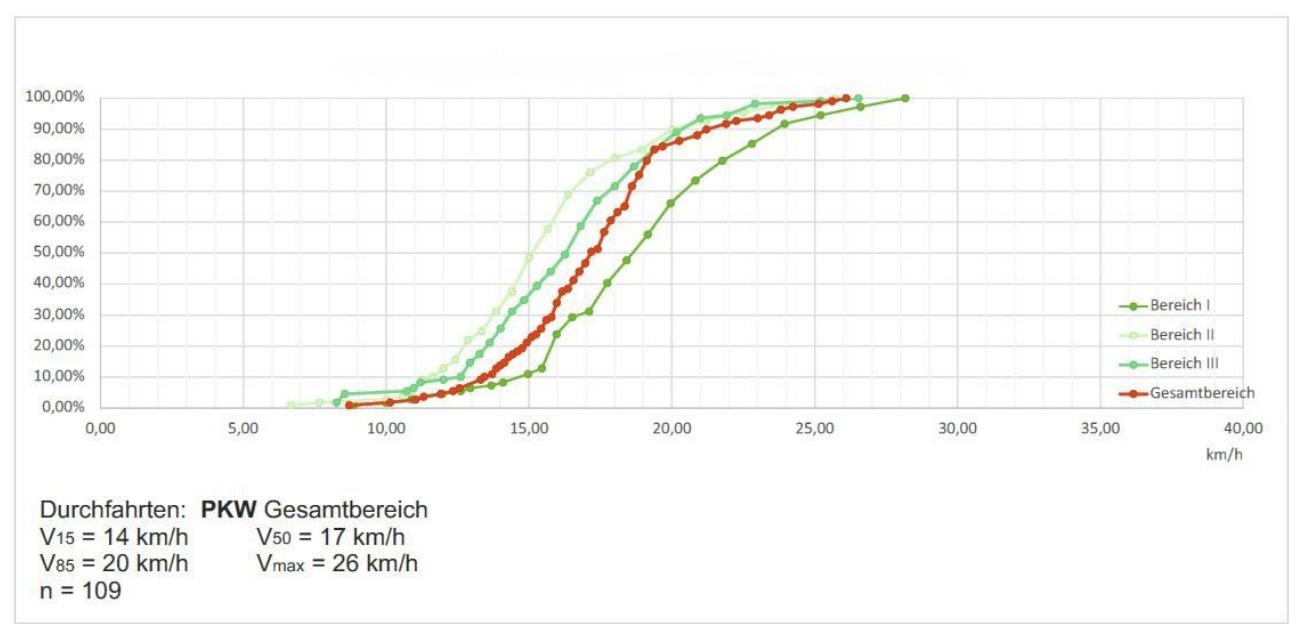

Abbildung 103: Geschwindigkeitsverteilung bei mehreren Bereichen (nur Pkw)

\subsection{Ergebnisse der Verkehrsbeobachtung}

Bei der nachfolgenden Darstellung der Ergebnisse folgen als erstes allgemeine Informationen zu Querschnittsbelastungen und danach zur Flächennutzung. Im Anschluss daran werden die Ergebnisse zum Geschwindigkeitsniveau und den Interaktionen zusammengefasst.

\subsubsection{Querschnittszählung}

Insgesamt wurden bei allen 50 VBB mindestens ein Querschnitt, zumeist aber zwei Querschnitte, und vereinzelt auch mehrere Querschnitte innerhalb eines VBB gezählt. So ergab sich eine Gesamtsumme von mehr als 100 gezählten Querschnitten. Es ist zu beachten, dass die Querschnitte zwar vorrangig an den Übergangsbereichen gezählt wurden, allerdings wurde auch in einigen Fällen an einem Knotenpunkt innerhalb eines VBB gezählt. Insbesondere in diesen Fällen können die einzelnen Querschnittswerte deutlich voneinander abweichen, da die Verkehrsbelastung an einem Knotenpunkt innerhalb eines VBBs durch ein- und abbiegende Fahrzeuge/Verkehrsteilnehmer anders sein kann als im Übergangsbereich.

In Abbildung 104 ist die Anzahl der Querschnitte in den verkehrsberuhigten Bereichen dargestellt, an denen eine festgelegte Menge an Pkw innerhalb der vier Beobachtungsstunden erfasst wurde. Dabei wurden aufgrund der geringen Verkehrsbelastungen in Wohnstraßen die Verkehrsmengen bis $200 \mathrm{Pkw}$ in vier Stunden in Klassenbreiten von $50 \mathrm{Pkw}$ unterteilt und darüber wurde eine Klassenbreite von 100 Pkw in vier Stunden gewählt.

Im Folgenden werden die Querschnittszählungen je Straßenfunktion kommentiert. Eine detaillierte Auswertung für die einzelnen Untersuchungsbeispiele getrennt für die Verkehrsarten und die einzelnen Querschnitte können dem separaten Anlagenband entnommen werden. 


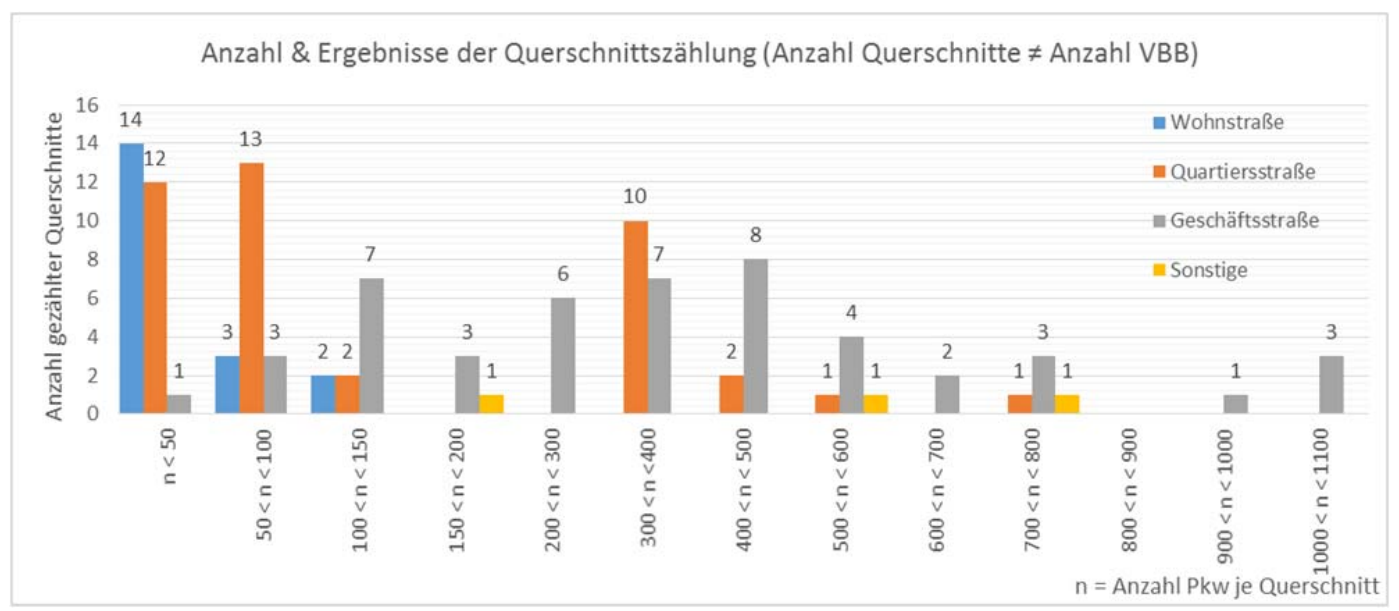

Abbildung 104: Anzahl und Ergebnisse der Querschnittszählung für Pkws in vier Stunden von 14 bis 16 Uhr

\section{Wohnstraßen:}

Bei den neun beobachteten Verkehrsberuhigten Bereich der Kategorie „Wohnstraßen“, wurden an insgesamt neunzehn Querschnitte die Verkehrsmengen erhoben, wobei die Belastung der Pkw zwischen fünf und $148 \mathrm{Pkw}$ in vier Stunden liegt. Vierzehn Querschnittswerte lagen bei unter 50 Pkw in vier Stunden, drei Querschnitte zwischen 50 und $100 \mathrm{Pkw}$ und nur zwei über $100 \mathrm{Pkw}$ in vier Stunden (143 und 148 Pkw in 4 Std) (vgl. Abbildung 104).

Die Anzahl der Radfahrer und Fußgänger lag an allen Querschnitten unterhalb der gezählten Anzahl an Pkw. Insgesamt wurden in Wohnstraßen, an allen Querschnitten zusammen 920 Pkw, 655 Fußgänger und 400 Radfahrer gezählt.

Die Verkehrsbelastungen in allen beobachteten Wohnstraßen sind insgesamt sehr gering. Die geschätzten durchschnittlichen Tagesverkehrsbelastungen (DTV) im Kfz-Verkehr liegen bei maximal 500 Fahrzeugen am Tag. Somit ist in allen Wohnstraßen der Kfz-Verkehr als sehr gering einzustufen. Die ausgewiesenen Verkehrsberuhigten Bereiche entsprechen in den beobachteten Untersuchungsabschnitten somit alle den Vorschriften der StVO bzw. der VwV-StVO.

\section{Quartiersstraßen:}

An 41 Querschnitte wurden bei den 20 beobachteten Verkehrsberuhigten Bereichen der Kategorie "Quartiersstraße“ gezählt. Die Verteilung über die Anzahl der Pkw je Querschnitt (in 4 Std.) variierte bei den Quartiersstraßen im Vergleich zu den „Wohnstraßen“ deutlicher. Auch hier lag zwar bei 27 Querschnitten die Pkw-Anzahl unter 150 Pkw in vier Stunden. Dem gegenüber waren aber auch 10 Querschnitte mit einer Pkw-Anzahl zwischen 300 und 400 Pkw in 4 Stunden vertreten. Weitere 4 Querschnitte wiesen 400-800 Pkw in vier Stunden auf (vgl. Abbildung 104).

Die Anzahl der Radfahrer und Fußgänger lag an allen Querschnitten unterhalb der gezählten Anzahl an Pkw. Insgesamt wurden in Quartiersstraßen, an allen Querschnitten zusammen 7.000 Pkw, 4.500 Fußgänger und 3.000 Radfahrer gezählt.

Die Verkehrsbelastungen an 27 Querschnitten der Quartiersstraßen waren sehr gering und lagen bei weniger als 500-600 Pkw pro Tag. Diese Bereiche entsprechen eindeutig den Vorschriften der StVO bzw. der VwV-StVO.

Die 10 Querschnitte, an denen eine 4-Stunden-Belastung von 300-400 Pkw erhoben wurde, weisen entsprechend einen geschätzten Tageswert von 1.000-1.500 Pkw pro 24h auf. Dieser Wert kann ebenfalls als noch sehr geringes Kfz-Verkehrsaufkommen eingestuft werden. Damit würden auch 
diese Querschnitte den Anforderungen der Vorschriften der StVO bzw. der VwV-StVO entsprechen.

Die 4 Querschnitte, an denen eine Verkehrsbelastung von 400-800 Pkw in vier Stunden erfasst wurde, weisen hingegen einen geschätzten Tagesverkehrswert von 1.500-3.000 Pkw pro 24h auf. Damit kann an diesen Querschnitten nicht mehr von einem sehr geringen Kfz-Verkehrsaufkommen gesprochen werden. Zwar ist eine Verkehrsbelastung zwischen 1.500 und $3.000 \mathrm{Kfz}$ pro Tag immer noch als niedrig einzustufen, aber ob dieser gemäß den Vorschriften der VwV-StVO als sehr geringes Kfz-Verkehrsaufkommen zu bewerten ist, kann zumindest bezweifelt werden. Eine abschließende Beurteilung kann allerdings erst erfolgen, wenn die Festlegung der VwV-StVO „der Fahrzeugverkehr eine untergeordnete Bedeutung" quantifiziert wird.

\section{Geschäftsstraßen:}

An 48 Querschnitte wurde innerhalb der 20 Geschäftsstraßen das Verkehrsaufkommen erhoben. Die Werte verteilten sich über fast alle definierten Belastungsbereiche (vgl. Abbildung 104). So wurde einerseits an 11 Querschnitten eine Pkw-Belastung von maximal 150 Fahrzeugen in den vier Stunden erfasst, andererseits konnten an 3 Querschnitten über $1.000 \mathrm{Pkw}$ in den vier Stunden erhoben werden.

Insgesamt wurden in Geschäftsstraßen, an allen Querschnitten zusammen 19.100 Pkw, 34.400 Fußgänger und 10.720 Radfahrer gezählt.

Legt man die Belastungsgrenze, die eine Übereinstimmung mit den Vorschriften der StVO bzw. der VwV-StVO zulässt, mit maximal $400 \mathrm{Pkw}$ in vier Stunden zugrunde, so konnte an insgesamt 27 Querschnitten eine Unterschreitung dieser Grenze festgestellt werden. Somit entsprechen etwas mehr als die Hälfte der betrachteten Querschnitte den Einsatzgrenze der StVO für einen Verkehrsberuhigten Bereich gemäß VZ 325.

An 17 betrachteten Querschnitten wurde ein Pkw-Verkehrsaufkommen von 400-800 Pkw erfasst, was wie zuvor erläutert zwar noch einen geringes $\mathrm{Kfz}$-Verkehrsaufkommen darstellt, allerdings ggfs. nicht mehr den Anforderungen der VwV-StVO an den Einsatzbereich der Verkehrsberuhigten Bereiche entspricht.

Die Pkw-Verkehrsbelastungen lagen an 4 Querschnitten mit über $800 \mathrm{Pkw}$ in 4 Stunden eindeutig über dem Wert einer Kfz-Verkehrsmenge, die eine Einstufung des Fahrzeugverkehrs von geringer Bedeutung, zulässt.

Ergänzend ist bei den Geschäftsstraßen festzustellen, dass das Fußgängerverkehrsaufkommen mengenmäßig das Pkw-Verkehrsaufkommen überschreitet. Entsprechend sind die Fußgänger in diesen Bereichen die dominierende Verkehrsart. Um die dominierende Verkehrsart zu betonen und gleichzeitig dem Kfz-Verkehr den Zugang zu den vom Fußgänger dominierten Bereich zu ermöglichen, wurde vermutlich in den entsprechenden Bereichen ein Verkehrsberuhigter Bereich gemäß VZ 325 ausgewiesen.

\section{Sonstige:}

In dem einen beobachteten Sonstigen Bereich, wurde an drei Querschnitten gezählt. Hierbei wichen die drei Querschnittswerte, aufgrund der Straßenführung, relativ stark voneinander ab. Während der niedrigste Wert, am Anfang einer Sackgasse lag (173 Pkw in 4 Std.), zeigten die anderen beiden Querschnitte mit Durchgangsverkehr, Werte von 519 und 785 Pkw in 4 Stunden an (Abbildung 104).

\section{Fazit:}

Zusammenfassend lässt sich bei den erfassten Verkehrsmengen festhalten, dass in den Wohnstraßen und in einem Großteil der Quartiersstraßen und der Geschäftsstraßen, der Fahrzeugverkehr mit einer Belastungen von weniger als $400 \mathrm{Kfz}$ in den vier betrachteten Stunden 
von untergeordneter Bedeutung ist. Im Bereich der Pkw-Belastungen von 400-800 Pkw in den vier beobachteten Stunden kann nicht eindeutig festgelegt werden, ob der Fahrzeugverkehr hier eine untergeordnete Rolle spielt oder nicht. Hier bedarf es in der StVO bzw. den VwV-StVO einer klaren Definition. Während die Verkehrsmengen bei den Quartiersstraßen, die nicht unterhalb der Schwelle von $400 \mathrm{Kfz}$ in den vier Stunden liegen, die Pkw-Belastungen im Bereich von 400-800 $\mathrm{Pkw}$ in den vier Stunden liegen, liegen in einzelnen Bereichen der Geschäftsstraßen die Belastungen oberhalb von $800 \mathrm{Pkw}$. Bei einer Belastung von mehr als $800 \mathrm{Pkw}$ in vier Stunden kann sicher nicht mehr davon ausgegangen werden, dass der Fahrzeugverkehr nur eine untergeordnete Rolle spielt. Daher scheint die Anwendung des VZ 325 zumindest bei diesen Geschäftsstraßen als überprüfungswürdig.

\subsubsection{Flächennutzungen}

Während der Durchführung der Querschnittszählungen ist gleichzeitig auch erhoben worden, auf welchen Flächen sich die Verkehrsteilnehmer bewegten. Dabei wurde zwischen Fußgängern, Radfahrern und verschiedenen Kfz-Arten unterschieden, wobei sich die drei repräsentativsten Kollektive aus den Fußgängern, Radfahrern und Pkw bildeten.

Zunächst wurden die drei wesentlichen Gestaltungsarten der Flächenaufteilung in den Verkehrsberuhigten Bereichen betrachtet. Dabei wurden zunächst nur diejenigen Untersuchungsobjekte betrachtet, deren Flächenaufteilung durchgängig ist, d.h. die entweder eine bauliche oder optische Trennung der Verkehrsflächen aufweisen oder nach dem Prinzip des Mischverkehrs niveaugleich ohne optische oder bauliche Trennelemente gestaltet sind. Im Anschluss wurde auf die möglichen Kombinationen der Gestaltungsarten eingegangen.

Abschließend erfolgte eine Betrachtung der Flächennutzung unter Bezug auf die einzelnen Straßenfunktionen. Für die folgenden Darstellungen sind jeweils die Ausnutzung der mittleren Fläche (Fahrgasse) nach den verschiedenen Verkehrsteilnehmern und Straßenkategorien ausgewählt worden.

Ziel der Untersuchung war es, herauszufinden, ob sich die Verkehrsteilnehmer durch eine vorgegebene Verkehrsführung beeinflussen lassen oder nicht. Dies wurde ebenfalls bei Mischverkehrsflächen untersucht, da es auch hier von Interesse ist, ob die Fußgänger das gleiche Verhalten aufweisen, wie bei einer optischen oder baulichen Trennung. Daher war es sinnvoll, auch die Mischverkehrsflächen in 3 Flächen einzuteilen, um herauszufinden zu können ob Mischverkehrsflächen von (allen) Teilnehmern in der ganzen Breite genutzt wurden oder nicht.

In der Auswertung bzw. in den folgenden Abbildungen 105, 107 und 108 beschreibt die Kenngröße $\mathrm{n}$ die Anzahl an Untersuchungsobjekten und in den Abbildungen 109 bis 111 die jeweilige Verkehrsstärke, nach Art des Verkehrsteilnehmers.

\section{Einheitlich gestaltete Flächen:}

In der vorliegenden Studie wurden drei Grundprinzipien der Straßenraumaufteilung in ausgewiesenen Verkehrsberuhigten Bereichen berücksichtigt. Das erste Prinzip ist die niveaugleiche Gestaltung des gesamten Straßenraumes ohne irgendwelche Trennungselemente, d.h. grundsätzlich ist die gesamte Straßenraumbreite durch alle Verkehrsteilnehmer nutzbar. Ausnahmen hierbei bilden einzelne Einbauten (z.B. Bäume) oder gekennzeichnete Parkstände, die den Straßenquerschnitt lokal einengen. Das zweite Prinzip umfasst ebenfalls einen niveaugleichen Ausbau der gesamten Straßenraumfläche, allerdings sind durch Veränderungen der Straßenbeläge oder durch Markierungen oder durch Rinnensteine optische Separationen des Straßenquerschnittes vorhanden, die ein Vorhandensein eines „Gehwegs“ oder einer „Fahrbahn“ 
suggerieren. Das dritte Prinzip umfasst die bauliche Trennung des Straßenraums in Gehwege und Fahrbahnen durch einen Bord (vgl. Kapitel 9.4).

In der nachfolgenden Betrachtung wurden nur VBB $(n=22)$ berücksichtigt, deren Straßenraum durchgängig nach einem der zuvor genannten drei Prinzipien gestaltet war.

Die Pkw nutzen in baulich getrennt gestalteten Bereichen wie zu erwarten nahezu ausschließlich die vorgegebene Fahrbahn. Lediglich in den Bereichen, in denen ein nur niedriger Bordstein, der von den Pkw problemlos überfahren werden kann, vorhanden ist, wurden von einzelnen PkwFahrern auch die seitlichen Straßenraumbereiche genutzt. Allerdings wurde der mittlere Straßenraumbereich auch bei den anderen beiden Gestaltungsprinzipien von mehr als $80 \%$ aller Pkw-Fahrer genutzt. Auffällig ist hierbei zunächst, dass der Anteil der Nutzung der mittleren Flächen bei den Straßenräumen mit dem Prinzip "Mischverkehr" höher ist, als bei den Straßenräumen mit dem Prinzip „optische Trennung“ (vgl. Abbildung 105). Ursächlich hierfür ist allerdings ein Untersuchungsbeispiel, dessen Anteil der Nutzung der mittleren Flächen bei nur rd. $40 \%$ liegt. Betrachtet man die Straßenraumaufteilung dieses Beispiels, dann wird deutlich, warum die Pkw nicht durchgehend die mittleren Straßenraumflächen befahren konnten (vgl. Abbildung 106). Betrachtet man nur die drei übrigen Beispiele mit optischer Trennung, so liegt die Nutzung der mittleren Straßenraumflächen bei deutlich über 90\% und somit über dem Anteil, der bei den Mischverkehrsflächen zu verzeichnen ist.

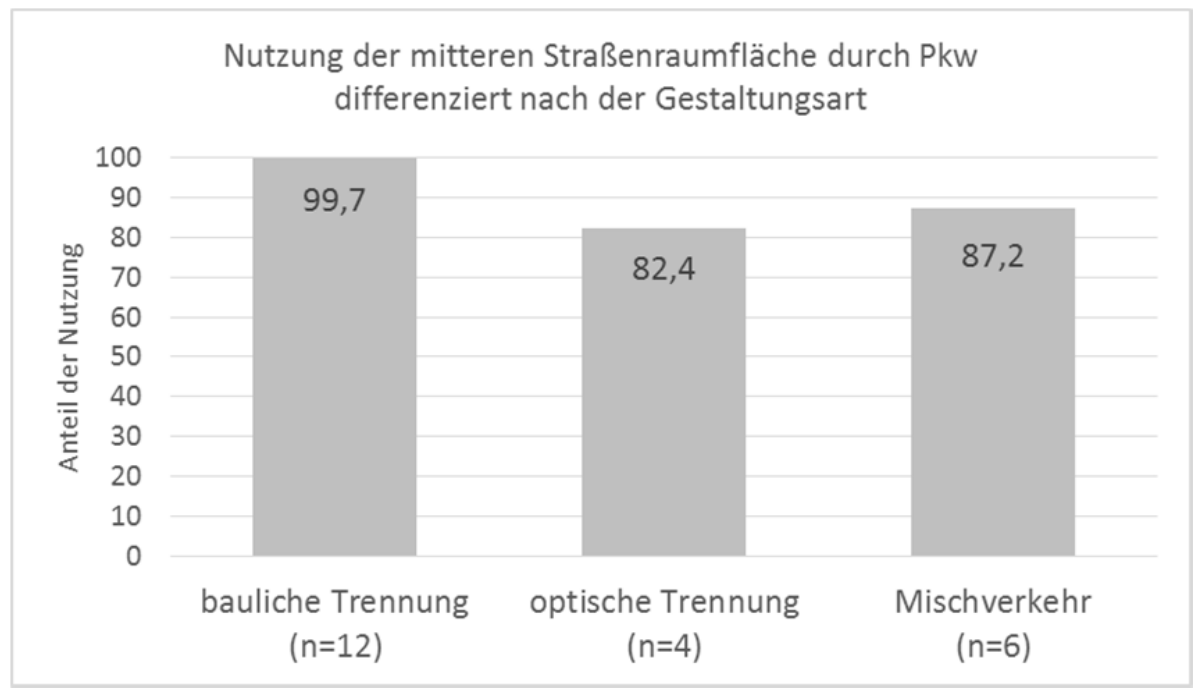

Abbildung 105: Ausnutzung der mittleren Verkehrsfläche durch Pkw in \%

Betrachtet man die Nutzung der Straßenraumflächen durch die Radfahrer in den ausgewiesenen Verkehrsberuhigten Bereichen, so ergibt sich hier ein anderes Bild als bei den Pkw. Der niedrigste Anteil der Nutzung der mittleren Straßenraumflächen liegt hier bei den Bereichen mit baulicher Trennung. Hier liegt der Nutzungsanteil bei den Radfahrern bei nur rd. $60 \%$. Während bei den Bereichen mit Mischverkehrsflächen der Nutzungsanteil der mittleren Straßenraumflächen durch die Radfahrer bei fast $75 \%$ liegt. Auffällig hoch ist der Nutzungsanteil der mittleren Straßenraumflächen bei den VBB mit optischer Trennung der Straßenräume. Dieser liegt bei über 90\% und weist den größten Anteil an allen drei Prinzipien aus (vgl. Abbildung 107). 


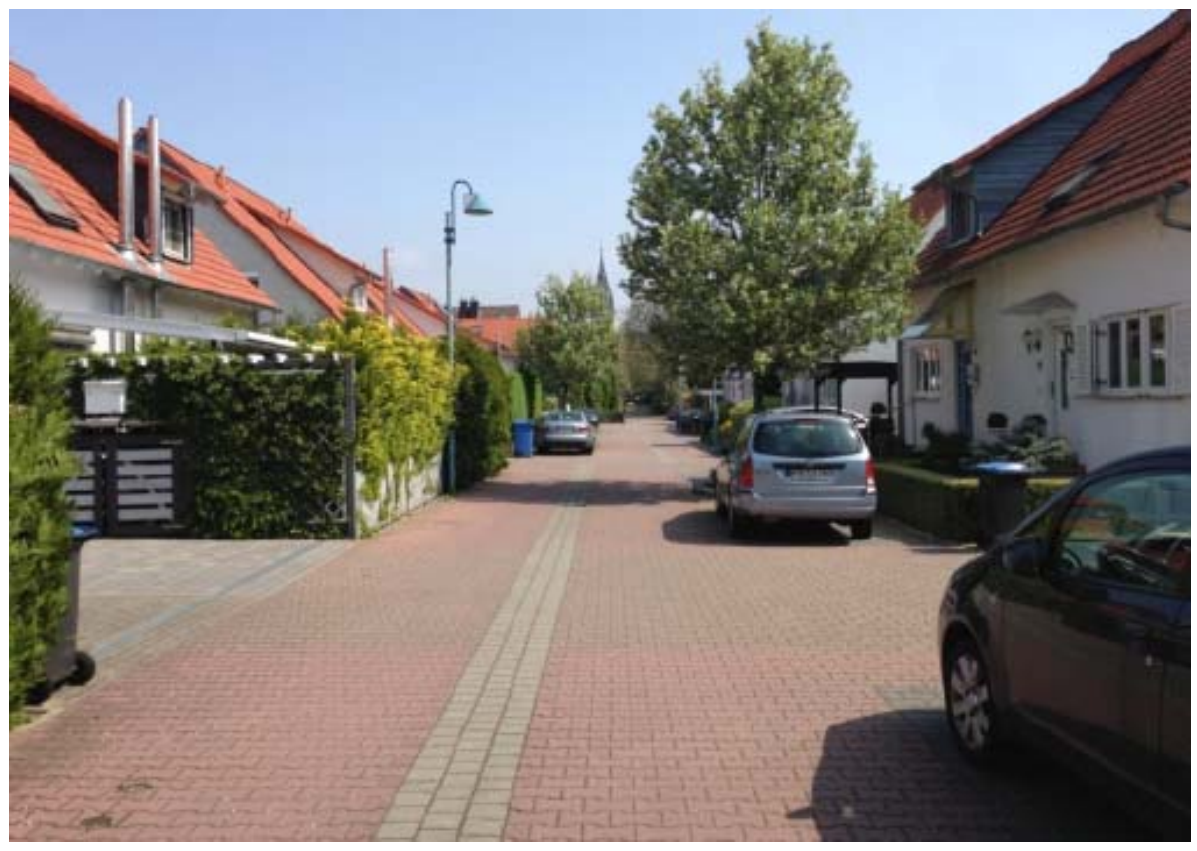

Abbildung 106: Querschnittsaufteilung mit optischer Trennung und Parkflächen am Rand

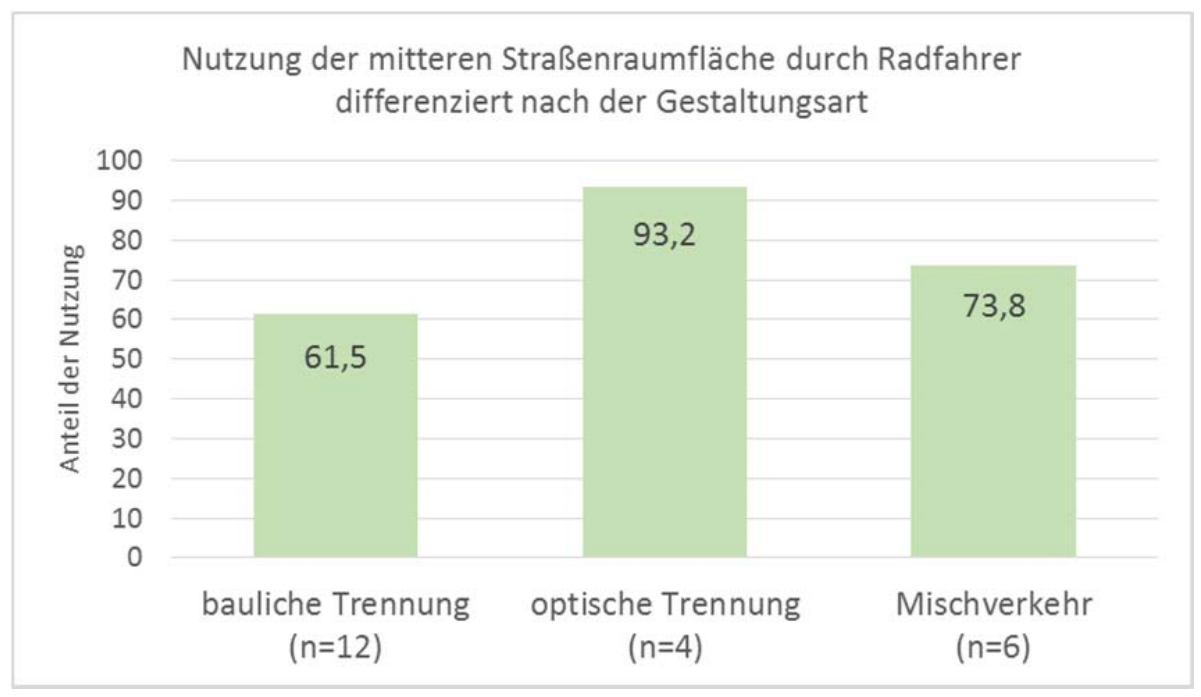

Abbildung 107: Ausnutzung der mittleren Verkehrsfläche durch Radfahrer in \%

Erwartungsgemäß deutlich geringer fällt die Nutzung der mittleren Straßenraumflächen durch die Fußgänger aus. Hier liegen die Anteile je nach Gestaltungsprinzip zwischen rd. 15\% und rd. 45\%. Den geringsten Anteil mit 15\% weisen dabei die ausgewiesenen Verkehrsberuhigten Bereiche mit baulicher Trennung im Straßenraum auf. In VBB mit Mischverkehrsflächen ohne Trennungselementen liegt der Anteil bei rd. 38\%. Etwas höher fällt der Anteil mit knapp 46\% in den Bereichen aus, deren Straßenraum durch optische Trennelemente gestaltet ist (vgl. Abbildung 108). 


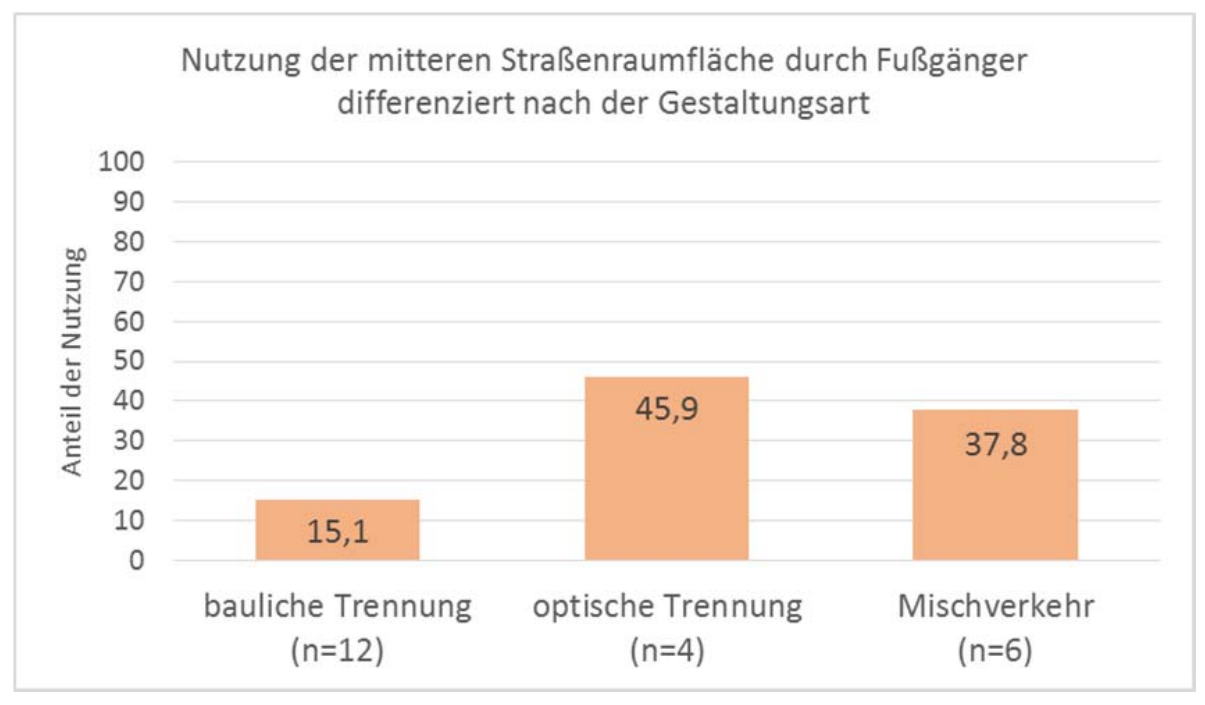

Abbildung 108: Ausnutzung der mittleren Verkehrsfläche durch Fußgänger in \%

Mehrheitlich gestaltete Flächen:

Während in 22 untersuchten Bereichen die Flächen durchgängig nach einem der oben genannten Prinzipien gestaltet wurden, lagen in 28 der untersuchten Bereiche Kombinationen der Gestaltungselemente vor. Dabei tauchte 24-mal die Kombination einer baulichen Trennung und einer optischen Trennung der Verkehrsflächen auf. Der Anteil der Nutzung der mittleren Straßenraumflächen durch den Pkw lag bei diesen 24 Untersuchungsobjekten bei etwas über 96\%. Bei den Radfahrern lag dieser Anteil bei rd. 83\% und bei den Fußgängern bei knapp 26\%.

\section{Wohnstraßen:}

33\% der untersuchten Wohnstraßen wiesen eine Mischverkehrsfläche auf und die restlichen 67\% eine optische Trennung, eine bauliche Trennung oder die Kombination aus beiden auf. Die Fußgänger benutzten zu rd. 36\% in allen beobachteten Wohnstraßen die mittlere Fläche. Die Radfahrer benutzten zu 64\% die mittlere Fläche und die Kfz zu 92\% (vgl. Abbildung 109). Der hohe Anteil der Nutzung der mittleren Flächen durch die Fußgänger liegt wie zuvor gezeigt, am hohen Anteil an Mischverkehrsflächen oder Straßenräumen mit optischer Trennung in Wohnstraßen.

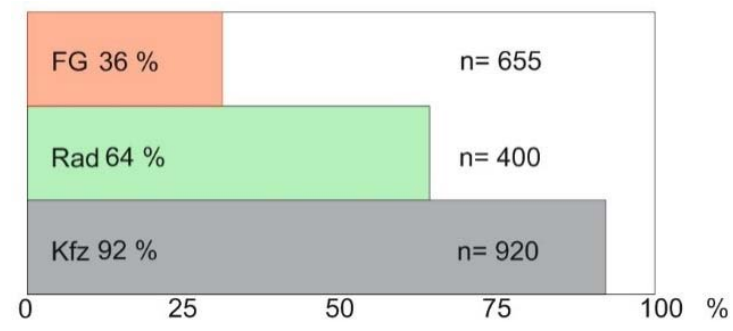

Abbildung 109: Ausnutzung der mittleren Verkehrsfläche in Wohnstraßen 


\section{Quartiersstraßen:}

Rund 90\% der beobachteten Quartiersstraßen wiesen eine optische Trennung, bauliche Trennung oder eine Kombination der Trennungsarten auf. Auch hier zeigte sich, dass die Verkehrsteilnehmer das Angebot der zugeordneten Flächen (Separation) annahmen. Es wurde versucht die Querschnitte so zu wählen, dass diese repräsentativ für den ganzen VBB stehen. Es zeigte sich, dass nur 25\% der Fußgänger die Fahrgasse an den erhobenen Querschnitten benutzen. Für die Radfahrer schien es eindeutig zu sein, dass sie die mittlere Fläche eher nutzen sollten (79\%) und die Pkw nutzten fast ausschließlich ebenfalls nur die vorgegebene Fahrgasse (97\%) (vgl. Abbildung 110).

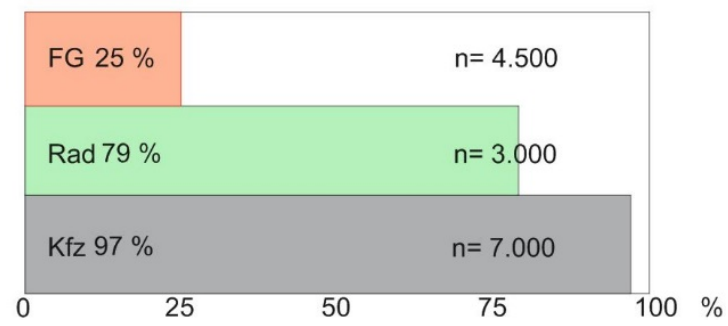

Abbildung 110: Ausnutzung der mittleren Verkehrsfläche in Quartiersstraßen

\section{Geschäftsstraßen:}

In den Geschäftsstraßen kam zu 95\% eine weiche bis harte Separation (optische oder bauliche Trennung) der Verkehrsteilnehmer vor. Von rund $19.100 \mathrm{Pkw}$ nutzten $100 \%$ die vorgegebene Fahrgasse. Weitere 74\% der Radfahrer nutzten ebenfalls die Fahrbahn. Auch wenn Fußgänger 1,75-mal so häufig in Geschäftsstraßen vorkamen wie Pkw, bewegten sich nur $20 \%$ auf der mittleren Fläche des VBBs (Abbildung 111).

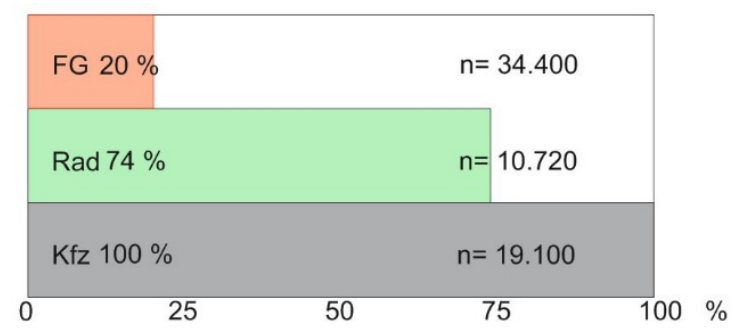

Abbildung 111: Ausnutzung der mittleren Flächen in Geschäftsstraßen

\section{Weitere Auswertungen zur Flächennutzung Kollektiv}

Zusätzlich wurde untersucht, wie sich die Nutzung der Verkehrsteilnehmer auf den Flächen der VBB zwischen den unfallauffälligeren und unfallunauffälligeren Bereichen verhält. Im unfallauffälligen Kollektiv nutzten weniger Fußgänger die mittlere Fläche als im unfallunauffälligen Kollektiv. Zu erwähnen ist, dass viele der unfallauffälligen VBB eine bauliche Trennung aufwiesen, die von den Fußgängern angenommen wurde.

Eine weitere Auswertung der 50 Untersuchungsobjekte, die sich mit der Verteilung der Unfalldichte und der aufkommenden Verkehrsmenge je Bereich beschäftigte zeigte aber, dass kein Zusammenhang zwischen der Unfallauffälligkeit und der Verkehrsmenge bestand (Abbildung 112). 


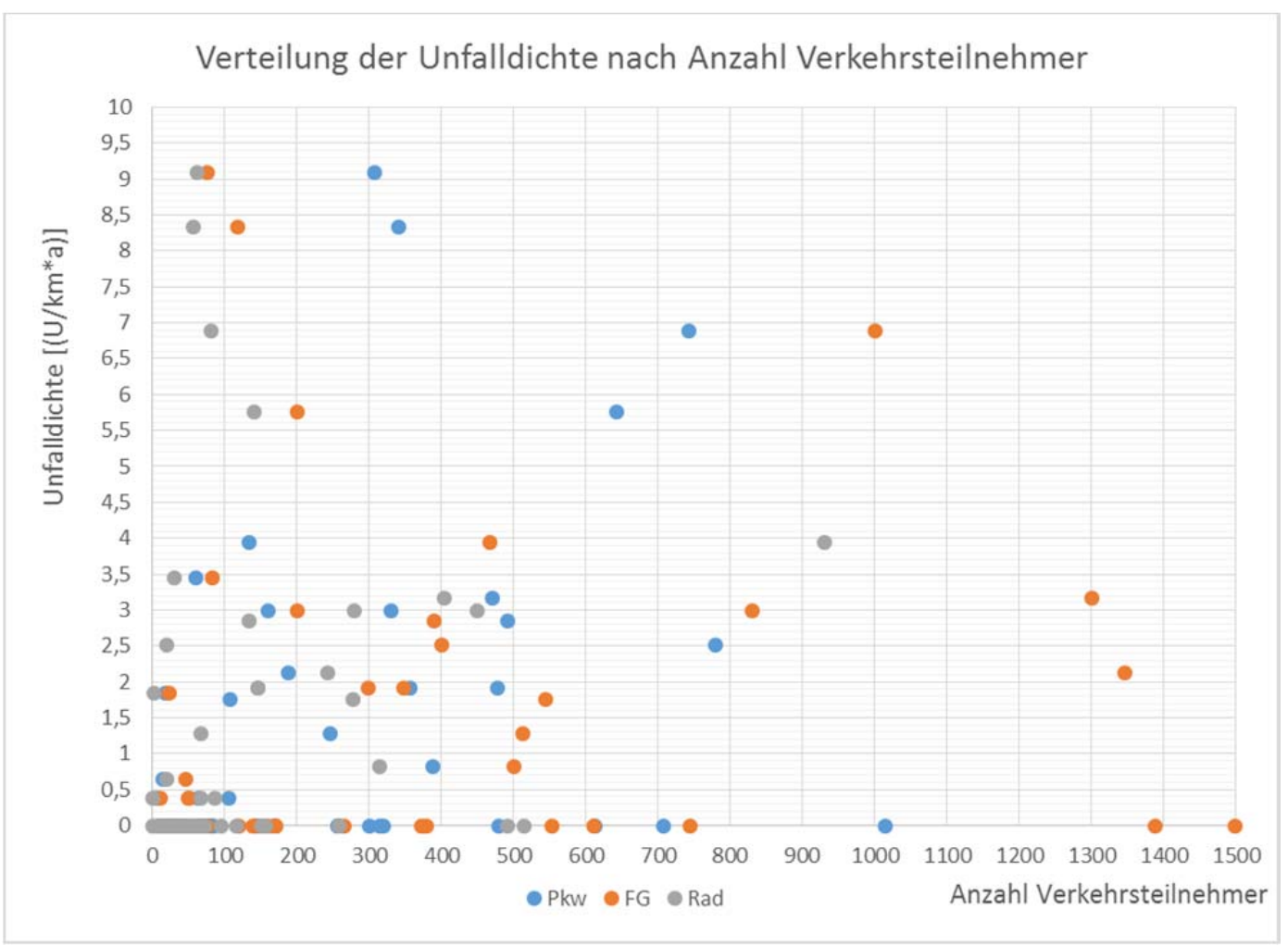

Abbildung 112: Verteilung der Unfalldichte in Abhängigkeit von der Anzahl der Verkehrsteilnehmer

\section{Fazit:}

Zusammenfassen lässt sich festhalten, dass sich Fußgänger immer dann, wenn sie die Möglichkeit hatten, in Richtung der Randbereiche orientierten. Dieses Verhalten wurde auch dann beobachtet, wenn Fußgänger aufgrund von Hindernissen „gezwungen“ wurden, die mittlere Fläche zu nutzen. Im Anschluss daran orientierten sie sich zurück in die Randbereiche. Die Mitte wurde hingegen dann benutzt, wenn sich dadurch kürzere Wege ergaben oder die Seitenränder so schmal ausgebaut waren, dass es keine geeignete Fläche zum Laufen gab (Abbildung 113). Der Pkw-Verkehr nutze fast ausschließlich die mittleren Straßenraumflächen, wenn diese ihm zur Verfügung standen. Der Radverkehr nutze je nach Gestaltungsform die mittleren Straßenraumflächen zu 60-90\%.
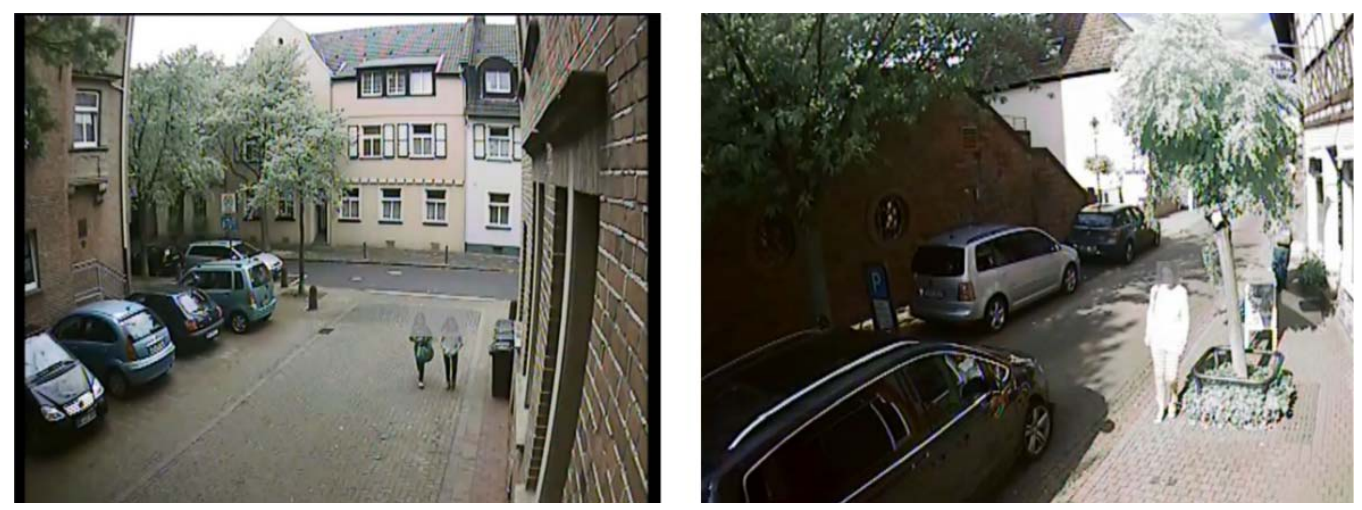

Abbildung 113: Nutzung der mittleren Verkehrsflächen 


\subsubsection{Geschwindigkeiten}

Die Durchfahrtgeschwindigkeiten wurden in den untersuchten Verkehrsberuhigten Bereichen abschnittsweise gemessen. Nur für die Kollektive Radfahrer und Pkw konnten dabei aussagekräftige Ergebnisse gewonnen werden. Wie im Kapitel $\mathbf{1 2 . 3}$ beschrieben wurde, wurden je aufgenommener Bereich die Kontrollgrößen V15, V50, V85 und Vmax ausgewertet. Auffällig war, dass die Durchfahrtgeschwindigkeiten unabhängig von der Art des VBBs waren und ungefähr gleich ",hoch“ lagen (vgl. Tabelle 35).

Tabelle 35: Durchfahrtgeschwindigkeiten von Verkehrsberuhigten Bereichen

\begin{tabular}{|c|c|c|c|c|c|c|}
\hline Straßenfunktion & $\begin{array}{l}\text { Verkehrs- } \\
\text { teilnehmer }\end{array}$ & $\mathrm{V} 15[\mathrm{~km} / \mathrm{h}]$ & V50 $[\mathrm{km} / \mathrm{h}]$ & V85 [km/h] & $\begin{array}{l}\text { Vmax } \\
{[\mathrm{km} / \mathrm{h}]}\end{array}$ & $\mathrm{n}$ \\
\hline \multirow{2}{*}{ Wohnstraße } & Pkw & 13 & 18 & 23 & 32 & 339 \\
\hline & Rad & 11 & 13 & 18 & 26 & 46 \\
\hline \multirow{2}{*}{ Quartiersstraße } & Pkw & 13 & 19 & 25 & 35 & 2.088 \\
\hline & Rad & 11 & 14 & 18 & 26 & 269 \\
\hline \multirow{2}{*}{ Geschäftsstraße } & Pkw & 12 & 16 & 22 & 35 & 5.123 \\
\hline & Rad & 9 & 13 & 18 & 34 & 2.219 \\
\hline \multicolumn{7}{|c|}{$\mathrm{n}=$ Anzahl der Durchfahrten } \\
\hline
\end{tabular}

Auch bei dem Vergleich der beiden Kollektive „unfallauffällige VBB“ mit den „unfallunauffälligen VBB", fielen keine Besonderheiten auf.

Die Gesamtbetrachtung über die gefahrenen Geschwindigkeiten zeigte, dass das gesamte Niveau, unabhängig der Straßenfunktion, relativ niedrig war. Bei genauerer Betrachtung der Ergebnisse je VBB fiel auf, dass Geschwindigkeiten dann niedriger waren, wenn allgemein „Hindernisse“ im Bereich vorhanden warten. Die Hindernisse ließen sich in unterschiedliche Kategorien einteilen, ob sie z.B. dauerhaft oder temporär waren und ob sie geeignete Mittel zur Geschwindigkeitsreduzierung darstellten oder nicht. Dauerhafte Gestaltungsmaßnahmen bestanden bspw. aus Pollern, Versätzen oder Pflanzkästen.

Zusätzlich wirkten auch Einflüsse auf den Verkehrsablauf als geschwindigkeitsdämpfend, die nur unregelmäßig und kurzfristig also temporär auftreten. Dies waren bspw. häufig genutzte Querungsstellen für den Fußgängerverkehr, wo höhere Fußgängerverkehrsmengen den KfzVerkehr und auch den Radverkehr in seiner Geschwindigkeit beeinflussten. Ebenso wirkten Bushaltestellen oder häufig frequentierte Stellplätze geschwindigkeitsmindernd.

Dauerhafte Hindernisse erfüllten nur ihren Zweck, wenn sie in regelmäßigen Abständen angebracht waren, denn dann wirkten sie linienhaft ${ }^{14}$. Im Rahmen der Bearbeitung war es zwar nicht möglich lokale Geschwindigkeiten zu messen, allerdings ließ sich beobachten, ob zwischen zwei Gestaltungsmaßnahmen eine Beschleunigung vorgenommen wurde oder nicht. Ein einmaliges Hindernis hatte zwar Einfluss auf die gesamte Durchfahrtzeit, allerdings war sie nicht konstant über den Bereich und wirkte somit nur lokal. Die folgende Abbildung 114 zeigt zwei Beispiele für dauerhafte Hindernisse, allerdings wirkten diese nur punktuell geschwindigkeitsreduzierend.

14 Unter linienhaften Hindernissen ist bspw. die wechselseitige Anordnung von Stellplätzen oder eine regelmäßig in kurzen Abständen wiederkehrende Einengung des Straßenraumquerschnittes bspw. durch Bäume zu verstehen, so dass eine durchgehende gradlinige Befahrbarkeit des Verkehrsberuhigten Bereiches nicht möglich ist. 

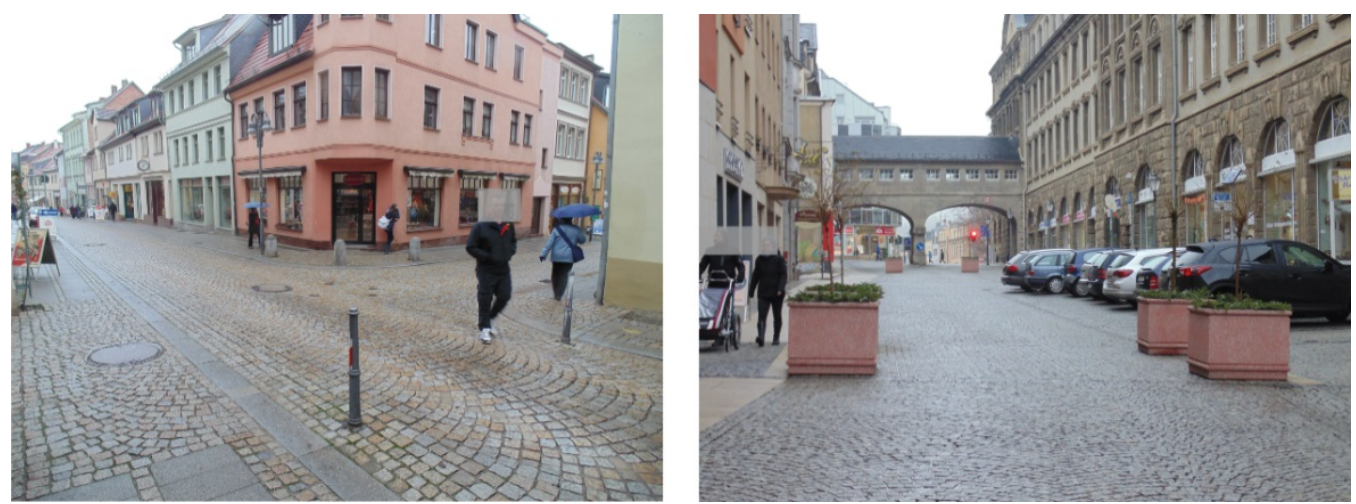

Abbildung 114: punktuelle dauerhafte Hindernisse

\section{Zusatz: Geschwindigkeitsmessung über mehrere Abschnitte}

Zusätzlich zu der Geschwindigkeitsmessung über eine Strecke eines Verkehrsberuhigten Bereichs, erfolgten auch Geschwindigkeitsmessungen über mehrere Abschnitte des VBBs. Damit konnten zum einen die Wirkung der Gestaltung und zum anderen die Einfahrtsgeschwindigkeiten gegenüber der Ausfahrtsgeschwindigkeit überprüft werden. Untersucht wurde, ob die Verkehrsteilnehmer z.B. ihr Geschwindigkeitsverhalten während der Durchfahrt veränderten oder nicht. Die Auswertung zeigt dabei nur die Durchfahrtgeschwindigkeit im ersten Bereich/Übergangsbereich und/oder im mittleren Bereich auf aber keine lokale Eintrittsgeschwindigkeit.

Für elf VBB konnte das Geschwindigkeitsniveau je Abschnitt während der Durchfahrt untersucht werden. Da die Kameras nur an geeigneten Masten angebracht werden konnten, variierten die einzelnen Abschnittslängen bei der Aufnahme der Geschwindigkeiten. In insgesamt zwei Wohnstraßen, fünf Geschäftsstraßen und vier Quartiersstraßen war es möglich die Untersuchung durchzuführen.

Bei sieben VBB wurden die Pkw während ihrer Durchfahrt „verfolgt“. Hierbei sind nur solche Pkw erfasst worden, die den ganzen Bereich durchfuhren. Pkw, die zwischendurch ab- oder einbogen, wurden nicht berücksichtigt. In den übrigen vier VBB war es zum Teil nicht möglich die Pkw zu verfolgen, so dass alle Pkw je Abschnitt erhoben worden sind, auch wenn sie den Bereich nicht gänzlich durchfuhren. Aus der Auswertung (vgl. Anlagenband) ist ablesbar, dass die Bereiche zumeist nicht mit der gleichen Geschwindigkeit durchfahren wurden und die Geschwindigkeiten je Abschnitt variierten. Wird nur nach den Kriterien Straßenfunktion, Anzahl Verkehrsteilnehmer und Länge des Bereichs ausgewertet, ergeben sich zunächst keine eindeutigen Aussagen darüber, ob Pkw in den Übergangsbereichen schneller fahren als innerhalb des Bereichs oder umgekehrt.

Für die Beurteilung der Ergebnisse, wurden daher die Bereiche noch einmal nach ihrer Abschnittsanzahl unterteilt. Daraus resultierte, dass es sieben VBB gab, die in zwei Abschnitte unterteilt wurden, zwei in drei Abschnitte und zwei in vier Abschnitte. Hier konnte festgestellt werden, dass die gefahrenen Geschwindigkeiten eng mit der Gestaltung in Verbindung standen. Denn bei den sieben VBB, die "nur" in zwei Abschnitte eingeteilt wurden, bestand Zweirichtungsverkehr, sodass es sich bei beiden Untersuchungsabschnitten um Übergangsbereiche handelte. Zu untersuchen war nun, warum im einen Teil schneller gefahren wurde als im anderen.

Hierbei wurden zunächst die Abschnittslängen betrachtet. Diese spielten keine Rolle bei den gefahrenen Geschwindigkeiten. In einem weiteren Schritt wurden die Gestaltungsmaßnahmen genauer betrachtet. Es fiel auf, dass in Bereichen langsamer gefahren wurde, in denen deutlich mehr Hindernisse vorzufinden waren. Die Hindernisse gestalteten sich, wie bereits angesprochen, ganz unterschiedlich. Von dauerhaft existierenden Gestaltungsmaßnahmen, wie Aufpflasterungen, Versätzen, Pollern oder Blumenkübel bis hin zu zeitlich unbeständigen und veränderlichen (zufälligen) Hindernissen wie ein erhöhtes Verkehrsaufkommen (Fußgänger, Kfz, Busse, etc.). 
Beobachtet wurde auch, dass die Pkw langsamer fuhren, wenn die Straßenraumbreite schmaler und/oder kurviger wurde.

Bei den VBB, die in mehrere Abschnitte eingeteilt wurden, ließen sich keine allgemeinen Aussagen über das Geschwindigkeitsniveau treffen. Es handelte sich dabei um drei Einbahnstraßen (Geschäftsstraßen) und einen Bereich mit Zweirichtungsverkehr (Wohnstraße), die bis auf die Wohnstraße alle im Innenstadt-/Altstadtbereich lagen. Bei allen vier VBB ließ sich zwar feststellen, dass in den mittleren Bereichen langsamer gefahren wurde, allerdings waren die Bedingungen so unterschiedlich, dass sich kein eindeutiges Ergebnis ergab. Schmale Straßenraumbreiten, hohes Fußgängeraufkommen, Kurven und Straßenneigungen wichen in den vier Bereichen zu stark voneinander $\mathrm{ab}$.

Festzustellen blieb jedoch, dass, auch wenn die Bereiche unterschiedlich gestaltet waren, langsamer gefahren wurde, so lange Hindernisse vorlagen. Die Geschwindigkeiten waren somit höher, wenn keine Gestaltungsmaßnahmen vorlagen. Anhand der Videobeobachtungen konnte an

dauerhaften Gestaltungsmaßnahmen/“Hindernissen" Aufpflasterungen, Poller, Pflanzkästen (unbepflanzt und bepflanzt), Versätze (kleine und große) sowie Parkplatzmarkierungen festgestellt werden, dass diese sich als geschwindigkeitsreduzierend erwiesen. Die „Hindernisse“ müssen den Belangen der anderen Verkehrsteilnehmer genügen, wobei schlechte Sichtbeziehungen und eine Gefährdung aller Verkehrsteilnehmer in jedem Falle ausgeschlossen werden müssen.

\subsubsection{Interaktionen}

In 200 Beobachtungsstunden wurden in den 9 Wohnstraßen insgesamt rd. 2.000 Verkehrsteilnehmer, in den 20 Quartiersstraßen rd. 14.500 Verkehrsteilnehmer und in den 20 Geschäftsstraßen insgesamt rd. 64.000 Verkehrsteilnehmer beobachtet, die den Bereich betraten oder verließen. Dies bedeutete bei den Wohnstraßen ein durchschnittliches Verkehrsaufkommen von etwa 55 Verkehrsteilnehmern pro Stunde, in den Quartiersstraßen von etwa 180 Verkehrsteilnehmern pro Stunde und bei den Geschäftsstraßen von etwa 800 Verkehrsteilnehmern pro Stunde.

Während der gesamten Beobachtungsdauer von 200 Stunden geschahen zwei Unfälle. Bezogen auf die geringe Beobachtungsdauer von 4 Stunden je Untersuchungsobjekt entspricht das hochgerechnet auf das Jahr knapp 90 Unfälle pro Jahr und Untersuchungsbereich, wenn davon ausgegangen wird, dass das Unfallgeschehen über den Tages- und Jahreszeitraum gleichverteilt wäre. Nimmt man hingegen an, dass einerseits das Unfallgeschehen von der Anzahl der Verkehrsteilnehmer abhängig ist und andererseits, dass das größte Verkehrsaufkommens des Tages in den beobachteten vier Tagesstunden stattfindet, dann entsprechen die zwei beobachteten Unfälle einem hochgerechneten Wert von immer noch etwa 40 Unfällen pro Jahr und Untersuchungsraum. Diese Zahl liegt deutlich über den Werten, die in der Unfallanalyse der Verkehrsberuhigten Bereiche festgestellt werden konnten. Daher wurden im Folgenden die beiden Unfälle näher betrachtet.

Bei dem ersten Unfall handelte es sich um einen Alleinunfall eines Kindes mit seinem Fahrrad. Dieses hat bei der Einfahrt in einen Verkehrsberuhigten Bereich (Wohnstraße) mit seinem Fahrrad das Verkehrszeichen touchiert, das den Beginn eines Verkehrsberuhigten Bereiches markiert, dabei kam das Kind samt Fahrrad zu Fall. Nachdem das Kind festgestellt hat, dass das Fahrrad keine Schaden durch den Sturz erlitten hat und es selbst auch nicht verletzt war, setzte es seine Fahrt mit dem Fahrrad fort. Das Verkehrsschild war augenscheinlich nicht beschädigt. Die Polizei wurde nicht über den Unfall in Kenntnis gesetzt. Der Unfall kann somit der Unfallkategorie 5 zugeordnet werden.

Der zweite Unfall ereignete sich im Zusammenhang mit dem ruhenden Verkehr (Quartiersstraße). Hierbei setzte ein Fahrzeug rückwärts aus einer Stellplatzfläche (senkrecht zur Fahrbahn) zurück. Dabei übersah der Fahrzeugführer ein weiteres Fahrzeug, was auf der Fahrbahn fuhr. Bei der Rückwärtsfahrt fuhr der Pkw in die Seite des Pkws auf der Fahrbahn. Es entstand augenscheinlich 
ein Sachschaden an beiden Fahrzeugen. Beide Fahrzeuge waren allerdings offensichtlich noch fahrbereit. Nach einem Gespräch zwischen beiden Fahrzeugführern haben beide ihre Fahrt mit ihren Fahrzeugen fortgesetzt. Die Polizei wurde vor Ort nicht über den Unfall in Kenntnis gesetzt. Der Unfall kann der Unfallkategorie 5 zugeordnet werden.

Beide Unfälle wurden anscheinend der Polizei nicht mitgeteilt, so dass beide Unfälle nicht in der amtlichen Statistik auftauchen werden. Diese Beobachtungen lassen ebenso wie die Aussagen verschiedener zuständiger Personen bei den Polizeidienststellen darauf schließen, dass es eine hohe Anzahl an nicht gemeldeter Unfälle in Verkehrsberuhigten Bereichen gibt. Dementsprechend ließe sich auch in Übereinstimmung mit der Anzahl der tatsächlich gemeldeten Unfälle die zuvor hochgerechnete Anzahl von rd. 40 Unfällen pro Jahr und Bereich erklären.

Neben den zwei zuvor beschriebenen Unfällen konnte in den 200 Beobachtungsstunden keine kritische Situation beobachtet werden. In Wohnstraßen war dies aufgrund der geringen Verkehrsbelastungen zu erwarten, da bei einer Verkehrsbelastung von weniger als einem Verkehrsteilnehmer in der Minute nahezu keine Interaktionen vorhanden sind. In den Quartierstraßen und insbesondere in den Geschäftsstraßen ließen die hohen Verkehrsteilnehmerzahlen jedoch vermehrt Interaktionen zwischen Verkehrsteilnehmern erwarten. Allerdings lagen in diesen Bereichen auch häufig bauliche oder zumindest optische Trennungen der Verkehrsarten vor, so dass eine Interaktion zwischen den Verkehrsteilnehmern nicht zustande kam.

Kam es insbesondere in den Geschäftsstraßen zu Interaktionen zwischen einem Fußgänger und einem Pkw, so konnten durchweg konfliktfreie Interaktionen beobachtet werden.

In den Bereichen, in denen ein erhöhter Überquerungsbedarf der Fußgänger bestand, konnte unabhängig von der Art der Gestaltung ebenfalls keine konfliktbehaftete Interaktion beobachtet werden.

\subsection{5 Übergangsbereiche}

Als Übergangsbereiche sind die Abschnitte des Straßenraumes im Rahmen dieser Studie definiert, die unmittelbar an die mit dem VZ325 ausgewiesenen Verkehrsberuhigten Bereiche angrenzen. Diesen Bereichen wurde im Rahmen der vorliegenden Studie besondere Bedeutung beigemessen, da rd. $40 \%$ der den verkehrsberuhigten Bereichen zugewiesenen Unfälle in diesen Übergangsbereichen stattfinden (vgl. Kapitel 11.2.7). Auch wenn in diesen Bereichen nicht mehr die Regeln des ausgewiesenen Verkehrsberuhigten Bereiches gemäß StVO gelten, so sind bei der Ausfahrt aus einem VBB jedoch die Regelungen des $\$ 10$ StVO zu berücksichtigen. Somit stellen diese Übergangsbereiche eine Besonderheit dar, die in unmittelbarem Zusammenhang mit dem VZ325 steht.

Befindet sich das VZ325 auf einem Straßenabschnitt, so stellt der Straßenabschnitt bis rd. 30m hinter dem VZ325 den Übergangsbereich dar. Mündet der Straßenabschnitt, der aufgrund des VZ325 einen Verkehrsberuhigten Bereich darstellt, in einen Knotenpunkt mit anderen Straßen, so stellt der Knotenpunkt den Übergangsbereich dar.

Nahezu alle im Rahmen dieser Studie untersuchten Verkehrsberuhigten Bereiche schließen unmittelbar an Knotenpunkte mit anderen Straßen an. In wenigen Fälle gehen diese in Fußwege über (vgl. Abbildung 115). 


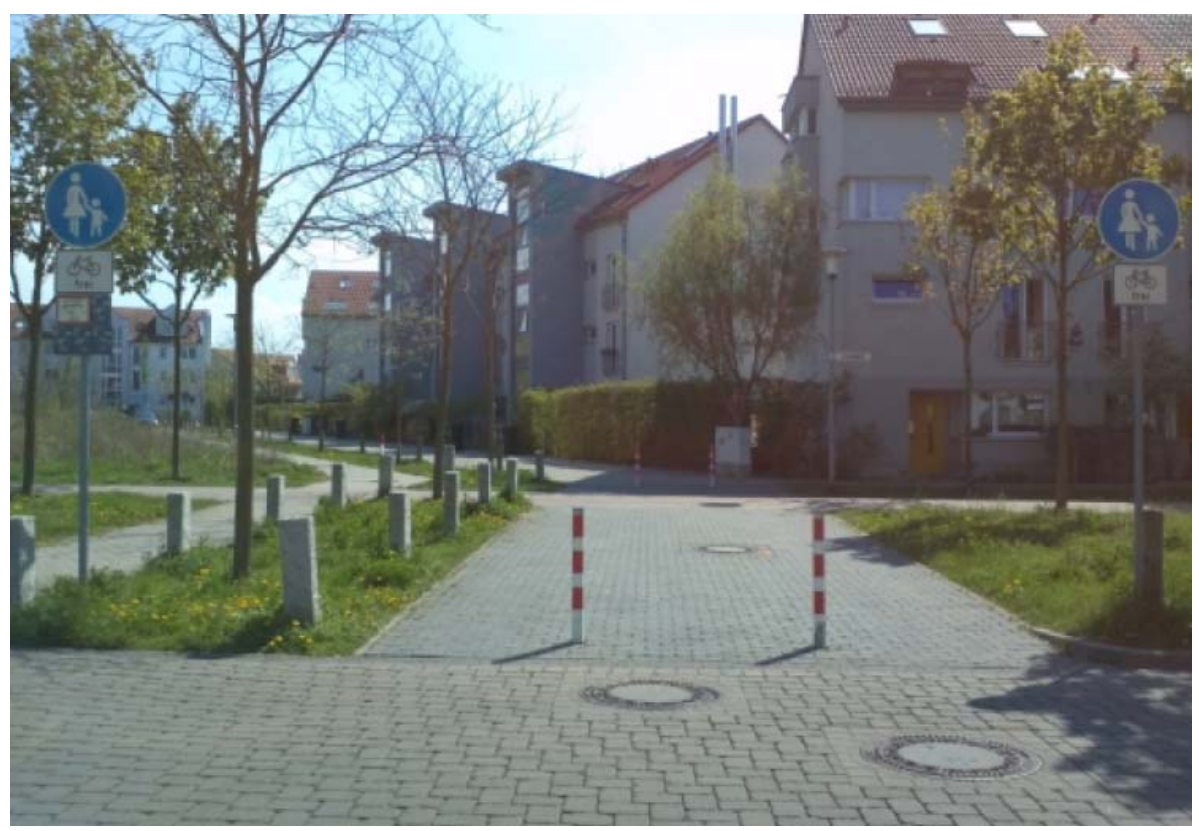

Abbildung 115: Übergang eines Verkehrsberuhigten Bereiches in einen Fußweg

Aufgrund der ursprünglichen Zielsetzung der Studie waren die Übergangsbereiche kein Schwerpunkt der vorliegenden Untersuchung. Ausgangspunkt der Studie waren ausschließlich die Bereiche zwischen den VZ325. Entsprechend wurde auch das Erhebungskonzept zur Beobachtung des Verkehrsablaufes entwickelt. Daher konnte der Verkehrsablauf in den Übergangsbereichen entweder gar nicht oder nur eingeschränkt beobachtet werden (vgl. Abbildung 116).

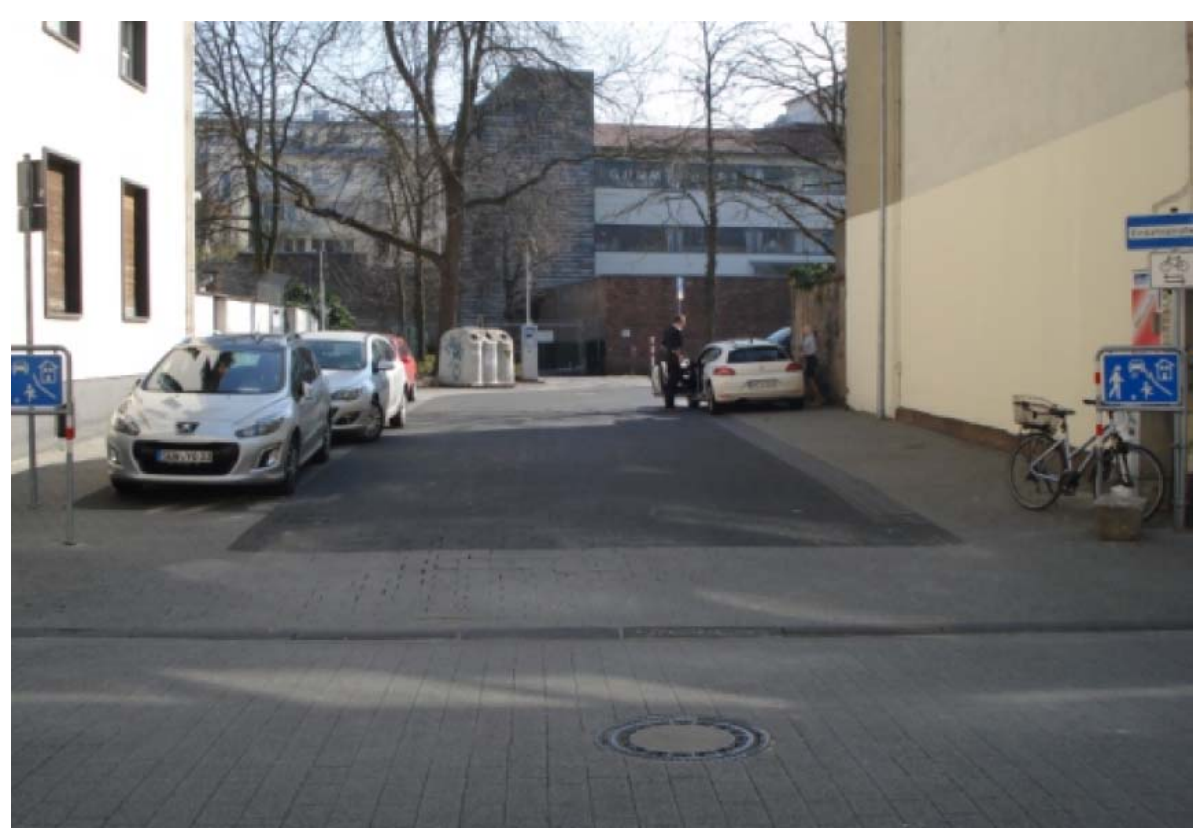

Abbildung 116: Übergang eines Verkehrsberuhigten Bereiches mit abgesenktem Bordstein

Auch wenn keine belastbaren Beobachtungen der Verkehrsabläufe in den Übergangsbereichen durchgeführt werden konnten, konnte zumindest prinzipiell festgestellt werden, dass die 
vorhandene Vorfahrtsregelung in der Ausfahrt aus den Verkehrsberuhigten Bereichen den Verkehrsteilnehmern häufig nicht klar war. Häufig wurde, wenn kein abgesenkter Bordstein den Übergangsbereich markierte, nach der Regelung „Rechts-vor-Links“ gehandelt. Es wird daher empfohlen, die Übergangsbereiche von Verkehrsberuhigten Bereichen durch eine weitere Untersuchung des Verkehrsablaufes gezielt zu betrachten, da hier einerseits rund $40 \%$ aller Unfälle im Zusammenhang mit den Verkehrsberuhigten Bereichen stattfanden und andererseits eine Unsicherheit bei den Verkehrsteilnehmern bezogen auf die geltenden Vorfahrtsregelungen in diesen Übergangsbereichen augenscheinlich vorhanden sind.

Derzeit ist die Vorfahrt an Übergangsbereichen von ausgewiesenen VBB generell durch den §10 der StVO geregelt. Hier wird beschrieben, dass die besonderen Sorgfaltspflichten bei der Ausfahrt und nicht die Vorfahrtregelungen (nach §8) gelten. Das bedeutet, dass der Ausfahrende anderen Verkehrsteilnehmern gegenüber Vorrang zu gewähren hat. Die Regelung gilt auch dann noch, wenn das Verkehrszeichen 325.2 bis zu $30 \mathrm{~m}$ in den Straßenraum eingerückt ist. Endet der ausgewiesene Bereich mit Zeichen $325.2 \mathrm{mehr}$ als $30 \mathrm{~m}$ vorher, gelten an der folgenden Einmündung die Vorfahrtregeln des §8. Es sollte aber davon ausgegangen werden, dass sich kein „normaler Verkehrsteilnehmer" der 30m-Regelung bewusst ist bzw. sie überhaupt kennt.

\subsection{Zusammenfassung der Ergebnisse}

Inhalte der Verkehrsbeobachtungen, die innerhalb der 50 untersuchten Verkehrsberuhigten Bereiche stattgefunden haben, sind Querschnittszählungen, Ermittlung über die Nutzung der zur Verfügung stehenden Flächen im Straßenraum, Geschwindigkeiten sowie Interaktionen der Verkehrsteilnehmer untereinander.

Es kann festgehalten werden, dass:

- das Geschwindigkeitsniveau unabhängig von Straßenfunktion des Verkehrsberuhigten Bereiches ist und die Geschwindigkeit $\bigvee_{85}$ im Durchschnitt bei $23 \mathrm{~km} / \mathrm{h}$ liegt.

- in Wohnstraßen die Verkehrsmenge mit 150 Fahrzeugen in vier Untersuchungsstunden am niedrigsten ist. Gefolgt werden diese von den Quartiersstraßen mit bis zu $800 \mathrm{Kfz}$ in vier Stunden und Geschäftsstraßen mit bis zu $1200 \mathrm{Kfz} / 4 \mathrm{~h}$, in Wohn- und Quartiersstraßen mind. genauso viele Fußgänger und Radfahrer vorkommen wie $\mathrm{Kfz}$ und in Geschäftsstraßen sogar doppelt so viele Fußgänger und Radfahrer vorkommen wie Kfz.

- sich Fußgänger in die Randbereiche der ausgewiesenen Verkehrsberuhigten Bereiche orientieren und Radfahrer sowie Kfz-Nutzer hauptsächlich bzw. ausschließlich die mittleren Flächen nutzen. Dabei ist es irrelevant, welche Verkehrsführung vorliegt.

- die Gestaltung Auswirkung auf das Geschwindigkeitsniveau hat. Sind temporäre oder dauerhafte „Hindernisse“ im Straßenraum vorhanden, sind die Geschwindigkeiten niedriger.

\section{Empfehlung von Gestaltungsmaßnahmen für ausgewiesene VBB}

Werden die Ergebnisse aus der aktuellen Studie mit Ergebnissen aus Studien der 1980er Jahre verglichen, in denen Verkehrsberuhigte Bereiche in Wohnstraßen zuletzt untersucht wurden, können damalige Aussagen zum Teil bestätigt werden, in anderen Teilen widersprechen sie sich jedoch.

Die Studie aus 1981 (BfS, 1981) ist zwar nur bedingt mit der aktuellen Studie vergleichbar, da sie sich hauptsächlich mit lokalen Geschwindigkeiten beschäftigt hat, trotzdem lassen sich die aktuellen Empfehlungen auf den Ergebnissen von 1981 aufbauen. 
Grundsätzlich lässt sich festhalten, dass Verkehrsberuhigte Bereiche in ihrer heutigen Anwendung funktionieren. Das Unfallgeschehen ist mit durchschnittlich 0,15 Unfällen je Jahr und Bereich unauffällig, obwohl das Unfallgeschehen seit einigen Jahren stagniert. Zum einen muss allerdings erwähnt werden, dass ein Fehler innerhalb der aufgenommenen Unfalldaten vorliegt und nicht jeder verzeichnete Unfall in ausgewiesenen Verkehrsberuhigten Bereichen geschieht (vgl. Kapitel 7.3). Zum anderen lässt sich vermuten, dass zudem die Einrichtung von Verkehrsberuhigten Bereichen deutschlandweit zugenommen hat und damit mehr ausgewiesene Bereiche vorliegen, die Unfallanzahl aber nicht gestiegen ist (vgl. Kapitel 7).

Der Einsatzort ausgewiesener Verkehrsberuhigter Bereiche hat sich seit 1981 von der Wohnstraße auch in Quartiers-, Erschließungs- und Geschäftsstraßen verlagert. Die Studie zeigt, dass die vorherrschenden Verkehrsmengen in ausgewiesenen Verkehrsberuhigten Bereichen je Straßenfunktion zwar variieren, jedoch keinen Einfluss auf das Unfallgeschehen haben. Auch wenn die festgestellten Verkehrsmengen in Geschäftsstraßen von bis zu $1.200 \mathrm{Kfz} / 4 \mathrm{~h}$ den Forderungen der VwV-StVO zu ausgewiesenen Verkehrsberuhigten Bereichen für "sehr geringe“ Verkehrsmengen vermutlich nicht nachkommen, kann keine Beeinträchtigung verzeichnet werden. Es wird jedoch aufgrund der kaum zu vermeidenden baulichen Gestaltung (bauliche Trennung) empfohlen, eine Alternative für Geschäftsstraßen (mit VZ325) zu finden, in der die Bedürfnisse des nichtmotorisierten Verkehrs ebenfalls berücksichtigt werden können.

1981 wurde zusätzlich eine geringe Ausdehnung der Verkehrsberuhigten Bereiche von ca. 60m empfohlen. Diese Empfehlungen konnten nicht bestätigt werden, da auch die Länge der untersuchten Verkehrsberuhigten Bereiche keinen messbaren Einfluss auf das Unfall- und Verkehrsgeschehen nimmt.

Auffälliger ist hingegen das Geschwindigkeitsniveau in Verkehrsberuhigten Bereichen. Liegt in ausgewiesenen Bereichen statt eines niveaugleichen Ausbaus eine bauliche Trennung vor, sind die Geschwindigkeiten im Vergleich zu 1981 auch heute noch am höchsten. Allerdings besteht kaum ein Unterschied zu Mischverkehrsflächen oder Bereichen mit optischer und baulicher Trennung. Wie bereits 1981 festgestellt, werden aber auch aktuell auf Mischverkehrsflächen statt der geforderten Schrittgeschwindigkeit von $7 \mathrm{~km} / \mathrm{h}$, deutlich höhere Geschwindigkeiten von ca. 22 $\mathrm{km} / \mathrm{h}\left(\mathrm{V}_{85}\right)$ gefahren.

Das Geschwindigkeitsniveau kann nur dann kontinuierlich gesenkt werden, wenn fortwährend „Hindernisse“ in den Straßenraum des Verkehrsberuhigten Bereichs eingebaut werden. Aufgrund dessen wird empfohlen eine entsprechende bauliche Gestaltung zu fordern. Dabei müssen die "Hindernisse" den Belangen der anderen Verkehrsteilnehmer insbesondere hinsichtlich der Barrierefreiheit genügen, wobei schlechte Sichtbeziehungen und eine Gefährdung aller Verkehrsteilnehmer ausgeschlossen werden müssen.

Um zudem eine qualitativ hochwertige Aufenthaltsfunktion in Verkehrsberuhigten Bereichen zu schaffen, darf der motorisierte Verkehr keine besondere Rolle spielen. Die Verkehrsstärke des motorisierten Verkehrs sollte im Verkehrsberuhigten Bereich nicht höher liegen als die des nichtmotorisierten Verkehrs. Dies muss im Vorfeld der Einrichtung eines Verkehrsberuhigten Bereiches oder durch Kontrollen nach der Ausweisung geprüft werden.

Die nachfolgenden Hinweise zur Gestaltung der Mischverkehrsflächen, die schon in den Empfehlungen aus dem Jahr 1980 stehen (BfS, 1980), sollten weiterhin angewendet werden:

- „Die Befestigungen der Mischflächen sind deutlich von dem anderer Straßen zu unterscheiden;

- die von Kraftfahrzeugen befahrbaren Bereiche sollen nicht länger als auf einer Strecke von maximal 30m bis $40 \mathrm{~m}$ geradeaus verlaufen; ggf. sind auch Schwellen einzurichten;

- den Fußgängern vorbehaltene Bereiche können bepflanzt oder möbliert werden; dabei empfiehlt es sich aber, mit der Möblierung sparsam zu sein und noch ausreichende Freiflächen, die Kinderspiele ermöglichen, offen zu lassen; 
- bei der Möblierung und Bepflanzung ist dafür Sorge zu tragen, dass keine gefährlichen Sichthindernisse geschaffen werden;

- der Verlauf des befahrbaren Bereiches in einer Mischfläche muss dem Fahrer z.B. durch farbliche Gestaltung der Oberfläche deutlich gemacht werden; es darf nur eine einzige Fahrgasse vorhanden sein;

- Anfang und Ende von Mischflächen sind durch entsprechende Ausführung als solche kenntlich zu machen."

Von folgender Empfehlung sollte jedoch abgesehen werden:

- „Diejenigen Flächen, die nicht als befahrbarer Bereich oder als Stellplätze dienen, sollen farblich hervorgehoben und durch Poller oder Bordsteine abgegrenzt werden."

Da rund 40\% der Unfälle an den Übergangsbereichen geschehen, wird ergänzend empfohlen, eindeutige Regelungen (auch in der StVO) für die Vorfahrtsituation aufzustellen. Damit die Bedeutung des Verkehrszeichens 325.2 (Ende des VBB) nicht verloren geht und die Vorfahrtsregelung gegenüber den geltenden Regel nach §10 StVO vereinfacht wird, werden zwei Gestaltungsvarianten empfohlen:

\section{Gestaltungsvariante 1:}

Endet ein ausgewiesener Verkehrsberuhigter Bereich unmittelbar an einer Einmündung in eine übergeordnete Straße (Knotenpunkt), so muss ein „abgesenkter Bordstein“ baulich vorhanden sein und den Übergang eindeutig hervorheben. Es ist bei der Gestaltung darauf zu achten, dass ein einwandfrei erkennbarer „abgesenkter Bordstein“ vorhanden ist, sowie eine ungehinderte Sicht auf die Einmündung und die Beschilderung zum Verkehrsberuhigten Bereich besteht. Von einer Einrückung des Verkehrszeichens 325 um wenige Meter in den Straßenraum ist unbedingt abzusehen. Unter Umständen ist eine nach $\S 10$ zulässige Zusatzbeschilderung aufgrund der örtlichen Situation erforderlich.

Bei einem „abgesenktem Bordstein“ gilt die besondere Sorgfaltspflicht bei der Ausfahrt nach $\S 10$ ebenso wie bei einer Ausfahrt aus einem VBB (VZ 325), kann jedoch im Gegensatz zur Regelung für das VZ 325 als „allgemein bekannte“ Regelung angesehen werden.

\section{Gestaltungsvariante 2:}

Endet ein ausgewiesener Verkehrsberuhigter Bereich nicht unmittelbar an der Einmündung in eine übergeordnete Straße (Knotenpunkt), so muss das Verkehrszeichen $325 \mathrm{immer}$ mindestens $30 \mathrm{~m}$ vom Rand der übergeordneten Straße in den Straßenraum eingerückt werden. Des Weiteren soll die bauliche Gestaltung an der Einmündung eine eindeutige Vorfahrtsregelung ausdrücken. Es darf demnach kein „abgesenkter Bordstein“ oder eine Mischverkehrsfläche direkt an der Kreuzung angebracht sein. 


\section{ANHANG 1 Unfallursachenverzeichnis}

(Destatis 2011)

01

02

03

04
Verkehrstüchtigkeit

Alkoholeinfluss

Einfluss anderer berauschender Mittel (z.B. Drogen, Rauschgift)

Übermüdung

Sonstige körperliche oder geistige Mängel

\section{Fehler der Fahrzeugführer:}

\section{Straßenbenutzung}

Benutzung der falschen Fahrbahn (auch Richtungsfahrbahn) oder verbotswidrige Benutzung anderer Straßenteile Verstoß gegen das Rechtsfahrgebot

\section{Geschwindigkeit}

Nicht angepasste Geschwindigkeit mit gleichzeitigem Überschreiten der zulässigen Höchstgeschwindigkeit

in anderen Fällen

\section{Abstand}

Ungenügender Sicherheitsabstand (Sonstige Ursachen, die zu einem Verkehrsunfall führen, sind den zutreffenden Positionen, wie Geschwindigkeit, Übermüdung usw. zuzuordnen)

Starkes Bremsen des Vorausfahrenden ohne zwingenden Grund

\section{Überholen}

Unzulässiges Rechtsüberholen

Überholen trotz Gegenverkehrs

Überholen trotz unklarer Verkehrslage

Überholen trotz unzureichender Sichtverhältnisse

Überholen ohne Beachtung des nachfolgenden Verkehrs und/oder ohne rechtzeitige und deutliche Ankündigung des Ausscherens

Fehler beim Wiedereinordnen nach rechts

Sonstige Fehler beim Überholen (z.B. ohne genügenden Seitenabstand; an Fußgängerüberwegen s. Pos. 38, 39)

Fehler beim Überholtwerden

\section{Vorbeifahren}

Nichtbeachten des Vorranges entgegenkommender Fahrzeuge beim Vorbeifahren an haltenden Fahrzeugen, Absperrungen oder Hindernissen (§6) (ausgenommen Pos. 32). Nichtbeachten des nachfolgenden Verkehrs beim Vorbeifahren an haltenden Fahrzeugen, Absperrungen oder Hindernissen und/oder ohne rechtzeitige und deutliche Ankündigung des Ausscherens

\section{Nebeneinanderfahren}

Fehlerhaftes Wechseln des Fahrstreifens beim Nebeneinanderfahren oder Nichtbeachten des Reißverschlussverfahrens (§ 7) (ausgenommen Pos. 20, 25). 


\section{Vorfahrt, Vorrang} Nichtbeachten der Regel "rechts vor links" Nichtbeachten der die Vorfahrt regelnden Verkehrszeichen (§ 8) (ausgenommen Pos. 29)

Nichtbeachten der Vorfahrt des durchgehenden Verkehrs auf Autobahnen oder Kraftfahrstraßen (§ 18, Abs. 3)

Nichtbeachten der Vorfahrt durch Fahrzeuge, die aus Feld- und Waldwegen kommen

Nichtbeachten der Verkehrsregelung durch Polizeibeamte oder Lichtzeichen (ausgenommen Pos. 39)

Nichtbeachten des Vorranges entgegenkommender Fahrzeuge

(Zeichen 208 StVO)

Nichtbeachten des Vorranges von Schienenfahrzeugen an Bahnübergängen

\section{Abbiegen, Wenden, Rückwärtsfahren, Ein- und Anfahren}

Fehler beim Abbiegen (§ 9) (ausgenommen Pos. 33, 40)

Fehler beim Wenden oder Rückwärtsfahren

Fehler beim Einfahren in den fließenden Verkehr (z.B. aus einem Grundstück, von einem anderen Straßenteil oder beim Anfahren vom Fahrbahnrand)

\section{Falsches Verhalten gegenüber Fußgängern}

an Fußgängerüberwegen

an Fußgängerfurten

beim Abbiegen

an Haltestellen (auch haltenden Schulbussen mit eingeschaltetem Warnblinklicht)

an anderen Stellen

\section{Ruhender Verkehr, Verkehrssicherung}

Unzulässiges Halten oder Parken

Mangelnde Sicherung haltender oder liegengebliebener Fahrzeuge und von

Unfallstellen sowie von Schulbussen, bei denen Kinder ein- oder aussteigen

Verkehrswidriges Verhalten beim Ein- oder Aussteigen, Be- oder Entladen

Nichtbeachten der Beleuchtungsvorschriften (ausgenommen Pos. 50)

\section{Ladung, Besetzung}

Überladung, Überbesetzung

Unzureichend gesicherte Ladung oder Fahrzeugzubehörteile

Andere Fehler beim Fahrzeugführer

\section{Technische Mängel, Wartungsmängel:}

$\begin{array}{ll}50 & \text { Beleuchtung } \\ 51 & \text { Bereifung } \\ 52 & \text { Bremsen } \\ 53 & \text { Lenkung } \\ 54 & \text { Zugvorrichtung } \\ 55 & \text { Andere Mängel }\end{array}$


Falsches Verhalten der Fußgänger:

Falsches Verhalten beim Überschreiten der Fahrbahn:

60 an Stellen, an denen der Fußgängerverkehr durch Polizeibeamte oder Lichtzeichen geregelt war

61 auf Fußgängerüberwegen ohne Verkehrsregelung durch Polizeibeamte oder Lichtzeichen

62 in der Nähe von Kreuzungen oder Einmündungen, Lichtzeichenanlagen oder Fußgängerüberwegen bei dichtem Verkehr an anderen Stellen:

63 durch plötzliches Hervortreten hinter Sichthindernissen

64 ohne auf den Fahrzeugverkehr zu achten

65 durch sonstiges falsches Verhalten

$66 \quad$ Nichtbenutzen des Gehweges

67 Nichtbenutzen der vorgeschriebenen Straßenseite

68 Spielen auf oder neben der Fahrbahn

69 Andere Fehler der Fußgänger

\section{allgemeine Unfallursachen:}

\section{Straßenverhältnisse}

Glätte oder Schlüpfrigkeit der Fahrbahn

70 Verunreinigung durch ausgeflossenes Öl

71 Andere Verunreinigungen durch Straßenbenutzer

72 Schnee, Eis

73 Regen

74 Andere Einflüsse (u.a. Laub, angeschwemmter Lehm)

\section{Zustand der Straße}

75 Spurrillen, im Zusammenhang mit Regen, Schnee oder Eis

76 Anderer Zustand der Straße

77 Nicht ordnungsgemäßer Zustand der Verkehrszeichen oder -einrichtungen

78 Mangelhafte Beleuchtung der Straße

79 Mangelhafte Sicherung von Bahnübergängen 


\section{Witterungseinflüsse}

Sichtbehinderung durch:

80 Nebel

81 Starken Regen, Hagel, Schneegestöber usw.

82 Blendende Sonne

83

Seitenwind

84

Unwetter oder sonstige Witterungseinflüsse

\section{Hindernisse}

85 Nicht oder unzureichend gesicherte Arbeitsstelle auf der Fahrbahn

86 Wild auf der Fahrbahn

87 Anderes Tier auf der Fahrbahn

88 Sonstiges Hindernis auf der Fahrbahn (ausgenommen Pos. 43, 44)

89 Sonstige Ursachen

(mit kurzer Beschreibung aufführen) 


\section{ANHANG 2 Fragebogen zur Umsetzungspraxis von Verkehrsberuhigten Bereichen}

\section{- Informationen über das Projekt}

Im Auftrag der Unfallforschung der Versicherer (UDV) wird derzeit durch die IGS Ingenieurgesellschaft Stolz $\mathrm{mbH}$ und die Hochschule Darmstadt eine Studie zur Ermittlung der Auswirkungen der Gestaltung Verkehrsberuhigter Bereiche auf das Unfallgeschehen erstellt. Die Bedeutung der Verkehrsberuhigung nahm in den letzten Jahren wieder zu. Verkehrsberuhigte Bereiche werden in Deutschland wieder vermehrt umgesetzt und in unseren Nachbarländern werden Shared Space Projekte (Niederlanden) und Begegnungszonen (Schweiz) erfolgreich betrieben. Häufig werden Verkehrsberuhigte Bereiche mit dem Ziel umgesetzt, das Wohnumfeld zu verbessern (insbesondere für Kinder und ältere Menschen), Konflikte zwischen den verschiedenen Verkehrsteilnehmern (Fußgänger, Radfahrer, Kfz-Verkehr) zu minimieren und die Verkehrssicherheit zu erhöhen.

Unumstritten ist, dass das geringe Geschwindigkeitsniveau und die attraktive Gestaltung der Verkehrsberuhigten Bereiche ein deutlich günstigeres Unfallgeschehen aufweisen als Stadtstraßen (Tempo 50). Die Unfallzahlen in den Verkehrsberuhigten Bereichen selbst stagniert jedoch in den letzten Jahren.

Ziel der Studie ist es, die bauliche Gestaltung, belastbare Unfallanalysen und aktuelle Erkenntnisse zum Verkehrsablauf von Verkehrsberuhigten Bereichen zu erheben, um daraus Zusammenhänge zwischen baulicher Gestaltung einerseits und dem Geschwindigkeitsniveau und dem Unfallgeschehen andererseits ableiten zu können. Die Ergebnisse sollen in der Erstellung einer aktuellen Planungshilfe zur Gestaltung und Planung von Verkehrsberuhigten Bereichen münden.

Um dieses Ziel zu erreichen, werden verschiedene Erhebungen im gesamten Bundesgebiet durchgeführt. U.a. wird dabei mit dem hier vorliegenden Fragebogen eine Umfrage zur Umsetzungspraxis von Verkehrsberuhigten Bereichen in den Kommunen durchgeführt. Hierfür benötigen wir Ihre Mithilfe und bitten Sie, uns einige Angaben zu Verkehrsberuhigten Bereichen in Ihrer Kommune zu übermitteln.

Im Fokus stehen dabei Verkehrsberuhigte Bereiche, die gemäß STVO mit den VZ 325/326 ausgeschildert sind. Verkehrsberuhigte Geschäftsbereiche gemäß STVO §45, Abs. 1d und Tempo30 Zonen (STVO VZ 274/275) sind nicht Gegenstand der Studie.

Ihre Angaben werden selbstverständlich vertraulich behandelt und im Rahmen der Studie nur in anonymisierter Form verarbeitet.

Für Rückfragen stehen wir Ihnen gerne zur Verfügung:

Dipl.-Ing. Michael Vieten

Ingenieurgesellschaft Stolz $\mathrm{mbH}$

Tel.: 02131/791892-0

Fax: 02131/791892-30

Email: vieten@igs-ing.de
Dipl.-Ing. Mark-Simon Krause

Hochschule Darmstadt

Tel.: 06151/16-8165

Fax: 06151/16-8170

Mark-Simon.Krause@h-da.de 


\section{Allgemeine Informationen}

\begin{tabular}{l}
\hline Stadt \\
\hline Ansprechpartner \\
\hline Telefon \\
\hline Abteilung \\
\hline Adresse \\
\hline Email \\
\hline Datum \\
\hline
\end{tabular}

\section{Verkehrsberuhigte Bereiche - Allgemein}

Wie viele Verkehrsberuhigte Bereiche existieren in Ihrer Kommune?

punktuell / Streckenbezogen gemäß VZ325 StVO

$\square<5 \quad \square<10 \quad \square<15 \quad \square<20 \quad \square<25 \quad \square>25 \quad \square$ keine

Flächenhaft / Gebiete - gemäß VZ325 StVO

$\square<5 \quad \square<10 \quad \square<15 \quad \square<20 \quad \square<25 \quad \square>25 \quad \square$ keine

Verkehrsberuhigte

Geschäftsbereiche gemäß §45

StVO,

$\square<5 \quad \square<10 \quad \square<15 \quad \square<20 \quad \square<25 \quad \square>25 \quad \square$ keine

Bereiche mit Shared Space- oder Begegnungszonen-Charakter

$\square<5 \quad \square<10 \quad \square<15 \quad \square<20 \quad \square<25 \quad \square>25 \quad \square$ keine

Wo werden bei Ihnen

Verkehrsberuhigte Bereiche gemäß

VZ325 StVO umgesetzt?

$\square$ in Wohngebieten

$\square$ in Geschäftsstraßen

Sonstiges:

In welchem Zeitraum wurden bei Ihnen Verkehrsberuhigte Bereiche

$\square 1980-1989 \quad \square 1990-1999$ umgesetzt?

$\square 2000-2009 \quad \square 2010$ - heute 


\section{Hintergründe und Ziele}

Mit welchem Ziel werden bei Ihnen Verkehrsberuhigte Bereiche eingerichtet?

\begin{tabular}{|c|c|}
\hline $\begin{array}{l}\text { zur Verbesserung der } \\
\text { Verkehrssicherheit }\end{array}$ & $\square$ unwesentlich $\square$ eher unwesentlich $\square$ wesentlich $\square$ hauptsächlich \\
\hline $\begin{array}{l}\text { zur Verbesserung der } \\
\text { Aufenthaltsqualität / Verringerung } \\
\text { der Dominanz des Kfz-Verkehrs } \\
\text { im Straßenraum }\end{array}$ & $\square$ unwesentlich $\square$ eher unwesentlich $\square$ wesentlich $\square$ hauptsächlich \\
\hline $\begin{array}{l}\text { Verbesserung der Straßen- und } \\
\text { Stadtgestaltung }\end{array}$ & $\square$ unwesentlich $\square$ eher unwesentlich $\square$ wesentlich $\square$ hauptsächlich \\
\hline $\begin{array}{l}\text { Verbesserung der } \\
\text { Querungsmöglichkeiten für } \\
\text { Fußgänger }\end{array}$ & $\square$ unwesentlich $\square$ eher unwesentlich $\square$ wesentlich $\square$ hauptsächlich \\
\hline $\begin{array}{l}\text { Verbesserung der gemeinsamen } \\
\text { Abwicklung von nicht } \\
\text { motorisiertem Verkehr und Kfz- } \\
\text { Verkehr }\end{array}$ & $\square$ unwesentlich $\square$ eher unwesentlich $\square$ wesentlich $\square$ hauptsächlich \\
\hline $\begin{array}{l}\text { Reduzierung der } \\
\text { Lärmbelastungen }\end{array}$ & $\square$ unwesentlich $\square$ eher unwesentlich $\square$ wesentlich $\square$ hauptsächlich \\
\hline $\begin{array}{l}\text { Verdrängung vom Kfz- } \\
\text { (Durchgangs-)Verkehr }\end{array}$ & $\square$ unwesentlich $\square$ eher unwesentlich $\square$ wesentlich $\square$ hauptsächlich \\
\hline Sonstige wesentlichen Ziele: & \\
\hline
\end{tabular}

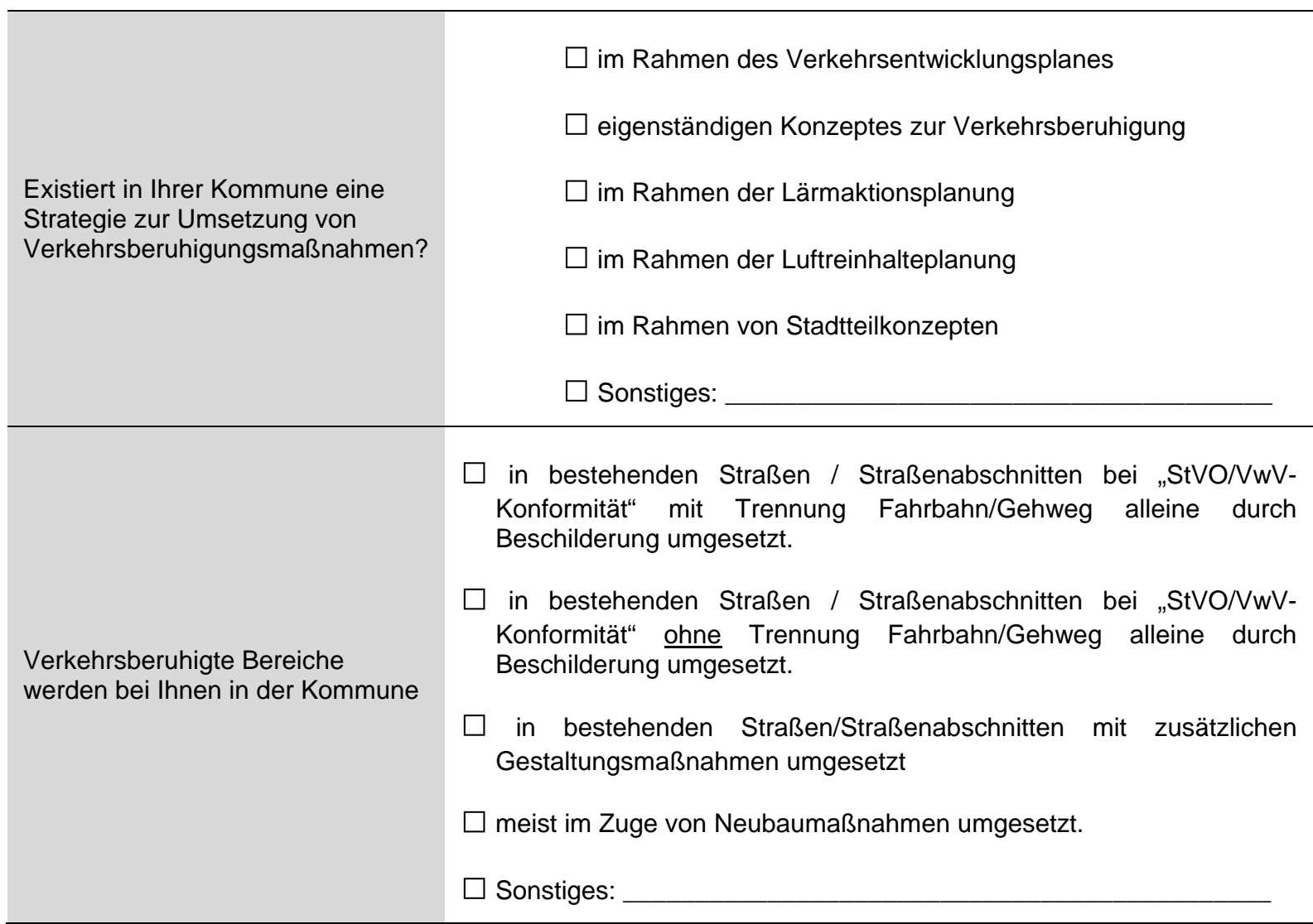




\section{Erfahrungen}

Haben Sie besondere Erfahrungen (positive sowie negative) mit einzelnen Gestaltungs-Maßnahmen zur Verkehrsberuhigung in Ihrer Kommune?

\begin{tabular}{ll}
\hline $\begin{array}{l}\text { Auswirkungen auf das } \\
\text { Unfallgeschehen/Verkehrssicherheit } \\
\text { Auswirkungen auf das } \\
\text { Geschwindigkeitsniveau }\end{array} \quad \square$ positiv $\square$ negativ $\square$ keine \\
\hline Auswirkungen auf den & $\square$ keine \\
Durchgangsverkehr & $\square$ positiv $\square$ negativ $\square$ keine \\
\hline Auswirkungen auf den & $\square$ positiv $\square$ negativ $\square$ keine \\
Unterhaltungsbedarf & $\square$ positiv $\square$ negativ $\square$ keine \\
\hline Auswirkungen auf die & $\square$ positiv $\square$ negativ $\square$ keine \\
Aufenthaltsqualität & \\
\hline Auswirkungen auf die & \\
Querungsmöglichkeiten & \\
\hline
\end{tabular}

Wurden in Ihrer Kommune schon einmal Wirksamkeitsuntersuchungen zu Verkehrsberuhigungsmaßnahmen $\square$ ja $\square$ nein durchgeführt?

Wenn ja, können Sie uns diese zur Verfügung stellen? 


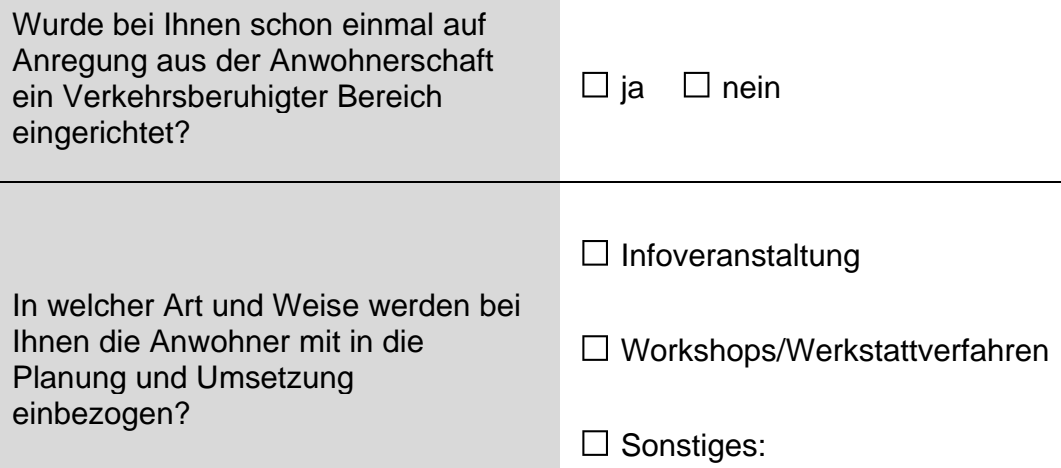

Sonstiges:

\section{Weiterführende Unterstützung}

Wurde in Ihrer Kommune

Verkehrsberuhigte Bereiche

eingerichtet, die überwiegend zur

Erhöhung der Verkehrssicherheit

beitragen sollten? $\square$ ja $\square$ nein
Wenn ja, können Sie uns diese

Bereiche benennen? $\square$ ja $\square$ nein

Im Rahmen der Studie werden rd. 200 Umsetzungsbeispiele von Verkehrsberuhigten Bereichen detaillierter untersucht. Hierzu benötigen wir ebenfalls Ihre Mithilfe. Daher möchten wir Sie fragen, ob Sie an einer detaillierteren Befragung zur Thematik teilnehmen würden:

$$
\square \text { ja } \square \text { nein }
$$

Unabhängig von Ihrer Bereitschaft, an einer detaillierteren Befragung teilzunehmen, möchten wir Sie bitten, uns eine Liste mit aus Ihrer Sicht besonders geeigneter Beispiele von Verkehrsberuhigten Bereichen zu nennen.

Benennung

geeigneter Beispiele: 


\section{ANHANG 3 Unfallanalysen „Kollektiv 2011“ Kategorie 1-6}

Dieser Teil der Unfallanalyse beschäftigte sich mit den unfallauffälligsten Untersuchungsobjekten des „Kollektivs Kat. 1-4“ aus Kapitel 10.1. In der vorliegenden Untersuchung galt ein Untersuchungsobjekt als unfallauffällig, wenn in einem Verkehrsberuhigten Bereich mind. 3 oder mehr Unfälle der Kat. 1-4 in 6 Jahren vorlagen. Insgesamt lagen 32 Untersuchungsobjekte vor, die als unfallauffällig eingestuft werden konnten (vgl. Tabelle 14). Für dieses Kollektiv wurden die Unfälle der Kategorie 5 und 6 für das Jahr 2011 ergänzt. Im Folgenden wird es deshalb als „Kollektiv 2011“ bezeichnet. Im „Kollektiv 2011“ sind alle Unfälle des Jahres 2011 (Kat. 1-6) enthalten, die in den jeweiligen Untersuchungsobjekten auftraten. Das Ziel dieser Auswertung war, einen Überblick zu erhalten, wie sich das Unfallgeschehen aller Unfälle der Untersuchungsobjekte verteilte und ob es wesentliche Unterschiede im Vergleich zum Unfallgeschehen des „Kollektivs Kat. 1-4" und dem innerörtlichen Unfallgeschehen der Kategorie 1-4 gab.

Wie auch bei den Auswertungen zum „Kollektiv Kat. 1-4“ (Kapitel 10), wurden beim „Kollektiv 2011" die Analysen weitestgehend in zwei Schritten durchgeführt:

\section{Schritt:}

Die in den Überschriften benannten Unfallattribute (Unfalltypen, Unfallarten, etc.) des „Kollektivs 2011“ wurden ausgewertet und mit dem „Kollektiv Kat. 1-4" verglichen.

\section{Schritt:}

Lagen Auffälligkeiten in Bezug auf ein bestimmtes Unfallmerkmal vor, so wurde dieses noch einmal genauer betrachtet. An diesen Stellen kommt es daher vor, dass auch weitere Unfallattribute ausgewertet wurden, die nicht in der Überschrift der Kapitel benannt sind. Anzumerken ist, dass nicht in jedem Kapitel detaillierte Auswertungen (2. Schritt) durchgeführt wurden, z.B. wenn es keine Auffälligkeiten gab oder eine weitere Untergliederung - auch aus Gründen der Übersichtlichkeit - nicht sinnvoll waren.

\section{Datengrundlage „Kollektiv 2011“}

Für die Betrachtung der Unfälle mit der Unfallkategorie 1-6 wurden die Daten für 2011 ausgewertet, da nur für das Jahr 2011 vollständige Unfalldaten vorlagen. Neun der insgesamt 32 unfallauffälligeren Beispiele besaßen im Jahr 2011 keinen Unfall. Für diese Fälle wurde überprüft, ob es in den anderen Jahren auffällig viele Unfälle der Kategorie 5 und 6 gab. Dies konnte aber ausgeschlossen werden. In vier der neun Beispiele gab es in keinem Jahr Unfälle der Kategorie 5-6 (es handelte sich dabei um Quartiersstraßen oder sonstige Bereiche). Die anderen 5 Beispiele wiesen wenig Unfälle der Kategorie 5-6 bzw. für vereinzelte Jahre ebenfalls gar keine Unfälle auf, so dass auch hier von einer korrekten Datengrundlage ausgegangen wurde.

Für das „Kollektiv 2011“ der Unfälle mit Kategorie 1-6 lagen insgesamt 110 Unfälle vor. Die folgende Tabelle zeigt den prozentualen Anteil der 278 Untersuchungsobjekte des „Kollektivs Kategorie 1-4" verglichen mit den 32 Untersuchungsobjekten des „Kollektivs 2011“, bezogen auf die jeweiligen Bundesländer. Daraus ist erkennbar, dass sich die 32 Untersuchungsobjekte vermehrt aus den Bundesländern zusammensetzen, in denen gezielt über die Unfalldatenbanken nach unfallauffälligen ausgewiesenen Verkehrsberuhigten Bereichen recherchiert wurde (BadenWürttemberg, Hessen und Sachsen-Anhalt). 
Tabelle 36:

Verteilung der Untersuchungsobjekte des „Kollektivs Kat. 1-4“ im Vergleich zum „Kollektiv 2011“

\begin{tabular}{|l|c|c|c|c|}
\hline \multirow{2}{*}{ Bundesland } & \multicolumn{4}{|c|}{ Untersuchungsobjekte } \\
\cline { 2 - 5 } & \multicolumn{2}{|c|}{ "Kollektiv Kat. 1-4" } & "Kollektiv 2011" \\
\cline { 2 - 5 } & Anzahl & Anteil & Anzahl & Anteil \\
\hline Baden-Württemberg & 49 & $18 \%$ & 10 & $21 \%$ \\
\hline Hessen & 136 & $49 \%$ & 10 & $31 \%$ \\
\hline Niedersachsen & 2 & $1 \%$ & 0 & $0 \%$ \\
\hline Nordrhein-Westfahlen & 18 & $6 \%$ & 0 & $0 \%$ \\
\hline Rheinland-Pfalz & 23 & $8 \%$ & 3 & $9 \%$ \\
\hline Sachsen & 19 & $7 \%$ & 2 & $6 \%$ \\
\hline Sachsen-Anhalt & 19 & $7 \%$ & 7 & $22 \%$ \\
\hline Schleswig-Holstein & 8 & $3 \%$ & 0 & $0 \%$ \\
\hline Thüringen & 4 & $1 \%$ & 0 & $0 \%$ \\
\hline Summe & $\mathbf{2 7 8}$ & $\mathbf{1 0 0 \%}$ & $\mathbf{3 2}$ & $\mathbf{1 0 0 \%}$ \\
\hline
\end{tabular}

Bei Betrachtung der Verteilung der Straßenfunktionen im „Kollektiv 2011“ fiel auf, dass der Anteil der Geschäftsstraßen hier wesentlich höher lag als beim „Kollektiv Kat. 1-4“ (Abbildung 117). Das umgekehrte Verhältnis zeigt sich bei den Wohnstraßen. Das Unfallgeschehen in Geschäftsstraßen wird demnach von leichten Unfällen dominiert. In Wohnstraßen sind es hingegen Unfälle mit Verletzten.

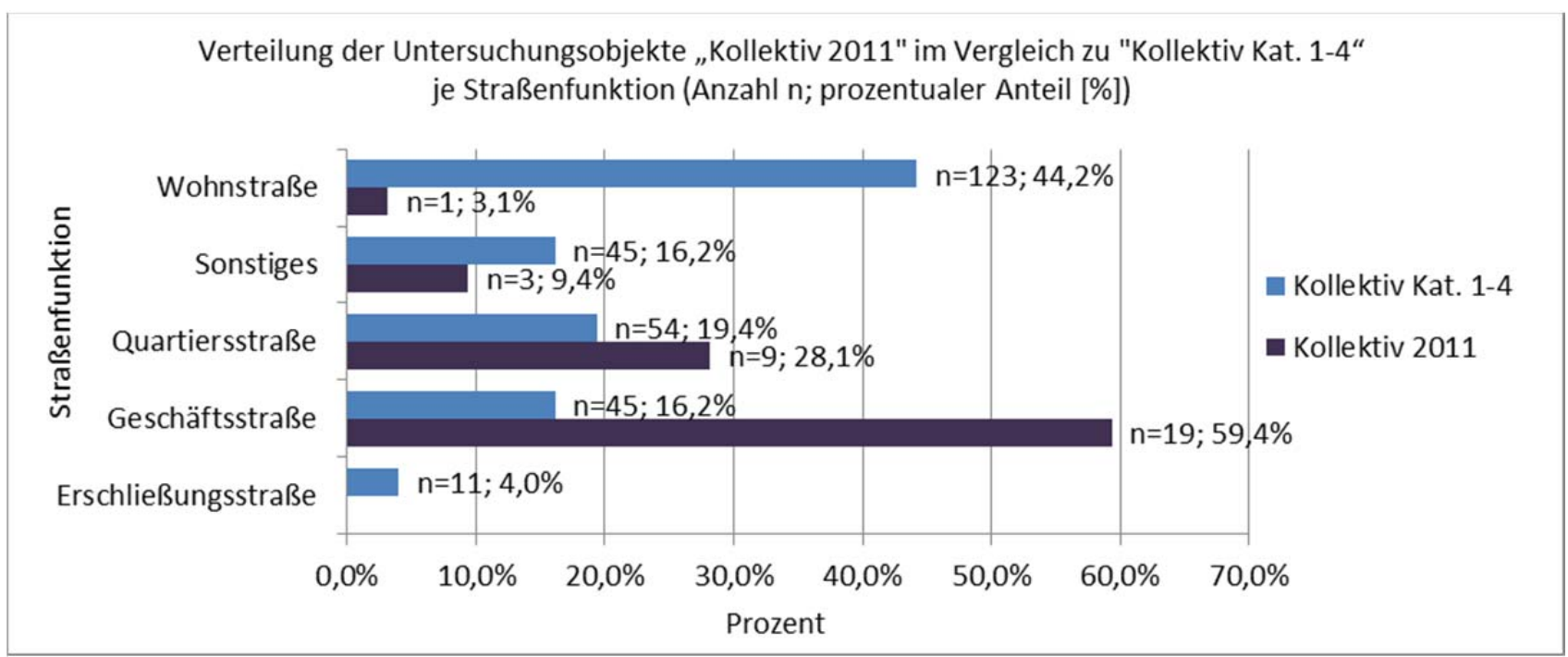

Abbildung 117: Verteilung der Untersuchungsobjekte des „Kollektivs 2011“ im Vergleich zum „Kollektiv Kat. 14 “ je Straßenfunktion

Auch die Anzahl der Untersuchungsobjekte je Verkehrsführung des „Kollektivs 2011“ variierte im Vergleich zum „Kollektiv Kat. 1-4“. In Abbildung 118 ist die prozentuale Verteilung zu erkennen. Während bei den 278 Beispielen (Kollektiv Kat. 1-4) die Mischverkehrsfläche (MV) zu 23\% vertreten war, kam sie in den 32 unfallreichsten Bereichen (Kollektiv 2011) nur zu 6\% vor. Die unfallträchtigsten Bereiche traten hauptsächlich in Bereichen mit baulicher Trennung (44\%) oder optischer und baulicher Trennung (28\%) auf. 


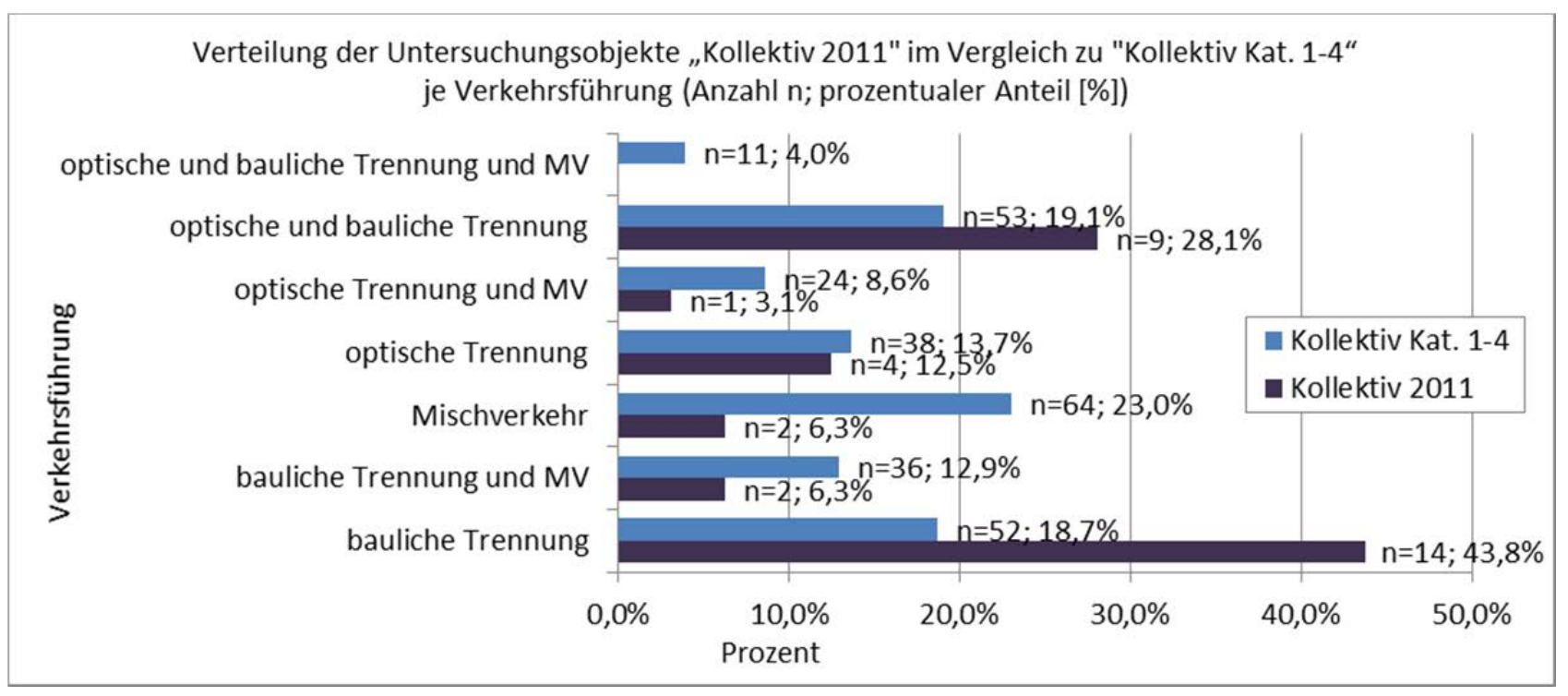

Abbildung 118: Verteilung der Untersuchungsobjekte des „Kollektivs Kat. 1-4“ im Vergleich zum „Kollektiv 2011“ je Verkehrsführung

Das Streckennetz der erhobenen 278 Steckbriefe umfasste insgesamt 79,3km. Die 32 Bereiche wiesen eine Netzlänge von 10,0km auf. Das waren 12,5\% der gesamten Abschnittslängen, bei einer "Beteiligung“ von ca. $11 \%$ der Untersuchungsobjekte. Die Anteile zu den einzelnen Straßenfunktionen innerhalb der beiden Kollektive verhielten sich, außer bei den Geschäfts- und Quartiersstraßen, gleich. Im „Kollektiv 2011“ war der Streckennetzanteil der Geschäftsstraßen ca. viermal so hoch wie im „Kollektiv Kat. 1-4“. Auch der Streckennetzanteil der Quartiersstraßen („Kollektiv 2011“) war im Vergleich doppelt so lang (Tabelle 37).

Tabelle 37: Strecken- und Durchschnittslängen der Untersuchungsobjekte des „Kollektivs Kat. 1-4“ im Vergleich zum „Kollektiv 2011“

\begin{tabular}{|l|c|c|c|c|}
\hline \multirow{2}{*}{ Straßenfunktion } & \multicolumn{4}{|c|}{ Untersuchungsobjekte } \\
\cline { 2 - 5 } & \multicolumn{3}{|c|}{ "Kollektiv Kat. 1-4" } & „Kollektiv 2011“ \\
\cline { 2 - 5 } & Längen [m] & Anteil & Längen [m] & Anteil \\
\hline Erschließungsstraße & 4.983 & $6,3 \%$ & 0 & $0,0 \%$ \\
\hline Geschäftsstraße & 11.208 & $14,1 \%$ & 5.679 & $56,6 \%$ \\
\hline Quartiersstraße & 12.301 & $15,5 \%$ & 3.046 & $30,4 \%$ \\
\hline Sonstige & 10.197 & $12,9 \%$ & 1.161 & $11,6 \%$ \\
\hline Wohnstraße & 40.581 & $51,2 \%$ & 145 & $1,4 \%$ \\
\hline Summe & $\mathbf{7 9 . 2 7 0}$ & $\mathbf{1 0 0 , 0 \%}$ & $\mathbf{1 0 . 0 3 1}$ & $\mathbf{1 0 0 , 0 \%}$ \\
\hline Durchschnittslänge & $\mathbf{2 8 5}$ & & $\mathbf{3 1 3}$ & \\
\hline
\end{tabular}

\section{Unfallkategorie}

In einem ersten Schritt wurden zunächst die Unfälle der Kategorien 1-4 der beiden Kollektive verglichen. D.h. für das „Kollektiv 2011“ wurden bei dieser Auswertung die Unfälle der Kategorie 5-6 Unfälle nicht berücksichtigt. Der Vergleich sollte aufzeigen, ob sich die Unfallkategorien 1-4 der beiden Unfallkollektive ähnlich verteilten, oder es erhebliche Unterschiede gab. Aufgrund der unterschiedlichen Anzahl der Betrachtungsjahre kann es zu Unterschieden kommen. Da sich schwere Unfälle wesentlich seltener ereignen und eine geringere Anzahl aufweisen, empfiehlt sich 
immer eine Betrachtung über mehrere Jahre. Beim Vergleich der beiden Kollektive war zu erkennen, dass im „Kollektiv Kat. 1-4“ die Kategorie 3 zu 77,5\% ausgeprägt war und beim „Kollektiv 2011“ nur zu 66,7\% (Abbildung 119). Die Schwankung lassen sich zum einen durch die unterschiedlichen Beobachtungszeiträume erklären, aber auch darüber, dass im „Kollektiv 2011“ die unfallauffälligeren Bereiche betrachtet wurden, die ggf. auch eine schwerere Unfallfolge besaßen. Die Unfälle der Kategorie 2 waren beim „Kollektiv 2011“ auch stärker vertreten.

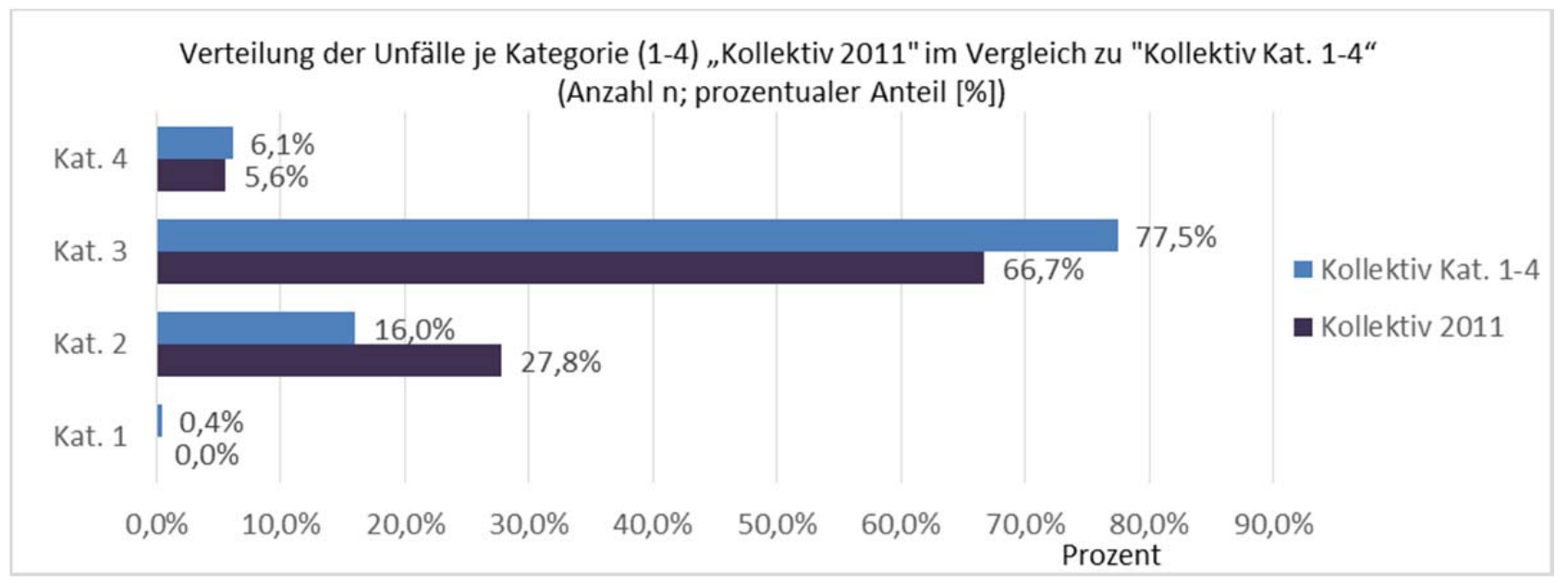

Abbildung 119: Verteilung der Unfallkategorien Kat. 1-4 des „Kollektivs Kat. 1-4“ im Vergleich zum „Kollektiv 2011“

Bei einer weiteren Betrachtung wurden alle 110 Unfälle des „Kollektivs 2011“ betrachtet. Abbildung 120 zeigt, dass, wenn Unfälle der Kategorie 5-6 mitbetrachtet wurden, es zu einer deutlichen Umverteilung der Unfallkategorien kam. Sofort fiel auf, dass die Unfälle der Kategorie 5 dominierten. Über zwei Drittel der Unfälle (67\%) waren „Sonstige Unfälle“ mit Sachschaden. Bedenkt man, dass die Dunkelziffer der Unfälle der Kategorie 5 sehr hoch ist (Kapitel 7.1), so würde das Ergebnis noch deutlicher ausfallen. Im Kollektiv waren keine Unfälle der Kategorie 6 (Sachschadensunfälle unter Einfluss berauschender Mittel) vorhanden.

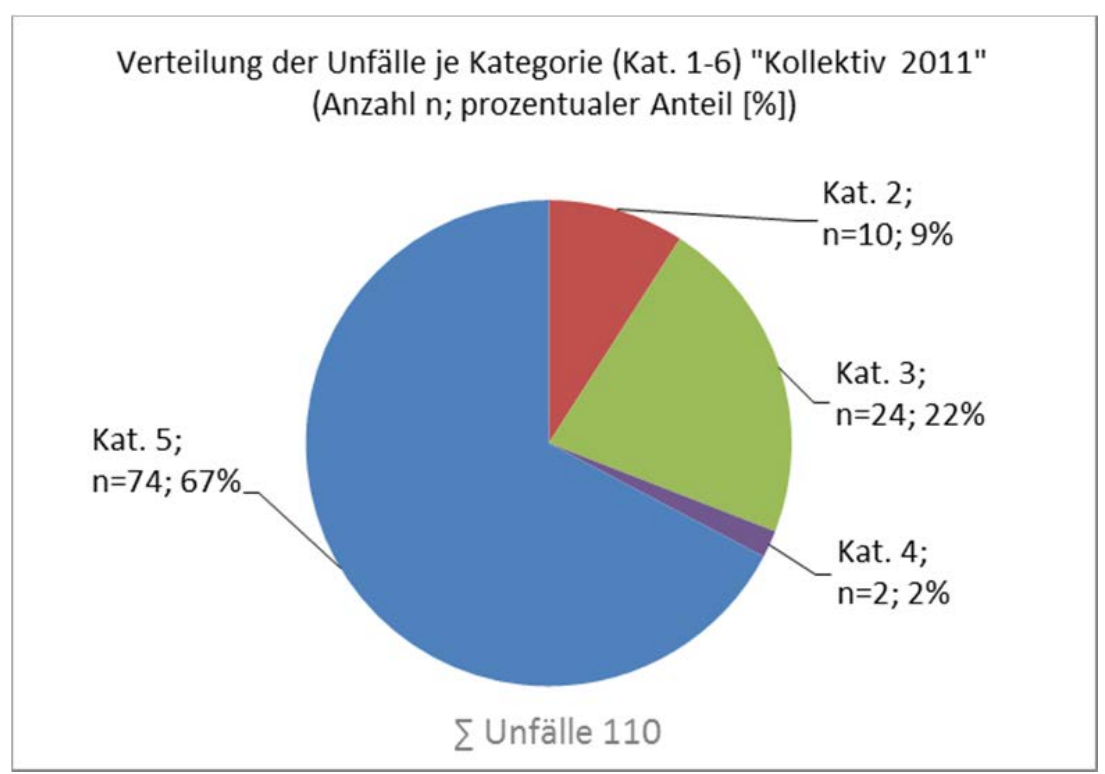

Abbildung 120: Verteilung der Unfälle des „Kollektivs 2011“ 


\section{Unfalltyp}

1. Schritt:

In der Abbildung 121 ist die Verteilung der Unfalltypen innerhalb der Kollektive dargestellt. Hierzu wurden das „Kollektiv Kat. 1-4“ mit dem „Kollektiv 2011“ verglichen. Hierbei sollte wieder der Unterschied zum „Kollektiv 2011“ aufgezeigt werden, auch wenn beim „Kollektiv 2011“ nur die Unfälle der Kategorie 1-4 betrachtet werden.

Im „Kollektiv Kat. 1-4“ war der Unfalltyp „Sonstiger Unfall“ (24\%) sowie der Unfalltyp „Einbiegen/Kreuzen-Unfall“ (31\%) am stärksten vertreten. In der Abbildung ist dieses Kollektiv durch blaue Balken gekennzeichnet.

Das „Kollektiv 2011 (Kat. 1-4)“ zeigte, dass die Unfalltypen der Bereiche mit mindestens drei Unfällen ebenfalls bei Unfalltyp „Einbiegen/Kreuzen-Unfall“ (22\%) und Unfalltyp „Sonstiger Unfall“ (19\%) stärker vertreten waren (hell lila Balken). Aber auch „Unfälle durch den ruhenden Verkehr" (14\%), „Überschreiten-Unfälle“ (17\%) und „Unfälle im Längsverkehr (17\%)“ machten rund die Hälfte der Unfälle aus.

Bei der Betrachtung des dritten „Kollektivs 2011“ (lila Balken), fiel auf, dass prozentual der Unfalltyp „Sonstiger Unfall“ (47\%) und der Unfalltyp „Unfälle durch den ruhenden Verkehr“ (19\%) den größten Anteil ausmachten.

Die Unfälle der Kategorie 5-6 waren also im Wesentlichen Unfälle des Unfalltyps „Sonstige Unfälle“. Dies veranschaulicht auch noch mal die Abbildung 122, in der die Verteilung der Unfalltypen für (ausschließlich) die Unfälle der Kategorie 5 des „Kollektivs 2011“ dargestellt ist.

Daraus ist zu erkennen, dass der Unfalltyp 7 (SO) mit 61\% am stärksten ausgeprägt war. Unfälle durch ruhenden Verkehr geschahen zu $21 \%$ und Einbiegen/Kreuzen-Unfälle waren der dritthäufigste vorkommende Unfalltyp (8\%).

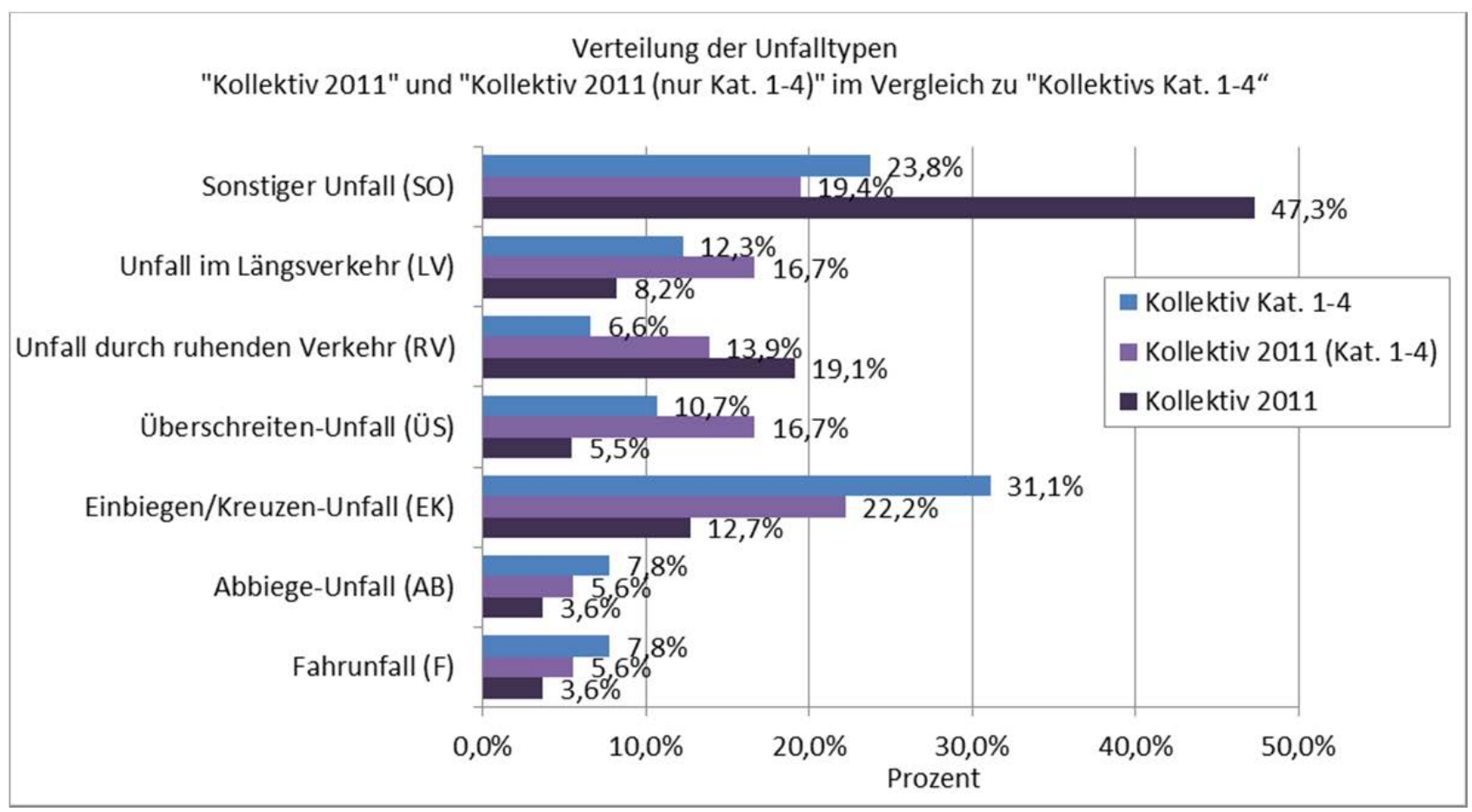

Abbildung 121: Verteilung der Unfalltypen des „Kollektivs Kat. 1-4“ in Vergleich zum „Kollektiv 2011“ 
Verteilung der Unfalltypen der Unfälle der Kat. 5 des "Kollektivs 2011" (Anzahl n; prozentualer Anteil [\%])

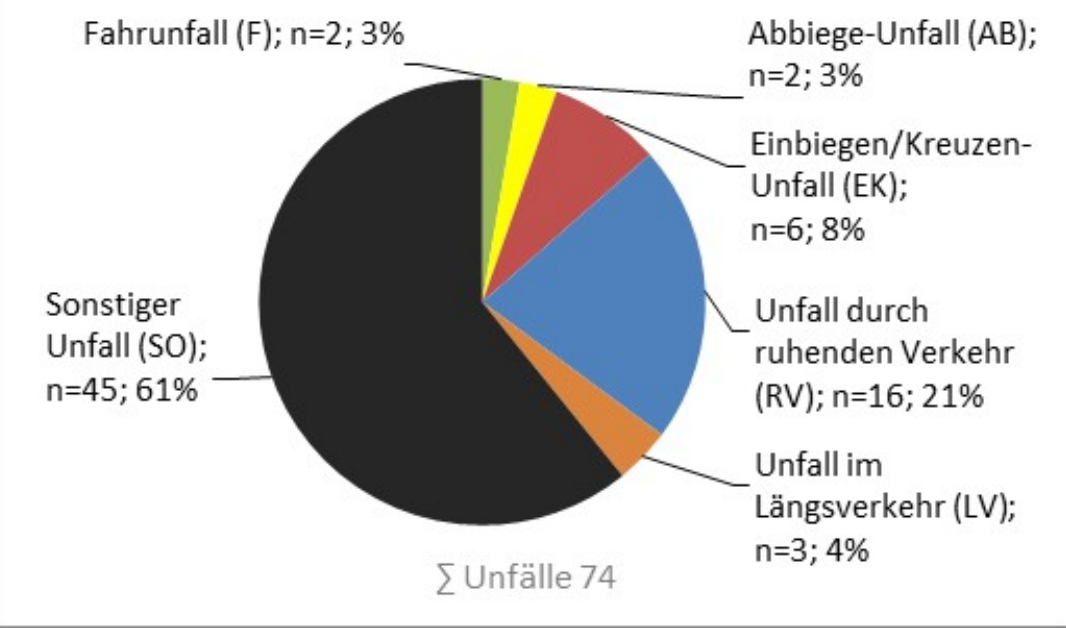

Abbildung 122: Verteilung der Unfalltypen der Unfälle der Kat. 5 des „Kollektivs 2011“

\section{Schritt:}

Im Folgenden wurden die Unfallarten der Unfälle der Kategorie 5 des „Kollektivs 2011“ für den Unfalltyp „Sonstiger Unfall“ und „Unfall durch ruhender Verkehr" genauer untersucht, um ggf. Auffälligkeiten und Unterschiede im Vergleich zum „Kollektiv Kat. 1-4“ aufzeigen zu können.

Auffällig war, dass beim Unfalltyp „Sonstiger Unfall“ im Wesentlichen nur „Zusammenstöße mit anderem Fahrzeug, das anfährt, anhält oder im ruhenden Verkehr steht" sowie „Unfälle anderer Art" als Unfallarten vorlagen (Tabelle 38).

Im Vergleich zu den Unfallarten des „Kollektiv Kat. 1-4“ war das ein erheblicher Unterschied, denn hier lagen die Unfallursachen in knapp der Hälfte der Sonstigen Unfälle bei einem "Zusammenstoß zwischen Fahrzeug und Fußgänger". Auch hier wurde die Vermutung wieder bestätigt, dass die Unfälle der Kategorie 5 mit einer Kollision zwischen Fußgängern und Kfz in der Regel nicht polizeilich erfasst werden, wenn kein Fußgänger-Personenschaden vorliegt. Bei der Auswertung der Kategorie 5 Unfälle des Unfalltyps „Sonstiger Unfall“ nach den Beteiligten fiel auf, dass kein Fußgänger beteiligt war.

Tabelle 38: Unfallarten des Unfalltyps „Sonstiger Unfall“ der Unfälle der Kategorie 5 des „Kollektivs 2011“

\begin{tabular}{|l|c|}
\hline Unfallart & Anzahl \\
\hline Nr. 1 Zusammenstoß mit anderem Fz, das anfährt, anhält oder im ruhenden Verkehr steht & 30 \\
\hline Nr. 9 Abkommen von der Fahrbahn nach links & 1 \\
\hline Nr. 10 Unfall anderer Art & 14 \\
\hline Summe & 45 \\
\hline
\end{tabular}

Aus der Betrachtung der Unfallarten des Unfalltyps „Unfall durch ruhender Verkehr“ der Kat. 5 Unfälle des „Kollektivs 2011“ war zu erkennen, dass auch hier hauptsächlich die Unfallart „Zusammenstoß mit anderem Fahrzeug, das anfährt, anhält oder im ruhenden Verkehr steht“ vorlag (Tabelle39). 
Tabelle39: Unfallursachen des Unfalltyps „Unfall ruhender Verkehr“ der Unfälle der Kategorie 5 des „Kollektivs 2011“

\begin{tabular}{|l|c|}
\hline Unfallart & Anzahl \\
\hline Nr. 1 Zusammenstoß mit anderem Fz, das anfährt, anhält oder im ruhenden Verkehr steht & 14 \\
\hline Nr. 10 Unfall anderer Art & 2 \\
\hline Summe & 16 \\
\hline
\end{tabular}

\section{Unfallart}

Während im „Kollektiv Kat. 1-4“ die Unfallarten „Zusammenstoß zwischen Fahrzeug und Fußgänger" und „Zusammenstoß mit anderem Fahrzeug, das einbiegt oder kreuzt“ jeweils mit 28\% vorkamen, machten sie im „Kollektiv 2011“ zusammen nur noch $19 \%$ aus. Dafür lag die Unfallart „Zusammenstoß mit anderem Fahrzeug, das anfährt, anhält oder im ruhenden Verkehr steht" bei 45,5\% und auch der „Unfall anderer Art“ fiel mit 24,5\% anteilig im „Kollektiv 2011“ hoch aus.

Die Auswertung der Unfallarten bestätigte die Aussage, die schon bei den Unfalltypen gezogen werden konnte, dass hauptsächlich Unfälle durch den ruhenden Verkehr oder Sonstige Unfälle (Wenden/Rückwärtsfahren) beim „Kollektiv 2011“ auftraten, allerdings ohne Beteiligung von Fußgängern.

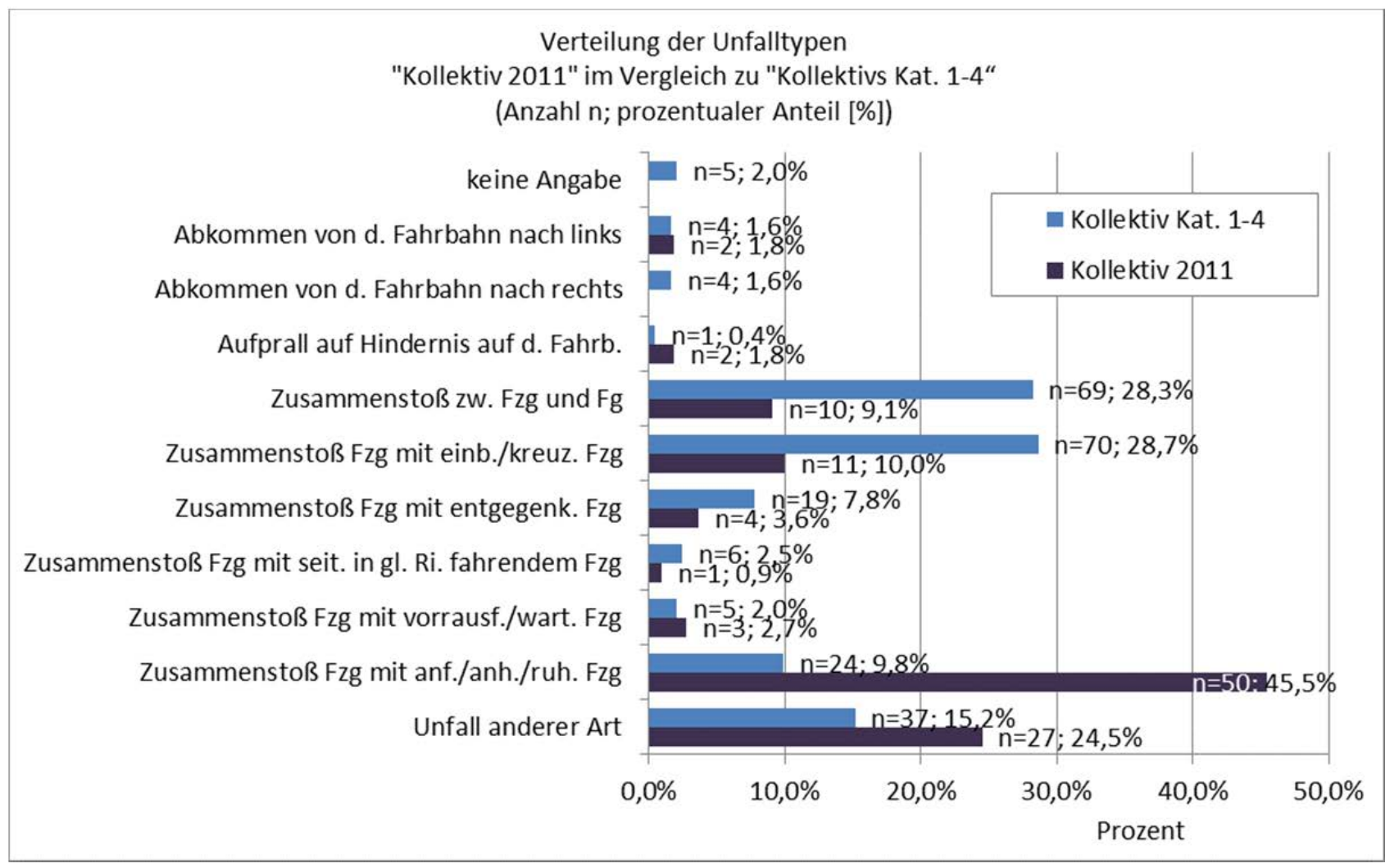

Abbildung 123: Verteilung der Unfallarten des „Kollektivs 2011“ im Vergleich zum „Kollektiv Kat. 1-4“ 


\section{Beteiligte}

Zunächst wurde ausschließlich das „Kollektiv 2011“ auf die Unfallbeteiligten hin untersucht. Für das „Kollektiv 2011“ lagen insgesamt 203 Beteiligte vor. Davon sind 110 Hauptverursacher (Beteiligte 1), 92 Beteiligte 2 und ein Beteiligter 3. An den 110 Unfällen waren im Wesentlichen beteiligt:

- 101 Pkw (75 Hauptverursacher, 26 Beteiligte 2),

- 13 sonstige/unbekannte Fahrzeuge (13 Hauptverursacher) und

- 18 Fahrradfahrer (10 Hauptverursacher, 8 Beteiligte 2).

Die Gruppe der Pkw dominierte bei den Beteiligten des „Kollektivs 2011“ und machte rund 50\% der Beteiligten aus (Abbildung 124). Die Gruppe der Fußgänger war bei den Hauptverursachern nicht vertreten, machte aber dennoch einen Anteil von 4\%, bezogen auf alle Beteiligten, aus. Mit insgesamt $9 \%$ waren Radfahrer in einen Unfall verwickelt.

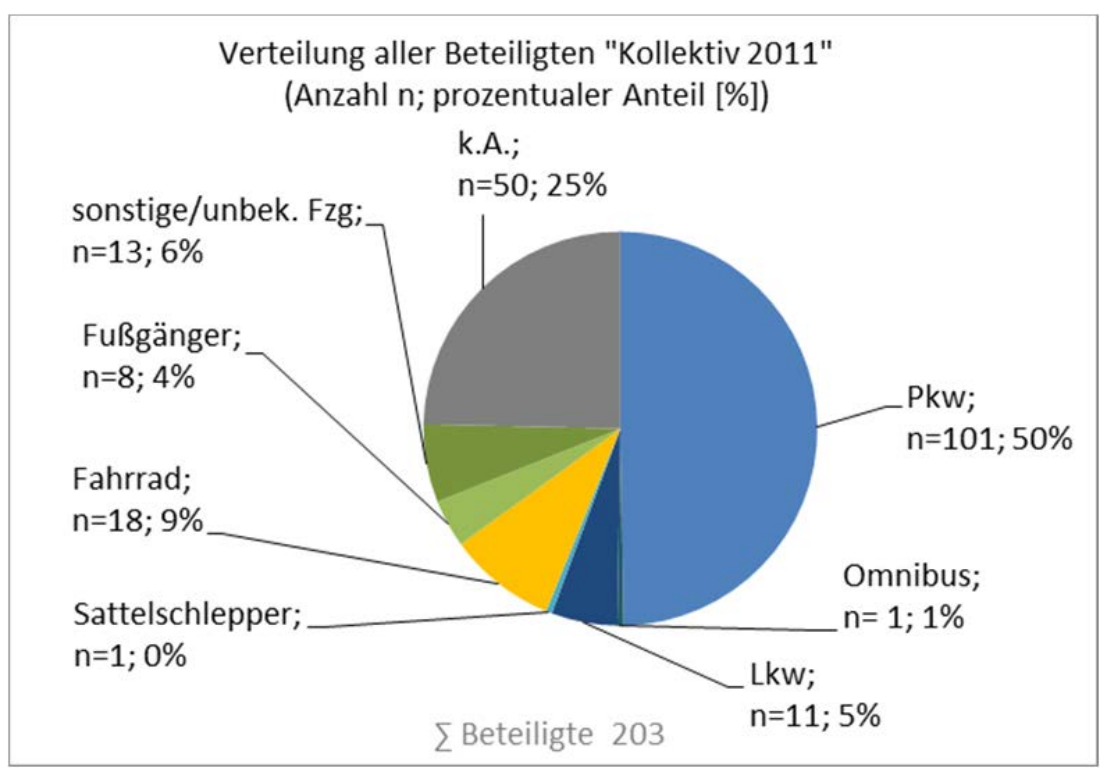

Abbildung 124: Verteilung der Beteiligten des „Kollektivs 2011“

Bei dem Vergleich der Beteiligten aus den beiden Kollektiven „Kollektiv 2011“ und „Kollektiv Kat. 14 “ fiel auf, dass beim „Kollektiv Kat. 1-4“ wesentlich häufiger Radfahrer und Fußgänger in Unfälle verwickelt waren (Abbildung 125). Beim „Kollektiv 2011“ lagen häufig zum Beteiligten 2 keine Angaben vor. In diesen Fällen lagen die Unfallart nicht bei „Zusammenstoß zwischen Fahrzeug und Fußgänger“ oder die Unfallursachen beim „Fehler gegenüber Fußgängern“, so dass zu mindestens ausgeschlossen werden kann, dass der zweite Beteiligte ein Fußgänger war.

Unter den 110 Unfällen des „Kollektiv 2011“ fanden 18 Alleinunfälle statt (9 von Pkw, fünf von Lkw/Sattelschlepper, ein von Fahrradfahrer und drei von sonstigen, unbekannten Fahrzeugen). Der Hauptunfalltypen der Alleinunfälle von Pkws war der "Sonstige Unfall“. Die häufigsten Unfallursachen lagen dreimal beim „Wenden/Rückwärtsfahren“ und viermal bei einem „anderen Fehler beim Fahrzeug-Führer". Die Unfalltypen und Unfallursachen deuten darauf hin, dass die Alleinunfalle der Kategorie 5 eher auf Unfälle beim Wenden, Rückwärtsfahren und Parken untereinander erfolgten. Die Alleinunfälle der Kategorie 1-4 von Pkws erfolgten eindeutig am häufigsten aufgrund von nichtangepassten oder überhöhten Geschwindigkeiten. 


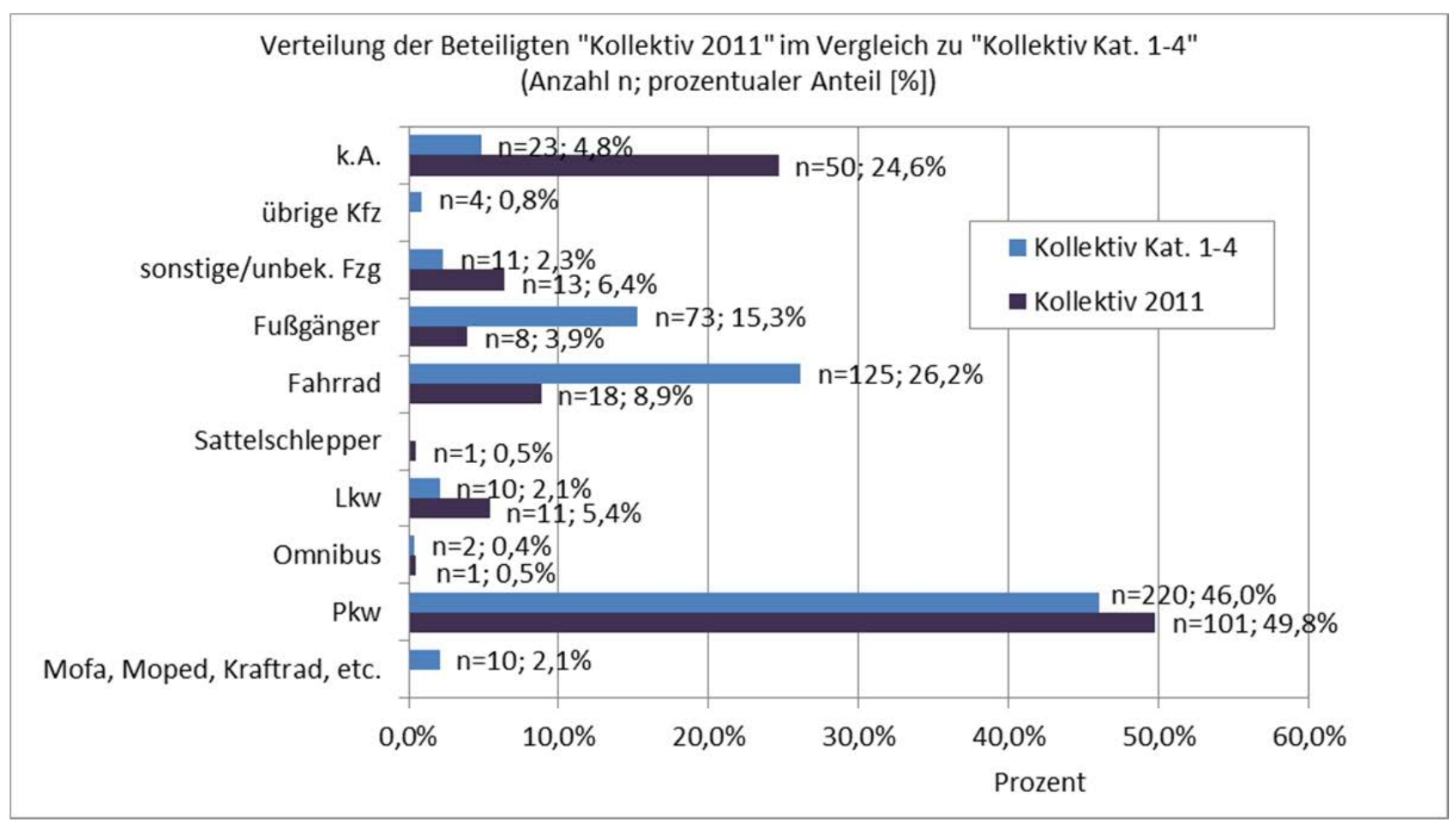

Abbildung 125: Verteilung der Beteiligten des „Kollektivs2011“ im Vergleich zum „Kollektiv Kat. 1-4“

\section{Unfallursache}

Die Hauptunfallursache in ausgewiesenen Verkehrsberuhigten Bereichen lag beim „Kollektiv Kat.14“ zu 25\% an der Ursache „Abbiegen, Wenden, Rückwärtsfahren, Ein- und Anfahren“. Beim „Kollektiv 2011" lagen die Hauptunfallursachen ebenfalls beim „Abbiegen, Wenden, Rückwärtsfahren, Ein- und Anfahren“. Der Anteil war mit 40\% nur wesentlich höher als beim „Kollektiv Kat. 1-4“. Auch die Unfallursache „Sonstige Fehler beim Fahrzeugführer" lagen mit 25\% deutlich höher als beim „Kollektiv Kat. 1-4“. Dafür waren die Unfallursachen „Vorfahrt/Vorrang“ (16\%) sowie „falsches Verhalten gegenüber Fußgängern“ (16\%) im „Kollektiv Kat. 1-4" deutlich höher als im „Kollektiv 2011“ (5\% bzw. 8\%). Die Unfallursache „Falsches Verhalten gegenüber Fußgängern“ spielte im „Kollektiv 2011“ keine nennenswerte Rolle mehr. Die Verteilung der Unfallursachen je Kollektiv kann der Abbildung 126 entnommen werden.

Im „Kollektiv Kat. 1-4“ geschahen rund ein Zehntel der Unfälle aufgrund der Ursache „Geschwindigkeit“. Im „Kollektiv 2011“ lag die Ursache der Unfälle nur noch zu 4\% an überhöhten oder nicht angepassten Geschwindigkeiten. 
Verteilung der Unfallursachen der Beteiligten 1 "Kollektiv 2011" im Vergleich zum "Kollektiv Kat. 1-4" (Anzahl n; prozentualer Anteil [\%])

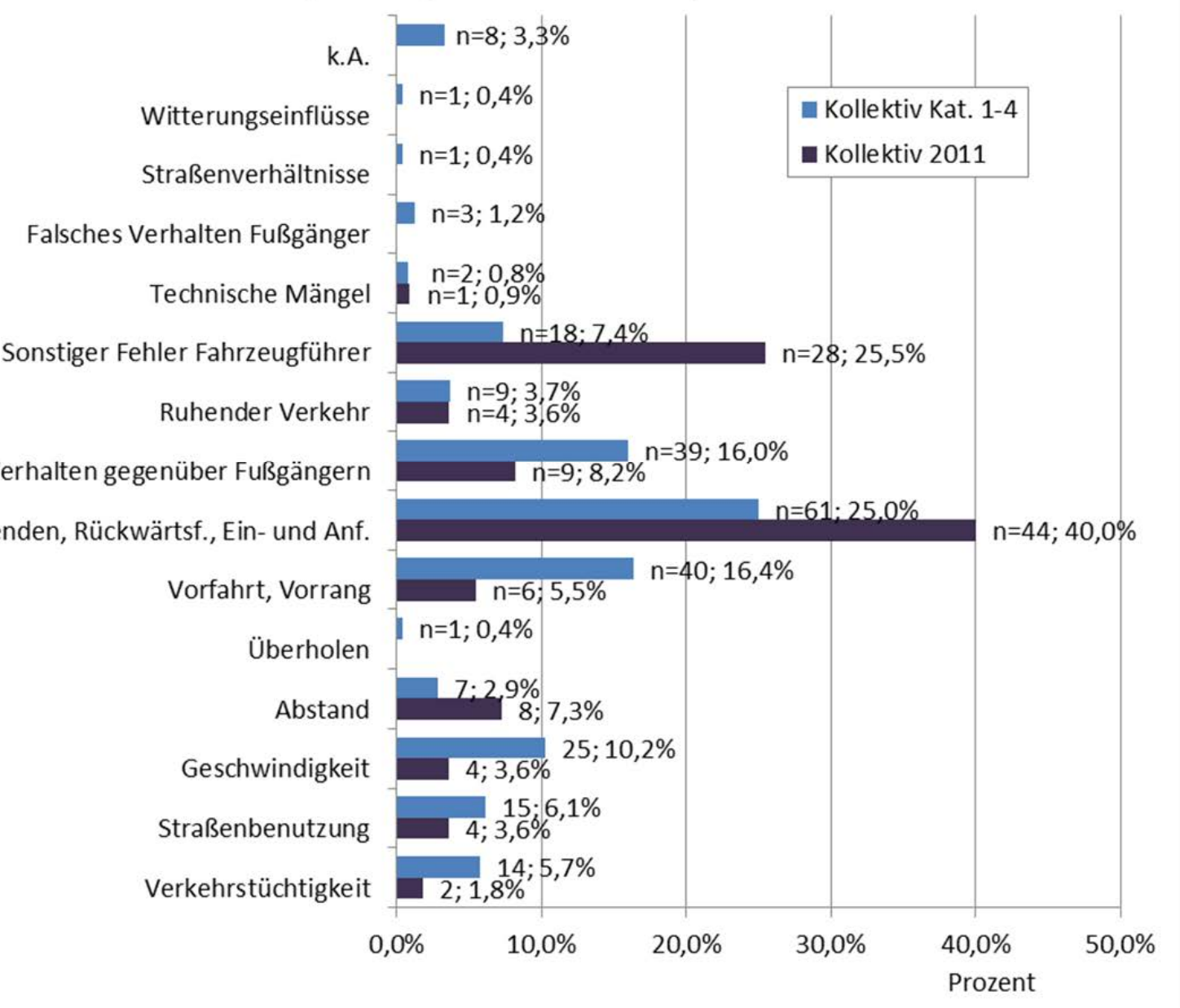

Abbildung 126: Verteilung der Unfallursache des „Kollektivs Kat. 1-4“ im Vergleich zum „Kollektiv 2011“

\section{Unfallumstände}

Wie auch bei dem „Kollektiv Kat. 1-4“ konnten bei dem „Kollektiv 2011“ keine Auffälligkeiten in den Unfallumständen abgeleitet werden. Der Abbildung 127 kann ein Vergleich der beiden Kollektive mit dem Erwartungswert $(\varnothing)$ Innerorts entnommen werden. Die Ausprägung der Unfallumstände war in fast allen Merkmalen beim „Kollektiv Kat. 1-4“ höher. Daraus ließ sich ableiten, dass Unfälle mit schwerwiegenderen Folgen häufiger bei Nässe/Glätte, in der Nacht, in Spitzenstunden oder am Wochenende auftraten. 


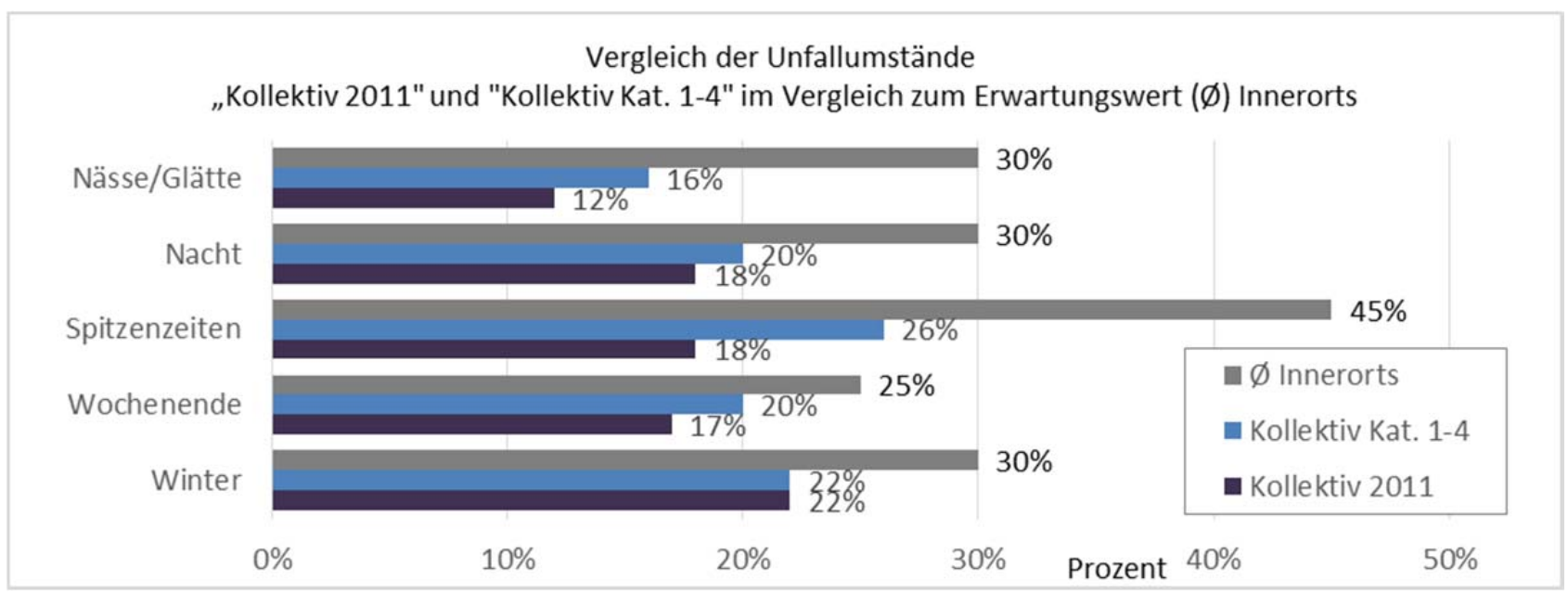

Abbildung 127: Vergleich der Unfallumstände des "Kollektivs 2011" und des "Kollektiv Kat. 1-4" im Vergleich zum Erwartungswert (Ø) Innerorts

\section{Zusammenfassung der Unfallanalyse für das „Kollektiv 2011“}

Zusammengefasst kann für das Unfallgeschehen des „Kollektivs 2011“ von ausgewiesenen Verkehrsberuhigten Bereichen folgendes festgehalten werden:

\section{Unfallkategorie:}

- Im „Kollektiv 2011“ waren die Unfälle der Kategorie 1-4 ähnlich verteilt wie im „Kollektiv Kat. 1-4“. Tendenziell lagen aber im „Kollektiv 2011“ (mit den unfallauffälligeren Bereichen) schwerere Unfallfolgen vor.

- Der Anteil der Unfallkategorie 5 betrug $67 \%$ am gesamten Unfallgeschehen des „Kollektivs 2011“. Die Unfallkategorien 1-4 teilten sich unter den übrigen 33\% auf. Kategorie 6 Unfälle waren nicht vertreten.

\section{Unfalltyp:}

- Hauptunfalltyp in ausgewiesenen Verkehrsberuhigten Bereichen des „Kollektivs 2011“ war der „Sonstige Unfall“. Im Vergleich zum „Kollektiv Kat. 1-4“ (24\%) war der „Sonstige Unfall“ mit einem Anteil von 47\% vergleichsweise hoch vertreten. Zweithäufigster Unfalltyp des „Kollektiv 2011“ war der „Unfall durch den ruhenden Verkehr“. Mit 19\% lag er zudem viel höher (+12\%) als beim „Kollektiv Kat. 1-4“.

- Die Anzahl der „Überschreiten-Unfälle“ spielten im „Kollektiv 2011“ keine wesentliche Rolle.

- Wurden nur die Unfalltypen der Unfälle der Kategorie 5 betrachtet, so fiel auf, dass die Unfalltypen „Sonstiger Unfall“ (47\%) und „Unfall durch ruhenden Verkehr" (19\%) insgesamt $66 \%$ ausmachten.

- Der Unfalltyp „Überschreiten Unfall“ war bei den Unfällen der Kategorie 5 nur mit 5\% vertreten. Die Unfalltypen „Fahrunfall“ und „Abbiegeunfall“ kamen jeweils zu 4\% vor.

\section{Unfallart:}

- Hauptunfallart im „Kollektiv 2011“ war mit 45\% der „Zusammenstoß mit anderem Fahrzeug, das anfährt, anhält oder im ruhenden Verkehr steht“. Die zweithäufigste Unfallart waren „Unfälle anderer Art" (25\%). Die Auswertung der Unfallarten zeigte auf, dass hauptsächlich Unfälle durch den ruhenden Verkehr oder Sonstige Unfälle (Wenden / Rückwärtsfahren) auftraten. 
- Die Unfallartarten im „Kollektiv 2011“ „Zusammenstoß zwischen Fahrzeug und Fußgänger“ und „Zusammenstoß mit anderem Fahrzeug, das einbiegt oder kreuzt“, kamen beide insgesamt mit 19\% vor. Im Vergleich zum „Kollektiv Kat. 1-4“ traten sie wesentlich seltener auf, im Gesamtkollektiv lag inr Wert bei jeweils 28\% (insg. 56\%).

\section{Beteiligte:}

- An Unfällen des "Kollektivs 2011“ waren Pkw etwas stärker vertreten als im „Kollektiv Kat. 1-4“. Im „Kollektiv 2011“ sind sie auch häufiger Hauptverursacher gewesen.

- Fußgänger und Radfahrer waren wesentlich seltener in Unfälle des „Kollektivs 2011“ involviert (-11\% und $-17 \%)$.

\section{Unfallursachen:}

- Hauptunfallursache von Unfällen des „Kollektivs 2011“ waren zu 40\% „Abbiegen, Wenden, Rückwärtsfahren, Ein- und Anfahren“. Im Vergleich zum „Kollektiv Kat. 1-4“ kam die Unfallursache dort zu 25\% vor.

- Die zweit häufigste Unfallursache war der „Sonstige Fehler beim Fahrzeugführer“ (25\%) und diese lag ebenfalls im Vergleich zum „Kollektiv Kat. 1-4“ (7\%) höher.

- Die Unfallursachen „Vorfahrt/Vorrang“ (5\%) sowie „falsches Verhalten gegenüber Fußgängern“ (8\%) spielten im „Kollektiv 2011“ keine große Rolle. Im „Kollektiv Kat. 1-4“ waren sie wesentlich häufiger vertreten.

\section{Unfallumstände:}

- Es lagen keine gravierenden Auffälligkeiten hinsichtlich der Unfallumstände im „Kollektiv 2011"vor.

- Tendenziell lag die Ausprägung der Unfallumstände in fast allen Merkmalen beim „Kollektiv Kat. 1-4" höher. Daraus ließ sich ableiten, dass Unfälle mit schwerwiegenderen Folgen häufiger bei Nässe/Glätte, in der Nacht, in Spitzenstunden oder am Wochenende auftraten. 


\section{ANHANG 4 Unfallanalysen „Kollektiv 2011“ nach Straßenfunktionen}

\section{Unfallkategorien / Unfalldichten / Unfallkostendichte}

Bei den vorliegenden Auswertungen wurden die Unfälle des „Kollektivs 2011“ je Straßenfunktion und ggf. auch je Verkehrsführung analysiert. Da das Unfallkollektiv nur aus 110 Unfällen und 32 Beispielen bestand, lagen selten Untergruppen vor, in denen eine ausreichend große Anzahl an Beispielen, Streckenabschnitten und Unfällen vorlag, um aussagekräftige Erkenntnisse gewinnen zu können.

Aus der Tabelle 40 ist erkennbar, dass die Untergruppen, wenn man sie je Verkehrsführung aufteilte, teilweise nur noch aus Einzelfällen bestanden. Als ausreichend groß und aussagekräftig konnte dann nur noch die Gruppe "Geschäftsstraßen mit baulicher Trennung" angesehen werden (in der Tabelle grün hinterlegt). Alle anderen Untergruppen waren nicht repräsentativ, da sie entweder zu wenige Untersuchungsobjekte oder zu kurze Abschnittslängen beinhalteten (Kapitel 11.2.1). Deshalb beschränkten sich die nachfolgenden Auswertungen ausschließlich auf die Differenzierung nach Straßenfunktionen. Selbst in diesem Fall mussten die "Sonstigen Straßen“ und "Wohnstraßen" aufgrund von Einzelfällen aus den nachfolgenden Untersuchungen ausgeschlossen werden.

Tabelle 40: Abschnittslängen, Anzahl der Unfälle und Untersuchungsobjekte des „Kollektivs 2011“ je Straßenfunktion und Verkehrsführung

\begin{tabular}{|c|c|c|c|c|}
\hline 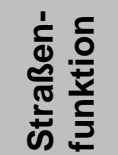 & Verkehrsführung & $\begin{array}{l}\text { Länge } \\
\text { [m] }\end{array}$ & $\begin{array}{c}\text { Unfälle Kat. } \\
1-6 \\
\text { U(ges) }\end{array}$ & $\begin{array}{c}\text { Anzahl } \\
\text { Untersuchungs } \\
\text {-objekte }\end{array}$ \\
\hline \multirow{6}{*}{ 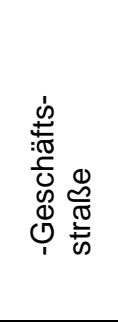 } & Bauliche Trennung & 2.788 & 60 & 10 \\
\hline & Mischverkehr & 235 & 0 & 1 \\
\hline & Optische Trennung & 1125 & 1 & 3 \\
\hline & Optische und bauliche Trennung & 1300 & 22 & 4 \\
\hline & Bauliche Trennung und Mischverkehr & 231 & 0 & 1 \\
\hline & Summe & 5.679 & 83 & 19 \\
\hline \multirow{5}{*}{ 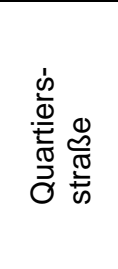 } & Bauliche Trennung & 767 & 4 & 2 \\
\hline & Mischverkehr & 110 & 2 & 1 \\
\hline & Optische und bauliche Trennung & 1661 & 17 & 5 \\
\hline & Bauliche Trennung und Mischverkehr & 508 & 0 & 1 \\
\hline & Summe & 3046 & 23 & 9 \\
\hline \multirow{4}{*}{ 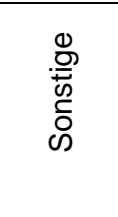 } & Bauliche Trennung & 436 & 1 & 1 \\
\hline & Optische Trennung & 69 & 3 & 1 \\
\hline & Bauliche Trennung und Mischverkehr & 656 & 0 & 1 \\
\hline & Summe & 1161 & 4 & 3 \\
\hline \multirow{2}{*}{$\begin{array}{l}\text { Wohn- } \\
\text { straße }\end{array}$} & Bauliche Trennung & 145 & 0 & 1 \\
\hline & Summe & 145 & 0 & 1 \\
\hline \multicolumn{2}{|c|}{ Summe } & 10.031 & 110 & 32 \\
\hline
\end{tabular}


In der Abbildung $\mathbf{1 2 8}$ ist die prozentuale Verteilung der Unfallkategorien in den jeweiligen Straßenfunktionen dargestellt. Hieraus war erkennbar, dass die Kategorie 5 Unfälle anteilig am häufigsten in Geschäftsstraßen auftraten. Hier machten sie einen Anteil von $71 \%$ des Unfallgeschehens der Kategorie 1-6 aus. Während der Anteil der Kategorie 5 Unfälle in Quartiersstraßen ca. 61\% ausmachte.

In Quartiersstraßen lag der Anteil der Kategorie 3 Unfälle dafür höher. Die Kategorie 1-3 Unfälle (Unfälle mit Personenschaden) besaßen in Quartiersstraßen einen Anteil von insgesamt 39\%, in Geschäftsstraßen lag er bei $28 \%$. Leider reichte die Datengrundlage nicht aus, um die Aussage zu bestätigen, dass die Dunkelziffer von Kategorie 5 Unfällen in Wohn- bzw. Quartiersstraßen besonders hoch war. Denn laut Aussagen von den Polizeibeamten wird bei den Kategorie 5 Unfällen in verkehrsberuhigten Wohnstraßen die Polizei selten zur Aufnahme des Unfalls gerufen. Hier kennen sich die Unfallbeteiligten in der Regel und wickeln den Unfall selbstständig ab (Kapitel 10.1.1). Die Unfälle der Kategorie 4 spielten eine untergeordnete Rolle und traten ausschließlich in Geschäftsstraßen auf.

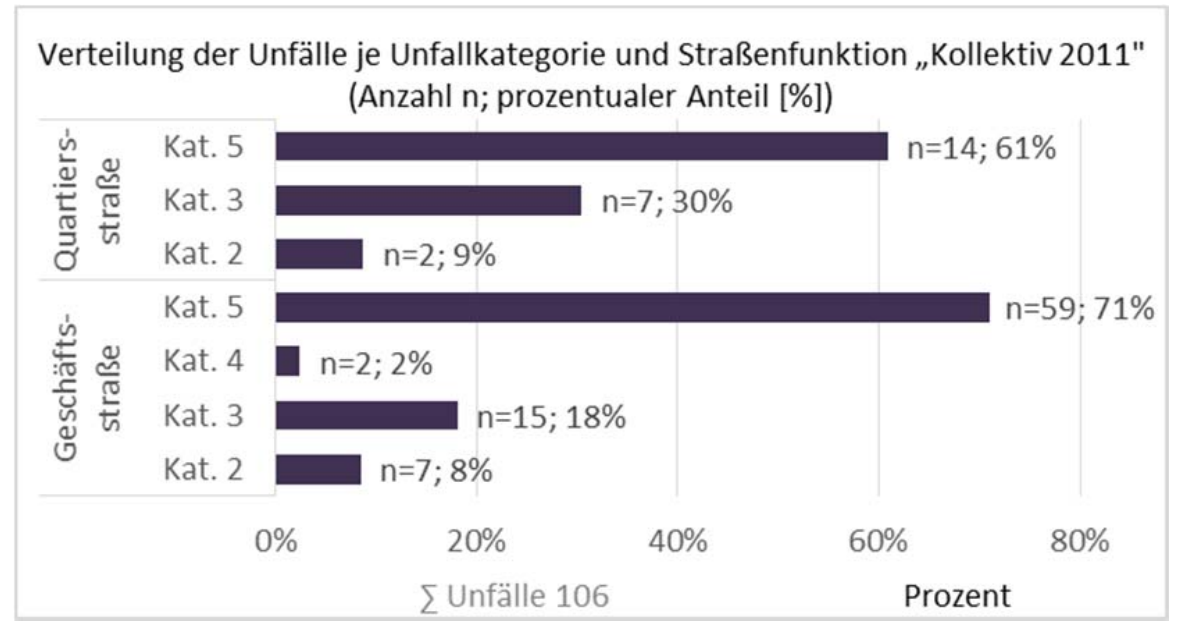

Abbildung 128: Verteilung der Unfälle je Unfallkategorie und Straßenfunktion des „Kollektivs 2011“

Die durchschnittliche Unfalldichte des „Kollektivs 2011“ der Verkehrsberuhigten Bereiche lag bei 10,97 Unfällen je km und Jahr (Abbildung 129). Die Unfalldichte der Geschäftsstraßen lag mit $14,62 \mathrm{U} /\left(\mathrm{km}^{*} \mathrm{a}\right)$ mit Abstand am höchsten. Auch die Unfalldichte der Quartiersstraßen war mit 7,55 U/(km*a) relativ hoch. Die anderen beiden Straßenfunktionen werden - auch im Folgenden ohne weiter darauf hinzuweisen - aus den o.g. Gründen nicht kommentiert.

Beim „Kollektiv Kat. 1-4“ lag im Schnitt nur ein Unfall der Kategorie 1-4 je 2 km vor (Abbildung 76). Beim „Kollektiv 2011“, welches die Kategorie 5-6 Unfälle mit berücksichtigte, lagen natürlich mehr Unfälle je Kilometer vor (11 Stück). Nicht nur, weil die Kategorie 5-6 Unfälle additiv hinzukamen, sondern auch, weil das Kollektiv die besonders unfallauffälligen Bereiche beinhaltete. Deshalb konnten die Unfalldichten in ihrer Anzahl nicht direkt mit einem anderen Kollektiv verglichen werden.

Resümierend kann festgehalten werden, dass Geschäftsstraßen auch im „Kollektiv 2011“ die höchsten Unfalldichten aufwiesen. 


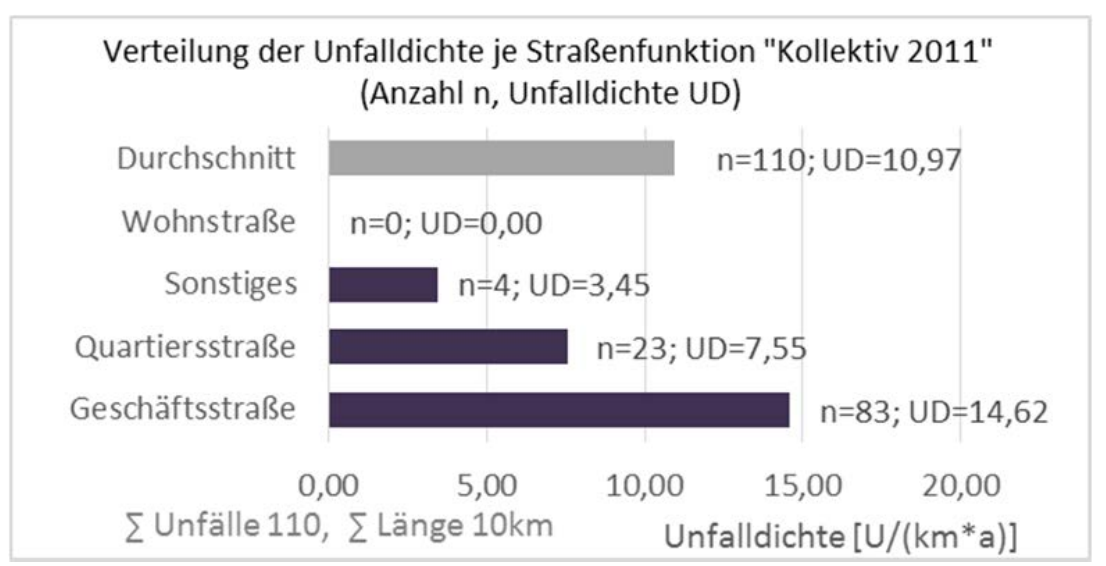

Abbildung 129: Verteilung der Unfalldichte je Straßenfunktion des "Kollektivs 2011"

\section{Unfallkostendichte}

Bei der Berechnung der Unfallkosten wurde wie im Kapitel 11.2.1 auf die pauschalen Unfallkostensätze aus dem Jahr 2009 zurückgegriffen. Für die Kategorie 5 Unfälle wurde zusätzlich der pauschale Unfallkostensatz für einen Unfall mit leichtem Sachschaden WU(LS) mit $5.930 €$ in Erschließungsstraßen in die Berechnungen integriert.

Die durchschnittliche Unfallkostendichte für Unfälle der Kategorie 1-6 in den unfallauffälligen verkehrsberuhigten Bereichen erschien auf den ersten Blick mit $235.000 € / \mathrm{km}$ sehr hoch zu sein (Abbildung 130). Zu vermuten wäre, dass aufgrund der geringeren Anzahl der Kategorie 1-4 Unfälle, auch die Unfallkosten geringer ausfallen würden. Die Anzahl der Kategorie 5 Unfälle war jedoch so erheblich hoch, dass auch die Unfallkosten aus den oben genannten Gründen nicht mit dem Wert des „Kollektivs Kat. 1-4“ verglichen werden könnten.

Die Unfallkostendichten verteilten sich ähnlich wie die Unfalldichten. Die Unfallkostendichte der Geschäftsstraßen lag mit $294.000 € /(\mathrm{km} * a)$ mit Abstand auch hier am höchsten.

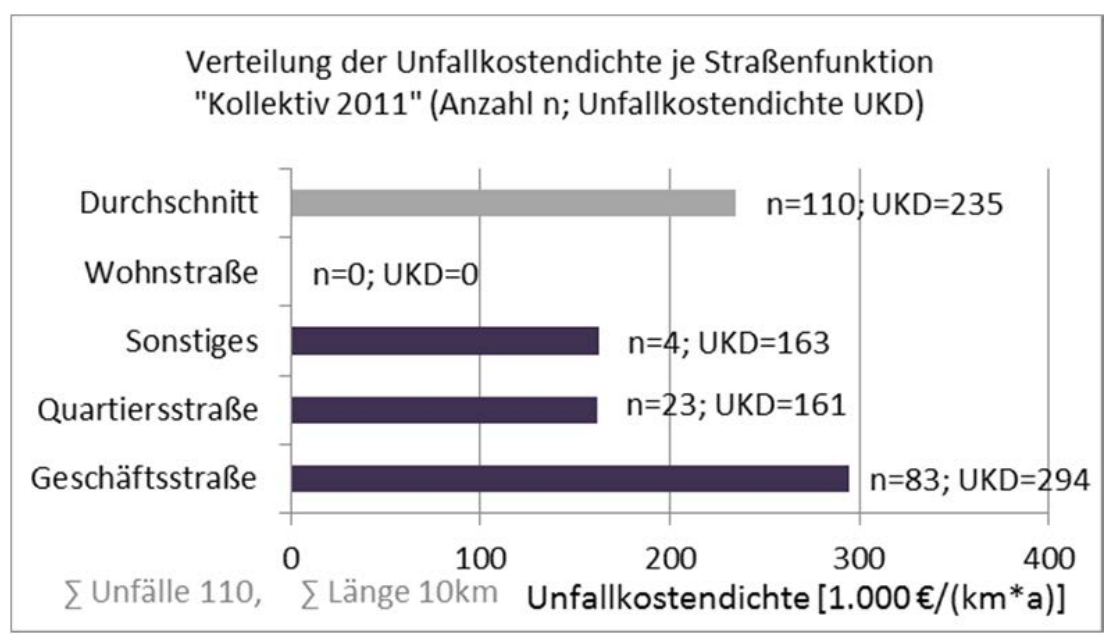

Abbildung 130: Verteilung der Unfallkostendichte je Straßenfunktion des "Kollektivs 2011"“, Preisstand 2009 


\section{Unfalltypen}

Die Verteilung der Unfalltypen je Straßenfunktion des „Kollektivs 2011“ kann der Abbildung 131 entnommen werden.

Auffällig war, dass in Geschäftsstraßen der Haupt-Unfalltyp „sonstiger Unfall“ mit 52\% und der zweithäufigste Unfalltyp „Unfall durch ruhendem Verkehr" mit 22\% war, während in den Quartiersstraßen sich die Anteile über die Unfalltypen „Einbiegen/Kreuzen“ und „sonstiger Unfall“ zu jeweils 35\% verteilten. „Unfälle durch ruhenden Verkehr" kamen in Quartiersstraßen nur in $13 \%$ der Fälle vor.

Bei dem Vergleich der Verteilung der Unfalltypen des „Kollektivs 2011“ mit denen des „Kollektivs Kat. 1-4" fiel auf, dass sich die Verteilung der Unfalltypen in Quartiersstraßen nicht wesentlich änderte. Bei den Geschäftsstraßen war jedoch eindeutig eine Verschiebung der Haupt-Unfalltypen zu erkennen. Durch die Berücksichtigung der Kategorie 5 Unfälle war der Haupt-Unfalltyp eindeutig der „Sonstige Unfall“. Die „Einbiegen/Kreuzen-Unfälle“ waren mit einem Anteil von 5\% bedeutend niedriger. Der zweithäufigste Unfalltyp war im „Kollektiv 2011“ bei den Geschäftsstraßen der „Unfall durch ruhenden Verkehr".

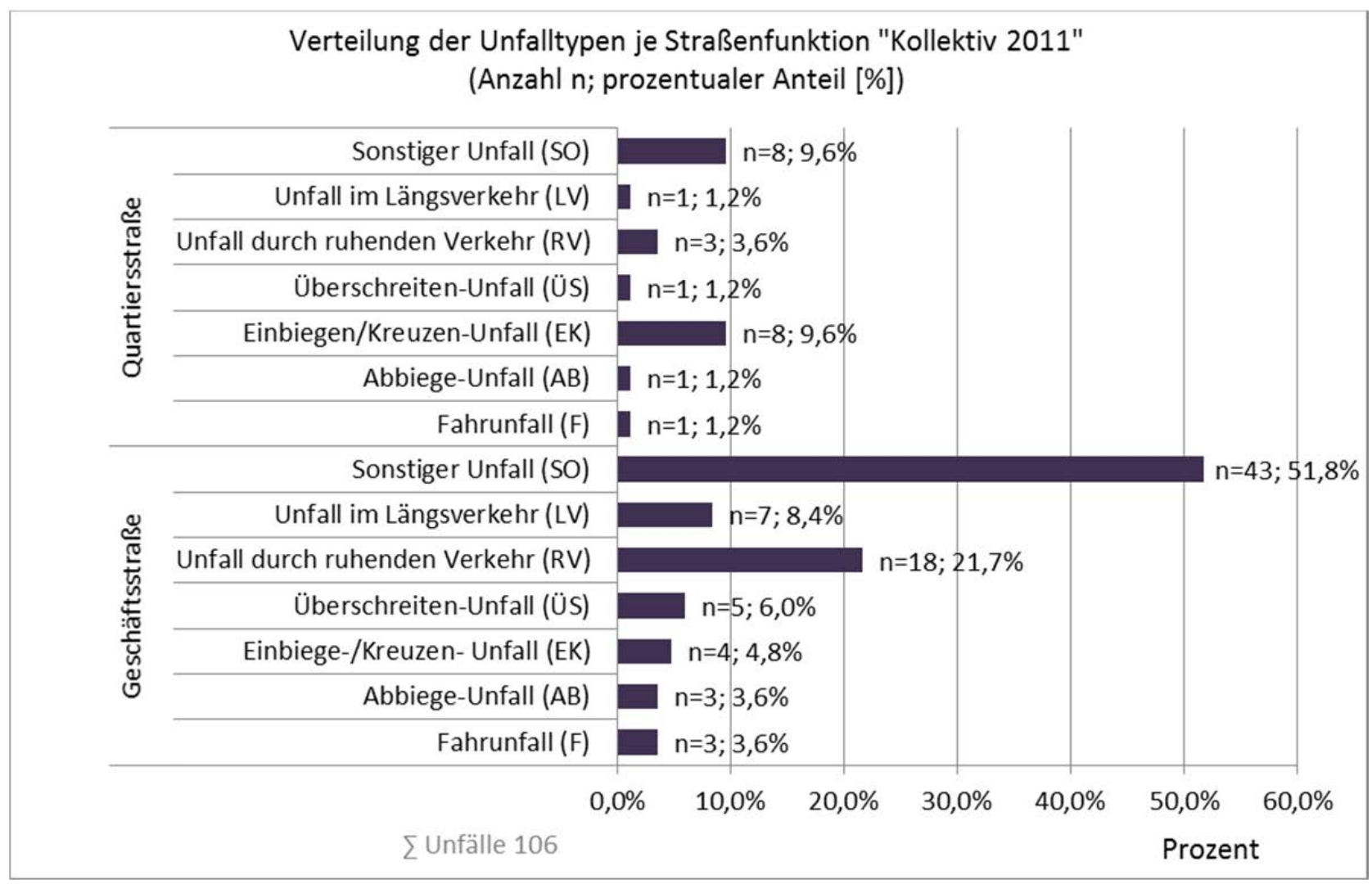

Abbildung 131: Verteilung der Unfalltypen je Straßenfunktion des „Kollektivs 2011“

Eine weitergehende differenzierte Betrachtung, an welchen Unfalltypen Fußgänger und Radfahrer beteiligt sind, war nicht sinnvoll, da in den jeweiligen Straßenfunktionen die Anzahl der beteiligten Fußgänger und Radfahrer sehr gering war. 


\section{Unfallart}

Bei den Quartiersstraßen lag der Anteil der „Zusammenstöße mit anderem Fahrzeug, das anfährt, anhält oder im ruhenden Verkehr steht“ mit 35\% höher als der Anteil der „Zusammenstöße mit anderem Fahrzeug, das einbiegt oder kreuzt“ (30\%) oder einem „Unfall anderer Art“ (17\%) (Abbildung 132).

Die Haupt-Unfallart in Geschäftsstraßen war der „Zusammenstöße mit anderem Fahrzeug, das anfährt, anhält oder im ruhenden Verkehr steht" (51\%). In Geschäftsstraßen waren rund 27\% der Unfälle „Unfälle anderer Art“.

Vergleicht man die Haupt-Unfallarten des „Kollektivs 2011“ mit denen des „Kollektivs Kat. 1-4“, so änderten sich diese in beiden betrachteten Straßenfunktionen. In Quartiersstraßen und Geschäftsstraßen des „Kollektivs 2011“ spielte die im „Kollektiv Kat. 1-4“ sehr häufig vorkommende Unfallart „Zusammenstoß zwischen Fahrzeug und Fußgänger" nur noch eine untergeordnete Rolle.

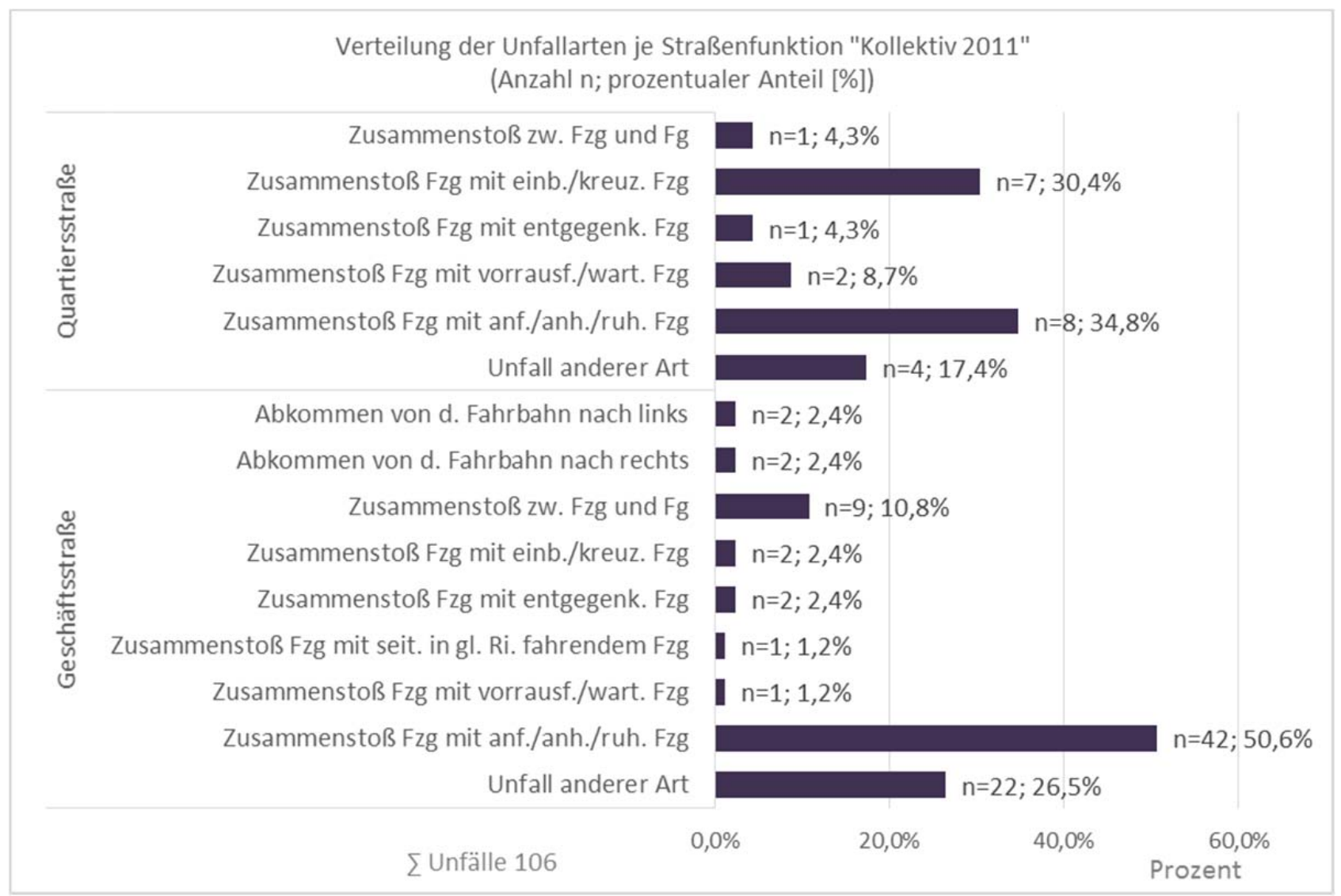

Abbildung 132: Verteilung der Unfallarten je Straßenfunktion des „Kollektivs 2011“

\section{Beteiligte}

An dieser Stelle wurden aus Gründen der Übersichtlichkeit nur die drei Beteiligten Fußgänger, Radfahrer und Pkw untersucht. Zu bedenken war, dass im „Kollektiv 2011“ wesentlich häufiger Kfz beteiligt waren und die nicht motorisierten Verkehrsteilnehmer sehr selten in Unfälle verwickelt wurden. Zudem lagen in fast der Hälfte der vorliegenden Unfalldaten des „Kollektivs 2011“ keine Angaben zum Beteiligten 2 vor. Diese wurden deshalb zahlenmäßig nicht berücksichtigt. 
Aus Tabelle 41 ist zu entnehmen, dass die Beteiligung von Radfahrern an Unfällen des „Kollektivs 2011“ in Quartiersstraßen höher war, als in Geschäftsstraßen. Fußgänger waren sehr selten an Unfällen der Kategorie 1-6 in Verkehrsberuhigten Bereichen verwickelt. Am häufigsten waren hier Pkws in Unfälle involviert.

Beim Vergleich der Beteiligung der Verkehrsteilnehmer des „Kollektivs 2011“ mit der des „Kollektivs Kat. 1-4" fiel auf, dass Radfahrer in Geschäftsstraßen unter Beachtung der Kategorie 5 Unfälle wesentlich seltener in einem Unfall involviert waren.

Eine vertiefende Betrachtung der Beteiligten je Verkehrsführung ist aufgrund der geringen Anzahl von Daten in den Untergruppen an dieser Stelle nicht sinnvoll gewesen.

Tabelle 41: Beteiligte Radfahrer, Fußgänger und Pkw je Straßenfunktion des „Kollektivs 2011“

\begin{tabular}{|c|c|c|c|c|c|c|c|}
\hline \multirow{3}{*}{ Straßenfunktion } & \multicolumn{6}{|c|}{ Beteiligte } & \multirow{3}{*}{$\begin{array}{c}\text { Anzahl } \\
\text { Unfälle } \\
\text { U(Kat.1-6-) }\end{array}$} \\
\hline & \multicolumn{2}{|c|}{ Radfahrer } & \multicolumn{2}{|c|}{ Fußgänger } & \multicolumn{2}{|c|}{ Pkw } & \\
\hline & Anzahl & Anteil & Anzahl & Anteil & Anzahl & Anteil & \\
\hline Geschäftsstraße & 8 & $10 \%$ & 7 & $8 \%$ & 65 & $78 \%$ & 83 \\
\hline Quartiersstraße & 7 & $29 \%$ & 1 & $4 \%$ & 19 & $79 \%$ & 24 \\
\hline Sonstiges & 1 & $33 \%$ & 0 & $0 \%$ & 2 & $67 \%$ & 3 \\
\hline Summe & 16 & & 8 & & 86 & & 110 \\
\hline
\end{tabular}

\section{Unfallursachen}

Wie auch in den vorherigen Auswertungen zu den Unfallursachen wurden nur die (Haupt-) Unfallursachen bzw. die ersten Beteiligten (Hauptverursacher) näher betrachtet. Insbesondere im vorliegenden Kollektiv fehlten in mehr als der Hälfte der Unfälle Angaben zum zweiten Beteiligten.

Die Haupt-Unfallursache von Quartiersstraßen und Geschäftsstraßen war „Abbiegen, Wenden, Rückwärtsfahren, Ein- und Anfahren" (Abbildung 133). Sie stachen eindeutig mit einem Anteil von $35 \%$ und $42 \%$ hervor. Sie war auch im „Kollektiv Kat. 1-4“ die Haupt-Unfallursache in den Straßenfunktionen, wobei hier die Anteile etwas niedriger ausfielen, so um die $25 \%$. 


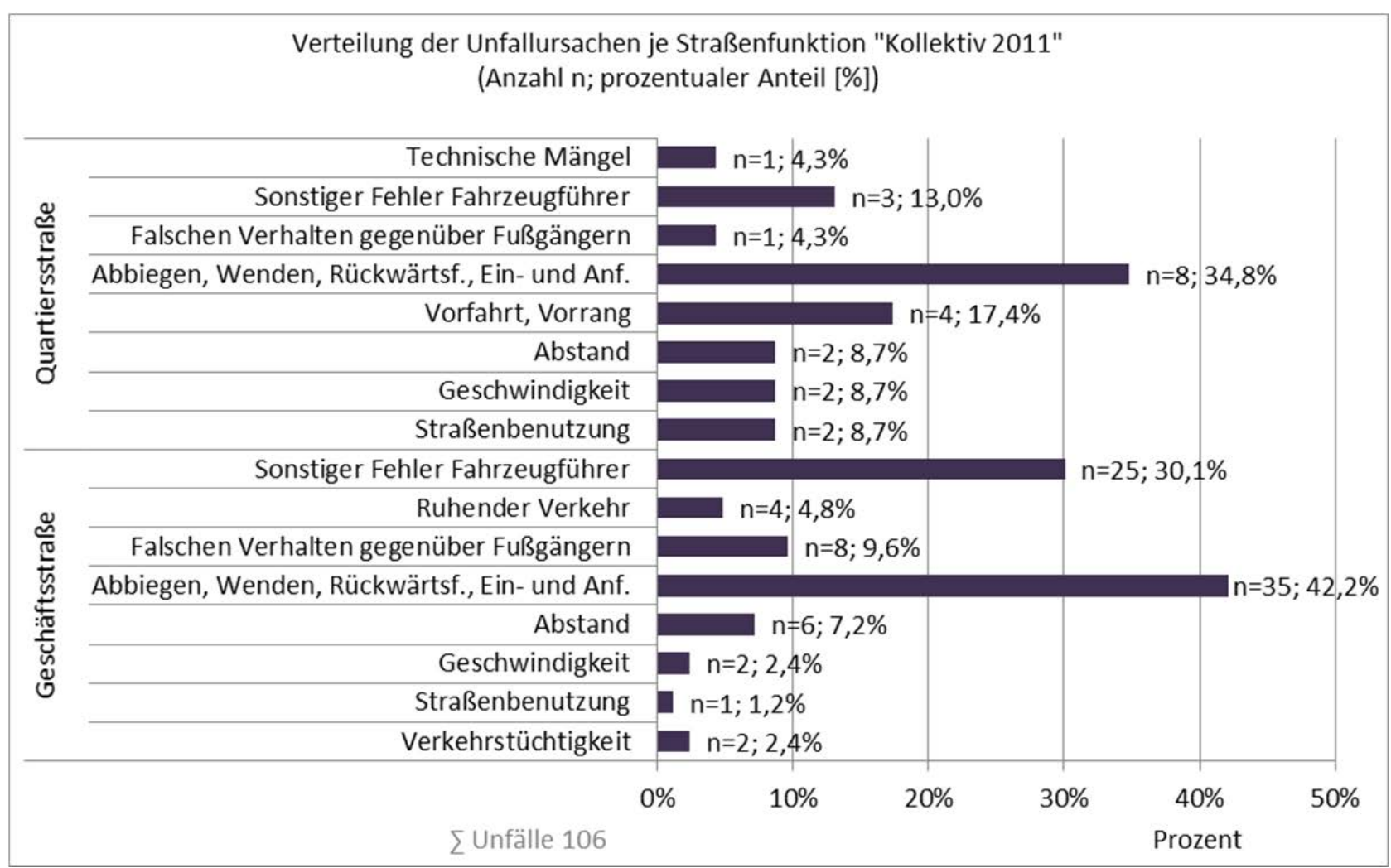

Abbildung 133: Verteilung der Unfallursachen je Straßenfunktion des „Kollektivs 2011“

\section{Unfallumstände}

Die Unfallumstände in den einzelnen Straßenfunktionen wiesen keine Auffälligkeiten im „Kollektiv 2011" auf (Abbildung 134).

Im Vergleich zum „Kollektiv Kat. 1-4“ traten in Geschäftsstraßen seltener Unfälle der Kategorie 1-6 in den Spitzenzeiten auf. In den Quartiersstraßen lagen die Unfälle nicht nur in den Spitzenzeiten deutlich unter den Werten des „Kollektivs Kat. 1-4“ sondern auch im Winter.

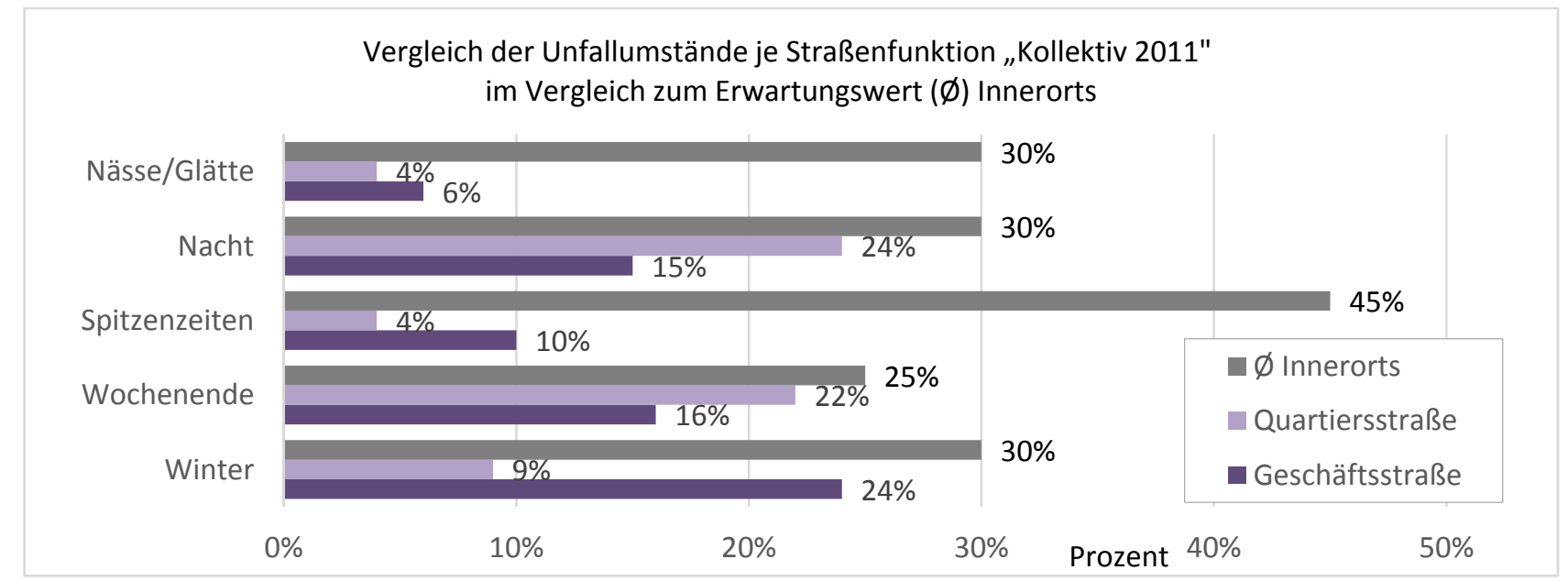

Abbildung 134: Vergleich der Unfallumstände je Straßenfunktion des "Kollektivs 2011" im Vergleich zum Erwartungswert (Ø) Innerorts 


\section{Übergangsbereiche}

Für die Unfälle der Kategorie 5 wurden aufgrund des Umfangs keine Verortungen in den Steckbriefen durchgeführt, so dass eine Auswertung hinsichtlich des Kriteriums „Übergangsbereich“ nicht möglich war. Für einen großen Teil der Unfälle der Kategorie 5 wurde aber überschlägig recherchiert, dass diese häufig nicht in Übergangsbereichen lagen. Dies passte auch zu den Auswertungen bezüglich der Haupt-Unfalltypen der Kategorie 5 Unfälle. Gemäß Abbildung 122 lag in 61\% der Kategorie 5 Unfälle der Unfalltyp „Sonstiger Unfall“ vor, bei dem die Haupt-Unfallart der „Zusammenstoß mit anderem Fahrzeug, das anfährt, anhält oder im ruhenden Verkehr steht“ war. Und in weiteren 21\% waren die Kategorie 5 Unfälle vom Typ „Unfall durch ruhenden Verkehr". Dies waren alles keine typischen Unfälle, die in Übergangsbereichen (häufig Einmündungen oder Kreuzungen) existieren.

\section{Streckenabschnittslängen}

Die Auswertung der Streckenabschnittlängen erfolgte analog zu Kapitel 11.2.8. Die Unfalldichte der Kategorie 1-6 Unfälle je Abschnittslänge sollte Aufschluss darüber geben, ob mehr Unfälle je $\mathrm{km}$ in kürzeren oder längeren Streckenabschnitten vorlagen. Aus diesem Grund wurden die Unfalldichten je Abschnittslänge untersucht. Hierzu wurden die Beispiele des „Kollektivs 2011“ je Abschnittslänge wieder in Intervallen zusammengefasst (0m bis $99 \mathrm{~m}, 100 \mathrm{~m}$ bis $199 \mathrm{~m}$, etc.).

Aus Abbildung 135 ist zu erkennen, dass tendenziell der Trend so aussah, dass längere Abschnitte geringere Unfalldichten aufwiesen. Dies konnte auch schon beim „Kollektiv Kat. 1-4“ festgestellt werden.

Ebenfalls ist aus der Abbildung zu erkennen, dass die Verkehrsberuhigten Bereiche mit einer Länge zwischen $200 \mathrm{~m}$ und $300 \mathrm{~m}$ wieder die geringsten Unfalldichten aufwiesen (Abbildung 90). Verkehrsberuhigte Bereiche mit einer Länge von 0-100m und 4-500m besaßen die höchsten Unfalldichten.

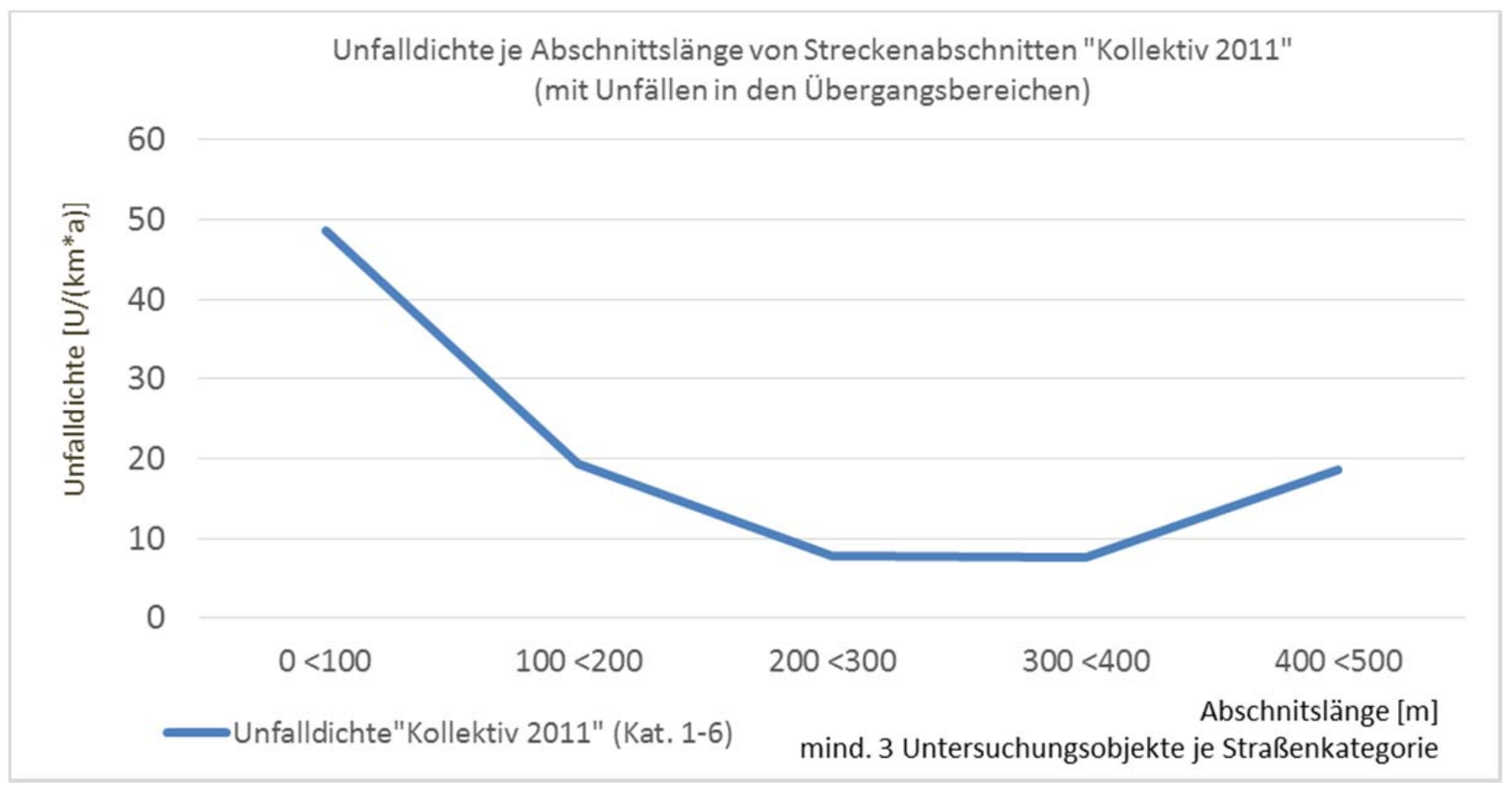

Abbildung 135: Unfalldichte je Abschnittslänge des "Kollektivs 2011" 


\section{Zusammenfassung der Ableitung von Zusammenhänge zwischen Unfällen des „Kollektivs 2011" und der Straßenfunktion}

Resümierend werden folgende Zusammenhänge zwischen Unfällen der Kategorie 1-6 in Verkehrsberuhigten Bereichen und der Gestaltung festgehalten:

\section{Unfallkategorien / Unfalldichten / Unfallkostendichten}

- Der Anteil der Kategorie 5 Unfälle war in Geschäftsstraßen mit $71 \%$ am höchsten. In Quartiersstraßen lag er mit 61\% etwas niedriger.

- Die Unfalldichten und Unfallkostendichten der Kategorie 1-6 Unfälle lagen in Geschäftsstraßen am höchsten (dies verhält sich ebenso beim „Kollektiv Kat. 1-4“).

\section{Unfalltyp}

- In Geschäftsstraßen war der Haupt-Unfalltyp mit 52\% der „Sonstige Unfall“. Der zweithäufigste Unfalltyp war der „Unfall mit ruhendem Verkehr“ (22\%).

- In den Quartiersstraßen lagen zwei Haupt-Unfalltypen vor. Zum einen „Einbiegen/KreuzenUnfälle“ und zum anderen „Sonstige Unfälle“ (jeweils zu 35\%). „Unfälle durch ruhenden Verkehr" kamen in Quartiersstraßen nur in 13\% der Fälle vor.

- Beim Vergleich des „Kollektivs 2011“ mit dem „Kollektiv Kat. 1-4“ fiel auf, dass sich die Verteilung der Unfalltypen in Quartiersstraßen nicht wesentlich änderte. Bei den Geschäftsstraßen war jedoch eindeutig eine Verschiebung der Haupt-Unfalltypen zu erkennen. Durch die Berücksichtigung der Kategorie 5 Unfälle war einer der HauptUnfalltypen nicht mehr der „Einbiegen/Kreuzen-Unfall“ sondern der „Unfall durch ruhenden Verkehr".

\section{Unfallart}

- Die Haupt-Unfallart in Geschäftsstraßen war der „Zusammenstoß mit anderem Fahrzeug, das anfährt, anhält oder im ruhenden Verkehr steht" (51\%).

- Bei den Quartiersstraßen lag der Anteil der „Zusammenstöße mit anderem Fahrzeug, das anfährt, anhält oder im ruhenden Verkehr steht" mit 35\% am höchsten.

- Beim Vergleich der Haupt-Unfallarten des „Kollektivs 2011“ mit denen des „Kollektivs Kat. 14" fiel auf, dass sich diese unterscheiden. In Quartiersstraßen und Geschäftsstraßen des „Kollektivs 2011“ spielte die im „Kollektiv Kat. 1-4“ sehr häufig vorkommende Unfallart „Zusammenstoß zwischen Fahrzeug und Fußgänger" nur noch eine untergeordnete Rolle.

\section{Beteiligte}

- Die Beteiligung von Radfahrern an Unfällen des „Kollektivs 2011“ war in Quartiersstraßen höher als in Geschäftsstraßen.

- Fußgänger sind sehr selten an Unfällen der Kategorie 1-6 in Verkehrsberuhigten Bereichen verwickelt gewesen.

- Bei dem Vergleich der Beteiligung der Verkehrsteilnehmer des „Kollektivs 2011“ mit der des „Kollektivs Kat. 1-4“ fiel auf, dass Radfahrer in Geschäftsstraßen unter Beachtung der Kategorie 5 Unfälle wesentlich seltener in einem Unfall der Kategorie 1-6 involviert waren.

\section{Unfallursachen}

- Die Hauptunfallursache von Quartiersstraßen und Geschäftsstraßen war „Abbiegen, Wenden, Rückwärtsfahren, Ein- und Anfahren“. Sie liegt mit 35\% und 42\% deutlich über den Anteilen des „Kollektivs Kat. 1-4“. Hier lag die Haupt-Unfallursache auch in 
„Abbiegen, Wenden, Rückwärtsfahren, Ein- und Anfahren“, wobei die Anteile etwas niedriger ausfielen, so um die $25 \%$.

\section{Unfallumstände}

- Die Unfallumstände in den einzelnen Straßenfunktionen wiesen keine Auffälligkeiten im „Kollektiv 2011“ auf.

\section{Streckenabschnittslängen}

- Verkehrsberuhigte Bereiche mit einer Länge zwischen 200 und 300m wiesen (wie auch im „Kollektiv Kat. 1-4) die geringsten Unfalldichten auf.

- Die höchsten Unfalldichten lagen in sehr kurzen Verkehrsberuhigten Bereichen vor (bis zu $100 \mathrm{~m}$ ) und solchen, deren Abschnittslänge zwischen 400 und 500m lang war (ebenfalls wie im „Kollektiv Kat. 1-4“). 


\section{ANHANG 5 Unfallkosten}

Es wurde untersucht, ob aufgrund einer ggf. unterschiedlichen Verunglücktenstruktur bei den Unfällen der Kat. 1-4 von ausgewiesenen VBB, es sinnvoll gewesen wäre, angepassten Unfallkostensätzen anzuwenden.

Bei der Verwendung der pauschalen Unfallkostensätze können sich Ungenauigkeiten durch die Änderungen der Struktur im Unfallgeschehen und/oder der Verunglücktenstruktur ergeben. Grundsätzlich ist es daher sinnvoll, angepasste Unfallkostensätze anzuwenden, die die Verunglücktenstruktur des vorliegenden Kollektivs berücksichtigen. Angepasste Unfallkostensätze entstehen auf der Grundlage der Kostensätze für Verunglückte und der Sachschäden bei Unfällen. Diese liegen im Merkblatt für die Auswertungen von Straßenverkehrsunfällen Teil 2 (FGSV 316/2, 2001) jedoch nur für das Jahr 2000 vor. Um zu untersuchen, ob eine Anpassung der Unfallkostensätze des vorliegenden Kollektivs zu wesentlichen Unterschiede im Vergleich zu den pauschalen Unfallkostensätzen führt, wurde eine indirekte ${ }^{15}$ Anpassung der Unfallkostensätze zum Preisstand 2000 durchgeführt und mit den pauschalen Unfallkostensätzen zum Preisstand 2000 verglichen.

Die angepassten Unfallkostensätze wurden wie folgt ermittelt (FGSV 316/2, 2001, S. 141):

\begin{tabular}{|c|c|}
\hline $\begin{aligned} \mathrm{WUa}(\mathrm{SP}) & =(\mathrm{V}(\mathrm{GT}) \cdot \mathrm{WV}(\mathrm{GT})+\mathrm{V}(\mathrm{SV}) \cdot \mathrm{WV}(\mathrm{SV}) \\
& +\mathrm{V}(\mathrm{LV}) \cdot \mathrm{W}(\mathrm{LV})+\mathrm{U}(\mathrm{SP}) \cdot \mathrm{WUS}(\mathrm{SP}) / \mathrm{U}(\mathrm{SP})\end{aligned}$ & [Euro/U] \\
\hline $\mathrm{WUa}(\mathrm{LV})=(\mathrm{V}(\mathrm{LV}) \cdot \mathrm{W}(\mathrm{LV})+\mathrm{U}(\mathrm{LV}) \cdot \mathrm{WUS}(\mathrm{LV})) / \mathrm{U}(\mathrm{LV})$ & [Euro/U] \\
\hline
\end{tabular}

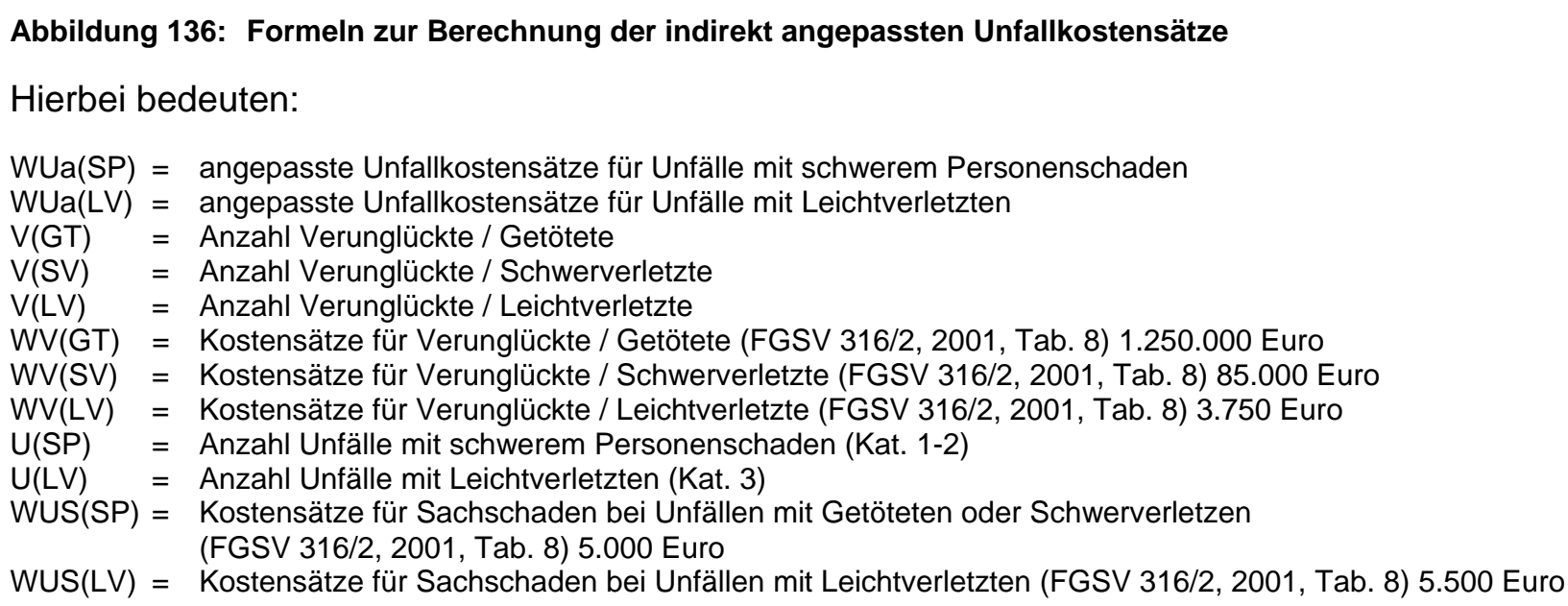

Das Ergebnis ist Tabelle 42 zu entnehmen. Die angepassten Unfallkostensätze unterscheiden sich nicht wesentlich voneinander. Daraus lässt sich schlussfolgern, dass die Struktur der Verunglückten der ausgewiesenen Verkehrsberuhigten Bereiche sich nicht wesentlich von den Erschließungsstraßen unterscheidet.

15 Das vorliegende Kollektiv konnte nur indirekt angepasst werden, da eine direkte Anpassung erst bei einem Kollektiv mit mindestens 400 Unfällen mit Personenschaden sinnvoll ist (MAS T2, Anhang 2). 
Tabelle 42: $\quad$ Indirekt angepasste und pauschale Unfallkostensätze Preisstand 2000

\begin{tabular}{|l|c|c|}
\hline & U(SP) & U(LV) \\
\hline $\begin{array}{l}\text { Indirekt angepasste Unfallkostensätze WUa für } \\
\text { Verkehrsberuhigte Bereiche Preisstand 2000 }\end{array}$ & $137.348 € / \mathrm{U}$ & 9.586 €/U \\
\hline $\begin{array}{l}\text { Pauschale Unfallkostensätze WU für } \\
\text { Erschließungsstraßen Preisstand 2000 }\end{array}$ & $130.000 € / \mathrm{U}$ & $10.000 € / \mathrm{U}$ \\
\hline
\end{tabular}

Einen wesentlichen Unterschied machte es hingegen, wenn die pauschalen Unfallkostensätze aus dem Jahr 2009 angewendet werden. Diese liegen deutlich über den Kostensätzen aus dem Jahr 2000 (Tabelle 43). Der Preisstand 2009 wurde aus dem durchschnittlichen Unfallgeschehen der Jahre 2005-2009 in Deutschland berechnet und passt somit zeitlich besser zum vorliegenden Unfallkollektiv der ausgewiesenen Verkehrsberuhigten Bereiche. Daher wurden die pauschalen Unfallkostensätze zum Preisstand 2009 in der vorliegenden Studie angewendet.

Tabelle 43: $\quad$ Pauschale Unfallkostensätze Preisstand 2009

\begin{tabular}{|l|c|c|c|}
\hline & U(SP) & U(LV) & U(SS) \\
\hline $\begin{array}{l}\text { Pauschale Unfallkostensätze WU für } \\
\text { Erschließungsstr. Preisstand 2009 }\end{array}$ & $154.000 € / U$ & $14.400 € / U$ & $14.100 € / U$ \\
\hline
\end{tabular}




\section{ANHANG 6 Beschreibung der Kameratechnik und Fehleranfälligkeit}

Die Verkehrsbeobachtung sowie die -erhebung erfolgten mittels Videokameras. Die Videokameras hatten eine so geringe Auflösung, dass weder die Kennzeichen der Fahrzeuge, noch Gesichter der Fahrer, Fahrradfahrer oder Fußgänger erkannt werden konnten. Die Auflösung der eingesetzten Kameras und Rekorder waren:

- Kamera: 752x582 Pixel D1,

- Rekorder: 704x576 Pixel,

- Bildrate: 10 Bilder pro Sekunde.

Die Kameras/Rekorder hatten eine Festbrennweite und einen Blickwinkel von $60^{\circ}$ bis $92^{\circ}$. Während der Videobeobachtung wurde kein Zoom eingesetzt.

Nach dem Bundesdatenschutzgesetz (und beispielsweise dem Datenschutzgesetz NordrheinWestfalen) sind personenbezogene Daten Einzelangaben über persönliche oder sachliche Verhältnisse einer bestimmten oder bestimmbaren natürlichen Person. Im Rahmen der durchgeführten Studie wurden keine dieser personenbezogenen Daten erhoben. Einzelangaben über persönliche oder sachliche Verhältnisse sind beispielsweise:

- Name, Alter, Familienstand, Geburtsdatum;

- Anschrift, Telefon-Nr., E-Mail-Adresse;

- Konto-Nr., Kreditkarten-Nr.;

- Kraftfahrzeug-Nr., Kfz-Kennzeichen

Die Kameras wurden an einem Fremdmast, in der Regel ein Beleuchtungsmast, auf einer Höhe von etwa $4 \mathrm{~m}$ bis $5 \mathrm{~m}$ angebracht. Zum Schutze der Kameras wurden sie zusammen mit einem schwarzen Kunststoffgehäuse, welches sich außerhalb der Kameras befand, aufgehängt. Befestigt wurde das Gehäuse inklusive Kamera mit einem sogenannten „SpeedyTie“, welches eine wieder lösbare, flexible und äußerst belastbare Halterung darstellt. Durch das „SpeedyTie" wurden die Fremdmasten nicht beschädigt. Der „Messfehler“ bei einer solchen Geschwindigkeitserhebung liegt auf $100 \mathrm{~m}$ bei $\pm 1 \mathrm{~km} / \mathrm{h}$ und wurde als hinreichend genau anerkannt, da es um Einstufungen in Größenordnungen ging und nicht um eine exakte km/h-Angabe.
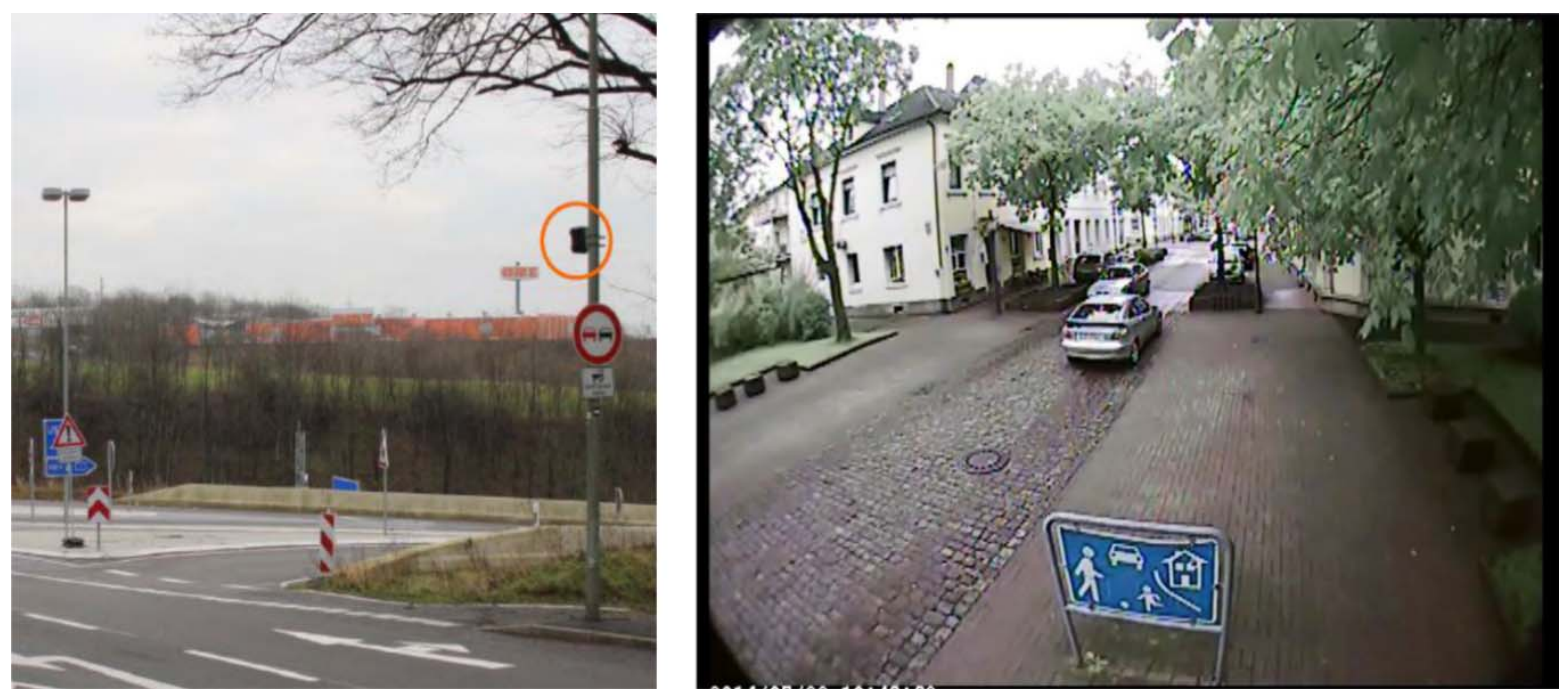

Abbildung 137: Beispielanbringung Kamera zur Verkehrsbeobachtung (links; Foto stammt nicht aus dem Projekt), sowie ein beispielhafter Blickwinkel (rechts) 


\section{Literaturverzeichnis}

BfS, 1980

Beratungsstelle für Schadensverhütung - Verband der Autoversicherer

Verkehrsberuhigung in Wohnbereichen

Empfehlungen Nr. 1 der Beratungsstelle für Schadensverhütung

Köln, Februar 1980

BfS, 1981

Beratungsstelle für Schadensverhütung - Verband der Autoversicherer

Erfahrungen mit „Verkehrsberuhigten Bereichen“ (Zeichen 325/326 StVO)

Information der Beratungsstelle für Schadenverhütung

Köln, März 1981

BfS, 1986

Beratungsstelle für Schadensverhütung - Verband der Autoversicherer

Verkehrserschließung von Wohnbereichen

Empfehlungen Nr. 6 der Beratungsstelle für Schadensverhütung,

Köln, 1986

BRBS 1992

Bundesministerium für Raumordnung, Bauwesen und Städtebau, u.a. [Hrsg.]

Forschungsvorhaben „Flächenhafte Verkehrsberuhigung - Folgerung für die Praxis“

Bonn, März 1992

Destatis 2013

Statistisches Bundesamt [Hrsg.]

Verkehrsunfälle - Zeitreihen

Wiesbaden, Juli 2013

Destatis, 2012

Statistisches Bundesamt [Hrsg.]

Fachserie 8, Reihe 7

Wiesbaden, 2012

Destatis 2011

Statistisches Bundesamt [Hrsg.]

Fachserie 8, Reihe 7

Wiesbaden, 2011

FGSV 220, 2012

Forschungsgesellschaft für Straßen- und Verkehrswesen (FGSV) [Hrsg.]

Begriffsbestimmung - Teil: Verkehrsplanung, Straßenentwurf und Straßenbetrieb

FGSV Nr. 220; Ausgabe 2012 
FGSV 316/1, 2012

Forschungsgesellschaft für Straßen- und Verkehrswesen (FGSV)

Merkblatt zur Örtlichen Unfalluntersuchung in Unfallkommissionen (M Uko)

FGSV Nr. 316/1, Ausgabe 2012

FGSV 316/2, 2001

Forschungsgesellschaft für Straßen- und Verkehrswesen (FGSV)

Merkblatt für die Auswertung von Straßenverkehrsunfällen, Teil 2: Maßnahmen gegen

Unfallhäufungen

FGSV Nr. 316/2, Ausgabe 2001

FGSV 200, 2006

Forschungsgesellschaft für Straßen- und Verkehrswesen (FGSV)

Richtlinie für die Anlage von Stadtstraßen RASt

FGSV Nr. 200, Ausgabe 2006

Henschel, König, Dauer, 2013

Straßenverkehrsrecht

Kommentar

42. Auflage, 2013

Müller, 2009

Dieter Müller

Kommentierung der Straßenverkehrsordnung

in: Ferner, Bachmeier, Müller: Fachanwaltskommentar Verkehrsrecht

Köln, 2009

MWMV 1979

Ministerium für Wirtschaft, Mittelstand und Verkehr des Landes NRW [Hrsg.]

Großversuch „Verkehrsberuhigung in Wohngebieten“

Schlussbericht der Beratergruppe

Köln, 1979, Kirschbaumverlag

Stvo 2013

Neuerlass der Straßenverkehrsordnung (StVO) vom 06.03.2013

Bundesgesetzblatt I, S. 367

Topp 2011

Hartmut H. Topp

Die Verkehrsberuhigung geht weiter!

In Straßenverkehrstechnik, Heft 5, 2011, S. 314-321

\section{VWV-StVO 2009}

Allgemeine Verwaltungsvorschrift zur Straßenverkehrsordnung (VWV-StVO) in der Fassung vom 22. Oktober 1998, in der Fassung vom 26. Januar 2001, Änderung vom 18. Januar 2001, vom 11. August 2005, vom 10. April 2006, vom 20. März 2008, vom 17. Juli 2009 


\section{Abbildungsverzeichnis}

Abbildung 1: $\quad$ Verkehrszeichen 325.1 und 325.2 gemäß StVO, Stand 2013 (Beginn und Ende eines Verkehrsberuhigten Bereichs)

Abbildung 2: $\quad$ Verkehrszeichen 274.1 und 274.2 gemäß StVO, Stand 2013 (Beginn und Ende einer Tempo 30-Zone)

Abbildung 3: $\quad$ Beispielhafte Darstellung einer Mischfläche (Foto: IGS mbH 2013)

Abbildung 4: $\quad$ Beispielhafte Darstellung einer punktuellen Einengung im Straßenraum zur Reduzierung der Fahrgeschwindigkeit (Foto: IGS mbH 2013)

Abbildung 5: Beispielhafte Darstellung eines Verkehrsberuhigten Bereiches mit hohem Parkdruck

Abbildung 6:

Abbildung 7:

Abbildung 8:

Abbildung 9:

Untersuchungsmethodik

Definition der Unfallkategorien (Quelle: UDV)

Definition der Unfalltypen (Quelle: UDV)

Ausprägung der Unfallumstände (Durchschnittswerte Deutschland) (Quelle MUko)

Abbildung 10: Darstellung von Verkehrsberuhigten Bereichen (grau dargestellt) im Kartendienst OpenStreetMap

Abbildung 11: Entwicklung der Anzahl der Unfälle mit Personenschaden und der dabei Verunglückten in GRB im Zeitraum 1995 bis 2012

Abbildung 12: Entwicklung der Anzahl der Unfälle mit Personenschaden und der dabei Verunglückten Innerorts im Zeitraum 1995 bis 2012

Abbildung 13: Entwicklung des prozentualen Anteils von Unfällen mit Personenschaden und der Zahl der Verunglückten in GRB am Unfallgeschehen mit Personenschaden innerorts gesamt

Abbildung 14: Entwicklung Anzahl der Unfälle mit Personenschaden in GRB im Zeitraum 2007 bis 2011, unterschieden nach (Flächen-)Bundesländern (Indexwert 100 für Jahr 2007)

Abbildung 15: Verteilung der Unfälle je Kategorie (Kat. 1-4) in GRB in BadenWürttemberg im Zeitraum 2007 bis 2012 (Angabe der Anzahl der Unfälle in Absolutzahlen; Unfälle Kat. 1-4 gesamt: 1.208)

Abbildung 16: Verteilung der Unfälle der Kat. 1-4 bzw. Kat. 1-3 je Unfalltyp in GRB in Baden-Württemberg im Zeitraum 2007 bis 2012 (Angabe des prozentualen Anteils des jeweiligen Unfalltyps an der Gesamtzahl der Unfälle und Angabe der Anzahl der Unfälle in Absolutzahlen; Unfälle Kat. 1-4 gesamt: 1.208; Unfälle Kat. 1-3 gesamt: 1.086)

Abbildung 17: $\quad$ Verteilung der Unfälle der Kat. 1-4 je Unfalltyp Innerorts bundesweit im Jahr 2011 (Angabe des prozentualen Anteils des jeweiligen Unfalltyps an der Gesamtzahl der Unfälle und Angabe der Anzahl der Unfälle in Absolutzahlen; Unfälle Kat. 1-4 gesamt: 210.427)

Abbildung 18: Beteiligte von Unfällen der Kat. 1-4 nach Verkehrsarten in GRB in BadenWürttemberg im Zeitraum 2007 bis 2012 (Angabe des Anteils der Beteiligung nach Verkehrsart an der Gesamtzahl an Unfällen; Unfälle Kat. 1-4 gesamt: 1.025)

Abbildung 19: Verteilung der Unfälle je Kategorie (Kat. 1-4) in GRB in Hessen im Zeitraum 2007 bis 2012 (Angabe der Anzahl der Unfälle in Absolutzahlen; Unfälle Kat. 1-4 gesamt: 517 )

Abbildung 20: Verteilung der Unfälle der Kat. 1-4 bzw. Kat. 1-3 je Unfalltyp in GRB in Hessen im Zeitraum 2007 bis 2012 (Angabe des prozentualen Anteils des jeweiligen Unfalltyps an der Gesamtzahl der Unfälle und Angabe der Anzahl der Unfälle in Absolutzahlen; Unfälle Kat. 1-4 gesamt: 517; Unfälle Kat. 1-3 gesamt: 466) 
Abbildung 21: Beteiligte von Unfällen der Kat. 1-4 nach Verkehrsarten in GRB in Hessen im Zeitraum 2007 bis 2012 (Angabe des Anteils der Beteiligung nach Verkehrsart an der Gesamtzahl an Unfällen; Unfälle Kat. 1-4 gesamt: 438)

Abbildung 22: Verteilung der Unfälle je Kategorie (Kat. 1-4) in GRB in NRW im Zeitraum 2007 bis 2011 (Angabe der Anzahl der Unfälle in Absolutzahlen; Unfälle Kat. 1-4 gesamt: 2.261)

Abbildung 23: Verteilung der Unfälle der Kat. 1-4 bzw. Kat. 1-3 je Unfalltyp in GRB in NRW im Zeitraum 2007 bis 2011 (Angabe des prozentualen Anteils des jeweiligen Unfalltyps an der Gesamtzahl der Unfälle und Angabe der Anzahl der Unfälle in Absolutzahlen; Unfälle Kat. 1-4 gesamt: 2.200; Unfälle Kat. 1-3 gesamt: 1.982)

Abbildung 24: $\quad$ Beteiligte von Unfällen der Kat. 1-4 nach Verkehrsarten in GRB in NRW im Zeitraum 2007 bis 2011 (Angabe des Anteils der Beteiligung nach Verkehrsart an der Gesamtzahl an Unfällen; Unfälle Kat. 1-4 gesamt: 2.200)

Abbildung 25: Verteilung der Unfälle je Kategorie (Kat. 1-4) in GRB in Sachsen-Anhalt im Zeitraum 2007 bis 2012 (Angabe der Anzahl der Unfälle in Absolutzahlen; Unfälle Kat. 1-4 gesamt: 218)

Abbildung 26: Verteilung der Unfälle der Kat. 1-4 bzw. Kat. 1-3 je Unfalltyp in GRB in Sachsen-Anhalt im Zeitraum 2007-2012 (Angabe des prozentualen Anteils des jeweiligen Unfalltyps an der Gesamtzahl der Unfälle und Angabe der Anzahl der Unfälle in Absolutzahlen; Unfälle Kat. 1-4 gesamt: 109; Unfälle Kat. 1-3 gesamt: 73)

Abbildung 27: Beteiligte von Unfällen der Kat. 1-4 nach Verkehrsarten in GRB in Sachsen-Anhalt im Zeitraum 2007 bis 2012 (Angabe des Anteils der Beteiligung nach Verkehrsart an der Gesamtzahl an Unfällen; Unfälle Kat. 1-4 gesamt: 109)

Abbildung 28: Verteilung der Unfälle je Kategorie (Kat. 1-4) in GRB in Thüringen im Zeitraum 2007 bis 2012 (Angabe der Anzahl der Unfälle in Absolutzahlen; Unfälle Kat. 1-4 gesamt: 128)

Abbildung 29: Verteilung der Unfälle der Kat. 1-4 bzw. Kat. 1-3 je Unfalltyp in GRB in Thüringen im Zeitraum 2007-2012 (Angabe des prozentualen Anteils des jeweiligen Unfalltyps an der Gesamtzahl der Unfälle und Angabe der Anzahl der Unfälle in Absolutzahlen; Unfälle Kat. 1-4 gesamt: 128; Unfälle Kat. 1-3 gesamt: 118)

Abbildung 30: Unfallbeteiligung nach Verkehrsarten in GRB in Sachsen-Anhalt im Zeitraum 2007 bis 2012 (nur Unfälle der Kategorie 1 bis 4) (Angabe des Anteils der Beteiligung nach Verkehrsart an der Gesamtzahl an Unfällen; Unfälle gesamt: 128)

Abbildung 31: Übersicht über die befragten Kommunen

Abbildung 32: Ziel, das mit der Einrichtung von VBB mit VZ 325 StVO bei den Kommunen verbunden ist

Abbildung 33: $\quad$ Angaben zu positiven (1), neutralen (2) und negativen (3) Erfahrungen mit Gestaltungsmaßnahmen in ausgewiesenen Verkehrsberuhigten Bereichen durch die befragten Kommunen

Abbildung 34: Steckbrief für die erhobenen Untersuchungsobjekte

Beispiel einer Wohnstraße mit ausgewiesenem Verkehrsberuhigtem Bereich

Abbildung 36: Beispiel einer Quartiersstraße mit ausgewiesenem Verkehrsberuhigtem Bereich

Abbildung 37: Beispiel einer Sammelstraße mit ausgewiesenem Verkehrsberuhigtem Bereich

Abbildung 38

Beispiel einer Erschließungsstraße

mit ausgewiesenem 
Abbildung 39: Beispiel einer Geschäftsstraße mit ausgewiesenem Verkehrsberuhigtem Bereich

Abbildung 40: Beispielbereich Sonstige Straße mit ausgewiesenem Verkehrsberuhigtem Bereich

Abbildung 41: Aufteilung der erhobenen Untersuchungsobjekte je Straßenfunktion

Abbildung 42: Beispiele für bauliche Trennungen (Fotos: IGS mbH 2013)

Abbildung 43: Beispiele für optische Trennungen (Fotos: IGS mbH 2013)

Abbildung 44: Beispiele für Mischverkehrsflächen (Fotos: IGS mbH 2013)

Abbildung 45: Verteilung der Untersuchungsobjekte des „Kollektivs Kat. 1-4“ je Verkehrsführung

Abbildung 46: Verteilung der Untersuchungsobjekte des "Kollektivs Kat. 1-4" je Straßenfunktion

Abbildung 47: Untersuchungsobjekte „Sonstige Straßen“ (Sackgasse zum Park) (Foto: IGS mbH 2013)

Abbildung 48: Verteilung der Unfälle je Kategorie (Kat. 1-4) des "Kollektivs Kat. 1-4" im Zeitraum 2006-2013

Abbildung 49: Unfälle der Kat. 1-4 innerhalb von Ortschaften (Quelle: Destatis, Reihe 7, 2012)

Abbildung 50: Verteilung der Unfälle je Kategorie (Kat. 1-4) „Kollektivs Kat. 1-4“

Abbildung 51: Prozentuale Verteilung der Unfälle je Kategorie (Kat. 1-4), „Kollektivs Kat. 1-4“ im Vergleich zu innerörtlichen Unfällen

Abbildung 52: Prozentuale Verteilung der Unfälle je Kategorie (Kat. 1-3), „Kollektivs Kat. 1-4“ im Vergleich zu innerörtlichen Unfällen

Verteilung der Unfalltypen des „Kollektivs Kat. 1-4“

Abbildung 53

Abbildung 54: Prozentuale Verteilung der Unfalltypen des „Kollektivs Kat. 1-4“ im Vergleich zu innerörtlichen Unfällen

Abbildung 55: Verteilung der Unfallarten des Unfalltyps 7 „Sonstiger Unfall“ des „Kollektivs Kat. 1-4" Abbildung 56: Verteilung der Unfallarten des „Kollektivs Kat. 1-4“

Abbildung 57: Prozentuale Verteilung der Unfallarten des „Kollektivs Kat. 1-4“ im Vergleich zu innerörtlichen Unfällen

Abbildung 58: Verteilung der Unfallursachen der Unfallart „Zusammenstoß zwischen Fahrzeug und Fußgänger" des „Kollektivs Kat. 1-4“

Abbildung 59: Verteilung der Unfallursachen der Unfallart „Zusammenstoß mit einbiegendem/- kreuzendem Fahrzeug“ des „Kollektivs Kat. 1-4“

Abbildung 60: Verteilung der Beteiligten an Unfällen des „Kollektivs Kat. 1-4“

Abbildung 61: Verteilung der Beteiligten an innerörtlichen Unfällen Kat. 1-4

Abbildung 62: Verteilung der Hauptverursacher und Beteiligte von Unfällen des „Kollektivs Kat. 1-4"

Abbildung 63: Verteilung der Hauptverursacher und Beteiligte von innerörtlichen Unfällen 2011

Abbildung 64: Prozentuale Verteilung der Beteiligten Verkehrsarten (für Fußgänger, Radfahrer und Pkw-Fahrer) je Kategorie des „Kollektivs Kat. 1-4“ (478 Beteiligte insgesamt)

Abbildung 65: Prozentuale Verteilung der Beteiligten Verkehrsarten (für Fußgänger, Radfahrer und Pkw-Fahrer) je Unfalltyp des „Kollektivs Kat. 1-4“ (478 Beteiligte insgesamt)

Abbildung 66: Prozentuale Verteilung der Beteiligten Verkehrsarten (für Fußgänger, Radfahrer und Pkw-Fahrer) je Unfallart des „Kollektivs Kat. 1-4“ (478 Beteiligte insgesamt)

Abbildung 67: Kombination der Beteiligten (Verkehrsarten) des „Kollektivs Kat. 1-4“

Abbildung 68: Kombination der Beteiligten (Verkehrsarten) von innerörtlichen Unfällen Kat. 1-4, Jahr 2011

Abbildung 69: Verteilung der Unfallursachen für den Beteiligten 1 des „Kollektivs Kat. 1-4“ 
Abbildung 70: Prozentuale Verteilung der Unfallursachen des Beteiligten 1 des „Kollektivs Kat. 1-4“ im Vergleich zu innerörtlichen Unfällen

Abbildung 71: Verteilung der Unfalltypen der Unfallursache „Vorrang, Vorfahrt“ des „Kollektivs Kat. 1-4“

Abbildung 72: Verteilung der Unfalltypen der Unfallursache „Abbiegen, Wenden, Rückwärtsfahren, Ein- und Anfahren“ des „Kollektivs Kat. 1-4“

Abbildung 73: Verteilung der Unfalltypen der Unfallursache „Falsches Verhalten gegenüber Fußgängern“ des „Kollektivs Kat. 1-4“

Abbildung 74: Verteilung der Unfallkategorien je Straßenfunktion des „Kollektivs Kat. 1-4“ (Anzahl)

Abbildung 75: Verteilung der Unfallkategorien je Straßenfunktionen „Kollektivs Kat. 1-4“ 90

Abbildung 76: Verteilung der Unfalldichte je Straßenfunktion des „Kollektivs Kat. 1-4“ 90

Abbildung 77: Verteilung der Unfalldichte je Straßenfunktion und Verkehrsführung des „Kollektivs Kat. 1-4“

Abbildung 78: Verteilung der Unfallkostendichte je Straßenfunktion des „Kollektivs Kat. 14", Preisstand 2009

Abbildung 79: Verteilung der Unfallkostendichte je Straßenfunktion und Verkehrsführung des „Kollektivs Kat. 1-4“, Preisstand 2009

Abbildung 80: Verteilung der Unfalltypen je Straßenfunktion des „Kollektivs Kat. 1-4“ Verteilung der Unfallarten je Straßenfunktion des „Kollektivs Kat. 1-4“ Verteilung der Unfallursachen je Straßenfunktion des „Kollektivs Kat. 1-4“

Abbildung 82:

Abbildung 83: Beispiel für einen Übergangsbereich (Foto: IGS mbH; Kartengrundlage: OpenStreetMap)

Abbildung 84: Verteilung der Unfallkategorien in Übergangsbereichen und innerhalb von VBB des „Kollektivs Kat. 1-4“

Abbildung 85: Verteilung der Unfallkategorien in Übergangsbereichen und innerhalb von VBB je Straßenfunktion des „Kollektivs Kat. 1-4“

Abbildung 86: Verteilung der Unfalltypen in Übergangsbereichen und innerhalb von VBB des „Kollektivs Kat. 1-4“

Abbildung 87: Verteilung der Unfalltypen in Übergangsbereichen und innerhalb von VBB je Straßenfunktion des „Kollektivs Kat. 1-4“

Abbildung 88: Beteiligte in Übergangsbereichen \& innerhalb von VBB des „Kollektivs Kat. 1- 4"

Beteiligte in Übergangsbereichen und innerhalb von VBB des „Kollektivs

$\begin{array}{ll}\text { Abbildung 89: } & \text { Beteiligte in Übergangsbereich } \\ \text { Kat. 1-4" je Straßenfunktion }\end{array}$

Abbildung 90: Unfalldichte je Straßenfunktion und Abschnittslänge von Streckenabschnitten des „Kollektivs Kat. 1-4“

Abbildung 91: Übersichtskarte über die Städte in denen insg. 50 Bereiche untersucht wurden

Abbildung 92:

Flächeneinteilung eines Verkehrsberuhigten Bereichs (hier mit einer Fahrgasse)

Abbildung 93: Flächeneinteilung eines Verkehrsberuhigten Bereichs (hier mit einer Mischverkehrsfläche)

Abbildung 94: Flächeneinteilung eines Verkehrsberuhigten Bereichs (hier mit einem einseitigen Rand)

Abbildung 95: Querschnitt, Einteilung des Flächenrasters

Ausschnitt Zählformular

Beispiel Geschwindigkeitsmessung

Abbildung 97:

Abbildung 98:

Beispiel zur Anbringung von Kameras innerhalb der VBB

Abbildung 99:

Schematische Übersicht der Gestaltung und der Streckenführung eines VBB

Abbildung 100:

Infos Untersuchungsobjekt und schematische Übersichtskarte

Abbildung 101: Darstellung zur Auswertung der Querschnittszählung und Flächennutzung

Abbildung 102: Geschwindigkeitsverteilung auf einem Abschnitt nach Verkehrsteilnehmern 
Abbildung 104: Anzahl und Ergebnisse der Querschnittszählung für Pkws in vier Stunden von 14 bis 16 Uhr

Abbildung 105: Ausnutzung der mittleren Verkehrsfläche durch Pkw in \%

Abbildung 106: Querschnittsaufteilung mit optischer Trennung und Parkflächen am Rand

Abbildung 107:

Ausnutzung der mittleren Verkehrsfläche durch Radfahrer in \%

Abbildung 108: Ausnutzung der mittleren Verkehrsfläche durch Fußgänger in \%

Abbildung 109:

Ausnutzung der mittleren Verkehrsfläche in Wohnstraßen

132

132

Ausnutzung der mittleren Verkehrsfläche in Quartiersstraßen

133

Abbildung 111:

Ausnutzung der mittleren Flächen in Geschäftsstraßen

133

Abbildung 112:

Verteilung der Unfalldichte in Abhängigkeit von der Anzahl der

Verkehrsteilnehmer

134

Abbildung 113:

Abbildung 114:

Abbildung 115:

Abbildung 116:

Abbildung 117:

Nutzung der mittleren Verkehrsflächen

134

punktuelle dauerhafte Hindernisse

136

Übergang eines Verkehrsberuhigten Bereiches in einen Fußweg

Übergang eines Verkehrsberuhigten Bereiches mit abgesenktem Bordstein

139

139

Verteilung der Untersuchungsobjekte des „Kollektivs 2011“ im Vergleich zum „Kollektiv Kat. 1-4“ je Straßenfunktion

Abbildung 118: Verteilung der Untersuchungsobjekte des „Kollektivs Kat. 1-4“ im Vergleich zum „Kollektiv 2011“ je Verkehrsführung

Abbildung 119: Verteilung der Unfallkategorien Kat. 1-4 des „Kollektivs Kat. 1-4“ im Vergleich zum „Kollektiv 2011“

Abbildung 120: Verteilung der Unfälle des „Kollektivs 2011“

Abbildung 121: Verteilung der Unfalltypen des „Kollektivs Kat. 1-4“ in Vergleich zum „Kollektiv 2011“

Abbildung 122:

Verteilung der Unfalltypen der Unfälle der Kat. 5 des „Kollektivs 2011“

Abbildung 123: Verteilung der Unfallarten des „Kollektivs 2011“ im Vergleich zum „Kollektiv Kat. 1-4"

Abbildung 124: Verteilung der Beteiligten des „Kollektivs 2011“

Abbildung 125: Verteilung der Beteiligten des „Kollektivs2011“ im Vergleich zum „Kollektiv Kat. 1-4“

Abbildung 126: Verteilung der Unfallursache des „Kollektivs Kat. 1-4“ im Vergleich zum „Kollektiv 2011“

Abbildung 127: Vergleich der Unfallumstände des "Kollektivs 2011" und des "Kollektiv Kat. 1-4" im Vergleich zum Erwartungswert ( $\varnothing$ ) Innerorts

Abbildung 128: Verteilung der Unfälle je Unfallkategorie und Straßenfunktion des „Kollektivs 2011“

Abbildung 129:

Verteilung der Unfalldichte je Straßenfunktion des "Kollektivs 2011"

Abbildung 130:

Verteilung der Unfallkostendichte je Straßenfunktion des "Kollektivs 2011"'“, Preisstand 2009

Abbildung 131: Verteilung der Unfalltypen je Straßenfunktion des „Kollektivs 2011“

Abbildung 132: Verteilung der Unfallarten je Straßenfunktion des „Kollektivs 2011“

Verteilung der Unfallursachen je Straßenfunktion des „Kollektivs 2011“

Vergleich der Unfallumstände je Straßenfunktion des "Kollektivs 2011" im

Vergleich zum Erwartungswert ( $\varnothing$ ) Innerorts

Abbildung 135: Unfalldichte je Abschnittslänge des "Kollektivs 2011" 


\section{Tabellenverzeichnis}

Tabelle 1: $\quad$ Definition Unfallarten $\quad 23$

Tabelle 2: $\quad$ Unfallursachen (in Gruppen zusammengefasst) 25

Tabelle 3: $\quad$ Datengrundlage für die Unfallanalysen 26

Tabelle 4: Gegenüberstellung der Unfälle Kat. 1-3 mit dem Merkmal „VBB“ aus den Unfalldatenbanken im Vergleich zu OpenStreetMap beispielhaft für Kommunen in Baden-Württemberg

Tabelle 5: Gegenüberstellung der Unfälle Kat. 1-3 mit dem Merkmal „VBB“ aus den Unfalldatenbanken im Vergleich zu OpenStreetMap beispielhaft für Kommunen in Hessen

Tabelle 6: Gegenüberstellung der Unfälle Kat. 1-3 mit dem Merkmal „VBB“ aus den Unfalldatenbanken im Vergleich zu OpenStreetMap beispielhaft für Kommen in Sachsen-Anhalt

Tabelle 7: Gegenüberstellung der Unfälle Kat. 1-3 mit dem Merkmal „VBB“ aus den Unfalldatenbanken im Vergleich zu OpenStreetMap beispielhaft für Kommen in Thüringen

Tabelle 8: Gegenüberstellung aller Unfälle der Kat. 1-3 mit dem Merkmal „VBB“ aus den Unfalldatenbanken im Vergleich zu OpenStreetMap (durchschnittliche Abweichung)

Tabelle 9: Unfälle mit Personenschaden und schwerwiegende Unfälle mit Sachschaden in GRB im Zeitraum 1995-2012 (Quelle: Destatis, 2013)

Tabelle 10: Verunglückte in GRB im Zeitraum 1995-2012 (Quelle: Destatis, 2013)

Tabelle 11: Unfallzahlen der Unfälle mit Personenschaden in GRB Unterschieden nach Bundesländern im Zeitraum 2007 bis 2011 (Quelle: Destatis 2013)

Tabelle 12: Zusammensetzung der Untersuchungsobjekte je Bundesland

Tabelle 13: Durchschnittliche Länge der Untersuchungsobjekte des "Kollektivs Kat. 1-4" je Straßenfunktion

Tabelle 14: Anzahl der Unfälle des "Kollektivs Kat. 1-4" je Untersuchungsobjekt

Tabelle 15: Anzahl und Anteil von Unfällen der Kategorie 1-4 von GRB im Vergleich zu allen innerörtlichen Unfällen (Quelle: Destatis, Reihe 7, 2012)

Tabelle 16: Unfallursachen der Unfallart „Zusammenstoß zwischen Fahrzeug und Fußgänger“ des Unfalltyps 7 „Sonstiger Unfall“ des „Kollektivs Kat. 1-4“

Tabelle 17: Unfallursachen der Unfallart „Unfälle anderer Art“ des Unfalltyp 7 „Sonstiger Unfall“ des „Kollektivs Kat. 1-4“

Tabelle 18: Prozentuale Verteilung der Hauptverursacher und Beteiligten des „Kollektivs Kat. 1-4"

Tabelle 19: Prozentuale Verteilung der Hauptverursacher und Beteiligten an innerörtlichen Unfällen

Tabelle 20: Häufigste Kombinationen der Beteiligten (Verkehrsarten) des „Kollektivs Kat. 1$4^{\prime \prime}$

Tabelle 21: Häufigste Kombinationen der Beteiligten (Verkehrsarten) von Innerörtlichen Unfällen Kat. 1-4

Tabelle 22: Pkw, Rad und Motorrad Alleinunfälle des „Kollektivs Kat. 1-4“

Tabelle 23: Pkw, Rad und Motorrad Alleinunfälle von innerörtlichen Unfällen

Tabelle 25: Unfallursachen der Alleinunfälle von Pkw-Fahrern des „Kollektivs Kat. 1-4

Tabelle 26: Unfallanzahl, Abschnittslängen und Unfalldichten je Straßenfunktion und Verkehrsführung des „Kollektivs Kat. 1-4“ 
Tabelle 27: Beteiligte Radfahrer und Fußgänger je Straßenfunktion und Unfalltyp des „Kollektivs Kat. 1-4“

Tabelle 28: Beteiligte Radfahrer und Fußgänger des „Kollektivs Kat. 1-4“ je Straßenfunktion und Unfallart

Tabelle 29: Beteiligte Radfahrer, Fußgänger und Pkw an Unfällen je Straßenfunktion des „Kollektivs Kat. 1-4“

Tabelle 30: Beteiligte Radfahrer, Fußgänger und Pkw an Unfällen des „Kollektivs Kat. 1-4“ je Straßenfunktion und Verkehrsführung

Tabelle 31: Beteiligte Radfahrer und Fußgänger des „Kollektivs Kat. 1-4“ je Straßenfunktion und Unfallursache

Tabelle 32: Ausprägung der Unfallumstände des „Kollektivs Kat. 1-4“ je Straßenfunktion

Tabelle 33: Unfälle Kat.1-4 in Übergangsbereichen und innerhalb von VBB des „Kollektivs Kat. 1-4"

Tabelle 34: Übersicht Untersuchungsobjekte

Tabelle 35: Durchfahrtgeschwindigkeiten von Verkehrsberuhigten Bereichen

Tabelle 36: Verteilung der Untersuchungsobjekte des „Kollektivs Kat. 1-4“ im Vergleich zum „Kollektiv 2011“

Tabelle 37: Strecken- und Durchschnittslängen der Untersuchungsobjekte des „Kollektivs Kat. 1-4" im Vergleich zum "Kollektiv 2011"

Tabelle 38: Unfallarten des Unfalltyps „Sonstiger Unfall“ der Unfälle der Kategorie 5 des „Kollektivs 2011“

Tabelle39: Unfallursachen des Unfalltyps „Unfall ruhender Verkehr" der Unfälle der Kategorie 5 des „Kollektivs 2011“

Tabelle 40: Abschnittslängen, Anzahl der Unfälle und Untersuchungsobjekte des „Kollektivs 2011“ je Straßenfunktion und Verkehrsführung

Tabelle 41: Beteiligte Radfahrer, Fußgänger und Pkw je Straßenfunktion des „Kollektivs $2011^{\prime \prime}$

Tabelle 42: Indirekt angepasste und pauschale Unfallkostensätze Preisstand 2000

Tabelle 43: Pauschale Unfallkostensätze Preisstand 2009 
DIE DEUTSCHEN VERSICHERER

\section{Gesamtverband der Deutschen Versicherungswirtschaft e. V.}

Wilhelmstraße 43 / 43 G, 10117 Berlin

Postfach 0802 64, 1002 Berlin

Tel. 030/20 20 -50 00, Fax $030 / 2020$ - 6000

www.gdv.de, www.udv.de 